\title{
Pavlovian conditioning and binge eating : some empirical explorations
}

Citation for published version (APA):

Overduin, B. J. J. (1996). Pavlovian conditioning and binge eating : some empirical explorations. [Doctoral Thesis, Maastricht University]. Rijksuniversiteit Limburg. https://doi.org/10.26481/dis.19960705bo

Document status and date:

Published: 01/01/1996

DOI:

10.26481/dis. 19960705 bo

Document Version:

Publisher's PDF, also known as Version of record

\section{Please check the document version of this publication:}

- A submitted manuscript is the version of the article upon submission and before peer-review. There can be important differences between the submitted version and the official published version of record. People interested in the research are advised to contact the author for the final version of the publication, or visit the DOI to the publisher's website.

- The final author version and the galley proof are versions of the publication after peer review.

- The final published version features the final layout of the paper including the volume, issue and page numbers.

Link to publication

\footnotetext{
General rights rights.

- You may freely distribute the URL identifying the publication in the public portal. please follow below link for the End User Agreement:

www.umlib.nl/taverne-license

Take down policy

If you believe that this document breaches copyright please contact us at:

repository@maastrichtuniversity.nl

providing details and we will investigate your claim.
}

Copyright and moral rights for the publications made accessible in the public portal are retained by the authors and/or other copyright owners and it is a condition of accessing publications that users recognise and abide by the legal requirements associated with these

- Users may download and print one copy of any publication from the public portal for the purpose of private study or research.

- You may not further distribute the material or use it for any profit-making activity or commercial gain

If the publication is distributed under the terms of Article $25 \mathrm{fa}$ of the Dutch Copyright Act, indicated by the "Taverne" license above, 


\section{Pavlovian Conditioning and Binge Eating}

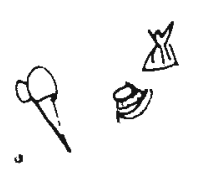


Cover Design: Mariëtte Strik

Printed by: Quick Print, Nijmegen

Overduin, Barend Jan Joost

Pavlovian conditioning and Binge Eating: Some Empirical Explorations/ Barend Jan Joost Overduin.

Thesis University of Limburg, Maastricht. - With ref. With summary in Dutch.

ISBN $90-9009-606-X$

Subject headings: binge eating/ classical conditioning lexperimental psychopathology. 


\title{
Pavlovian Conditioning and Binge Eating Some Empirical Explorations
}

\author{
PROEFSCHRIFT \\ ter verkrijging van de graad van doctor \\ aan de Rijksuniversiteit Limburg te Maastricht, \\ op gezag van de Rector Magnificus, Prof. Mr. M.J. Cohen, \\ volgens het besluit van het College van Dekanen, \\ in het openbaar te verdedigen op \\ vrijdag 5 juli 1996 om 14.00 uur
}

door

Barend Jan Joost Overduin 


\section{Promotor:}

Prof. Dr. M.A. van den Hout

\section{Co-promotor:}

Dr. A. Jansen

\section{Beoordelingscommissie:}

Prof. Dr. H. Merckelbach (voorzitter)

Prof. Dr. N.H. Frijda (Universiteit van Amsterdam)

Prof. Dr. J. Jolles

Dr. N.A. Nicolson

Dr. J.H. Strubbe (Rijksuniversiteit Groningen)

The research in this thesis was financially supported by grant no. 560-268-052 from the Netherlands Organization for Scientific Research, section Behavioural Sciences (NWO-SGW)

The following organizations are gratefully acknowledged for financially supporting the publication of this thesis:

- Faculty of Health Sciences, University of Limburg, Maastricht

- Hoechst-Marion-Roussel, Hoevelaken

- Holland Sweetener Company, Maastricht

- N.W.O., Stichting Gedragswetenschappen, Den Haag

- Quest International, Naarden

- Suikerstichting Nederland, Baarn 
Aan mijn ouders 



\section{Contents}

1.1 On the experimental psychopathology approach

types of investigation - rationale of modelling psychopathology

1.2 Binge eating: definition and characteristics

diagnosistic criteria - prevalence - binge size - binge frequency binge ingredients - mood and binge eating - impact on social functioning and well-being - summary

1.3 Herman \& Polivy's counterregulation model of binge eating description of the counterregulation model - critique of the counterregulation model - final remarks

Chapter 2: A Pavlovian Conditioning Theory of Binge Eating (CBE)

2.1 Description of the conditioning theory of binge eating

five assumptions

2.2 Overview of relevant data

binge cues - homeostatic regulation of blood sugar level -

anticipatory responses to sensory food cues - classical conditioning

of food intake and physiological responses - hyperinsulinaemial

hypoglycaemia experienced as craving - extinction of conditioned physiological responses and binge eating

2.3 Summary and implications for the present thesis

Summary - implications for the empirical work of this thesis

\section{Part II: Empirical Studies}

Chapter 3: Conditioned Insulin and Blood Sugar Responses in Humans in Relation to Binge Eating

Chapter 4: Introduction and commentary to Mityushov (1954):

Conditioned reflex secretion of insulin

Chapter 5: Cue Reactivity to Food-and Body-Related Stimuli in Restrained and Unrestrained Eaters 
Chapter 6: Food Cue Reactivity in Fasting and Non-fasting Subjects

Chapter 7: Stroop Interference and Food Intake

Chapter 8: A New Scale for Use in Non-clinical Research into

Disinhibitive Eating

\section{Part III: Conclusion}

Chapter 9: Conclusions and General Discussion

9.1 Overview of the empirical work in this thesis

9.2 Quantitative reappraisal of the CBE theory

five hypotheses reanalyzed

9.3 The conditioning theory of binge eating: final qualification

9.4 Future research: theoretical considerations maintaining a blood sugar/insulin-related explanation - learning perspective based on post-meal deprivation

9.5 Future research: practical considerations necessity of modelling - three models of binge eating - I. the cue-reactivity model - II. the conditioned hypoglycaemia model III. the post-meal deprivation model

References

Appendix: Mityushov (1954): "Conditioned Reflex Secretion of Insulin" (translation)

Summary

Samenvatting

Dankwoord 


\section{Chapter 1 \\ EXPERIMENTAL PSYCHOPATHOLOGY AND BINGE EATING}

This thesis is an investigation into binge eating. The term "binge eating" refers to a pathology consisting of repeated bursts of excessive food intake. The approach that will be taken throughout this thesis goes under the name of experimental psychopathology. Experimental psychopathology involves the development and empirical testing of hypotheses about underlying mechanisms in psychopathology (Abramson \& Seligman, 1977; Jansen, Merckelbach \& Van den Hout, 1992). After a brief introduction of methods and rationale of the experimental psychopathology approach (section 1.1), essential features of binge eating will be described (section 1.2). The epithet "experimental" by no means implies that experimental psychopathology is non-theoretical: in fact, theories play a pivotal role in experimenting. Section 1.3 contains a critical discussion of counterregulation, a paradigm that has dominated binge eating research in the last two decades. In chapter two, a conditioning theory of binge eating will be described. It is this theory which has inspired the empirical work found in subsequent chapters.

\subsection{ON THE EXPERIMENTAL PSYCHOPATHOLOGY APPROACH}

\subsubsection{Types of investigation}

Within experimental psychopathology, investigations may take numerous forms, three of which will be described here as a frame of reference. In "type P" investigations, the behaviour of patients and non-patients is compared in structured situations, so as to demonstrate or identify crucial parameters of the pathology. An example in the field of eating disorders would be to provide bulimia nervosa patients and healthy subjects with various foods and compare their food choice or rate of ingestion in the lab (e.g. Hadigan, Kissileff \& Walsh, 1989). A problem with this type of research is that recruitment of large samples of well-defined patients is often time consuming and meets various practical difficulties. For pragmatic reasons, researchers may choose to switch to "type Q" investigations, in which two groups of non-clinical subjects are compared. The two groups differ in their extra-experimental history, as for for example reflected by low or high scores on questionnaires tapping habits or traits related to the pathology. An example would be the comparison of the eating behaviour of frequent dieters vs. nondieters in a structured lab situation. A problem with type $P$ and $Q$ research is that individual history may escape rigorous experimental control. Hence a third type of 


\section{Chapter 1}

investigation exists, "type R", involving the study of lab models of the pathology". "Modelling" refers to the "production, under controlled conditions, of phenomena analogous to naturally occurring mental disorders" (Abramson \& Seligman, 1977). Thus, a model is a lab-analogue, or a reversible "miniature version" of the real pathology, to be evoked in non-clinical subjects. A lab-model of binge eating may be at hand as soon as non-clinical subjects have been found repeatedly to increase their food intake after some experimental manipulation. Although the evocation of the lab behaviour may tell us something about the origins of the real pathology, it is not the final goal of modelling (Willner, 1991b): important knowledge derives from subsequent investigation of how the lab model is influenced by various manipulations. For instance, it might be tested whether prodigious food intake by non-clinical subjects in the lab can be suppressed by having the subject smelling the food for a prolonged time. As the development and testing of models is central to experimental psychopathology research, its rationale will be more closely discussed next.

\subsubsection{Rationale of modelling psychopathology}

Ultimate goals of experimental psychopathology are to promote understanding and, perhaps, to suggest new treatments of psychopathology. As such, working with lab models requires a more formal approach than does clinical observation, which admittedly is also a source of knowledge and ideas (cf. Beck et al., 1979). There are advantages and disadvantages to the lab approach.

Many workers have received the endeavour of developing and testing lab models of psychopathology rather coolly. To them, modelling appears to lead researchers astray, that is away from the "full blown pathology of real life". For example, learned helplessness, often used as a lab model of depression, was criticized by the noted British clinician and scientist I. Marks as follows:

\section{"..) present behavioral approaches are not specially promising for the} management of depression.(.) it has not so far been shown that learned helplessness is associated with anything more than mild mood change without the concomitants of serious clinical depression such as guilt, nihilism, suicidal ideas, anorexia, and insomnia lasting at least several weeks. " (cited in Peterson, Maier \& Seligman, 1993, p. 13)

1 The term "model" will be used here only in connection with the experimental procedure of producing miniature psychopathological behaviour in the lab. For the theoretical model of pathological behaviour guiding the experiment, the term "theory" will be reserved. 
Admittedly, lab models can involve stylized forms of behaviour, which only remotely resemble the clinical phenomenon. However, the similarity of lab model and pathology is one, but not always the major concern in the development and testing of models of pathological behaviour, as M.E.P. Seligman, the major proponent of the learned helplessness paradigm, noted in his reply to Marks' criticism:

(the thought that) ". any model of depression in the laboratory must somehow produce suicide, crying, weight loss, guilt and nihilism (..) does not bear scientific scrutiny. Model airplanes do not need to make transatlantic flights; they only need to embody the essence of flying in an airplane. A laboratory model of anxiety need not produce screaming, defecation, and panic antacks - it need only isolate the essential properties of anxiety" (ibid.)

In his reply, Seligman describes the isolation of essential properties of the psychopathology as the crux to model building and testing. The advantage of the isolation of symptoms is understandable if one considers that a given psychopathological symptom hardly ever comes alone. Comorbidity is common and often it remains unclear which symptom precedes, modulates or maintains the other in a given patient. For example, depression and anxiety often coincide with binge eating (APA, 1994; Beebe, 1994; Cooper, 1995; Garner et al., 1990). Thus, isolation of specific symtoms for study becomes especially difficult in patients. Hence, studying a lab model in non-clinical subjects may allow a more efficient investigation of mechanisms underlying the pathology.

Abramson and Seligman (1977) present a list of criteria to evaluate lab models of psychopathology. The important questions to be asked are: a. Are the essential features of pathology's causes as well as its prevention and cures validly described by the model? b. To what extent is there similarity of symptoms between the model and "naturally occurring" psychopathology? $\underline{c}$. To what extent is there similarity between pathology and model regarding physiology, cause, cure and prevention? $\mathrm{d}$. Is the lab analogue a model of one specific psychopathology or of a feature occurring in a range of psychopathologies? Importantly, it should be noted that if all the above criteria are fully met, then so much knowledge is available that testing the model would become superfluous (Jansen et al., 1992a).

In conclusion, there are both limitations and advantages in studying lab models. The limitations arise from the consideration that the functioning of humans is embedded in a complex social cultural context. Hence, the isolation of putatively "essential" characteris- 


\section{Chapter 1}

tics reduces ecological validity. On the other hand, modelling of isolated aspects of the pathology, may elucidate causal mechanisms, which might otherwise remain hidden amongst a myriad of intertwined factors.

\subsection{BINGE EATING: DEFINITION AND CHARACTERISTICS}

"Binge eating", the object of investigation in this thesis, is not to be understood as simple overeating. In this section, the definition, main characteristics and concomitants of binge eating will be described.

\subsubsection{Definition and diagnosis}

The fourth edition of the highly influential Diagnostic and Statistical Manual of Mental Disorders (DSM IV) includes the following definition of binge eating (APA, 1994):

1. eating, in a discrete period of time (e.g., within an two-hour period), an amount of food that is definitely larger than most people would eat during a similar period of time under similar circumstances; 2 . a sense of lack of control over eating during the episode (e.g., a feeling that one cannot stop eating or control what or how much one is eating).

Binge eating is an important symptom in bulimia nervosa, binge eating disorder ${ }^{2}$ and in anorexia nervosa, binge-eating/purging type. For the diagnosis of bulimia nervosa an average binge frequency of at least twice weekly for a period of three months is required; for the diagnosis of binge eating disorder the binge episodes should occur on at least two days a week for a period of six months (APA, 1994). Especially because of its quality of control-loss, binge eating cannot simply be equated with gluttony in obese individuals: less than half of the obese suffer from binge eating (Marcus, 1993; 1995).

Binge eaters with a diagnosis of bulimia nervosa or anorexia nervosa (binge eating/purging type) try to counter the caloric effects of binges by dieting, fasting, and/or doing sports excessively. Self-induced vomiting or using laxatives are also among the means used to lose the weight. In bulimic patients who present for treatment, $80-90 \%$ engage in self-induced vomiting. Because of these behaviours, bulimic binge eaters are not especially obese (APA, 1994). In both bulimia and anorexia nervosa, the weightreducing behaviours coincide with morbid, often unwarranted fears of being fat and getting fatter. Moreover, self-esteem is seen in terms of slimness and amount of body

${ }^{2}$ The diagnose Binge-Eating Disorder has been introduced only recently. In contrast to bulimics, patients diagnosed with Binge-Eating Disorder do not display so-called inappropriate compensatory hehaviours such as self-induced vomiting, misuse of laxatives, fasting and/or excessive exercise (APA, 1994). 
fat. Irrational ideas about eating and distorted body images (to be addressed only marginally in this dissertation) indeed form an important target of successful cognitive behavioural therapeutic interventions in eating disorders (Fairburn, 1995).

\subsubsection{Prevalence of binge eating}

Throughout the 1980 s, different studies estimated the point prevalence of binge eating in the female population ${ }^{3}$ at between 1 and $79 \%$ (Fairburn \& Beglin, 1990; Jansen, 1990). However, these wide ranging figures clearly reflect different definitions of binge eating that have been adopted. Importantly, if one relies on subjective criteria, individuals may refer to intake of merely one chocolate biscuit as "binge eating" (e.g. Rosen, Leitenberg, Fisher \& Khazam, 1986; Rossiter \& Agras, 1990). It is only logical that with the gradual development of more stringent criteria (e.g. the DSM-IV definition) the reported prevalences of binge eating have shown a decline through the years (Jansen, 1990). To get a full picture one should combine recent prevalence estimates for bulimia nervosa, binge eating disorder and anorexia nervosa (binge eating/purging type). Firstly, for bulimia nervosa the prevalence in the adult female population was estimated to lie at around $1 \%$. (Fairburn \& Beglin, 1990; Hoek, 1993; 1995). Secondly, the prevalence of anorexia nervosa (binge eating/purging type) should be expected to be about half of that for anorexia nervosa, which has been estimated at $0.3 \%$ (Hoek, 1993; 1995) or 0.5-1 $\%$ (APA, 1994). Thirdly, binge eating disorder has a prevalence between 0.7 and $4 \%$ (APA, 1994) or $2 \%$ (Marcus, 1993). These figures add up to a total prevalence of binge eating in the female population of somewhere between 1.85 and $5.5 \% .^{4}$.

${ }^{3}$ The prevalence of eating disorders among males has been reported as only a tenth or a twentieth of that for females (Hoek, 1993).

${ }^{4}$ It should be noted that the present calculations are provisional. Fairburn \& Beglin (1990) bave voiced doubts about the possibility of ever obtaining the "true" prevalence of binge eating (at least in bulimia nervosa) in the general population. Furthermore, indications that only a small subset of binge eaters bas been detected and is receiving treatment mean that patient groups may not be representative of binge eaters in the general population (Fairburn \& Beglin, 1990). Therefore, the data on binge eating presented in this section should be considered with some caution. 


\section{Chapter 1}

\subsubsection{Binge sizes}

The DSM-IV criteria for binge eating do not specify an absolute caloric value per binge. This has to do with the large variability of binge size both inter-individually (range: more than 5000 kcalories; Rossiter \& Agras, 1990; Rossiter et al, 1992) and intraindividually (range: approximiately 2000 kcalories; Rosen et al., 1986; Rossiter \& Agras, 1990) in the amount of food ingested during self-reported binges. Whilst there is no doubt that huge quantities of food can be ingested in binge eating, several factors may hamper precise estimations of binge size. First, patients may be prone to refer to any intake of "forbidden food" as a binge, even if only 50 kcalories (the caloric value of one chocolate biscuit) are involved (e.g. Rosen et al., 1986; Rossiter \& Agras, 1990). Inclusion of these so-called "subjective binges" (Fairburn \& Wilson, 1993) may lead to underestimation of the problem. As a provisional solution of this issue, Fairburn (1987) has proposed to count only "uncontrollable" eating episodes larger than $1000 \mathrm{kcal}$ as binges. It appears that about $45-50 \%$ of the self-reported binges (in bulimic patients) conform to this criterion (Rossiter \& Agras, 1990; Walsh, 1993).

Another difficulty in establishing binge size is the underreporting: diary studies typically revealed average binge size of slightly above $1000 \mathrm{kcal}$,, while in lab or otherwise controlled circumstances typically average values of between 1500 and $5000 \mathrm{kcal}$ have been found (Walsh, 1993).

In bulimia patients who do practice self-induced vomiting after bingeing, the average binge size is about twice that of those who do not vomit (Lacey \& Gibson, 1985; Rossiter \& Agras, 1990; Rossiter et al., 1992).

\subsubsection{Binge frequency}

Included in the DSM-IV criteria for the bulimia nervosa and binge eating disorders is the requirement that bingeing should occur at an average frequency of twice a week (bulimia nervosa) or on two different days per week (binge eating disorder) for a period of three and six months respectively (APA, 1994). In spite of these criteria it appears to be difficult to make accurate estimates of binge frequency. As with binge size estimations, frequency estimates are hampered by the phenomenon of "subjective bingeing", i.e. labelling of small or normal meals involving "forbidden foods", as a binge (Fairburn

${ }^{5}$ Note that the following data were mostly drawn from studies on bulimic patients. For reference, the caloric value of one candy bar is between 200 and 250 kilocalories, 200 grams of potato crisps contain about $1080 \mathrm{kcal}$, and mean daily intake is about $2000 \mathrm{kcal}$ for adults, males somewhat more than females (Oettle et al., 1987; Rossiter et al., 1992). The largest binge reported has been 55000 $\mathrm{kcal}$ (this binge included several pauses in which self-induced vomiting occurred; Walsh, 1993). 
\& Wilson, 1993). Several studies in bulimics have reported an average binge frequency of 10-11 per week (Walsh, 1993; Rosen et al., 1986; Rossiter \& Agras, 1990). It should be emphasized that these figures reflect both individuals who binge at least daily and those that do so less often. When counting only binges exceeding $1000 \mathrm{kcal}$ (Fairburn, 1987), the picture becomes different: Rosen et al. (1986) followed a group of 20 Bulimia patients (with bingeing and self-induced vomiting more than three times a week) for one week, and found an average of five binges above 1100 calories. Rossiter and Agras (1990) asked 32 Bulimia patients of the purging type to keep a food intake diary, and found an average of about 4.8 binges above 1000 kcalories in one week (Rossiter \& Agras, 1990).

\subsubsection{Ingredients of a binge}

Often it is reported that during binges, the choice of foods is no longer guided by pleasant taste: raw wheat or pure gravy are ingested if nothing else is available to satisfy the craving. The sensory quality of the binge food does, however, play a role, especially at the commencement of the binge: food types with a special mouth feel, i.e., sweet, soft and creamy, appear to have a special attraction (Abraham \& Beumont, 1982). This might also have to do with the ease with which such food can be expelled after bingeing (Beumont, 1995).

More importantly, binges tend to consist of snacks like chocolate, ice-cream, cakes and pie, and other "forbidden foods" for anyone with the ambition to lose weight (e.g. Abraham \& Beumont, 1982; Rosen et al., 1986; Walsh, 1993). This fact has lead to the idea that binges may gratify a carbohydrate-specific hunger aimed at replenishing brain serotonin (e.g. Pirke, 1995). However, in the early 1980s the alleged overrepresentation of carbohydrate in binges was already discarded (Abraham \& Beumont, 1982). Furthermore, subsequent lab and self-report studies have not found a different macro-nutrient composition in binges and normal meals (Elmore \& de Castro, 1991; Jansen, Van den Hout \& Griez, 1990; Rossiter et al., 1992; Walsh, 1993). Clearly, this does not deny the fact that large amounts of all macro-nutrients may be ingested during binges.

\subsubsection{Mood and binge eating}

As to the affective states surrounding binge episodes, it might be best to say that binge episodes constitute a mixed blessing. First, tension, depression, frustration, loneliness, anxiety and boredom are among the most frequently mentioned precipitants of binges (Abraham \& Beumont, 1982; Bruce \& Agras, 1992; Mitchell, Hatsukami, Eckert \& Pyle, 1985; Beebe, 1994). Once bingeing starts, levels of reported anxiety, depression, etc., appear to be lowered in comparison with the pre binge state (Beebe, 1994). 


\section{Chapter 1}

Second, however, bingeing itself also gives rise to negative moods (Beebe, 1994; Mitchell et al., 1985). Indeed, a range of studies reviewed by Beebe (1994) shows that what is "gained" in mood during bingeing (i.e. lower anxiety, depression) is "lost" by the emergence of new negative feelings, like guilt, loss of control, helplessness, and again depression after the binge, a negative outcome which may lead the patient into purging. Bingeing in patients can thus be said to be consoling for a very limited time.

\subsubsection{Relationship of bingeing to social functioning and well-being}

Whatever the particular background of the individual, it is clear that chronic binge eating co-occurs with impaired social functioning and well-being. Binge eating and purging, which are often kept secret from close relatives and spouse, negatively affect social relationships (Beumont, 1995; Mitchell et al., 1985). Also work impairment as well as financial problems caused by the high cost of binge foods have been reported (Mitchell et al., 1985). Often, bulimia nervosa coincides with depression. There has been ample debate about the causal status of depression in binge eating (Beebe, 1994). An important observation is that overall severity of psychopathology seems unpredictive of treatment success in bulimia nervosa, and that psychopathology diminishes significantly after treatment of the bulimic symptoms (Garner et al., 1990). This may indicate that psychopathological symptoms are secondary to the binge eating/purging itself (Beumont, 1995; Cooper, 1995).

\subsubsection{Summary}

The diagnosis of binge eating includes frequent large food intakes accompanied by feelings of loss of control. Phenomena closely related to binge eating are self-imposed food restriction and post-binge vomiting, related to morbid fears of gaining weight. Binge eating is more prevalent in the female than in the male population. The prevalence of binge eating in the adult female population has been estimated to lie between one and five percent. Binge size varies widely intra- and inter-individually, but in different studies averages have been reported of between 600 and 3000 kilocalories. Average frequency of substantial binge episodes (i.e., $1000 \mathrm{kcal}$ or more) in bulimics have been reported to lie at five per week. Although typical binge food consists for an important part of high-caloric snacks, they do not appear to satisfy specific hungers for carbohydrates: binges do not differ significantly from normal meals in their macro-nutrient composition. Affective consequences of bingeing are mixed: binges appear temporarily to alleviate depression and tension, but also cause shame, guilt and feelings of controlloss. Binge eating and vomiting coincide with impaired well being and social functioning of the individual. There is some consensus among researchers that depression and other 
psychopathology are a consequence rather than a cause of binge eating.

\subsection{HERMAN \& POLIVY'S COUNTERREGULATION MODEL OF B BINGE EATING}

As counterregulation has been the predominant lab model in research of human binge eating for many years, it will be described and evaluated below. The conclusions will provide a background for our decision to adopt a different (conditioning) paradigm in chapter 2 .

\subsubsection{Description of the counterregulation model}

In the mid 1970s, hunger and weight were no longer seen as the only factors governing food intake. Research on normal and deranged eating turned towards the concept of eating style. Two prominent workers in the new research area were C.P. Herman and J. Polivy at the University of Toronto psychology department, who developed the "preload-counterregulation" paradigm (Herman \& Polivy, 1980; 1984; Ruderman, 1986). The basic procedure is as follows: subjects participate in a "taste test" involving a questionnaire-guided comparison of different sorts of ice cream. Each subject has ad lib access to plates with abundant ice cream for a period of, for example, 10 minutes, and is kept unaware of the fact that her/his intake will be measured. There are two conditions: in the "preload" condition a high caloric item (e.g. a milkshake) must be eaten prior to the taste test, whereas the control condition involves no preloading. Furthermore, in the basic design the ice cream intake of dieters is compared with that of non-dieters. Dieting is identified by high scores on a questionnaire, the Herman and Polivy's Restraint Scale $^{6}$. The pattern of food intake that has been repeatedly observed by Herman and Polivy is as follows. Unrestrained eaters (the "non-dieters") act reasonably by diminishing their food when they have already eaten a preload. That is, they "regulate" their food intake. Surprisingly, restrained eaters (i.e. the "dieters") have been observed to "counterregulate: they eat more in the preload than in the control condition?

The explanation given by Herman and Polivy $(1980 ; 1984)$ is based on two elements. First, restrained eaters are said to impose a deliberate, "cognitive" restraint on their food intake. In the no-preload condition, this prompts them to eat less ice cream than

\footnotetext{
${ }^{6}$ For a more detailed discussion of the Restraint Scale see chapter 8 of this thesis.
}

${ }^{7}$ Note that an important criterion of a lab model of binge eating thus was fulfilled: after some experimental manipulation, an increase in food intake was observed. 


\section{Chapter 1}

unrestrained eaters. Second, restrained eaters are likely to see their restraint broken by the "forced" ingestion of the high-caloric preload. Herman and Polivy have reasoned that broken restraint prompts catastrophic thoughts (e.g. "I've blown my diet, so I might as well go on eating"), that will pave the way for excessive eating. Contributing to the larger food intake in these dieters are "biological pressures" acting to compensate for previous chronic restriction of food intake ${ }^{8}$.

Later studies have extended the basic findings by demonstrating broken restraint after other experimental manipulations. It was shown that restrained eaters also increase their food intake after low-mood inductions, or after information that an already eaten preload was of high caloric content. Also the mere prospect of forced ingestion of high caloric food items has been found to trigger increases in food intake (see Herman \& Polivy 1984; Ruderman, 1986). Although the original purpose of the preload/counterregulation paradigm was to investigate dieters' eating behaviour, counterregulation has increasingly been labelled as a form of disinhibition (Herman \& Polivy, 1988; Polivy \& Herman, 1989).

\subsubsection{Critique of the counterregulation model}

Twenty years have past since the concepts of restraint and counterregulation were introduced. The gathered data and related theorizing have been effective in drawing researchers' attention from mere hunger and body weight towards cognitions and behavioural mechanisms active in the disinhibiting eater. Also, this line of research has underscored the multitude of problems encountered by people attempting to lose weight. It has become clear that various stimuli, mood states, thoughts and ideas may have an instantaneous and facilitating effect on food intake.

Despite these advantages, several data suggest that the mechanism of (broken) restraint cannot fully explain why people counterregulate or, more generally, indulge in binge eating. Also, methodological criticisms have been raised about key explanations in counterregulation research. Some of these critical notes (see for example Charnock, 1989 a,b; Dritschel, Cooper \& Chamock, 1993; Jansen, 1990; Lowe, 1993; for replies see Heatherton, Herman, Polivy, King \& McGree, 1988; Polivy \& Herman, 1989) will be briefly discussed below, followed by an overall evaluation.

Counterregulation as a lab-analogue of overeating The fact that in counterregulation experiments the preload manipulation (ingestion of a 420 -gram milkshake) has been

8 The "biological pressure" explanation of urge was only initially defended by Herman \& Polivy. Later, it was discarded (compare the two papers by Herman \& Polivy from 1980 and 1984); see also section 1.3.2 of this thesis). 
between 6 and 13.5 times as large as the increase in ice-cream consumption by restrained eaters (i.e., 31 grams (Herman \& Mack, 1975), and 68 grams (Herman, Polivy \& Esses, 1987) respectively) should not be considered a major blow to the validity of the paradigm: counterregulation is a model that need only embody the essence of overeating (section 1.1), and indeed, the preload manipulation appears to lead to an increase of subsequent food intake. But is this reliably the case? Some authors have claimed that it is not (Chamock, 1989a; 1989b; Dritschel et al., 1993; Jansen, Oosterlaan, Merckelbach \& Van den Hout, 1988). The result often presented under the heading "counterregulation" is based statistically on a restraint $x$ preload interaction effect. However, closer inspection often reveals that the restraint $x$ preload interaction effect is caused by unrestrained eaters eating less when preloaded than when unpreloaded (a reliable, but trivial finding), and restrained eaters eating equal amounts of food in both conditions. Clearly, for counterregulation to reflect "overeating", it would be required that restrained eaters eat more when preloaded than when unpreloaded, but this crucial test has seldomly been conducted. In sum, counterregulation proper seems to be a somewhat less robust finding than has often been realized. Although non-regulation admittedly is an interesting phenomenon, it does not strike one as a convincing analogue of binge eating.

Broken restraint as a prerequisite for counterregulation In a critical test of the claim that breaking cognitive restraint is responsible for lab disinhibition, Jansen et al. (1988b) monitored the thought stream of restrained and unrestrained eaters in a preload study. A 26-item questionnaire with thoughts like "I've blown my diet so I might as well continue to eat" and "I feel out of control", etc., was completed by subjects. The results showed that, although ad lib ice-cream intake showed the usual restraint $x$ preload interaction effect, preloaded restrained eaters showed as many catastrophic thoughts as preloaded unrestrained eaters.

Another issue concerns the identification of the diet boundary, i.e. the critical amount that should be eaten in order to break cognitive restraint, leading to disinhibitive eating (Herman \& Polivy, 1984). Herman and Polivy themselves have experienced considerable difficulty in objectifying the diet boundary. In their 1984 article (p. 151) they suggest that a 210 -gram milkshake preload was too small to break the restraint in many dieters; preloads of 420 grams would be more effective. However, three years later Herman, Polivy \& Esses (1987) set out to determine the critical preload size required for breaking restraint, by simply asking their subjects. It appeared that restrained eaters were unable to estimate their diet boundary, a fact which led the investigators to drop this theme from investigation. In retrospect, Herman et al. (1987) suggested that "an increasing number of dieters consider their diet to be broken by any forced preload at all" (p. 164). What is more, two subsequent studies (Rogers \& Hill, 1989; Jansen \& 


\section{Chapter 1}

Van den Hout, 1991) have shown that mere smelling of palatable food (i.e. zero intake) was sufficient to lead to subsequent disinhibition. It is hard to see why restraint would be broken by smelling palatable food. All in all, disinhibitive eating may well occur without a preceding break of "cognitive restraint".

Explanation of the urge to eat Disinhibitive eating has been said to originate in a loss of cognitive restraint (Herman \& Polivy, 1980; 1984). When analysing this statement, one notices that it does not clarify why the loss of cognitive restraint should induce overeating: no additional urge-mechanism is postulated. The authors (Herman \& Polivy, 1984) remain remarkably uninformative on this point, when they state that their counterregulating subjects ate until their "satiety boundary" was reached. The satiety boundary is described as a state in which further food intake would give rise to feelings of discomfort. To explain counterregulation, Herman and Polivy (1984) claim that the satiety boundary in restrained eaters lies at higher level than that in unrestrained eaters, thus "allowing if nor guaranteeing (..) prodigious consumption" (p.150) once restraint has been broken. One substantiation of the higher satiety boundary alluded to (albeit rather unpersistingly), is that restraint eaters, as a consequence of their dieting might experience an increased biological pressure to eat, so as to restore their biological set point of weight (p. 145). In addition to ideal weight being an ill-defined construct (Greeno \& Wing, 1994), empirical findings make this explanation less likely. First, only between $37 \%$ and $50 \%$ of high restraint subjects report they are currently dieting (Dritschel et al., 1993 Lowe, 1993; chapter 5 and 8 this thesis). Second, a study in which dieting status was checked showed that currently dieting restrained eaters did not counterregulate after a preload, whereas non-dieting restrained eaters did. This finding has been replicated (Lowe, 1993, p.104), and is contrary to the below-set point explanation of counterregulation'. Third, there is the robust finding that restrained eaters generally have a higher BMI than unrestrained eaters do (see the studies in Jansen, 1990; this thesis chapters 5, 7, and 8). Apparently, the "higher satiety boundary" is no explanation of urge, but rather a stately way of saying that more food has been eaten (Jansen, 1990).

9 Apparently in line with a set point explanation of counterregulation is the finding that in the strongest example of counterregulation reported (Herman \& Mack, 1975) normal weight but not overweight restrained eaters did counterregulate (for the reanalysis of these data, see Ruderman, 1986). However, if disinhibition were caused by being below the biological set point, it would follow that 1 . the counterregulating restrained eaters' biological set point is at overweight level; 2 . overweight restrained eaters do not show disinhibitive eating in daily life. These (rather unlikely) allegations still need to be proven. 


\subsubsection{Final remarks}

In sum, the preload-counterregulation paradigm has led to increased knowledge and has set the issues in experimental research on overeating. However, the underlying notion of the counterregulation theoreticians has always been that disinhibitive eating can only occur if prior inhibition ("restraint") existed (Heatherton et al., 1988; Herman \& Polivy, 1990). Of course, when taken literally, this notion is overwhelmingly logical. In practice, however, it is becoming increasingly clear that the boundaries of knowledge growth have been reached in counterregulation research. A striking illustration is the recent report that restrained eaters will only counterregulate if they score high on a scale measuring "tendency to overeat" (Westenhoeffer, Broeckmann, Muench \& Pudel, 1994). In one of their more recent papers, Herman and Polivy $(1990 ;$ p. 123) themselves have suggested that researchers of binge eating "(..) are not in a sirong position to posit causes or mechanisms, since we are not sure what exactly we are trying to explain" (..). To be sure, it appears that the view that binge eating is a lifting of inhibition may discourage the search for mechanisms that explain the urge. This has been a reason for our adopting a different approach in the present thesis: in chapter two, a conditioning theory of binge eating will be described. This theory involves an urge mechanism that may be the "final common pathway" of the different ways in which stimuli may trigger binge eating. Additionally, it describes a mechanism through which binge eating may be self-maintaining. 



\section{A CONDITIONING THEORY OF BINGE EATING}

A conditioning theory of binge eating will be described in section 2.1 , followed by relevant empirical data (section 2.2). Section 2.3 contains an introduction to hypotheses derived from the conditioning theory. These hypotheses will be tested in subsequent chapters.

\subsection{DESCRIPTION OF THE THEORY}

The conditioning theory of binge eating (henceforth CBE theory; Jansen, 1990; 1994a) posits that bingeing is a learned and self-perpetuating behaviour. CBE theory derives from addiction research, where classical (i.e., Pavlovian) conditioning has been accredited an important role in tolerance and relapse (e.g. Childress et al., 1992; Siegel, 1983; Tiffany, 1995a). In short, cues (CS) that signal large food intake (UCS) will, through a conditioning process, become triggers of physiological responses (CRs). These CRs are experienced subjectively as food craving, thereby encouraging binge eating. A subtle version of this mechanism is well-known to us all: when walking past a bakery and sensing the fragrance of freshly baked pies, we will often feel the water running into our mouths, suddenly experiencing an appetite that was absent only moments ago. In binge eating the mechanism has got considerably out of hand. While salivation is one of the CRs, emphasis will be placed on conditioned blood sugar decrease and insulin secretion. Underlying the CBE theory are five basic assumptions, which will be described next.

\subsubsection{First assumption: binges are signalled by cues}

The conditioned stimuli (CSs) are formed by cues that invariably precede binge eating. Examples are the sight, smell, and taste of typical binge foods. The association between between binge cues and binge eating is especially strong because binge eaters tend to restrict their food intake in between binges, thereby actively avoiding the high-caloric "forbidden" binge foods. Binge cues may also be affective and situational: feelings of loneliness, anger, depression or boredom all may reliably precede bingeing, as may certain times of the day or the environment in which binges occur (cf. being home alone, in the kitchen near the fridge, etc.).

1 Related, but not formally developed ideas can be found in Wardle (1990); see also Woods (1991) for an eloquent and inspiring review of related ideas in the field of normal food intake. 


\subsubsection{Second assumption: food intake provokes corrective physiological responses}

Food ingestion leads to a temporary disturbance of the internal physiological state. However, because the body generally strives towards stability of its internal milieu, it will emit regulatory, homeostaric responses. For example, a meal generally causes blood sugar levels to rise considerably. As a reaction, the hormone insulin is secreted from the pancreas, with the effect that blood sugar levels are redirected towards the pre-meal baseline. In conditioning terminology, the physiological disturbance following food intake is the unconditioned stimulus (UCS), and the homeostatic responses form the unconditioned responses (UCRs).

\subsubsection{Third assumption: through conditioning, binge cues come to trigger anticipatory} physiological responses

By means of a conditioning process, binge cues become conditioned stimuli (CS) that will trigger conditioned salivation, insulin secretion, blood sugar decreases, etc., (CRs) before food intake has taken place. The conditioned physiological responses are called anticipative and compensatory because they counter the physiological disturbance that usually follows food intake. Moreover, the intensity of the CRs will be proportional to the amount of expected food intake. Specifically, the CBE theory predicts large insulin secretion and large hypoglycaemia to cues that signal binge eating.

\subsubsection{Fourth assumption: conditioned physiological responses are subjectively experienced as food craving}

According to CBE theory, perception of binge cues induces insulin secretion and a fall in blood sugar level. The CBE theory posits that this body state will be subjectively experienced as food craving. Consequently, food ingestion, serving as "drug-taking behaviour" becomes more likely. A somewhat picturesque way of describing this would be that bingeing is an attempt to "eat away" the conditioned hypoglycaemia.

\subsubsection{Fifth assumption: binge eating is a self-maintaining process}

Evidently, craving heightens the likelihood of bingeing. In binge eaters, it could be said that each binge acts as a conditioning trial in which binge cues (CS) are paired with a binge (UCS), thus strengthening the cue-binge association. From the body's "viewpoint", the homeostatic "necessity" of emitting strong compensatory CRs to binge cues is "reconfirmed" each time a binge occurs. The individual thus may enter a vicious circle: a next encounter with binge cues will again elicit the "necessary" conditioned responses that again, through craving, facilitate bingeing. 


\subsection{OVERVIEW OF RELEVANT DATA}

\subsubsection{Existence of binge cues}

In binge eating research, the probability relationship between binge cues (CS) and binges (UCS) has received little systematic attention. This is probably due to its apparent triviality: there seems little need to argue that food-intrinsic cues, like sight, smell and taste of binge food, precede bingeing. As a matter of fact, retrospective self-reports never fail to include food stimuli as important precipitants of binge eating (Abraham \& Beumont, 1982; Bruce \& Agras, 1992; Mitchell et al., 1985) and of temptation and relapse in dieters (Grilo, Shiffman \& Wing, 1989). However, as far as the present author knows, no quantitative reports exist about the probability relationship between specific cues and bingeing at the individual level. Nevertheless, some indications can be found that binge eating is generally preceded by cues unique to binge episodes. First, it has been found that bingers with bulimia or anorexia show intermediate dieting that is often accomplished by avoiding "forbidden" high-caloric foods that are typically ingested during binges (Walsh, 1993). Therefore, especially cues pertaining to binge food rather than other foods could be expected to be uniquely confined to binge contexts. Second, it has become clear that in therapeutic contexts, patients can, when asked, identify typically tempting binge foods, circumstances and locations. These cues may be used in cue-exposure procedures (e.g. Jansen et al., 1992).

To find evidence that affective binge precipitants (low moods, negative thoughts and stressful social interactions) act as "cues" seems more problematic. Although emotional states are among the most frequently mentioned precipitants of bingeing by patients (Abraham \& Beumont, 1982; Bruce \& Agras, 1992; Mitchell et al., 1985), it remains unclear whether mood states act as the actual binge cues, or as so-called "occasion setters", i.e. the background against which the food cue-binge (CS-UCS) association is established (Catania, 1991; Davidson, 1993).

\subsubsection{Homeostatic regulation of blood sugar level}

The idea of homeostatic responses correcting disturbances of the internal milieu is, of course, not new. It was developed in the 1920s by W.B. Cannon, the father of 20th century physiology. The concept of homeostasis offered an explanatory framework for better understanding the multitude of co-occurring and interacting body processes (Cannon, 1939; Guyton, 1991, ch. 1). A comprehensive treatment of homeostatic physiology related to food intake is beyond the scope of this thesis (and of its author too). A bird eye's view will only be given of blood sugar regulation. When ingested 


\section{Chapter 2}

food has passed through the stomach, it will appear in the small intestine, where its various nutrients are absorbed and appear in the blood stream. Consequently, the level of blood glucose (i.e. blood sugar) begins to rise. However, blood sugar is tightly regulated: several homeostatic responses are emitted to counter the blood sugar rise. One of these responses is an excessive insulin release by the pancreas. Insulin, sometimes also called "the hormone of plenty", lowers blood sugar levels by, for example, making cell walls more permeable for glucose. As a consequence, the glucose formerly contained in the food will enter the various body cells and tissues where it is stored for later use or utilized at once ${ }^{2}$. In sum, whatever the disturbance imposed on the body's internal milieu, the interaction between nutrients, nervous activity and hormones will act to keep blood sugar within a restricted range. For reviews see for example Brodsky \& Devlin (1994), Cryer (1992), Guyton (1991), Hardy (1981), Steffens et al. (1991).

\subsubsection{Existence of anticipatory responses to sensory food cues}

Description. Food-anticipatory responses to cues (as described in the CBE theory) abound in the mammalian body, and have first been described as "psychic reflexes" by Pavlov (1910). In the last twenty years, the term cephalic phase responses (cephalos = head) has come into general use. Cephalic phase response (Berthoud et al., 1981; Giduck, Threatte \& Kare, 1987; Le Blanc, 1992; Powley \& Berthoud 1985, Teff et al., 1993) is a general term referring to physiological responses that are triggered by foodrelated, visual, olfactory, gustatory or oropharyngal-mechanical cues. Thus, cephalic responses may occur both before and during food ingestion. Besides salivation, studied so thoroughly by Pavlov, secretion of gastric and pancreatic juice and of a range of hormones like insulin, pancreatic polypeptide, glucagon can be secreted cephalically. Also other responses have been reported, like increases in metabolic rate (i.e. the body's energy production) and glucose absorption by the liver (Le Blanc, 1992). Many, but not all of these cephalic responses peak at about five minutes and return to baseline at ten minutes after food cue perception (Le Blanc, 1992; Berthoud \& Powley, 1985). Physiologically speaking, the early cephalic phase responses should be distinguished from post-absorptive, homeostatic (see section 2.2.2) reactions to nutrients entering the blood. In particular, the hormonal response to food intake often shows two phases: a smaller cephalic wave in the first few minutes after intake followed by the onset of a post-absorptive response at 5-15 minutes (e.g. Guyton, 1991; Teff et al., 1993). This is

\footnotetext{
2 A reverse mechanism comes into play if the blood sugar level becomes too low, or fuel demands increase, e.g., during stress or vigorous exercise. In these cases, various blood sugat enhancing hormones (glucagon, growth hormone, catecholamines and corticosteroids) are secreted. Generally, they suppress insulin secretion, and mobilize sugars and fat previously stored in cells and tissues.
} 
not to say that the impact of cephalic responses could not extend to longer periods than 10 minutes; the functioning of organs and tissues can be altered for more prolonged periods, when the initial cephalically triggered hormones no longer circulate in the blood (Le Blanc, 1992; Thompson et al., 1988; Bruce et al., 1987). Moreover, cephalic responding is not confined to the pre-ingestive period; it may also occur when new food items are introduced during an ongoing meal (Louis-Sylvestre, 1984). It is generally held that the efferent nervous pathway of cephalic responses is primarily parasympathetic, involving the different branches of the vagus nerve connected to the various glands and organs (Powley \& Berthoud, 1985; Louis-Sylvestre, Giachetti \& Le Magnen, 1983; Strubbe \& Steffens, 1975; Strubbe \& Steffens, 1993).

Functions Because of the very existence of cephalic responses, the issue arises about their possible biological function (e.g. Powley \& Berthoud, 1985). Several functions have been proposed.

First, cephalic responses may attenuate the physiological disturbances that follow the ingestion of food (Powley \& Berthoud, 1985; Woods, 1991). In fact, it has been shown that the nutrient intake leads to larger physiological disturbances when the cephalic phase is by-passed (e.g. by infusion of nutrients) whereas, conversely, the blood sugar rise in response to glucose infusions is decreased if a cephalic response is evoked concurrently (Bruce et al., 1987, Le Magnen, 1992; Strubbe \& Steffens, 1993).

Second, cephalic responses may prepare the various body structures for the breaking up, storage and metabolism of food (Le Blanc, 1992; Powley \& Berthoud, 1985; Woods, 1991). In addition, drawing upon the finding of increased metabolic rate during the first 30 minutes after the beginning of cephalic responses, Le Blanc (1992) has speculated that cephalic responses might aid in mobilizing energy for the pursuit and capture of prey.

A third function of cephalic responses may be to induce or enhance appetite. This might be no less advantageous in an evolutionary sense than the regulatory functions mentioned earlier: appetite instigates food intake at the very moments food is available (Woods, 1991). This is neatly expressed in the French proverb L'appetit vient en mangeant (Le Magnen, 1992).

The fourth function, proposed by Louis-Sylvestre (1984), is that cephalic responses may stimulate versatility of food intake, a function which becomes apparent during meals. In rats turning from one food item to another, an additional insulin secretion super-imposed on the normal post-prandial insulin level is seen, with the effect of lowering postprandial blood sugar rise, and probably, maintaining the hedonic value of intake (Louis- 
Sylvestre, 1984) ${ }^{3}$. A perhaps related effect has been shown in humans. After having eaten a lunch of considerable size, subjects were sensorily primed by a small bite of either pizza or ice cream. A subsequent ad lib intake test with both foods abundantly available showed that intake of the primed food item always exceeded that of the unprimed, although both items had been regarded equally highly by the subjects (Cornell, Rodin \& Weingarten, 1989).

Modulating factors Cephalic phase responses appear in different forms and intensities both intra- and inter-individually. Several sources of variation in cephalic responding exist, mainly related to the nature of the signalled food, and the learning history of the subject.

First, the chemical composition of the anticipated food may matter. Already Pavlov (1910) pointed at the adaptiveness of cephalic responses. In his dogs, the chemical characteristics of the various anticipatory secretions were specifically attuned to those of the expected intake. For example, thickness, amount and chemical composition of the cephalically aroused saliva and pancreatic juice were most appropriate to deal with physiological consequences of the ingestive, even if the (learned) food cue was so alien to food as the ticking of a metronome or the sight of a bottle (see Babkin, 1949; Dworkin, 1993).

A second factor influencing the intensity of cephalic responses is the palatability and perceived palatability of the expected food. Apparently, the most potent elicitors of salivary and insulin responses are sensorily rich food items such as odorous apple pie or spicy pizza, especially when they are brought in contact with the oral cavity (Christensen \& Navazesh, 1984; Teff et al., 1991). The role of visual attractiveness of food items has been neatly demonstrated by Lucas et al. (1987). Half of a freshly baked quiche was mashed in a food processor, after which it was mixed with an odourless blue dye. Both this item and an original (attractive) piece of quiche was presented to normal weight subjects. The cephalic insulin response to the attractive dish was more intense than that to the unattractively mashed variant.

Third, the anticipated amount of food or the expected metabolic consequences play a role in the intensity of the cephalic insulin response (Powley \& Berthoud, 1985): in rats sipping from a saccharin solution, the intensity of the cephalic insulin response has been found to increase proportionally with the quantity ingested, a remarkable finding as saccharin is a metabolically inert artificial sweetener. The effect might be dependent

3 Parenthetically, one should be careful to attribute such behavioural effects to the action of insulin alone, because cephalically induced increases of insulin secretion are usually small compared with those found after food intake (see also sections 9.2 and 9.3 of this thesis). 
upon the rats' previous intake experience with real sugars. When drinking the sweet saccharin, the rats "anticipated" a blood sugar rise proportional to the amount of saccharin ingested. Accordingly, for a larger intake of the sweet solution, a proportionally larger insulin response appeared.

Fourth, cognitive factors have been proposed to account for occurrence or non-occurrence of cephalic responses (e.g. Sjöström et al., 1980). The expectation that food items presented will be actually ingested appears to be especially important (e.g. Pavlov, 1910). Bruce et al. (1987) found a 0.90 correlation between a questionnaire score of intake expectation and the cephalic hypoglycaemic response to food cues. An interesting example showing the impact of cognition has been provided by Goldfine, Abraira, Gruenewald and Goldstein (1970), who were able to induce a cephalic insulin rise in their subjects through hypnotical suggestions of a palatable meal.

Finally, habitual eating pattern appears to modulate the intensity of the cephalic responses. A robust finding is that obese persons show larger cephalic insulin responses than do normal weight persons (Simon et al., 1980; Sjöström et al., 1980). In absolute levels the intensity of cephalic responses has been found to be nearly four times as large in the obese; expressed in percentage increase from baseline levels (obese humans are known to have increased baseline levels of insulin), the obese showed twice as strong cephalic insulin responses (Sjöström et al., 1980). Another study found a trend towards larger cephalic insulinaemic response in obese (Johnson et al., 1983). Although these results are most likely due to hypertrophy of the insulin producing Beta-cells of the pancreas (Dr. Strubbe, personal communication), there remains an additional possibility (which would be in line with CBE theory) that the larger average meal size of the obese in daily life contributes to enlarged cephalic insulin responses.

There is also some indication that cephalic salivation is modified by food intake experience. Le Goff, Leichner \& Spigelman (1988) compared salivary flow to food cues in bulimics and anorexics before and after therapy. Before therapy salivation to food stimuli was more intense in bulimics than in anorexics. After therapy, a re-assessment showed that bulimics salivated less, and anorexics salivated more to food cues than they had originally. This could be explained accordingly by assuming that a bulimic eating style, involving occasional large food intake (binge UCSs), results in stronger salivation (CR), whereas in anorexic patients, food cues predicting small food intake, lead to modest salivary responses (Jansen, Boon, Nauta \& Van den Hout, 1992).

In contrast with the above results, Broberg and Bernstein (1989) and Moyer, Rodin and Cummings (1993) did not find enlarged cephalic insulin responses or hypoglycaemia in bulimic patients exposed to palatable foods. In these cases the CBE predictions were not confirmed. 


\subsubsection{Classical conditioning of food intake and related physiological responses}

Although it is true that cephalic responses to food cues, especially those occurring during food ingestion, may be understood partly as expressions of "hard-wired" reflex loops which are activated when the ingested food strikes various receptors while travelling through the mouth, throat and stomach (Berthoud et al., 1981; Nicolaïdis, 1977), this does not at all imply that the influence of learning history should be discarded (e.g. Dworkin, 1993; Jansen, 1994a; Jansen, Boon, Nauta \& Van den Hout, 1992; Woods, 1981; 1991). On the physiological level, learning may act in at least two ways: by modulation of the existing hard-wired reflex loops (Dworkin, 1993), and by bringing the efferent part of the reflex loop under arbitrary stimulus control (Woods \& Kulkosky, 1976). On the behavioural level, learned cues may activate food seeking and intake. Clearly, the strongest support for these allegations (at the heart of the CBE theory) would be provided if acquisition of the responses described could be demonstrated by means of a classical conditioning procedure. In the following overview such studies will be discussed. It has indeed proven possible to bring meal initiation, meal size, salivation, insulin secretion and hypoglycaemia under the control of arbitrary cues.

Conditioned meal initiation and meal size increase Weingarten $(1984 ; 1985)$ has provided evidence that meal initiation in rats can be classically conditioned to arbitrary cues. In his procedure, which extended over several days, feeding in rats was always preceded by a 4-minute CS (light-buzzer compound), after which a food tray was filled. Then, during the test session, rats were first given ad lib access to food. After they gave signs of being satiated (cessation of intake; grooming), the light-buzzer CS was switched on. It was observed that the rats started eating again, and doing so ingested an amount equalling about $20 \%$ of their daily intake prior to the experiment. It seems that conditioned physiological responses may have contributed to the meal initiation. In a similar procedure to Weingarten's, Strubbe (1992) used the slamming of a food tray as a CS for food delivery. At the test session, conditioned insulin appeared to the slamming of the tray. In humans, surprisingly little research has been conducted in this field. Birch et al. (1989) found in children an increase in candy intake in presence of a candy-predicting light, but only if the children had been aware of the CS-UCS (light-candy) contingency. One might wonder what purpose would be served by increasing food intake after perception of signals predictive of food. Zamble (1973) has provided clues to this problem. He showed that rats that were allowed one-hour food access daily (each day at a different time) ate more if their meals were signalled 15 minutes in advance, than if food was unsignalled. The fact that these eating behaviours resulted in smaller weight loss in the signalled rats, suggests that conditioned enhancement of food intake may be 
of survival value if food is scarce and/or "patchily" distributed in the organism's environment (see Collier, 1986).

Conditioned hypoglycaemia. Blood sugar level can be brought under arbitrary CS control of odours and injection rituals (for reviews see Dworkin, 1993; Woods \& Kulkosky, 1976). In many but not all instances, insulin injections have been used as the UCS, yielding mixed results of either conditioned hypo- or hyperglycaemia, depending on the doses of insulin used and the specifics of the procedure (for detailed discussions see Dworkin, 1993; Woods, 1983; Chapter 4 of this thesis).

More relevant for the CBE-model are procedures in which pure glucose or normal food have been used as a UCS. Some will be discussed here (see also chapters 3 and 4 of this thesis. A strong demonstration of learning in hypoglycaemia using a glucose UCS has been provided by Deutsch (1974) who fed his rats with the sweetener saccharin and observed a hypoglycaemia although this chemical, as noted earlier, does not contribute directly to blood sugar level. Deutsch suggested the observed hypoglycaemia was a compensatory response reflecting the rats' learning history with natural (real) sugars in which sweet taste always had predicted a blood sugar rise. To test this suspicion, Deutsch first investigated whether the hypoglycaemic response could be extinguished. This indeed happened after offering the rats free access to saccharin for a prolonged time. Next, Deutsch argued that it should be possible to established conditioned hypoglycaemia to an arbitrary taste CS as well. Thus, he repeatedly presented his rats with a (non-sweet) cafeine solution to drink followed by the UCS, intubation of glucose into the rats'stomachs. After ten such pairings, the taste of the cafeine itself was able to elicit a hypoglycaemia. Matysiak \& Green (1984) have also found conditioned hypoglycaemia in rats after a learning procedure involving glucose injection UCSs. In contrast, Woods (1976) failed to find this result. This was probably due to the small glucose dose involved. In humans and dogs, a strong example of hypoglycaemic conditioning, leading to about $15 \%$ fall in blood sugar using UCS glucose injections, was reported by Russian physiologist Mityushov (1954; see Chapter 4 and the appendix of this thesis).

Conditioned insulin secretion The blood sugar conditioning studies referred to above, did not include direct measurements of insulin. Nevertheless, it has often been suggested that the observed blood sugar fall was secondary to a conditioned insulin secretion (Dworkin, 1993; Mityushov, 1954; Woods, 1983). Direct evidence has come from studies by Woods et al. (1977) and Strubbe (1992) who demonstrated insulin secretion to meal predicting cues. These authors meal-fed rats at a limited and fixed number of times each day. Woods et al. (1977), giving their rats unlimited excess to food for one hour daily, found an increased insulin secretion at the time of day the rats were usually fed. Strubbe 


\section{Chapter 2}

(1992) used slamming of a food tray as the CS. Two procedures were tested: rats had either two or six times daily free access to food (UCS). On the test day, slamming the food tray alone evoked significant insulin secretion, but only so in rats who had been receiving two meals daily. One of the explanations was that the meal size (i.e. UCS intensity) during the conditioning procedure had been larger in rats fed twice, than in those fed six times daily. Also deprivation in itself might have played a facilitating role (see Weingarten, 1985).

The data presented are in line with the CBE notion that meal initiation and food intakerelated physiological responses can be conditioned to learned cues in people and animals. Food deprivation is not a prerequisite for these effects to occur.

\subsubsection{Hyperinsulinaemia/hypoglycaemia experienced as craving}

The CBE theory assumes that conditioned insulin secretion and hypoglycaemia will be subjectively experienced as craving for food. This assumption receives support from different sources. First, research on hypoglycaemic episodes in diabetic patients shows that hypoglycaemia is often accompanied by feelings of hunger (Hepburn, 1993; Service, 1995). Second, Rodin, Bach, Peranini \& De Fronzo (1985) used the clamp technique, enabling them to keep insulin and blood sugar at fixed levels. Several combinations of blood glucose/insulin levels were established in human subjects who were asked each time to report the intensity of their hunger feelings. It appeared that hunger scores were highest during combined hyperinsulinaemia/hypoglycaemia. Equally, large (Grossmann, 1986) and small (Campfield et al., 1996) insulin injections in humans have led to increases in reported hunger. Fourth, in rats, a short-lived insulin secretion followed by a hypoglycaemia has been found to reliably precede meal intake (Campfield \& Smith, $1990 \mathrm{a} ; 1990 \mathrm{~b})$. Finally, there are indications that conditioned meal initiation in rats (see section 2.2.4) is accompanied by a blood sugar decline in rats (Weingarten et al., quoted in Le Magnen, 1992 p. 84).

\subsubsection{Extinction of conditioned physiological responses and binge eating}

The CBE-hypothesis that craving and binge eating follow the acquisition of conditioned insulin secretion and hypoglycaemia, has not been tested directly. However, animal and human data are available about the reverse mechanism, that is, extinction of conditioned responses by repeated CS presentations not followed by the UCS.

Animal studies First, Deutsch (1974) has observed that rats' cephalic hypoglycaemic response to the sweet taste of saccharin disappeared after 60 days of ad lib access to the 
(metabolically inert) saccharin ${ }^{4}$. Second, extinction of explicitly conditioned hypoglycaemias has been demonstrated after repeatedly exposing the rats to the gustatory or olfactory CS (Deutsch, 1974; Woods, 1976).

Studies in humans Exposure to binge food cues (CS) with prevention of subsequent food intake (UCS) has been demonstrated to reduce binge eating. The procedure is as follows. First, therapist and patient will map cues and acts which are characteristic of binge episodes. This information is used during the exposure sessions when the binge situation is mimicked as realistically as possible. Under supervision of the therapist, the patient is encouraged to go through the ritual of selecting and preparing the typical binge food, thereby handling, smelling or licking the food, and tasting morsels of it. To increase realism, the exposure will be often carried out at the typical binge location, i.e. the patient's home. Although strong food craving arises, the patient is prevented from ingesting food. Prolonged exposure, for example 45 minutes or more, will result in the craving being significantly decreased. Several studies have been published about the effects of the cue exposure procedure in bulimic patients. Overall, the results have been promising in that a reduction of binge frequency was found (Hansen \& De Haan, 1995; Jansen, Van den Hout, De Loof, Zandbergen \& Griez, 1989, Jansen, Broekmate \& Heymans, 1992; Kennedy et al., 1995; Schmidt \& Marks, 1988, 1989) which appeared to have persisted at 6-months (Schmidt \& Marks, 1989) or 1-year (Jansen et al., 1992b) follow-up. Interestingly, also levels of depression and unrealistic cognitions about food and body shape had diminished after treatment (Jansen et al., 1992b). Taken together, these findings fit well into the CBE theory's conceptualization of binge cues as CS and binge eating as a UCS.

4 The issue of whether saccharin-induced hypoglycaemia is learned or inborn has been much debated. For instance, Berthoud et al. (1981) have claimed that saccharin-induced insulin secretion in rats should be regarded an inborn response, because 10 -fold exposure to mere saccharin did not lead to its extinction. In a verbatim commentary on Dr. Berthoud's paper, Woods (1981), referring to Deutsch's results, has argued that 10 extinction trials are insufficient to refute a learning interpretation. Dr. Berthoud's reply that Deutsch's results were not to the point, because Deutsch only measured blood sugar at two hours after saccharin presentation, is valid. Indeed, more convincing data should involve measurement at five minutes after exposure to saccharin, and such measurements still need to be done. Note that already in Pavlov's times, essentially the same debate evolved about the allegedly learned nature of salivation to meat in dog puppies (Joravsky, 1989). 


\subsection{SUMMARY AND IMPLICATIONS FOR THE PRESENT THESIS}

\subsubsection{Summary}

A conditioning theory of binge eating (CBE theory) was presented in this chapter. CBE theory regards binge eating as an acquired behaviour. Binge cues acting as conditioned stimuli (CSs) that reliably predict large food intake (UCS) will eventually acquire the power to elicit physiological responses (CRs). The CRs are subjectively experienced as craving. Once established, binge eating is a self-maintaining process. The binge eating individual may enter a vicious circle: each binge implies a pairing of binge cues (CS) with large food intake (UCS). Consequently, future encounters with binge cues will trigger intense CRs, that in their turn favour binge eating, and so on. Although different physiological CRs are triggered by the CS, especially insulin secretion and hypoglycaemia are hypothesized to be important in craving. Data relevant to the assumptions of the CBE theory were discussed. Although it remains to be demonstrated whether binge eaters are conditioned in the hypothesized way, a cue exposure procedure, implied by CBE theory, appears to be an effective treatment of binge eating. The procedure, involving presentation of binge cues with simultaneous prevention of food ingestion, has been reported more than once to reduce binge eating. These findings are compatible with a hypothesized extinction of the learned CS-UCS (cue-binge) association.

\subsubsection{Implications for the research in this thesis}

So far, little direct research of CBE-predictions has been carried out. The non-clinical lab investigations to be presented in the following chapters have been inspired by the CBE theory and focus on various aspects of the theory.

The hypothesis of the investigation in chapter 3 is directed at the etiological level: if a set of cues CS is made to predict a considerably large intake of sugar (UCS), then eventually the cues themselves will elicit conditioned insulin and blood sugar responses, craving and heightened sugar intake. Chapter 4 discusses the results of a remarkable, but not widely known Russian study (first published in 1954) on conditioned hypoglycaemia. The data from this study are re-analysed, and put into the perspective of modern research (including the $\mathrm{CBE}$ theory). Chapter 5 contains an investigation of the proximal stimuli of bingeing: restrained eaters (i.e. unsuccessful dieters who occasionally binge) and unrestrained eaters (normals) are compared with regard to their psychophysiological reactions to food cues. This study tries to identify relevant parameters of food cue reactivity, while testing the hypotheses that restrained eaters, because of their 
learning history show increased food cue reactivity. A related study is reported in chapter 6. Here it is hypothesized that fasting subjects will show larger psychophysiological reactivity to food cues than non-fasting subjects do. Once again, the principal aim is to identify sensitive parameters of psychophysiological cue reactivity, the fasting manipulation being an attempt to induce maximal food cue reactivity. Chapter 7 focuses on the cognitive level. Restrained and unrestrained eaters are exposed to an appetizer (i.e. the food cue), and subsequently compared with regard to their attentional bias for food words in the Stroop task. As restrained eaters are hypothesized to show disinhibitive eating after the appetizer, it is hypothesized that they will display larger attentional bias for food words then do unrestrained eaters. Also, the relationship between attentional bias and subsequent ice cream intake in an ad lib taste test is assessed. Finally, Chapter 8 serves a more methodological purpose. It describes the development of a new disinhibitive eating questionnaire which may be helpful in selecting non-clinical binge eaters, and in the development of new "lab-models" of binge eating (section 1.1.). 



\section{Chapter 3}

\section{CONDITIONED INSULIN AND BLOOD SUGAR RESPONSES IN HUMANS IN RELATION TO BINGE EATING ${ }^{1}$}

\section{INTRODUCTION}

An important feature of bulimia nervosa is recurrent binge eating. During binge eating, an amount of food is ingested that is definitely larger than most people would eat during a similar period of time under similar circumstances (APA, 1994). A typical characteristic of binge eating also included in the DSM IV binge definition is a sense that control over food intake is lacking (APA, 1994). Furthermore, bingers often report an irresistable food craving just before a binge (Abraham \& Beumont, 1982; Jansen, Van den Hout \& Griez, 1990; Mitchell, Hatsukami, Eckert \& Pyle, 1985).

Hence, binge eating can be said to bear resemblance to alcohol and drug addiction: both are characterized by the intake of large amounts of substances, craving and loss of control over intake. Addiction research of the last two decades has elucidated the role of conditioning in drug tolerance and craving (Siegel, 1983; Childress et al., 1992). Specific cues (CS) that signal drug intake (UCS) eventually become able to elicit physiological responses (CR) that compensate for the anticipated bodily effects of drug intake. Thus a behavioral tolerance to the drug develops, guided by cues like the injection ritual or the usual environment in which the drug is used. Additionally, the drug-signalling cues are supposed to trigger craving and drug seeking behavior. Recently, an analogous conditioning theory of binge eating was proposed (Jansen 1990; 1994a; Wardle, 1990). In the present article the conditioning model of binge eating (CBE model) is outlined and a first test of it reported.

The CBE model (Jansen, 1990; 1994a) is based on five assumptions: (1) each episode of (binge) eating disrupts the body's internal milieu; (2) to counter the disruption, physiological, homeostatic reactions occur; (3) binges are preceded by specific cues; (4) these cues can become elicitors of the homeostatic responses by a process of classical conditioning; (5) the conditioned reactions are subjectively experienced as craving for food.

1 Submitted for publication by J. Overduin \& A. Jansen. The authors gratefully acknowledge Mrs. Annie Raven for her assistance in carrying out the present experiment, Dr. Paul Menheere for his biotechnical advice, Mr. Ton Teerling (Quest International, Naarden) for supplying us with the benzaldehyde and maltol flavors, and the Holland Sweetener Company (Mastricht) for providing us with aspartame. Dr. Barry Dworkin (Penn State University) is acknowledged for commenting on some of our data. 
The first two assumptions imply that bingeing (as food intake in general) has disruptive effects on the internal milieu of the body (for an overview see Woods, 1991). The body tries to restore equilibrium by regulatory, homeostatic reactions. As an important example, a meal causes blood sugar levels to rise, and this prompts the pancreas to release the blood sugar lowering hormone insulin. The third assumption is that binges tend to be preceded by so-called binge cues like the sight, smell, and taste of typical binge food. One could also think of times of the day, particular moods, or the environment in which binges usually occur (cf. being home and feeling lonely in the afternoon). The basic notion is that the person has learned that these cues uniquely and reliably predict binges. Fourthly, the $\mathrm{CBE}$ model proposes that through classical conditioning binge cues can acquire the power to trigger responses like insulin secretion before any food intake has taken place. In conditioning terms, the cues come to act as a CS triggering an insulin secretion (CR) that leads to a drop in blood sugar level. The blood sugar fall is called both anticipative and compensatory because it occurs prior to food intake and compensates for expected physiological disturbances (i.e. post-prandial blood sugar rise). The last assumption in the CBE model is that the cue elicited responses like hyperinsulinemia and hypoglycemia are subjectively experienced as craving. Evidently, craving heightens the chances that a binge will take place. When a binge indeed occurs, the association between cues (CS) and binges (UCS) is strengthened. In this manner the individual can enter a vicious circle, in that the next encounter with binge cues will elicit larger CRs again leading to bingeing and so forth.

Data relevant to the last two assumptions of the CBE model, implying that homeostatic responses and craving can be conditioned to food predicting cues, can be found scattered throughout the literature. The sight, smell and taste of food are known to evoke secretion of insulin and other hormones known under the name cephalic phase responses (e.g. Le Blanc, 1992; Powley \& Berthoud, 1985). In some cases a hypoglycemia has been observed as well (Louis-Sylvestre, 1976). The intensity of cephalic phase responses are said to be correlated to the palatability of the food and the amount of subsequently ingested food (Louis-Sylvestre, 1984; Lucas, Bellisle \& Di Maio, 1987).

Explicit conditioning procedures have shown that insulin secretion hypoglycemia and meal initiation can become conditioned to arbitrary cues like odors, an injection procedure and the usual time of feeding. The UCS consisted of either food or a glucose solution (Deutsch, 1974; Mityushov, 1954; Strubbe, 1992; Woods \& Kulkosky, 1976; Woods et al., 1977; Weingarten, 1984). Finally, the CBE model has been clinically applied to form a therapy for binge eating. The procedure, involving exposure to binge cues with simultaneous prevention of bingeing, appears to be promising (Jansen, Broekmate \& Heymans, 1992).

The aim of the present study was to test the CBE model in normal healthy subjects. A conditioning procedure was run in which a peppermint odor/flavor CS was repeatedly 
paired with the ingestion of glucose solution. Responses were monitored on the physiological, behavioral and subjective level. The hypothesis under scrutiny was that after conditioning, presentation of the CS alone would trigger a) conditioned hypoglyce$\mathrm{mia} /$ hyperinsulinemia coinciding with b) increased subjectively experienced craving, leading subsequently to $\mathrm{c}$ ) increased ad lib intake of glucose drinks.

\section{METHOD}

\section{Design}

The experiment was run according to a 2 (condition: control vs. experimental) $\times 2$ (measurement: pre- vs. post-conditioning) design, and carried out in a double-blind fashion. In the experimental condition subjects were conditioned by sixfold pairing a peppermint smell/taste CS with a subsequent ingestion of a glucose dose (UCS). Glucose was chosen as the UCS, because its ingestion causes a sharp increase in blood sugar level, therewith provoking a strong insulin response (Rodin, 1990; Tse et al., 1983). In the control condition an aspartame solution was used as the UCS. Aspartame tastes sweet (like glucose) but is metabolically inert so that no blood sugar change occurs after ingestion (Rodin, 1990; Carlson \& Shah, 1989; Horwitz, McLane and Kobe, 1988). Two sessions were included to encourage the association of the UCS presentation only with the CS proper, and not with other stimuli related to the lab visit. To control for the non-associative effect of experience with the UCS, a random control procedure (Robbins \& Ehrman, 1992) was added: on each conditioning day all subjects received a take-away drink to be ingested at home. This drink contained aspartame for the experimental, and glucose for the control group. Due to this arrangement, at the time of the second test session (i.e. the last day of the experiment), subjects in both conditions had been exposed to same doses of aspartame and glucose drinks.

\section{Blood Parameters}

Blood sugar and insulin were monitored before and after CS presentation on test days in order to test the CBE hypotheses of conditioned hypoglycemia/hyperinsulinemia. Cpeptide was measured as a control on insulin secretion. C-Peptide and insulin are always secreted simultaneously in equimolar quantities, but $\mathrm{C}$-peptide circulates in the blood for a longer period than shorter-lived insulin. Thus C-peptide measurement could reveal rapid, otherwise unnoticed fluctuations in insulin (Polonsky \& Rubenstein, 1984). Finally, levels of the pancreatic hormone glucagon were measured. Glucagon increases the blood sugar level and is a powerful antagonist of insulin: its secretion is stimulated 
when levels of blood sugar become too low, and inhibited when, for example, sugar is ingested (Brodsky \& Devlin, 1994). The glucagon was measured mainly as a control hormone: heightened glucagon levels could provide an explanation if hyperinsulinemia but no simultaneous hypoglycemia should be found (a phenomenon not uncommon in insulin secretion to food stimuli (Bruce, Storlien, Furler \& Chisholm, 1987; Powley \& Berthoud, 1985). Glucagon was also monitored because it was speculated that conditioned hypoglycemia might not only be secondary to insulin secretion, but also to a conditioned inhibition of glucagon secretion.

\section{Subjects}

In a first step, 100 first- and second-year female university students who had responded to an advertisement in the university paper were given a selection questionnaire on personal health and taste preference. Eventually, 20 subjects (age $19.9 \pm 1.9$ years; BMI $20.1 \pm 1.2$ ) were selected using the following criteria: healthy state, no history of diabetes in the subject or her direct relatives, normal weight (i.e. a Body Mass Index between 18.5 and 23.5), current use of oral contraceptives (so as to blunt natural menstrual cyclic fluctuations of progesterone and appetite; Lissner et al., 1988), no history of bingeing, or dieting to lose weight (implying no eating disorders), and no extreme like or dislike of sweet substances and peppermint. It was announced that the research involved assessments of the impact of repeated tasting on sensitivity for subtle taste differences of sweet drinks, and that relevant blood parameters would be monitored. Allocation to either experimental $(\mathrm{N}=10)$ or control group $(\mathrm{N}=10)$ was done pseudo-randomly, taking care that the neither BMI nor age differed between groups. For each subject the procedure started between 6-12 days after the onset of the last menstruation, i.e. 1-7 days after contraceptive pill use was resumed. In this way, care was taken that the last day in the experiment would be at least 3 days before the onset of the next menstruation. At the end of the experiment each subject was paid the Dutch equivalent of US $\$ 300$.

\section{Materials}

Questionnaires Most questionnaires used Visual Analog Scales (VAS) with scores between 0 ("not at all") and 100 ("very much"). During the test sessions a 5-item VAS on the levels of hunger, thirst, tension, craving for salty as well as sweet food was administered 10 times (see table 3.1 for the time schedule of presentation). Further, the taste test questionnaire included ratings of the sensory qualities of the three test drinks. Some of these items were in fact "time fillers" (see procedure); only hedonic and sweetness ratings of the test drinks were considered as relevant data. The conditioning 
sessions each included two 5-item VASs identical to those described above, as well as questions on the sensory characteristics of the three UCS drinks and the pepper$\mathrm{mint} / \mathrm{mentholatum} \mathrm{CS}$. These questions were included solely to stimulate conscious identification of the CS and UCS; no further use was made of the data from these questionnaires.

Conditioned stimulus The conditioned stimulus was equal in both conditions and consisted of a compound of peppermint/mentholatum odor and flavor, presented in combination with a pre-ingestive ritual in an CS-room. The odor/flavor combination was used, as its "belongingness" with ingestion (UCS) was thought to accelerate the acquisition of the conditioned response (e.g. Schwartz \& Robbins, 1995; Woods, Makous \& Hutton, 1969). The peppermint-odor was dispersed by an electronic device in which peppermint/mentholatum oil was continuously evaporated. The flavor consisted of a solution of peppermint $(3 \mathrm{gr} / \mathrm{l})$ combined with mentholatum flavor $(1 \mathrm{gr} / \mathrm{l})$ in water. The subject would apply $2 \mathrm{ml}$ of this solution to the tongue resulting in the typically refreshing oral sensation of peppermint. The stereotyped acts the subject was instructed to perform before ingestion of the UCS (see procedure) completed the CS compound.

Unconditioned stimulus The UCS for the experimental condition was a solution of 50 grams of glucose (dextrose; Janssen Chimica, Tilburg) in $30 \mathrm{cl}$ of water. Fifty grams of oral glucose contain about $200 \mathrm{kcal}$ (Rodin, 1990) and generally lead to a blood sugar rise between 46 and $61 \mathrm{mg} / \mathrm{dl}$ with subsequent insulin levels between 60 and 110 micro Units/ml (Förster, Haslbeck \& Mehnert, 1972; Tse et al., 1983). In our control condition the UCS was an aspartame solution $(0.5$ grams dissolved in $30 \mathrm{cl}$ of water; Holland Sweetener Company, Maastricht). During a previous pilot study in other subjects, this aspartame solution had been rated as being equally sweet as the glucose UCS of the experimental condition. For both conditions a small amount $(3 \mathrm{ml} / \mathrm{l})$ of almond flavor (a $1 \%$ benzaldehyde-in-alcohol solution) was added for taste in the UCS solutions.

Test drinks The drinks presented during the taste test of the two test sessions were of equal composition in both conditions, namely a $150 \mathrm{~g} / \mathrm{l}$ glucose in water solution, again flavored with almond $(3 \mathrm{ml} / \mathrm{l})$. A total of 3 liters of the test drink was available to the subject, i.e., considerably more than the expected ad lib intake during the taste test.

Take-away drinks It was attempted to maximize the difference in appearance of the takeaway and lab drinks, so that CS perception would remain strictly limited to the lab situation. The quantity of sweeteners (glucose for the control group and aspartame for the experimental group) was the same as in the UCSs. Instead of almond, a "marshmallow" (maltol) flavor was added, and the drink was handed out in a dark-colored bottle (contents: $30 \mathrm{cl}$ ). 


\section{Chapter 3}

Urine jar This $25 \mathrm{cl}$ jar, made of transparent glass, was to be taken home for collection of the first morning urine, and brought back to the lab the next session as "a measure of metabolism of the take-away drinks". No further analysis was made of the urine samples (see procedure).

The drinking box The box from which all lab-beverages were drunk was made of whitecolored solid material. Its top contained three holes through which the straws (diameter $5 \mathrm{~mm}$; length $30 \mathrm{~cm}$ ) protruded. The straws did not require strong sucking to get the drink up from below. At conditioning sessions three cylinders, each filled with $10 \mathrm{cl}$ (i.e. one-third of the UCS), were placed in the box. However, for the ad lib taste tests during test sessions, three one liter jars filled with the test drinks were used. The subjects were not able to see the contents of the box or the color of the drinks.

The pipette The pipette, used for applying the peppermint flavor CS on the tongue, was an open glas laboratory tube (length $15 \mathrm{~cm}$ ) with a rubber balloon on top. The subject was to squeeze the balloon with the pipette tube below the fluid surface, and then let loose; thus the pipette would fill with approximately $2 \mathrm{ml}$ of the peppermint solution (CS), which was ingested by again squeezing the balloon while aiming at the tongue.

Biochemical analysis Blood glucose was measured in venous whole blood by an automated glucoseoxidase method performed on the EBIO analyzer (Eppendorf, Hamburg). Serum insulin and $C$-peptide were measured by radioimmunoassay after polyethyleneglycol (6000) pretreatment. For insulin materials were used provided by Pharmacia (Uppsala, Sweden), while the kit for C-peptide was manifactured by Byk-Sangtec (Dietzenbach, Germany). The interassay coefficients of variations were better than 8.4 \% for the insulin assay and better than $9.7 \%$ for the $C$-peptide assay at the concentration levels of interest in this article. Glucagon was measured in EDTA plasma by sequential radioimmunoassay using the glucagon double antibody kit from D.P.C. (Los Angeles, U.S.A.). Interassay coefficients of variations are better than $15 \%$ at the concentration level of $35 \mathrm{pmol} / \mathrm{l}$.

\section{Procedure}

A few days prior to the experiment an introductory meeting was held. The experimental procedure itself encompassed 10 daily sessions within a period of 12 days; between the fifth and sixth sessions there was a two-day weekend pause. There were two test sessions (day 1 and 10), two "no-conditioning" lab sessions to avoid association of UCS presentation and lab stimuli different from the proper CS (days 4 and 7) and 6 conditioning sessions (days 2,3,5,6,8 and 9). For each subject all sessions were planned at the same time of day. Blood parameters and ad lib glucose intake were measured only on test days. 
Introductory session This session was held to provide the subjects with preliminary information on the procedure and to get them acquainted with the lab, as well as handling the experimental attributes (pipette, straws, etc.). These materials were demonstrated in a room different from the CS room. Subjects were informed that on the first and the last days a longer procedure than normal would take place in which blood samples would be taken. The subject signed a statement in which she promised to refrain from ingesting peppermint or peppermint-related chewing gum, and sweetened liquor during the two weeks of the experiment. Furthermore, instructions were given to eat a meal of two sandwiches with one glass of drink three hours before each session, and to refrain from further eating and drinking until the session.

Conditioning sessions. CS+ (days 2,3.5.6.8 and 9) The subject was welcomed in a waiting room. It was pointed out to the subject that she was to enter a room in which a peppermint odor would be hanging in the air, and that a taste test involving sweet drinks would have to be carried out there. Upon entering the CS room the subject sat down at a table on which the drinking box and pipette were within reach. The experimenter was seated at an angle of 90 degrees within sight of the subject at a distance of 1.20 meters. Then the subject was instructed to apply the peppermint/mentholatum (CS) solution to her tongue. She was encouraged to taste the solution attentively and let it rinse through her mouth for one minute before actually swallowing it. During this minute the experimenter repeated the instruction to concentrate on the peppermint-taste, and added that soon the sweet beverage taste test would begin. It had been explained beforehand that the test would involve three slightly differently composed drinks, and that the subject had to detect the just noticeable differences between the drinks (in reality, each of the three straws was resting in $10 \mathrm{cl}$ of the same UCS solution). After the minute of tasting the peppermint flavor, the subject was instructed to swallow it, and there was a pause of 15 seconds. Then the experimenter told the subject to put the leftmost straw in to her mouth, but not yet start drinking. After 10 seconds the experimenter stood up, said "start drinking now; drink all three drinks from left to right in a steady tempo and concentrate on their taste differences", and left the room. In general the $30 \mathrm{cl}$ drink was finished in 2-3 minutes, after which the subject remained in the room for another 20 minutes. At regular intervals she was instructed by intercom to fill out a questionnaire (see materials). After 20 minutes the session was ended, the subject was given the take home drink, questionnaire and urine jar and then was allowed to leave. See table 3.1 for the time schedule of the conditioning sessions.

The "no-conditioning" sessions (day 4 and 7) During these sessions the subject was welcomed in the waiting room. It was announced that this time something different from usual would happen, i.e. that no taste test would be carried out. Next, the subject was 


\section{Chapter 3}

led to another room where a paper and pencil test was carried out of $2 \times 6$ minutes as "a check for concentration". After that the session was terminated.

Table 3.1. Schedule of conditioning and test trials

Conditioning trials

$\begin{array}{llllll} & |\operatorname{cs}| j \text { us } \mid & & & \\ -5 & 0 & 5 & 10 & 15 & 20 \text { minutes }\end{array}$

Test trials

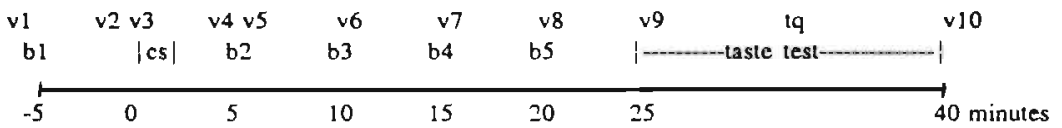

\footnotetext{
$\mathrm{cs}=$ conditioned stimulus (peppermint taste and odor)

us $=$ unconditioned stimulus (glucose or aspartame drinks)

$\mathrm{b}=$ blood sampling

$\mathrm{v}=$ visual analogue scale

tq $=$ tsste questionnaire
}

Home sessions The take-away drinks (see materials) were ingested at home between 7 and 10 p.m.. The instructions were to drink the beverage at a self chosen rate, on the condition that after the onset of drinking, the bottle should be emptied by the subject within 10 minutes. Subsequently, a questionnaire on which hedonic and sensory characteristics of the drinks were rated. No use was made of these data. The subject was instructed to collect and bring along a sample of morning urine at the next session for "analysis of the metabolism of the take-away drinks". In reality, the only purpose of this was to ensure that the subjects would drink the take-away drinks at home and no analyses were conducted.

Test-sessions (day 1 and 10 ) After a welcome the subject was seated in a nursing room where an indwelling catheter (venflon) was applied to the cubital vein of the left arm, a few centimeters distal of the elbow. Next, the subject was brought into the standard waiting room for adaptation and relaxation for a period of 30 minutes. Then the actual procedure began: the experimenter appeared, and explained that the forthcoming taste test would take place in the room where a peppermint odor would be hanging in the air. It was explained that after entering the room the taste test would begin after a pause the length of which might be $0,5,10,15$ or 20 minutes, depending on the outcome of a 
special "lottery". Then, the drawing of the first (baseline) blood samples was carried out, and experimenter and subject entered the CS room at about 4 minutes after the drawing of the first blood samples. After the subject was seated, the presentation of the CS proceded in exactly the same manner as during the conditioning sessions. However, after the subject had put the leftmosi straw in her mouth, the experimenter, instead of giving the "go" signal (as in the conditioning session), looked at his "lottery documents" and said: "no, you can't begin now, please leave the straw, fill out the next questionnaire, and wait". After the subject completed the questionnaire a second round of bloodsamples was taken (in reality the "lottery" was a sham procedure aimed at actively maintaining the subject's expectation of the UCS while blood samples were taken over a period of 20 minutes). The lottery was repeated four minutes later: the subject again had to put the left straw in her mouth and after 10 seconds she had to leave the straw, blood was taken. This action sequence was repeated 4 times. Matters were arranged in a way that blood samples were drawn at 5, 10,15 and 20 minutes after the presentation of the CS (according to Mityushov (1954; see also Woods \& Kulkosky, 1976) the hypoglycemia was expected to reach its nadir at $20-45$ minutes after CS presentation). At 24.5 minutes after entering in the $\mathrm{CS}$ room the subject again put the left straw in her mouth. This time the experimenter did indeed give the "go" signal, and told the subject to commence the taste test, and left the room. First one sip of each drink should be taken from the left to the right (this had been explained earlier). After that, the subject was allowed to drink as much as she wanted or needed to complete the questionnaire.

After 15 minutes the experimenter re-entered the room, and after removal of the venflon the subject was allowed to leave (first test session), or was taken to another room in which the subject was weighed, debrieved and thanked for participation (second test session). The amount of ingested glucose drink was weighed after the subject had left. See table 3.1 for the time schedule of these sessions.

\section{ANALYSIS OF DATA}

Statistical analyses were carried out using a SPSS-PC software program.

In a first step, an effect score was computed for each of the physiological parameters and the scores on all Visual Analog Scales of the two test sessions. For blood sugar, insulin and glucagon the effect score was defined as the difference between the mean level of samples 2-5 (i.e. the post-stimulus values) and the level of sample 1 (baseline). For C-peptide (which was analyzed only in the first and last blood samples) the difference between the post- and pre-stimulus sample was computed. 


\section{Chapter 3}

Similarly, effect scores for hunger and craving were defined as the difference between the mean of post-stimulus scores ( 8 scores) and the mean of baseline scores ( 2 scores). Further, for both the pre- and post-conditioning test session, the amount of ingested glucose test drink, and the mean sweetness and hedonical rating was computed.

Finally, all the computed physiological effect scores, quantities of drinks and subjective ratings were subjected to a 2 (experimental vs. control condition) $\times 2$ (pre/post conditioning) repeated measures ANOVA.

As one of the subjects (experimental condition) fainted during the blood sampling during both test sessions, her physiological ${ }^{2}$ and subjective data were exluded from further analysis. Furthermore, in one subject (control condition) the venflon jammed during the first test session, so that no blood data were acquired. Finally, one subject (control condition) showed a peculiar (exceptionally high and rapidly falling) baseline insulin level in the second test session. She was considered an outlier and her physiological data were excluded from further analysis.

\section{RESULTS}

Blood parameters (figures 3.1 to 3.4 ). For none of these parameters was the critical condition $x$ measurement interaction effect found. Furthermore, no significant main effect of condition or measurement appeared. A closer look at figure 3.2 shows that, after conditioning, the insulin response of the experimental group was slightly stronger than that of the control group. Exploratory t-tests revealed that the group difference was significant only at 5 minutes after CS presentation $(t(16)=2.52 ; p<.03$ ).

Subjective state during the test sessions Neither main nor interaction effects were found on hunger, thirst, tension and craving for either salty or sweet food. The craving-forsweet-food scores during the post-conditioning test session is depicted in figure 3.5.

Intake and subjective ratings of glucose test drinks For intake of the glucose test drinks, the critical measurement $x$ condition interaction effect was not found. However, a main effect was found for measurement $(F(1,16)=15.7 ; p=.001)$, reflecting increased intake of glucose after conditioning in both conditions (see figure 3.6). For hedonic rating a trend towards a condition $x$ measurement interaction effect was found $(F(1,16)$ $=3.36 ; \mathrm{p}<.09$ ) reflecting increased ratings after conditioning in the experimental condition (table 3.2). No effects were found on sweetness ratings of the test drinks.

${ }^{2}$ Analysis of the blood samples taken, however, revealed no abnormalities in both blood sugar and insulin levels. 


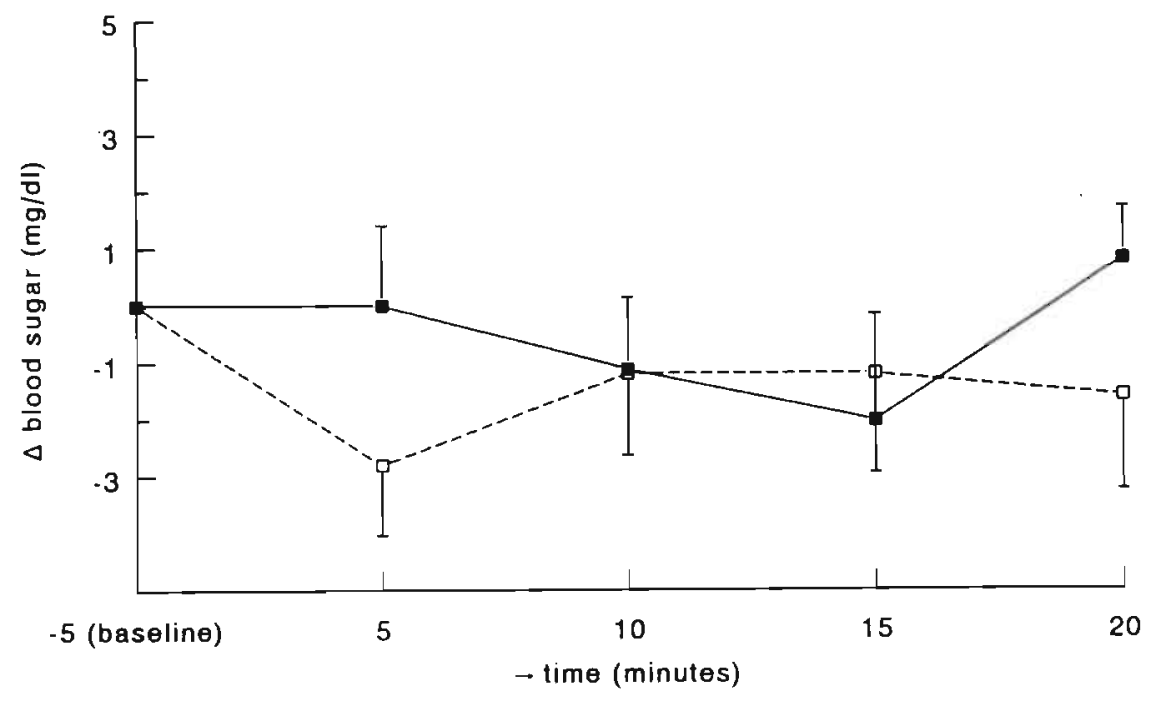

- - EXP. CONDITION --Q-- CONTR. CONDITION

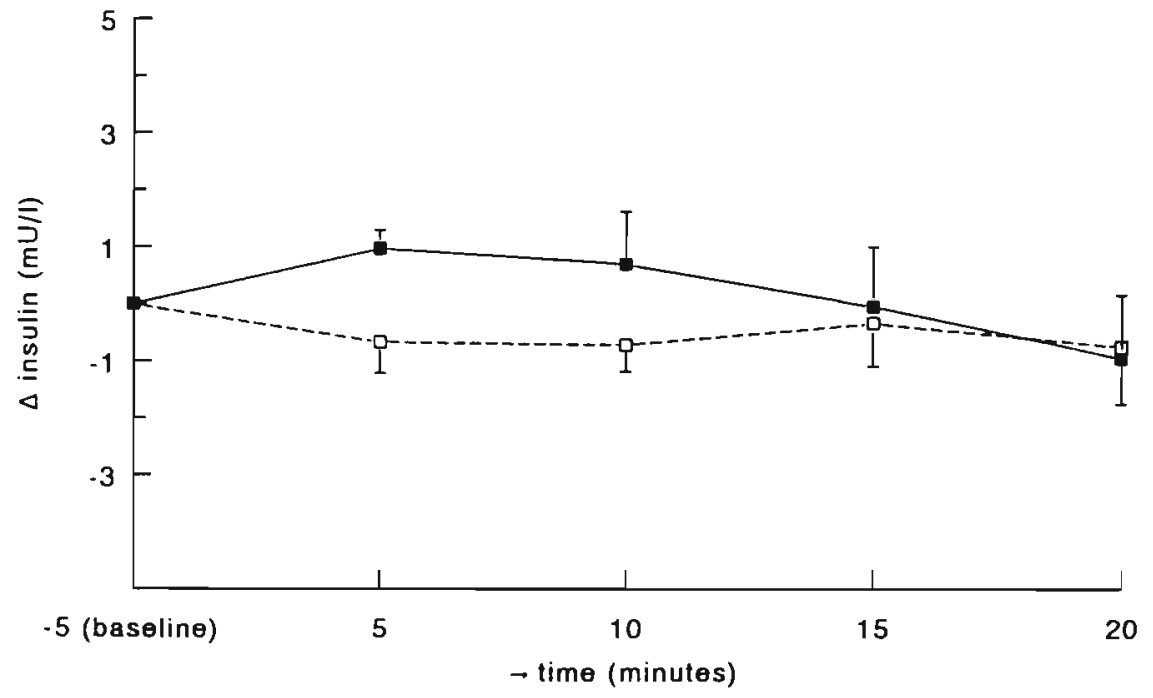

Figures 3.1. and 3.2. Blood sugar and insulin responses (mean \pm s.e.m) to CS presentation during the post-conditioning test session. The values represent deviation from baseline levels, which were $79.8 \pm 2.0 \mathrm{mg} / \mathrm{dl}$ for blood sugar and $9.4 \pm 1.2 \mathrm{mU} / \mathrm{l}$ for insulin. 


$$
\text { - - EXP. CONDITION } \quad--\square \text { CONTR. CONDITION }
$$

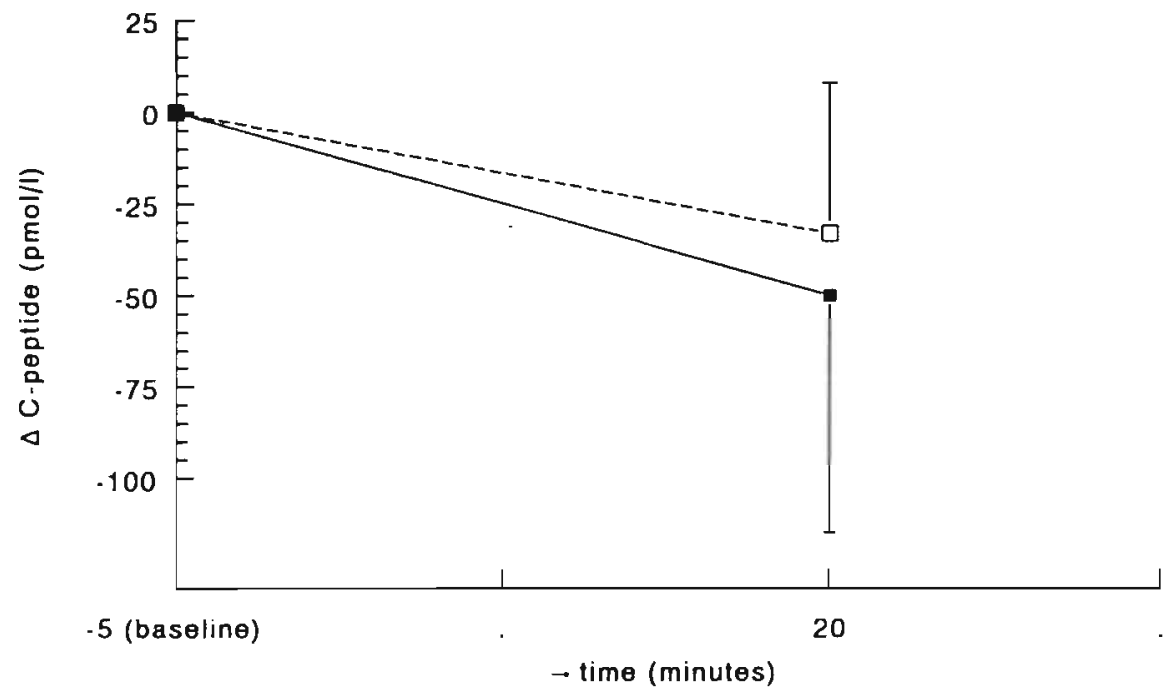

- - EXP. CONDITION --口-- CONTR. CONDITION

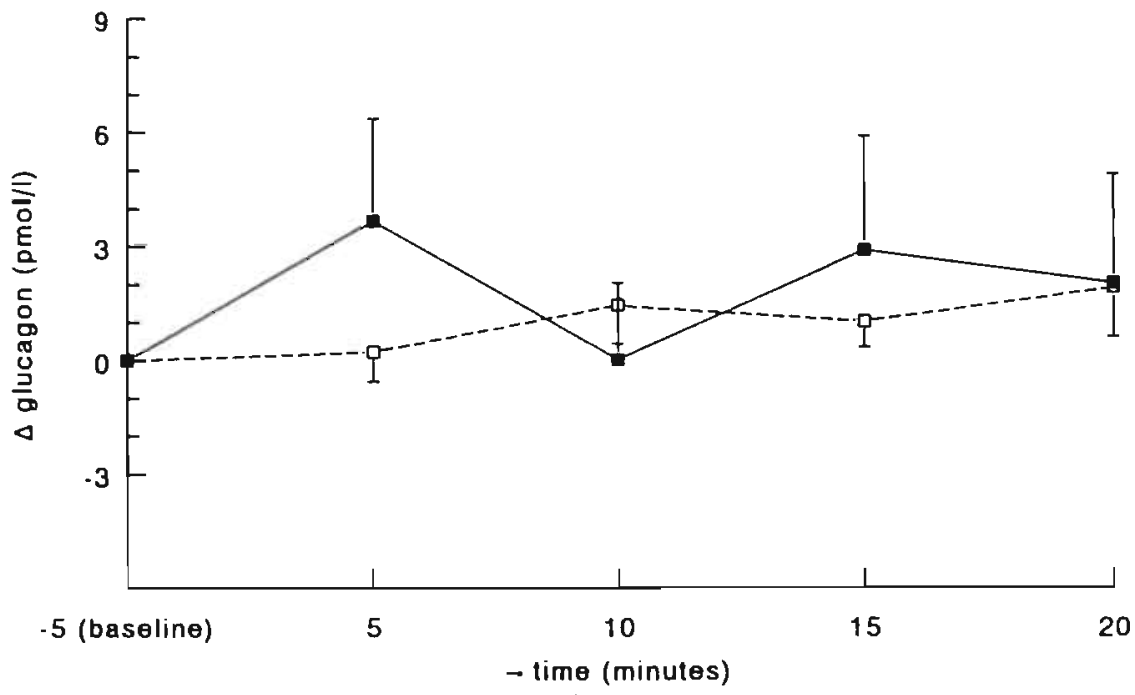

Figures 3.3. and 3.4. C-Peptide and glucagon responses (mean \pm s.e.m) to CS presentation during the post-conditioning test session. The values represent deviation from baseline levels, which were $830 \pm 90 \mathrm{pmol} / \mathrm{l}$ for C-Peptide, and 54.2 $\pm 2.2 \mathrm{pmod} / \mathrm{l}$ for glucagon. 


\section{Preliminary discussion and data from an added condition}

By and large, the CBE prediction of conditioned physiological responses in the experimental group was not confirmed. Nevertheless, some interesting results were found in the experimental condition: a larger insulin response in the experimental condition at 5 minutes after CS presentation, and a trend towards increased hedonic rating of the glucose drinks. However, the mean size of the insulin response in the experimental condition (approximately $1 \mathrm{mUnit} / \mathrm{l}$ ) was not impressive, given the fact that the range of spontaneously occurring fluctuations in insulin level tends to be larger than this (see e.g. Lucas, Bellisle \& de Maio, 1987). To investigate the possibility that the increased insulin secretion co-occurred with the trend towards increased hedonic ratings of the glucose test drinks, the correlation between the shifts in insulin secretion and hedonic rating was computed. This correlation turned out to be -0.32 (n.s.), suggesting a discordance between insulin secretion and hedonic value. Furthermore, a second series of ANOVAs was employed to account for possible inter-individual differences in the peak latency of the putative conditioned responses. In these analyses the baseline level was compared with the most extreme of the post-stimulus values in the direction of our hypotheses, i.e. maximum values for insulin level, and minimal values of blood sugar and glucagon levels post-stimulus and these values were subjected to the same $2 \times 2$ ANOVA as above. None of these analyses revealed significant effects. Further, the expected larger levels of craving and glucose intake in the experimental than in the control condition failed to be confirmed too.

Table 3.2. Hedonic and sweetness ratings (mean \pm sd) of test drinks before and after conditioning (range: $0-100$ )

\begin{tabular}{cccc}
$\begin{array}{c}\text { hedonic value } \\
\text { before } \\
\text { after }\end{array}$ & $34.2(23.5)$ & $31.5(24.4)$ & water condition \\
& $46.7(21.3)$ & $31.1(17.4)$ & $40.8(19.1)$ \\
$\begin{array}{c}\text { sweetness } \\
\text { before } \\
\text { after }\end{array}$ & $52.4(15.7)$ & $67.6(17.7)$ & $32.1(18.4)$ \\
\hline
\end{tabular}

The exact reason for the striking increase in glucose intake found at the post-conditioning test session in nearly all subjects remained unclear: it might be an effect related either to experience with sweet taste or acquaintance with laboratory procedure. In order to further elucidate this issue, a third condition was run: 9 subjects (BMI: 21.0, sd 1.4; 


\section{Chapter 3}

age: 23.1 , sd 2.9) were selected according to the same criteria as in the previous conditions. The procedure was equal to that in the other conditions except for the following: 1) in the conditioning sessions $30 \mathrm{cl}$ of plain water served as the "UCS"; 2) no blood samples were drawn 3) no random control procedure was carried out, i.e. no take away drinks were given.

Once again, it appeared that the glucose intake after conditioning was larger than before conditioning in all but one of the subjects (figure 3.6). On the average, glucose intake was $69 \%$ larger after than before conditioning (paired $t(8)=-3.36 ; p=.01$ ). After adding the intake data of this condition to those of the other two conditions, a 3 (condition) $\times 2$ (pre-vs post conditioning) repeated measurements ANOVA was run. This test did not reveal a condition $x$ measurement interaction effect, whereas the significant main effect of measurement $(F(1,24)=25.0 ; p<.001)$ reflected the increased intake in all three conditions. This result suggests that mere exposure to sweet drinks was not the critical factor in the increased glucose intake observed in the experimental (glucose UCS) and control (aspartame UCS) conditions.

\section{DISCUSSION}

The present procedure involved sixfold pairing of a peppermint flavor/fragrance CS with a UCS ingestion of glucose (experimental condition) or aspartame (control condition) drinks in human female subjects. In contrast with the hypothesis, during the postconditioning test session, the experimental subjects did not show significant conditioned blood sugar and glucagon decreases or $\mathrm{C}$-peptide increases relative to either the preconditioning session or the control subjects. Separate t-tests showed that the insulin response at 5 minutes after CS presentation was larger in the experimental than in the control condition. This difference was in the direction predicted by the CBE model, although the effect size did not exceed the range of spontaneous occurring baseline fluctuations. The prediction of especially increased craving for sweet substances in experimental subjects after CS presentation after conditioning was not confirmed. Mean hedonic rating of glucose drinks increased somewhat more in the experimental than in the control condition, but the condition $x$ measurement interaction did not reach significance. The most impressive effect, found in both conditions as well as a later added condition in which plain water was presented as the UCS, was that ad lib glucose intake during the post-conditioning test session was $78 \%$ larger than during the pre-conditioning test.

To start with the most impressive effect, the increase in ad lib glucose intake would fit 


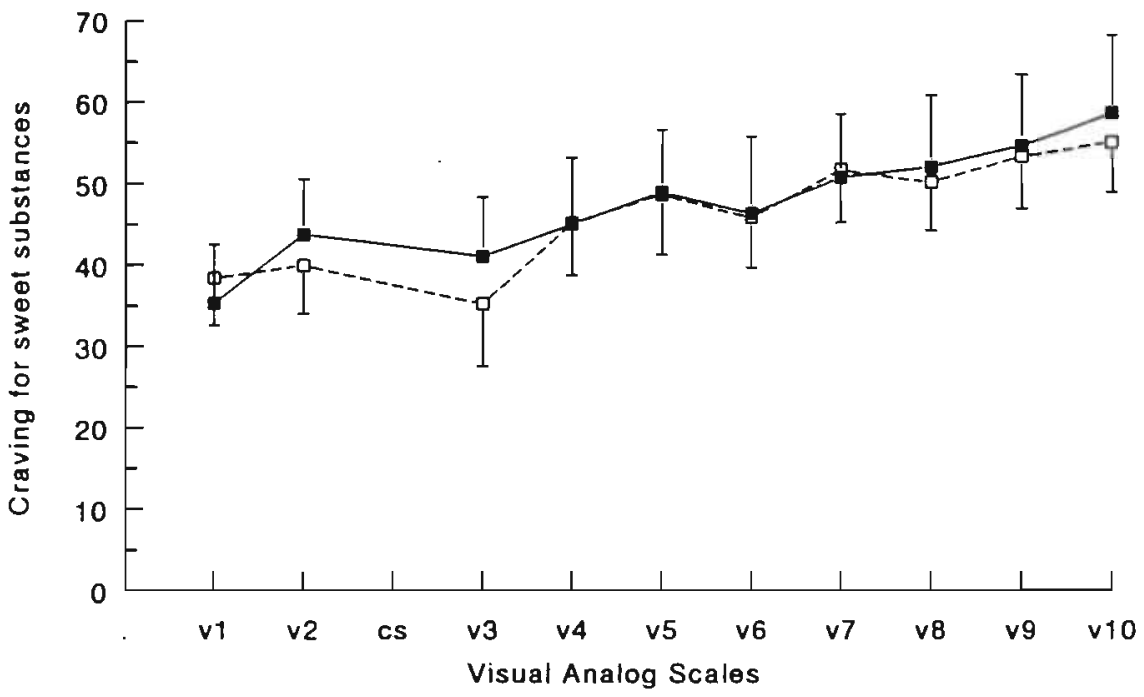

Figure 3.5. Mean \pm s.e.m. of subjective craving scores for sweet foods during the post-conditioning test session. CS presentation was between $v 2$ and $v 3$.

BEFORE CONDITIONING

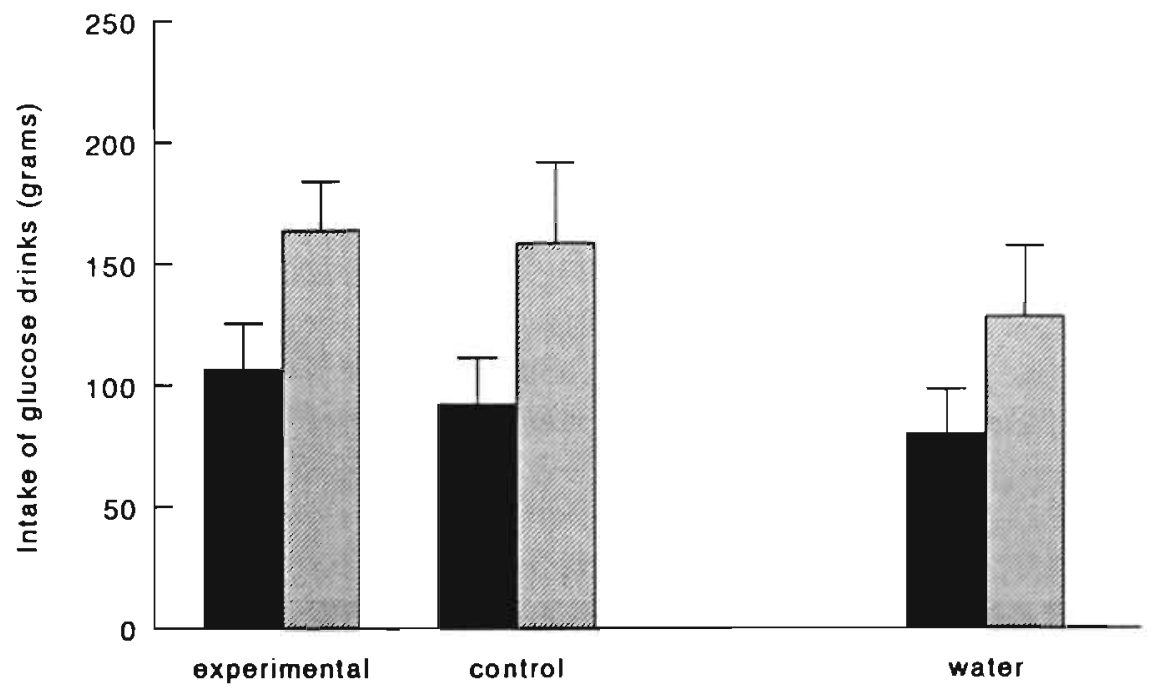

Figure 3.6. Mean \pm s.e.m. of grams of ingested glucose test drinks during the preand post-conditioning test sessions. 


\section{Chapter 3}

the predictions of the CBE model, were it not that the aspartame and plain water conditioned control subjects showed a comparable increase. It is therefore plausible that another behavioral mechanism was at work, for example "neophobia" or "bait shyness", a well-known phenomenon from animal research. Although, in general, neophobia refers to initial reluctance to ingest unfamiliar foods, it may also occur whenever animals are placed in a new environment. An initial decrease or inhibition of spontaneous food intake is shown which only gradually disappears; it can take days before intake has returned to the prior level (Barnett, 1958; Williams, 1968; Woods, 1991). Such a phenomenon may have occurred during our experiment: at the first test session (day 1) the subjects were still unadapted to the lab and procedure, and they might have been "reluctant" to drink. On the second test session ( 9 sessions later), however, the initial reluctance could have faded, thus clearing the way for the observed larger glucose intake. A further physiological explanation of the faded neophobia, i.e. by higher insulin levels (Woods, 1991) seems not warranted in the present case because of the weak insulinemic effects found. Admittedly, however, the possibility that exaggerated insulin secretion occurred after oral contact with the glucose cannot be ruled out (blood samples were taken only before but not during the glucose taste test).

As to the absent physiological and subjective effects of the present conditioning procedure, one might conclude that the CBE model has been refuted. However, the basic prediction we made was that once conditioned hypoglycemia had occurred, craving and increased intake of sweet drinks would follow. Unfortunately, since the first step, i.e., evoking conditioned glycemic and insulinemic changes, failed, the present data are inconclusive with regard to the hypothesized impact of (learned) hypoglycemia/hyperinsulinemia on craving.

The remainder of this discussion will focus on characteristics of the CS, UCS and the conditioning procedure in the present study, that may have underlain the present results and deserve consideration in future glycemic conditioning research.

First, it might be argued that our subjects' pre-experimental experience with the peppermint CSs like tooth paste, chewing gum, and peppermint, i.e. stimuli associated with only weak internal physiological effects ${ }^{3}$, might have slowed down their rate of conditioning through latent inhibition (Schwartz \& Robbins, 1995). However, in as far as latent inhibition acts by means of reduced attention to the presented stimuli (Miller et al., 1995; Schwartz \& Robbins, 1995), the inclusion of both the unique pre-ingestive ritual and explicit identification of the CS, UCS and their contingency will have

\footnotetext{
${ }^{3}$ Note that our subjects did not have an exceptional like of peppermint (see subject selection criteria); thus experience with larger peppermint intake was probably absent.
} 
diminished such an effect during our procedure. Moreover, it has been shown that in a successful instance of glycemic conditioning with large UCSs, latent inhibition by preexposure to the CS failed to thwart rapid acquisition of the conditioned response (Mityushov, 1954).

Second, one might wonder whether the UCS intensity (i.e. 50 grams of glucose) was sufficiently strong to lead to conditioning. When looking at the literature on the physiological effects of glucose, this suspicion seems unjustified. The blood sugar rise in response to 50 grams of glucose (i.e. between 46 and $61 \mathrm{mg} / \mathrm{dl}$; Förster et al., 1972; Tse et al., 1983) overwhelmingly larger than the usual "spontaneous" baseline fluctuations (being between 1 and $2 \mathrm{mg} / \mathrm{dl}$; Lucas, Bellisle \& Di Maio, 1987). Also, though glucose is a powerful blood sugar raiser, ingestion of doses above 50 grams would not have led to a large additional blood sugar rise (e.g. Förster et al., 1972). Still, previous successful instances of glycemic conditioning used an intravenous glucose UCS, involving larger blood sugar rises (up to $200 \mathrm{mg} / \mathrm{dl}$; Mityushov, 1954) and leading to more rapid acquisition of a significant $C R$ than when oral glucose UCS are used. Therefore, in the present study, it may be not so much the UCS, but its combination with a limited number of conditioning trials that has been responsible for the present failure to find conditioned hypoglycemia.

Finally, it might be that both extinction and blocking have occurred during the present procedure, and were relatively important, given the small number of conditioning trials. Extinction of the conditioned response could have occurred as a result of the random control procedure (ingesting the take-away drink) in the experimental group. Although care was taken to maximize its sensory difference with the lab-UCS drinks, the takeaway drink may still have been perceived as a "tin full of sweet drink" CS, and, as it contained metabolically inert aspartame, its ingestion may have extinguished conditioned responses already established during lab-sessions. A perhaps more important explanation of our finding of no conditioning may have been blocking (Schwartz \& Robbins, 1995): prior to the experiment our subjects (like anybody) may have had an already firmly established association between oral sweetness (CS) and subsequent blood sugar increases (UCS). Our conditioning procedure involved association of an odor/fragrance CS with oral glucose. Thus, during conditioning sessions two CSs may have been operating: the experimental, "intended" CS (CS1), and the sweet taste of the UCS glucose beverage itself (CS2). Hence, during the conditioning trials, relative to CS2, CS1 did not provide additional information about an impending blood sugar rise. Hence, it might be argued that the CSI alone would not able to trigger conditioned responses during the post-conditioning test session. Although we had good reason for not applying a glucose probe to the tongue as a CS on test days (oral glucose is known to elicit a 


\section{Chapter 3}

(mainly unconditioned) insulin secretion through glucose sensitive receptors located in the mouth and upper gastrointestinal tract (Grill, Berridge \& Ganster, 1984; Nicolaïdis 1977), it may be valuable to present a small sweet tasting stimulus (e.g. aspartame solution, Bruce et al., 1987) at the test session.

In conclusion, research remains to be done which elucidates the role of conditioned glycemic responses in craving for food. As a first step, procedures must be developed to which human subjects acquire significant conditioned glycemic and insulin responses. Then, using these procedures, while controlling for neophobic interference, one could assess the contribution of physiological learning to craving and binge eating. 


\section{Chapter 4}

\section{INTRODUCTION AND COMMENTARY TO: M.I. MITYUSHOV (1954), "CONDITIONED REFLEX SECRETION OF INSULIN"1}

As has been known for decades now, the physiological impact of Pavlovian conditioning extends beyond salivation, skin conductance and heartrate (Ádám, 1967; Dworkin, 1993; Razran, 1961). Current research on the role of conditioned responses in homeostatic regulation ${ }^{2}$ (e.g. Dworkin, 1993) has once again demonstrated the potential importance of the orginal studies on classical conditioning by the Eastem European Pavlovians, carried out several decades ago. The present article contains the translation of a Russian paper on blood sugar conditioning, which still appears to be highly relevant for today's conditioning research. The paper was first published in 1954 by the physiologist M.I. Mityushov. Before presenting the paper, we will describe its relevance for current research. The paper will be followed by a reanalysis and discussion of the presented data.

\section{INTRODUCTION}

At first glance, it seems as if today's Pavlovian conditioning research has become a science of stimuli rather than responses. For example, in a recent review of the predominant RescorlaWagner theory, the terms CS and US are used approximately 560 times, whereas the response terms CR or UR are altogether lacking (Miller et al., 1995) ${ }^{3}$. The frequent use of "CS" and "UCS" can be attributed to the description of conditioning principles pertaining to stimulus interactions, for instance, latent inhibition, blocking, and overshadowing. Meanwhile, the roles

1 This paper, together with the translation of Mityushov (1954; see Appendix of this thesis) will be submitted for publication by J. Overduin, B.R. Dworkin \& A. Jansen. The authors gratefully acknowledge Professor Gyōrgy Ádám, Eōtvōs University, Budapest, for his helpful comments on both our article and the translation of Mityushov's paper.

${ }^{2}$ Strikingly, the potential importance of conditioning for "The Wisdom of the Body" appears to have been overlooked by W.B. Cannon, the father of modern regulatory physiology, notwithstanding the fact that Pavlov and Cannon were acquainted and met each other on several occasions (sce Cannon, 1939; Dworkin, 1993).

${ }^{3}$ Garcia et al. (1985) already noted that in the classic paper by Rescorla (1967), the terms CS and US were mentioned 130 times, whereas "CR" was only used ten times, and "UR" not once. Hence, it appears that the situation has grown even more asymmetrical ever since. 


\section{Chapter 4}

of CR and UCR have received less attention. The few discussions about the nature of conditioned responses have focussed on the "survival value" for the organism. Nevertheless, the prevalent view is that nothing is gained by alluding to functionality of conditioned responses. For instance, it may often seem gratuitous to argue that an increase in skin conductance CRs serves the "purpose" of preventing the organism from injury to the skin (e.g. Furedy, 1989; Turkann, 1989). Overall, such skepticisms may be justified in the case where the CR measured is peripheral or multi-faceted (e.g., skin conductance fluctuations, gross skeletal activity; Dworkin, 1989; 1993).

However, in the domain of homeostatic regulation, reference to the adaptive value of conditioned responses may certainly be elucidating (Dworkin, 1989; 1993). Pavlov himself held this opinion. His earlier physiological work (1897/1910) has been fully open to the possibility of conditioned responses serving regulatory purposes ${ }^{4}$. After describing the digestive system, with its secretions so well-attuned to the chemical compostion of the ingestive, Pavlov goes on to say that also the composition of conditioned salivation and other secretions is in accordance with the composition of the signalled substance (UCS). For example, CSs signalling the presentation of dry meat powder (UCS) trigger a more abundant watery salivation than CSs predictive of moist real meat (Babkin, 1949; Pavlov, 1897/1910). Today, the regulatory view of classical conditioning is especially in vogue in the field of drug conditioning. According to the prevalent view, drug intake (UCS) poses a disturbance of the internal milieu, which prompts regulatory physiological responses (UCRs). If the drug intake is reliably signalled by cues like the injection ritual in a specific environment, these CSs acquire the power to trigger conditioned responses that attenuate the physiological disturbance caused by intake of the drug (Eikelboom \& Stewant, 1982; MacRae, Scoles \& Siegel, 1987; Siegel, 1989). In most cases, the CR appears earlier than, or simultaneously with the UCR. In this way, the CR may enhance physiological regulation by mitigating a physiological disturbance in its very early stages, or by strengthening the homeostatic UCR (see Dworkin, 1993). It will be proposed here that conditioning of blood sugar level can be understood by similar principles.

\section{Principles of blood sugar conditioning}

Before introducing contemporary blood sugar conditioning research, a brief overview of blood

${ }^{4}$ In his later years (from 1903 onwards), Pavlov began to regard salivation rather as a tool to measure the strength of conditioning. As appears from his writings, Pavlov less and less considered the chemical composition or physiological impact of his dogs" conditioned secretions as a research object to focus upon (Dworkin, 1993). 
sugar regulation is in place. Blood sugar (more precisely, blood glucose) is the main fuel of e.g. the brain and the heart. Not surprisingly, the level of blood sugar is tightly controlled by a multitude of the nervous and endocrine activities. Under normal circumstances, two organs, the pancreas and liver, play a predominant role in blood sugar regulation. The pancreas is able to secrete insulin, a powerful blood sugar lowering hormones. The trigger for insulin secretion can be a high level of blood sugar and/or activity in the parasympathetic vagus nerve which innervates the pancreas (Niijima, 1989; Strubbe \& Steffens, 1993). The liver is able to lower or heighten blood sugar level, depending on circumstances. In the former case, glucose is withdrawn from the blood and stored as glycogen. In the latter, e.g. glycogen is converted to glucose and released into the blood stream. The hepatic blood sugar regulation is under hormonal and nervous control: catecholamines, glucagon and sympathetic nervous activity increase glucose production, whereas insulin and parasympathetic nervous activity decreases the glucose output from the liver (Brodsky \& Devlin, 1994). Interestingly, the autonomic nervous influences on pancreas and liver tend to be coordinated and synergistic. The source of these coordinated actions apparently lies within the hypothalamus (Niijima, 1989). In general, blood sugar regulation, supported by many more physiological factors than described here, acts so as to limit fluctuations in blood sugar during normal state, mobilize blood sugar in case of need (e.g., during stress or vigorous exercise), and store sugars in case of abundance (e.g. after a meal).

Conditioning of blood sugar level appears to be possible, and has systematically been studied since the 1960s (for reviews see Dworkin, 1993; Woods \& Kulkosky, 1976). The studies were all carried out in rats, given repeated injections with insulin (the UCS) in the course of days or weeks. Generally, the conditioned stimuli used in these studies have been complex, consisting of a stereotyped injection ritual within a salient environment. From a simple homeostatic viewpoint, the expected result of these procedures would be a hyperglycemic CR, i.e., a conditioned blood sugar rise that counters the hypoglycemia caused by the insulin UCS. However, the results found within this line of research were more subtle. Presentation of the CS (i.e. a saline injection in the conditioning environment) led to hypoglycemic CRs in some studies, and hyperglycemic CRs in others. These results have been explained in two ways.

${ }^{5}$ Although somewhat beyond the focus of the present article, glucagon deserves mentioning as the most powerful blood sugar raising hormone. Like insulin, glucagon is a pancreatic hormone, and its rate of secretion is inversely proportional to that of insulin. Because of the major impact of these hormones, the insulin/glucagon ratio has been used as an index of physiological state in organisms (Brodsky \& Devlin, 1994). 


\section{Chapter 4}

First, the insulin dose used as UCS has not been equally large in different studies. In several studies, the UCS was a huge insulin dose (leading to blood insulin levels that were 200 times larger than baseline level; N.B. under normal circumstances, after a meal, insulin levels rise to a peak of between 2 to 20 times baseline, see e.g. Guyton, 1991). In these cases, the insulin was thought to stimulate certain brain receptors involved in a positive feedback loop leading to additional insulin secretion (UCR) ${ }^{6}$. The unconditioned insulin reflex eventually re-emerged as the conditioned response which in its turn was responsable for the observed hypoglycemia. In contrast, studies in which smaller insulin doses were used as the UCS, have found a conditioned hyperglycemia (e.g. Siegel, 1972; Woods \& Shogren, 1972). These studies may have more relevance for blood sugar regulation under normal physiological condition. The physiological disturbance, caused by these smaller insulin UCS was a moderate fall in blood sugar level, which prompts compensatory UCRs (e.g. glucagon and catecholamine response, inhibition of insulin secretion) in order to restore baseline levels of blood sugar. After conditioning, these UCRs apparently were copied in CRs which could be elicted by the CS. Consequently, CS presentation lead to a hyperglycemic response (see Dworkin, 1993).

A second explanation, put forward to account for the findings of both conditioned hyper- and hypoglycemia, has been that the procedures differed in the amount of stress imposed on the rats. Notably, handling, drawing blood, and using unfamiliar cages to run the conditioning trials might impose stress upon the animals. Stress may increase blood sugar (by e.g. catecholamine, corticosteroid and glucagon release), thus overshadowing possible hypoglycemic CRs. Indeed, it has appeared that conditioned hyperglycemia appeared if the conditioning environment differed strongly from the normal home cage. Conditioning in a cage resembling the home cage led to conditioned hypoglycemia (see Dworkin, 1993; Flaherty, Grigson \& Brady, 1987).

Although the above studies are certainly informative about the role of conditioning in blood sugar regulation, insulin injections do not strike as naturally occurring physiological UCSs. It might be that using UCSs containing glucose would be a more natural stimulus: after food intake the blood glucose level generally shows a distinctive rise, to which ample homeostatic reactions are emitted (Guyton, 1991). Therefore, it is puzzling that glucose has been seldomly used as the UCS.

We are aware of only four reports on conditioning procedures with pure glucose as the UCS.

${ }^{6}$ For further explanations of the physiological mechanisms probably involved, see Woods (1983) and Dworkin (1993). 
Deutsch (1974) who used rats, found. a $3.5 \%$ hypoglycemia to a caffein flavour CS, after repeatedly pairing of a flavour with a glucose dose (UCS) which was intubated into the stomach. Deutsch claimed that the observed conditioned hypoglycemia had been the result of a conditioned insulin secretion. Matysiak and Green (1984) camied out three experiments in which an injection ritual (CS) was repeatedly paired with intravenous glucose UCS in rats. Post conditioning testing in all three experiments showed a conditioned blood sugar decrease (CR) of approximately $3 \%$ in response to a saline injections; a control group of rats that had previously received saline UCSs showed a 4-6\% hyperglycemia. These data were somewhat problematic however, because baseline levels were measured on a day different from the test day. Woods (1976), after conditioning two groups of rats with either glucose or saline injections as UCS, did find an insignificant hyperglycemia on the test trial (injecting saline), but there were no differences between glucose and saline-conditioned rats. It is possible that the hyperglycemia resulted from stress. Woods attributed the lacking hypoglycemia to the glucose UCS dose not being large enough. Overduin and Jansen (submitted) used $50 \mathrm{gr}$ of oral glucose as a UCS in humans, and peppermint taste/odor as CS in humans, but found no significant hypoglycemia. This was probably explained by the limited number of six conditioning trials and the - comparatively - small physiological impact of oral dose of glucose which was used.

Taken together, procedures using glucose as the UCS have produced either hypo- or euglycemic, but not hyperglycemic CRs. Although none of these results contradict a homeostatic, i.e., regulatory explanation, replications of the conditioned hypoglycemic effect surely would add to make this explanation more convincing. In this respect, the study by Mityushov has an interesting contribution to make.

\section{The article}

Although M.I. Mityushov's article, of which a translation will follow next, was entitled Conditioned Reflex Secretion of Insulin, it only reported direct measurements of blood sugar. The article was first published in 1954 in the Russian Journal Zhurnal Visshey Nervnoi Deyatel'nosti, which can be translated as "Journal of Higher Nervous Activity". The Zhumal was the main Eastern-European journal of the Pavlovians and, in the 1950s and 1960s, excerpts from it were published in German and English as well. For reasons unknown, Mityushov's paper never appeared in translation.

Several features of Mityushov's experiment contribute to its relevance for contemporary research. First, a large glucose injection was used as a UCS and an (albeit indirect) attempt 
Chapter 4

was made to assess the influence of insulin in the conditioned hypoglycemia. Second, humans and dogs, rather than rodents served as subjects. Third, relatively many conditioning trials were conducted. Last, but not least, although Mityushov's article does not contain rigorous statistical analyses, it appears that unequivocal and interesting results were found.

TRANSLATION OF MITYUSHOV'S ARTICLE (SEE APPENDIX)

\section{COMMENTARY}

\section{Abstract}

In a conditioning procedure, Mityushov repeatedly administered intravenous glucose (UCS) to four dogs and nine humans. The injection procedure was carried out in a specific room served as the CS. At the post conditioning test session, a saline injection (CS) was given at $t=0$. The blood sugar response (CR) was measured by repeated blood sampling at $t=-10,-5,5,15$ and 45 minutes for humans, and at $-10,-5,5,15,30,60$ and 90 minutes for dogs. Explicit data were presented for several responses of 4 humans and 2 dogs. These subjects had been subjected to between 5 and 52 conditioning trials. In all cases, the saline injection elicited a conditioned blood sugar fall of between 5 and $20 \%$ below baseline. The nadir of the response occurred at 15 minutes after the saline injection. As the CR was measured repeatedly within subjects, it could be seen that $C R$ intensity tended to increase as a function of the number of previous conditioning trials. Although no direct insulin measurements could be made at the time, Mityushov collected evidence that the hypoglycemia observed in humans and dogs, was secondary to a conditioned secretion of insulin. Mice that were injected with blood that was drawn from dogs during their conditioned hypoglycemia, showed a subsequent fall in their blood sugar level. Mityushov claimed that he observed the conditioned hypoglycemic response in all of his human and canine subjects.

\section{Additional analyses}

Mityushov's article contains neither graphs nor statistical tests of the blood sugar data. Nevertheless, it was possible to further analyze the data depicted in tables A.1 to A.3 of Mityushov's article (see appendix). For this purpose, data for dogs and humans were treated separately. Only data were considered of the "correct" test trials, i.e., those taking place in the 


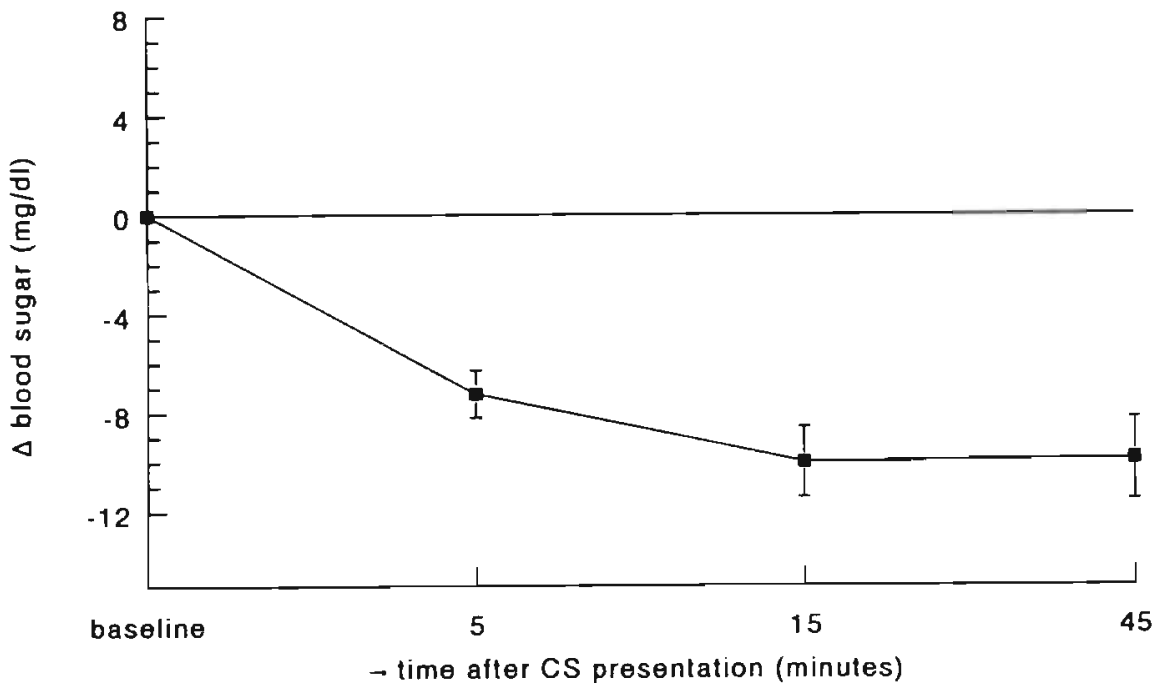

Figure 4.1. Post-conditioning blood sugar responses (mean \pm s.e.m.) to saline injections in 4 humans, based on a total of 17 responses. Responses expressed as deviation from baseline.
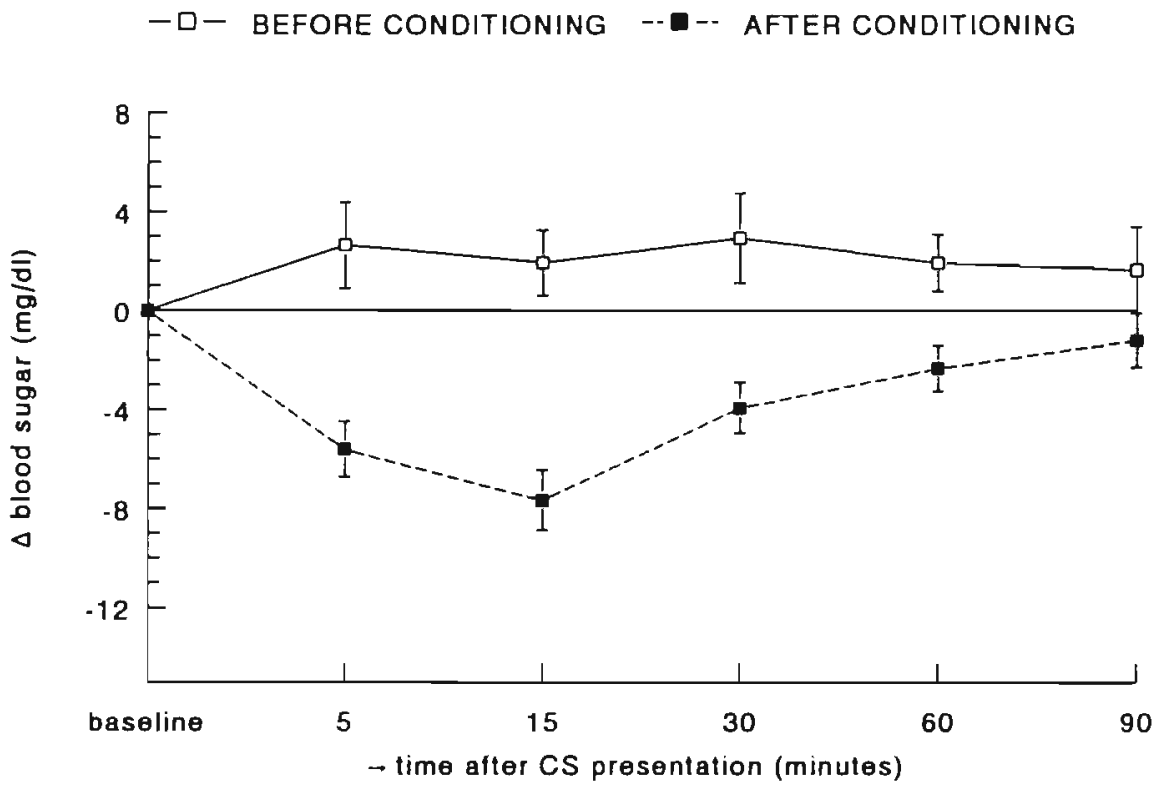

Figure 4.2. Pre- and post-conditioning blood sugar responses (mean \pm s.e.m.) to saline injections in 2 dogs, based on a total of 7 responses before, and 15 after conditioning. Responses expressed as deviation from baseline. 


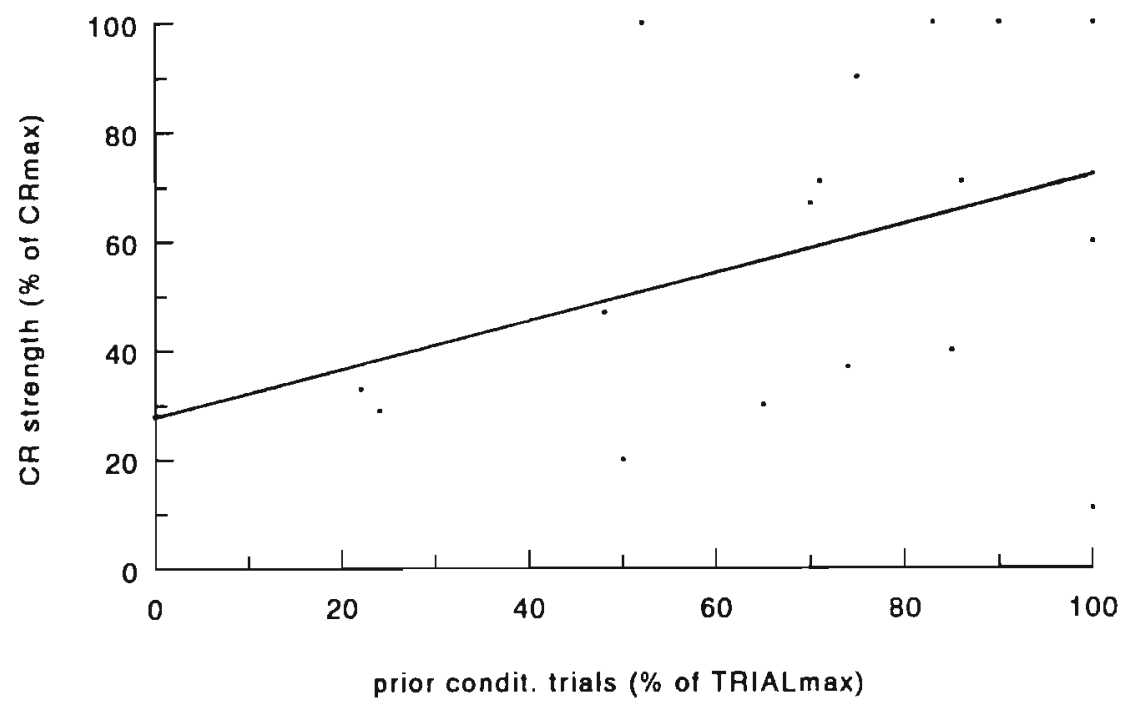

Figure 4.3. Acquisition curve of conditioned hypoglycemia in humans. For each subject, CR strength has been expressed as the percentage of the maximum CR intensity shown throughout the experiment.

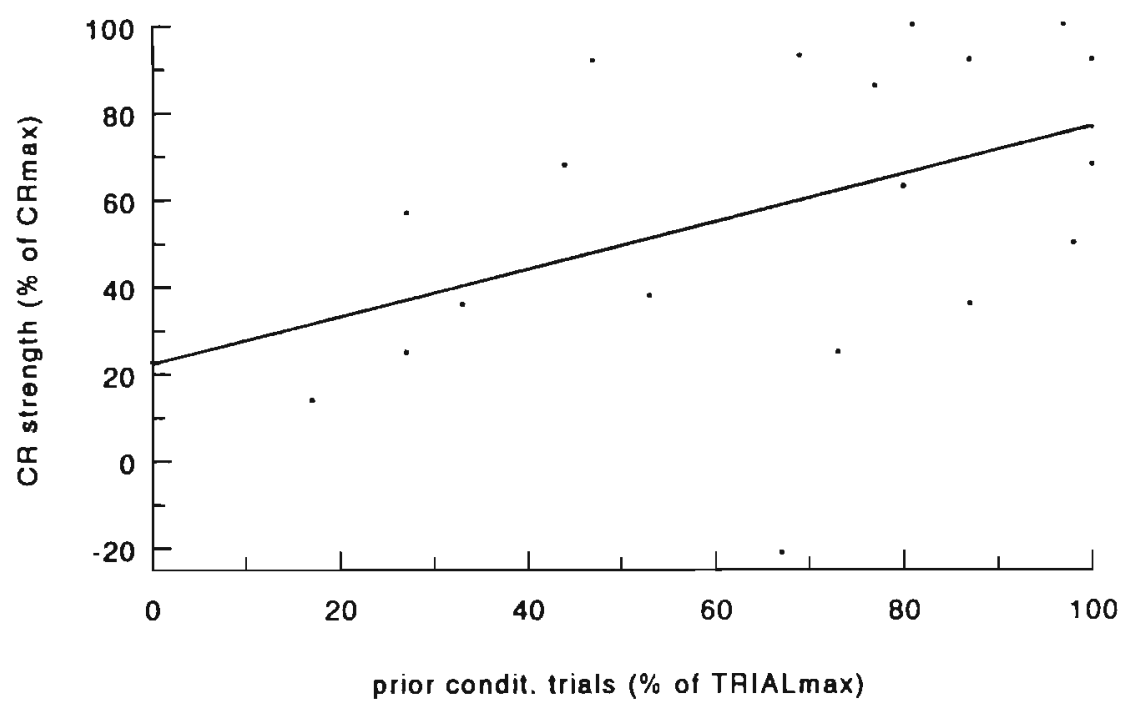

Figure 4.4. Acquisition curve of conditioned hypoglycemia in dogs. See figure 4.3. for further explanation. 
conditioning chamber with the usual experimenter.

Means Figures 4.1 and 4.2, depicting the glycemic responses to saline injections for humansand dogs respectively, were construed as follows. First, baseline level was computed as the mean of the two pre-injection baseline values. Then, the blood sugar values after each saline injection were expressed as the deviation from baseline. Finally, all responses of all subjects were taken together, mean and s.e.m. were computed and entered into the figures. For dogs, separate graphs were drawn for responses before and after conditioning.

The differences between baseline and post injection blood sugar levels were tested separately within three data subsets: for canine pre- and post-conditioning, and human post-conditioning data respectively. For each data set, a paired t-test was done comparing the mean baseline level with the mean blood sugar level after saline injection. For dogs' responses prior to conditioning (9 responses), no difference was found $(\mathrm{t}(8)=.17$; ns). After conditioning, for dogs $(\mathrm{t}(18)=$ 5.35; $\mathrm{p}<0.001 ; 19$ responses), and humans ( $\mathrm{t}(18)=7.83 ; \mathrm{p}<0.001 ; 19$ responses) highly significant differences were found, reflecting a hypoglycemia with its nadir at 15 minutes after the saline injection. Additional t-tests, comparing baseline with the individual post-injection blood sugar levels, revealed that after conditioning, all blood sugar levels measured within 45 minutes after saline injection, were significantly below baseline (table 4.1 ).

Table 4.1. Tests for differences between blood sugar levels before and after saline injection.

\begin{tabular}{cccc}
\hline & Before conditioning & \multicolumn{2}{c}{ After conditioning } \\
& dogs & $\operatorname{dogs}$ & humans \\
minutes & & & \\
$\mathbf{5}$ & $\mathrm{ns}$ & $<.001$ & $<.001$ \\
$\mathbf{1 5}$ & .05 & $<.001$ & $<.001$ \\
$\mathbf{3 0}$ & $<.05$ & $<.003$ & $<.001$ \\
$\mathbf{4 5}$ & & & \\
$\mathbf{6 0}$ & $\mathrm{ns}$ & $\mathrm{ns}$ & \\
$\mathbf{9 0}$ & $\mathrm{ns}$ & $\mathrm{ns}$ & \\
\hline
\end{tabular}

To summarize, both canine and human subjects consistently showed conditioned hypoglycemic responses to the saline injection. Prior to conditioning, saline responses lead to significantly elevated blood sugar level at 15 and 30 minutes for dogs (no human data were reported for these pre-conditioning trials).

Acquisition curves of the conditioned hypoglycemia Because the blood sugar response to saline 


\section{Chapter 4}

injections was assessed after different numbers of previous conditioning trials within subjects, an acquisition curve of the hypoglycemic response could be construed for humans and dogs (figures 4.3 and 4.4). Given the individual differences in the size of the largest $C R$ and the number of conditioning trials that were nn, the following method was adopted ${ }^{7}$. For each individual, the most intense hypoglycemic CR of all test trials (CRmax) was determined. Also the maximum number of conditioning trials for the given indiviudal was computed as Trialmax. Then, for each test trial, two values were computed: $x$, the number of conditioning trials run before the trial in question expressed as a percentage of Trialmax, and $y$, the strength of the CR expressed as a percentage of CRmax. After that, the values $(x, y)$ for all reported $C R s$ in the experiment were entered in a scatter plot $(x, y)$. Finally, a provisional leaming curve was constructed by drawing a linear regression line through the scatter plot $^{8}$. As can be seen from the positive slope of the learning curve, CR-strength tended to increase as more conditioning trials were run. The correlation between $x$ and $y$ were significantly different from zero: 0.43 for canine, and 0.35 for human subjects.

Habituation of the hyperglycemic response to repeated saline injections in dogs before conditioning As shown in figure 4.5, saline injections before conditioning evoked a slight hyperglycemic response in dogs. To investigate whether after repeated injections a waning of the hyperglycemic response did appear, the $(x, y)$ plot was made of glycemic responses at 15 minutes after injection, in the same way as was done above (see figures 4.3 and 4.4). As above, the glycemic responses were expressed as a percentage of CRmax for the given dog (i.e. his largest hypoglycemic response occurring after subsequent conditioning; see above). Also, a linear regression line was drawn through the plot. Given the positive slope of the regression line it appears that the hyperglycemic response tended to decline as more saline injections had been received by the dog. The correlation between number of previous saline trials and the strength of the hyperglycemia was -0.32 . Evidently, the hyperglycemic response did not disappear completely after many saline injections. As Mityushov observed, the hyperglycemia to saline may have been caused by stress related to the injection procedure. Modern research has confirmed that stress may increase blood sugar level e.g. by catecholamine and glucagon release as well as an inhibition of insulin secretion (Brodsky \& Devlin, 1994; Dworkin, 1993).

\footnotetext{
${ }^{7}$ Only blood sugar data at 15 minutes after saline injections were used.

${ }^{8}$ Because of the limited number of datapoints, no higher order curve fitting was attempted.
} 


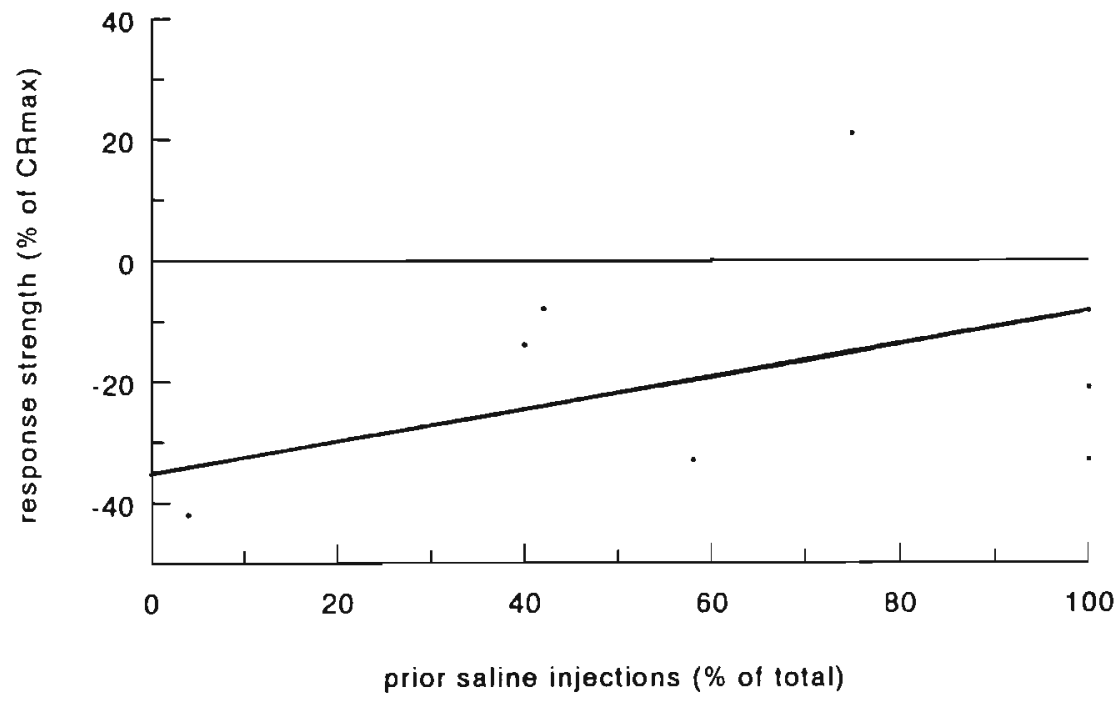

Figure 4.5. Habituation of the hyperglycemic response to repeated saline injections in 2 dogs, based on 7 responses. For each subject, response strength was expressed as percentage of the maximum $\mathrm{CR}$ shown throughout the experiment.

\section{DISCUSSION}

To our knowledge, Mityushov's study is the first successful demonstration of blood sugar conditioning in humans using a glucose UCS. The results are in line with other studies in which a glucose UCS was used (Deutsch, 1974; Matysiak \& Green, 1984). Furthermore, the data from this study are in line with a homeostatic explanation: a CS which has come to predict a blood sugar rise provokes a compensatory, hypoglycemic CR (see Dworkin, 1993).

Before discussing the results further, three problems of Mityushov's study should be mentioned. First, although Mityushov claims that he found a conditioned hypoglycemia in all subjects, he has only presented data for a small subset of his subjects. Unfortunately, overall means or statistical tests are lacking. A second problem is the lack of control group receiving e.g. repeated saline UCS injections. Of course, it can be said that this problem is mitigated because the blood sugar response to saline was measured also before conditioning began. In other words, the subjects served as their own controls. However, although Mityushov claims 


\section{Chapter 4}

that all subjects responded alike during these "baseline trials", his reporting only part of the data would not meet today's methodological standards. A third problem of Mityushov's procedure concerns the unnaturally high blood sugar levels $(250 \mathrm{mg} / \mathrm{dl}$ for humans; $420 \mathrm{mg} / \mathrm{dl}$ for dogs) provoked by the injected glucose UCS. One might wonder if the present results should be extrapolated to normal circumstances in which blood sugar levels do not exceed 190 $\mathrm{mg} / \mathrm{dl}$ (Guyton, 1991). However, observations in the literature suggest no discontinuities in blood sugar regulation under extreme hyperglycemia. Firstly, blood sugar levels of approximately $300 \mathrm{mg} / \mathrm{dl}$ after intravenous glucose injections in humans have been found to retum to baseline in slightly over one hour ${ }^{9}$, and this has been found to be related with increased insulin secretion and a suppression of glucagon release ${ }^{10}$ (Blouin et al., 1993). During sustained hyperglycemia in humans $(300 \mathrm{mg} / \mathrm{dl})$, insulin secretion rate remains at a high rate for hours (Polonsky et al., 1988). The rate of insulin secretion in dogs' and rats' pancreas is linearly correlated to at least blood sugar levels up to $300 \mathrm{mg} / \mathrm{dl}$ in dogs (Girardier et al., 1976), and $500 \mathrm{mg} / \mathrm{dl}$ in rats (Gerich, Charles \& Grodsky, 1976). Furthermore, blood sugar storage in the liver (through glycogen synthesis) increases linearly with blood sugar level, up to levels of $450 \mathrm{mg} / \mathrm{dl}$ in rats (Niijima, 1989). One might derive from these observations that the results found by Mityushov could be extrapolated to less extreme physiological conditions as well".

When looking at the data presented by Mityushov, it appears that his conditioning procedure has been effective in producing significant hypoglycemic conditioned responses after saline injection. As mentioned earlier, these results fit well with a homeostatic explanation. The CS (saline injection) that had come to signal a blood sugar increase (UCS), evoked CRs aimed at lowering blood sugar. That is, the CRs could be understood as adding to the nullification of the expected homeostatic disturbance. Which physiological pathways might then be involved? Mityushov suggests that insulin secretion was basic to the observed conditioned hypoglycemia (hence the title of his paper). This is a plausible explanation given the finding of a blood sugar fall in mice after an injection with blood drawn from the hypoglycemic dogs: some blood factor

${ }^{9}$ Mityushov reported the same blood sugar response in his subjects after the glucose UCS injection.

10 Like insulin, glucagon secretion is under the influence of autonomic nervous activity (Brodsky \& Devlin, 1994; Niijima, 1989).

11 This is of course in contrast with the conditioning studies using large insulin injections as the UCS (see the introduction of this article). 
may have been responsible, and insulin is one of the very few blood sugar lowering factors. Additionally, there is by now substantial evidence that the endocrine pancreas is capable of secreting insulin in response to naturally occurring food signals, like the smell, taste and sight of food (the cephalic responses, Bruce et al., 1987; Powley \& Berthoud, 1985) and learned signals predicting meals (Strubbe, 1992; Woods et al., 1977). These responses have been found to be mediated by parasympathetic nervous activity (Strubbe, 1992; Strubbe \& Steffens, 1993). What Mityushov probably did, is to bring insulin secretion under control of the injection ritual. A second mechanism underlying the hypoglycemia observed by Mityushov, might be a nervously regulated inhibition of glucagon secretion. Thirdly, the possibility exists that the saline injection (CS) induced a conditioned inhibition of the glucose production in the liver. This might have occurred through activity in the hepatic branch of the parasympathetic vagus nerve (Niijima, 1989). Whether this pathway is indeed susceptible to conditioning remains to be established.

Two alternative explanations of the observed hypoglycemia should be mentioned. First, the saline injection might have diluted the blood, and consequently blood sugar level would appear to have fallen (this problem appears in research in rats, see, for example, Deutsch, 1974). A second, alternative explanation might be that the hypoglycemia resulted from a temporary depletion of blood sugar, caused by a transient increase in utilization due to injection-related stress. However, both explanations are unlikely, because, as can be seen in figure 4.2 , saline control injections given to dogs before conditioning, in all cases led to slight hyperglycemias. Besides its physiological implications, the paper by Mityushov bears upon procedural issues in glycemic conditioning. Mityushov's finding of rapid blood sugar and insulin conditioning in humans (after 5 trials) is highly remarkable because of the lacking natural belongingness of an injection-CS to blood sugar level ${ }^{12}$. Moreover, no odors were added to the CS-compound, although odors and blood sugar level fluctuations can be said to have natural "belongingness" (consider food ingestion), and Woods et al. (1969) discovered that glycemic conditioning was considerably speeded up in rats if odors formed part of the CS. Another remarkable feature of the Mityushov report is that, whilst today's conditioning literature would not recommend preexposure to the CS, as this is generally understood to retard conditioning (e.g. MacRae, Scoles \& Siegel, 1987; Miller et al., 1995), Mityushov was able to find a rapid acquisition of the CR

12 Of course, also the appearance of the experimenter and the conditioning room seem to bave served as additional CSs, as Mityushov's hypoglycemic CRs failed to appear as soon as the saline injection took place in a different room or with a different experimenter (see the appendix of this thesis, tables A.2 and A.3). 


\section{Chapter 4}

after giving repeated saline injections before conditioning ${ }^{13}$.

The broader relevance of Mityushov's data is that the magnitude of the conditioned hypoglycemias (5-20\%) overlaps with that of the hypoglycemias that have been recently found to precede meal initiation in rats (Campfield \& Smith, 1990), and humans (Campfield et al., 1996). Although Campfield and Smith have always emphasized that the origin of the pre-meal hypoglycemias are endogenous, Mityushov's results suggest that the responses might be conditioned to external cues, a suggestion also made by Woods (1990). In fact, it has been recently proposed that conditioned insulin secretion and hypoglycemia play a role in normal and binge eating (Jansen, 1994a; Woods, 1991)

It is obvious that Mityushov's study on blood sugar conditioning deserves replication with control groups, direct insulin measurement, and statistic tests added. Furthermore this study may serve as an illustration of the potential importance of investigations carried out decades ago by Pavlovian physiologists in Eastern Europe, who found conditioned response interesting in their own right. When this large data base, embodied in several thousands of non-English publications (Dworkin, 1993; Razran, 1961) would be explored, provoking and interesting results might be found, many of which await replication and extension.

In the Eastera-European Pavovian tradition, pre-exposure to the CS used in subsequent conditioning was a standsrd procedure. Pre-exposure was meant to habiruate orienting reflex to the $C S$, because orienting was confidert antagonistic to conditioning, habituation was thought to be a prereģisite to successful conditioning. 
Chapter 5

\section{CUE REACTIVITY TO \\ FOOD- AND BODY-RELATED STIMULI \\ IN RESTRAINED AND UNRESTRAINED EATERS ${ }^{1}$}

\section{INTRODUCTION}

The symptomatology of bulimia nervosa includes recurrent binge eating and an overconcern regarding body shape and weight (APA, 1994). During binge eating, large amounts of food are ingested while the subject experiences an irresistable food craving and loss of control over food intake. Recently, classical conditioning models, derived from addiction research (e.g. Siegel, 1983; Drummond, Tiffany, Glautier \& Remington, 1995) have been applied to binge eating (Jansen, 1990, 1994; Wardle, 1990). In these models it is assumed that through a learning process, cues predictive of binge eating (e.g. the sight and smell of snacks, or states of depression and boredom) eventually come to elicit anticipatory physiological responses. One of these is a secretion of insulin which counters the post-binge blood sugar rise. According to the conditioned binge eating theory, anticipatory responses, when intense, are experienced as a craving for food.

In aldietion research, an interesting pperationalization of conditioning theories has emerged: cue reactivity measurement, a method in which drug-related cues are presented and psychophysiological measures are monitored. The intensity of these reactions (i.e. cue reactivity) is supposed to be a reflection of the responses underlying craving. For example, alcoholics and smokers display heart acceleration and increased skin conductance responses when presented with substance-related cues, an effect that was not seen in normals Niaura, Rohsenow, Binkoff, Monti, Pedraza, Abrams, 1988; Childress, Ehrman, Rohsenow, Robbins \& O'Brien, 1992). An important reason for assessing cue reactivity is that it probably forms a risk factor in relapse of treated addicts (Childress et al., 1992; Drumond et al., 1995). Moreover, measurement of cue reactivity could be used to study the effect of exposure therapy in addicts (Jansen, 1994). In contrast to addiction research, binge eaters" psychophysiological cue reactivity to food stimuli has been investigated rarely, perhaps with the exception of salivation (e.g. Klajner, Herman, Polivy o Chabra, 1981; LeGoff \& Spigelman, 1987).

In the present study, an experimental, non-clineal approach (see Abramson \& Seligman, 1977) was adopted by studying cue reachivity in restrained eaters. The restraint concept

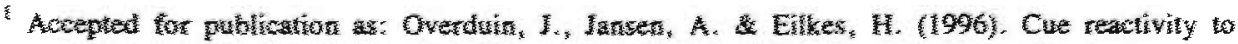

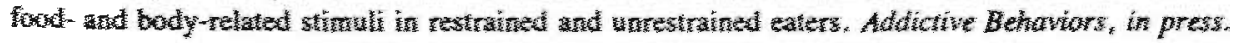


was originally developed to describe the occasionally disinhibitive eating behavior of dieters, and it has often been used as an analogue of binge eating tendency (Wardle \& Beinart, 1981; Herman \& Polivy, 1988). Two similarities between restrained eaters and bulimics can be mentioned.

The first is that the eating style of restrained subjects, selected by Herman \& Polivy's Restraint Scale (Herman \& Polivy, 1980) is characterized by unsuccessful attempts to restrict food intake and an increased frequency of binge eating (Wardle, 1980). Within the laboratory, restrained eaters have been found to increase their intake of high-caloric foods after a range of manipulations like, for example, ingestion of a "preload" (for example, a high-caloric pudding; Herman \& Polivy, 1980; Lowe, 1993), merely smelling a preload (Jansen \& Van den Hout, 1991; Rogers \& Hill, 1989), or induction of certain mood states (Greeno \& Wing, 1994; Lowe, 1993; Ruderman, 1986). In line with these findings, the present study was aimed at testing the hypothesis of increased cue reactivity to attractive food cues in high vs. low restrained eaters.

A second similarity between bulimics and restrained eaters is the existence of an overconcern (albeit to a different extent) with body shape and weight. As a rule, bulimics and restrained eaters consider themselves too fat and they are afraid of getting fatter (Herman \& Polivy, 1988). In this study, we tested the hypothesis that restrained eaters show more intense emotional responses than unrestrained eaters when viewing slides depicting their own body.

In the present study, food and body cue reactivity was assessed by monitoring autonomic physiological responses. These were considered relevant for three reasons: their role in anticipation and processing of ingested food (Woods, 1991), their being sensitive measures in cue reactivity research of various addictions (Niaura et al., 1988; Childress et al., 1992), and their suitability as indices of affective responses to stimuli (Lang, Greenwald, Bradley, \& Hamm, 1993).

In short, two hypotheses were tested in the present study. First, all subjects will show more intense appetitive responses to slides depicting binge food than to slides with a neutral content; restrained eaters show this effect to a larger extent than do unrestrained eaters. Second, restrained eaters will show more intense emotional arousal to pictures of their own body, than unrestrained eaters.

\section{METHOD}

\section{Subjects}

Female subjects were called for by means of an advertisement in a local newspaper. The 75 individuals that responded were invited to fill in the Restraint Scale (RS; Herman et 
al., 1978). Of these, 26 individuals having either a RS-score below 10 or above $15^{2}$ were asked to participate in the experiment. Characteristics of the two subject groups thus formed are shown in table 5.1. After the experiment, it appeared that two subjects did have an extreme Body Mass Index (BMI resp. 16.5 and 26.5). Data from these subjects were excluded from statistical analysis.

Table 5.1. Characteristics of Restrained and Unrestrained subjects in this study

\begin{tabular}{|c|c|c|c|c|c|c|}
\hline & \multicolumn{2}{|c|}{$\begin{array}{l}\text { Unrestrained } \\
\qquad(N=13)\end{array}$} & \multicolumn{2}{|c|}{$\begin{array}{l}\text { Restrained } \\
\quad(N=11)\end{array}$} & \multicolumn{2}{|c|}{ t-test } \\
\hline & mean & $\mathrm{sd}$ & mean & sd & $\mathrm{t}$ & p-value \\
\hline Body Mass Index & 19.9 & 1.4 & 21.8 & 1.7 & 2.90 & .01 \\
\hline Age & 20.3 & 1.7 & 21.5 & 3.1 & 1.14 & $\mathrm{~ns}$ \\
\hline Restraint Scale & 5.2 & 2.2 & 20.2 & 2.8 & 14.6 & $<.001$ \\
\hline
\end{tabular}

\section{Psychophysiological parameters as related to hypotheses}

Hypothesis one: larger reactivity to food cues than for neutral cues, a fortiori so in restrained eaters.

Skin conductance response, amplitude Enlarged SCRs have been found in alcohol and nicotine addicts who where presented substance related cues (Niaura et al., 1988; Childress et al., 1992). We expected all subjects to show larger SCR-amplitudes in response to bingefood than to neutral slides; furthermore this effect was expected to be stronger in restrained than in unrestrained eaters.

Heartrate (HR) The heart is innervated by both sympathetic and parasympathetic nerves, and reactions to stimuli are often multiphasic and not easily interpreted (Venables, 1991). So far, cue reactivity research has scarcely addressed this issue: heartrate data are usually averaged over longer time intervals. Overall HR-increase after cue presentation has been found in smoking-addicts, but responses in alcoholics have been contradictory (Childress et al., 1992). Kostarczyk (1986) has found HR acceleration in dogs, presented with a conditioned stimulus which had been previously paired with a food delivery. The accelaration occurred during the 10 post-stimulus seconds. Given the above findings, we expected a larger HR acceleration in restrained than in unrestrained eaters watching slides with binge

\footnotetext{
${ }^{2}$ That is, 0.5 standarddeviations below and above the mean RS-score of a group of 482 Dutch female students respectively; Overduin \& Jansen, 1996; Jansen \& Overduin, unpublished data).
} 


\section{Chapter 5}

foods.

Startle reflex, amplitude Lang and co-workers (Lang et al., 1990, Bradley et al., 1990) have demonstrated that changes in the intensity of startle eyeblink reflexes, elicited during picture viewing, covary with the subjective emotional valence of the picture. Viewing positive slides has an inhibitory, whereas viewing negative pictures has a facilitating effect on the reflex amplitude (Lang et al., 1990). Lang et al. have suggested that startle modulation is bases on the activation of either appetitive or defensive behavior systems. It should be noted that predictions about the reaction of restrained eaters to food slides are problematic. On one side, one would expect increases in startle amplitude because of negative emotional valence of the slide content (due to likely associations with guilt and loss of control), on the other one should expect less intense startle responses because food slides induce an appetitive state. Therefore, in the case of restrained subjects, no strict directional prediction was formulated for startle reflex modulation to food slides. Unrestrained subjects were expected to display a small decrease in startle amplitude, related to the craving elicited by the slides.

Startle reflex, onset latency The startle onset latency appears to covary inversely with startle amplitude (Bradley, Cuthbert \& Lang, 1990). That is, increases in amplitude go hand in hand with decreases in onset latency and vice versa. Thus, during presentations of binge food pictures we expected unrestrained eaters to show increased onset latencies, while for restrained eaters no specific predictions were made.

Facial EMG In emotion research, EMG activity of facial muscles has been demonstrated to be specifically indicative of emotional valence. Two important examples are the Zygomatic 'cheek-muscle', which contributes to the act of smiling and becomes increasingly active when a person views pictures with a positive emotional valence, and the Corrugator 'frownmuscle' which is active during experience of negative emotion (Lang et al., 1993; Dimberg, 1987). For the same reasons explained above for the startle response, no explicit predictions could be made for facial muscle EMG responses to food slides in restrained eaters.

Hypotheses two: Increased emotional arousal in restrained eaters to slides depicting their own body.

Skin conductance reaction, recovery time SCR-recovery time reflects the speed with which the SC-level retums to baseline after having reached its peak. Repeatedly, it has been suggested that increased SCR recovery time is indicative of a defensive response (Dimberg, 1987; Edelberg, 1972). Accordingly, in the present study we expected increased SCR recovery time in restrained, but not in unrestrained eaters presented with slides depicting 
their own body.

Heart rate HR acceleration at between 3-5 seconds after stimulus onset is due to sympathetic activity and has been interpreted as reflecting a defensive reaction (Venables, 1991). We therefore expected restrained eaters to show larger heart rate accelerations than unrestrained eaters during presentation of pictures of their own body.

Startle reflex. amplitude Because of the negative emotional valence of body slides, we expected these slides to induce a larger increase in startle amplitude in restrained than in unrestrained subjects (Lang et al., 1990).

Startle reflex, onset latency Presentation of body slides was expected to lead to larger decreases in startle onset (relative to responses to neutral slides) in restrained than in unrestrained subjects.

Facial EMG Because the negative emotional valence of body pictures, restrained eaters were expected to show larger decreases in zygomatic, and larger increases in corrugator EMG-activity than were unrestrained eaters.

\section{Equipment, and processing of the psychophysiological signals}

All psychophysiological signals were fed to Beckman couplers and subsequently stored on multichannel magnetic tape for further off-line analysis. One of the channels contained information about times and types of stimulus presentation. Only the Skin Conductance signal was recorded (and subsequently scored) on paper using of a Beckman R711 polygraph with multi-channel writer.

SCR was recorded using two $\mathrm{Ag}-\mathrm{AgCl}$ electrodes (diameter $8 \mathrm{~mm}$ ) attached to the middle fingers of the non-dominant hand. Two parameters were drawn from the SCR-signal: amplitude (SCR-amp) and recovery time (SCR-rec). SCR-amp was defined as the (baseline corrected) maximum SC-level within 6 seconds after slide onset (baseline was defined as the SC level before a significant post-stimulus increase began). SCR-rec was defined as the time interval between the moment the SC level reached its peak and the moment the signal had returned to a level of $(0.5 *$ SCR-amp) above baseline.

Startle reflexes were elicited by a pulse of $90 \mathrm{~dB}$ white noise with instantaneous rise time through headphones (Anthony, 1985). Part of the startle reflex is the eyeblink, and the relevant EMG signal was detected using two $\mathrm{Ag}-\mathrm{AgCl}$ electrodes applied on the orbicularis oculi, just beneath the lower left eyelid. The EMG-signal was sampled from tape with a frequency of $1000 \mathrm{~Hz}$ and filtered by a digital, 255-point, $60 \mathrm{~Hz}$ highpass FIR-filter. Then the signal was rectified and integrated using a time constant of 20 milliseconds. Under the condition that a typical reflex shape could be distinguished, two parameters were extracted from the signal: amplitude and onset latency. Startle amplitude was defined as the highest EMG-level within $150 \mathrm{msec}$ after presentation of the noise. The amplitude was corrected 


\section{Chapter 5}

for pre-stimulus baseline level, defined as the mean EMG level in the 50 pre-stimulus milliseconds. Startle onset latency was defined as the time interval between presentation of the startle stimulus and the moment when EMG level began to rise sharply and significantly above baseline level. Reactions with onset latency earlier than 18 or later than $70 \mathrm{msec}$ or with no apparent reflex shape were excluded from further data processing.

Facial muscle EMG activity from the zygomatic and corrugator muscles was recorded using two $\mathrm{Ag}-\mathrm{AgCl}$ electrodes with a diameter of $2 \mathrm{~mm}$, placed according to suggestions by Fridlund and Cacioppo (1986). Signals from both channels were treated likewise. After $1000 \mathrm{~Hz}$ sampling from the tape the signals were respectively lead through a digital, 255point, $90 \mathrm{~Hz}$ high-pass FIR-filter, rectified, and integrated by a contour-following integrator with a time constant of $500 \mathrm{msec}$ (Fridlund, 1979). Baseline EMG was defined as the mean intensity of the signal in the 5 seconds preceding slide presentation (Dimberg, 1987). The reaction was defined as the mean EMG level during the 5 seconds after stimulus onset, corrected for baseline.

Heartrate was recorded by means of two Blue Sensor disposable ECG electrodes (type R$00-S$ ) attached on a diagonal axis over the heart region and a reference electrode on the shoulder. From magnetic tape the signal was sampled with a frequency of $1000 \mathrm{~Hz}$ and led through a digital, 255-point, $35 \mathrm{~Hz}$ lowpass FIR-filter. After that, times of R-peaks in the signal were detected by a special devised computerprogram and checked for artifacts and electronic interference. In a next step second-to-second heartrate for the 12 post-stimulus seconds were computed by a specially devised software program employing the weighing procedure proposed by both Velden and Graham (1988). Baseline was defined as the mean heartrate in the 5 seconds preceeding stimulus onset. Eventually two values were extracted: 'overall acceleration', i.e. the mean second-to-second heartrate for the 12 seconds after stimulus onset, and defensive acceleration, defined as the highest of the second-to-second heartrate for seconds 3, 4 and 5 after stimulus onset (Laberg, Wilson, Eldredge \& Nordby, 1991).

\section{Materials}

All auditory stimuli (music and startle noises) were presented through Beyer DT 109 headphones. The music used for the depressive mood induction (see procedure) was a piece by the classical composer Prokoviev, entitled 'Russia under the Mongolian Yoke' played at half speed (Clark, 1983). Slide projection was carried out with a Kodak carousel projector, which was automatically driven by a computer program on a PDP Minc Microcomputer.

There were three slide categories, 'neutral', 'food' and 'body'. The neutral pictures were the same for all subjects and showed pieces of office equipment. Body and food slides were selected individually for each subject. Care was taken that the food stimuli would reflect 
each subject's preference. To achieve this, a rating scale was administered 3 weeks before the experiment on which the subjects had to rate 5 snack categories on preference and 'binge provoking characteristics'. From each of the three categories ranked highest by the subject, 2 slides were included in the slide series. The 5 categories were potato chips, chocolate bars, candy bars, licorice, and pies. The set of body slides consisted of pictures that had been taken of the subject three weeks prior to the experiment (see procedure). The subject was visible wearing a standard set of underwear that would leave belly, arms, legs, neck and face uncovered. In a set of six slides the body was shown from different standardized angles: two pictures were taken frontally, two en profil, and two from an angle of 45 degrees.

\section{Questionnaires of the experimental session}

During the experimental session subjective data were mostly collected by means of Visual Analog Scales (VASs) on which subjects score between 0 ("not at all") and 100 ("extremely").

Two times a VAS containing the following three items was administered (see procedure): "At this moment I feel tense", "At this moment I feel peckish" and "At this moment I feel depressed". Further, each member of the presented slide-series (see procedure) was evaluated by two questions: "how agreeable do you find this slide?" and "How much food craving does this slide evoke?" The final questionnaire contained questions about subjects' food intake prior to the experimental session and the questions: "How satisfied are you with your body appearance?", "Are you currently dieting in order to lose weight?"

\section{Procedure}

Pre-experimental session Three weeks before the experimental session, six pictures were taken of the subjects for use in the experiment (see materials). After the photo-session a questionnaire was handed out in which subjects could rate a number of food items on their craving arousing properties. These ratings were eventually used for selection of the food slides (see materials).

Experimental session Each subject was asked to eat a standard meal at 3 hours prior to the experimental session and to refrain from eating thereafter. The subject was welcomed and informed about the course of the experiment. The first VAS was handed out. Each subject was seated in a comfortable chair, electrodes were attached, and a headphone was put on. She was asked to remain in her chair quietly so as to not disturb the psychophysiological measurements. In order to ensure that full-fledged cue reactivity would be registred a mood induction procedure was employed, as craving in restrained eaters has been repeatedly reported to be mood dependent (Greeno \& Wing, 1994; Cools, Schotte \& McNally, 1992; 


\section{Chapter 5}

Ruderman, 1985). First, it was announced that music would be played. The subject was asked to concentrate on sad thoughts as long as the music would play. The experimenter left the room, lights were dimmed and the music began playing for 5 minutes. After the music the experimenter reentered the room and administered the second VAS. After that the slide series was announced. It was emphasized that the subject should watch the slides carefully, because later some questions would be asked about them. Then the experimenter left the room, which was darkened now and the slide presentation began. Two slides were used as a introduction, and after that the six neutral, six food and six body slides were presented in random order. Each slide was shown for a period of twelve seconds. Inter slide intervals differed and were either ten, fifteen, twenty or twenty-five seconds. During half of the slides a startle eliciting noise was presented. To preclude subjects' expectations on when the startle noise would sound the noise was presented at variable times (either 1, 3 or 7 seconds) after slide onset for the different slides. Following the first slide presentation all slides were shown again, and each slide was rated. After the removal of the electrodes the final questionnaire was administered. The subject was weighted and paid for her participation. The six personal body slides were handed over to the subject.

\section{RESULTS}

\section{Statistical method}

Data were processed using SPSS-PC statistical software. In most instances, repeated measurement ANCOVAs were conducted, with Body Mass Index as a covariate, as the two subject groups differed on this variable (see table 5.1). Due to malfunctioning of the psychophysiological equipment and interference, part of the data of some subjects were excluded from further analysis.

\section{Manipulation check of the mood induction}

The mood induction proved to be successful in both groups. A 2 (before/after mood induction) $\times 2$ (restraint) ANCOVA showed a main effect for mood induction, indicating a significant increase in negative $\operatorname{mood}$ scores $(F(1,22)=36.2, p<.001)$. Furthermore the mood induction led to decreased craving ratings $(F(1,22)=7.85 ; p=.01)$. No main effect of mood induction was found on subjective tension level. None of the subjective ratings showed a main effect of group or a group $x$ mood induction interaction effect. 


\section{Subjective and psychophysiological responses to the slides}

Results of the statistical tests are presented according to the main hypotheses of the experiment.

Hypothesis 1: cue reactivity is larger for food than for neutral slides in all subjects, but to a larger extent in restrained subjects.

Data on SCR amplitude, Heartrate, startle reflex parameters, and craving ratings for the food slides were subjected to separate 2 (restraint) $\times 2$ (slide type) repeated measurement ANCOVAs with Body Mass Index as the covariate. The predicted restraint $x$ slidetype interaction was found only for corrugator EMG activity $(F(1,17)=5.95 ; p<.03)$. The pattern of cell-means (not shown here) revealed that the average EMG response to both slide types was a decrease in corrugator (frown) activity, which suggests a positive emotional response in both subject groups.

Main effects of slidetype were found for food craving $(F(1,22)=133.9 ; p<.001)$ and startle onset latency $(F(1,22)=8.68 ; p<.008)$. These effects reflected a more intense appetitive response to foodslides than to neutral slides. No main effects of restraint were found.

In sum, the results showed that, although food slides triggered more intense psychophysiological reactions and craving than did neutral slides, restrained and unrestrained eaters differed only in corrugator EMG activity, albeit to a modest extent.

Hypothesis 2: body slides elicit more negative emotional arousal in restrained than in unrestrained subjects.

Data on SCR recovery time, HR during the 2-5 seconds post-stimulus time window, Corrugator and Zygomatic EMG changes, Startle reflex onset and amplitude, and emotional ratings of the slides were subjected to separate 2 (restraint) $\times 2$ (slide type) ANCOVAs with repeated measures and Body Mass Index as a covariate.

No main or interaction effect on emotional rating was found, reflecting that the restrained subjects did not rate their body-pictures more negatively than did unrestrained subjects. None of the psychophysiological parameters showed the predicted restraint $\mathrm{x}$ slide type interaction effect. Also no main effect was found for restraint. A main effect for slide type was found only for Heartrate acceleration $(F(1,14)=8.98 ; p<.01)$ and for SCR recovery time $(F(1,18)=5.09 ; p<.04)$. These effects reflected more intense negative emotional arousal during presentation of the body slides. 


\section{Chapter 5}

Again, it can be said that none of the predicted differences between restrained and unrestrained eaters was found.

\section{Body satisfaction and current dieting}

Restrained and unrestrained eaters did not differ in body satisfaction $(\mathrm{t}(20)=.47$; ns). This was somewhat surprising because the restrained eating style is often assumed to originate from body dissatisfaction. Of the unrestrained subjects, nobody was currently dieting, whereas of the restraints $38 \%$ was.

\section{DISCUSSION}

In the present investigation it was found that restrained and unrestrained eaters did not differ in psychophysiological reactivity to slides of either their preferred binge food or their own body. Moreover, no group differences were found on subjective ratings of food slides (craving), or body slides (negative emotional valence). Nevertheless, a main effect of slide type on several psychophysiological parameters was found. On the average, responses to the body slides were the most intense, followed by the food and neutral slides respectively. The present failure to find a group difference in psychophysiological reactivity to food and body cues may result either from peculiarities of the present procedure, or from more fundamental problems with a pure conditioning explanation of cue reactivity.

The former explanation is based on the assumption that the classical conditioning interpretation of cue reactivity is valid, but that the present procedure should be adjusted to demonstrate differential cue reactivity. This possibility should be taken seriously in the light of the small existing empirical data base on psychophysiological food cue reactivity in humans. Five adjustments of the experimental procedure might be thought of. First, the present study involved non-clinical subjects. It might be that significantly increased cue reactivity is confined to clinical binge eaters, because of their occasional huge food intake. As to body cue reactivity, a analogous argument could be made, based on the different body esteem of e.g. bulimia nervosa patients and non-clinical subjects. Second, the data indicate that the (individually selected) food slides did have had some arousing effect on the subjects. However, it might be that because of their mere visual nature, food slides lack in truly disinhibitive potency. Accordingly, investigation of the effects of real food items providing additional olfactory/gustatory stimulation should deserve attention. Third, the subjective ratings show that the food slides elicited increased arousal and increased craving in both restrained and unrestrained subjects. This might be the consequence of a basic characteristic in which food cue reactivity study differ from studies into addictions. Not only had both 
subject groups some learning experience with the presented food cues, also their habitual intake may have been comparably large (indeed, the slides were individually selected on craving arousing value). This might have lead to equal food cue reactivity. Future research might better collect quantitative data on subjects' usual craving and intake of food associated with the cues presented during the lab procedure rather than relying on a personality questionnaire (i.e. the Restraint Scale) only. Fourth, the psychophysiological parameters monitored in the present study may not have been sufficiently "food-specific". Heightened cue-reactivity to disinhibiting food cues might be found only in physiological domains more tightly linked to food ingestion, e.g. blood sugar or insulin responses (Jansen, 1994). And fifth, there is a possibility that notable differences between restrained and unrestrained eaters in food cue reactivity begin to appear at more than 12 seconds after cue perception, i.e. beyond the time window in which we monitored our subjects' responses.

The second explanation for our failure to find differential cue reactivity in restrained and unrestrained eaters may be more fundamental in nature: the conditioning account of cue reactivity may not be adequate. As a matter of fact, Tiffany (1995) has proposed that the expression of cue-reactivity (in addicts) should be understood as a reflection of behavioral and cognitive demands of a situation rather than as a mere residual of learning history. Specifically, cue-elicited responses might be especially intense in cases where an encounter with the substance itself is clearly forthcoming. This issue may not have been sufficiently addressed in the present procedure: although the subjects were to watch the slides attentively, no explicit attempts were included to arouse subjects' expectations of encounters with the depicted food items, e.g. of ingesting or abstaining from ingestion in the presence of the food). It might be that differential food cue reactivity may be demonstrated only after procedures in which the psychosocial concomitants described by Tiffany are explicitely manipulated.

Research on cue reactivity in the field of binge eating has only recently started. At present, assessment of the contributions of situational vs. conditioning influences, as well as identification of the appropriate response parameters appear to be the most central issues in food cue reactivity research. 



\section{FOOD CUE REACTIVITY IN FASTING AND NON-FASTING SUBJECTS ${ }^{1}$}

\section{INTRODUCTION}

A recently proposed theory of binge eating holds that cues that reliably precede intake of binge food can acquire the ability to elicit a range of anticipatory physiological responses. These classically conditioned responses facilitate bodily processing of food and help in maintaining homeostasis (Jansen, 1990; 1994a; Woods, 1991). Additionally, the theory states that conditioned physiological responses to binge cues are subjectively experienced as craving and as such can trigger food intake. Research on the nature of conditioned anticipatory responses could be helpful in both controlling them, and in preventing relapse in treated patients (Childress, Ehrman, Rohsenow, Robbins \& O'Brien, 1992; Glautier, 1994; Jansen, Broekmate \& Heijmans, 1992). Until now, however, the exact nature of the conditioned physiological responses to binge cues remains unclear (Overduin, Jansen \& Eilkes, 1996). A major aim of the present study was the determination of psychophysiological and subjective responses to food cues (sight and smell) in normal, non-clinical subjects. Moreover, as dietary restriction is supposedly linked to the development of binge eating (e.g. Polivy \& Herman, 1985; Tuschl, 1990) and short-term deprivation was expected to increase reactivity to food cues, also responses of 24-hour fasting subjects were studied. Studies in the addiction field show that increases in salivation, skin conductance and subjectively reported craving to cue presentation are the most consistent findings, whereas heart rate acceleration was found less often (Childress et al., 1992; Glautier, 1994). In the present study it was hypothesized that presentation of cues signalling desired food will elicit more salivation and larger increases in heartrate and frequency of skin conductance fluctuations than presentation of control (i.e. soap) stimuli. Furthermore, food cue reactivity was hypothesized to be larger in fasting than in non-fasting subjects.

1 Accepted for publication as: Overduin, J. \& Jansen, A. (1996). Food cue reactiviy in fasting and non-fasting subjects. European Eating Disorders Review, in press. Heiny Eilkes is gratefully acknowledged for her assistance in carrying out the present experiment. 


\section{METHOD}

\section{Design}

Each of the fasting and non-fasting subjects was exposed to both food- and soap stimuli. The order of stimulus presentation was counterbalanced within each condition. Psychophysiological cue reactivity was determined by measuring salivation, spontaneous skin conductance fluctuations and heartrate, before (baseline) and during stimulus presentations.

\section{Subjects}

Twenty-one healthy normal weight students without a history of eating disorders ( 9 females and 12 males; mean age 21.6 years) participated in the experiment. The subjects were randomly allocated to the fasting condition $(n=11)$ and to the non-fasting condition $(\mathrm{n}=10)$. Mean BMI in the fasting group $(21.9$, sd 2.8$)$ did not differ from the normal group $(21.4$, sd $2.0 ; \mathrm{t}(19)=.55 ; \mathrm{ns})$. It was told to the subjects that the experiment involved an investigation of "attentional and sensory processes".

\section{Questionnaires and psychophysiological parameters}

Questionnaires At the beginning of the experiment hunger and craving ratings were collected by means of on Visual Analogue Scales (VASs) ranging from 0 ("not at all") to 100 ("very much"). After the end of presentation of each stimulus a VAS was administered on food craving and hedonic evaluation of the stimulus1.

All electronic signals, including the markers for stimulus presentation, were registered by Beckman couplers and a Beckman R711 polygraph with a multi-channel writer.

Skin Conductance was recorded by means of two $\mathrm{Ag}-\mathrm{AgCl}$ electrodes (diameter $8 \mathrm{~mm}$ ) attached to the middle fingers of the non-dominant hand. The number of non-specific fluctuations in skin conductance level (further to be called "SC-fluctuations") were counted to acquire an index of the frequency of sympathetic nervous "bursts" both before and during presentation of the stimuli. They were defined as SC-level increases exceeding 0.05 micromhos within 2 seconds (Boucsein, 1992) and taking place at least 6 seconds after breathing artefacts or stimulus onset. The number of fluctuations was counted for each minute.

Heartrate was recorded by means of two Blue Sensor disposable ECG electrodes (type R-00-S) attached on a diagonal axis over the heart region and a reference electrode on the shoulder. For each minute the mean heartrate was established.

Saliva was collected by means of three dental rolls, according to the method of LeGoff, 
Leichner \& Spigelman (1988). The subject inserted a first roll underneath the tongue, and two rolls laterally, into each side of the lower jaw between teeth and gums. Salivation collection time was exactly three minutes. The mean salivation per minute was computed for statistical analysis.

Breathing amplitude Because the number of SC-fluctuations is influenced by irregular breathing (sighing, coughing and so on; Boucsein, 1992), breathing amplitude was monitored using a Beckman respiratory belt connected to a Beckman Voltage/Pressure Coupler. The mean breathing amplitude during the 6 second interval preceding each $\mathrm{SC}$-fluctuation was established and they were averaged for the minute under consideration. In this way the mean breathing amplitude preceding SC-fluctuation was computed for each of the 14 one-minute intervals of the experiment. Then, for each minute, control periods of 6 seconds not followed by SC-fluctuations were inspected and mean breathing amplitudes were computed in the same way as above. Finally, the difference between pre SC-fluctuation breathing amplitudes and the control breathing amplitudes was assessed by t-tests.

\section{Stimuli}

The food stimuli consisted of two candy bars (Twix) stripped from their paper and one for each subject freshly prepared healthy brown roll, with salat, cheese, ham and tomato.

The control stimuli consisted of four small pieces of round, lightly flowerishly perfumed bathing soap. Two of the pieces were white-, and two were green-coloured. All stimuli were presented on a plate with a blinded removable cover over it. After lifting the cover, the items, resting on colored paper napkins, became visible.

\section{Procedure}

The fasting group was asked to refrain from eating for a period of 24 hours prior to the experimental session. To ensure that fasting really would take place, the subjects were instructed to collect their morning urine and bring it with them, so as to "control for fasting". In reality no further analyses were carried out on these urine-samples. The non-fasting group was instructed to have a normal meal at 3 hours prior to the experimental session and to refrain from eating until after the experiment. After entering the lab, each subject first completed the hunger and craving VAS. Then she was informed about the general procedure and how to insert the dental rolls. She was seated in a comfortable chair and instructed not to move exaggerately during the experiment, so as not to disturb the measurements. The explanation of the procedure was repeated while the electrodes were attached. During the first two minutes of the experiment, 


\section{Chapter 6}

psychophysiological baseline measurements were carried out with the subject sitting still in the chair and the experimenter sitting in front of her. Thereafter the subject was asked to insert the dental rolls. Within 6 seconds after the insertion of the dental rolls, the first stimulus was presented in front of the subject and the cover of the dish was lifted by the experimenter. The instruction was given to attend (look and smell) to the food intensely so that the subject would be able to correctly complete the VASs later on. The dish was arranged so that the subject's nose was close to it, so that no bending down was required to smell and watch. Three minutes after the onset of stimulus presentation, the subject was told to remove the dental rolls from his mouth, to put them in the container and to close it. Stimulus presentation and physiological measurement continued for another two minutes after which the hedonic evaluation and craving VAS was administred with the stimulus still within sight. Then there was a pause of eight minutes in which they were distracted by the projection of six neutral slides with office equipment. The subject was told to watch them attentively and to memorize them as good as possible so as to be able to answer questions about them later on. After this pause the second stimulus was presented. The procedure was identical to the first stimulus presentation. Finally, the subject was weighed, measured, debriefed, paid for participation and offered the sandwich presented during the experiment.

\section{RESULTS}

\section{Data processing}

Because fasting has been found to lead to decreased tonic sympathetic nervous activity (Landsberg \& Young, 1978; Webber \& MacDonald, 1993), phasic (cue-elicited) physiological activity was corrected for tonic (baseline) levels: for heartrate and skin conductance the mean levels of seven (i.e. two pre-stimulus and five post-stimulus) minutes were computed. Then, tonic level was defined as the average of the two pre-stimulus minute levels. Phasic reactivity score was defined as the difference between the average of the 5 post-stimulus minutes levels and tonic level. The tonic and phasic reactivity scores for heartrate and skin conductance, salivation rate and the VAS-ratings of the stimuli were subjected to a 2 (condition; fasting vs non-fasting) $\times 2$ (stimulus-type; food vs soap) MANOVA with stimulus-type as a within-subjects factor.

Unfortunately, due to malfunctioning of the equipment, for some people skin conductance and heartrate data were lost. 
Table 6.1. Subjective state prior to presentation of stimuli (scoring range: $0-100$ )

\begin{tabular}{|c|c|c|c|c|c|c|}
\hline & \multicolumn{2}{|c|}{$\begin{array}{c}\text { non-fasting subjects } \\
\qquad(\mathrm{N}=10)\end{array}$} & \multicolumn{2}{|c|}{$\begin{array}{l}\text { fasting subjects } \\
\qquad(\mathrm{N}=11)\end{array}$} & \multicolumn{2}{|c|}{ t-test } \\
\hline & mean & sd & mean & sd & $t(23)$ & pule \\
\hline hunger-VAS & 50.4 & 20.0 & 84.2 & 10.5 & -4.9 & $<\infty \mathrm{ll}$ \\
\hline food craving-VAS & 47.6 & 19.6 & 83.6 & 16.9 & -4.6 & $<.001$ \\
\hline
\end{tabular}

\section{Manipulation checks}

t-Tests demonstrated that at the beginning of the experiment, the fasting subjects experienced significantly more hunger $(\mathrm{t}(19)=4.92 ; \mathrm{p}<.001)$ and craving for food $(\mathrm{t}(19)$ $=6.46 ; \mathrm{p}<.001$ ) than non-deprived subjects (see table 6.1). Also, the food stimuli were not only considered significantly tastier in appearance $[F(1,18)=148.2 ; p<.001]$ and more agreeable in smell $[\mathrm{F}(1,18)=6.58 ; \mathrm{p}<.02]$, but also leading to larger food craving $[F(1,18)=39.9 ; \mathrm{p}<.001]$ than the soap stimuli (table 6.2).

Thus, both manipulations succeeded: fasting subjects experienced more hunger and craving than non-fasting subjects and the food stimuli turned out to be considerably appetitive.

Table 6.2. Subjective ratings during food and soap stimulus presentation (scoring range: 0-100)

\begin{tabular}{|c|c|c|c|c|c|c|c|c|}
\hline & \multicolumn{3}{|c|}{ Non-fasting subjects } & \multicolumn{3}{|c|}{ Fasting subjects } & \multicolumn{2}{|c|}{ t-test } \\
\hline & mean & sd & $\mathrm{N}$ & mean & sd & $\mathrm{N}$ & i & p \\
\hline \multicolumn{9}{|l|}{ Hedonic rating of stimulus } \\
\hline food & 68.6 & 19.3 & 10 & 88.6 & 15.0 & 11 & 2.66 & $<.02$ \\
\hline soap & 15.0 & 19.8 & 10 & 19.3 & 30.2 & 11 & .38 & ns \\
\hline \multicolumn{9}{|l|}{ Craving for food } \\
\hline food & 62.7 & 17.8 & 10 & 91.4 & 9.8 & 10 & 4.47 & $<.001$ \\
\hline soap & 24.4 & 18.2 & 10 & 56.3 & 30.2 & 11 & 2.89 & $<.01$ \\
\hline
\end{tabular}

\section{Responses to stimulus presentation}

The minute-to-minute courses of heartrate and skin conductance fluctuations are shown in figures 6.1 and 6.2. Data on the tonic (baseline) levels showed that fasting subjects displayed a smaller number of SC-fluctuations $[F(1,17)=4.75 ; p<.05]$ and a trend towards lower heartrate $[F(1,18)=4.34 ; p<.06]$ than non-fasting subjects.

$t$-Tests on the difference between the mean breathing amplitude before SC-fluctuations and that of control periods (see method section) failed to reach significance. Thus it can be concluded that the SC-fluctuations did not result from breathing irregularities during stim- 
Table 6.3. Psychophysiological responses to food and soap stimulus presentation

\begin{tabular}{|c|c|c|c|c|c|c|c|c|}
\hline & \multicolumn{3}{|c|}{ Non-fasting subjects } & \multicolumn{3}{|c|}{ Fasting subjects } & \multicolumn{2}{|c|}{ t-test } \\
\hline & mean & sd & $\mathbf{N}$ & mean & sd & $\mathbf{N}$ & t & $p$ \\
\hline \multicolumn{9}{|l|}{ Salivation (mg/min) } \\
\hline food & 575 & 359 & 10 & 609 & 280 & 11 & .24 & ns \\
\hline soap & 651 & 397 & 10 & 536 & 272 & 10 & .76 & ns \\
\hline \multicolumn{9}{|l|}{$\Delta$ Heartrate (BPM) } \\
\hline food & 3.6 & 4.1 & 10 & 6.8 & 4.9 & 10 & 1.56 & $\mathbf{n s}$ \\
\hline soap & 4.0 & 3.3 & 10 & 4.4 & 6.4 & 9 & .17 & ns \\
\hline \multicolumn{9}{|c|}{$\Delta S C$-fluctuations/minute } \\
\hline food & 1.8 & 1.5 & 9 & 0.5 & 1.1 & 11 & 2.31 & $<.04$ \\
\hline soap & 0.6 & 2.2 & 10 & 0.0 & 1.2 & 10 & .81 & ns \\
\hline
\end{tabular}

ulus presentation.

On phasic reactivity scores (see table 6.3), a main effect of condition was found only for frequency of SC-fluctuations $[F(1,17)=4.44 ; p<.05]$, based on fasting subjects showing smaller increases in SC fluctuation frequency in response to both stimuli. However, the hypothesized condition $\mathrm{x}$ stimulus-type interaction did not reach significance. This implies that fasting subjects did not show especially strong reactivity to food cues. Finally, neither main effects of stimulus-type, nor stimulus-type $\mathrm{x}$ condition interaction effects were found for the other physiological parameters.

\section{DISCUSSION}

In the present study, food cue reactivity was examined in fasting and non-fasting subjects. Firstly, it was found that fasting subjects showed a lower baseline frequency of skin conductance fluctuations and a trend towards lower baseline heartrate. Secondly, although subjects gave higher appetitive ratings to food than to soap stimuli, this was not reflected in stronger psychophysiological reactivity (i.e. salivation, SC-fluctuations, heartrate). Finally, fasting subjects reported more craving for the food stimuli than did non-fasting subjects, but they did not show the hypothesized enlarged food cue reactivity. In fact, their SCfluctuations increase to food cue appeared to be even smaller than non-fasting subjects. The smaller baseline frequency of spontaneous skin conductance fluctuations and trend towards lower baseline heartrate in fasting subjects fits with earlier documented findings on lowered sympathetic nervous activity in fasting subjects (Schultz-Gambard, 1988; Landsberg 
\& Young, 1978; Webber \& MacDonald, 1993). Probably, this should be seen as a general bodily adaptation to deprivation that is aimed at sparing energy (Landsberg \& Young, 1978), rather than as a reflection of readiness for food intake.

The finding of no larger food cue reactivity to food than to soap, as well as the approximately equal cue reactivity in fasting and non-fasting subjects is surprising. Even if one takes into consideration that the exact nature of anticipatory responses to food stimuli (for example, the critical parameters and their directionality) is as yet largely unknown, one is struck by the finding that fasting subjects did not show stronger salivation. Salivation, a response obviously related to food ingestion, has been claimed to be an objective index of hunger (e.g. Le Goff et al., 1988). Indeed, besides being hungry, our fasting subjects craved and highly appreciated the food presented to them. But why, then, didn't they salivate more?

A first possibility might lie in the procedure of the present study. It might be that not all the subjects have complied with the fasting instruction, thereby obscuring differences in cue reactivity with the non-fasting subjects. Indeed, the present study did not involve objective checks on deprivation level (for example, increased levels of circulating ketone bodies; Hoffer, 1988). Nevertheless, relevant information pertaining to this issue can be drawn from the present psychophysiological data. If true cue reactivity after fasting were larger in fasting than in non-fasting subjects, and not all subject in the fasting condition were complying with their instructions, enlarged variability in responses would be expected among subjects in the fasting condition. However, the standard deviations in table 6.3 do not consistently confirm this prediction. Thus, although we have no waterproof evidence that all subjects in the fasting condition did stick to their regimen, no convincing indication could be found of heightened cue reactivity in a subset of our fasting subjects.

Secondly, it is possible that increased cue reactivity occurs only after fasting durations exceeding 24 hours. Data from the literature on appetitive parameters related to cue reactivity may be relevant in this respect. For humans, the plateau of perceived hunger intensity appears to occur after two days of fasting (Le Magnen, 1985). However, the additional impact of presenting food cues has, to our knowledge, not been investigated. For rats, the literature is inconclusive as to whether the size of the first meal after fasting is strongly correlated with fasting duration if fasting continues for more than 24 hours (Le Magnen, 1985). All in all, the possibility that increased cue reactivity could indeed be found after fasting periods longer than 24 hours deserves future attention.

A third explanation for the lack of differences in cue reactivity may be related to our subjects' learning history, which, unlike hunger level, was not manipulated in the present study. Jansen $(1990,1994)$ has argued that food cue reactivity will be particularly strong in cases of intermittent dieting and overeating, a common pattem among binge eaters. 


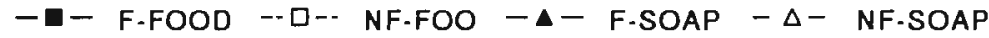

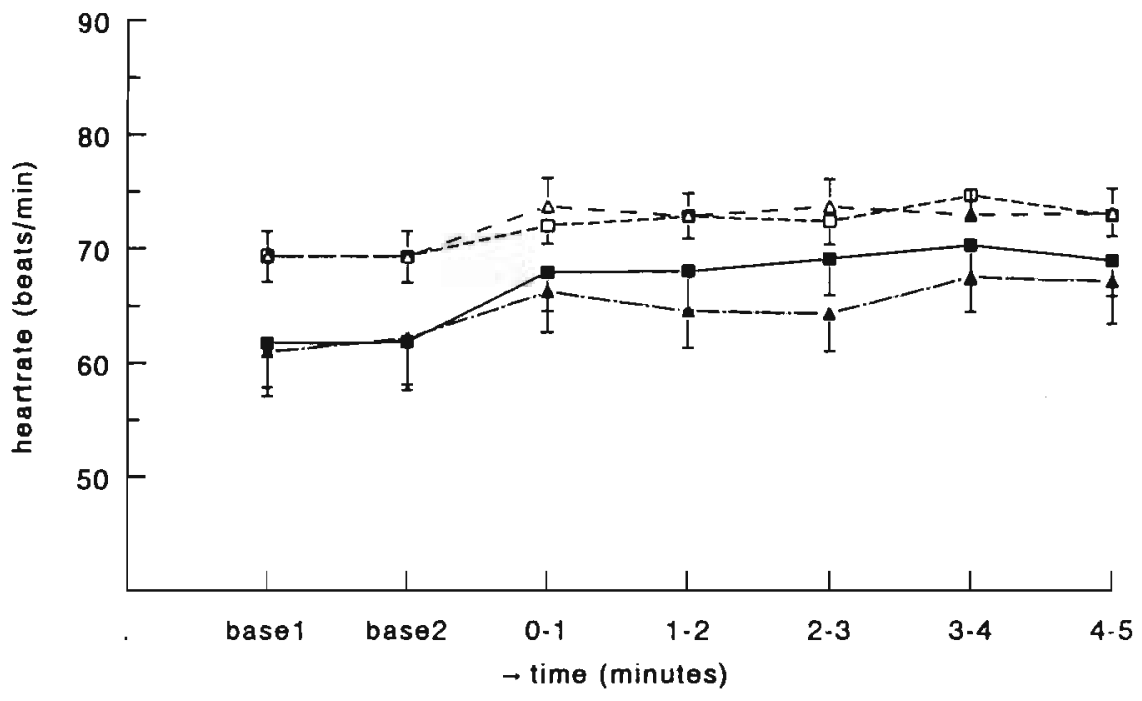

Figure 6.1. Minute-to-minute heartrates (mean \pm s.e.m.) before and during stimulus presentations.

-ם- F-FOOD --D-- NF-FOO - A- F-SOAP - $\triangle-$ NF-SOAP

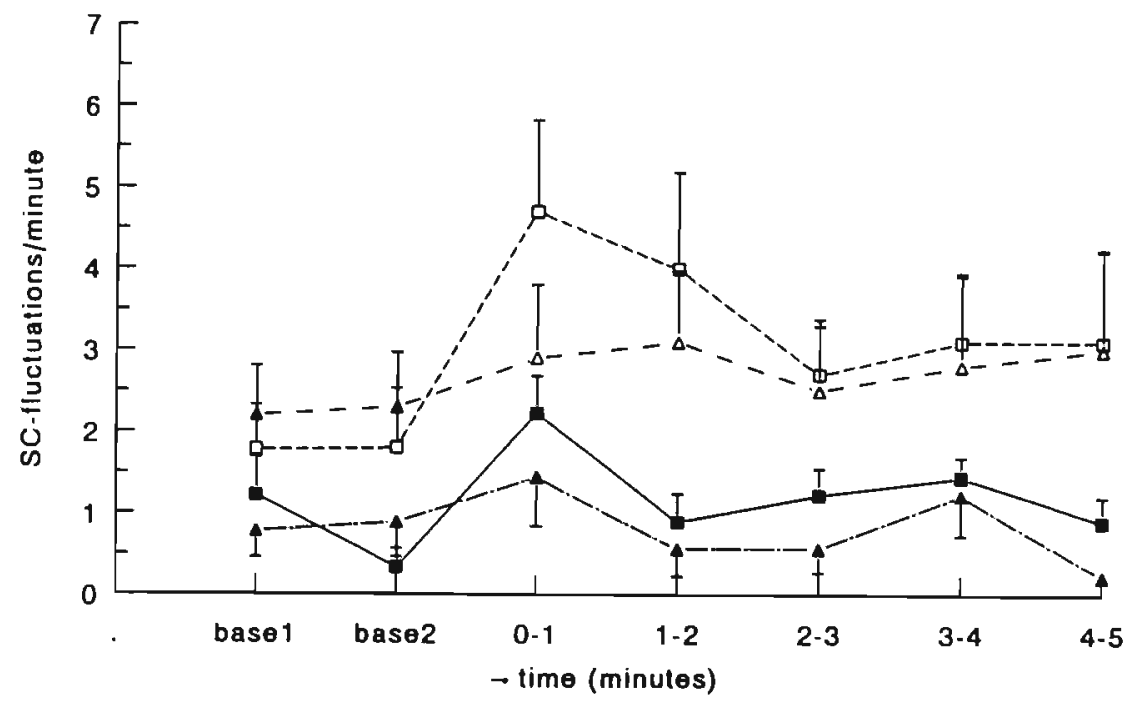

Figure 6.2. Minute-to-minute frequency of skin conductance fuctuations (mean \pm s.e.m.) before and during stimulus presentations. 
Indeed, animal studies show that a food intake scheme of one or two meals a day elicit significantly larger anticipatory responses to food cues than an intake scheme of six meals a day (Woods et al., 1977; Strubbe, 1992).

An experimental manipulation of learning history then, would require a conditioning procedure in which a set of food cues (CS) reliably predicts subsequent large intake (US). Also, it might critically be investigated whether deprivation level in its own right facilitates such learning, i.e., whether deprivation acts as an 'occasion setter' of the conditioning process (Davidson, 1993; Catania, 1991). Jansen, Boon, Nauta \& van den Hout (1992) have shown that such a Pavlovian conditioning procedure elicits increased salivation to the food cues in undeprived subjects. Altematively, it might be worthwhile to investigate whether cue reactivity intensity decreases if subjects are exposed repeatedly to food cues while being prevented actual intake. Prolonged exposure to binge cues with response prevention has been shown to be successful in decreasing craving and binge frequency in binge eaters (Jansen, Heymans \& Broekmate, 1992), but psychophysiological parameters were not monitored in these studies.

In sum, visual and olfactory cues related to food and non-food items have again been demonstrated to trigger various changes in subjective and psychophysiological parameters, but the logic underlying the intensity of these changes needs further exploration. We argue that future cue reactivity research may benefit from focusing more on the role of conditioning. It might be hypothesized that the intensity of cue reactivity covaries with the magnitude of the learned, hence expected, subsequent food ingestion. 



\section{Chapter 7 \\ STROOP INTERFERENCE AND FOOD INTAKE ${ }^{1}$}

\section{INTRODUCTION}

Preoccupation with food and eating, as well as the tendency to be overly concerned with one's body weight and shape, are key symptoms of the eating disorders bulimia nervosa and anorexia nervosa (APA, 1987; Walsh, 1992). Concerns about body shape and weight are considered pivotal to the maintenance of chaotic eating behaviour such as dieting, fasting and splurging (Fairburn, Cooper, Cooper, McKenna \& Anastasiades, 1991; Garfinkel, 1992).

In order to assess information processing in eating-disordered subjects some authors recently used the Stroop colour-naming task. In the Stroop task words printed in various colours are presented to the subject who then is required to name the colour as quickly as possible, while ignoring the meaning of the words. In studies on eating disorders reaction times to neutral words and words about food or body shape (so called target words) are compared. A relatively longer reaction time for target words indicates that the subject is distracted by the meaning of the target words, and this is supposed to be indicative of distorted information processing.

Until now several studies have shown Stroop interference for target words in eating disordered subjects. Significant interference for a combination of eating and shaperelated words were found in normal weight bulimic subjects (Fairburn et al., 1991; Cooper, Anastasiades \& Fairburn, 1992; Cooper \& Fairburn, 1993). Other studies report interference effects with separately presented eating-related words in anorexic and/or bulimic patients (Channon, Hemsley \& de Silva, 1988; Ben-Tovim, Walker, Fok \& Yap, 1989; Ben-Tovim \& Walker, 1991). Most of the studies undertaken document an interference effect for eating-related words in eating disordered subjects.

How can these data be explained? Ben-Tovim et al. (1989) hypothesized that the attentional bias for eating-related words reflects anxiety evoked by the meaning of these words and can thus be regarded as a measure of psychopathology. However, in the light of the finding that fasting subjects are hyperattentive to non-aversive appetitive stimuli (Channon \& Hayward, 1990; Lavy \& van den Hout, 1993), Stroop interference does not necessarily seem to indicate anxiety. In line with this, Channon and Hayward (1990) explained the observed attentional bias for food cues of fasting and eating-disordered subjects in terms of restriction. They state that the Stroop task provides "an objective

1 Published as: Overduin, J., Jansen, A. \& Louwerse, E. (1995). Stroop interference and food intake. International Journal of Eating Disorders 18, 277-285. 
index of current food restriction" (p. 451). But this conclusion might be a bit premature. For example, Green and Rogers (1993) found slower colournaming of foodwords in restrained eaters, irrespectively of whether the subjects were currently dieting or not. Therefore, although food restriction may result in hyperattention to food cues, hyperattention to food cues presumably isn't exclusively related to food restriction.

Another explanation of hyperattention was offered by Lavy and van den Hout (1993). According to them hyperattention might be related to an increased urge to act that may be reflected either in the avoidance of threatening, or in the approach of appetitive cues. From this one could derive the hypothesis that hyperattention to food words may not only be triggered by deprivation but, more generally, by stimuli that are desired strongly.

In the present study we wanted to investigate whether it is possible to instantaneously induce an attentional bias in subjects by providing them with an appetizer, i.e. a small amount of food that tastes "moreishly". Secondly, conceming the urge-to-act hypothesis, we wanted to assess the relation between Stroop interference and subsequent ad libitum food intake.

Results found on Stroop interference for separately presented body shape-related words appear to be more equivocal. Channon et al. (1988) failed to find an enlarged interference effect for shape-related words in anorexic subjects. Ben-Tovim et al. (1989) reported a trend for Stroop interference on shape-related words in anorexic and bulimic subjects whereas Ben-Tovim and Walker (1991) demonstrated a significant interference effect in bulimic as well as anorexic subjects. Mahamedi and Heatherton (1993) found that eating a preload lead to an increase in attentional bias for body shape words, especially in restrained eaters. However, after a comparison of the post-preload attentional bias in restrained and unrestrained eaters one cannot conclude that bias was reliably stronger in restrained eaters. Results from a within subjects study by Ogden and Greville (1993) in which attentional biases before and after a preload were assessed point in a similar direction.

The subjects in our experiment were non-clinical restrained and unrestrained eaters. If the emotional concerns explanation of Stroop interference for body shape words were true, one would expect larger reaction times for restrained eaters whether they would have eaten an appetizer or not. The urge-to-act explanation of attentional bias, on the other hand, is based on approach and withdrawal behavior and does not lead to strong expectations about Stroop reaction times for body shape words. 


\section{METHOD}

\section{Subjects}

Initially, around 100 female university students were approached to participate in a 'study on life-style' by filling out a 'life-style questionnaire'. Four key eating behaviour items were included in the list (e.g. "I repeatedly find it necessary to engage in dieting" and "My eating behaviour is normal when I'm in the presence of others but uncontrolled when I'm alone"). After that, 51 of the most extreme scorers (high and low) on these items were invited to participate in the experiment. At the end of the experiment the subjects completed the Restraint Scale (Herman, Polivy, Pliner, Threlkeld \& Munic, 1978) in order to prevent priming concerns about weight, shape and food intake. Every subject was termed high or low restrained on the basis of the median RS-score (being a score of 10 ) of the sample. Characteristics of the two groups are shown in Table 7.1.

Table 7.1. Characteristics of Restrained and Unrestrained subjects in this study

\begin{tabular}{lccccccc}
\hline & \multicolumn{2}{c}{ Unrestrained } & \multicolumn{2}{c}{ Restrained } \\
& \multicolumn{2}{c}{$(\mathrm{N}=25)$} & \multicolumn{2}{c}{$(\mathrm{N}=26)$} & \multicolumn{2}{c}{ t-test } \\
mean & sd & mean & sd & t & p-value \\
Body Mass Index & 20.7 & 1.3 & 22.3 & 2.7 & 2.64 & .01 \\
Age & 23.3 & 2.4 & 22.6 & 1.9 & 1.16 & ns \\
Restraint Scale & 6.8 & 2.5 & 15.0 & 3.5 & 9.66 & $<.001$ \\
\hline
\end{tabular}

\section{Materials}

Food The appetizer consisted of 60 grams of bavaroise pudding. Three flavours of ice cream (vanilla, strawberry, and chocolate) were used for the ad lib taste-test. The ice was arranged on a plate in such a way that eating would leave only a marginal visual trace.

Stroop program The Stroop task was run on a personal computer. In the middle of the screen words were shown one by one in 8 -mm block letters against a black background. The subject's voice was detected by a microphone connected with a sounddetector which registered response latencies in milliseconds. As soon as a sound was detected, the word disappeared from the screen. The time interval between presentation of consecutive words was 2 seconds. In case of wrong answers or disturbing sounds a 'missing value' was recorded.

Stroop words For every subject a total of 120 words was presented. The words were picked from four arrays (Table 7.2) in such a way that at the end of the task each of the 40 words had been shown three times. The colour of every word (either red, blue, green or yellow) was randomly chosen by the computer program. 


\section{Chapter 7}

There were two categories of 'neutral' words. The office equipment words had been added to establish a baseline with which reaction times for the target words were compared. The second category involved life-style words, so as to strengthen the impression that the experiment indeed was a study on life-style and perception. Response latencies for these words were not used in the data analysis.

Table 7.2. Words used in the modified Stroop task

\begin{tabular}{llll}
\hline \multicolumn{2}{c}{ TARGET WORDS } & \multicolumn{2}{c}{ NEUTRAL WORDS } \\
eating & shape & office & life-style \\
bun & balance & desk & ambition \\
cake & belly & envelope & appointment \\
candy & bikinis & felt-pen & bonus \\
chips & cheeks & file & career \\
chocolate & fat & paper & dedication \\
ice cream & hips & pen & manager \\
liquorice & legs & pencil & promotion \\
pie & slim & ruler & study \\
pudding & thighs & scissors & success \\
tart & thin & tape & working \\
\hline
\end{tabular}

\section{Procedure}

Subjects were told that we were studying influences of life-style on perceptual processes such as taste, smell, and visual perception. They were not informed that the test would involve highly palatable food.

The subjects were asked to have a standard meal of two sandwiches 3 hours before the experiment, and to refrain from eating in the period thereafter. They were randomly assigned to either the appetizer or the no-appetizer condition and were tested individually. After entering the laboratory, each subject received information about how to carry out the Stroop task. First, a short Stroop task with neutral words (the number one to ten) was run for practice. Then, in the appetizer condition, the pudding was presented and the subject was asked to eat it while concentrating on its colour, fragrance, and taste. Subjects in the control, no-appetizer-condition continued immediately with the Stroop task.

After the task three pre-weighed plates of ice cream were presented together with an icecream taste-evaluation questionnaire. The experimenter announced that he would leave the room for 15 minutes, during which the subject should complete the questionnaire. It was pointed out that tasting the ice cream would be helpful in completing the question- 
naire and that the subject was allowed to eat as much as she wanted or thought necessary. After the taste test the experimenter returned to administer the Restraint Scale. The ice cream that was left was taken to another room and re-weighed by the second experimenter. Finally, the subject was debriefed and paid for participation.

\section{RESULTS}

The Stroop interference scores for every target (i.e. eating and shape) category was defined as the median of response latencies for that category minus the median of response latencies for the neutral category. Reaction times above 1500 and below 250 milliseconds were excluded from the analysis. Standard deviations are shown between parentheses.

\section{Manipulation check}

Subjects scored the palatability of the bavaroise pudding (i.e. the appetizer, on a Visual Analogue Scale, ranging from 0 "not good at all" to 100 "extremely tasty". The overall mean score was 57.9 (s.d. 24.8) meaning that, although the pudding was not rated as extremely tasteful, at least it was considered reasonably palatable. No difference between restrained and unrestrained subjects in this respect was found $[\mathrm{t}(22)=1.08, \mathrm{NS})]$.

Table 7.3. Interference times for food words (milliseconds)

\begin{tabular}{|c|c|c|c|c|c|c|}
\hline & \multicolumn{3}{|c|}{ Unrestrained Eaters } & \multicolumn{3}{|c|}{ Restrained Eaters } \\
\hline & mean & sd & $\mathrm{N}$ & mean & sd & $\mathbf{N}$ \\
\hline No-appetizer & -6.0 & 30.3 & 13 & 17.1 & 29.4 & 14 \\
\hline Appetizer & 25.8 & 34.6 & 12 & 20.9 & 30.1 & 12 \\
\hline
\end{tabular}

\section{Food Words}

Table 7.3 shows the Stroop interference times for food words. A $2 \times 2$ ANOVA revealed a main effect of condition $(F(1,49)=4.18 ; p<.05)$, but no group effect. The condition by group interaction showed a weak trend $F(1,49)=2.57 ; p=.12$ ). It appeared that in the no-appetizer condition restrained subjects showed a significantly greater Stroop interference than unrestrained subjects $[\mathrm{t}(25)=2.01, \mathrm{p}<0.03]$.

Table 7.4. Ad lib intake of ice-cream (grams) 


\begin{tabular}{lcccccc}
\hline & \multicolumn{3}{c}{ Unrestrained Eaters } & \multicolumn{3}{c}{ Restrained Eaters } \\
& mean & sd & $\mathrm{N}$ & mean & sd & $\mathrm{N}$ \\
No-appetizer & 95.1 & 54 & 13 & 136.1 & 83 & 14 \\
Appetizer & 121.5 & 90 & 12 & 74.3 & 61 & 12 \\
\hline
\end{tabular}

It can be further seen that among unrestrained subjects, the ones in the appetizer condition showed significantly greater Stroop interference than those in the no-appetizer condition $(t(23)=2.44 ; p=.01$, one-tailed). In restrained subjects, no effect of condition was found $t(24)=.33$; NS).

\section{Food intake}

The food intake data are presented in Table 7.4. A $2 \times 2$ ANOVA showed no main effects for group or condition. However, a significant group by condition interaction was found $(F(1,49)=4.58 ; p<.04)$. Oddly enough, in the appetizer condition only the restrained subjects appeared to have lowered (i.e. regulated) their food intake relative to subjects in the no- appetizer condition $(\mathrm{t}(24)=2.19 ; \mathrm{p}=.04)$; among unrestrained subjects no effect of condition was found $(\mathrm{t}(23)=.88$; NS). For all subjects taken together, there was no strong $\mathrm{P}-\mathrm{M}$ correlation $(\mathrm{r}=0.27 ; \mathrm{n}=51$; NS) between the Stroop interference score for food words and ad libitum food intake. Interestingly, when correlation coefficients were calculated for the restrained and unrestrained subjects separately, the unrestrained group showed a firm correlation of 0.58 ( $\mathrm{n}=25$; $\mathrm{p}<$ $0.01)$, whereas in the restrained group no correlation was found $(r=-0.06 ; n=26$; NS).

Table 7.5. Interference times for shape-related words (milliseconds)

\begin{tabular}{lllllll}
\hline & \multicolumn{3}{c}{ Unrestrained Eaters } & \multicolumn{3}{c}{ Restrained Eaters } \\
& mean & sd & N & mean & sd & N \\
No-appetizer & 12.3 & 32.8 & 13 & 25.9 & 45.6 & 14 \\
Appetizer & 23.9 & 39.3 & 12 & 28.8 & 28.2 & 12 \\
\hline
\end{tabular}

\section{Body shape-related words}

In Table 7.5, Stroop interference times for shape-related words are presented. A $2 \times 2$ ANOVA did not show any main effects for condition and group or a condition $x$ group interaction. Clearly, restrained and unrestrained eaters did not differ in their level of 
Stroop interference for shape-related words. Moreover, for both unrestrained and restraint subjects eating the appetizer did not influence Stroop response latencies for shape-related words.

\section{DISCUSSION}

The main finding of the present study is that eating a small amount of palatable food triggers an attentional bias to eating-related stimuli in normal unrestrained subjects. This attentional bias strongly correlated with the amount of subsequent ad libitum food intake. In contrast to the unrestrained subjects, the restrained eaters were shown to be continually hyperattentive to eating-related words. Their subsequent food intake however appeared to be independent of hyperattention. Finally, restrained eaters did not show a larger attentional bias for body shape-related stimuli than non-restrained eaters.

What do these findings mean? In the first place, our data contradict the idea that an attentional bias to food related stimuli reflects a specific psychopathology of clinical eating disorders (Ben-Tovim \& Walker, 1991; Cooper \& Fairburn, 1993). Our non-clinical restrained subjects showed a permanent attentional bias to eating-related stimuli.

Secondly, the attentional bias in unrestrained subjects after ingestion of the appetizer suggests that Stroop interference per se does not reflect fear, emotional concerns, or deprivation. The firm correlation between Stroop latencies and food intake in unrestrained subjects suggests a selective attention to cues related to desires, cravings or urges. Interestingly, no correlation was found between Stroop food interference and food intake in restrained eaters. This could be because restrained subjects, by definition, normally tend to suppress their food intake. One could expect that Stroop interference scores and food intake will correlate strongly when restrained eaters show disinhibition. Unfortunately, in the present study restrained eaters in the appetizer condition seem to have regulated their intake because they ate less than those in the non appetizer condition. This lack of disinhibition might be explained by the size of our pudding, which was considerably smaller than the preloads in traditional counterregulation experiments (Jansen, Merckelbach, Oosterlaan, Tuiten \& van den Hout, 1988; Herman \& Mack, 1975; Hibscher \& Herman, 1977; Herman \& Polivy, 1980). It would thus be highly interesting to examine the relationship between Stroop interference and subsequent food intake in restrained eaters who indeed show disinhibited eating after a large preload.

A third interesting finding is that compared with unrestrained subjects, restrained subjects didn't show an especially large attentional bias toward body shape words, neither in the no-appetizer nor in the appetizer condition. These data seem to be in contrast with reports 


\section{Chapter 7}

of Stroop interference in bulimics (Fairburn et al., 1991; Cooper et al., 1992; Cooper \& Fairbum, 1993). In these studies however, it was not tested to which extend either shapeor the food-related target stimuli contributed to the effect. Also our findings are not entirely in accordance with findings of Ogden and Greville (1993) and Mahamedi and Heatherton (1993). The reason for this could have to do with the difference in methods. Mahamedi and Heatherton (like Fairburn et al.) used a card Stroop in which target words from the same category are presented together. In the Ogden and Greville study a computerized Stroop task was used, but again the target words were presented in blocks. Both methods introduce the danger that the subjects will ruminate about the meaning of the words during the task, and this could lead to longer reaction times, especially in restrained subjects who have just eaten a high-caloric milkshake. In the present study, words from target and neutral categories were presented one by one in a mixed fashion, which presumably diminishes the rumination effect. Undoubtedly, restrained eaters and eating-disordered subjects are overly concerned about their body shape. If interference as found by a computer-run Stroop task indeed reflects selective hyperattention to stimuli of personal or emotional valence, one would expect that restrained subjects show a greater Stroop interference for body shape-related words than unrestrained subjects do. However, the data do not support this hypothesis. If, however, the attentional bias reflects immediate avoidance or approach tendencies, one would not strongly expect an attentional bias for shape-related words: it is rather difficult to avoid or approach shape or body. In conclusion, it could be quite possible that card- or blocked versions of the Stroop measure rather emotional rumination on body words than attentional bias.

Finally, some remarks can be made about the value of the Stroop task in the assessment of treatment outcome for eating disordered patients. In spider phobics, Lavy, van den Hout and Amtz (1993) found a marginal correlation between pretreatment attentional bias and treatment (exposure) success. It might be hypothesized that a sustained post-treatment attentional bias for eating-related words could be a predictor of relapse in bulimic subjects. If that prediction is found to be true, the Stroop task may serve as an instrument for the assessment and predict of (long-term) treatment success. Recently, it has been shown that craving extinguishes during prolonged exposure with response prevention to cues that have become associated with the desired substance (Jansen, Broekmate \& Heymans, 1992; Powell, Gray \& Bradley, 1993). It is well-known too that craving can be triggered by exposure to appetitive cues (Weingarten \& Elston, 1990; Jansen et al., 1992) and therefore could constitute a causal factor in relapse. It could very well be that the conceptualization of craving as an approach behaviour leads to a promising application of the computerized Stroop technique in the study of eating disorders. 


\section{A NEW SCALE FOR USE IN NON-CLINICAL RESEARCH INTO DISINHIBITIVE EATING ${ }^{1}$}

\section{INTRODUCTION}

The food intake pattern of all bulimic and about half of the anorexic patients includes recurrent binge eating: during binges, control of food intake is lost and quantities of food are ingested that are larger than most people would eat under similar circumstances (Walsh, 1993). Lab paradigms of disinhibitive eating behaviour have been developed and studied in non-clinical subjects in order to unravel the underlying mechanisms (For the rationale behind this non-clinical approach, see Abramson \& Seligman, 1977). Questionnaire scores often provide the criterion of subject selection. In lab research into disinhibitive eating, the most widely used questionnaire has been the Restraint Scale (RS; Herman \& Polivy, 1980). This questionnaire has been shown to identify subjects prone to a form of disinhibitive eating called counterregulation. In the basic counterregulation paradigm subjects are "preloaded" by having them ingest a high-caloric food item, after which the food intake is assessed in an ad lib ice cream taste test. Subjects with high RS scores have been found to counterregulate, i.e. they show larger food intake when preloaded than when unpreloaded, whereas low scorers regulate their food intake and eat less when they have been preloaded previously (Herman \& Polivy, 1980). The replicable nature of counterregulation allowed for more extensive research on disinhibition through various modifications of the basic paradigm (for overviews see Lowe, 1993; Greeno \& Wing, 1994).

Although of all available questionnaires in the field, the Restraint Scale has been demonstrated the most effective predictor of counterregulation (Heatherton et al., 1988; Lowe, 1993), its use in disinhibition research has stirred up at least four critical issues.

The first issue relates to criticisms about some psychometric characteristics of the RS. Although the internal consistency of the scale is satisfactory, doubts have been raised about the stability of the internal structure of the scale. Different studies have shown factors to be composed of different items or items to be loading significantly on two factors at the same time. A most prominent example of the factorial instability is the collapse of the factor structure within a obese subject sample (e.g. Ruderman, 1983; for

${ }^{1}$ To be publisbed as: Overduin, J. \& Jansen, A. (1996). A new scale for use in non-clinical research into disinhibitive eating. Personality and Individual Differences, in press. Ellen Louwerse, Susan Bōgels and Peter de Jong are gratefully acknowledged for their aid in administering the questionnaires. Harrie Vorst (Methodology Department, University of Amsterdam) is thanked for commenting on some of our data. 
a reference of these criticisms and a reply see Heatherton et al., 1988). The instability of the factor structure may very well be a consequence of the wide array of themes tapped by relatively small numbers of items (i.e. weight fluctuations, diet frequency, negative evaluation of weight gain, binge eating). The factor instability suggests that not all constituent themes within the questionnaire are clearly mapped (see McDonald \& Mulaik, 1979). One solution to this problem might be to extend the scale with items highly specific to each of the themes; in this way each theme will eventually be covered specifically by one factor (Briggs et al., 1986). Indeed, such an approach has been employed in the development of new questionnaires, notably the Dutch eating Behaviour Questionnaire (DEBQ; van Strien, Frijters, Bergers \& Defares, 1986) and the Three factor eating behaviour Questionnaire (TFEQ; Stunkard \& Messick, 1985). However, although these questionnaires appear to have more clearly discernable subscales, recommendations have been given to use only one subscale at one time, either because the total scale was not intended to measure one construct (DBEQ; van Strien et al., 1986), or because inter-subscale correlations turned out to be insufficiently high (TFEQ; see Heatherton et al., 1988).

The second issue in using the RS bears upon the well-established finding that counterregulation of high RS-scorers appears mainly after ingestion of large, and not after small preloads. Importantly however, in recent years it has become clear that also small preloads or food cues (e.g. sight, smell and taste of food) can act as triggers of craving and increased subsequent food intake (Jansen \& van den Hout, 1991; Rogers \& Hill, 1989; Weingarten \& Elston, 1990). Analogously to developments in addiction research (e.g. Siegel, 1983; Childress, 1992; Drummond, Tiffany, Glautier \& Remington, 1995), new models of binge eating have been put forward emphasizing leamed cue reactivity as a maintenance factor (Jansen, 1990; 1994; Wardle, 1990). Cue reactivity means that stimuli predictive of food intake trigger a physiological state that is experienced as craving (Jansen, 1994). As to the predictive value of the Restraint Scale, disinhibitive eating after presentation of food cues has been found only in subjects with extreme RS scores (Jansen \& van den Hout, 1991). Hence, a subclass of high restrained eaters (i.e. those scoring slightly above the median RS score) did not show changes in eating behaviour after cue presentation. This may be a direct consequence of these subjects endorsing of the "concern for dieting" items in the Restrained scale (high RS scores can result from either strong concern for dieting or high disinhibition, or both; Westenhoeffer et al., 1994). Specific measures of concern for dieting, such as TFEQ and DEBQ have been found to identify successful dieters, i.e. those who do not frequently disininhibit (Heatherton et al., 1988; Lowe, 1993). This renders it likely that many high restrained eaters in this experiment deliberately inhibited their food intake during the taste test. Thus, although high scorers on the RS might possess considerable food cue reactivity, high "concern for dieting" may lessen chances on observable changes in food 
intake after presentation of food cues other than large preload.

Finally, neither in the RS nor in the DEBQ or TFEQ reference is made to self-esteem. Recently however, it has been shown that in particular high restrained eaters whose selfesteem is low do counterregulate (Polivy, Heatherton \& Herman, 1988). It has been proposed that the larger variability among high RS scorers in food intake within the lab can be attributed to different levels of self-esteem among individuals within this group.

The aim of the present study is to alleviate the above problems with using the Restraint Scale, which admittedly remains the questionnaire most predictive of at least one form of disinhibition, i.e. counterregulation. We attempted to develop a questionnaire for selection of subjects maximally prone to lab disinhibition to a broad range of cues, and which would have the following characteristics: a clearer factor structure than the RS, attenuation of the unwanted impact of concern for dieting items on food intake, and reference to self-esteem. Moreover, the scale should be internally consistent so that total scores could be used. We compared the new developed questionnaire with the Restraint Scale on two characteristics: psychometric properties, and characteristics of extreme groups selected on basis of scores.

\section{GENERAL METHODOLOGY}

All questionnaires were administered within a non-clinical population of students participating in courses, and volunteering university employees. All statistical analyses were carried out using the SPSS-X software program.

\section{DEVELOPMENT OF A NEW DISINHIBITION SCALE}

\section{Purpose and rationale}

A rational, construct-oriented approach (see Nunnally, 1978; Wilde, 1977) was adopted to develop a new scale for selecting individuals maximally susceptible to triggered disinhibitive eating in the lab. We reasoned that lab-disinhibition may be best predicted by items referring to the occurrence of disinhibition in daily life. Furthermore we considered that disinhibition occurs in three forms that should all be mapped to get a full picture: 1) eating, at times, too much "objectively" 2) eating too much "subjectively", i.e. cases in which the individual judges that self-imposed restrictions are overruled; 3 ) objective consequences of repeated disinhibition, i.e. weight fluctuations. To these three factors thought to predict lab disinhibition we added self-esteem as a fourth "risk" 


\section{Chapter 8}

factor. Our objective was to end up with a questionnaire containing subscales for each of the above factors clearly defined both semantically and psychometrically (see Briggs et al., 1986; Comrey, 1978; Nunnally, 1978).

\section{Construction of the initial item pool}

An initial pool of items addressing the above four themes (binge eating, failed restriction, weight fluctuations and self-esteem) was composed as follows.

Binge eating Item 6 of the Restraint Scale (see table 8.1), which evidently refers to binge eating practices, was extended with related items on overeating behaviours thought to be "socially somewhat unacceptable". Occurrence of binge eating in daily life, whatever its cause, was assumed to be a good predictor of disinhibition in the lab.

Failed restriction These items tapped on the inability to hold on to dietary restriction each time it's imposed. All of these items had a general format "If I try to impose restriction on my eating I fail". Our items deal very generally with the occurrence of disinhibition after attempts to restrict food intake. Putative causes of the disinhibition were not addressed. High scores on failed restriction items were assumed to contribute to disinhibition in the lab. Matters were arranged so that people who report to never self-impose eating restrictions received a zero score on these items.

Weight fluctuations High scores on weight fluctuation items of the Restraint Scale have been claimed to result from a chaotic eating pattern in which disinhibition follows attemps at dieting, a jojo-effect (Heatherton, Polivy, \& Herman, 1991; Herman \& Polivy, 1982; Lowe, 1993). Our weight fluctuations items were Restraint Scale items (nos. 2, 3 and 4), and a newly conceived item. A modification of Restraint scale item no. 10 was included as well: instead of the difference between prior maximum and currently desired weight, the difference between prior maximum and minimum weight was computed (see also Laessle et al., 1989). High scores on the weight fluctuations items were assumed to increase susceptibility to lab disinhibition.

Self-esteem Items on self-esteem were added not because self-esteem as such is part of the disinhibition concept, but because, as said earlier, low self-esteem has been found to facilitate the occurrence of disinhibitive eating in the lab (Polivy et al., 1988). The items were picked from the Rosenberg Self-esteem Scale (Rosenberg, 1965), and consisted of evaluations of the self in comparison with other people. The items were scored in such a way that answers in the direction of lower self-esteem received higher scores.

Answering format Most items were self-referring descriptions of behaviours or attitudes (self-esteem items). Respondents were to indicate the frequency with which each description applied to their daily situation. Except for items on weight fluctuations this was done on a 5-point Likert scale ranging from 0 points ("never") to 4 ("always"). To 
diminish the possibility of acquiescence (Nunnally, 1978) some items were phrased inversely to disinhibition and its scoring direction should be reversed. The "failed restriction" items had a sixth alternative added, to allow for the possibility that a respondent never self-imposes restrictive eating. This alternative was scored zero.

\section{Item analysis and construction of the Disinhibitive Eating Scale}

The item pool consisting of 36 items was administered to 350 female students and university employees who were approached within the area of the university building (Body Mass Index $20.93 \pm 2.2$; age $23.33 \pm 4.4$. A negligible proportion (about $3 \%$ ) of those approached, declined to participate in the investigation. Item analysis was conducted on basis of a principal components analysis with orthogonal rotation of the extracted factors, as well as assessment of item-total correlations. Items loading poorly on the extracted factors and/or having low item-total correlations were removed from the pool. Some of the binge eating items were removed to prevent relative overrepresentation of these items in the remaining item pool. Finally, the item phrasing were reconsidered and in some cases simplified. The thus formed Disinhibition Scale (DIS) consisted of 17 items divided within 4 subscales (see table 8.2 for the subscale structure and item phrasings): "binge eating" (5 items), "failed restriction" (4 items), "weight fluctuations" (4 items) and "self-esteem" (4 items). The 5-point Likert-format of items was maintained.

\section{TESTING THE RESTRAINT AND DISINHIBITION SCALES}

\section{Subjects and method}

Both Restraint Scale and the newly developed disinhibition scale (DIS) were administered to 269 females (BMI $21.03 \pm 2.4$; age $19.8 \pm 3.0$ ) who were participating in a freshman course at the psychology department. The Restraint Scale was scored according to the requirements introduced by Herman \& Polivy (1980), the DIS according to those in table 8.2. After the establishment of the normative data, three psychometric properties of the questionnaires were investigated: the factor structure (using a principal components analysis with subsequent rotation of the extracted factors confirming to the eigenvalue $>1$ as well as the scree criterion), level of internal consistency (as revealed by Cronbach's Alpha), and item-total correlations and correlations between subscales (only for the DIS). Then, the relationship between the two questionnaires was established by correlations.

Finally the relationship between dieting frequency and the DIS was explored. The 


\section{Chapter 8}

formulation of the DIS items on failed restriction is conditional: "if I try to restrict my eating then I fail". No explicit reference is made to the frequency with which attempts are made to restrict food intake, although "diet frequency" has been claimed to be influential in disinhibition after exposure to binge cues (Lowe, 1993). To elucidate how diet frequency fits the structure of the DIS, a new factor analysis was carried out on all DIS items extended with the Restraint Scale item no. 1 (i.e., the frequency of dieting item).

\section{Table 8.1. Factor structure of the Restraint Scale}

\begin{tabular}{|c|c|c|}
\hline & F-1 & F-2 \\
\hline 1. How often are you dieting? & .75 & .34 \\
\hline $\begin{array}{l}\text { 2. What is the maximum amount of weight (in kilos) } \\
\text { that you have ever lost in one month? }\end{array}$ & .15 & .65 \\
\hline 3. What is your maximum weight gain within a week? & .14 & .81 \\
\hline 4. In a typical week, how much does your weight fluctuate? & .07 & .79 \\
\hline $\begin{array}{l}\text { 5. Would a weight fluctuation of } 5 \mathrm{lbs} \text { affect the way } \\
\text { you live your life? }\end{array}$ & .76 & -.08 \\
\hline 6. Do you eat sensibly in front of others and splurge alone? & .39 & .44 \\
\hline 7. Do you give too much time and thought to food? & .67 & .32 \\
\hline 8. Do you have feelings of guilt after overeating? & .71 & .41 \\
\hline 9. How conscious are you of what you're eating? & .58 & .08 \\
\hline $\begin{array}{l}\text { 10. How many pounds over your desired weight were you at } \\
\text { your maximum weight? }\end{array}$ & .48 & .49 \\
\hline
\end{tabular}

\section{Results}

Restraint Scale The factor analysis revealed a structure with two factors with eigenvalue larger than 1, accounting for $54.0 \%$ of the common itemvariance, the structure of which is shown in table 8.1. As can be seen, three items had considerable (i.e. $>.40$ ) loadings on both factors. Cronbach's alpha for the total scale was .83 which reflects a fully satisfactory level of internal consistency. The item-total correlations ranged from .38 to .70 (mean .53).

Disinhibitive Eating Scale The factor structure (table 8.2) shows four factors with eigenvalue larger than 1 , accounting for $67.7 \%$ of the common itemvariance and having each item loading considerably (above .40) only on the factor prototypical of its subscale, and not on the other factors. Thus the factor pattern approached the Thurstonian 'simple' factor structure (Nunnally, 1978) Internal consistency of the total scale (alpha $=.88$ ) and subscales (alpha's ranging from .76 to .88 ) were good, as were the it- 
Subscale bingeing $(\alpha=0.88)$

2 . At times I lose control over my eating behaviour"

5. I eat sensibly when with others, but I gorge myself

$\begin{array}{llll}.67 & .29 & .27 & .23 \\ .75 & .22 & .25 & .08 \\ & & & \\ .82 & .15 & .17 & .19 \\ .77 & .13 & .19 & .06 \\ .81 & .14 & .06 & .24\end{array}$

8. If others saw how much I ate, then I'd feel ashamed

11. I eat so much at one go that it is actually disgusting other people

Subscale self-esteem $\quad(\alpha=0.85)$

3. I have the feeling that other people do things better than me

6. There are many things about myself that I would change if possible

9. I'd rather be a different person than 1 am now

12. I'm very satisfied with the way I am (-)

$\begin{array}{llll}.20 & .77 & -.03 & -.08 \\ .11 & .86 & .06 & -.02 \\ & & & \\ .21 & .84 & .12 & .06 \\ .17 & .75 & .13 & .18 \\ & & & \\ .16 & .07 & .77 & .11 \\ & & & \\ .14 & -.05 & .82 & .13 \\ .13 & .13 & .83 & .13 \\ .37 & .16 & .73 & .15\end{array}$

Subscale unsuccessful dieting $(\alpha=0.85)$

1. When I'm watching my weight, I succeed in not touching food items (-)

4. When I'm dieting, I manage not to eat between meals (-)

10. When I'm dieting, $I$ can't control myself and start eating 'forbidden foods'

\section{Subscale Weight fluctuations $(\alpha=0.76)$}

14. How strongly has your weight fluctuated in the past few years?

15. What is the maximum weight you have lost in one month?

16. What is the average variation in your weight in one week? I

Difference score of:

17. What has been your highest weight since you were 18 (excluding pregnancy)?

18. What has been your lowest weight since you were 18 ?

$\begin{array}{llll}.34 & .08 & .18 & .73 \\ .08 & -.07 & .08 & .83 \\ .10 & .05 & .19 & .60\end{array}$

(-) negatively keyed item

answering format: $0=$ never; $1=$ sometimes; $2=$ regularly; $3=$ often; $4=$ always; (items 1 through 13)

answering format: $0=$ not at all; $1=$ bardly at all; $2=$ moderately; $3=$ considerably; $4=$ extremely

answering format: $0=$ less than $0.5 \mathrm{~kg} ; 1=0.5$ to $1 \mathrm{~kg} ; 2=1$ to $2 \mathrm{~kg} ; 3=2$ to $4 \mathrm{~kg} ; 4=4 \mathrm{~kg}$ or more

I answering format: $0=$ less than $0.5 \mathrm{~kg} ; 1=0.5$ to $1 \mathrm{~kg} ; 2=1$ to $1.5 \mathrm{~kg} ; 3=1.5$ to $2.5 \mathrm{~kg} ; 4=2.5 \mathrm{~kg}$ or more

answering format: $0=$ less than $3 \mathrm{~kg} ; 1=3$ to $5 \mathrm{~kg} ; 2=5$ to $7 \mathrm{~kg} ; 3=7$ to $10 \mathrm{~kg} ; 4=10 \mathrm{~kg}$ or more 


\section{Chapter 8}

em-total correlations (range .33 to .69 , mean .51). In general, correlations between subscales were moderately positive (table 8.3 ).

Correlation and comparison of the Restraint and Disinhibition Scales The correlations between RS and DIS total scores was .78. Factor analysis of the DIS items together with the diet frequency showed the original DIS factor structure, with the diet frequency item loading .37 on the failed restriction, and .57 on the weight fluctuations factors. This shows that not so much the failed restriction subscale (with items phrased conditionally), but the weight fluctuations subscale accounted for the frequency of failed attempts at dietary restriction.

Table 8.3. Inter-subscale correlations of the Disinhibitive Eating Scale

\begin{tabular}{lccc} 
& Bingeing & Failed restriction & Self-esteem \\
Bingeing & - & & \\
Failed restriction & .49 & - & - \\
Self-esteem & .44 & .24 & .13 \\
Weight fluctuations & .41 & .35 & \\
\hline
\end{tabular}

\section{NORMATIVE DATA AND CHARACTERISTICS OF SUBJECT GROUPS WITH EXTREME DIS AND RS SCORES}

\section{Subjects and method}

First, we established the population distribution of DIS and RS total scores within our sample of 269 female students. Then, for each questionnaire three pairs of extreme scoring groups were formed according to the following criteria. 1) below vs. above the median; 2) below 25 th vs. above 75 th percentile; 3 ) below 10 th vs. above 90 th percentile.

For each of the three criteria, we assessed to what extent the groups formed on basis of DIS and RS consisted of the same individuals. This comparison bears upon divergent validity of the DIS: if the DIS would select the same individuals as the RS, then no purpose would be served by using it.

For each questionnaire separately, high and low scoring groups were compared on BMI and current dieting. The current dieting item was phrased simply: "are you presently dieting to lose weight?" and could be answered yes or no. Three such comparisons, i.e. one for each selection criterion, were made. Difference in BMI were tested with t-tests; percentage of individuals dieting by Chi square tests. 


\section{Results}

Normative data of DIS and RS total scores are presented in table 8.4. Mean score for the RS was 11.66 (sd 5.5) and for the DIS 22.93 (sd 10.9). Strikingly, the RS median was 11, i.e. considerably lower than in Northern American samples. The overlap of extreme groups formed by selection by DIS and RS are shown in figure 8.1. It is clear that the composition of groups selected by either DIS or RS differed, and that the difference was larger when more extreme criteria are used for selection.

Table 8.4. Population distribution of Restraint Scale and Disinhibitive Eating Scale total scores ( $N=269$ females)

\begin{tabular}{ccc}
\hline Percentile & RS & DIS \\
$\mathbf{5}$ & 3 & 7 \\
$\mathbf{1 0}$ & 4 & 9 \\
$\mathbf{2 0}$ & 6 & 12 \\
$\mathbf{2 5}$ & 7 & 14 \\
$\mathbf{3 0}$ & 8 & 15 \\
$\mathbf{4 0}$ & 9 & 18 \\
$\mathbf{5 0}$ & 10 & 21 \\
$\mathbf{6 0}$ & 12 & 24 \\
$\mathbf{7 0}$ & 13 & 27 \\
$\mathbf{7 5}$ & 14 & 29 \\
$\mathbf{8 0}$ & 16 & 31 \\
$\mathbf{9 0}$ & 19 & 37 \\
95 & 22 & 43 \\
\hline
\end{tabular}

Differences in current dieting an BMI of extremely high and low scoring groups on DIS and RS are shown in table 8.5. The results for DIS and RS were comparable and consistent: high scorers had larger BMI and higher prevalence of current dieting than low scorers. Interestingly, among individuals scoring above the RS median i.e. the group traditionally selected for lab research on counterregulation, less than half was currently dieting ${ }^{2}$. Even of the individuals with RS scores within the highest ten percentiles, only $65 \%$ reported to be currently dieting.

Also for BMI, significant and large group differences appeared irrespectively of the used group allocation criterion. The size of differences were as large as between 1.5 population standard deviations of BMI. The correlation of the BMI with RS and DIS scores were respectively 0.48 and 0.52 .

\footnotetext{
${ }^{2}$ Parenthetically, only $16.5 \%$ of all subjects reported to be currently dieting.
} 
Table 8.5. Comparisons of extreme scoring subject groups, using three criteria for Restraint Scale and Disinhibitive Eating Scale scores: a. median split; b. $<25$ th vs $>75$ th percentile; c. $<10$ th vs $>90$ th percentile. $(N=269$ lemales)

\begin{tabular}{|c|c|c|c|c|c|c|c|c|}
\hline \multirow{2}{*}{\multicolumn{3}{|c|}{$\begin{array}{l}\text { RESTRAINT SCALE } \\
\text { cut-off scores }\end{array}$}} & \multicolumn{4}{|c|}{ Body Mass index } & \multicolumn{2}{|c|}{$\%$ currently dieting } \\
\hline & & & \multicolumn{2}{|c|}{ low scorers } & \multicolumn{2}{|c|}{ high scorers } & \multirow{2}{*}{$\begin{array}{c}\text { low scorers } \\
4.7\end{array}$} & \multirow{2}{*}{$\begin{array}{c}\text { high scorers } \\
44.7^{4}\end{array}$} \\
\hline $\mathbf{a}$ & $\leq 11$ & $>11$ & 19.9 & 1.7 & 22.0 & $2.6^{\circ}$ & & \\
\hline b & $<8$ & $>14$ & 19.3 & 1.4 & 22.7 & $2.9^{-}$ & 1.6 & $56.9^{+}$ \\
\hline c & $<5$ & $>19$ & 19.0 & 1.3 & 22.1 & $2.7^{\circ}$ & 0 & $65.2^{+}$ \\
\hline \multicolumn{4}{|c|}{ DISINHIBITIVE EATING SCALE } & \multicolumn{3}{|c|}{ Body Mass index } & \multicolumn{2}{|c|}{ \% currently dieting } \\
\hline & \multicolumn{2}{|c|}{ cut-off scores } & \multicolumn{2}{|c|}{ low scorers } & \multicolumn{2}{|c|}{ high scorers } & low scorers & high scor \\
\hline $\mathbf{a}$ & $\leq 21$ & $>21$ & 20.0 & 1.7 & 22.1 & $2.6^{\circ}$ & 10.3 & $41.4^{+}$ \\
\hline b & $<15$ & $>29$ & 19.5 & 1.7 & 22.9 & $2.8^{\circ}$ & 2.9 & $44.1^{\dagger}$ \\
\hline c & $<10$ & $>37$ & 19.2 & 1.7 & 23.3 & $3.5^{\circ}$ & 3.6 & $57.1^{+}$ \\
\hline
\end{tabular}

- t-test for group differences: $\mathrm{p}<.001$

' chi-square test: $\mathrm{p}<.001$

\section{DISCUSSION}

The results of the psychometric comparison of DIS and the RS in a non-clinical sample of 269 female students showed comparably good internal consistency in the two questionnaires. Factor analyses yielded a two-factor solution in the RS, and a four-factor solution in the DIS. The RS total score distribution in the sample had a median of 11. The correlation between the two scales was 0.78 . It was found that high scorers on either RS or DIS have a considerably higher Body Mass Index and a higher prevalence of current dieting than did low scorers. Finally, it appeared that groups formed on basis of extreme DIS scores were partly composed of different individuals than groups formed on basis of extreme RS scores, and that this difference grew larger when more extreme scores were used as allocation criterion.

The psychometric analysis of the RS confirms earlier reports (for example, Heatherton et al., 1988) of a good value of Cronbach's alpha. Once again the existence of two factors has been demonstrated, which in this case do not differ from the concern for dieting and weight fluctuations factors. It should be noted however that it can be suspected on statistical grounds ( $46 \%$ of the item variance was not explained by the two factors; 3 items loaded > .40 on both factors) as well as on the relatively wide ranging item content, that the RS 
scores potentially encompasses more than two concepts, but that these concepts would emerge as factors if more items were added to the RS. Of course there is little news to this conclusion and discussions on the most fruitful interpretation of restraint scores continue (Charnock, 1989a, 1989b; Dritschel, Cooper \& Charnock, 1993; Heatherton et al., 1988; Lowe, 1993; Ogden, 1993; Polivy \& Herman 1989). Our finding of a median RS score of 11 in our sample of Dutch female students was a confirmation of our earlier experience (Overduin \& Jansen, unpublished data) and of other reports on European subject samples (Wardle, 1986; Laessle, 1989). The implication is that subject selection on basis of an assumed median score of 15 (as found in Northern American samples) may not be appropriate in research within the average European female population.

The DIS was found to show the psychometric characteristics for which it was developed. In the first place a good internal consistency while at the same time all items can be said to be referring to disinhibition or a risk factor for disinhibition. Said otherwise, the total DIS scores appear to be both statistically and semantically meaningful. Secondly, a fair level of common item variance $(67.7 \%$ ) was explained by a well-interpretable four-factor structure with each item loading considerably on only one factor. Importantly, all factors were concordant with the a priori conceived subscales. The resulting advantage is that the DIS total score is based upon a transparant set of meaningful subscale scores. The relative contribution of each subscale to the total DIS score can thus be analyzed for each individual. Finally, the added self-esteem subscale formed an independent factor and the correlation with the rest of the DIS turned out to be moderately positive $(r=0.35$ ). Thus, addition of self-esteem items, expected to heighten the predictive power of the DIS as to lab disinhibition succeeded without compromising the internal consistency or factor structure of the DIS.

Two similarities between the DIS and RS are evident. In the first place a high correlation between the two scales was found, which is not too surprising because of a considerable overlap of item content (e.g. weight fluctuations subscale and RS item no. 6). Secondly, both scales have moderate to strong positive correlations with Body Mass Index. As a consequence in comparisons of high and low scoring groups on food intake in the lab, these differences in BMI should not be neglected. The same applies for the prevalence of current dieting which differs for high and low scorers on both scales. This covariation once again appears to be a fundamental problem in research on disinhibitive eating. Moreover, the fact that among high scorers on DIS and RS only a slight majority (i.e. far from 100\%) is currently dieting, indicates the necessity of carefully distinguishing between general dieting frequency and current dieting when studying behaviours of subjects selected by means of these questionnaires (see e.g. Lowe, 1993).

Also differences between the DIS and RS were found, the most important of which is 


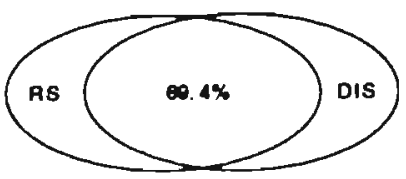

a

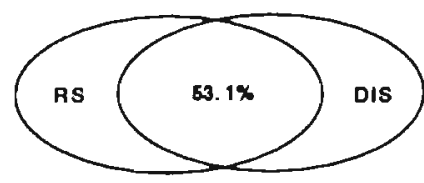

b

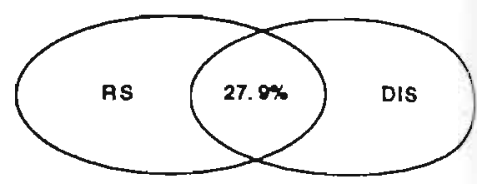

c

Figure 8.1. Percentage of subjects selected by both RS and DIS when applying different group selection criteria: a. median split; b. $<25$ vs. $>75$ th percentile; c. $<10$ th vs. $>90$ th percentile.

practical: in part, different individuals are selected by the two scales when extreme scores are used as a criterion. Both scales have appeared to be equally internally consistent, so that the reasons and possible consequences of selection on basis of DIS scores must be sought for in the item content. Firstly, in contrast to the RS, the DIS contains no concern for dieting items. The aim of the two scales differed: the RS was designed to identify dieters (Polivy \& Herman, 1989; Heatherton, 1988), whereas the DIS was designed for prediction of maximal proneness to lab disinhibition. Of course, the absence of explicit concern for dieting items in the DIS does not at all imply that high DIS-scorers are unconcerned with dieting. What it does imply however, is that relative to the RS, the impact of concern for dieting has been attenuated in the DIS total scores. And, as has been pointed out in the introduction, because individuals high on concern for dieting will have the inclination to inhibit their food intake in the lab, attenuation of its contribution to DIS-scores has the advantage that subjects are selected whose reactivity to food cues will be reflected in eating behaviour also in procedures in which small preloads or food cues are presented. In other words, the chances of false negative findings (i.e. inhibition in subjects with high cue reactivity) will be decreased. Secondly, in contrast to the RS, self-esteem items are included in the DIS. High DIS scorers will have low self-esteem, whereas among high restaint scorers there could be either high or low in self-esteem, thus accounting for different composition of extreme scoring groups. A last relevant issue is that of content validity: to what extent does the DIS address factors related to pathological eating behaviour? Content validity is relevant if one wishes to eventually apply lab results to clinical cases. The content validity of the DIS is suggested by research of Tobin, Johnson, Steinberg, Staats and Dennis (1991), who factor-analyzed a large and complete self-report battery on bulimic symptoms in patients: four factors of the six factors found, i.e., weight fluctuations, binge eating, self-esteem and failed restriction are also present within the DIS. The remaining two factors found by Tobin et al. were family history, and drive for thinness, the latter being similar to 
concern for dieting which was not included in the DIS for reasons explained carlier. All in all, personality factors important in pathological disinhibitive eating can be found in DIS scores to a reasonable extent.

The DIS has been shown to be a conceptually and psychometrically sound selection instrument which taps a construct that is related, but not equal to that measured by the RS. Different emphases exist in the DIS and RS, which partly makes for selection of subject groups of different composition. The DIS was constructed to alleviate some of the issues related to use of the RS in cue reactivity research. Naturally, its predictive power as to lab disinhibition remains to be demonstrated empirically. 



\section{Chapter 9}

\section{CONCLUSIONS AND GENERAL DISCUSSION}

In this final chapter, the results of the empirical studies of the present thesis will be summarized (9.1). After a reappraisal of its physiological assumptions (9.2), a final conclusion will be formulated about the classical conditioning theory of binge eating (9.3). Finally, theoretical and practical suggestions for future research will be made in sections 9.4 and 9.5 respectively.

\subsection{OVERVIEW OF THE EMPIRICAL WORK IN THIS THESIS}

After the description of the conditioning theory of binge eating (CBE) in chapter 2, subsequent chapters were devoted to empirical tests of related hypotheses. In two chapters, the results of conditioning procedures were described. Chapter 3 described a classical conditioning procedure that was run in order to evoke a physiological state which, according to $\mathrm{CBE}$ theory, precedes binge eating. On six occasions, olfactory/taste cues (i.e., the conditioned stimuli, CSs) were paired with glucose ingestion (the unconditioned stimulus, UCS). Subjects in the control condition received the metabolically inert sweetener aspartame as the UCS. At test trials (before and after the conditioning procedure) the CS compound was presented without the UCS, and levels of subjective craving, insulin and blood sugar measured. Subsequently, ad lib glucose intake was measured in a taste test. CBE hypotheses for the post-conditioning test day were that CS presentation would elicit the following responses: conditioned hypoglycaemia, conditioned hyperinsulinaemia, increased craving for sweet snacks, and increased glucose intake. These hypotheses could not be confirmed, except for a small group difference in insulin response, which, however, did not exceed baseline fluctuations. Furthermore, in both conditions, intake of glucose was considerably larger after than before conditioning. As this behaviour was not mirrored in ratings of subjective craving, it was attributed to a "neophobia" or "bait shyness" that had occurred during the pre-conditioning test session, but faded over the subsequent conditioning trials, a phenomenon which has been described previously in the animal literature. In order to investigate whether repeated exposure to sweet drinks (UCS) was a critical factor, a similar conditioning procedure was run additionally with plain water as a UCS. Also in this condition, glucose intake was increased after the procedure. It was therefore concluded that the disappearance of the subjects' "neophobia" had been related to their getting acquainted with the lab procedure, rather than with the taste of sweet drinks. Chapter 4 contained a comment on, as well as a re-analysis of a not widely known study on hypoglycaemic conditioning 


\section{Chapter 9}

by the Russian physiologist Mityushov (1954; Appendix, this thesis). In contrast to our conditioning study described in chapter three, Mityushov was able to demonstrate a statistically as well as physiologically significant conditioned hypoglycaemia in humans and dogs, after a procedure involving repeated pairing of an injection ritual (CS) with intravenous glucose (UCS). Further observations suggested that the hypoglycaemia was secondary to a conditioned secretion of insulin. Although Mityushov's report would not fully meet today's methodological standards (e.g. no control group was run; no overall statistics were reported), it was concluded that his conditioning procedure evoked a physiological state that, according to the CBE theory, occurs prior to bingeing.

The next two chapters investigated psychophysiological cue reactivity to food related stimuli. In CBE terms, cue reactivity could be considered as a residual of learning history. In chapter 5 reactions to slides with binge-related food items were studied. The CBE prediction in this study was that cue reactivity would be larger in restrained than in unrestrained eaters. This was hypothesized because restrained eaters can be considered "unsuccessful dieters" who regularly engage in (non-clinical) binge eating. Hence, they should display especially intense conditioned responses to food cues. A manipulation check revealed that subjective hedonic ratings of the food slides were higher than those for control slides (depicting office equipment). Nevertheless, no increased psychophysiological cue reactivity was found in restrained subjects, so that the CBE hypothesis was not confirmed. The study in chapter 6 assessed psychophysiological reactivity to the sight and smell of attractive food. Two groups of normal subjects were compared, one of which had been fasting for the previous 24 hours. It was thought that the fasting state would form a baseline to which food craving could be easily elicited by food cues, and that the related physiological responses would be more clearly discernible. The hypotheses were that subjective craving to food cues would be larger in fasting than nonfasting subjects, and, more specifically, that these differences would be mirrored in the intensity of the psychophysiological responses. As expected, it was found that food item presentation elicited more craving in fasted than in non-fasted subjects. However, no group differences were found in psychophysiological cue reactivity, a finding which was not in line with the CBE hypothesis.

Chapter 7 contained an experimental test of the cognitive effects of cue-elicited craving. Restrained and unrestrained eaters were compared on their attentional bias to food words measured in a modified Stroop colour-naming test. There were two conditions. In the appetizer condition, subjects had to eat a spoonful of palatable pudding, whereas in the control condition no appetizer was ingested. Then, the modified Stroop test was run, comparing, among other things, colour naming latency for food and neutral words. After the Stroop test, ad lib ice cream intake was measured during a "taste test". The 
hypotheses were that ingestion of the appetizer would result in stronger Stroop interference for food words, and larger food intake in restrained than in unrestrained subjects. This was not confirmed: in the appetizer condition, no especially strong Stroop interference or disinhibitive eating could be found in restrained subjects. However, it was also observed that whereas restrained subjects did not counterregulate, unrestrained subjects did. Interestingly, for unrestrained subjects, the magnitude of the Stroop interference for food words appeared to correlate 0.58 with the subsequent intake of ice-cream. This was taken to suggest that attentional bias to food words may be a potential predictor of increased eating after cue presentation.

Finally, in chapter $\mathbf{8}$, the development was described of the DIS, a new questionnaire that might be used as a selection tool in future research. The DIS aims at selecting persons who are maximally prone to disinhibitive eating. In this respect, the DIS differs from the widely used Restraint Scale, that was originally developed for identifying dieters. The psychometric analysis of the DIS showed good internal consistency, as well as a clear four-factor structure tapping binge eating, failed restriction, weight fluctuations, and self-esteem, i.e. a factor structure resembling that found in earlier studies of large item pools tapping a full spectrum of bulimic characteristics. It was concluded that the DIS could be used as a tool in the development of new models of binge eating.

Taken together, none of our novel studies specifically testing hypotheses derived from $\mathrm{CBE}$ theory produced confirmative results. What should the implications of these results be for the CBE theory? One might suppose that particulars of the studies were responsible. Our non-clinical restrained subjects may have represented too weak cases of binge eating; also, the food slides used as cues in our cue reactivity experiment may not have been overly "realistic"; finally, our conditioning study may have included too few trials to demonstrate conditioning.

However, one might also rebut these arguments with the following considerations. Of the available questionnaires in the field, the Restraint Scale has been generally recognized as the most predictive of lab-overeating and binge eating in daily life (Heatherton et al., 1988; Wardle, 1980). Further, our food slides have probably been sufficiently realistic, which is suggested by the fact that, for all subjects taken together, psychophysiological reactions and subjective craving were stronger for food than did for neutral (office equipment) slides (Chapter 5). Finally, in other contexts, conditioned hypoglycaemia (using intravenous UCSs) have been demonstrated after two to five conditioning trials (Fehm-Wolfsdorf et al., 1993; Mityushov, 1954; Appendix, this thesis; Woods et al., 1969), so that after our six trials (using oral UCSs) one should expect at least a significant trace of evolving hypoglycaemia. All in all, it appears that the present studies have been fairly critical and suggest reconsideration of the CBE theory. This reconsider- 


\section{Chapter 9}

ation can be found in the following section.

\subsection{QUANTITATIVE REAPPRAISAL OF THE CBE THEORY}

In the original formulations of the conditioning theory of binge eating (chapter 2 this thesis; Jansen, $1990 ; 1994 \mathrm{a}$ ), no quantitative estimates were given of the size of putative conditioned physiological responses. Considering the general lack of confirmation for the CBE theory in the present thesis, it seems worthwhile to reconsider CBE theory's physiological predictions in a quantitative and critical way. Below, five key physiological CBE hypotheses (see chapter 2 for background explanation) will be assessed, using data from general physiology, cephalic phase response studies, conditioning studies, and comparative studies of physiological responding in bulimics and normals.

\subsubsection{Hypothesis one: binge eating causes exceptionally large blood sugar rises (UCS)}

Analysis: The CBE line of reasoning is that bingeing provokes a very large blood sugar rise, requiring that the size of the (compensatory) conditioned hypoglycaemia (CR) be proportionally large. The large intensity $C R$ would then be reflected in the subjective experience of craving.

No direct measurements of blood sugar rises after bingeing are available. Nevertheless, it is doubtful that beyond moderate meal size, additional intake leads to higher blood sugar rises. With fasting baseline typically lying between 70 and $100 \mathrm{mg} / \mathrm{dl}$, a normal meal will lead to blood sugar levels of between 100-150 mg/dl (Bantle et al., 1983; Oettlé, Emmett \& Heaton, 1988; Shively, Apgar \& Tarka, 1986; Teff, Levin \& Engelman, 1993; Weingarten, Hendler \& Rodin, 1988). Empirical data show that the upper boundaries of blood sugar level do not lie considerably higher. First, ingestion of doses of glucose (i.e. $1200 \mathrm{kcal}$ of a extremely potent blood sugar raiser; Brand Miller, Pang \& Broomhead, 1995) have been found to induce blood sugar levels not exceeding ${ }^{1}$

1 The physiological reason for the limited blood sugar rise to large meals in healthy (non-diabetic) persons depends on at least four inborn, homeostatic mechanisms. First, large meals will slow down gastric emptying, meaning that the food stays longer in the stomach and is allowed slower access to the small intestine, the place whence nutrients appear in the blood (McHugh, 1983). Second, if food is eaten hurriedly (this may be seen in binge eaters; section 1.2) the subsequent blood sugar rise is blunted importantly, due to several slowening effects on digestion (Read et al, 1986). Third, as soon as the glucose enters the blood, an (unconditioned) post-absorptive insulin response directs sugar from the blood into cells and tissues. Fourth, if, despite the aforementioned mechanisms, blood sugar level did exceed $150-190 \mathrm{mg} / \mathrm{dl}$, the sugar will not be reabsorbed by the kidneys and appears in the urine (this so-called glucosuria is responsible for the large volumes of high sugar urine which can be found in diabetics; Guyton, 1991). 
$180 \mathrm{mg} / \mathrm{dl}$ (Förster et al., 1972). Second, the ingestion of large amounts of favourite "real food" items appears to lead to moderate blood sugar rises. Kaye, Gwirtsman and George (1988) encouraged normal subjects to eat a buffet of favourite foods until their limits were reached. The recorded intake was 1482 (sd 430) $\mathrm{kcal}$, whereas blood sugar had only risen to $122 \mathrm{mg} / \mathrm{dl}$. A third - specifically bulimic - factor blunting the postmeal blood sugar rise is self-induced vomiting. Johnson et al. (1994) observed a rapid and strong blood sugar fall in bulimics who expelled ingested food (Johnson et al., 1994). In the Kaye et al. (1988) study, bulimics were encouraged to binge on a buffet of favourite food items, and given the possibility to purge. After a mean intake of 3022 (sd 1084) kcal and self-induced vomiting, bulimcs' average blood sugar level was found to lie at $120 \mathrm{mg} / \mathrm{dl}$. After this, subsequent bingeing and purging was allowed, and eventually the mean amount of ingested food was 7688 (sd 3971) kcal. In spite of this huge intake, blood sugar levels at subsequent l-hour intervals remained between 90 and 100 $\mathrm{mg} / \mathrm{dl}$.

Conclusion: Although no direct data are available on binge eating bulimics, ingesting large amounts of foods is unlikely to provoke an extreme blood sugar rise, because of the action of various homeostatic controls and the effects of self-induced vomiting.

\subsubsection{Hypothesis two: The conditioned blood sugar fall compensates substantially for the anticipated post-binge blood sugar rise}

Analysis: The CBE theory posits that conditioned hypoglycaemia compensates for the expected blood sugar rise (UCRs) ${ }^{2}$. How effective is this compensation?

To quantify the amount of compensation provided by the $\mathrm{CR}$, the $|\mathrm{CR} / \mathrm{UCR}|$ ratio can be computed. For example, if this ratio is 1 , full compensation of the post meal blood sugar rise occurs; if the ratio is 0.10 , this would mean that the conditioned hypoglycaemia would compensate for $10 \%$ of the expected blood sugar rise. The successful blood sugar conditioning studies using glucose as the UCS (Deutsch, 1974; Mityushov, 1954/Appendix, this thesis) provide insight into the |CR/UCR | ratio. In Deutsch's study, the blood sugar rise (UCR) to the glucose UCS in rats was $31.3 \%$; whereas the CR hypoglycaemic response to placebo intubation was $3.3 \%$ from baseline. Thus the

${ }^{2}$ For the sake of simplicity, blood sugar rise and fall are called responses here. Physiologically speaking, however, this may not be entirely correct. The blood sugar rise to ingested food ("UCR") is the result of passive absorption of glucose from the gut. A similar consideration may apply to the conditioned hypoglycemia which probably results from an (active) insulin secretion (see chapter 4 , this thesis; Dworkin, 1993; Eikelboom \& Stewart, 1982, for further discussions). 


\section{Chapter 9}

|CR/UCR | ratio in this case was approximately $0.11 .^{3}$ In Mityushov's study, the observed hypoglycaemic $\mathrm{CR}$ in humans was $10 \mathrm{mg} / \mathrm{dl}$, whereas the blood sugar rise to the UCS in humans was about $165 \mathrm{mg} / \mathrm{dl}$, making the $|\mathrm{CR} / \mathrm{UCR}|$ ratio $10 / 165=0.06$. Conclusion: Judging from the scanty data available, hypoglycaemic CRs appear to be far from compensatory.

\subsubsection{Hypothesis three: cephalic insulin responses are, physiologically speaking, substantial responses}

A substantial part of the data basis underlying CBE theory concerns cephalic phase responses to food cues (section 2.2). These responses are put forward as illustrations of substantial responses causing a fall in blood sugar level and underlying feelings of craving.

Analysis The available data show that the magnitude of the cephalic insulin response are rather small when compared with either baseline fluctuations or post-meal insulin secretion (Bellisle et al., 1983; Le Magnen, 1992; Moyer, Rodin \& Cummings, 1993). Whereas the range of reported cephalic insulin increases from baseline has been between 0-250\% of baseline levels in rats (Louis-Sylvestre, 1976; Powley \& Berthoud, 1985; Strubbe \& Steffens, 1975), and between 0 and $95 \%$ for humans (Lucas, Bellisle \& de Maio, 1987; Bruce et al., 1987; Sjöstrom et al., 1980; Moyer et al., 1993; Powley \& Berthoud, 1985; Teff et al., 1991; 1993; 1995), the post-absorptive increase of insulin after moderate (i.e. $100-700 \mathrm{kcal}$ ) food intake in humans lies between 200 and $1000 \%$ of baseline (Bantle et al., 1983; Bellisle et al., 1983; Shively et al., 1986; Rodin, Reed \& Jamner, 1988; Weingarten et al., 1988). Importantly for the CBE theory, two studies (Broberg \& Bernstein, 1989; Moyer, Rodin \& Cummings, 1993) have been conducted in bulimic patients who were to smell palatable food attentively. Neither study reported cephalic insulin responses exceeding baseline; moreover, the standard deviations of responses reported by Broberg and Bernstein (1989) were too small to suggest outliers among the subjects. In their discussion these authors have suggested that these results may have been due to their subjects not being in the "proper mood" that usually accompanies binge episodes. This remark applies to the vast majority of lab studies with bulimic subjects.

Conclusion Cephalic insulin secretion to food cues appears to be modestly sized when

${ }^{3}$ Note that another provisional computation could be made which adjusts for hyperglycaemia that occurred because of the rats' stress during the stomach intubation procedure (Deutsch reported that prior to the conditioning procedure, the rats had shown a $3.1 \%$, stress-related blood sugar rise when intubated with water). If one subtracts this blood sugar rise from both UCR and CR, then the $\mid$ CR/UCR $\mid$ ratio becomes $(3.3+3.1) /(31.3-3.1)=6.4 / 28.2=0.23$. 
compared with either baseline fluctuations or with post-meal insulin secretion. This observation applies to bulimics as well.

\subsubsection{Hypothesis four: cues related to binge food trigger especially large conditioned hypoglycaemia/hyperinsulinaemia in bulimics}

Analysis: As noted earlier, a potent elicitor of anticipatory insulin secretion and hypoglycaemia is sweet taste (section 2.2; Deutsch, 1974; Louis-Sylvestre, 1976, Powley \& Berthoud, 1985). Furthermore, binge eaters have been found to avoid snacks and other high caloric food items in between binges (e.g. Rosen et al., 1986; Walsh, 1993). If, as predicted by CBE theory, considerable conditioned hypoglycaemia or hyperinsulinaemia to binge food cues (CS) occurred in binge eaters, it follows that these responses should be observed in binge eaters and normals after the ingestion some fixed amount of binge-related food. Specifically, bulimics should show a retardation and/or a blunting of post-ingestive blood sugar rise, as well as a simultaneously enlarged insulin secretion.

First of all, two studies have been carried out comparing insulin and blood sugar responses to intravenous glucose in bulimics and normals (i.e., no food cues were involved in these studies). Blouin et al. (1991) found a smaller peak blood sugar value, but also a smaller insulin/glugacon ratio in bulimics than in normals. These findings were not replicated by Blouin et al. (1993), who did not find differences between responses of bulimics and normals. Studies which did involve oral ingestion of carbohydrates showed no irregularities in responses of bulimia patients: after ingestion of a sweet glucose drink (Hohlstein et al., 1986; Casper et al., 1989), or a 1200 kcal lemonflavoured carbohydrate drink (Turner et al., 1991), equal blood sugar and insulin responses were found in bulimics and normals. Addition of further "binge-relevant" sensory cues did not alter this pattern of results. Weingarten et al. (1988) provided bulimics and normals with equicaloric $(334 \mathrm{kcal})$ meals in a forbidden version (milkshake, and french toast with sirup) and an unforbidden version (cottage cheese and fruit). Again no between-group differences were found in blood sugar and insulin response. Moreover, the unforbidden meal evoked a somewhat larger insulin response in both subject groups, and this is contrary to CBE prediction. In one study (Schweiger et al., 1987) a difference between bulimics and normals was found: ingestion of a pudding rich in carbohydrates led indeed to a larger insulin secretion in bulimics (in line with $\mathrm{CBE}$ predicitions). However, this enlarged insulin secretion in bulimics appeared to be secondary to an enlarged blood sugar rise, a result which is in contrast with the $\mathrm{CBE}$ predictions. Jansen (1994a) has questioned the relevance of the above studies for CBE theory, because this theory only predicts pre-meal responses (however, see section 9.2.5 
for a further discussion on this issue). Furthermore, the cited studies neither involved individually chosen binge foods, nor were they conducted in the usual bingeing environment of the binge eating subjects.

Conclusion In contrast with the CBE hypothesis, ingestion of sweet or binge relevant food items has not been found to result in enlarged insulin or (hypoglycaemic) blood sugar responses in bulimics.

\subsubsection{Hypothesis five: conditioned hypoglycaemia underlies the urge to binge}

Analysis: The CBE theory posits that conditioned hypoglycaemias to food cues are experienced subjectively as an urge to eat.

Importantly, any hypoglycaemia explanation of binge eating should take into account that food intake itself will increase blood sugar level as nutrients start being absorbed from the small intestine. If hypoglycaemia underlies the urge to eat, an important question concerns the speed with which the hypoglycaemia will subside once eating has started. Probably, hypoglycaemias of the largest reliable size reported in the conditioning literature (i.e. $10 \mathrm{mg} / \mathrm{dl}$; Mityushov, 1954; Broberg \& Bernstein, 1989) would dissipate soon after the commencement of eating. This can be concluded from the available literature on blood sugar rise after ingestion of typical binge foods (candy bars and potato crisps). Ingestion of one candybar and a cup of tea (296 kcal) was found to lead to a $24 \mathrm{mg} / \mathrm{dl}$ rise within 20 minutes after meal onset; a serving of potato crisps and some cola drink (292 kcal) lead to a $29 \mathrm{mg} / \mathrm{dl}$ rise within the same time (Oettle et al., 1987). Other important data are given by Broberg \& Bernstein (1989) who did indeed find a considerable cephalic hypoglycaemia of $8-9 \mathrm{mg} / \mathrm{dl}$ after having their subjects smell palatable cinnamon rolls. Both in their bulimic and dieting subjects subsequent intake of the cookies was allowed. The bulimics who ate 36 grams of the rolls (perhaps about $150-200 \mathrm{kcal}$ ) returned to blood sugar baseline at between 10 and 15 minutes after the beginning of eating. These data show that after merely moderate intake hypoglycaemia is lifted long before the ending of the average binge, which has been reported to lie at 55.7 (sd 32.4) minutes (Jansen, Van den Hout \& Griez, 1990). It is only to be expected that bingeing is even more effective in lifting hypoglycaemia ${ }^{4}$.

Conclusion Hypoglycaemias of the size reported in the conditioning literature can be expected to subside soon after meal onset. The fact that the duration of the average binge appears to be considerably longer than that of hypoglycaemia, suggests that the role of conditioned hypoglycaemia may be limited, and that additional mechanisms

4 Compare the $\pm 300 \mathrm{kcal}$ snack, used in the Oettle et al. (1987) investigation, with the rate of food intake in binge eaters (between 30 and $80 \mathrm{kcal} / \mathrm{min}$; Rossiter et al., 1992; Walsh, 1993). 
should be postulated to fully explain binge eating.

\subsubsection{Conclusions}

Of course, the above discussions give only hints about the size of conditioned responses predicted by CBE theory. The true response sizes await revelation by monitoring of patients during full-fledged binge attacks. Until further notice, the following can be concluded:

a. the presently available data do not endorse CBE theory's assumption of physiologically significant conditioned hypoglycaemias/hyperinsulinaemias that "should be eaten away";

b. in bulimic patients, no enlarged hyperinsulinaemias or hypoglycaemias ("CRs") have been demonstrated in response to the taste or smell of binge related foods ("CSs");

c. ingestion of snacks provokes a rise in blood sugar that can be expected to lift hypoglycaemias of the size reported in the conditioning literature within a considerably shorter time than the generally reported duration of binges.

In sum, on basis of the known empirical data, profound scepticism seems warranted about the role of conditioned hypoglycaemia/hyperinsulinaemia in binge eating.

\subsection{THE CONDITIONING THEORY OF BINGE EATING: FINAL QUALIFICATION}

A conditioning theory of binge eating (CBE theory) was described in chapter two. By and large, none of the $C B E$ hypotheses empirically tested in this thesis could be confirmed. Furthermore, a quantitative reappraisal of the physiological assumptions of the CBE theory (section 9.2) cast considerable doubt on the premise that conditioned hypoglycaemia/hyperinsulinaemia is central to binge eating. In sharp contrast, CBE theory also has inspired a cue exposure procedure that promises to be an effective treatment of binge eating (section 2.2.6). It is not immediately clear what should be concluded from a case where an (as yet) unproven theory inspires effective therapy. Interestingly enough, similar dilemmas have been harassing conditioning research of panic disorder and addiction. We will examine the commentaries made by two major workers in these fields, McNally (1994) and Siegel (1989), before drawing final conclusions for binge eating.

McNally (1994) views classical conditioning theory starkly as a "misleading metaphor for the mechanisms underlying panic" while admitting that "(..) it has inspired effective treatments of panic disorder" (p. 108). McNally's main criticism of the conditioning 


\section{Chapter 9}

account of panic is that theoreticians have failed to clearly identify stimuli (CS, UCS) and responses (CR, UCR), often with awkward implications such as bodily sensations in panic being CS,CR and UCS at the same time. McNally thinks that this vagueness does not allow conditioning accounts to be very fruitful in basic research of panic disorder.

Siegel (1989) comments on several studies in which conditioned drug tolerance was found in the absence of demonstrable conditioned physiological responses. He concurs with the view that "failures to demonstrate compensatory CRs do not represent major challenges to the conditioning account of tolerance" (p. 163), and points to present knowledge deficits in classically conditioned drug tolerance. More specifically, too little is known yet about when and how physiological responses are triggered by environmental and drug stimuli, and how physiological CRs exactly relate to behavioural phenomena like tolerance and drug intake.

To what extent do the above considerations apply to the conditioning theory of binge eating? Concerning McNally's remarks, it should be emphasized that, unlike the panic theories, CBE theory's stimuli and responses have been well-defined. Equally truly, however, other investigators of binge eating have proposed an entirely different learning mechanism in bingeing that, while involving different responses and stimuli, has also inspired a therapeutical procedure that leads to important reductions in binge eating (Leitenberg et al., 1984; Schmidt \& Marks, 1989; for explanation see section 9.4.2). Nevertheless, the "vagueness" intrinsic to the existence of two different theories may have less to do with misleading metaphors, than with the possible existence of more than one mechanism underlying binge eating (Schmidt \& Marks, 1989). Siegel's commentary distinguishes between the physiological and behavioural levels. As to the physiology related to binge eating, we would not adopt Siegel's opinion that failures to demonstrate compensatory responses are minor challenges to the conditioning theory of binge eating. The physiological events (if any) underlying the urge to eat might turn out to be entirely different from those hypothesized, prompting reformulation or even rejection of CBE theory ${ }^{5}$. Nevertheless, on the behavioural level CBE theory appears to be working well, given the success of cue exposure. There is by now accumulating data suggesting this (section 2.2.6), so that the situation for binge eating is no different than that for conditioned drug tolerance. For therapeutic purposes this situation need not be disastrous: CS (binge cues) and UCS (binge eating) and their relationship are transparent.

$S$ For example, it might be that the peripheral, conditioned compensatory responses that have been previously observed in addiction research (see the overview presented in Macrae, Scoles \& Siegel, 1987), can be understood as serving homeostatic regulation (see e.g. chapter 4, this thesis), whereas craving and other behavioural effects critically depend on as yet unknown central effects (occurring within the brain). 
The therapist's position could be compared with that of a radio owner who, although fairly skilled at operating the knobs, remains unaware of the radio's interior electronics. For both therapist and basic researcher, however, uncertainty remains about the mechanism through which the habit of binge eating was acquired in the first place. To summarize and conclude, the physiological mechanism of urge (if any) remains elusive; the major advance relative to the "urge-less" theory of binge eating (e.g. counterregulation theory, section 1.3), is that a maintenance factor appears to have been identified, that can be cast in conditioning terms and used for therapeutical purposes; the contribution of learning to binge eating has not been exhaustively studied. Important questions remain about the physiological and acquisition mechanisms in binge eating.

Next, theoretical (section 9.4) and practical (section 9.5) directions for future research will be proposed.

\subsection{FUTURE RESEARCH: THEORETICAL CONSIDERATIONS}

In this section, two directions ${ }^{6}$ in research on the role of learning in binge eating will be proposed. The first approach should be considered an update of the conditioned hypoglycaemia/hyperinsulinaemia explanation that was originally offered by CBE theory. In the second approach, repeated post-binge vomiting and restriction are identified as key factors in binge eating. It may well be that both mechanisms are simultaneously active in binge eaters, judging from reports that exposure therapies directed at either mechanism have been effective in reducing binge eating (Schmidt \& Marks, 1989). Practical research implications of the theorizing will be described in sections 9.5.3 and 9.5.4.

\subsubsection{Maintaining a blood sugar/insulin-related explanation}

Reliable pre-meal blood sugar fall: characteristics When one still looks for a role of learned insulin and glucose dynamics in craving and binge eating, a recent development may provide clues. Campfield and Smith (1990a; 1990b) have reported a robust role of insulin and blood sugar signals in meal initiation. Blood sugar levels were continuously monitored in free moving rats with ad lib food available. Campfield and Smith claim

6 A third, social learning approach, which is in current upswing in cue-reactivity research (Tiffany 1995a; 1995b) will not be elaborated further here. First, because it explicitly rejects the idea that craving is a central mechanism in relapse (Tiffany, 1995b, p. 155; this might be the case for drug and alcohol addictions, but is rather unlikely for binge eating), and second, because apparently, the social learning account of relapse has not yet reached a stage at which convincing lab models may be developed (Bradizza, Stasiewicz \& Maisto, 1994). 
that almost without any exception, ad lib meal intake in the rats was preceded by a transient blood sugar fall of at least $6 \%$ below baseline, lasting for more than 6 minutes. Meal initiation occurred at 12.1 (sd 1.7) minutes after the beginning of the blood sugar fall, i.e. 5.4 (sd 1.5) minutes after the nadir of the hypoglycaemia. Other characteristics of the robust hypoglycaemia-food intake sequence were revealed in numerous experiments: if food became available at 5 minutes after the hypoglycaemia had disappeared, no food intake occurred; if the hypoglycaemia was countered by a rapid glucose infusion, no feeding occurred; if the experimenters mimicked a hypoglycaemia by infusion of other biochemicals, food intake occurred if the characteristics of the glycaemic curve resembled those of the spontaneous hypoglycaemias; no within-subjects correlation was found between the depth of the hypoglycaemia and the amount of subsequent intake. One origin of the spontaneous hypoglycaemias was found to lie in a prior short-lived insulin increase (50\% above baseline).

The results have been confirmed in humans whose blood sugar level was monitored continuously for a prolonged period (Campfield et al., 1992; 1996). The subjects could require a meal if they wanted. It was found that hypoglycaemias of $6-10 \%$ below baseline preceded meal request. These blood sugar dynamics also correlated with selfreported hunger.

Commentary Exactly how the hypos described by Campfield and Smith might relate to learning and conditioning is not clear yet. The following comments can be made.

1. In contrast to the $\mathrm{CBE}$ theory account, the hypoglycaemias (and the preceding hyperinsulinaemias) described by Campfield and Smith are short-lived and signal meal initiation rather than compensate for homeostatic disturbances.

2. The size of cue elicited (cephalic and conditioned) hypoglycaemias reported in some of the human studies (Broberg \& Bernstein, 1989; Mityushov, 1954/Appendix this thesis) is within the range of the meal-preceding hypoglycaemias found by Campfield and Smith. Moreover, the hypos were preceded by a small 50\% insulin "spike", the size of which is that of the typical (cue-elicited) insulin responses (see sections 2.2 and 9.2, this thesis).

3. Are the observed hypoglycaemias endogenous and/or learned in nature? In his commentary, Woods (1990) has argued against Campfield and Smith's purely endogenous explanation. He points to the fact that the rats in the Campfield \& Smith studies were kept in a very stable monotonous environment, whereas in a sensorily richer environment the rats' behaviour (e.g. the observed hypoglycaemia and food intake!) might become more easily attuned to external (meal-related) events and other cues. Interestingly, the only meals not preceded by hypoglycaemia concerned foods that were novel to the rats. As the rats got acquainted with the food items, gradually the hypoglyc- 
aemias began to appear as with other foods (Dr. Campfield, personal communication). This suggests a role of learning in the meal preceding hypoglycaemias.

4. Now that the moderately sized, short-lived hypoglycaemias found by Campfield and Smith appear to be playing a role in normal food intake, it still remains elusive in what way these responses might contribute to binge eating. Two possibilities may be mentioned. First, one might speculate that in binge eaters, for some reason the frequency of transient hypoglycaemias exceeding some critical threshold is increased (relative to healthy normals) before or during binge eating. Frequent hypoglycaemic dips might be experienced subjectively as "waves of craving" leading to temptation to binge (or continuing the binge if the hypos were superimposed on the post-prandial blood sugar rise $^{7}$ ). Considering the turmoil reigning in the blood sugar and insulin domain after food intake, the latter speculation remains problematic. A related possibility might be that the hypoglycaemic mechanism only induces binge-initiation, and that during the binge another principle is at work (see for instance section 9.4.2).

5. Finally, it also could turn out that hypoglycaemias occur equally in normals and binge eaters, but that binge eaters are especially inclined to interpret small hypoglycaemias as hunger. In fact, from research on hypoglycaemic episodes in diabetic patients, it is known that there is an individual stereotype in the experienced symptoms. There are "trembling/sweating" individuals, whereas others report predominantly hunger during hypoglycaemia (Hepburn, 1993; Service, 1995). Nevertheless, an explanation based merely on differential interpretation of body signals is likely to be too weak to account for the particular vigour with which binge eating proceeds (see section 1.2).

\subsubsection{Learning perspective based on post-meal deprivation and vomiting}

There is consensus among food intake researchers that meal size is correlated not only with momentary, but also with expected level of deprivation (see Collier, 1986; Davey, 1989; Le Magnen, 1992; Weingarten, 1985; Woods \& Strubbe, 1994). Moreover, binges of bulimics and anorexics of the binge eating/purging type (APA, 1994) are part of a eating pattern of restriction, fasting and vomiting. While these behaviours are meant to be "rational" means of weight restriction, the possibility of their becoming maintenance factors in binge eating should not be ruled out. This section will review various studies to suggest that yet another learning mechanism may contribute to bingeing: learned cues that reliably predict the occurrence of deprivation after a meal might

7 As noted in section 2.2, Louis-Sylvestre (1984) did demonstrate short insulin bursts after introduction of new food items during meals in rats. It is equally true that changing the foods during a meal increases total intake. 
increase the size of that meal. The data presented below will be used as a starting-point for a new lab model of binge eating (section 9.5.3).

Repeated fasting after meals may increase meal size Although no systematic data have been collected, it seems safe to say that binge eating episodes are frequently followed by a period of self-imposed restriction and/or vigorous exercise (APA, 1994). There is one report that suggests that meal size might increase if deprivation reliably follows that meal (Le Magnen, 1992, ch. 1). Rats were adjusted to a diet of 3 daily one-hour meals a day at fixed times for 18 days. Then one of the three meals was omitted daily, thus creating the following schedule: meal I - 7 hours - meal II -- 15 hours -- meal I, and so on. Food intake was monitored for another 18 days. After several days, a clear meal size pattern developed: meal II, i.e. the meal preceding the 15 -hour deprivation, became considerably larger than meal I, and ultimately increased with about $90 \%$ of the calories of the now omitted third meal. This effect, found irrespectively of the time of day, was taken to suggest that rats adjust their meal size by using information of future availability of food. Meal size increases when post-meal deprivation is expected. At least until recently, no human research had been done on this mechanism (Le Magnen, 1992). The implication is that if bingeing is reliably followed by prolonged restriction or fasting, this might lead to a learned increase of the pre-fasting (i.e. binge) meal.

Repeated vomiting after meals may increase meal size Binges are often followed by vomiting in $80-90 \%$ of the bulimic patients (APA, 1994; Rosen et al., 1986). Post-binge vomiting appears to have an enhancing impact on bingeing itself. Firstly, the speed of eating appears to be larger in purging than in non-purging bulimics (compare Rossiter et al., 1992 with Walsh, 1993). Secondly, the binge size of purging bulimics is twice of that of non-purging bulimics (compare Rossiter \& Agras, 1990 with Rossiter et al., 1992) ${ }^{8}$. It will be suggested here that, if bingeing is reliably followed by vomiting, a learning mechanism might induce learned increase of the fast-preceding meal (i.e. the binge) itself. This allegation will be substantiated by data on physiological state after vomiting, operant conditioning, conditioned sham feeding, and conditioned desatiation studies.

First, post-binge vomiting may lead to a temporary state of deprivation that bears upon food intake. Johnson et al. (1994) had 12-hour fasted bulimic subjects binge and vomit subsequently during which blood sugar and insulin levels were monitored. After

${ }^{8}$ Almost certainly, this can be explained by the simple fact that vomiting creates space for yet more binge food. Hence, it becomes understandable how the largest binge sizes reported in the literature could have been in the $20,000-55,000 \mathrm{kcal}$ range, i.e., between 10 and 28 times the average daily food intake in the normal population and, literally, impossible to stomach (Russell, 1979; Walsh, 1993). 
vomiting a steep decline of blood sugar level was found (12 $\mathrm{mg} / \mathrm{dl}$ within 15 minutes) bringing blood sugar back at baseline level. Concurrently, however, the insulin level remained twice as high as baseline ${ }^{9}$. A similar overshoot of insulin level after a 3000 kcal binge and subsequent vomiting in bulimics was found by Kaye et al. (1989), and it might imply a momentary glycaemic state of deprivation ${ }^{10}$. Direct evidence that expected insulin overshoot may produce instrumental food seeking and eating comes from a study by Siegel and Nettleton (1970). Four groups of rats were housed in Skinner boxes. Group A received repeated insulin injections and had the possibility to bar-press for food, while Group B received insulin injections without this possibility. Control groups $C$ and $D$ received saline injections with and without bar pressing possibility respectively. During the post-conditioning test session, saline was injected in all groups and bar pressing frequency was monitored: group A rats clearly showed the largest bar pressing rate of all groups, while at a pre-conditioning test session the rates had been equal for all groups. Siegel and Nettleton (1970) concluded that the rats of group $\mathrm{A}$ had acquired an instrumental feeding response to reduce expected "insulininduced distress".

Second, sham feeding studies may be relevant as an analogue of post-binge vomiting: large food-intake is signalled orally, but the amount of nutrients subsequently absorbed does not follow suit. Van Vort and Smith (1987) have shown that flavours associated with sham feeding enhance meal size. Rats were provided with a fistula that could be opened (sham feeding) as well as closed (real feeding). During a conditioning procedure, the rats were given a diet of milk. In alternating sequence, the milk had either an added flavour which signalled sham feeding (CS-) or a flavour that preceded real feeding $(\mathrm{CS}+)$. A post-conditioning test with fistulas closed (the real feeding situation) showed that the amount of milk flavoured with the CS- taste was drunk in larger amounts (33$50 \%$ more) than the previous CS + flavoured milk. The effect was immune to extinction for at least three post-conditioning test trials. Similar results have been found in other

${ }^{9}$ Methodologically unfortunately, in the Johnson et al. procedure, the vomiting was followed by renewed ingestion so that no data are available of completed binge-purge cycle that ends with purgeing.

${ }^{10}$ See also Davidson (1993). Rats were trained to display a set of motor acts. Each motor act was acquired under a unique and fixed deprivation level. After training, insulin injections led the rats to display the motor act acquired during 24-hour deprivation. As a comparison, injections with the satiety hormone cholecystokinine led the rats to display the motor act which had been learned under 0 -hour deprivation. These observations are in line with the idea that an excess of insulin (or its physiological consequences) and deprivation may share a common interoceptive quality. 


\section{Chapter 9}

studies as well (see Le Magnen, 1992, ch. 6). For binge eating this might imply that oral stimulation by binge food ("CS"), reliably followed by disproportionally small nutrient absorption (because of vomiting), may lead to conditioned meal enlargement.

A final data source is conditioned desatiation research in rats. Booth $(1985 ; 1991)$ has advocated that meal size is under influence of expected post-meal signals of satiety (e.g. nutrients appearing in the blood). After repeatedly pairing a flavour with carbohydrate dense food (strongly satiating), Booth added this flavour to different foods and saw decreased meal size in rats. In contrast, a dramatic increase in meal size was found in rats when during a meal involving high caloric food, a flavour was introduced that had been previously associated with low post-ingestive satiety. This increased intake was coined "conditioned desatiation"11 (Booth, 1985; 1991; Le Magnen, 1992, ch. 6). For binge eaters, the taste of binge foods might become associated with relatively low satiation (due to vomiting relatively little is actually absorbed ${ }^{12}$ ). Hence, conditioned desatiation to binge cues might act to increase intake of typical binge foods.

Breaking the binge-deprivation association decreases binge eating This has become apparent from the results of a therapeutic procedure called binge-exposure with prevention of vomiting (Rosen \& Leitenberg, 1982; Leitenberg et al., 1984; Schmidt \& Marks, 1989). The procedure involves several sessions in which the patient ingests binge food past the point where the urge to vomit develops. However, the patient is prevented from vomiting and the therapist remains with the patient until the urge to vomit has declined. Several studies have reported that this procedure leads to important reductions of binge eating ${ }^{13}$ (Rosen \& Leitenberg, 1982; Leitenberg et al., 1984; Schmidt \& Marks, 1989;

11 Two critical remarks are in place here. A vast literature exists in which flavour CSs predicting bigh nutrient value (UCS) increase preference of the flavour (for a review see Sclafani, 1995). Just how to reconcile these robust findings with the data supporting conditioned desatiation constitutes an as yet unresolved issue. Second, conditioned desatiation certainly must have its limits. The implication that non-nutritive sweeteners, as used in diet drinks, should lead to an ever-increasing intake of these drinks, does not stand closer scrutiny (e.g. Jansen, 1994b).

12 There are at least four indications suggesting a modest intestinal absorption of nutrients from binge foods expelled by self-induced vomiting. Firstly, vomiting generally takes place soon after the binge, before the food has entered the small intestine. Secondly, when large amounts of foods are ingested, gastric emptying (i.e. the transit of foods from the stomach into the small intestine) slowens down (McHugh, 1983). Thirdly, rapid eating (such as especially bulimics of the purging type do (Rossiter et al., 1992; Walsh, 1993) with little chewing slowens down the speed with which ingested nutrients appear in the blood (Read et al, 1986). Fourthly, bulimic patients' gastric emptying of small amounts of food has been found to be slower than that of healthy individuals (Geliebter et al., 1992).

${ }^{13}$ Parenthetically, the surplus value of exposure with prevention of vomiting relative to cognitive behavioural treatments of binge eating has been the subject of continuing discussion (Agras et al., 1989; Leitenberg \& Rosen, 1989; Cooper \& Steere, 1995). 
Cooper \& Steere, 1995). Note that these results are contrary to predictions of CBE theory, because, according to CBE theory, substantial ingestion of binge foods would be equal to a conditioning trial in which binge cues (CS) are paired with bingeing (UCS; see section 2.2, this thesis). A common characteristic of this therapy and CBE inspired cue-exposure with binge prevention procedure (section 2.2.6) is that the unrolling of a stereotypic behaviour chain during the binge episode is thwarted. Although the rationale of the Rosen and Leitenberg procedure was derived from anxiety research (Leitenberg et al., 1984), it might be considered an example of extinction of a learned association between binge cues (or bingeing itself) ("CS") and post-binge deprivation state ("UCS"). Additional remarks If the mechanism of signalled post-binge deprivation indeed exists and contributes to binge eating, it might be enhanced by pre-binge deprivation. Repeated deprivation experience may induce learned increase in food intake to deprivation-related cues (Weingarten, 1985; Revusky \& Garcia, 1970). Theoretically, the classical conditioning terminology may not be entirely appropriate in this case: intake of certain foods (a response accompanied by sensory stimulation) is regarded as a cue (the CS), and postmeal deprivation (a state following active responses) is considered the UCS. It may be that the mechanism might be better described by regarding binge eating as an instrumental response, performed in anticipation of deprivation, i.e., an "economic" property of the feeding system that can be found in most animals (Davey, 1989, ch. 7). Because of the present lack of rigorous terminology, and for practical purposes (e.g. the modelling of binge eating; section 9.5.3), we will, until further notice, stick to the CS-UCS terminology derived from classical conditioning.

\subsection{FUTURE RESEARCH: PRACTICAL CONSIDERATIONS}

Three lab models of binge eating will be presented in this final section. As will be explained, the models serve two practical purposes. Firstly, to provide a framework for basic research on learning mechanisms in binge eating. Secondly, as procedures to establish "miniature binges" in the lab, to be utilized for pre-clinical research on the effectiveness of newly developed treatments.

\subsubsection{The necessity of modelling}

In chapter one, it was explained that experimental psychopathology involves the hypothesizing and investigation of mechanisms that underlie behavioural disorders. This is the fundamental approach. Additionally, a more practical mission of experimental 


\section{Chapter 9}

psychopathology research is (or rather, could be) the testing of new behavioural techniques proposed by the therapeutic or scientific community. Not unlike the screening of newly developed psychopharmaceuticals (Willner, 1991a), behavioural techniques might be tested for their preventive or curative value. But how might this be accomplished in practice? One method that will be proposed here is the development of "lab models". As explained in chapter one, "modelling" refers to the reliable production of behaviours in the lab that can be considered a "miniature" analogue of the psychopathology under study.

In this final section, three possible lab models of binge eating will be described: the cue reactivity model, the conditioned hypoglycaemia model, and the post-meal deprivation model. All rely on the theoretical CBE notion that binge eating is a learned, cue-driven, and self-perpetuating behaviour ${ }^{14}$. Each lab model requires fundamental, preparatory research (see section 1.1). First, some essential parameters of binge eating should be identified in patients (type $P$ research) and/or in specifically selected non-clinical subjects (type $Q$ research). Once type $Q$ experiments have demonstrated replicable responses related to binge eating, a potential lab model may be within reach. Modelling the disorder is not a final goal, but the beginning of the practical stage (Willner, 1991b): in subsequent type $\mathrm{R}$ research, one attempts to "treat" the lab bingeing using the new behavioural technique. If proven effective in this way, the behavioural technique might be passed on for use in clinical trials.

Importantly, a workable lab model of binge eating should include some core manipulation that produces a significant and reliable increase of craving and/or food intake. In more lively terms, the proof of a binge eating model, like the proverbial pudding, should be in the eating (Herman \& Polivy, 1980; Jansen, 1990), and interpretation of the effects of the basic manipulation should be straightforward and require no "harking" whatsoever ${ }^{15}$.

Note that the empirical data in the present thesis have not been very encouraging as to the feasibility of model development on the basis of $C B E$ principles. Thus, the three models presented next should also be considered as coordinated and ultimate attempts to test the validity of $\mathrm{CBE}$ related theorizing.

${ }^{14}$ Human models of binge eating, especially if inspired by learning theory, have been rare: the counterregulation model has dominated the field (see section 1.3). In general, models relevant for binge eating have been largely confined to animal research: they are stress models (Greeno \& Wing, 1994), nutritional reward models (Sclafani, 1995) and biochemical and surgical models of overeating (Montgomery, 1991).

${ }^{15}$ Harking = Hypothesizing After the Results are Known (van der Heijden, 1995). 


\subsubsection{Model I: the cue reactivity model of binge eating}

Backeround One interesting aspect of psychophysiological/cognitive cue reactivity is its possible use as a diagnostic index of relapse danger in treated patients. In basic research there are three facets of cue reactivity to be studied: its dependency on learning history, its occurrence after cue presentation, and its influence on food intake. A practical advantage of this type of approach in modelling is that demonstration of cue reactivity requires no painstaking learning procedures. For this reason cue reactivity experiments are sometimes referred to as "opportunistic" experiments (Glautier \& Tiffany, 1995) Type $\mathrm{P}$ research Comparing patients and controls; searching for parameters.

* Cognitive changes after cue presentation: Stroop food word interference has already been amply demonstrated in binge eaters (see Chapter 7); However, such interference has not yet been demonstrated in clinical subjects after exposure to cues, and/or before the onset of a binge ${ }^{16}$.

* Increased psychophysiological food cue reactivity should be demonstrated in binge eaters. Parenthetically, it might well turn out to be intrinsically impossible to disentangle appetitive and emotional factors in responses to food cues in bulimic patients (compare e.g. Niaura et al., 1988).

* It should be demonstrated that food cue presentation increases subsequent ad lib food intake in patients. This is relevant even if no sensitive psychophysiological cue reactivity parameters might be identified. It could also be investigated whether high reactivity to food cues influences food intake of the items itself or also generalizes to the food item itself, or generalizes to other foods as well (compare Cornell, Rodin \& Weingarten, 1989).

Type $\mathrm{Q}$ research Comparing non-clinical subjects with different learning history; withinsubject comparisons of reactivity to foods differing in habitual intake.

* Subjects should be selected on the basis of either scores on scales specifically tapping disinhibitive eating (e.g. the DIS, see chapter 8), or more rigorously, on the basis of explicit self-reports on eating habits. These might include frequency estimates of bingeing and specification of meal size and particular foods involved.

* Foods could be allocated to a small number categories according to the frequency and/or habitual amount that is ingested. Cue reactivity to food cues belonging to these

${ }^{16}$ If the urge-to-act explanation of Stroop interference (Chapter 7, this thesis) is valid, it might be interesting to use an adapted version (with weight concern/vomiting-related words) within the context of Rosen \& Leitenberg's binge food exposure procedure (section 9.4.2). In patients who have just eaten typical binge foods, such Stroop tests might be used to assess suddenly aroused weight concems and tendencies towards self-induced vomiting. 


\section{Chapter 9}

categories could be compared in a within-subjects design.

* Sensitive physiological parameters of food cue reactivity should be sought for.

* As in type P research, another next step is to assess the impact on intake of a subsequently offered food. Intake should be larger after cue-presentation than after no cue presentation.

* If possible, at least two separate sessions (one with and one without cue presentation) should be held, to ensure equal deprivation level during cue presentation. Care should then be taken that no intake neophobia appears (Chapter 3). A prerequisite would be balancing of the order of cue and non-cue sessions. Furthermore, as intake-neophobia appears to fade due to exposure to the lab environment (Chapter 3 ), it might also be considered to run one or several habituation sessions, before running the experimental sessions.

Type $\mathrm{R}$ research Establishing and using the cue reactivity model of binge eating.

* Core manipulation: cues are presented and food intake and craving measured. A within-subject design might be employed with non-clinical binge prone subjects. Responses to different categories of food cues (see under type $Q$ research), or between food cues and neutral or no cues are compared.

* Core results: food intake and craving should be larger after cues predicting large food intake, than after cues predicting small or no food intake.

* Using the model: once the model is established, practical questions about binge eating might be answered by introducing additional manipulations on the normal subjects (e.g. instructions, mood inductions, prolonged cue exposure, instructed eating patterns, homework), and an assessment of their impact on the lab-overeating produced by the core manipulation.

\subsubsection{Model II: the conditioned hypoglycaemia model of binge eating}

Background In contrast to the cue reactivity model, the conditioned hypoglycaemia model of binge eating requires an explicit learning procedure. A starting point for model development might be a procedure resembling Mityushov's (1954; chapter 4). Before describing the model, a practical consideration is in place here. Conditioning procedures like Mityushov's are laborious and time-consuming. Because, at this moment, the role of hypoglycaemia/hyperinsulinaemia in binge eating remains to be established (see section 9.3), top priority should be given to research which demonstrates reliable and significant (conditioned) hypoglycaemia or hyperinsulinaemia during fully-fledged binge attacks in patients. If these responses cannot be demonstrated, then hypoglycaemia/hyperinsulinaemia clearly do not tap the essence of binge eating and there would seem little point - at least for binge eating research - to make the extensive effort of modelling binge eating in 
humans by glycaemic conditioning. Alternatively, if binge eating attacks are indecd preceded by significant hypoglycaemia/hyperinsulinaemia, then a powerful model of binge eating might be within reach.

Type $\mathrm{P}$ research Studies in patients.

* Blood sugar and insulin levels should be monitored on line during binge attacks. The same should be done when, after exposure to binge foods, craving is high up. These measurements should preferably be carried out at patients' typical binge locations.

Type Q research Comparing subject groups with different learning history.

* Possible research would resemble cue reactivity research type II, but in this case blood sugar and insulin responses are measured. The Broberg and Bernstein (1989) study, which as far as the present author is aware, is the only convincing report of cephalic hypoglycaemia in a sample of healthy individuals (albeit dieting), should be replicated.

* The relationship between cephalic hypoglycaemia and subsequent food intake should be established.

Type $\mathrm{R}$ research Establishing and using the conditioned hypoglycaemia model of binge eating.

* Core manipulation: the unconditioned stimuli must involve a strong blood sugar rise. Conditioning with oral glucose has failed (Chapter 3). Effective conditioning with food would probably require even more trials, given the moderate glycaemic index of common foods (Brand Miller et al., 1995). The procedure used in the study by Mityushov (1954; appendix A) might offer more possibility and should be replicated. If the hypoglycaemic state could be conditioned, the procedure should first be extended to food. This could be done by introducing intake-related CS to the injection ritual, and measure ad lib food intake during the hypoglycaemic state.

* Core results: a robust binge eating model would exist if $\mathrm{CS}^{*}$ presentation were found to enhance craving and food intake (via hypoglycaemia), relative to a $\mathrm{CS}^{-}$or neutral situation, and/or unconditioned subjects.

* Using the model: see under the cue reactivity model type $\mathrm{R}$ research.

\subsubsection{Model III: the post-meal deprivation model of binge eating}

Background In section 9.4.2, an overview was given of data suggesting that meal size can be increased by associating meal cues with post-meal deprivation. These data suggests practical ways in which a lab analogue of bingeing in non-clinical subjects might be evoked after an explicit conditioning procedure.

Type $\mathrm{P}$ research Studies in patients.

* Data are needed on the probability relationship between bingeing and fasting in patients on the individual level. What is the probability that bingeing is followed by 


\section{Chapter 9}

prolonged fasting? Are there specific cues (i.e. certain foods, moods or circumstances) that predict whether binge eaters go on a prolonged fast after bingeing? Such data might be collected more systematically through the use of intake diaries.

* Similar data to that above must be acquired on the probability relationship between bingeing and self-induced vomiting within individuals.

* The short-term physiological consequences of self-induced vomiting after bingeing have received too little attention. To what extent does a metabolic state resembling deprivation occur after expelling large food quantities?

Type $O$ research Studies in non-clinical subjects.

* no strong suggestion can be made at this point.

Type $\mathrm{R}$ research Establishing and using the signalled deprivation model of binge eating.

* core manipulation: this procedure would consist of repeatedly pairing cues $\left(\mathrm{CS}^{+}\right)$with a "compound UCS" of a meal followed by an 8-9 hour deprivation. A within-subject design could be employed, comparing craving and food intake after $\mathrm{CS}^{+}$with no $\mathrm{CS}$ or a $\mathrm{CS}^{-}$. The $\mathrm{CS}^{-}$should be a signal for "no post-meal deprivation", for example by pairing the same meal followed by a period of 8-9 hours in which food is repeatedly ingested by subjects. The CSs could lie within the food itself (flavouring, colouring) or in the way it has to be ingested (typical room, small spoons; strange plates).

* Core results: after conditioning, ad lib ingestion of test food should be robustly larger after $\mathrm{CS}^{+}$than after $\mathrm{CS}^{-}$presentation.

* Using the model: see the cue reactivity model, type $\mathrm{R}$ research.

\section{Finis}






\section{REFERENCES}

A.P.A. (1994). Diagnostic and Statistical Manual of Mental Disorders 4th ed. Washington: American Psychiatric Association.

Abraham, S.F. \& Beumont, P.J.V. (1982). How patients describe bulimia or binge eating. Psychological Medicine 12, 625-635.

Abramson, L.Y. \& Seligman, M.E.P. (1977). Modeling psychopathology in the laboratory. In: J.D. Maser \& M.E. Seligman (Eds.), Psychopathology: Experimental Models, pp 1-26. San Francisco, W.H. Freeman.

Ádám, G. (1967). Interoception and Behaviour. Budapest: Hungarian Academy of Sciences.

Agras, W.S., Schneider, J.A., Arnow, B., Raeburn, S.D. \& Telch, C.F. (1989). Cognitive-behavioral and response prevention treatments for bulimia nervosa. Journal of Consulting and Clinical Psychology 57, 215-221.

American Psychiatric Association (1987). Diagnostic and Statistical Manual of Mental Disorders (3rd Revised. Ed.). Washington, DC: Author.

Anthony, B.J. (1985). In the blink of an eye: implications of reflex modification for information processing. Advances in Psychophysiology 1, 167-218.

Babkin, B.P. (1949). Pavlov: a Biography. Chicago/London: University of Chicago Press

Bantle, J.P., Laine, D.C., Castle, G.W., Thomas, J.W., Hoogwerf, B.J. \& Goetz, F.G. (1983). Post prandial glucose and insulin responses to meals containing different carbohydrates in normal and diabetic subjects. New England Journal of Medicine 309, 7-12.

Barnett, S.A. (1958). Experiments on "neophobia" in wild and laboratory rats. British Journal of Psychology 49, 195-201.

Beck, A.T, Rusch, A.J., Shaw, B.F. \& Emery, G. (1979). Cognitive Therapy of Depression. New York: Guilford Press.

Beebe, D.W. (1994). Bulimia nervosa and depression: a theoretical and clinical appraisal in light of the binge-purge cycle. British Journal of Clinical Psychology 33, 259-276.

Bellisle, F., Louis-Sylvestre, J. Demozay, F., Blsazy, D. \& Le Magnen, J (1983). Reflex insulin response associated to food intake in human subjects. Physiology and Behavior 31, 515-521.

Ben-Tovim, D.I., Walker, M.K., Fok, D. \& Yap, E. (1989). An adaptation of the Stroop Test for measuring shape and food concerns in eating disorders: a quantitative measure of psychopathology? International Journal of Eating disorders 8, 681-687.

Ben-Tovim, D.I. \& Walker, M.K. (1991). Further evidence for the Stroop Test as a quantitative measure of psychopathology in eating disorders. International Journal of Eating Disorders 10, $609-613$.

Berthoud, H.-R., Bereiter, D.A., Trimble, E.R., Siegel, E.G. \& Jeanrenaud, B. (1981). Cephalic phase, reflex insulin secretion: neuroanatomical and physiological characterization. Diabetologia $20,393-401$.

Beumont, P.J.V. (1995). The clinical presentation of anorexia and bulimia nervosa. In, K.D. Brownell \& Ch. G. Fairburn (Eds), Eating Disorders and Obesity: a Comprehensive Handbook, pp. 151-158. New York, London: Guilford Press.

Birch, L.L., McPhee, L., Sullivan, S. \& Johnson, S. (1989). Conditioned meal initiation in young children. Appetise 13, 105-113.

Blouin, A.G., Blouin, J.H., Braaten, J.T., Sarwar, G., Bushnik, T. \& Walker, J. (1991). Physiological and psychological responses to a glucose challenge in bulimia. International Journal of Easing Disorders 10, 285-296.

Blouin, A.G., Blouin, J., Bushnik, T., Braaten, J., Goldstein, C. \& Sarwar, G. (1993). A doubleblind placebo-controlled glucose challenge in bulimia nervosa: psychological effects. Biological Psychiarry 33, 160-168.

Booth, D.A. (1985). Food-conditioned eating preferences and aversions with interoceptive elements: conditioned appetites and satieties. Annals of the New York Academy of Sciences 443, 22-41. 


\section{References}

Booth, D.A. (1991). Protein- and carbohydrate specific cravings: neuroscience and sociology. In, M.I. Friedman, M.G. Tordoff and M.R. Kare (Eds.), Chemical Senses, Vol. 4: Appetile and Nurrition, pp. 261-276. New York: Marcel Dekker.

Boucsein, W. (1992). Electrodermal Activity. New York: Plenum Press.

Bouwmeester, D. (1995). Quantum Mechanics and Classical Optics. Thesis. Leiden: University of Leiden.

Bradizza, C.M., Stasiewicz, P.R. \& Maisto, S.A. (1994). A conditioning reinterpretation of cognitive events in alcohol and drug cue exposure. Journal of Behavior Therapy and Experimental Psychiatry 25, 15-22.

Bradley, M.M., Cuthbert, B.N. \& Lang, P.J. (1990). Startle reflex modification: emotion or attention? Psychophysiology 27, 513-522.

Brand Miller, J., Pang, E. \& Broomhead, L. (1995). The glycaemic index of foods containing sugars: comparison of foods with naturally-occurring vs. added sugars. British Journal of Nutrition $73,613-623$.

Briggs, S.R. \& Cheek, J.M. (1986). The role of factor analysis in the development and evaluation of personality scales. Journal of Personality 54, 106-148.

Broberg D.J., Bernstein, I.L. (1989). Preabsorptive insulin release in bulimic women and chronic dieters. Appetite 13, 161-169.

Brodsky, I.G. \& Devlin, J.T. (1994). Hormone and nutrient interactions. In, M.E. Shils, J.A. Olson \& M. Shike (Eds), Modern Nutrition in Health and Disease, 8th ed., 603-622. Philadelphia Baltimore, etc: Lea \& Febiger.

Bruce, B. \& Agras, W.S. (1990). Binge eating in females: a population-based investigation. International Journal of Eating Disorders 12, 365-373.

Bruce. D.G., Storlien, L.H., Furler, S.M. \& Chisholm, D.J. (1987). Cephalic phase metabolic responses in normal weight adults. Metabolism 36, 721-725.

Campfield, L.A. \& Smith, F.J. (1990a). Transient declines in blood glucose signal meal initiation. International Journal of Obesity 14 (suppl 3), 15-31.

Campfield, L.A. \& Smith, F.J. (1990b). Systemic factors in the control of food intake: evidence for patterns as signals. In E.M. Stricker (Ed), Handbook of Behavioral Neurobiology vol. 10: Neurobiology of Food and Fluid Intake, pp. 183-206.

Campfield, L.A., Smith, F.J., Rosenbaum, M. \& Geary, N. (1992). Human hunger: is there a role for blood glucose dynamics? Appetite 18, 244.

Campfield, L.A., Smith, F.J., Rosenbaum, M. \& Hirsch, J. (1996). Human eating: evidence for a physiological basis using a modified paradigm. Neuroscience and Biobehavioral Reviews 20, 133 . 137.

Cannon, W.B. (1939). The Wisdom of the Body 2nd ed. New York: W.W. Norton.

Carlson, H.E. \& Shah, J.H. (1989). Aspartame and its constituent amino acids, effects on prolactin, cortisol, growth hormone, insulin, and glucose in normal humans. American Journal of Clinical Nutrision 49, 427-432.

Casper, R.C., Pandy, G.N., Jaspan, J.B. \& Rubenstein, A.H. (1988). Hormone and metabolite plasma levels after oral glucose in bulimia and healthy controls. Biological Psychiarry 24, 663-674.

Catania, A.C. (1991). Glossary. In I.H. Iversen \& K.A. Lattal (Eds.), Experimental Analysis of Behavior, vol. 2. Amsterdam etc: Elsevier.

Channon, S., Hemsley, D. \& de Silva, P. (1988). Selective processing of food words in anorexia nervosa. British Journal of Clinical Psychology 27, 259-260.

Channon, S. \& Hayward, A. (1990). The effect of short-term fasting on processing of food cues in normal subjects. International Journal of Earing Disorders 9, 447-452.

Charnock, D.J.K. (1989a). A comment on the role of dietary restraint in the development of bulimia nervosa. British Journal of Clinical Psychology 28, 329-340.

Charnock, D.J.K. (1989b). Exercising restraint: a response to Polivy \& Herman. British Journal of 
Clinical Psychology 28, 343-346.

Childress, A.R, Ehrman, R., Rohsenow, D.J., Robbins, S.J. \& O'Brien, Ch.P., 1992. Classically conditioned factors in drug dependence. In J.H. Lowinson, P. Ruiz, R.B. Millman \& J.G. Langrod (Ed.), Substance Abuse: a Comprehensive Texibook, 2nd Ed. pp 56-69. Baltimore etc: Williams \& Wilkins.

Christensen, C.M. \& Navazesh, M. (1984). Anticipatory salivary flow to the sight of different foods. Appetite 5, 307-315.

Clark, D.M. (1983). On the induction of depressed mood in the laboratory: evaluation and comparison of the Velten and musical procedures. Advances in Behaviour Research \& Therapy 5, 27-49.

Collier, G. (1986). The dialogue between the house economist and the resident physiologist. Nutrition \& Behavior 3 9-26.

Comrey, A.L. (1978). Common methodological problems in factor analytic studies. Journal of Counseling and Clinical Psychology 46, 648-659.

Cools, J., Schotte, D.E. \& McNally, R.J. (1992). Emotional arousal and overeating in restrained eaters. Journal of Abnormal Psychology 101, 348-351.

Cooper, M.J., Anastasiades, P. \& Fairburn, C.G. (1992). Selective processing of eating-, shape-, and weight-related words in persons with bulimia nervosa. Journal of Abnormal Psychology 101, 352 . 355.

Cooper, M.J. \& Fairburn, C.G. (1993). Demographic and clinical correlates of selective information processing in patients with bulimia nervosa. International Journal of Eating Disorders 13, 109 116.

Cooper, P.J. (1995). Eating disorders and their relationship to mood and anxiety disorders. In, K.D. Brownell \& Ch. G. Fairburn (Eds), Eating Disorders and Obesiry: a Comprehensive Handbook, pp. 159-164. New York, London: Guilford Press.

Cooper, P.J. \& Steere, J. (1995). A comparison of two psychological treatments for bulimia nervosa: implications for models of maintenance. Behaviour Research \& Therapy 33, 875-885.

Cornell, C.E., Rodin, J. \& Weingarten, H. (1989). Stimulus-induced eating when satiated. Physiology \& Behavior 45, 695-704.

Cryer, Ph. (1992). Glucose homeostasis and hypoglycemia. In, J.D. Wilson \& D.W. Forster (Eds.), Williams Textbook of Endocrinology 8th ed., 1223-1253. Philadelphia etc.: W.B. Saunders.

Davey, G. (1989). Ecological Learning Theory. Londen, New York: Routledge.

Davidson, R.L. (1993). The nature and function of interoceptive signals to feed: toward integration of physiological and leaming perspectives. Psychological Review 100, 640-657.

Deutsch, R. (1974). Conditioned hypoglycemia: a mechanism for saccharin-induced sensitivity to insulin in the rat. Journal of Comparative and Physiological Psychology 86, 350-358.

Dimberg, U. (1987). Facial reactions, autonomic activity and experienced emotion: a three component model of emotional conditioning. Biological Psychology 24, 105-122.

Dritschel, B., Cooper, P.J. \& Charnock, D. (1993). A problematic counter-regulation experiment: implications for the link between dietary restraint and overeating. International Journal of Eating Disorders 13, 297-304.

Drummond, D.C., Tiffany, S.T., Glautier, S. \& Remington, B. (Eds.) (1995). Addictive Behaviour: Cue Exposure, Theory and Practice. Chichester etc: Wiley \& Sons.

Dworkin, B.R. (1989). Learning and functional utility. Behavioral and Brain Sciences 12, 139-141.

Dworkin, B.R. (1993). Learning and Physiological Regulation. Chicago, London: University of Chicago Press.

Edelberg, R. (1972) Electrodermal recovery rate, goal-orientation, and aversion. Psychophysiology 9, 512-520.

Eikelboom, R. \& Stewart, J. (1982). Conditioning of drug-induced physiological responses. Psychological Review 89, 507-528.

Elmore, D.K. \& de Castro, J.M. (1991). Meal patterns of normal, untreated bulimia nervosa and recove- 
red bulimic women. Physiology and Behavior 49, 99-105.

Fairburn, C.G. (1987). The definition of bulimia nervosa: guidelines for clinicians and research workers. Annals of Behavioral Medicine 9, 3-7.

Fairburn, C.G. \& Beglin, S.J. (1990). Studies of the epidemiology of bulimia nervosa. American Journal of Psychiarry 147, 401-408.

Fairburn, C.G., Cooper, P.J., Cooper, M.J., McKenna, F.P. \& Anastasiades, M. (1991). Selective information processing in bulimia nervosa. International Journal of Eating Disorders 10, 415-422.

Fairburn, C.G. \& Wilson, G.T. (1993). Binge eating: definition and classification. In, C.G. Fairburn \& G.T. Wilson (Eds.). Binge Eating: Nature, Assessment, and Treatment, 3-14. New York, London: Guilford Press.

Fairburn, C.G. (1995). Short-term psychological treatments for bulimia nervosa. In, K.D. Brownell \& Ch. G. Fairburn (Eds), Eating Disorders and Obesin: a Comprehensive Handbook, pp. 344-348. New York, London: Guilford Press.

Fehm-Wolfsdorf, G., Gnadler, M., Kern, W., Klosterhalfen, W., \& Kerner, W. (1993). Classically conditioned changes of blood glucose level in humans. Physiology and Behavior 54, 155-160.

Flaherty, C.F., Grigson, P.S. \& Brady, A. (1987). Relative novelty of conditioning context influences directionality of glycemic conditioning. Journal of Experimental Psychology 13, 144-149.

Förster, H., Haslbeck, M. \& Mehnert, H., 1972. Metabolic studies following the oral ingestion of different doses of glucose. Diabetes 21, 1102-1108.

Fridlund, A.J. (1979). Contour-following integrator for dynamic tracking of electromyographic data. Psychophysiology 16, 491-493.

Fridlund, A.J. \& Cacioppo, J.T. (1986). Guidelines for human electromyographic research. Psychophysiology 23, 567-589.

Furedy, J.J. (1989). Flights of teleological fancy about classical conditioning do not produce valid science or useful technology. Behavioral and Brain Sciences 12, 142-143.

Garcia, J., Lasiter, P.S., Bermudez-Rattoni, F. \& Deems, D.A. (1985). A general theory of aversion learning. Annals of the New York Academy of Sciences 443, 8-22.

Garfinkel, P.E. (1992). Evidence in support of attitudes to shape and weight as a diagnostic criterion of bulimia nervosa. International Journal of Eating Disorders, 4, 321-325.

Garner, D.M, Olmsted, M.P., Davis, R., Rockert, W., Goldbloom, D. \& Eagle, M. (1990). The association between bulimic symptoms and reported psychopathology. Insernational Journal of Earing disorders 9, 1-15.

Geliebter, A., Melton, P.M., McCray, R.S., Gallagher, D.R., Gage, D. \& Hashim, S.A. (1992). Gastric capacity, gastric emptying, and test-meal intake in normal and bulimic women. American Journal of Clinical Nutrition 56, 656-661.

Gerich, J.E., Charles, M.A., Grodsky, G.M. (1976). Regulation of pancreatic insulin and glucagon secretion. Annual Review of Physiology 38, 353-388.

Giduck, S.A., Threatte, R.M. \& Kare, M.R. (1987). Cephalic reflexes: their role in digestion and possible roles in absorption and metabolism. Journal of Nutrition 117, 1191-1196.

Girardier, L., Seydoux J. \& Campfield, L.A. (1976). Control of A and B cells in vivo by sympathetic nervous input and selective hyper- or hypoglycemia in dog pancreas. Journal of Physiology (Paris), $801-814$.

Glautier, S. (1994). Classical conditioning, drug cues and drug addiction. In, Ch. R. Legg \& D. Booth (Eds.), Appetite: Neural and Behavioural Bases, pp 165-192. Oxford etc, Oxford University Press.

Glautier, S. \& Tiffany, S.T. (1995). Methodological issues in cue reactivity research. In, D.C. Drum mond, S.T. Tiffany, S. Glautier \& B. Remington (Eds.), Addictive Behaviour: Cue Exposure Theory and Practice, 75-98. Chichester etc.: Wiley.

Goldfine, I.D., Abraira, C., Gruenewald, D. \& Goldstein, M.S. (1970). Plasma insulin levels during imaginary food ingestion under hypnosis. Proceedings of the Society for Experimental Biology and 
Medicine, 133, 274-276.

Green, M.W. \& Rogers, P.J. (1993). Selective attention to food and body shape words in dieters and restrained nondieters. International Journal of Eating Disorders 14, 515-517.

Greeno, C.G. \& Wing, R.R. (1994). Stress-induced eating. Psychological Bulletin 115, 444-464.

Grill, H.J., Berridge, K.C., Ganster, D.J., 1984. Oral glucose is the prime elicitor of preabsorptive insulin secretion. American Journal of Physiology 248, R88-R95.

Grilo, C.M, Shiffman, S. \& Wing, R.R. (1989). Relapse crises and coping among dieters. Journal of Consulting and Clinical Psychology 57, 488-495.

Grossman, S.P. (1986). The role of glucose, insulin and glucagon in the regulation of food intake and body weight. Neuroscience \& Biobehavioral Reviews 10, 295-315.

Guyton, A.C. (1991). Textbook of Medical Physiology, 8th ed. Philadelphia etc: Saunders.

Hadigan, C.M., Kissileff, H.R. \& Walsh, B.T. (1989). Patterns of food selection during meals in women with bulimia. American Journal of Clinical Nutrition 50, 759-766.

Hansen, A. \& de Haan, E. (1995). De behandeling van bulimia nervosa met cue-exposure; ervaringen uit de praktijk. Directieve Therapie 15, 279-291.

Hardy, R.N. (1981). Endocrine Physiology. London: Edward Arnold.

Heatherton, T.F., Herman, C.P., Polivy, J., King, G.A., \& McGree, S.T., 1988. The (mis)measure ment of restraint: an analysis of conceptual and psychometric issues. Journal of Abnormal Psychology 97, 19-28.

Hepburn, D.A. (1993). Symptoms of hypoglycaemia. In, B.M. Frier \& B.M. Fisher (Eds.), Hypoglycaemia and Diaberes: Clinical and Physiological Aspects, 93-103. London, etc.: Edward Arnold.

Herman, C.P. \& Mack, D. (1975). Restrained and unrestrained eating. Journal of Personaliry, 43. 647-660.

Herman, C.P., Polivy, J., Pliner, P., Threlkeld, J. \& Munic, D. (1978). Distractability in dieters and nondieters: an alternative view of "externality". Journal of Personaliry and Social Psychology 36, 536-548.

Herman, C.P. \& Polivy, J. (1980). Restrained eating. In, A.B. Stunkard (Ed)., Obesity, pp. 208-225. Philadelphia: Saunders.

Herman, C.P. \& Polivy, J. (1982). Weight change and dietary concern in the overweight: are they really independent? Appetite 3, 280-281.

Herman, C.P. \& Polivy, J. (1984). A boundary model for the regulation of eating. In A.B. Stunkard \& E.Stellar (Eds). Eating and its disorders, pp. 141-156. New York: Raven Press.

Herman, C.P., Polivy, J. \& Esses, V.M. (1987). The illusion of counter-regulation. Appetite 9, 161 169.

Herman, C.P. \& Polivy, J. (1988). Restraint and excess in dieters and bulimics. In K.M. Pirke, W. Vandereycken and D. Ploog (Eds.), The Psychobiology of Bulimia Nervosa, pp 33-41. Berlin etc: Springer-Verlag.

Herman, C.P. \& Polivy, J. (1990). From dietary restraint to binge eating: attaching causes to effects. Appetite 14, 123-125.

Hibscher, J.A. \& Herman, C.P. (1977). Obesity, dieting, and the expression of 'obese' characteristics. Journal of Comparative Physiology and Psychology 91, 374-380.

Hoek, H.W. (1993). Review of the epidemiological studies of eating disorders. International Review of Psychiarry 5, 61-74

Hoek, H.W. (1995). The distribution of eating disorders. In, K.D. Brownell \& Ch. G. Fairburn (Eds.), Easing Disorders and Obesiry: a Comprehensive Handbook, pp. 207-211. New York, London: Guilford Press.

Hoffer, L.J. (1988). Starvation. In, M.E. Shils \& V.R. Young (eds.). Modern Nutrition in Health and Disease, 7th ed., pp. 774-794. Philadelphia: Lea \& Febriger.

Hohlstein, L.A., Gwirtsman, H.E., Whalen, F. \& Enns, M.P. (1986). Oral glucose tolerance in bulimia. International Journal of Eating Disorders 5, 157-160. 


\section{References}

Horwitz, D.L., McLane, M. \& Kobe, P., 1988. Response to a single dose of aspartame or saccharin by niddm patients. Diabetes Care 11, 230-234.

Jansen, A. (1990). Binge earing: Notes and Data. Doctoral dissertation. Maastricht: University of Limburg.

Jansen, A. (1994a). The learned nature of binge eating. In Ch. R. Legg \& D.A. Booth (Ed.), Apperire: Neural and Behavioural Bases, pp 193-211. Oxford: Oxford University Press.

Jansen, A. (1994b). Notitie over het aanleren en veranderen van smaakpreferenties. Suikerstichting Nederland.

Jansen, A., Oosterlaan, J., Merckelbach, H. \& Van den Hout, M.A. (1988a). Non-regulation of food intake in restrained, emotional, and external eaters. Journal of Psychopashology and Behavioral Assessment 10, 345-354.

Jansen, A., Merckelbach, H., Oosterlaan, J., Tuiten, A. \& Van den Hout, M.A. (1988b). Cognitions and self-talk during food intake of restrained and unrestrained eaters. Behaviour Research and Therapy, 26, 393-398.

Jansen, A., Van den Hout, M.A., De Loof, C. Zandbergen, J. \& Griez, E. (1989). A case of Bulimia successfully treated by cue exposure. Journal of Behaviour Therapy and Experimental Psychiatry $20,327-332$.

Jansen, A., Van den Hout, M.A. \& Griez, E. (1990). Clinical and non-clinical binges. Behaviour Research and Therapy 28, 439-444.

Jansen, A. \& Van den Hout, M.A. 1991. On being led into temptation: "counterregulation" of dieters after smelling a "preload". Addictive Behaviors 5, 247-253.

Jansen, A., Merckelbach, H. \& Van den Hout, M.A. (1992a). Experimentele Psychopathologie. As sen, Maastricht: van Gorcum.

Jansen, A., Boon, B., Nauta, H. \& van den Hout, M.A. (1992b). Salivation discordant with hunger. Behaviour Research and Therapy 30, 163-166.

Jansen, A., Broekmate, J. \& Heymans, M. (1992c). Cue-exposure vs self-control in the treatment of binge eating: a pilot study. Behaviour Research and Therapy 30, 235-241.

Johnson, W.G. \& Wildman, H.E. (1983). Influence of external and covert food stimuli on insulin secretion in obese and normal persons. Behavioral Neuroscience 97, 1025-1028.

Johnson, W.G., Jarrell, M.P., Chupurdia, K.M. \& Williamson, D.A. (1994). Repeated binge/purge cycles in bulimia nervosa: Role of glucose and insulin. International Journal of Eating Disorders $15,331-341$.

Joravsky, D. (1989). Russinn Psychology: A Critical Appraisal. Oxford etc.: Blackwell.

Kaye, W.H., Gwirtsman, H.E. \& George, D.T. (1989). The effect of bingeing and vomiting on hormonal secretion. Biological Psychiatry 25, 768-780.

Kennedy, S.H., Katz, R., Neitzer, C.S., Ralevski, E. \& Mendlowitz, S. (1995). Exposure with response prevention treatment of anorexia nervosa bulimic subtype and bulimia nervosa. Behaviour Research and Therapy 33, 685-689.

Klajner, F., Herman, P.H., Polivy, J. \& Chhabra, R. (198I). Human obesity, dieting, and anticipatory salivation to food. Physiology and Behavior 27, 195-198.

Kostarczyk, E., 1986. Autonomic correlates of alimentary conditioned and unconditioned reactions in the dog. Journal of the Ausonomic Nervous System 817, 279-288.

Laberg, J.C., Wilson, G.T., Eldredge, K. \& Nordby, H. (1991). Effects of mood on heart rate reactivity in bulimia nervosa. International Journal of Eating Disorders 10, 169-178.

Lacey, J.H. \& Gibson, E. (1985). Controlling weight by purgation and vomiting: a comparative study of bulimics. Journal of Psychiarric Research 19, 337-341

Laessle, R.G., Tuschl, R.J, Kotthaus, B.C. \& Pirke, K.M. (1989). A comparison of the validity of three scales for the assessment of dietary restraint. Journal of Abnormal Psychology 98, 504-507.

Landis, C. \& Hunt, W.A. (1939). The Startle Pantern. New York: Farrar \& Rinehart.

Landsberg, L. \& Young, J.B. (1978) Fasting, feeding and regulation of the sympathetic nervous system. 
New England Journal of Medicine 298, 1295-1301.

Lang, P.J., Bradley, M.M. \& Cuthbert, B.N. (1990). Emotion, attention, and the startle reflex. Psychological Review 97, 377-398.

Lang, P.J., Greenwald, M.K., Bradley, M.M. \& Hamm, A.O. (1993). Looking at pictures: affective, facial visceral and behavioral reactions. Psychophysiology 30, 261-273.

Lavy, E., van den Hout, M. \& Amtz, A. (1993). Attentional bias and spider phobia: conceptual and clinical issues. Behaviour Research and Therapy 31, 17. 24.

Lavy, E. \& van den Hout, M. (1993). Attentional bias for appetitive cues: effects of fasting in normal subjects. Behavioural and Cognitive Psychotherapy 21, 297-310.

LeGoff, D.B. \& Spiegelman, M.N. (1987). Salivary response to olfactory food stimuli as a function of dietary restraint and body weight. Appetite 12, 83-94.

LeGoff, D.B., Leichner, P. \& Spigelman, M.N. (1988). Salivary response to olfactory stimuli in anorexics and bulimics. Apperite $11,15-25$.

Le Magnen, J. (1985). Hunger. Cambridge etc: Cambridge University Press.

Le Magnen, J. (1992). Neurobiology of Feeding and Nutrision. San Diego etc: Academic Press.

LeBlanc, J. (1992). Cephalic phase response to food and its effect on feeding behavior. In, G.H. Anderson and S.H. Kennedy (Eds), The Biology of Feast and Famine: Relevance to Eating Disorders, 4760. San Diego etc., Academic Press.

Leitenberg, H., Gross, J., Peterson, J. \& Rosen, J.C. (1984) Analysis of an anxiety model and the process of change during exposure plus response prevention treatment of bulimia nervosa. Behavior Therapy 15, 3-20.

Leitenberg, H. \& Rosen, J. (1989). Cognitive-behavioral therapy with and without exposure plus response prevention in treatment of bulimia nervosa: comment on Agras, Schneider, Arnow, Raeburn, and Telch. Journal of Consulting and Clinical Psychology 57, 776-777.

Louis-Sylvestre, J. (1976). Preabsorptive insulin release and hypoglycemia in rats. American Journal of Physiology 230, 56-60.

Louis-Sylvestre, J. \& Le Magnen J. (1983). Vagotomy abolishes the differential palatability of food. Appetite 4, 295-299.

Louis-Sylvestre, J. (1984). Meal size: role of reflexly induced insulin release. Journal of the Autonomic Nervous System 10, 317-324.

Lowe, M.R. (1993). The effects of dieting on eating behavior: a three-factor model. Psychological Bulletin 114, 100-121.

Lucas, F., Bellisle, F. \& Di Maio, A. (1987). Spontaneous insulin fluctuations and the preabsorptive insulin response to food ingestion in humans. Physiology and Behavior 40, 631-636.

MacRae, J.R., Scoles, M.T. \& Siegel, S. (1987). The contribution of Pavlovian conditioning to drug tolerance and dependence. British Journal of Addicrion 82, 371-380.

Mahamedi, F. \& Heatherton, T.F. (1993). Effects of high calorie preloads on selective processing of food and body shape stimuli among dieters and nondie ters. International Journal of Eating Disorders 13, 305-314.

Marcus, M.D. (1993). Binge eating in obesity. In, C.G. Fairburn \& G.T. Wilson (Eds.). Binge Earing: Nature, Assessment, and Treatment, 77-96. New York, London: Guilford Press.

Marcus, M.D. (1995). Binge eating and obesity. In, K.D. Brownell \& Ch. G. Fairburn (Eds), Eating Disorders and Obesity: a Comprehensive Handbook, pp. 441-444. New York, London: Guilford Press.

Matysiak, J. \& Green, L. (1984). On the directionality of classically-conditioned glycemic responses. Physiology and Behavior 32, 5-9.

McDonald, R.P. \& Mulaik, S.A. (1979). Determinacy of common factors: a nontechnical review. Psychological Bulletin 86, 297-306.

McHugh, P.R. (1983). The control of gastic emptying. In, J.G. Kral, T.L. Powley \& C.McC. Brooks (Eds.), Vagal Nerve Function: Behavioural and Methodological Considerations, 221-231. 


\section{References}

Amsterdam, etc.: Elsevier.

McNally, R.J. (1994). Panic Disorder: a Critical Analysis. New York etc.: Guilford Press.

Miller, R.R., Barnet, R.C. \& Grahame, N.J. (1995). Assessment of the Rescorla-Wagner Model. Psychological Bullerin 117, 363-386.

Mitchell, J.E., Hatsukami, D., Eckert, E.D. \& Pyle, R.L. (1985). Characteristics of 275 patients with Bulimia. American Journal of Psychiatry 142, 482-485.

Montgomery, A.M.J. (1991). Animal models of eating disorders. In, P. Willner (Ed.), Behavioural Models in Psychopharmacology, pp. 177-214. Cambridge etc.: Cambridge University Press.

Moyer, A., Rodin, J. \& Cummings, N. (1993). Cephalic phase insulin release in Bulimia. International Journal of Eating Disorders 14, 331-339.

Mityushov, M.I. (1954). Conditioned reflex insulin secretion. Journal of Higher Nervous Activiry (in Russian) 4, 206-212.

Niaura, R.S., Rohsenow, D.J., Binkoff, J.A., Monti, P.M., Pedraza, M. \& Abrams, D.B. (1988). Relevance of cue reactivity to understanding alcohol and smoking relapse. Journal of Abnormal Psychology 97, 133-152.

Nicolaĩdis, S. (1977). Sensory-neuroendocrine reflexes and their anticipatory and optimizing role on metabolism. In M.R. Kare and O. Maller (Eds.), The Chemical Senses and Nurrition, 123-143. New York etc.: Academic Press.

Niijima, A. (1989). Nervous regulation of metabolism. Progress in Neurobiology 33, 135-147.

Nunnally, J.C. (1978). Psychometric Theory, 2nd ed. New York etc. McGraw-Hill.

Oettle, G.J., Emmett, P.M. \& Heaton, K.W. (1987). Glucose and insulin responses to manufactured and whole-food snacks. American Journal of Clinical Nutrition 45, 86-91.

Ogden, J. (1993). The measurement of restraint: confounding success and failure? International Journal of Ealing Disorders 13, 69-76.

Ogden, J. \& Greville, L., (1993). Cognitive changes to preloading in restrained and unrestrained eaters as measured by the Stroop task. International Journal of Eating Disorders 14, 185-195.

Overduin, J. (1993). Het voordeel van de schrik: over het onvolledig gebruik van de reflex in emotieonderzoeken. De Psycholoog 28, 193-200.

Overduin, J. \& Jansen, A. (1996). A new scale for use in non-clinical research into disinhibitive eating. Personaling and Individual Differences, in press.

Overduin, J., Jansen, A. \& Eilkes, H. (1996). Cue reactivity to food- and body stimuli in restrained and uarestrained eaters. Addictive Behaviors in press.

Overduin, J. \& Jansen, A. submitted. Conditioned insulin and blood sugar responses in bumans in relation to binge eating.

Pavlov, I.P. (1897/1910). The Work of the Digestive Glands. London: Griffin.

Peterson, C., Maier, S.F. \& Seligman, M.E.P., 1993. Learned Helplessness: a Theory for the Age of Personal Control. New York, Oxford: Oxford University Press.

Pirke, K.M. (1995). Physiology of Bulimia Nervosa. In, C.G. Fairburn \& G.T. Wilson (Eds.). Binge Eating: nature, assessment, and trearment, 261-265. New York, London: Guilford Press.

Polivy, J. \& Herman, C.P. (1985). Dieting and binging: a causal analysis. American Psychologist 40, 193-201.

Polivy, J., Heatherton, T.F. \& Herman, C.P. (1988). Self-esteem, restraint, and eating behavior. Journal of Abnomal Psychology 97, 354-356.

Polivy, J. \& Herman, C.P. (1989). Dietary restraint and binge eating: Response to Charnock. British Journal of Clinical Psychology 28, 341-343.

Polonsky, K.S., Given, D., Hirsch, L. et al. (1988). Quantitative study of insulin secretion and clear ance in normal and obese subject. Journal of Clinical Investigation 81, 435-441.

Polonsky, K.S. \& Rubenstein, A.H., 1984. C-peptide as a measure of the secretion and hepatic extraction of insulin. Diabetes 33, 486-494.

Powell, J., Gray, J. \& Bradley, B. (1993). Subjective craving for opiates: evaluation of a cue exposure 
protocol for use with detoxified opiate addicts. British Journal of Clinical Psychology 32, 39-53.

Powley, T.L. \& Berthoud, H-R. (1985). Diet and cephalic phase insulin responses. American Journal of Clinical Nutrition 42, 991-1002.

Razran, R. (1961). The observable unconscious and the inferable conscious in current Soviet psychophysiology. Psychological Review 68, 81-147.

Read, N.W., Welch, I. McL., Austen, C.J., Barnish, C. et al. (1986). Swallowing food without chewing: a simple way to reduce postprandial glycaemia. British Journal of Nutrition 55, 43-47.

Rescorla, R.A. (1967). Pavlovian conditioning and its proper control procedures. Psychological Review $74,71-80$.

Revusky, S. \& Garcia, J. (1970). Leamed associations over long delays. In, G.H. Bower (Ed), The Psychology of Learning and Motivation vol 4., pp. 1-84. New York, London: Academic Press.

Robbins, S.J. \& Ehrman, R.N. (1992). Designing studies of drug conditioning in humans. Psychopharmacology $106,143-153$.

Rodin, J., Bach, J., Peranini, E. \& De Fronzo, F. (1985). Effect of insulin and glucose on feeding behavior. Metabolism 34, 827-833.

Rodin, J., Reed, R. \& Jamner, L. (1988). Metabolic effects of fructose and glucose: implications for food intake. American Journal of Clinical Nutrition 47, 683-689.

Rodin, J. (1990). Comparative effects of fructose, aspartame, glucose, and water preloads on calorie and macronutrient intake. American Journal of Clinical Nutrition, 51, 428-435.

Rogers, P.J. \& Hill, A.J. (1989). Breakdown of dietary restraint following mere exposure to food stimuli: interrelationships between restraint, hunger, salivation, and food intake. Addictive Behaviors 14, 387-397.

Rosen, J.C. \& Leitenberg, H. (1982). Bulimia Nervosa, treatment with exposure and response prevention. Behavior Therapy 13,117-124.

Rosen, J.C., Leitenberg, H., Fisher, C. \& Khazam, C. (1986). Binge-eating episodes in bulimia nervosa: the amount and type of food consumed. International Journal of Eating Disorders 5, 255-267.

Rosenberg, M. (1965). Society and the Adolescent Self-image. Princeton, N.J.: Princeton University Press.

Rossiter, E.M, \& Agras, W.S. (1990). An empirical test of the DSM-III-R definition of binge. Inter national Journal of Eating Disorders 9, 513-518.

Rossiter, E.M., Agras, W.S., Telch, C.F. \& Bruce, B. (1992). The eating patterns of non-purging bulimic subjects. International Journal of Eating Disorders 11, 111-120.

Ruderman, A.J. (1983). The Restraint Scale: a psychometric investigation. Behaviour Research and Therapy 21, 253-258.

Ruderman, A.J. (1985). Dysphoric mood and overeating: a test of restraint theory's disinhibition hypothesis. Journal of Abnormal Psychology, 94, 78-85.

Ruderman, A. J. (1986). Dietary restraint: a theoretical and empirical review. Psychological Bulletin $99,247-262$.

Russell, G. (1979). Bulimia nervosa: an ominous variant of anorexia nervosa. Psychological Medicine 9, 429-448.

Sapolsky, R.M. (1994). Why Zebras Don't Get Ulcers: A Guide To Stress, Stress-related Diseases, and Coping. New York: Freeman \& Co.

Schmidt, U. \& Marks, I.M. (1988). Cue exposure to food plus response prevention of binges for bulimia: a pilot study. International Journal of Eating Disorders 7, 663-672.

Schmidt, U. \& Marks, I.M. (1989). Exposure plus prevention of bingeing vs. exposure plus prevention of vomiting in bulimia nervosa: a crossover study. Journal of Nervous and Menial Disease 177, 259-266.

Schultz-Gambard, E. (1988). Indikatoren von Hunger: Psychophysiologische Untersuchung zur Wirkung einer 24-stündigen Nahrungsdeprivation. Doctoral Dissertation. Bielefeld: University of Bielefeld.

Schwartz, B. \& Robbins (1995). Psychology of Learning \& Behavior, 4th ed. New York etc: Norton.

Schweiger, U., Poellinger, J., Laessle, RF., Wolfram, G., Fichter, M.M. \& Pirke K.-M. (1987). 


\section{References}

Altered insulin response to a balanced test meal in bulimic patients. International Journal of Eating Disorders 6, 551-556.

Sclafani, A. (1995). How food preferences are learned: laboratory animal models. Proceedings of the Nutrition Society 54, 419-427.

Service, F.J. (1995). Hypoglycemic disorders. New England Journal of Medicine 332, 1144-1152.

Shively, C.A., Apgar, J.L. \& Tarka, S.M. (1986). Postprandial glucose and insulin responses to various snacks of equivalent carbohydrate content in normal subjects. American Journal of Clinical Nurrition 43, 335-342.

Siegel, S. (1972). Conditioning of insulin-induced glycemia. Journal of Compararive and Physiological Psychology 78, 233-241.

Siegel, S. (1983). Classical conditioning, drug tolerance and drug dependence. In, R. Smart, F. Glaser, Y. Israel, H. Kalant, R. Popham, and W. Schmidt (Eds.), Research Advances in Alcohol and Drug Problems, pp 207-246. New York: Plenum Press.

Siegel, S. (1989). Pharmacological conditioning and drug effects. In, A.J. Goudie \& M.W. EmmettOglesby (Eds.), Psychoactive Drugs: Tolerance and Sensitization, pp 115-180. Clifton, New Jersey: Humana Press.

Siegel, S. \& Nettleton, N. (1970). Conditioning of insulin-induced hyperphagia. Journal of Comparative and Physiological Psychology 72, 390-393.

Simon, C., Schlienger, J.L, Sapin, R. \& Imler, M. (1986). Cephalic phase insulin secretion in relation to food presentation in normal and overweight subjects. Physiology and Behavior 36, 463-469.

Sjōstrōm, L., Garellick, G., Krotkiewski, M. \& Luyckx, A. (1980). Peripheral insulin in response to the sight and smell of food. Metabolism 29, 901-909.

Steffens, A.B., Strubbe, J.H., Scheurink, A.J.W. \& Balkan, B. (1991). Neuroendocrine activity during food intake modulates secretion of the endocrine pancreas and contributes to the regulation of body weight. In: M.I. Friedman, M.G. Tordoff and M.R. Kare (Eds.), Chemical Senses Vol. 4: Apperite and Nutrition, pp 405-426. New York: Marcel Dekker.

Strubbe, J.H. (1992). Parasympathetic involvement in rapid meal-associated conditioned insulin secretion in the rat. American Journal of Physiology 263, R615-R618.

Strubbe, J.H. \& Steffens, A.B. (1975). Rapid insulin release after ingestion of a meal in the unanes thetized rat. American Journal of Physiology 229, 1019-1022.

Strubbe, J.H. \& Steffens, A.B. (1993). Neural control of insulin secretion. Hormones and Metabolic Research 25, 507-512.

Stunkard, A.J. \& Messick, S. (1985). The Three-Factor Eating Questionnaire to measure dietary restraint, disinhibition and hunger. Journal of Psychosomatic Research 29, 71-83.

Teff, K.L., Mattes, R.D. \& Engelman, K. (1991). Cephalic phase insulin release in normal weight males: verification and reliability. American Journal of Physiology 261, E430-E436.

Teff, K.L., Levin, B.E. \& Engelman, K. (1993). Oral sensory stimulation in men: effects on insulin, C-peptide, and catecholamines. American Journal of Physiology 265, R1223-R1230.

Teff, K.L., Devine, J., Engelman, K. (1995). Sweet taste: effect on cephalic phase insulin release in men. Physiology and Behavior 57, 1089-1095.

Thompson, J.P., Palmer, R.L. \& Petersen, S.A. (1988). Is there a metabolic component to counter regulation? International Journal of Eating Disorders 7, 307-319.

Tiffany, S.T. (1995a). Potential functions of classical conditioning in drug addiction. In, D.C. Drummond, S.T. Tiffany, S. Glautier \& B. Remington (Eds.), Addictive Behaviour: Cue Exposure Theory and Practice, pp 47-71. Chichester etc: Wiley.

Tiffany, S.T. (1995b). The role of cognitive factors in reactivity to drug cues. In, D.C. Drummond, S.T. Tiffany, S. Glautier \& B. Remington (Eds.), Addictive Behaviour: Cue Exposure Theory and Practice, pp 137-165. Chichester etc: Wiley.

Tobin, D.L., Johnson, C, Steinberg, S., Staats, M. \& Dennis, A.B. (1991). Multifactorial assessment of bulimia nervosa. Journal of Abnormal Psychology 100, 14-21. 
Tse, Th. F., Clutter, W.E., Shah, S.D., Miller, J. Ph. \& Cryer, Ph. E., 1983. Neuroendocrine responses to glucose ingestion in man. Journal of Clinical Investigation 72, 270-277.

Turkkan, J.S. (1989). Classical conditioning: the new hegemony. Behavioral and Brain Sciences 12 , 121-179.

Turner, M.St.J., Foggo, M., Bennie, J., Carroll, S., Dick, H. \& Goodwin, G.M. (1991). Psychological, hormonal and biochemical changes following carbohydrate bingeing: a placebo controlled study in bulimia nervosa and matched controls. Psychological Medicine 21, 123-133.

Tuschl, R.J. (1990). From dietary restraint to binge eating: some theoretical considerations. Apperite 14, 105-109.

Van Der Heijden, P.G.M. (1995). Hoe te leven met statistiek. Nederlands Tijdschriff voor de Psychologie $50,134-146$.

Van Het Reve, K. (1979). Een Dag uit het Leven van de Reuzenkoeskoes. Amsterdam: G.A. van Oorschot.

Van Strien, T., Frijters, J.E.R., Bergers, G.P.A. \& Defares, P.B. (1986). The Dutch Eating Behaviour Questionnaire (DEBQ) for assessment of restrained, emotional, and external eating behavior. International Journal of Eating Disorders 2, 295-315.

Van Vort, W. \& Smith, G.P. (1987). Sham feeding experience produces a conditioned increase of meal size. Appetite 9, 21-29.

Velden, M \& Graham, F.K. (1988). Depicting heart rate over real time: two procedures that are mathematically identical. Journal of Psychophysiology 2, 291-292.

Venables, P.H. (1991). Autonomic activity. Annals of the New York Academy of Sciences 620, 191207.

Walsh, B.T. (1992). Diagnostic criteria for eating disorders in DSM-IV: work in progress. Internasional Journal of Eating Disorders, 4, 301-304.

Walsh, B.T. (1993). Binge eating in bulimia nervosa. In: C.G. Fairburn \& G.T. Wilson (Eds), Binge Eating: Nature, Assessment, and Treatment, pp 37-49. New York, London: Guilford Press.

Wardle, J. (1980). Dietary restraint and binge eating. Behavioural Analysis and Modification 4, 201 209.

Wardle, J. (1986). The assessment of restrained eating. Behaviour Research and Therapy 24, 213-215.

Wardle, J. (1990). Conditioning processes and cue exposure in the modification of excessive eating. Addictive Behaviors 15, 387-393.

Wardle, J. \& Beinart, H. (1981). Binge eating: a theoretical review, British Journal of Clinical Psychology $20,97-109$.

Webber, J. \& MacDonald, I.A. (1993). Metabolic actions of catecholamines in man. Bailliere's Clinical Endocrinology and Metabolism 7, 393-413.

Weingarten, H.P. (1984). Meal initiation controlled by learned cues: basic behavioral properties. Appetise $5,147-158$.

Weingarten, H.P. (1985). Stimulus control of eating: implications for a two-factor theory of hunger. Appetite 6, 387-401.

Weingarten, H.P., Hendler, R. \& Rodin, J. (1988). Metabolism and endocrine secretion in response to a test meal in normal-weight bulimic women. Psychosomatic Medicine 50, 273-285.

Weingarten, H.P. \& Elston, D. (1990). The phenomenology of food cravings. Appetile 15, 231-246.

Westenhoeffer, J. (1991). Dietary restraint and disinhibition: is restraint a homogeneous construct? Appetite 16, 45-55.

Westenhoeffer, J., Broeckmann, P., Muench, A-K. \& Pudel, V. (1994). Cognitive control of eating behaviour and the disinhibition effect. Appetite 23, 27-41.

Wilde, G.J.S. (1977). Trait description and measurement by personality questionnaires. In, R.B. Cartell \& R.M. Dreger (Eds.), Handbook of Modern Personality Theory, 69-103. New York, etc: Wiley \& Sons.

Williams, R.A. (1968). Effects of repeated food deprivations and repeated feeding tests on feeding 


\section{References}

behavior. Journal of Comparative and Physiological Psychology 65, 222-226.

Willner, P. (1991a). Behavioural Models in Psychopharmacology: Theoretical, Industrial and Clinical Perspectives. Cambridge etc: Cambridge University Press.

Willner, P. (1991b). Methods for assessing the validity of animal models of human psychopathology. In, A.A. Boulton, G.B. Baker \& M.T. Martin-Iverson (Eds.), Animal Models in Psychiarry vol. I. , 1-23. Clifton, N.J.: Humana Press.

Woods, S.C. (1976). Conditioned hypoglycemia. Journal of Comparative and Physiological Psychology $90,1164-1168$.

Woods, S.C. (1981). Abstract from discussion with H.-R. Berthoud. Diabetologia 20, 401.

Woods, S.C. (1983). Conditioned hypoglycemia and conditioned insulin secretion. Advances in Metabolic Disorders 10, 485-495.

Woods, S.C. (1990). On blood glucose and eating. International Journal of Obesity 14 (suppl 3), $33-$ 34.

Woods, S.C. (1991). The eating paradox: how we tolerate food. Psychological Review 98, 488-505.

Woods, S.C. \& Kulkosky, P.J. (1976). Classically conditioned changes of blood glucose level. Psychosomatic Medicine 38, 201-219.

Woods, S.C., Makous, W. \& Hutton, R.A. (1969). Temporal parameters of conditioned hypoglycemia. Journal of Comparative and Physiological Psychology 69, 301-307.

Woods, S.C. \& Shogren, R.E. (1972). Glycemic responses following conditioning with different doses of insulin in rats. Journal of Comparative and Physiological Psychology, 81, 220-235.

Woods, S.C., Vasselli, J.R., Kaestner, E., Szakmary, G.A., Milburn, P. \& Vitiello, M.V. (1977). Conditioned insulin secretion and meal feeding in rats. Journal of Comparative and Physiological Psychology 91, 128-133.

Woods, S.C. \& Strubbe, J.H. (1994). The psychobiology of meals. Psychonomic Bulletin \& Review 1, $141-155$.

Wooley O.W., Wooley S.C. \& Williams B.S. (1976). Salivation as a measure of appetite: studies of the anorectic effects of calories and amphetamine. In, D. Novin, W. Wyrwicka \& G.A. Bray, Hunger: Basic Mechanisms and Clinical Implications, ppn 421-429).

Zamble, E. (1973). Augmentation of eating following a signal for feeding in rats. Learning and Motivation 4, 138-147. 


\section{Appendix}

\section{TRANSLATION ${ }^{1}$ OF M.I. MTTYUSHOV ${ }^{2}$ (1954) \\ "CONDITIONED REFLEX SECRETION OF INSULIN"}

From the viewpoint of today's Pavlovian physiology, the internal secretory glands form a powerful lever which enables the nervous system to change all physiological functions over a broad range. The central nervous system regulates various functions of the organism, a process that not only involves nervous impulses through specific pathways to the organs, but also a hormonal, viz. humoral link.

The mechanism by which endocrine glands participate in the nervous regulation of physiological functions, has until now been scantily studied. The main reason for this lies in the fact that until recently, even in the Soviet Union, a majority of the endocrine physiologists has adopted a methodologically unsound approach towards the study of internal secretory glands.

The internal glands were considered in isolation from the nervous system and ascribed an autonomous and often predominant role in physiological regulation. K.M. Bykov (2) in his speech at the plenary session of the Academy of Sciences of the USSR and the Academy of Medical Sciences, dedicated to the problems in Pavlovian physiological studies has stated: "In recent years, the study of hormones has secluded itself from general physiology and pathology into the specific discipline of endocrinology. The consequent isolation of specific subproblems from the more general study of organisms has lead many endocrinologists and medics to the erroneous conclusion that the various hormones play a self-supporting, autonomous role in the life of the organism".

After the plenary session the attention of many researchers was drawn towards the study of nervous regulation of endocrine glands. The study of these issues opens new vistas for the understanding of the mechanisms as well as the significance of the various glandular contributions to the physiological regulation in the organism.

Up until now the nervous regulation of the endocrine glands has been very scantly investigated. In the literature one can find a large number of facts about nervous regulation of the pituitary, the adrenal cortex as well as of the thyroid gland. The nervous regulation

\footnotetext{
'Originally published as: Матошов, М. И. (1954). Усповнорефлекторнан инкреция инсулнна. Журвал Высшеа Нервнов Деятельвостн 4, 206-212. Translation: Joost Overduin, Limburg University, Maastricht, The Netherlands; Prof. Gyōrgy Ádám, Eōtvōs University, Budapest, Hungary. About a dozen idioms have been adopted from an earlier translation by L.J. Shein. Prof. Shepard Siegel, McMaster University, Hamilton, Ontario is acknowledged for providing us with this translation.
}

${ }^{2}$ M.I. Mityushov, Laboratory of Nervous Regulation of Endocrine Functions, I.P. Pavlov Physiological lnstitute, USSR Academy of Sciences. 
of other endocrine glands, specifically the pancreatic islets, has until now received considerably less attention. Different authors have made quite contradictory assertions on this subject.

The insular apparatus of the pancreas plays an exceptionally important role in the regulation of carbohydrate metabolism. That is why the study of the nervous regulation of this activity is of great interest. The purpose of our study is an investigation of the cortical regulation of insulin secretion.

In the literature we found very few data on the cortical regulation of the insular apparatus of the pancreas. Observations by many clinical scientists suggest a role of psychic trauma in the development of diabetes in humans. As an example, the case described by B.G. Baranov can be quoted, who observed the development of severe form of diabetes in a woman who had suffered a nervous breakdown. Moreover, experimental research by N.S. Sedina (7) demonstrated a conditioned hypoglycemia to subcutaneous and intraperitoneal injections of saline, following previous repeated glucose administration through subcutaneous and intraperitoneal injections. She attributes the development of hypoglycemia to a conditioned increase of insulin production. Her attempt to induce conditioned hypoglycemia through intravenous glucose injections did not succeed however.

In our research we used the conditioned reflex method likewisely. The experiments were carried out on 4 dogs as well as on 9 human subjects. The unconditioned stimulus in our experiment was a sharp change in blood sugar following the intravenous glucose injection. As is well-known, a change in blood sugar level is an adequate stimulus for the insular apparatus including all further mechanisms regulating blood sugar level. In our opinion, the intravenous glucose injection appears to be the most rational means of influencing the insular apparatus, because intravenous injections allow for precise adjustments of the stimulus intensity (glucose instantaneously enters the blood stream in a concentration unhampered by a slow rate of absorption such as seen after subcutaneous and intraperitoneal injections.

The absence of conditioned reflex hypoglycemia in the study of N.S. Sedina (7) who used intravenous glucose injections as a UCS, does not seem crucial to the issue of whether conditioned reflex hypoglycemia is possible. Sedina conducted her experiments in two dogs. In one of them, a total of 10 trials was run. The CS was paired with the UCS merely in 7 cases within a two-month period. In the second dog, 9 trials were run in which CS and US were paired only 6 times in the course of 11 days. Clearly, after such a small number of pairings it would be unfeasible to conclude the impossibility of producing a conditioned reflex.

The conditioned stimulus in our study consisted of the intravenous injection procedure as well as the circumstances under which it took place (the glucose was injected regularly at 
Table A.1. Control trials (saline injections) in dogs

Data (1952)

Number of before the injection previous saline (2 measurements with injections $10 \mathrm{~min}$. in between)

$$
\text { minutes after the saline injection }
$$

$\begin{array}{lllll}5 & 15 & 30 & 60 & 90\end{array}$

Dog Baikal

11 III

18 III

22 III

27 III

3 IV

9 IV

$\begin{array}{lllll}99 & 102 & 102 & 97 & - \\ 107 & 103 & 105 & 96 & 98 \\ 94 & 89 & 89 & 91 & 89 \\ 103 & 108 & 108 & 106 & 99 \\ 92 & 89 & 89 & 91 & 94 \\ - & 88 & 88 & 85 & 83\end{array}$

$97: 97$
$94 ; 96$
$91 ; 89$
$103 ; 105$
$92 ; 91$
$85 ; 83$

$74 ; 74$

$77 ; 77$

$70 ; 72$

Dog Dik

$\begin{array}{llllllll}8 \mathrm{I} & 2 & 74 ; 74 & 76 & 76 & 80 & 80 & 80 \\ 10 \mathrm{I} & 3 & 77 ; 77 & 75 & 77 & 75 & 75 & 75 \\ 12 \mathrm{I} & 5 & 70 ; 72 & 74 & 74 & 77 & 77 & 79\end{array}$

Table A.2. Conditioned changes in blood sugar level after repeated glucose injections

\begin{tabular}{|c|c|c|c|c|c|c|c|}
\hline \multirow{3}{*}{ Data (1952) } & \multicolumn{7}{|c|}{ BLOOD SUGAR LEVEL $(\mathrm{mg} / \mathrm{dl})$} \\
\hline & \multirow{2}{*}{$\begin{array}{l}\text { Number of } \\
\text { previous glu- } \\
\text { cose injections }\end{array}$} & \multirow{2}{*}{$\begin{array}{l}\text { before the injection } \\
\text { ( } 2 \text { measurements with } \\
10 \text { min. in between) }\end{array}$} & \multicolumn{5}{|c|}{ minutes after the saline injection } \\
\hline & & & 5 & 15 & 30 & 60 & 90 \\
\hline \multicolumn{8}{|l|}{ Dog Baikal } \\
\hline $9 \mathrm{IV}$ & 8 & $83 ; 83$ & 82 & 80 & 82 & 83 & 87 \\
\hline $15 \mathrm{IV}$ & 14 & $96 ; 96$ & 94 & 85 & 87 & 92 & 90 \\
\hline $22 \mathrm{~V}$ & 16 & $87 ; 92$ & 79 & 85 & 87 & 88 & 90 \\
\hline $3 \mathrm{VI}$ & 20 & $94 ; 95$ & 92 & 97 & 99 & 97 & 92 \\
\hline $10 \mathrm{VI}$ & 22 & $95 ; 97$ & 91 & 93 & 99 & 100 & - \\
\hline $19 \mathrm{VI}$ & 24 & $97 ; 100$ & 95 & 91 & 88 & 97 & \\
\hline $26 \mathrm{VI}$ & 26 & $96 ; 96$ & 90 & 85 & 90 & 96 & 96 \\
\hline 8 VII & 29 & $92 ; 92$ & 87 & 80 & 87 & 89 & 98 \\
\hline $14 \mathrm{VII}$ & 30 & $88 ; 88$ & 86 & 77 & 88 & 104 & - \\
\hline \multicolumn{8}{|l|}{ Dog Dik } \\
\hline $5 \Pi$ & $9^{3}$ & $77 ; 79$ & 72 & 76 & 79 & 77 & 77 \\
\hline 19 II & 14 & $79 ; 77$ & 72 & 70 & 76 & 74 & 72 \\
\hline $28 \mathrm{II}$ & 17 & $76 ; 78$ & 72 & 72 & 74 & 78 & 74 \\
\hline $13 \mathrm{III}$ & 23 & $81 ; 80$ & 74 & 71 & 78 & 78 & 8 \\
\hline $23 \mathrm{~V}$ & 36 & $88 ; 96$ & 85 & 79 & 83 & 85 & 8 \\
\hline $3 \mathrm{VI}$ & 40 & $90 ; 92$ & 79 & 79 & 86 & 88 & 88 \\
\hline $10 \mathrm{VI}$ & 42 & $88 ; 86$ & 73 & 73 & 81 & 75 & 81 \\
\hline $19 \mathrm{VI}$ & 45 & $90 ; 84$ & 90 & 82 & 84 & 88 & 82 \\
\hline 8 VII & 51 & $85 ; 85$ & 80 & 78 & 83 & 83 & - \\
\hline 14 VII & 52 & $82 ; 85$ & 82 & 74 & 82 & 82 & \\
\hline
\end{tabular}

${ }^{3}$ From the first to the fiftheenth trial the UCS was an injection of $100 \mathrm{ml} 40 \%$ glucose solution. From the 15 th trial onwards, the UCS became $150 \mathrm{ml} 40 \%$ glucose solution. 
Table A.3. Conditioned changes in blood sugar level after injection of saline in humans

Data (1952)

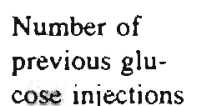

before the injection

BLOOD SUGAR LEVEL ( $\mathrm{mg} / \mathrm{dl}$ )

cose injections

(2 measurements with

minutes after the saline injection remarks

$10 \mathrm{~min}$. in between)

$5 \quad 15 \quad 45$

Subject S.G.

$\begin{array}{llllll}7 \text { III } & 5 & 93 ; 93 & 86 & 88 & 77 \\ \text { I4 III } & 10 & 80 ; 78 & 76 & 71 & 76 \\ 21 \text { III } & 15 & 83 ; 85 & 78 & 72 & 72 \\ 25 \text { III } & 17 & 89 ; 78 & 84 & 78 & 84 \\ & & & & & \\ 28 \text { III } & 18 & 99 ; 87 & 85 & 81 & 79 \\ \text { 3I III } & 19 & 105 ; 103 & 91 & 87 & 83 \\ 4 \text { IV } & 21 & 83 ; 87 & 83 & 85 & 83\end{array}$

Subject G.A.

7 III

$82 ; 82$

$\begin{array}{lll}79 & 73 & 66\end{array}$

77

$60 \mathrm{ml} 40 \%$ glucose

solution as a US

14 III

$74 ; 78$

$73 ; 69$

$91 ; 85$

$101 ; 101$

$83 ; 87$

$71 \quad 71$

$61 \quad 63$

7
8

$84 \quad 78$

$85 \quad 74$

$82 \quad 82$

76

72

84 injection occurred

in other room

28 III

19

90; 92

$\begin{array}{ll}88 & 88\end{array}$

injection occurred

in other room

4 IV

23

91; -

$\begin{array}{lll}82 & 76 & 78\end{array}$

$40 \mathrm{ml} 40 \%$ glucose

injection as US

25 IV

17

84; -

$\begin{array}{lll}76 & - & 78\end{array}$

$81 ; 83$

$\begin{array}{lll}74 & 72 & 80\end{array}$

$28 \mathrm{~V}$

19
27

94; 94

$\begin{array}{lll}87 & 85 & 85\end{array}$

Subject P.F.

14 IV

10

74; -

$74 \quad 72$

69

$40 \mathrm{ml} 40 \%$ glucose

21 IV

15

83; -

$\begin{array}{lll}74 & 74 & 76\end{array}$

25 IV

91; -

28 lV

20

$81 ; 79$

$\begin{array}{lll}84 & 87 & 89\end{array}$

$\begin{array}{lll}70 & 70 & 72\end{array}$ 
identical hours of the day by the same persons). On certain days, instead of glucose injections, intravenous saline of equal volume was given in the same manner, i.e., only the $\mathrm{CS}$ was presented. Prior to the glucose injections, several control trials were run to ascertain whether injections of $40-150 \mathrm{ml}$ saline in itself would have any effect. It was shown that the intravenous saline injections either did not change the blood sugar level, or did cause an unsignificant rise of $5-7 \mathrm{mg} \%$. More often, an increase in bloodsugar was seen, and this may be explained by certain emotional arousal that results from the injections procedure. This blood sugar rise in response to saline injections does not disappear even if intravenous injection have been given more often (in our experiment until 25 times, in dog Baikal).

The sharp rise of bloodsugar level after intravenous glucose injections appears to be a strong stimulus to which the insular apparatus must respond with an enhanced release of insulin. Repeated injections of glucose under definite stereotyped circumstances had to yield conditioned reflex secretion of insulin in response to intravenous saline injected under the same circumstances. Daily, or every second day, we infused from 40 to $150 \mathrm{ml} 40 \%$ glucose solution in the $v$. saphena of the dogs. Intravenous injections of the above glucose doses caused an increase of blood sugar concentration up to $550 \mathrm{mg} \%$ (during 5 minutes after the injection) that generally returned to baseline levels during the first hour. Blood sugar levels were assessed by means of the method of Hagedorn and Jensen. The blood to be analyzed was drawn from the marginal vein of the ear. Our experiments have shown that it is sufficient to present the dogs 8 to 10 times with the abovementioned dose of glucose, before saline injections carried out in the same manner started to cause a blood sugar fall of $5-15 \%$. The experimental results in all 4 dogs were of the same type. For the purpose of brevity we will only present the results of 2 dogs.

Dog Baikal, male, weight $10.5 \mathrm{~kg}$, received intravenous injections of saline in 25 sessions that were run before the start of sessions with glucose presentation. On six occasions bloodsugar concentrations were determined, and not once a lowering of blood sugar level was found after the intravenous saline injection (table A.1). After 8 glucose injections (40 $\mathrm{ml} 40 \%$ solution), i.e., 8 pairings of CS and UCS, saline injections in the same manner started to cause decreases in blood sugar level. The blood sugar decrease now was elicited by the action of the CS alone. Usually the blood sugar level returned to its initial value during the first hour. The level of blood sugar in the glucose injection trials rose to 380 $420 \mathrm{mg} \%$.

Dog Dik, male, weight $26,5 \mathrm{~kg}$. Prior to the administration of glucose, control injections with saline did not cause a noticeable decrease in bloodsugar level. Six trials were conducted with saline injections, and in three cases the blood sugar levels were determined (table A.1). After 9 glucose injections (100 $\mathrm{ml} 40 \%$ solution) causing increases in blood 
sugar level up to $450-520 \mathrm{mg} \%$, saline injections carried out in the same manner started to elicit a fall in blood sugar level (table A.2). In these sessions, a return to baseline levels of blood sugar occurred in the course of the first hour, just as had been the case in dog Baikal. The experimental results in the other two dogs, Zhuchka and Bek, were completely analogous to the cases described above. We carried out 39 trials with the dogs, in which after an initial 7-11 glucose injections, a saline injection was given instead, i.e. only the CS was presented by us. In 35 of these trials we got a noticeable fall of blood sugar level after saline injection, in 4 trials the aforementioned effect was not seen (in 2 of the latter 4 sessions a violation of the usual procedure had occurred: the regular experimenter was absent, and had been replaced by another person. Our observations on humans ( 9 in fact healthy subjects aged between 31 and 56) confirm the results of the trials run with animals. We injected $40-60 \mathrm{ml} 40 \%$ glucose solution, in the $v$. cubitalis.

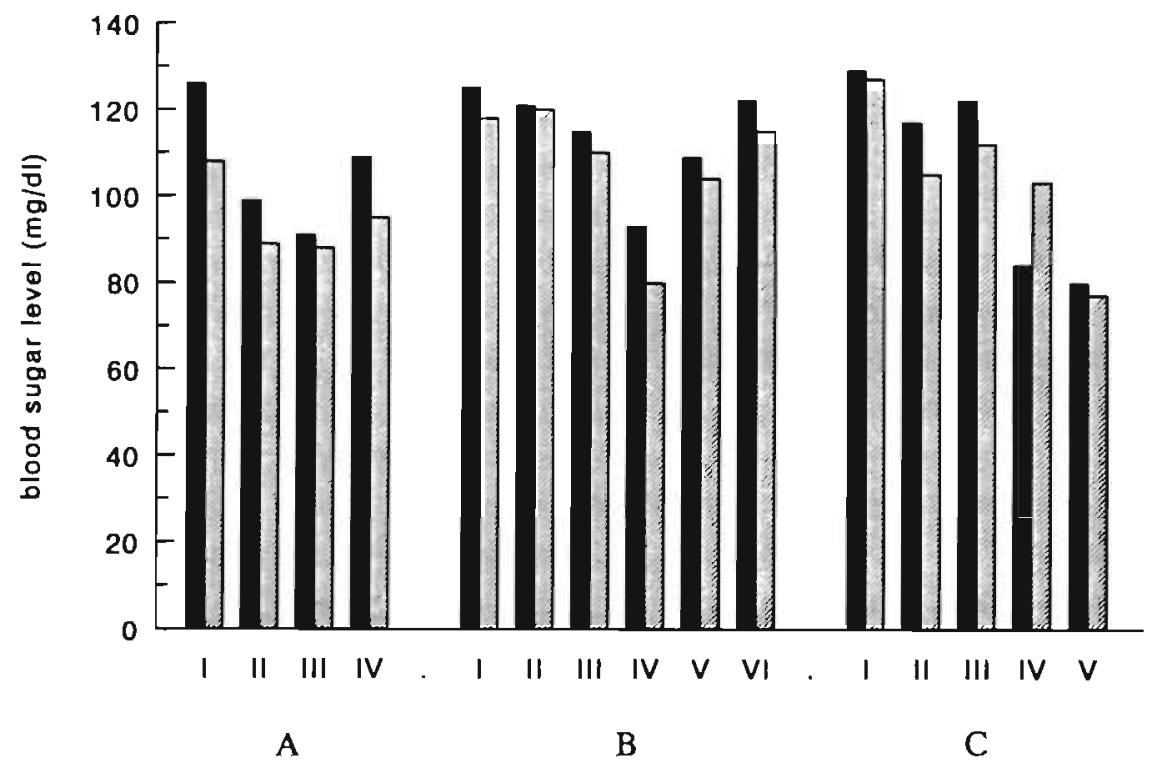

figure A.1: Determination of insulin content in dogs' blood by the bloodsugar test in white mice.

- black-colored bars: blood sugar concentration in milligram\% in the mice at 2 hours after injecting them with dog blood taken just before the saline injection;

- gray-colored bars: the same, but laken from dogs at 15 minutes after the saline injection, being the CS to insulin secretion (The US consisted of a glucose injection). Each bar represents the mean blood sugar level in mice after infusion of $0,6 \mathrm{ml}$ blood of the dogs A-Dik; B- Baikal; C- Bek. For each dog, blood was tested on a different number of occasions. 
At five minutes after the injection, the blood sugar level increased to values up to $250 \mathrm{mg} \%$ and a return to baseline level appeared in the course of the first hour. Blood for the analyses was drawn from the finger according to the usual method.

Preliminary control trials showed that an intravenous saline injection caused either no noticeable change or a non-significant increase in blood sugar level. After 5-10 fold repeated glucose injections, saline injections, i.e. presentation of the CS alone, began to cause a decrease in blood sugar level of between 5 and $20 \mathrm{mg} \%$ (table A.3). The data collected in all 9 subjects appeared to be alike: therefore, in table A. 3 we only show the results of 4 subjects. In humans, 33 sessions were run in which, after a preceding 5 - to 14 fold glucose administration, the same quantity of saline was injected under the same circumstances, i.e. the CS was presented alone. In 30 of these sessions we found a clear drop in blood sugar level after the saline injections. In 3 sessions the blood sugar level did not change. In those cases we had not followed the usual experimental procedure: for reasons beyond our influence, the saline injections had to be given in a different room. Thus, in the overwhelming majority of investigations in both animals and humans we found a conditioned fall in blood sugar in response to a saline injection. As indicated above, saline by itself did not evoke similar changes in blood sugar level.

The conditioned fall in blood sugar found by us, as well as that found by H.C. Sedina (7) with subcutaneous and intraperitoneal glucose injections, may depend on two factors: first, on a conditioned insulin secretion, and second, on a conditioned increase of glucose utilization by the tissues of the organism. In the literature there are reports by a number of authors on conditioned lowering of blood sugar level after of systematic subcutaneous injections of insulin in a stereotyped manner (J.A. Povorinski (5), V.A. Savchenko (6), I.J. Maleva (4), and C.M Leites (3). In the investigations of these authors the conditioned decrease of blood sugar appeared to be based exclusively on a conditioned enhancement of glucose utilization by the tissues.

We would be interested in first place in the degree in which the insular apparatus contributed to the observed conditioned fall in blood sugar. Therefore, we decided to carry out a series of experiments which included an assessment of insulin levels in the animals' blood. Only changes of insulin concentration in blood would be able to demonstrate a clearcut contribution of glandular secretion to conditioned reflex lowering of blood sugar level. Unfortunately, we do not yet possess a reliable method of chemical determination of insulin in the blood and presently the most suitable method appears to be the biological test.

We used the method by Brooks and Horsters, widely used in laboratory of E.S. London. We drew $0.6 \mathrm{ml}$ blood of a dog and administred $0,2 \mathrm{ml}$ to each of three mice (subcutaneously to the belly skin). After 2 hours an analysis was conducted on the blood sugar concentration of the mice. In the laboratory of E.S. London, it was established that $0.2 \mathrm{ml}$ 


\section{Appendix}

of the alien blood had not changed the blood sugar level in the mice, but that larger quantities did evoke changes.

To render the conditions of the experiment as constant as possible, the mice in our experiment were moved to a separate cage and deprived of food at one hour before the start of the experiment. In dogs, two blood samples were drawn from the marginal vein of the ear. The first was drawn immediately before the saline (control) injection, and the second at 15 minutes after the injection, i.e. at the time of the conditioned decrease of blood sugar. The latter time was chosen because in the majority of our experimental sessions the conditioned decrease of blood sugar had been the strongest then (see tables).

The results of this series of experiments are shown in figure A.1 from which one can see that in the majority of cases (14 out of 15) the blood sugar level in the mice that had been given the dog's blood from the period of the conditioned blood sugar decrease was less then of mice who had received the blood of the dogs collected before the saline injection.

Consequently, the dogs' blood during the conditioned decrease of blood sugar level contained considerably more insulin than the blood collected prior to CS presentation.

Thus, the biological tests of insulin level show that in the conditioned fall in blood sugar level as found by us, an important role is played by the secretion of insulin. Consequently, on basis of all experimental data from our investigations on animals and humans, we conclude that after systematic (8-10 times repeated) intravenous glucose injections, a conditioned fall in blood sugar level occurred when instead of glucose saline was given in the same manner.

This fall in blood sugar level was caused to a certain degree by a conditioned secretion of insulin, as confirmed by our results with tests of the insulin level in the blood.

received 6-X-1952

\section{References}

1. B.G. Baranov (1952). Sovietskaya Vrachebnaya Gazeta 13;1.

2. K.M. Bykov (1950). Stenographic report of the united scientific session of the USSR Academy of Sciences and Academy of Medical Sciences SSSR, devoted to the problems of Pavlovian Physiological Studies.

3. C.M. Leites (1951). Abstract of the lecture at the united meeting of the All-Soviet and Ukrainian Institutes of Experimental Endocrinology 97.

4. I.J. Maleva (1951). Klinicheskaya Meditsina 29, 9, 41.

5. J.A. Povorinski (1939). Reports of the IX. Scientific Meeting of the Central Psychoneurological Institute, Kharkov.

6. Savchenko, V.A. (1946). On the mechanism of action of insulin and adrenalin.

7. Sedina, N.A. (1949). Mechanisms of pathological reactions, issue 11-15, 267. 
. 



\section{SUMMARY OF THIS THESIS}

Probably between one and five percent of the female adult population is suffering from binge eating. Binge eating is part of the DSM-IV diagnoses bulimia nervosa, anorexia nervosa (binge-eating/purging subtype) and binge-eating disorder, and is characterized by recurrent ingestion, within a discrete period of time (e.g. within a 2-hour period), of an amount of food that is definitely larger than most people would eat during a similar period of time and under similar circumstances. During binges there is a sense that control over eating is lacking. Chapter 1 highlights the prevalence, size and frequency of binge eating, as well as its social impact. Strikingly, binge eating often occurs in women who attempt to lower their body weight. In the past two decades, binge eating has been the object of much theorizing and research. Notably, the influential restraint theory maintains that binge eating can be explained by the existence of "disinhibitors" (for example, low moods or catastrophic thoughts) breaking an ongoing attempt to restrict food intake (the "restraint"). A laboratory model of overeating, called "counterregulation", has been put forward as the operationalization of this theory. However, doubts can be raised about the validity of the restraint theory, firstly, because counterregulation has been shown to be a behavior that is only modestly replicable, and secondly, because the restraint theory does not postulate an explicit urge-to-eat mechanism.

In chapter 2, the Conditioning Theory of Binge Eating ( $\mathrm{CBE}$ theory), pivotal to the work in this thesis, is described. $\mathrm{CBE}$ theory posits that underlying binge eating are classically (Pavlovian) conditioned responses. These conditioned responses (CR) occur in response to "binge cues" (CSs; conditioned stimuli), which, for the individual, have previously been reliable predictors of a binge (UCS, unconditioned stimulus). Binge cues may be, for example, the sight, smell or taste of certain foods, or the individual's presence in an environment in which binges usually occur. The perception of binge cues is supposed to lead to a conditioned secretion of the hormone insulin, and an accompanying fall in blood sugar level (CR). Although these responses are, homeostatically speaking, adaptive because they attenuate the post-prandial blood sugar rise, according to $\mathrm{CBE}$ theory they are subjectively experienced as a craving for food. A review of the relevant literature is given. Interestingly, CBE theory has given rise to the development of a therapeutic procedure for binge eating called cue-exposure. In this procedure, patients are presented with the odour and sight (CS) of binge foods for a prolonged time (for example one hour long), without the possibility of ingesting these foods (UCS). The extinction of craving (and bingeing), predicted by CBE theory, has indeed been demonstrated in several studies. 


\section{Summary}

Subsequent chapters report empirical studies in which several CBE hypotheses have been tested.

Chapter 3 describes a test of the CBE hypothesis that perception of stimuli that reliably predict a blood sugar rise will lead to insulin secretion, hypoglycaemia and increased craving for sweet substances. In six conditioning trials, subjects were asked to ingest a glucose drink (UCS) in a room with a peppermint odour (CS) hanging in the air. Glucose was chosen as the UCS because it leads to a large blood sugar rise and prompts a strong insulin response. During test sessions, before and after conditioning, only the CS was presented, after which blood samples were taken, and craving was monitored by questionnaire. Subsequently, subjects participated in a "taste test", during which they were to compare several glucose drinks and in which the amount of ingested glucose was measured. Subjects in a control condition, in which the inert sweetener aspartame was presented during conditioning, were hypothesized to show no conditioned responses. The results showed no differences between conditions on blood sugar level or subjective craving. A slightly increased secretion of insulin was observed in the experimental condition. However, the size of the observed difference did not exceed the range of normal spontaneous fluctuations. Interestingly, in both conditions, a strong increase in the amount of ingested glucose was observed after conditioning. This finding was explained as a consequence of a neophobia or "bait shyness" during the initial test session: the subjects would be reluctant to drink freely in the novel lab situation. Subsequently, repeated lab-sessions could have led to a gradual fading of the reluctance to drink. To investigate the phenomenon further, an additional condition was run. This time, the conditioning procedure involved a normal tap water UCS (instead of sweet drinks). Once again, a large increase in glucose intake was observed after conditioning. It was concluded that a key factor in the increased glucose intake was an increased experience with carrying out a lab-procedure, rather than repeated exposure to sweet drinks. These results may have implications for future food intake research using repeated measurements.

The CBE prediction that blood sugar conditioning is possible using a glucose-containing UCS is corroborated in chapter 4. A commentary and re-analysis is given of a 1954 study by the Russian physiologist Mityushov (see also the appendix of this thesis). Mityushov found a physiologically significant hypoglycaemia after injecting saline in humans and dogs who had been given repeated glucose injections previously. The observed results suggest a physiological state which, according to CBE theory, occurs prior to bingeing. Mityushov's procedure might, in case of replication of the results, be employed in future research into binge eating.

The next two chapters test CBE hypotheses about food cue reactivity, i.e., the intensity 
with which people respond to presentation of food-related cues. The CBE theory predicts that cue reactivity is especially intense in individuals with occasional large food intake. In chapter 5, slides depicting subjects' favourite binge foods were used as cues. During presentation of the slides, psychophysiological responses (e.g. skin conductance, heartrate and facial EMG) were monitored. Two groups of subjects, restrained and unrestrained eaters (selected by Herman \& Polivy's Restraint Scale) were compared. Because high Restraint scores suggest a food intake pattern characterized by alternate dieting and overeating, the CBE-derived prediction was that restrained eaters would show larger cue reactivity than unrestrained eaters. The results did not confirm this prediction. Whereas slides with binge food were indeed rated higher on craving-arousing property than were control slides (with office equipment), no differences were found in psychophysiological responses of restrained and unrestrained subjects.

In chapter 6, real food items (sandwiches and candy bars) were presented to subjects given the instruction to smell and look towards these food items attentively. Skin conductance responses, heart rate and salivation were monitored in subjects who had fasted either for 3 , or for 24 hours. The CBE-related hypothesis was that 24-hour fasting subjects would show larger craving and (accordingly) cue reactivity and craving than 3hour fasting subjects. The results showed no group differences in cue reactivity, although, as expected, the 24-hour fasting subjects reported increased craving and a higher hedonic rating of the presented food items.

Chapter 7 features a comparison between restrained and unrestrained eaters (as selected by the Restraint Scale, see chapter 5). Given the CBE theory, it was predicted that ingestion of an appetizer would lead restrained eaters to disinhibit during a subsequent taste test with ice-cream. The present study tried to assess the preoccupation (attentional bias) for food-related stimuli in disinhibiting individuals by means of the Stroop colournaming test. In the control condition, no appetizer was presented. The results showed that, contrary to expectations, no disinhibitive eating was found among the restrained subjects. A remarkable finding was the significant correlation between the level of attentional bias and the amount of subsequently ingested ice-cream in unrestrained eaters. In restrained eaters the correlation was near zero. A possible clinical application of the Stroop test as a predictor of relapse in treated bulimics is discussed.

In Chapter 8 some problems are noted in using the Restraint Scale in lab studies of binge eating. Doubts are raised about the ability of the Restraint Scale (which was originally developed to identify dieters) to optimally predict overeating in the lab (see also section 1.3, and chapters 5 and 7 of this thesis). Furthermore, the Restraint Scale has been criticized for some psychometric weaknesses. Given these problems, a new selection instrument was developed the Disinhibitive Eating Scale (DIS), which contains 
four subscales: failing diets, binge eating, weight fluctuations and self-esteem. A psychometric analysis of the 17-item DIS revealed generally satisfying characteristics. Although DIS and RS total scores intercorrelated strongly, it was shown that both scales select subject groups which differ in composition. It remains to be established whether the DIS indeed possesses the predictive validity for disinhibition in the lab, for which it was developed.

Chapter 9 concludes the present thesis, with the observation that none of the novel studies reported here have produced results confirmative of the hypotheses derived from $\mathrm{CBE}$ theory. In an attempt to elucidate why, a critical and quantitative re-assessment was conducted of the literature considered as supportive of the CBE assumptions (see chapter 2 ). The following picture emerged. First, there is little support for the existence of physiologically speaking significant conditioned blood sugar and insulin responses. Second, at present there are no indications that bulimics show increased insulin and blood sugar reactions to food-related cues. Finally, ingestion of only one candy bar or a few biscuits results in a blood sugar rise that will nullify conditioned hypoglycaemias of the size reported thus far within 10-15 minutes; nevertheless the average binge has been reported to last two to three times longer than that.

Based on these considerations, profound scepticism seems warranted about the role of conditioned blood sugar and insulin responses in binge eating. Considering the success of the cue exposure therapy which was derived from $\mathrm{CBE}$ theory (section 2.2.6), a curious, but not disastrous situation has arisen.

The present dissertation concludes with theoretical and practical suggestions for future research on binge eating. Two important aspects of $C B E$ theory that have not been rejected, i.e., the role of learning, and stimulus-driven character of binge eating, are given emphasis.

Two theoretical directions are discussed. In the first, a possible (and modest) role of learned blood sugar and insulin responses is proposed for the minutes immediately preceding a binge. In the second, it is proposed that an additional mechanism is active in binge eating. Perception of cues that have come to reliably predict a period of fasting and/or self-induced vomiting will lead to increased food intake. In this view, people would not so much binge in order to lift a momentary deprivation (i.e. the hypoglycemia predicted by CBE theory), but rather to preclude a forthcoming deprivation. Several studies, in line with the existence of such a mechanism, are reviewed.

To conclude, several practical suggestions are made for future binge eating research. Emphasis is put on the development of convincing and robust lab models of binge eating. A model of binge eating can be said to exist if some core manipulation reliably produces increased craving and food intake. Subsequent knowledge growth may then 
occur by introducing additional manipulations, and evaluating their effects on eating behaviour. Three possible lab models of binge eating are described. First, the cue reactivity model, in which food intake and craving are increased by presentation of stimuli related to food items previously ingested in daily life. Second, the conditioned hypoglycaemia model, in which, after a conditioning procedure resembling that of Mityushov (see chapter 4; appendix), cue presentation leads to increased food intake, mediated by a conditioned hypoglycaemia. And third, the post-meal deprivation model, in which food intake is increased by presentation of stimuli that, in a previous learning procedure, have become predictive of a fasting period. Relevant experiments, and results needed for the development of the models are described in some detail. The research proposals may give food for research activities for many years to come. 



\section{SAMENVATTING}

Vermoedelijk lijdt tussen de één en vijf procent van de vrouwelijke volwassenen op dit ogenblik aan eetbuien (Engels: binge eating). Het vertonen van eetbuien vormt een onderdeel van de DSM-IV diagnoses 'Bulimia Nervosa', 'Anorexía Nervosa (vreetbuien/purgerende type)', en de 'Vreetbuienstoornis', en wordt omschreven als het frequent, binnen een beperkte tijd (bijvoorbeeld twee uur) eten van een hoeveelheid voedsel die beslist groter is dan wat de meeste mensen in eenzelfde periode en onder dezelfde omstandigheden zouden eten. Karakteristiek daarbij is het gevoel de beheersing over de voedselinname kwijt te zijn. In hoofdstuk 1 worden prevalentie, grootte, frequentie, samenstelling en sociale gevolgen van eetbuien nader beschreven. Opvallend is dat eetbuien vaak voorkomen bij vrouwen die het uiterst belangrijk vinden om slank te zijn. De vraag werpt zich dan ook op hoe het optreden van eetbuien kan worden verklaard. De invloedrijke restraint-theorie stelt dat overeten wordt veroorzaakt door 'ontremmers', (zoals bijvoorbeeld somberheid of catastrofale gedachten) die een tot dan toe bewust volgehouden lijnpoging (de restraint) torpederen. Deze theorie werd geoperationaliseerd en in het lab bestudeerd onder de naam counterregulatie. Twijfel is echter gerezen over de houdbaarheid van de restraint/counterregulatie theorie: ten eerste blijkt counterregulatie matig repliceerbaar in het lab, ten tweede geeft de restraint theorie geen expliciete verklaring voor de eetdrang.

In hoofdstuk 2 is de Conditioning Theory of Binge Eating (CBE-theorie) beschreven, welke centraal staat in deze dissertatie. De CBE-theorie stelt dat er bij eetbuien sprake is van aangeleerde, zgn. klassiek (of: Pavloviaans) geconditioneerde responsen. De geconditioneerde responsen (CR) treden op zodra een persoon geleerd heeft dat bepaalde stimuli (CS, ook wel eetbui-cues) betrouwbare voorspellers zijn van eetbuien (ongeconditioneerde stimuli, UCS). Eetbui-cues kunnen zijn het zien, ruiken of proeven van voedsel, of het verblijf in ruimtes waar eetbuien gewoonlijk plaatsvinden. De waarneming van eetbui-cues leidt automatisch tot afscheiding van het hormoon insuline en een bloedsuikerdaling (CR), die anticiperen op de grote bloedsuikerstijging na de eetbui. Enerzijds is dit een zinvolle, natuurlijke reactie (het lichaam streeft een evenwichtige bloedsuiker huishouding na). Anderzijds wordt de anticipatieve bloedsuikerdaling volgens de CBE-theorie subjectief beleefd als een drang tot eten. Een Iiteratuuroverzicht beschrijft de evidentie voor CBE-assumpties. Interessant is dat intussen enkele succesvolle effectstudies zijn verricht naar een therapie genaamd cue exposure. Hierbij worden patiënten langdurig geconfronteerd met de geur en uiterlijk (CS) van het eetbuien voedsel, echter zonder de mogelijkheid het voedsel (UCS) in te nemen. De door de CBE-theorie voorspelde uitdoving van de eetdrang bleek in deze studies uit te komen. 
In de volgende hoofdstukken worden verscheidene $\mathrm{CBE}$-hypotheses empirisch getoetst. In Hoofdstuk 3 wordt de CBE-voorspelling getest dat insuline secretie, bloedsuikerdaling en toegenomen drang tot inname van zoetigheid kunnen worden opgewekt door een stimulus die een glucose inname voorspelt. Gedurende zes conditioneringssessies dronken proefpersonen een glucosedrank in een kamer waar een pepermuntgeur (CS) was verspreid. Glucose verhoogt het bloedsuikerniveau aanzienlijk en leidt tot insulineafscheiding. In twee sessies, één voorafgaand en één volgend op de reeks conditioneringssessies, vonden de gedragsmetingen plaats. Alleen de CS werd gepresenteerd, vervolgens werden bloed en vragenlijsten afgenomen. Hierna kregen de proefpersonen een smaaktest, waarbij glucose dranken moesten worden vergeleken; de hoeveelheid daarbij ingenomen drank werd gemeten. Voor de controle groep - geconditioneerd met de inerte zoetstof aspartaam - werden geen effecten van conditionering verwacht. De resultaten van deze studie toonden niet de verwachte effecten op bloedsuiker en craving. Wel werd er een iets vergrote insulinerespons gevonden in proefpersonen van de experimentele groep op vijf minuten na CS-aanbieding. Op basis van de relevante literatuur werd echter geconcludeerd dat de grootte van het gevonden effect die van spontane fluctuaties niet oversteeg. Opvallend resultaat, in beide condities, was de sterk toegenomen hoeveelheid gedronken glucosedrank na conditionering. Gehypothetiseerd werd dat dit resultaat samenhing met zgn. neophobia en bait shyness tijdens de eerste, onwennige testsessie, waardoor proefpersonen terughoudend waren bij het drinken van de glucose drank. De dagenlange conditioneringsprocedure zou hebben geleid tot het wegebben van de terughoudendheid. Teneinde dit verschijnsel nader te onderzoeken werd een derde conditie toegevoegd, waarbij proefpersonen deze keer werden geconditioneerd met kraanwater in plaats van zoete drankjes. Na conditionering bleek ook in deze conditie de consumptie van glucosedrank toegenomen. Geconcludeerd werd dat, meer nog dan toegenomen vertrouwdheid met de lab procedure, de ervaring met zoete drankjes hiervoor verantwoordelijk was. Deze conclusie is relevant voor toekomstig onderzoek waarin herhaalde metingen van voedselinname aan de orde zijn.

De CBE-assumptie dat bloedsuikerconditionering met behulp van glucose bevattende UCS-sen wel degelijk mogelijk is, wordt in Hoofdstuk 4 ondersteund. Dit bevat een commentaar op, en heranalyse van een studie van de Russische fysioloog Mitjoesjov uit 1954 (zie de Appendix). Dr. Mitjoesjov slaagde erin een fysiologisch significante hypoglykaemie aan te tonen na het injecteren van een 'placebo' (saline) bij mensen en honden die eerder herhaaldelijk glucose injecties hadden ontvangen. De resultaten suggereren een fysiologische toestand die volgens de CBE theorie voorafgaat aan eetbuien. Mitjoesjov's procedure zou - na replicatie van de uitkomsten - wellicht benut kunnen worden in toekomstig eetbui-onderzoek. 
De volgende twee hoofdstukken bevatten studies over zgn. cue-reactiviteit: d.w.z. de intensiteit van psychofysiologische reacties tijdens het waarnemen van aan voedsel gerelateerde stimuli. Uit de CBE-theorie volgt dat voedselcue-reactiviteit vergroot is bij personen met een geschiedenis van grote voedselinnames.

In hoofdstuk 5 werden als stimuli dia's gebruikt met daarop voor elke proefpersoon geselecteerd 'eetbuien-voedel'. Tijdens het dia-kijken werden psychofysiologische reacties als huidgeleiding, hartslagfrequentie, en gezichtsspierspanningen gemeten. De reacties van twee groepen proefpersonen - geselecteerd met behulp van Herman en Polivy's Restraint Scale - werden met elkaar vergeleken. Aangezien een hoge score op de Restraint Scale duidt op een eetpatroon met afwisselend lijnen en overeten, was de CBE-hypothese dat hoge scorers (lijners) sterkere cue-reactiviteit zouden vertonen dan lage scorers (niet-lijners). Hoewel een manipulatiecheck toonde dat de dia's met eetbuivoedsel als smakelijker werden gewaardeerd dan controle dia's (met kantoorartikelen), werden geen verschillen gevonden in psychofysiologische reacties van lijners en nietlijners.

In hoofdstuk 6 werden de proefpersonen voedingswaren (broodjes gezond en candybars) voorgezet, met de opdracht er verscheidene minuten aandachtig aan te blijven ruiken en naar te kjjken. Huidgeleiding, hartslag en salivatie werden geregistreerd van proefpersonen die vooraf drie dan wel vierentwintig uur hadden gevast. De CBE hypothese was de waarneming van het voedsel een intensere eetdrang en (hiermee) psychofysiologische reacties zou teweegbrengen bij degenen die vierentwintig, dan bij degenen die drie uur hadden gevast. Hoewel de vierentwintig uur inderdaad een vergrote subjectieve eetdrang (en waardering van het voedsel) rapporteerden, kon de gestelde hypothese niet worden bevestigd. In dit geval was door het voedsel opgewekte eetdrang dus niet zichtbaar in intense fysiologische reacties.

In Hoofdstuk 7 werd opnieuw het gedrag vergeleken van lijners en niet-lijners (geselecteerd mbv de Restraint Scale, zie hoofdstuk 5). Uit de CBE-theorie en de bestaande literatuur werd de voorspelling afgeleid dat het aanbieden van een 'appetizer' bij lijners zou leiden tot disinhibitief eetgedrag tijdens een smaaktest met roomijs. In dit experiment werden hierbij optredende cognitieve effect onderzocht, met behulp van de Stroop reactietaak. Met deze taak werd gehoopt inzicht te krijgen in de mate van preoccupatie (ook wel attentionele bias) voor voedselstimuli. In de controle conditie werd geen appetizer aangeboden. Tegen de hypothese in bleek dat lijners in de appetizer conditie geen disinhibitief eetgedrag vertoonden. Opmerkelijk was de significante correlatie tussen de attentionele bias voor voedselwoorden en de vervolgens genuttigde hoeveelheid ijs bij niet-lijners. Bij lijners was deze correlatie afwezig. Gewezen werd op een mogelijke klinische toepassing van de Stroop taak als voorspeller van terugval bij 


\section{Samenvatting}

bulimische patiënten.

Hoofdstuk 8 gaat in op enkele problemen bij het gebruik van de Restraint Scale als selectieinstrument voor eetbui-onderzoek in het lab. Men kan betwijfelen of de Restraint Scale, orspronkelijk ontwikkeld ter selectie van lijners, optimaal geschikt is om overeten in het lab te voorspellen (zie ook 1.3, en hoofdstukken 5 en 7). Daarenboven is de Restraint Scale in het verleden wegens enkele psychometrische zwakheden bekritiseerd. Vandaar dat een nieuw selectie-instrument wordt gepresenteerd, de Disinhibitive Eating Scale (DIS), met vier subschalen: mislukkende dieten, eetbuien, gewichtsfluctuaties en self-esteem. Een psychometrische analyse van de 17 items tellende DIS leverde alleszins bevredigende resultaten. Hoewel de DIS en Restraint Scale vrij sterk correleerden, werd aangetoond dat beide vragenlijsten verschillende groepen proefpersonen selecteren. Het wachten is nu op een bevestiging van de voorspellende waarde van de DIS voor (over)eetgedrag in het lab.

In hoofdstuk 9 wordt de slotbalans opgemaakt. Geconstateerd wordt dat geen van de hier gerapporteerde, nieuw uitgevoerde studies resultaten hebben opgeleverd die bevestigend waren voor de CBE-theorie. In een poging tot nadere verklaring wordt de literatuur die eerder als ondersteuning voor de CBE-assumpties is gepresenteerd (hoofdstuk 2) kritisch en quantitatief hergeëvalueerd. Het volgende beeld rijst hieruit op. Ten eerste blijkt in de literatuur weinig ondersteuning te vinden voor het bestaan van fysiologisch gezien - aanzienlijke geconditioneerde bloedsuiker en insuline responsen. Ten tweede is uit onderzoek tot nog toe niet geleken dat bulimia nervosa patiënten in versterkte mate insuline en bloedsuikerresponsen vertonen na waarneming van aan voedsel gerelateerde stimuli. Tot slot blijkt dat inname van slechts één candy bar of een paar koekjes na 10-15 minuten tot een bloedsuikerstijging leidt die geconditioneerde hypoglykaemieën van de in de literatuur beschreven grootte volledig opheft; tegelijkertijd is uit ander onderzoek gebleken dat eetbuien gemiddeld twee tot drie keer zo lang duren. Op basis van bovenstaande overwegingen wordt geconcludeerd dat omtrent de rol van geconditioneerde bloedsuikerdaling en insuline secretie in eetbuien diepgaand scepticisme gerechtvaardigd lijkt. Gezien de vermoedelijke effectiviteit van uit de CBE-theorie afgeleide cue-exposure therapie (zie paragraaf 2.2 .6 ) is een merkwaardige, doch nietdesastreuze situatie ontstaan.

De disseratie sluit af met theoretische en practische suggesties voor toekomstig eetbuionderzoek. Twee aspecten van de CBE-theorie die niet zijn weerlegd (nl. de rol van conditionering en aan voedsel gerelateerde stimuli) worden hierbij benadrukt.

Een tweetal theoretische benaderingen wordt geschetst. In de eerste wordt gewezen op een mogelijke (bescheiden) rol van aangeleerde bloedsuiker en insuline reacties, vooral gedurende de minuten voorafgaand aan een eetbui. In de tweede benadering is een 
mechanisme geschetst waarin de rol van vasten en zelf-opgewekt braken na een eetbui centraal staat. Waarneming van eetbui-gerelateerde signalen die braken of een periode van vasten voorspellen, zou de voedselinname doen toenemen. Eenvoudig gezegd zou een eetbui niet zozeer voortduren teneinde een momentane deprivatie (zie het lage bloedsuiker-niveau uit de CBE-theorie) 'weg te eten', maar om een toekomstige deprivatie te voorkómen. Een literatuuroverzicht gaat in op aanwijzingen voor het bestaan van dit mechanisme.

Tot slot worden practische aanbevelingen voor toekomstig eetbui-onderzoek gedaan. Gesteld wordt dat de ontwikkeling van overtuigende en robuuste laboratorium modellen van eetbuien bij mensen cruciaal is. Van een eetbui-model is sprake indien men beschikt over een procedure waarmee betrouwbaar eetdrang en vergrote voedselinname worden bewerkstelligd. Door het vervolgens toevoegen van extra manipulaties aan deze basisprocedure, en evaluatie van de consequenties voor de voedselinname, kunnen eetbuien verder in het lab worden onderzocht.

Drie mogelijke eetbui modellen worden beschreven, het cue reactivity model, waarbij de eetdrang en voedselinname worden vergroot door aanbieding van stimuli gerelateerd aan voedsel dat de proefpersoon kent uit het dagelijks leven, het condirioned hypoglycaemia model, waarbij, volgend op een conditioneringsprocedure à la Mitjoesjov (zic hoofdstuk 4; appendix), presentatie van een CS via een geconditioneerde hypoglykaemie leidt tot een toegenomen voedselinname, en het post-meal deprivation model, waarbij de voedselinname wordt vergroot door aanbieding van stimuli die in een voorafgaande leerprocedure een periode van vasten voorspelden. Relevant onderzoek en resultaten, nodig voor de ontwikkeling van bovenstaande modellen, worden in enig detail beschreven. De uitvoering van deze onderzoeksvoorstellen kan voor vele jaren werk opleveren. 



\section{DANKWOORD}

Hoewel op het omslag slechts mijn eigen naam prijkt, hebben ook anderen een rol gespeeld bij het voltooien van deze dissertatie. Welke rol? Lastige vraag. Men denke eens aan alle krachten die een pingpongballetje de oceaan doen oversteken: niet alleen stromingen in de eindrichting, ook zijstromingen, tegenstroming en stiltes in de stroming hebben onderweg bijgedragen (zie Karel van het Reve, 1979). Zo voel ik het ook een beetje bij dit proefschrift. Enkelen wil ik met name genoemd hebben.

Anita Jansen, 'cheffin', jouw intrigerende theorie vormde de basis voor het project waaraan ik heb mogen werken. Onze samenwerking was voor mij een boeiende, prikkelende, soms echter ook onzachte ervaring. Leerzaam was het zeker: ik voel me thans niet langer 'in opleiding'. Ik hoop dat de toekomstige eetbui-wetenschap nog vaak door nieuwe ideeën van jou zal worden opgefrist.

Marcel van den Hout, promotor, bedankt voor de gastvrijheid van jouw instituten DEP en EPP, en voor je bereikbaarheid die ik als buitengewoon practisch heb ervaren. Steeds weer als ik klopte, bleek jouw deur niet gesloten.

Heiny Eilkes, Ellen Louwerse en Annie Raven: hartelijke dank voor jullie inzet en zorgvuldigheid bij het helpen uitvoeren van de experimenten die in dit proefschrift staan. Hetzelfde wil ik mutatis mutandis kwijt aan alle proefpersonen die hebben meegewerkt, maar die geen exemplaar van mijn proefschrift hebben gekregen.

Theo van Aerts, dank voor het programmeren van de reeks psychofysiologische signaalverwerkers, een puike familie; Erik Schouten, je was een stoïcijns beantwoorder van telkens weer dezelfde vragen over mijn Manova-uitdraai; Bob Wilkinson, vriendelijke dank voor je bijdrage aan mijn Engels. Angela Verweij, vaak wist je op onnavolgbare wijze te informeren of ik wel goed had geslapen; Marja Hortulanus, dank-je voor het verscheuren van dat bierviltje. Alle collega's van DEP/EPP met wie ik heb gepingpongd, alsmede degenen met wie ik niet heb gepingpongd: het ga jullie goed. Welmoet Gerritsen, bedankt voor enkele vriendschappelijke telefoongesprekken; Casper Schoemaker, dat ik me jouw volkomen terechte commentaren nooit voor $100 \%$ ter harte heb genomen, komt ongetwijfeld doordat ik mijn arikels al gedeeltelijk af meende te hebben. Mijn paranimfen: Sabine Kroeze, ik heb echt geboft met jouw kamergenoot- en kameraadschap. Instemmend wil ik je hier citeren: "Gén gepeiger meer!" ; Bart Leunissen, een frequent en langdurig Horecabezoek - voor zover Maastricht dit toeliet - was ons niet vreemd. Ik vertrouw erop dat we een vorm zullen vinden om deze belangwekkende bezigheid in de toekomst voort te zetten.

Mijn ouders: lieve Ma en $\mathrm{Pa}$, dit boekwerk is aan jullie opgedragen. Opvoeden is vast veeleisender dan het schrijven van wel XX dissertaties. Mochten jullie enkele zaken uit deze dissertatie niet geheel kunnen navoelen, wees dan niet bezorgd: het is waarschijnlijk een teken van mijn nu aangebroken 'Jaren des Onderscheids'. 



\section{CURRICULUM VITAE}

Barend Jan Joost Overduin werd op 25 oktober 1964 geboren in Enschede. Daar doorliep hij het Atheneum-B van het Kottenpark College. Vervolgens ging hij natuurkunde studeren aan de Rijksuniversiteit Leiden totdat hij in september 1984 zijn propedeuse diploma (met genoegen) ontving. Aansluitend studeerde hij psychologie, eerst aan de Rijksuniversiteit Utrecht (richtingen Klinische Psychologie en Functieleer), later aan de Universiteit van Amsterdam waar hij in september 1991 cum laude afstudeerde bij de vakgroep Psychonomie, afdeling Emotieleer (Prof. N.H. Frijda). Vijf dagen later begon hij als onderzoeker in opleiding te werken bij de vakgroep Differentiële en Experimentele Psychologie (toen nog GGK) van de Rijksuniversiteit Limburg. Deze dissertatie is een neerslag van dit werk. Momenteel wordt hij dermate geboeid door de combinatie van pharmacologie en ethologie, dat hij overweegt om weer te gaan studeren.

\section{Publicaties}

Overduin, J. (1992). Passie. Bespreking van 'Dieptepsychologie' van J.H. van den Berg. De Psycholoog 27, 450.

Overduin, J. (1993). Het voordeel van de schrik. Over het onvolledige gebruik van de reflex in emotieonderzoeken. De Psycholoog 28, 193-201.

Merckelbach, H. \& Overduin, J. (1993). Een Nederlandse Pavloviaan: J. ten Cate (1887-1967). De Psycholoog 29 67-71.

Overduin, J. (1994). Leidens Ontzet in de ethologie. Herwaardering van de dierlijke subjectiviteit. De Psycholoog 29, 279-284.

Overduin, J., Jansen, A. \& Louwerse, E. (1995). Stroop interference and food intake. International Journal of Eating Disorders 18, 277-285. (Chapter 7, this thesis)

Overduin, J. \& Jansen, A. (1995). The conditioning model of binge eating. In: I. Florin \& B. Tuschen (eds), Current Research in Eating Disorders, 16-27. Münster: Verlag für Psychotherapie.

Jansen, A., Overduin, J. \& Meijboom, A. (1995). Controlled and automatic cognitive processes in eating disorders. In: 1. Florin \& B. Tuschen (eds), Current Research in Eating disorders, 74-81. Münster: Verlag für Psychotherapie.

Overduin, J. (1996). Empirisch onderzoek van de eetbui: uitgangspunten en enkele verkenningen. Psychoscoop 7, 4-11.

Overduin, J. \& Jansen, A. (1996). A new scale for use in non-clinical research into disinhibitive eating. Personality and Individual Differences, in press. (Chapter 8 , this thesis)

Overduin, J. \& Jansen, A. (1996). Food cue reactivity in fasting and non-fasting subjects. European Eating Disorders Review, in press. (Chapter 6, this thesis)

Overduin, J., Jansen, A. \& Eilkes, H. (1996). Cue-reactivity to food- and body stimuli in restrained and unrestrained eaters. Addictive Behaviors, in press. (Chapter 8 , this thesis) 



\section{Pavlovian Conditioning and Binge Eating}

Some Empirical Explorations

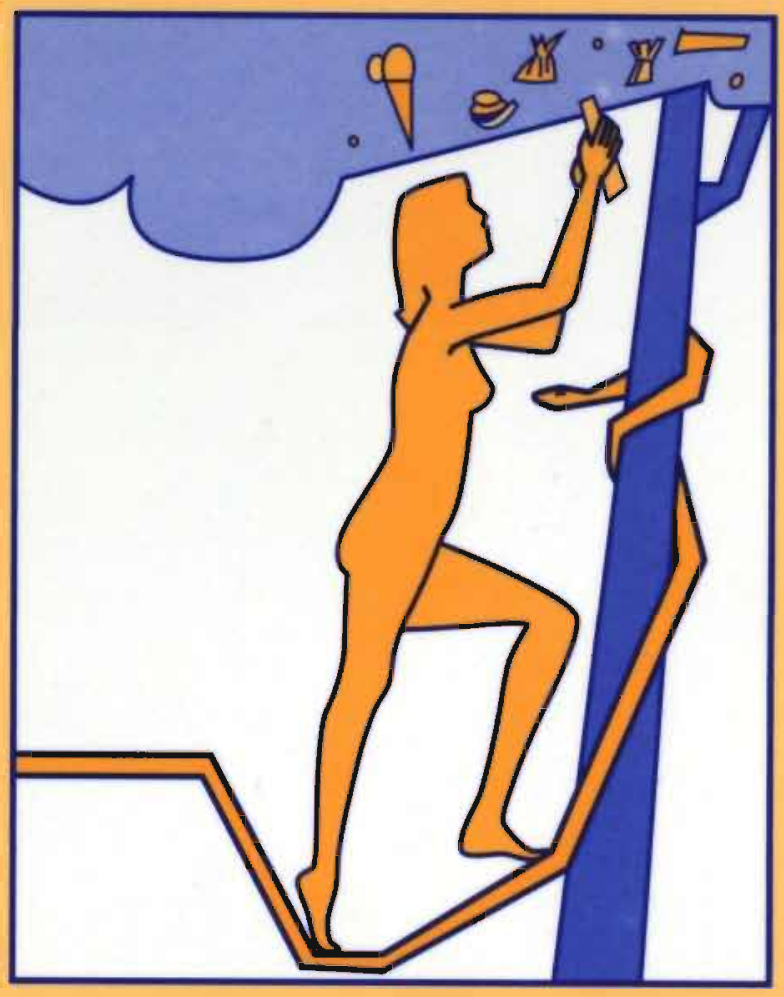

\section{Joost Overduin}




\section{Pavlovian Conditioning and Binge Eating}

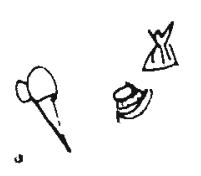


Cover Design: Mariëtte Strik

Printed by: Quick Print, Nijmegen

Overduin, Barend Jan Joost

Pavlovian conditioning and Binge Eating: Some Empirical Explorations/ Barend Jan Joost Overduin.

Thesis University of Limburg, Maastricht. - With ref. With summary in Dutch.

ISBN $90-9009-606-X$

Subject headings: binge eating/ classical conditioning lexperimental psychopathology. 


\title{
Pavlovian Conditioning and Binge Eating Some Empirical Explorations
}

\author{
PROEFSCHRIFT \\ ter verkrijging van de graad van doctor \\ aan de Rijksuniversiteit Limburg te Maastricht, \\ op gezag van de Rector Magnificus, Prof. Mr. M.J. Cohen, \\ volgens het besluit van het College van Dekanen, \\ in het openbaar te verdedigen op \\ vrijdag 5 juli 1996 om 14.00 uur
}

door

Barend Jan Joost Overduin 


\section{Promotor:}

Prof. Dr. M.A. van den Hout

\section{Co-promotor:}

Dr. A. Jansen

\section{Beoordelingscommissie:}

Prof. Dr. H. Merckelbach (voorzitter)

Prof. Dr. N.H. Frijda (Universiteit van Amsterdam)

Prof. Dr. J. Jolles

Dr. N.A. Nicolson

Dr. J.H. Strubbe (Rijksuniversiteit Groningen)

The research in this thesis was financially supported by grant no. 560-268-052 from the Netherlands Organization for Scientific Research, section Behavioural Sciences (NWO-SGW)

The following organizations are gratefully acknowledged for financially supporting the publication of this thesis:

- Faculty of Health Sciences, University of Limburg, Maastricht

- Hoechst-Marion-Roussel, Hoevelaken

- Holland Sweetener Company, Maastricht

- N.W.O., Stichting Gedragswetenschappen, Den Haag

- Quest International, Naarden

- Suikerstichting Nederland, Baarn 
Aan mijn ouders 



\section{Contents}

1.1 On the experimental psychopathology approach

types of investigation - rationale of modelling psychopathology

1.2 Binge eating: definition and characteristics

diagnosistic criteria - prevalence - binge size - binge frequency binge ingredients - mood and binge eating - impact on social functioning and well-being - summary

1.3 Herman \& Polivy's counterregulation model of binge eating description of the counterregulation model - critique of the counterregulation model - final remarks

Chapter 2: A Pavlovian Conditioning Theory of Binge Eating (CBE)

2.1 Description of the conditioning theory of binge eating

five assumptions

2.2 Overview of relevant data

binge cues - homeostatic regulation of blood sugar level -

anticipatory responses to sensory food cues - classical conditioning

of food intake and physiological responses - hyperinsulinaemial

hypoglycaemia experienced as craving - extinction of conditioned physiological responses and binge eating

2.3 Summary and implications for the present thesis

Summary - implications for the empirical work of this thesis

\section{Part II: Empirical Studies}

Chapter 3: Conditioned Insulin and Blood Sugar Responses in Humans in Relation to Binge Eating

Chapter 4: Introduction and commentary to Mityushov (1954):

Conditioned reflex secretion of insulin

Chapter 5: Cue Reactivity to Food-and Body-Related Stimuli in Restrained and Unrestrained Eaters 
Chapter 6: Food Cue Reactivity in Fasting and Non-fasting Subjects

Chapter 7: Stroop Interference and Food Intake

Chapter 8: A New Scale for Use in Non-clinical Research into

Disinhibitive Eating

\section{Part III: Conclusion}

Chapter 9: Conclusions and General Discussion

9.1 Overview of the empirical work in this thesis

9.2 Quantitative reappraisal of the CBE theory

five hypotheses reanalyzed

9.3 The conditioning theory of binge eating: final qualification

9.4 Future research: theoretical considerations maintaining a blood sugar/insulin-related explanation - learning perspective based on post-meal deprivation

9.5 Future research: practical considerations necessity of modelling - three models of binge eating - I. the cue-reactivity model - II. the conditioned hypoglycaemia model III. the post-meal deprivation model

References

Appendix: Mityushov (1954): "Conditioned Reflex Secretion of Insulin" (translation)

Summary

Samenvatting

Dankwoord 


\section{Chapter 1 \\ EXPERIMENTAL PSYCHOPATHOLOGY AND BINGE EATING}

This thesis is an investigation into binge eating. The term "binge eating" refers to a pathology consisting of repeated bursts of excessive food intake. The approach that will be taken throughout this thesis goes under the name of experimental psychopathology. Experimental psychopathology involves the development and empirical testing of hypotheses about underlying mechanisms in psychopathology (Abramson \& Seligman, 1977; Jansen, Merckelbach \& Van den Hout, 1992). After a brief introduction of methods and rationale of the experimental psychopathology approach (section 1.1), essential features of binge eating will be described (section 1.2). The epithet "experimental" by no means implies that experimental psychopathology is non-theoretical: in fact, theories play a pivotal role in experimenting. Section 1.3 contains a critical discussion of counterregulation, a paradigm that has dominated binge eating research in the last two decades. In chapter two, a conditioning theory of binge eating will be described. It is this theory which has inspired the empirical work found in subsequent chapters.

\subsection{ON THE EXPERIMENTAL PSYCHOPATHOLOGY APPROACH}

\subsubsection{Types of investigation}

Within experimental psychopathology, investigations may take numerous forms, three of which will be described here as a frame of reference. In "type P" investigations, the behaviour of patients and non-patients is compared in structured situations, so as to demonstrate or identify crucial parameters of the pathology. An example in the field of eating disorders would be to provide bulimia nervosa patients and healthy subjects with various foods and compare their food choice or rate of ingestion in the lab (e.g. Hadigan, Kissileff \& Walsh, 1989). A problem with this type of research is that recruitment of large samples of well-defined patients is often time consuming and meets various practical difficulties. For pragmatic reasons, researchers may choose to switch to "type Q" investigations, in which two groups of non-clinical subjects are compared. The two groups differ in their extra-experimental history, as for for example reflected by low or high scores on questionnaires tapping habits or traits related to the pathology. An example would be the comparison of the eating behaviour of frequent dieters vs. nondieters in a structured lab situation. A problem with type $P$ and $Q$ research is that individual history may escape rigorous experimental control. Hence a third type of 


\section{Chapter 1}

investigation exists, "type R", involving the study of lab models of the pathology". "Modelling" refers to the "production, under controlled conditions, of phenomena analogous to naturally occurring mental disorders" (Abramson \& Seligman, 1977). Thus, a model is a lab-analogue, or a reversible "miniature version" of the real pathology, to be evoked in non-clinical subjects. A lab-model of binge eating may be at hand as soon as non-clinical subjects have been found repeatedly to increase their food intake after some experimental manipulation. Although the evocation of the lab behaviour may tell us something about the origins of the real pathology, it is not the final goal of modelling (Willner, 1991b): important knowledge derives from subsequent investigation of how the lab model is influenced by various manipulations. For instance, it might be tested whether prodigious food intake by non-clinical subjects in the lab can be suppressed by having the subject smelling the food for a prolonged time. As the development and testing of models is central to experimental psychopathology research, its rationale will be more closely discussed next.

\subsubsection{Rationale of modelling psychopathology}

Ultimate goals of experimental psychopathology are to promote understanding and, perhaps, to suggest new treatments of psychopathology. As such, working with lab models requires a more formal approach than does clinical observation, which admittedly is also a source of knowledge and ideas (cf. Beck et al., 1979). There are advantages and disadvantages to the lab approach.

Many workers have received the endeavour of developing and testing lab models of psychopathology rather coolly. To them, modelling appears to lead researchers astray, that is away from the "full blown pathology of real life". For example, learned helplessness, often used as a lab model of depression, was criticized by the noted British clinician and scientist I. Marks as follows:

\section{"..) present behavioral approaches are not specially promising for the} management of depression.(.) it has not so far been shown that learned helplessness is associated with anything more than mild mood change without the concomitants of serious clinical depression such as guilt, nihilism, suicidal ideas, anorexia, and insomnia lasting at least several weeks. " (cited in Peterson, Maier \& Seligman, 1993, p. 13)

1 The term "model" will be used here only in connection with the experimental procedure of producing miniature psychopathological behaviour in the lab. For the theoretical model of pathological behaviour guiding the experiment, the term "theory" will be reserved. 
Admittedly, lab models can involve stylized forms of behaviour, which only remotely resemble the clinical phenomenon. However, the similarity of lab model and pathology is one, but not always the major concern in the development and testing of models of pathological behaviour, as M.E.P. Seligman, the major proponent of the learned helplessness paradigm, noted in his reply to Marks' criticism:

(the thought that) ". any model of depression in the laboratory must somehow produce suicide, crying, weight loss, guilt and nihilism (..) does not bear scientific scrutiny. Model airplanes do not need to make transatlantic flights; they only need to embody the essence of flying in an airplane. A laboratory model of anxiety need not produce screaming, defecation, and panic antacks - it need only isolate the essential properties of anxiety" (ibid.)

In his reply, Seligman describes the isolation of essential properties of the psychopathology as the crux to model building and testing. The advantage of the isolation of symptoms is understandable if one considers that a given psychopathological symptom hardly ever comes alone. Comorbidity is common and often it remains unclear which symptom precedes, modulates or maintains the other in a given patient. For example, depression and anxiety often coincide with binge eating (APA, 1994; Beebe, 1994; Cooper, 1995; Garner et al., 1990). Thus, isolation of specific symtoms for study becomes especially difficult in patients. Hence, studying a lab model in non-clinical subjects may allow a more efficient investigation of mechanisms underlying the pathology.

Abramson and Seligman (1977) present a list of criteria to evaluate lab models of psychopathology. The important questions to be asked are: a. Are the essential features of pathology's causes as well as its prevention and cures validly described by the model? b. To what extent is there similarity of symptoms between the model and "naturally occurring" psychopathology? $\underline{c}$. To what extent is there similarity between pathology and model regarding physiology, cause, cure and prevention? $\mathrm{d}$. Is the lab analogue a model of one specific psychopathology or of a feature occurring in a range of psychopathologies? Importantly, it should be noted that if all the above criteria are fully met, then so much knowledge is available that testing the model would become superfluous (Jansen et al., 1992a).

In conclusion, there are both limitations and advantages in studying lab models. The limitations arise from the consideration that the functioning of humans is embedded in a complex social cultural context. Hence, the isolation of putatively "essential" characteris- 


\section{Chapter 1}

tics reduces ecological validity. On the other hand, modelling of isolated aspects of the pathology, may elucidate causal mechanisms, which might otherwise remain hidden amongst a myriad of intertwined factors.

\subsection{BINGE EATING: DEFINITION AND CHARACTERISTICS}

"Binge eating", the object of investigation in this thesis, is not to be understood as simple overeating. In this section, the definition, main characteristics and concomitants of binge eating will be described.

\subsubsection{Definition and diagnosis}

The fourth edition of the highly influential Diagnostic and Statistical Manual of Mental Disorders (DSM IV) includes the following definition of binge eating (APA, 1994):

1. eating, in a discrete period of time (e.g., within an two-hour period), an amount of food that is definitely larger than most people would eat during a similar period of time under similar circumstances; 2 . a sense of lack of control over eating during the episode (e.g., a feeling that one cannot stop eating or control what or how much one is eating).

Binge eating is an important symptom in bulimia nervosa, binge eating disorder ${ }^{2}$ and in anorexia nervosa, binge-eating/purging type. For the diagnosis of bulimia nervosa an average binge frequency of at least twice weekly for a period of three months is required; for the diagnosis of binge eating disorder the binge episodes should occur on at least two days a week for a period of six months (APA, 1994). Especially because of its quality of control-loss, binge eating cannot simply be equated with gluttony in obese individuals: less than half of the obese suffer from binge eating (Marcus, 1993; 1995).

Binge eaters with a diagnosis of bulimia nervosa or anorexia nervosa (binge eating/purging type) try to counter the caloric effects of binges by dieting, fasting, and/or doing sports excessively. Self-induced vomiting or using laxatives are also among the means used to lose the weight. In bulimic patients who present for treatment, $80-90 \%$ engage in self-induced vomiting. Because of these behaviours, bulimic binge eaters are not especially obese (APA, 1994). In both bulimia and anorexia nervosa, the weightreducing behaviours coincide with morbid, often unwarranted fears of being fat and getting fatter. Moreover, self-esteem is seen in terms of slimness and amount of body

${ }^{2}$ The diagnose Binge-Eating Disorder has been introduced only recently. In contrast to bulimics, patients diagnosed with Binge-Eating Disorder do not display so-called inappropriate compensatory hehaviours such as self-induced vomiting, misuse of laxatives, fasting and/or excessive exercise (APA, 1994). 
fat. Irrational ideas about eating and distorted body images (to be addressed only marginally in this dissertation) indeed form an important target of successful cognitive behavioural therapeutic interventions in eating disorders (Fairburn, 1995).

\subsubsection{Prevalence of binge eating}

Throughout the 1980 s, different studies estimated the point prevalence of binge eating in the female population ${ }^{3}$ at between 1 and $79 \%$ (Fairburn \& Beglin, 1990; Jansen, 1990). However, these wide ranging figures clearly reflect different definitions of binge eating that have been adopted. Importantly, if one relies on subjective criteria, individuals may refer to intake of merely one chocolate biscuit as "binge eating" (e.g. Rosen, Leitenberg, Fisher \& Khazam, 1986; Rossiter \& Agras, 1990). It is only logical that with the gradual development of more stringent criteria (e.g. the DSM-IV definition) the reported prevalences of binge eating have shown a decline through the years (Jansen, 1990). To get a full picture one should combine recent prevalence estimates for bulimia nervosa, binge eating disorder and anorexia nervosa (binge eating/purging type). Firstly, for bulimia nervosa the prevalence in the adult female population was estimated to lie at around $1 \%$. (Fairburn \& Beglin, 1990; Hoek, 1993; 1995). Secondly, the prevalence of anorexia nervosa (binge eating/purging type) should be expected to be about half of that for anorexia nervosa, which has been estimated at $0.3 \%$ (Hoek, 1993; 1995) or 0.5-1 $\%$ (APA, 1994). Thirdly, binge eating disorder has a prevalence between 0.7 and $4 \%$ (APA, 1994) or $2 \%$ (Marcus, 1993). These figures add up to a total prevalence of binge eating in the female population of somewhere between 1.85 and $5.5 \% .^{4}$.

${ }^{3}$ The prevalence of eating disorders among males has been reported as only a tenth or a twentieth of that for females (Hoek, 1993).

${ }^{4}$ It should be noted that the present calculations are provisional. Fairburn \& Beglin (1990) bave voiced doubts about the possibility of ever obtaining the "true" prevalence of binge eating (at least in bulimia nervosa) in the general population. Furthermore, indications that only a small subset of binge eaters bas been detected and is receiving treatment mean that patient groups may not be representative of binge eaters in the general population (Fairburn \& Beglin, 1990). Therefore, the data on binge eating presented in this section should be considered with some caution. 


\section{Chapter 1}

\subsubsection{Binge sizes}

The DSM-IV criteria for binge eating do not specify an absolute caloric value per binge. This has to do with the large variability of binge size both inter-individually (range: more than 5000 kcalories; Rossiter \& Agras, 1990; Rossiter et al, 1992) and intraindividually (range: approximiately 2000 kcalories; Rosen et al., 1986; Rossiter \& Agras, 1990) in the amount of food ingested during self-reported binges. Whilst there is no doubt that huge quantities of food can be ingested in binge eating, several factors may hamper precise estimations of binge size. First, patients may be prone to refer to any intake of "forbidden food" as a binge, even if only 50 kcalories (the caloric value of one chocolate biscuit) are involved (e.g. Rosen et al., 1986; Rossiter \& Agras, 1990). Inclusion of these so-called "subjective binges" (Fairburn \& Wilson, 1993) may lead to underestimation of the problem. As a provisional solution of this issue, Fairburn (1987) has proposed to count only "uncontrollable" eating episodes larger than $1000 \mathrm{kcal}$ as binges. It appears that about $45-50 \%$ of the self-reported binges (in bulimic patients) conform to this criterion (Rossiter \& Agras, 1990; Walsh, 1993).

Another difficulty in establishing binge size is the underreporting: diary studies typically revealed average binge size of slightly above $1000 \mathrm{kcal}$,, while in lab or otherwise controlled circumstances typically average values of between 1500 and $5000 \mathrm{kcal}$ have been found (Walsh, 1993).

In bulimia patients who do practice self-induced vomiting after bingeing, the average binge size is about twice that of those who do not vomit (Lacey \& Gibson, 1985; Rossiter \& Agras, 1990; Rossiter et al., 1992).

\subsubsection{Binge frequency}

Included in the DSM-IV criteria for the bulimia nervosa and binge eating disorders is the requirement that bingeing should occur at an average frequency of twice a week (bulimia nervosa) or on two different days per week (binge eating disorder) for a period of three and six months respectively (APA, 1994). In spite of these criteria it appears to be difficult to make accurate estimates of binge frequency. As with binge size estimations, frequency estimates are hampered by the phenomenon of "subjective bingeing", i.e. labelling of small or normal meals involving "forbidden foods", as a binge (Fairburn

${ }^{5}$ Note that the following data were mostly drawn from studies on bulimic patients. For reference, the caloric value of one candy bar is between 200 and 250 kilocalories, 200 grams of potato crisps contain about $1080 \mathrm{kcal}$, and mean daily intake is about $2000 \mathrm{kcal}$ for adults, males somewhat more than females (Oettle et al., 1987; Rossiter et al., 1992). The largest binge reported has been 55000 $\mathrm{kcal}$ (this binge included several pauses in which self-induced vomiting occurred; Walsh, 1993). 
\& Wilson, 1993). Several studies in bulimics have reported an average binge frequency of 10-11 per week (Walsh, 1993; Rosen et al., 1986; Rossiter \& Agras, 1990). It should be emphasized that these figures reflect both individuals who binge at least daily and those that do so less often. When counting only binges exceeding $1000 \mathrm{kcal}$ (Fairburn, 1987), the picture becomes different: Rosen et al. (1986) followed a group of 20 Bulimia patients (with bingeing and self-induced vomiting more than three times a week) for one week, and found an average of five binges above 1100 calories. Rossiter and Agras (1990) asked 32 Bulimia patients of the purging type to keep a food intake diary, and found an average of about 4.8 binges above 1000 kcalories in one week (Rossiter \& Agras, 1990).

\subsubsection{Ingredients of a binge}

Often it is reported that during binges, the choice of foods is no longer guided by pleasant taste: raw wheat or pure gravy are ingested if nothing else is available to satisfy the craving. The sensory quality of the binge food does, however, play a role, especially at the commencement of the binge: food types with a special mouth feel, i.e., sweet, soft and creamy, appear to have a special attraction (Abraham \& Beumont, 1982). This might also have to do with the ease with which such food can be expelled after bingeing (Beumont, 1995).

More importantly, binges tend to consist of snacks like chocolate, ice-cream, cakes and pie, and other "forbidden foods" for anyone with the ambition to lose weight (e.g. Abraham \& Beumont, 1982; Rosen et al., 1986; Walsh, 1993). This fact has lead to the idea that binges may gratify a carbohydrate-specific hunger aimed at replenishing brain serotonin (e.g. Pirke, 1995). However, in the early 1980s the alleged overrepresentation of carbohydrate in binges was already discarded (Abraham \& Beumont, 1982). Furthermore, subsequent lab and self-report studies have not found a different macro-nutrient composition in binges and normal meals (Elmore \& de Castro, 1991; Jansen, Van den Hout \& Griez, 1990; Rossiter et al., 1992; Walsh, 1993). Clearly, this does not deny the fact that large amounts of all macro-nutrients may be ingested during binges.

\subsubsection{Mood and binge eating}

As to the affective states surrounding binge episodes, it might be best to say that binge episodes constitute a mixed blessing. First, tension, depression, frustration, loneliness, anxiety and boredom are among the most frequently mentioned precipitants of binges (Abraham \& Beumont, 1982; Bruce \& Agras, 1992; Mitchell, Hatsukami, Eckert \& Pyle, 1985; Beebe, 1994). Once bingeing starts, levels of reported anxiety, depression, etc., appear to be lowered in comparison with the pre binge state (Beebe, 1994). 


\section{Chapter 1}

Second, however, bingeing itself also gives rise to negative moods (Beebe, 1994; Mitchell et al., 1985). Indeed, a range of studies reviewed by Beebe (1994) shows that what is "gained" in mood during bingeing (i.e. lower anxiety, depression) is "lost" by the emergence of new negative feelings, like guilt, loss of control, helplessness, and again depression after the binge, a negative outcome which may lead the patient into purging. Bingeing in patients can thus be said to be consoling for a very limited time.

\subsubsection{Relationship of bingeing to social functioning and well-being}

Whatever the particular background of the individual, it is clear that chronic binge eating co-occurs with impaired social functioning and well-being. Binge eating and purging, which are often kept secret from close relatives and spouse, negatively affect social relationships (Beumont, 1995; Mitchell et al., 1985). Also work impairment as well as financial problems caused by the high cost of binge foods have been reported (Mitchell et al., 1985). Often, bulimia nervosa coincides with depression. There has been ample debate about the causal status of depression in binge eating (Beebe, 1994). An important observation is that overall severity of psychopathology seems unpredictive of treatment success in bulimia nervosa, and that psychopathology diminishes significantly after treatment of the bulimic symptoms (Garner et al., 1990). This may indicate that psychopathological symptoms are secondary to the binge eating/purging itself (Beumont, 1995; Cooper, 1995).

\subsubsection{Summary}

The diagnosis of binge eating includes frequent large food intakes accompanied by feelings of loss of control. Phenomena closely related to binge eating are self-imposed food restriction and post-binge vomiting, related to morbid fears of gaining weight. Binge eating is more prevalent in the female than in the male population. The prevalence of binge eating in the adult female population has been estimated to lie between one and five percent. Binge size varies widely intra- and inter-individually, but in different studies averages have been reported of between 600 and 3000 kilocalories. Average frequency of substantial binge episodes (i.e., $1000 \mathrm{kcal}$ or more) in bulimics have been reported to lie at five per week. Although typical binge food consists for an important part of high-caloric snacks, they do not appear to satisfy specific hungers for carbohydrates: binges do not differ significantly from normal meals in their macro-nutrient composition. Affective consequences of bingeing are mixed: binges appear temporarily to alleviate depression and tension, but also cause shame, guilt and feelings of controlloss. Binge eating and vomiting coincide with impaired well being and social functioning of the individual. There is some consensus among researchers that depression and other 
psychopathology are a consequence rather than a cause of binge eating.

\subsection{HERMAN \& POLIVY'S COUNTERREGULATION MODEL OF B BINGE EATING}

As counterregulation has been the predominant lab model in research of human binge eating for many years, it will be described and evaluated below. The conclusions will provide a background for our decision to adopt a different (conditioning) paradigm in chapter 2 .

\subsubsection{Description of the counterregulation model}

In the mid 1970s, hunger and weight were no longer seen as the only factors governing food intake. Research on normal and deranged eating turned towards the concept of eating style. Two prominent workers in the new research area were C.P. Herman and J. Polivy at the University of Toronto psychology department, who developed the "preload-counterregulation" paradigm (Herman \& Polivy, 1980; 1984; Ruderman, 1986). The basic procedure is as follows: subjects participate in a "taste test" involving a questionnaire-guided comparison of different sorts of ice cream. Each subject has ad lib access to plates with abundant ice cream for a period of, for example, 10 minutes, and is kept unaware of the fact that her/his intake will be measured. There are two conditions: in the "preload" condition a high caloric item (e.g. a milkshake) must be eaten prior to the taste test, whereas the control condition involves no preloading. Furthermore, in the basic design the ice cream intake of dieters is compared with that of non-dieters. Dieting is identified by high scores on a questionnaire, the Herman and Polivy's Restraint Scale $^{6}$. The pattern of food intake that has been repeatedly observed by Herman and Polivy is as follows. Unrestrained eaters (the "non-dieters") act reasonably by diminishing their food when they have already eaten a preload. That is, they "regulate" their food intake. Surprisingly, restrained eaters (i.e. the "dieters") have been observed to "counterregulate: they eat more in the preload than in the control condition?

The explanation given by Herman and Polivy $(1980 ; 1984)$ is based on two elements. First, restrained eaters are said to impose a deliberate, "cognitive" restraint on their food intake. In the no-preload condition, this prompts them to eat less ice cream than

\footnotetext{
${ }^{6}$ For a more detailed discussion of the Restraint Scale see chapter 8 of this thesis.
}

${ }^{7}$ Note that an important criterion of a lab model of binge eating thus was fulfilled: after some experimental manipulation, an increase in food intake was observed. 


\section{Chapter 1}

unrestrained eaters. Second, restrained eaters are likely to see their restraint broken by the "forced" ingestion of the high-caloric preload. Herman and Polivy have reasoned that broken restraint prompts catastrophic thoughts (e.g. "I've blown my diet, so I might as well go on eating"), that will pave the way for excessive eating. Contributing to the larger food intake in these dieters are "biological pressures" acting to compensate for previous chronic restriction of food intake ${ }^{8}$.

Later studies have extended the basic findings by demonstrating broken restraint after other experimental manipulations. It was shown that restrained eaters also increase their food intake after low-mood inductions, or after information that an already eaten preload was of high caloric content. Also the mere prospect of forced ingestion of high caloric food items has been found to trigger increases in food intake (see Herman \& Polivy 1984; Ruderman, 1986). Although the original purpose of the preload/counterregulation paradigm was to investigate dieters' eating behaviour, counterregulation has increasingly been labelled as a form of disinhibition (Herman \& Polivy, 1988; Polivy \& Herman, 1989).

\subsubsection{Critique of the counterregulation model}

Twenty years have past since the concepts of restraint and counterregulation were introduced. The gathered data and related theorizing have been effective in drawing researchers' attention from mere hunger and body weight towards cognitions and behavioural mechanisms active in the disinhibiting eater. Also, this line of research has underscored the multitude of problems encountered by people attempting to lose weight. It has become clear that various stimuli, mood states, thoughts and ideas may have an instantaneous and facilitating effect on food intake.

Despite these advantages, several data suggest that the mechanism of (broken) restraint cannot fully explain why people counterregulate or, more generally, indulge in binge eating. Also, methodological criticisms have been raised about key explanations in counterregulation research. Some of these critical notes (see for example Charnock, 1989 a,b; Dritschel, Cooper \& Chamock, 1993; Jansen, 1990; Lowe, 1993; for replies see Heatherton, Herman, Polivy, King \& McGree, 1988; Polivy \& Herman, 1989) will be briefly discussed below, followed by an overall evaluation.

Counterregulation as a lab-analogue of overeating The fact that in counterregulation experiments the preload manipulation (ingestion of a 420 -gram milkshake) has been

8 The "biological pressure" explanation of urge was only initially defended by Herman \& Polivy. Later, it was discarded (compare the two papers by Herman \& Polivy from 1980 and 1984); see also section 1.3.2 of this thesis). 
between 6 and 13.5 times as large as the increase in ice-cream consumption by restrained eaters (i.e., 31 grams (Herman \& Mack, 1975), and 68 grams (Herman, Polivy \& Esses, 1987) respectively) should not be considered a major blow to the validity of the paradigm: counterregulation is a model that need only embody the essence of overeating (section 1.1), and indeed, the preload manipulation appears to lead to an increase of subsequent food intake. But is this reliably the case? Some authors have claimed that it is not (Chamock, 1989a; 1989b; Dritschel et al., 1993; Jansen, Oosterlaan, Merckelbach \& Van den Hout, 1988). The result often presented under the heading "counterregulation" is based statistically on a restraint $x$ preload interaction effect. However, closer inspection often reveals that the restraint $x$ preload interaction effect is caused by unrestrained eaters eating less when preloaded than when unpreloaded (a reliable, but trivial finding), and restrained eaters eating equal amounts of food in both conditions. Clearly, for counterregulation to reflect "overeating", it would be required that restrained eaters eat more when preloaded than when unpreloaded, but this crucial test has seldomly been conducted. In sum, counterregulation proper seems to be a somewhat less robust finding than has often been realized. Although non-regulation admittedly is an interesting phenomenon, it does not strike one as a convincing analogue of binge eating.

Broken restraint as a prerequisite for counterregulation In a critical test of the claim that breaking cognitive restraint is responsible for lab disinhibition, Jansen et al. (1988b) monitored the thought stream of restrained and unrestrained eaters in a preload study. A 26-item questionnaire with thoughts like "I've blown my diet so I might as well continue to eat" and "I feel out of control", etc., was completed by subjects. The results showed that, although ad lib ice-cream intake showed the usual restraint $x$ preload interaction effect, preloaded restrained eaters showed as many catastrophic thoughts as preloaded unrestrained eaters.

Another issue concerns the identification of the diet boundary, i.e. the critical amount that should be eaten in order to break cognitive restraint, leading to disinhibitive eating (Herman \& Polivy, 1984). Herman and Polivy themselves have experienced considerable difficulty in objectifying the diet boundary. In their 1984 article (p. 151) they suggest that a 210 -gram milkshake preload was too small to break the restraint in many dieters; preloads of 420 grams would be more effective. However, three years later Herman, Polivy \& Esses (1987) set out to determine the critical preload size required for breaking restraint, by simply asking their subjects. It appeared that restrained eaters were unable to estimate their diet boundary, a fact which led the investigators to drop this theme from investigation. In retrospect, Herman et al. (1987) suggested that "an increasing number of dieters consider their diet to be broken by any forced preload at all" (p. 164). What is more, two subsequent studies (Rogers \& Hill, 1989; Jansen \& 


\section{Chapter 1}

Van den Hout, 1991) have shown that mere smelling of palatable food (i.e. zero intake) was sufficient to lead to subsequent disinhibition. It is hard to see why restraint would be broken by smelling palatable food. All in all, disinhibitive eating may well occur without a preceding break of "cognitive restraint".

Explanation of the urge to eat Disinhibitive eating has been said to originate in a loss of cognitive restraint (Herman \& Polivy, 1980; 1984). When analysing this statement, one notices that it does not clarify why the loss of cognitive restraint should induce overeating: no additional urge-mechanism is postulated. The authors (Herman \& Polivy, 1984) remain remarkably uninformative on this point, when they state that their counterregulating subjects ate until their "satiety boundary" was reached. The satiety boundary is described as a state in which further food intake would give rise to feelings of discomfort. To explain counterregulation, Herman and Polivy (1984) claim that the satiety boundary in restrained eaters lies at higher level than that in unrestrained eaters, thus "allowing if nor guaranteeing (..) prodigious consumption" (p.150) once restraint has been broken. One substantiation of the higher satiety boundary alluded to (albeit rather unpersistingly), is that restraint eaters, as a consequence of their dieting might experience an increased biological pressure to eat, so as to restore their biological set point of weight (p. 145). In addition to ideal weight being an ill-defined construct (Greeno \& Wing, 1994), empirical findings make this explanation less likely. First, only between $37 \%$ and $50 \%$ of high restraint subjects report they are currently dieting (Dritschel et al., 1993 Lowe, 1993; chapter 5 and 8 this thesis). Second, a study in which dieting status was checked showed that currently dieting restrained eaters did not counterregulate after a preload, whereas non-dieting restrained eaters did. This finding has been replicated (Lowe, 1993, p.104), and is contrary to the below-set point explanation of counterregulation'. Third, there is the robust finding that restrained eaters generally have a higher BMI than unrestrained eaters do (see the studies in Jansen, 1990; this thesis chapters 5, 7, and 8). Apparently, the "higher satiety boundary" is no explanation of urge, but rather a stately way of saying that more food has been eaten (Jansen, 1990).

9 Apparently in line with a set point explanation of counterregulation is the finding that in the strongest example of counterregulation reported (Herman \& Mack, 1975) normal weight but not overweight restrained eaters did counterregulate (for the reanalysis of these data, see Ruderman, 1986). However, if disinhibition were caused by being below the biological set point, it would follow that 1 . the counterregulating restrained eaters' biological set point is at overweight level; 2 . overweight restrained eaters do not show disinhibitive eating in daily life. These (rather unlikely) allegations still need to be proven. 


\subsubsection{Final remarks}

In sum, the preload-counterregulation paradigm has led to increased knowledge and has set the issues in experimental research on overeating. However, the underlying notion of the counterregulation theoreticians has always been that disinhibitive eating can only occur if prior inhibition ("restraint") existed (Heatherton et al., 1988; Herman \& Polivy, 1990). Of course, when taken literally, this notion is overwhelmingly logical. In practice, however, it is becoming increasingly clear that the boundaries of knowledge growth have been reached in counterregulation research. A striking illustration is the recent report that restrained eaters will only counterregulate if they score high on a scale measuring "tendency to overeat" (Westenhoeffer, Broeckmann, Muench \& Pudel, 1994). In one of their more recent papers, Herman and Polivy $(1990 ;$ p. 123) themselves have suggested that researchers of binge eating "(..) are not in a sirong position to posit causes or mechanisms, since we are not sure what exactly we are trying to explain" (..). To be sure, it appears that the view that binge eating is a lifting of inhibition may discourage the search for mechanisms that explain the urge. This has been a reason for our adopting a different approach in the present thesis: in chapter two, a conditioning theory of binge eating will be described. This theory involves an urge mechanism that may be the "final common pathway" of the different ways in which stimuli may trigger binge eating. Additionally, it describes a mechanism through which binge eating may be self-maintaining. 



\section{A CONDITIONING THEORY OF BINGE EATING}

A conditioning theory of binge eating will be described in section 2.1 , followed by relevant empirical data (section 2.2). Section 2.3 contains an introduction to hypotheses derived from the conditioning theory. These hypotheses will be tested in subsequent chapters.

\subsection{DESCRIPTION OF THE THEORY}

The conditioning theory of binge eating (henceforth CBE theory; Jansen, 1990; 1994a) posits that bingeing is a learned and self-perpetuating behaviour. CBE theory derives from addiction research, where classical (i.e., Pavlovian) conditioning has been accredited an important role in tolerance and relapse (e.g. Childress et al., 1992; Siegel, 1983; Tiffany, 1995a). In short, cues (CS) that signal large food intake (UCS) will, through a conditioning process, become triggers of physiological responses (CRs). These CRs are experienced subjectively as food craving, thereby encouraging binge eating. A subtle version of this mechanism is well-known to us all: when walking past a bakery and sensing the fragrance of freshly baked pies, we will often feel the water running into our mouths, suddenly experiencing an appetite that was absent only moments ago. In binge eating the mechanism has got considerably out of hand. While salivation is one of the CRs, emphasis will be placed on conditioned blood sugar decrease and insulin secretion. Underlying the CBE theory are five basic assumptions, which will be described next.

\subsubsection{First assumption: binges are signalled by cues}

The conditioned stimuli (CSs) are formed by cues that invariably precede binge eating. Examples are the sight, smell, and taste of typical binge foods. The association between between binge cues and binge eating is especially strong because binge eaters tend to restrict their food intake in between binges, thereby actively avoiding the high-caloric "forbidden" binge foods. Binge cues may also be affective and situational: feelings of loneliness, anger, depression or boredom all may reliably precede bingeing, as may certain times of the day or the environment in which binges occur (cf. being home alone, in the kitchen near the fridge, etc.).

1 Related, but not formally developed ideas can be found in Wardle (1990); see also Woods (1991) for an eloquent and inspiring review of related ideas in the field of normal food intake. 


\subsubsection{Second assumption: food intake provokes corrective physiological responses}

Food ingestion leads to a temporary disturbance of the internal physiological state. However, because the body generally strives towards stability of its internal milieu, it will emit regulatory, homeostaric responses. For example, a meal generally causes blood sugar levels to rise considerably. As a reaction, the hormone insulin is secreted from the pancreas, with the effect that blood sugar levels are redirected towards the pre-meal baseline. In conditioning terminology, the physiological disturbance following food intake is the unconditioned stimulus (UCS), and the homeostatic responses form the unconditioned responses (UCRs).

\subsubsection{Third assumption: through conditioning, binge cues come to trigger anticipatory} physiological responses

By means of a conditioning process, binge cues become conditioned stimuli (CS) that will trigger conditioned salivation, insulin secretion, blood sugar decreases, etc., (CRs) before food intake has taken place. The conditioned physiological responses are called anticipative and compensatory because they counter the physiological disturbance that usually follows food intake. Moreover, the intensity of the CRs will be proportional to the amount of expected food intake. Specifically, the CBE theory predicts large insulin secretion and large hypoglycaemia to cues that signal binge eating.

\subsubsection{Fourth assumption: conditioned physiological responses are subjectively experienced as food craving}

According to CBE theory, perception of binge cues induces insulin secretion and a fall in blood sugar level. The CBE theory posits that this body state will be subjectively experienced as food craving. Consequently, food ingestion, serving as "drug-taking behaviour" becomes more likely. A somewhat picturesque way of describing this would be that bingeing is an attempt to "eat away" the conditioned hypoglycaemia.

\subsubsection{Fifth assumption: binge eating is a self-maintaining process}

Evidently, craving heightens the likelihood of bingeing. In binge eaters, it could be said that each binge acts as a conditioning trial in which binge cues (CS) are paired with a binge (UCS), thus strengthening the cue-binge association. From the body's "viewpoint", the homeostatic "necessity" of emitting strong compensatory CRs to binge cues is "reconfirmed" each time a binge occurs. The individual thus may enter a vicious circle: a next encounter with binge cues will again elicit the "necessary" conditioned responses that again, through craving, facilitate bingeing. 


\subsection{OVERVIEW OF RELEVANT DATA}

\subsubsection{Existence of binge cues}

In binge eating research, the probability relationship between binge cues (CS) and binges (UCS) has received little systematic attention. This is probably due to its apparent triviality: there seems little need to argue that food-intrinsic cues, like sight, smell and taste of binge food, precede bingeing. As a matter of fact, retrospective self-reports never fail to include food stimuli as important precipitants of binge eating (Abraham \& Beumont, 1982; Bruce \& Agras, 1992; Mitchell et al., 1985) and of temptation and relapse in dieters (Grilo, Shiffman \& Wing, 1989). However, as far as the present author knows, no quantitative reports exist about the probability relationship between specific cues and bingeing at the individual level. Nevertheless, some indications can be found that binge eating is generally preceded by cues unique to binge episodes. First, it has been found that bingers with bulimia or anorexia show intermediate dieting that is often accomplished by avoiding "forbidden" high-caloric foods that are typically ingested during binges (Walsh, 1993). Therefore, especially cues pertaining to binge food rather than other foods could be expected to be uniquely confined to binge contexts. Second, it has become clear that in therapeutic contexts, patients can, when asked, identify typically tempting binge foods, circumstances and locations. These cues may be used in cue-exposure procedures (e.g. Jansen et al., 1992).

To find evidence that affective binge precipitants (low moods, negative thoughts and stressful social interactions) act as "cues" seems more problematic. Although emotional states are among the most frequently mentioned precipitants of bingeing by patients (Abraham \& Beumont, 1982; Bruce \& Agras, 1992; Mitchell et al., 1985), it remains unclear whether mood states act as the actual binge cues, or as so-called "occasion setters", i.e. the background against which the food cue-binge (CS-UCS) association is established (Catania, 1991; Davidson, 1993).

\subsubsection{Homeostatic regulation of blood sugar level}

The idea of homeostatic responses correcting disturbances of the internal milieu is, of course, not new. It was developed in the 1920s by W.B. Cannon, the father of 20th century physiology. The concept of homeostasis offered an explanatory framework for better understanding the multitude of co-occurring and interacting body processes (Cannon, 1939; Guyton, 1991, ch. 1). A comprehensive treatment of homeostatic physiology related to food intake is beyond the scope of this thesis (and of its author too). A bird eye's view will only be given of blood sugar regulation. When ingested 


\section{Chapter 2}

food has passed through the stomach, it will appear in the small intestine, where its various nutrients are absorbed and appear in the blood stream. Consequently, the level of blood glucose (i.e. blood sugar) begins to rise. However, blood sugar is tightly regulated: several homeostatic responses are emitted to counter the blood sugar rise. One of these responses is an excessive insulin release by the pancreas. Insulin, sometimes also called "the hormone of plenty", lowers blood sugar levels by, for example, making cell walls more permeable for glucose. As a consequence, the glucose formerly contained in the food will enter the various body cells and tissues where it is stored for later use or utilized at once ${ }^{2}$. In sum, whatever the disturbance imposed on the body's internal milieu, the interaction between nutrients, nervous activity and hormones will act to keep blood sugar within a restricted range. For reviews see for example Brodsky \& Devlin (1994), Cryer (1992), Guyton (1991), Hardy (1981), Steffens et al. (1991).

\subsubsection{Existence of anticipatory responses to sensory food cues}

Description. Food-anticipatory responses to cues (as described in the CBE theory) abound in the mammalian body, and have first been described as "psychic reflexes" by Pavlov (1910). In the last twenty years, the term cephalic phase responses (cephalos = head) has come into general use. Cephalic phase response (Berthoud et al., 1981; Giduck, Threatte \& Kare, 1987; Le Blanc, 1992; Powley \& Berthoud 1985, Teff et al., 1993) is a general term referring to physiological responses that are triggered by foodrelated, visual, olfactory, gustatory or oropharyngal-mechanical cues. Thus, cephalic responses may occur both before and during food ingestion. Besides salivation, studied so thoroughly by Pavlov, secretion of gastric and pancreatic juice and of a range of hormones like insulin, pancreatic polypeptide, glucagon can be secreted cephalically. Also other responses have been reported, like increases in metabolic rate (i.e. the body's energy production) and glucose absorption by the liver (Le Blanc, 1992). Many, but not all of these cephalic responses peak at about five minutes and return to baseline at ten minutes after food cue perception (Le Blanc, 1992; Berthoud \& Powley, 1985). Physiologically speaking, the early cephalic phase responses should be distinguished from post-absorptive, homeostatic (see section 2.2.2) reactions to nutrients entering the blood. In particular, the hormonal response to food intake often shows two phases: a smaller cephalic wave in the first few minutes after intake followed by the onset of a post-absorptive response at 5-15 minutes (e.g. Guyton, 1991; Teff et al., 1993). This is

\footnotetext{
2 A reverse mechanism comes into play if the blood sugar level becomes too low, or fuel demands increase, e.g., during stress or vigorous exercise. In these cases, various blood sugat enhancing hormones (glucagon, growth hormone, catecholamines and corticosteroids) are secreted. Generally, they suppress insulin secretion, and mobilize sugars and fat previously stored in cells and tissues.
} 
not to say that the impact of cephalic responses could not extend to longer periods than 10 minutes; the functioning of organs and tissues can be altered for more prolonged periods, when the initial cephalically triggered hormones no longer circulate in the blood (Le Blanc, 1992; Thompson et al., 1988; Bruce et al., 1987). Moreover, cephalic responding is not confined to the pre-ingestive period; it may also occur when new food items are introduced during an ongoing meal (Louis-Sylvestre, 1984). It is generally held that the efferent nervous pathway of cephalic responses is primarily parasympathetic, involving the different branches of the vagus nerve connected to the various glands and organs (Powley \& Berthoud, 1985; Louis-Sylvestre, Giachetti \& Le Magnen, 1983; Strubbe \& Steffens, 1975; Strubbe \& Steffens, 1993).

Functions Because of the very existence of cephalic responses, the issue arises about their possible biological function (e.g. Powley \& Berthoud, 1985). Several functions have been proposed.

First, cephalic responses may attenuate the physiological disturbances that follow the ingestion of food (Powley \& Berthoud, 1985; Woods, 1991). In fact, it has been shown that the nutrient intake leads to larger physiological disturbances when the cephalic phase is by-passed (e.g. by infusion of nutrients) whereas, conversely, the blood sugar rise in response to glucose infusions is decreased if a cephalic response is evoked concurrently (Bruce et al., 1987, Le Magnen, 1992; Strubbe \& Steffens, 1993).

Second, cephalic responses may prepare the various body structures for the breaking up, storage and metabolism of food (Le Blanc, 1992; Powley \& Berthoud, 1985; Woods, 1991). In addition, drawing upon the finding of increased metabolic rate during the first 30 minutes after the beginning of cephalic responses, Le Blanc (1992) has speculated that cephalic responses might aid in mobilizing energy for the pursuit and capture of prey.

A third function of cephalic responses may be to induce or enhance appetite. This might be no less advantageous in an evolutionary sense than the regulatory functions mentioned earlier: appetite instigates food intake at the very moments food is available (Woods, 1991). This is neatly expressed in the French proverb L'appetit vient en mangeant (Le Magnen, 1992).

The fourth function, proposed by Louis-Sylvestre (1984), is that cephalic responses may stimulate versatility of food intake, a function which becomes apparent during meals. In rats turning from one food item to another, an additional insulin secretion super-imposed on the normal post-prandial insulin level is seen, with the effect of lowering postprandial blood sugar rise, and probably, maintaining the hedonic value of intake (Louis- 
Sylvestre, 1984) ${ }^{3}$. A perhaps related effect has been shown in humans. After having eaten a lunch of considerable size, subjects were sensorily primed by a small bite of either pizza or ice cream. A subsequent ad lib intake test with both foods abundantly available showed that intake of the primed food item always exceeded that of the unprimed, although both items had been regarded equally highly by the subjects (Cornell, Rodin \& Weingarten, 1989).

Modulating factors Cephalic phase responses appear in different forms and intensities both intra- and inter-individually. Several sources of variation in cephalic responding exist, mainly related to the nature of the signalled food, and the learning history of the subject.

First, the chemical composition of the anticipated food may matter. Already Pavlov (1910) pointed at the adaptiveness of cephalic responses. In his dogs, the chemical characteristics of the various anticipatory secretions were specifically attuned to those of the expected intake. For example, thickness, amount and chemical composition of the cephalically aroused saliva and pancreatic juice were most appropriate to deal with physiological consequences of the ingestive, even if the (learned) food cue was so alien to food as the ticking of a metronome or the sight of a bottle (see Babkin, 1949; Dworkin, 1993).

A second factor influencing the intensity of cephalic responses is the palatability and perceived palatability of the expected food. Apparently, the most potent elicitors of salivary and insulin responses are sensorily rich food items such as odorous apple pie or spicy pizza, especially when they are brought in contact with the oral cavity (Christensen \& Navazesh, 1984; Teff et al., 1991). The role of visual attractiveness of food items has been neatly demonstrated by Lucas et al. (1987). Half of a freshly baked quiche was mashed in a food processor, after which it was mixed with an odourless blue dye. Both this item and an original (attractive) piece of quiche was presented to normal weight subjects. The cephalic insulin response to the attractive dish was more intense than that to the unattractively mashed variant.

Third, the anticipated amount of food or the expected metabolic consequences play a role in the intensity of the cephalic insulin response (Powley \& Berthoud, 1985): in rats sipping from a saccharin solution, the intensity of the cephalic insulin response has been found to increase proportionally with the quantity ingested, a remarkable finding as saccharin is a metabolically inert artificial sweetener. The effect might be dependent

3 Parenthetically, one should be careful to attribute such behavioural effects to the action of insulin alone, because cephalically induced increases of insulin secretion are usually small compared with those found after food intake (see also sections 9.2 and 9.3 of this thesis). 
upon the rats' previous intake experience with real sugars. When drinking the sweet saccharin, the rats "anticipated" a blood sugar rise proportional to the amount of saccharin ingested. Accordingly, for a larger intake of the sweet solution, a proportionally larger insulin response appeared.

Fourth, cognitive factors have been proposed to account for occurrence or non-occurrence of cephalic responses (e.g. Sjöström et al., 1980). The expectation that food items presented will be actually ingested appears to be especially important (e.g. Pavlov, 1910). Bruce et al. (1987) found a 0.90 correlation between a questionnaire score of intake expectation and the cephalic hypoglycaemic response to food cues. An interesting example showing the impact of cognition has been provided by Goldfine, Abraira, Gruenewald and Goldstein (1970), who were able to induce a cephalic insulin rise in their subjects through hypnotical suggestions of a palatable meal.

Finally, habitual eating pattern appears to modulate the intensity of the cephalic responses. A robust finding is that obese persons show larger cephalic insulin responses than do normal weight persons (Simon et al., 1980; Sjöström et al., 1980). In absolute levels the intensity of cephalic responses has been found to be nearly four times as large in the obese; expressed in percentage increase from baseline levels (obese humans are known to have increased baseline levels of insulin), the obese showed twice as strong cephalic insulin responses (Sjöström et al., 1980). Another study found a trend towards larger cephalic insulinaemic response in obese (Johnson et al., 1983). Although these results are most likely due to hypertrophy of the insulin producing Beta-cells of the pancreas (Dr. Strubbe, personal communication), there remains an additional possibility (which would be in line with CBE theory) that the larger average meal size of the obese in daily life contributes to enlarged cephalic insulin responses.

There is also some indication that cephalic salivation is modified by food intake experience. Le Goff, Leichner \& Spigelman (1988) compared salivary flow to food cues in bulimics and anorexics before and after therapy. Before therapy salivation to food stimuli was more intense in bulimics than in anorexics. After therapy, a re-assessment showed that bulimics salivated less, and anorexics salivated more to food cues than they had originally. This could be explained accordingly by assuming that a bulimic eating style, involving occasional large food intake (binge UCSs), results in stronger salivation (CR), whereas in anorexic patients, food cues predicting small food intake, lead to modest salivary responses (Jansen, Boon, Nauta \& Van den Hout, 1992).

In contrast with the above results, Broberg and Bernstein (1989) and Moyer, Rodin and Cummings (1993) did not find enlarged cephalic insulin responses or hypoglycaemia in bulimic patients exposed to palatable foods. In these cases the CBE predictions were not confirmed. 


\subsubsection{Classical conditioning of food intake and related physiological responses}

Although it is true that cephalic responses to food cues, especially those occurring during food ingestion, may be understood partly as expressions of "hard-wired" reflex loops which are activated when the ingested food strikes various receptors while travelling through the mouth, throat and stomach (Berthoud et al., 1981; Nicolaïdis, 1977), this does not at all imply that the influence of learning history should be discarded (e.g. Dworkin, 1993; Jansen, 1994a; Jansen, Boon, Nauta \& Van den Hout, 1992; Woods, 1981; 1991). On the physiological level, learning may act in at least two ways: by modulation of the existing hard-wired reflex loops (Dworkin, 1993), and by bringing the efferent part of the reflex loop under arbitrary stimulus control (Woods \& Kulkosky, 1976). On the behavioural level, learned cues may activate food seeking and intake. Clearly, the strongest support for these allegations (at the heart of the CBE theory) would be provided if acquisition of the responses described could be demonstrated by means of a classical conditioning procedure. In the following overview such studies will be discussed. It has indeed proven possible to bring meal initiation, meal size, salivation, insulin secretion and hypoglycaemia under the control of arbitrary cues.

Conditioned meal initiation and meal size increase Weingarten $(1984 ; 1985)$ has provided evidence that meal initiation in rats can be classically conditioned to arbitrary cues. In his procedure, which extended over several days, feeding in rats was always preceded by a 4-minute CS (light-buzzer compound), after which a food tray was filled. Then, during the test session, rats were first given ad lib access to food. After they gave signs of being satiated (cessation of intake; grooming), the light-buzzer CS was switched on. It was observed that the rats started eating again, and doing so ingested an amount equalling about $20 \%$ of their daily intake prior to the experiment. It seems that conditioned physiological responses may have contributed to the meal initiation. In a similar procedure to Weingarten's, Strubbe (1992) used the slamming of a food tray as a CS for food delivery. At the test session, conditioned insulin appeared to the slamming of the tray. In humans, surprisingly little research has been conducted in this field. Birch et al. (1989) found in children an increase in candy intake in presence of a candy-predicting light, but only if the children had been aware of the CS-UCS (light-candy) contingency. One might wonder what purpose would be served by increasing food intake after perception of signals predictive of food. Zamble (1973) has provided clues to this problem. He showed that rats that were allowed one-hour food access daily (each day at a different time) ate more if their meals were signalled 15 minutes in advance, than if food was unsignalled. The fact that these eating behaviours resulted in smaller weight loss in the signalled rats, suggests that conditioned enhancement of food intake may be 
of survival value if food is scarce and/or "patchily" distributed in the organism's environment (see Collier, 1986).

Conditioned hypoglycaemia. Blood sugar level can be brought under arbitrary CS control of odours and injection rituals (for reviews see Dworkin, 1993; Woods \& Kulkosky, 1976). In many but not all instances, insulin injections have been used as the UCS, yielding mixed results of either conditioned hypo- or hyperglycaemia, depending on the doses of insulin used and the specifics of the procedure (for detailed discussions see Dworkin, 1993; Woods, 1983; Chapter 4 of this thesis).

More relevant for the CBE-model are procedures in which pure glucose or normal food have been used as a UCS. Some will be discussed here (see also chapters 3 and 4 of this thesis. A strong demonstration of learning in hypoglycaemia using a glucose UCS has been provided by Deutsch (1974) who fed his rats with the sweetener saccharin and observed a hypoglycaemia although this chemical, as noted earlier, does not contribute directly to blood sugar level. Deutsch suggested the observed hypoglycaemia was a compensatory response reflecting the rats' learning history with natural (real) sugars in which sweet taste always had predicted a blood sugar rise. To test this suspicion, Deutsch first investigated whether the hypoglycaemic response could be extinguished. This indeed happened after offering the rats free access to saccharin for a prolonged time. Next, Deutsch argued that it should be possible to established conditioned hypoglycaemia to an arbitrary taste CS as well. Thus, he repeatedly presented his rats with a (non-sweet) cafeine solution to drink followed by the UCS, intubation of glucose into the rats'stomachs. After ten such pairings, the taste of the cafeine itself was able to elicit a hypoglycaemia. Matysiak \& Green (1984) have also found conditioned hypoglycaemia in rats after a learning procedure involving glucose injection UCSs. In contrast, Woods (1976) failed to find this result. This was probably due to the small glucose dose involved. In humans and dogs, a strong example of hypoglycaemic conditioning, leading to about $15 \%$ fall in blood sugar using UCS glucose injections, was reported by Russian physiologist Mityushov (1954; see Chapter 4 and the appendix of this thesis).

Conditioned insulin secretion The blood sugar conditioning studies referred to above, did not include direct measurements of insulin. Nevertheless, it has often been suggested that the observed blood sugar fall was secondary to a conditioned insulin secretion (Dworkin, 1993; Mityushov, 1954; Woods, 1983). Direct evidence has come from studies by Woods et al. (1977) and Strubbe (1992) who demonstrated insulin secretion to meal predicting cues. These authors meal-fed rats at a limited and fixed number of times each day. Woods et al. (1977), giving their rats unlimited excess to food for one hour daily, found an increased insulin secretion at the time of day the rats were usually fed. Strubbe 


\section{Chapter 2}

(1992) used slamming of a food tray as the CS. Two procedures were tested: rats had either two or six times daily free access to food (UCS). On the test day, slamming the food tray alone evoked significant insulin secretion, but only so in rats who had been receiving two meals daily. One of the explanations was that the meal size (i.e. UCS intensity) during the conditioning procedure had been larger in rats fed twice, than in those fed six times daily. Also deprivation in itself might have played a facilitating role (see Weingarten, 1985).

The data presented are in line with the CBE notion that meal initiation and food intakerelated physiological responses can be conditioned to learned cues in people and animals. Food deprivation is not a prerequisite for these effects to occur.

\subsubsection{Hyperinsulinaemia/hypoglycaemia experienced as craving}

The CBE theory assumes that conditioned insulin secretion and hypoglycaemia will be subjectively experienced as craving for food. This assumption receives support from different sources. First, research on hypoglycaemic episodes in diabetic patients shows that hypoglycaemia is often accompanied by feelings of hunger (Hepburn, 1993; Service, 1995). Second, Rodin, Bach, Peranini \& De Fronzo (1985) used the clamp technique, enabling them to keep insulin and blood sugar at fixed levels. Several combinations of blood glucose/insulin levels were established in human subjects who were asked each time to report the intensity of their hunger feelings. It appeared that hunger scores were highest during combined hyperinsulinaemia/hypoglycaemia. Equally, large (Grossmann, 1986) and small (Campfield et al., 1996) insulin injections in humans have led to increases in reported hunger. Fourth, in rats, a short-lived insulin secretion followed by a hypoglycaemia has been found to reliably precede meal intake (Campfield \& Smith, $1990 \mathrm{a} ; 1990 \mathrm{~b})$. Finally, there are indications that conditioned meal initiation in rats (see section 2.2.4) is accompanied by a blood sugar decline in rats (Weingarten et al., quoted in Le Magnen, 1992 p. 84).

\subsubsection{Extinction of conditioned physiological responses and binge eating}

The CBE-hypothesis that craving and binge eating follow the acquisition of conditioned insulin secretion and hypoglycaemia, has not been tested directly. However, animal and human data are available about the reverse mechanism, that is, extinction of conditioned responses by repeated CS presentations not followed by the UCS.

Animal studies First, Deutsch (1974) has observed that rats' cephalic hypoglycaemic response to the sweet taste of saccharin disappeared after 60 days of ad lib access to the 
(metabolically inert) saccharin ${ }^{4}$. Second, extinction of explicitly conditioned hypoglycaemias has been demonstrated after repeatedly exposing the rats to the gustatory or olfactory CS (Deutsch, 1974; Woods, 1976).

Studies in humans Exposure to binge food cues (CS) with prevention of subsequent food intake (UCS) has been demonstrated to reduce binge eating. The procedure is as follows. First, therapist and patient will map cues and acts which are characteristic of binge episodes. This information is used during the exposure sessions when the binge situation is mimicked as realistically as possible. Under supervision of the therapist, the patient is encouraged to go through the ritual of selecting and preparing the typical binge food, thereby handling, smelling or licking the food, and tasting morsels of it. To increase realism, the exposure will be often carried out at the typical binge location, i.e. the patient's home. Although strong food craving arises, the patient is prevented from ingesting food. Prolonged exposure, for example 45 minutes or more, will result in the craving being significantly decreased. Several studies have been published about the effects of the cue exposure procedure in bulimic patients. Overall, the results have been promising in that a reduction of binge frequency was found (Hansen \& De Haan, 1995; Jansen, Van den Hout, De Loof, Zandbergen \& Griez, 1989, Jansen, Broekmate \& Heymans, 1992; Kennedy et al., 1995; Schmidt \& Marks, 1988, 1989) which appeared to have persisted at 6-months (Schmidt \& Marks, 1989) or 1-year (Jansen et al., 1992b) follow-up. Interestingly, also levels of depression and unrealistic cognitions about food and body shape had diminished after treatment (Jansen et al., 1992b). Taken together, these findings fit well into the CBE theory's conceptualization of binge cues as CS and binge eating as a UCS.

4 The issue of whether saccharin-induced hypoglycaemia is learned or inborn has been much debated. For instance, Berthoud et al. (1981) have claimed that saccharin-induced insulin secretion in rats should be regarded an inborn response, because 10 -fold exposure to mere saccharin did not lead to its extinction. In a verbatim commentary on Dr. Berthoud's paper, Woods (1981), referring to Deutsch's results, has argued that 10 extinction trials are insufficient to refute a learning interpretation. Dr. Berthoud's reply that Deutsch's results were not to the point, because Deutsch only measured blood sugar at two hours after saccharin presentation, is valid. Indeed, more convincing data should involve measurement at five minutes after exposure to saccharin, and such measurements still need to be done. Note that already in Pavlov's times, essentially the same debate evolved about the allegedly learned nature of salivation to meat in dog puppies (Joravsky, 1989). 


\subsection{SUMMARY AND IMPLICATIONS FOR THE PRESENT THESIS}

\subsubsection{Summary}

A conditioning theory of binge eating (CBE theory) was presented in this chapter. CBE theory regards binge eating as an acquired behaviour. Binge cues acting as conditioned stimuli (CSs) that reliably predict large food intake (UCS) will eventually acquire the power to elicit physiological responses (CRs). The CRs are subjectively experienced as craving. Once established, binge eating is a self-maintaining process. The binge eating individual may enter a vicious circle: each binge implies a pairing of binge cues (CS) with large food intake (UCS). Consequently, future encounters with binge cues will trigger intense CRs, that in their turn favour binge eating, and so on. Although different physiological CRs are triggered by the CS, especially insulin secretion and hypoglycaemia are hypothesized to be important in craving. Data relevant to the assumptions of the CBE theory were discussed. Although it remains to be demonstrated whether binge eaters are conditioned in the hypothesized way, a cue exposure procedure, implied by CBE theory, appears to be an effective treatment of binge eating. The procedure, involving presentation of binge cues with simultaneous prevention of food ingestion, has been reported more than once to reduce binge eating. These findings are compatible with a hypothesized extinction of the learned CS-UCS (cue-binge) association.

\subsubsection{Implications for the research in this thesis}

So far, little direct research of CBE-predictions has been carried out. The non-clinical lab investigations to be presented in the following chapters have been inspired by the CBE theory and focus on various aspects of the theory.

The hypothesis of the investigation in chapter 3 is directed at the etiological level: if a set of cues CS is made to predict a considerably large intake of sugar (UCS), then eventually the cues themselves will elicit conditioned insulin and blood sugar responses, craving and heightened sugar intake. Chapter 4 discusses the results of a remarkable, but not widely known Russian study (first published in 1954) on conditioned hypoglycaemia. The data from this study are re-analysed, and put into the perspective of modern research (including the $\mathrm{CBE}$ theory). Chapter 5 contains an investigation of the proximal stimuli of bingeing: restrained eaters (i.e. unsuccessful dieters who occasionally binge) and unrestrained eaters (normals) are compared with regard to their psychophysiological reactions to food cues. This study tries to identify relevant parameters of food cue reactivity, while testing the hypotheses that restrained eaters, because of their 
learning history show increased food cue reactivity. A related study is reported in chapter 6. Here it is hypothesized that fasting subjects will show larger psychophysiological reactivity to food cues than non-fasting subjects do. Once again, the principal aim is to identify sensitive parameters of psychophysiological cue reactivity, the fasting manipulation being an attempt to induce maximal food cue reactivity. Chapter 7 focuses on the cognitive level. Restrained and unrestrained eaters are exposed to an appetizer (i.e. the food cue), and subsequently compared with regard to their attentional bias for food words in the Stroop task. As restrained eaters are hypothesized to show disinhibitive eating after the appetizer, it is hypothesized that they will display larger attentional bias for food words then do unrestrained eaters. Also, the relationship between attentional bias and subsequent ice cream intake in an ad lib taste test is assessed. Finally, Chapter 8 serves a more methodological purpose. It describes the development of a new disinhibitive eating questionnaire which may be helpful in selecting non-clinical binge eaters, and in the development of new "lab-models" of binge eating (section 1.1.). 



\section{Chapter 3}

\section{CONDITIONED INSULIN AND BLOOD SUGAR RESPONSES IN HUMANS IN RELATION TO BINGE EATING ${ }^{1}$}

\section{INTRODUCTION}

An important feature of bulimia nervosa is recurrent binge eating. During binge eating, an amount of food is ingested that is definitely larger than most people would eat during a similar period of time under similar circumstances (APA, 1994). A typical characteristic of binge eating also included in the DSM IV binge definition is a sense that control over food intake is lacking (APA, 1994). Furthermore, bingers often report an irresistable food craving just before a binge (Abraham \& Beumont, 1982; Jansen, Van den Hout \& Griez, 1990; Mitchell, Hatsukami, Eckert \& Pyle, 1985).

Hence, binge eating can be said to bear resemblance to alcohol and drug addiction: both are characterized by the intake of large amounts of substances, craving and loss of control over intake. Addiction research of the last two decades has elucidated the role of conditioning in drug tolerance and craving (Siegel, 1983; Childress et al., 1992). Specific cues (CS) that signal drug intake (UCS) eventually become able to elicit physiological responses (CR) that compensate for the anticipated bodily effects of drug intake. Thus a behavioral tolerance to the drug develops, guided by cues like the injection ritual or the usual environment in which the drug is used. Additionally, the drug-signalling cues are supposed to trigger craving and drug seeking behavior. Recently, an analogous conditioning theory of binge eating was proposed (Jansen 1990; 1994a; Wardle, 1990). In the present article the conditioning model of binge eating (CBE model) is outlined and a first test of it reported.

The CBE model (Jansen, 1990; 1994a) is based on five assumptions: (1) each episode of (binge) eating disrupts the body's internal milieu; (2) to counter the disruption, physiological, homeostatic reactions occur; (3) binges are preceded by specific cues; (4) these cues can become elicitors of the homeostatic responses by a process of classical conditioning; (5) the conditioned reactions are subjectively experienced as craving for food.

1 Submitted for publication by J. Overduin \& A. Jansen. The authors gratefully acknowledge Mrs. Annie Raven for her assistance in carrying out the present experiment, Dr. Paul Menheere for his biotechnical advice, Mr. Ton Teerling (Quest International, Naarden) for supplying us with the benzaldehyde and maltol flavors, and the Holland Sweetener Company (Mastricht) for providing us with aspartame. Dr. Barry Dworkin (Penn State University) is acknowledged for commenting on some of our data. 
The first two assumptions imply that bingeing (as food intake in general) has disruptive effects on the internal milieu of the body (for an overview see Woods, 1991). The body tries to restore equilibrium by regulatory, homeostatic reactions. As an important example, a meal causes blood sugar levels to rise, and this prompts the pancreas to release the blood sugar lowering hormone insulin. The third assumption is that binges tend to be preceded by so-called binge cues like the sight, smell, and taste of typical binge food. One could also think of times of the day, particular moods, or the environment in which binges usually occur (cf. being home and feeling lonely in the afternoon). The basic notion is that the person has learned that these cues uniquely and reliably predict binges. Fourthly, the $\mathrm{CBE}$ model proposes that through classical conditioning binge cues can acquire the power to trigger responses like insulin secretion before any food intake has taken place. In conditioning terms, the cues come to act as a CS triggering an insulin secretion (CR) that leads to a drop in blood sugar level. The blood sugar fall is called both anticipative and compensatory because it occurs prior to food intake and compensates for expected physiological disturbances (i.e. post-prandial blood sugar rise). The last assumption in the CBE model is that the cue elicited responses like hyperinsulinemia and hypoglycemia are subjectively experienced as craving. Evidently, craving heightens the chances that a binge will take place. When a binge indeed occurs, the association between cues (CS) and binges (UCS) is strengthened. In this manner the individual can enter a vicious circle, in that the next encounter with binge cues will elicit larger CRs again leading to bingeing and so forth.

Data relevant to the last two assumptions of the CBE model, implying that homeostatic responses and craving can be conditioned to food predicting cues, can be found scattered throughout the literature. The sight, smell and taste of food are known to evoke secretion of insulin and other hormones known under the name cephalic phase responses (e.g. Le Blanc, 1992; Powley \& Berthoud, 1985). In some cases a hypoglycemia has been observed as well (Louis-Sylvestre, 1976). The intensity of cephalic phase responses are said to be correlated to the palatability of the food and the amount of subsequently ingested food (Louis-Sylvestre, 1984; Lucas, Bellisle \& Di Maio, 1987).

Explicit conditioning procedures have shown that insulin secretion hypoglycemia and meal initiation can become conditioned to arbitrary cues like odors, an injection procedure and the usual time of feeding. The UCS consisted of either food or a glucose solution (Deutsch, 1974; Mityushov, 1954; Strubbe, 1992; Woods \& Kulkosky, 1976; Woods et al., 1977; Weingarten, 1984). Finally, the CBE model has been clinically applied to form a therapy for binge eating. The procedure, involving exposure to binge cues with simultaneous prevention of bingeing, appears to be promising (Jansen, Broekmate \& Heymans, 1992).

The aim of the present study was to test the CBE model in normal healthy subjects. A conditioning procedure was run in which a peppermint odor/flavor CS was repeatedly 
paired with the ingestion of glucose solution. Responses were monitored on the physiological, behavioral and subjective level. The hypothesis under scrutiny was that after conditioning, presentation of the CS alone would trigger a) conditioned hypoglyce$\mathrm{mia} /$ hyperinsulinemia coinciding with b) increased subjectively experienced craving, leading subsequently to $\mathrm{c}$ ) increased ad lib intake of glucose drinks.

\section{METHOD}

\section{Design}

The experiment was run according to a 2 (condition: control vs. experimental) $\times 2$ (measurement: pre- vs. post-conditioning) design, and carried out in a double-blind fashion. In the experimental condition subjects were conditioned by sixfold pairing a peppermint smell/taste CS with a subsequent ingestion of a glucose dose (UCS). Glucose was chosen as the UCS, because its ingestion causes a sharp increase in blood sugar level, therewith provoking a strong insulin response (Rodin, 1990; Tse et al., 1983). In the control condition an aspartame solution was used as the UCS. Aspartame tastes sweet (like glucose) but is metabolically inert so that no blood sugar change occurs after ingestion (Rodin, 1990; Carlson \& Shah, 1989; Horwitz, McLane and Kobe, 1988). Two sessions were included to encourage the association of the UCS presentation only with the CS proper, and not with other stimuli related to the lab visit. To control for the non-associative effect of experience with the UCS, a random control procedure (Robbins \& Ehrman, 1992) was added: on each conditioning day all subjects received a take-away drink to be ingested at home. This drink contained aspartame for the experimental, and glucose for the control group. Due to this arrangement, at the time of the second test session (i.e. the last day of the experiment), subjects in both conditions had been exposed to same doses of aspartame and glucose drinks.

\section{Blood Parameters}

Blood sugar and insulin were monitored before and after CS presentation on test days in order to test the CBE hypotheses of conditioned hypoglycemia/hyperinsulinemia. Cpeptide was measured as a control on insulin secretion. C-Peptide and insulin are always secreted simultaneously in equimolar quantities, but $\mathrm{C}$-peptide circulates in the blood for a longer period than shorter-lived insulin. Thus C-peptide measurement could reveal rapid, otherwise unnoticed fluctuations in insulin (Polonsky \& Rubenstein, 1984). Finally, levels of the pancreatic hormone glucagon were measured. Glucagon increases the blood sugar level and is a powerful antagonist of insulin: its secretion is stimulated 
when levels of blood sugar become too low, and inhibited when, for example, sugar is ingested (Brodsky \& Devlin, 1994). The glucagon was measured mainly as a control hormone: heightened glucagon levels could provide an explanation if hyperinsulinemia but no simultaneous hypoglycemia should be found (a phenomenon not uncommon in insulin secretion to food stimuli (Bruce, Storlien, Furler \& Chisholm, 1987; Powley \& Berthoud, 1985). Glucagon was also monitored because it was speculated that conditioned hypoglycemia might not only be secondary to insulin secretion, but also to a conditioned inhibition of glucagon secretion.

\section{Subjects}

In a first step, 100 first- and second-year female university students who had responded to an advertisement in the university paper were given a selection questionnaire on personal health and taste preference. Eventually, 20 subjects (age $19.9 \pm 1.9$ years; BMI $20.1 \pm 1.2$ ) were selected using the following criteria: healthy state, no history of diabetes in the subject or her direct relatives, normal weight (i.e. a Body Mass Index between 18.5 and 23.5), current use of oral contraceptives (so as to blunt natural menstrual cyclic fluctuations of progesterone and appetite; Lissner et al., 1988), no history of bingeing, or dieting to lose weight (implying no eating disorders), and no extreme like or dislike of sweet substances and peppermint. It was announced that the research involved assessments of the impact of repeated tasting on sensitivity for subtle taste differences of sweet drinks, and that relevant blood parameters would be monitored. Allocation to either experimental $(\mathrm{N}=10)$ or control group $(\mathrm{N}=10)$ was done pseudo-randomly, taking care that the neither BMI nor age differed between groups. For each subject the procedure started between 6-12 days after the onset of the last menstruation, i.e. 1-7 days after contraceptive pill use was resumed. In this way, care was taken that the last day in the experiment would be at least 3 days before the onset of the next menstruation. At the end of the experiment each subject was paid the Dutch equivalent of US $\$ 300$.

\section{Materials}

Questionnaires Most questionnaires used Visual Analog Scales (VAS) with scores between 0 ("not at all") and 100 ("very much"). During the test sessions a 5-item VAS on the levels of hunger, thirst, tension, craving for salty as well as sweet food was administered 10 times (see table 3.1 for the time schedule of presentation). Further, the taste test questionnaire included ratings of the sensory qualities of the three test drinks. Some of these items were in fact "time fillers" (see procedure); only hedonic and sweetness ratings of the test drinks were considered as relevant data. The conditioning 
sessions each included two 5-item VASs identical to those described above, as well as questions on the sensory characteristics of the three UCS drinks and the pepper$\mathrm{mint} / \mathrm{mentholatum} \mathrm{CS}$. These questions were included solely to stimulate conscious identification of the CS and UCS; no further use was made of the data from these questionnaires.

Conditioned stimulus The conditioned stimulus was equal in both conditions and consisted of a compound of peppermint/mentholatum odor and flavor, presented in combination with a pre-ingestive ritual in an CS-room. The odor/flavor combination was used, as its "belongingness" with ingestion (UCS) was thought to accelerate the acquisition of the conditioned response (e.g. Schwartz \& Robbins, 1995; Woods, Makous \& Hutton, 1969). The peppermint-odor was dispersed by an electronic device in which peppermint/mentholatum oil was continuously evaporated. The flavor consisted of a solution of peppermint $(3 \mathrm{gr} / \mathrm{l})$ combined with mentholatum flavor $(1 \mathrm{gr} / \mathrm{l})$ in water. The subject would apply $2 \mathrm{ml}$ of this solution to the tongue resulting in the typically refreshing oral sensation of peppermint. The stereotyped acts the subject was instructed to perform before ingestion of the UCS (see procedure) completed the CS compound.

Unconditioned stimulus The UCS for the experimental condition was a solution of 50 grams of glucose (dextrose; Janssen Chimica, Tilburg) in $30 \mathrm{cl}$ of water. Fifty grams of oral glucose contain about $200 \mathrm{kcal}$ (Rodin, 1990) and generally lead to a blood sugar rise between 46 and $61 \mathrm{mg} / \mathrm{dl}$ with subsequent insulin levels between 60 and 110 micro Units/ml (Förster, Haslbeck \& Mehnert, 1972; Tse et al., 1983). In our control condition the UCS was an aspartame solution $(0.5$ grams dissolved in $30 \mathrm{cl}$ of water; Holland Sweetener Company, Maastricht). During a previous pilot study in other subjects, this aspartame solution had been rated as being equally sweet as the glucose UCS of the experimental condition. For both conditions a small amount $(3 \mathrm{ml} / \mathrm{l})$ of almond flavor (a $1 \%$ benzaldehyde-in-alcohol solution) was added for taste in the UCS solutions.

Test drinks The drinks presented during the taste test of the two test sessions were of equal composition in both conditions, namely a $150 \mathrm{~g} / \mathrm{l}$ glucose in water solution, again flavored with almond $(3 \mathrm{ml} / \mathrm{l})$. A total of 3 liters of the test drink was available to the subject, i.e., considerably more than the expected ad lib intake during the taste test.

Take-away drinks It was attempted to maximize the difference in appearance of the takeaway and lab drinks, so that CS perception would remain strictly limited to the lab situation. The quantity of sweeteners (glucose for the control group and aspartame for the experimental group) was the same as in the UCSs. Instead of almond, a "marshmallow" (maltol) flavor was added, and the drink was handed out in a dark-colored bottle (contents: $30 \mathrm{cl}$ ). 


\section{Chapter 3}

Urine jar This $25 \mathrm{cl}$ jar, made of transparent glass, was to be taken home for collection of the first morning urine, and brought back to the lab the next session as "a measure of metabolism of the take-away drinks". No further analysis was made of the urine samples (see procedure).

The drinking box The box from which all lab-beverages were drunk was made of whitecolored solid material. Its top contained three holes through which the straws (diameter $5 \mathrm{~mm}$; length $30 \mathrm{~cm}$ ) protruded. The straws did not require strong sucking to get the drink up from below. At conditioning sessions three cylinders, each filled with $10 \mathrm{cl}$ (i.e. one-third of the UCS), were placed in the box. However, for the ad lib taste tests during test sessions, three one liter jars filled with the test drinks were used. The subjects were not able to see the contents of the box or the color of the drinks.

The pipette The pipette, used for applying the peppermint flavor CS on the tongue, was an open glas laboratory tube (length $15 \mathrm{~cm}$ ) with a rubber balloon on top. The subject was to squeeze the balloon with the pipette tube below the fluid surface, and then let loose; thus the pipette would fill with approximately $2 \mathrm{ml}$ of the peppermint solution (CS), which was ingested by again squeezing the balloon while aiming at the tongue.

Biochemical analysis Blood glucose was measured in venous whole blood by an automated glucoseoxidase method performed on the EBIO analyzer (Eppendorf, Hamburg). Serum insulin and $C$-peptide were measured by radioimmunoassay after polyethyleneglycol (6000) pretreatment. For insulin materials were used provided by Pharmacia (Uppsala, Sweden), while the kit for C-peptide was manifactured by Byk-Sangtec (Dietzenbach, Germany). The interassay coefficients of variations were better than 8.4 \% for the insulin assay and better than $9.7 \%$ for the $C$-peptide assay at the concentration levels of interest in this article. Glucagon was measured in EDTA plasma by sequential radioimmunoassay using the glucagon double antibody kit from D.P.C. (Los Angeles, U.S.A.). Interassay coefficients of variations are better than $15 \%$ at the concentration level of $35 \mathrm{pmol} / \mathrm{l}$.

\section{Procedure}

A few days prior to the experiment an introductory meeting was held. The experimental procedure itself encompassed 10 daily sessions within a period of 12 days; between the fifth and sixth sessions there was a two-day weekend pause. There were two test sessions (day 1 and 10), two "no-conditioning" lab sessions to avoid association of UCS presentation and lab stimuli different from the proper CS (days 4 and 7) and 6 conditioning sessions (days 2,3,5,6,8 and 9). For each subject all sessions were planned at the same time of day. Blood parameters and ad lib glucose intake were measured only on test days. 
Introductory session This session was held to provide the subjects with preliminary information on the procedure and to get them acquainted with the lab, as well as handling the experimental attributes (pipette, straws, etc.). These materials were demonstrated in a room different from the CS room. Subjects were informed that on the first and the last days a longer procedure than normal would take place in which blood samples would be taken. The subject signed a statement in which she promised to refrain from ingesting peppermint or peppermint-related chewing gum, and sweetened liquor during the two weeks of the experiment. Furthermore, instructions were given to eat a meal of two sandwiches with one glass of drink three hours before each session, and to refrain from further eating and drinking until the session.

Conditioning sessions. CS+ (days 2,3.5.6.8 and 9) The subject was welcomed in a waiting room. It was pointed out to the subject that she was to enter a room in which a peppermint odor would be hanging in the air, and that a taste test involving sweet drinks would have to be carried out there. Upon entering the CS room the subject sat down at a table on which the drinking box and pipette were within reach. The experimenter was seated at an angle of 90 degrees within sight of the subject at a distance of 1.20 meters. Then the subject was instructed to apply the peppermint/mentholatum (CS) solution to her tongue. She was encouraged to taste the solution attentively and let it rinse through her mouth for one minute before actually swallowing it. During this minute the experimenter repeated the instruction to concentrate on the peppermint-taste, and added that soon the sweet beverage taste test would begin. It had been explained beforehand that the test would involve three slightly differently composed drinks, and that the subject had to detect the just noticeable differences between the drinks (in reality, each of the three straws was resting in $10 \mathrm{cl}$ of the same UCS solution). After the minute of tasting the peppermint flavor, the subject was instructed to swallow it, and there was a pause of 15 seconds. Then the experimenter told the subject to put the leftmost straw in to her mouth, but not yet start drinking. After 10 seconds the experimenter stood up, said "start drinking now; drink all three drinks from left to right in a steady tempo and concentrate on their taste differences", and left the room. In general the $30 \mathrm{cl}$ drink was finished in 2-3 minutes, after which the subject remained in the room for another 20 minutes. At regular intervals she was instructed by intercom to fill out a questionnaire (see materials). After 20 minutes the session was ended, the subject was given the take home drink, questionnaire and urine jar and then was allowed to leave. See table 3.1 for the time schedule of the conditioning sessions.

The "no-conditioning" sessions (day 4 and 7) During these sessions the subject was welcomed in the waiting room. It was announced that this time something different from usual would happen, i.e. that no taste test would be carried out. Next, the subject was 


\section{Chapter 3}

led to another room where a paper and pencil test was carried out of $2 \times 6$ minutes as "a check for concentration". After that the session was terminated.

Table 3.1. Schedule of conditioning and test trials

Conditioning trials

$\begin{array}{llllll} & |\operatorname{cs}| j \text { us } \mid & & & \\ -5 & 0 & 5 & 10 & 15 & 20 \text { minutes }\end{array}$

Test trials

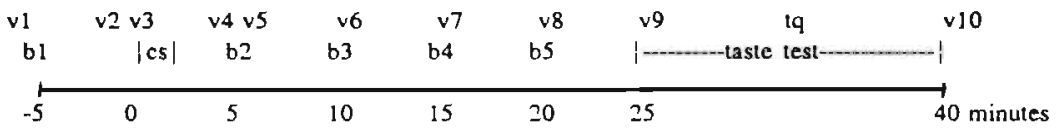

\footnotetext{
$\mathrm{cs}=$ conditioned stimulus (peppermint taste and odor)

us $=$ unconditioned stimulus (glucose or aspartame drinks)

$\mathrm{b}=$ blood sampling

$\mathrm{v}=$ visual analogue scale

tq $=$ tsste questionnaire
}

Home sessions The take-away drinks (see materials) were ingested at home between 7 and 10 p.m.. The instructions were to drink the beverage at a self chosen rate, on the condition that after the onset of drinking, the bottle should be emptied by the subject within 10 minutes. Subsequently, a questionnaire on which hedonic and sensory characteristics of the drinks were rated. No use was made of these data. The subject was instructed to collect and bring along a sample of morning urine at the next session for "analysis of the metabolism of the take-away drinks". In reality, the only purpose of this was to ensure that the subjects would drink the take-away drinks at home and no analyses were conducted.

Test-sessions (day 1 and 10 ) After a welcome the subject was seated in a nursing room where an indwelling catheter (venflon) was applied to the cubital vein of the left arm, a few centimeters distal of the elbow. Next, the subject was brought into the standard waiting room for adaptation and relaxation for a period of 30 minutes. Then the actual procedure began: the experimenter appeared, and explained that the forthcoming taste test would take place in the room where a peppermint odor would be hanging in the air. It was explained that after entering the room the taste test would begin after a pause the length of which might be $0,5,10,15$ or 20 minutes, depending on the outcome of a 
special "lottery". Then, the drawing of the first (baseline) blood samples was carried out, and experimenter and subject entered the CS room at about 4 minutes after the drawing of the first blood samples. After the subject was seated, the presentation of the CS proceded in exactly the same manner as during the conditioning sessions. However, after the subject had put the leftmosi straw in her mouth, the experimenter, instead of giving the "go" signal (as in the conditioning session), looked at his "lottery documents" and said: "no, you can't begin now, please leave the straw, fill out the next questionnaire, and wait". After the subject completed the questionnaire a second round of bloodsamples was taken (in reality the "lottery" was a sham procedure aimed at actively maintaining the subject's expectation of the UCS while blood samples were taken over a period of 20 minutes). The lottery was repeated four minutes later: the subject again had to put the left straw in her mouth and after 10 seconds she had to leave the straw, blood was taken. This action sequence was repeated 4 times. Matters were arranged in a way that blood samples were drawn at 5, 10,15 and 20 minutes after the presentation of the CS (according to Mityushov (1954; see also Woods \& Kulkosky, 1976) the hypoglycemia was expected to reach its nadir at $20-45$ minutes after CS presentation). At 24.5 minutes after entering in the $\mathrm{CS}$ room the subject again put the left straw in her mouth. This time the experimenter did indeed give the "go" signal, and told the subject to commence the taste test, and left the room. First one sip of each drink should be taken from the left to the right (this had been explained earlier). After that, the subject was allowed to drink as much as she wanted or needed to complete the questionnaire.

After 15 minutes the experimenter re-entered the room, and after removal of the venflon the subject was allowed to leave (first test session), or was taken to another room in which the subject was weighed, debrieved and thanked for participation (second test session). The amount of ingested glucose drink was weighed after the subject had left. See table 3.1 for the time schedule of these sessions.

\section{ANALYSIS OF DATA}

Statistical analyses were carried out using a SPSS-PC software program.

In a first step, an effect score was computed for each of the physiological parameters and the scores on all Visual Analog Scales of the two test sessions. For blood sugar, insulin and glucagon the effect score was defined as the difference between the mean level of samples 2-5 (i.e. the post-stimulus values) and the level of sample 1 (baseline). For C-peptide (which was analyzed only in the first and last blood samples) the difference between the post- and pre-stimulus sample was computed. 


\section{Chapter 3}

Similarly, effect scores for hunger and craving were defined as the difference between the mean of post-stimulus scores ( 8 scores) and the mean of baseline scores ( 2 scores). Further, for both the pre- and post-conditioning test session, the amount of ingested glucose test drink, and the mean sweetness and hedonical rating was computed.

Finally, all the computed physiological effect scores, quantities of drinks and subjective ratings were subjected to a 2 (experimental vs. control condition) $\times 2$ (pre/post conditioning) repeated measures ANOVA.

As one of the subjects (experimental condition) fainted during the blood sampling during both test sessions, her physiological ${ }^{2}$ and subjective data were exluded from further analysis. Furthermore, in one subject (control condition) the venflon jammed during the first test session, so that no blood data were acquired. Finally, one subject (control condition) showed a peculiar (exceptionally high and rapidly falling) baseline insulin level in the second test session. She was considered an outlier and her physiological data were excluded from further analysis.

\section{RESULTS}

Blood parameters (figures 3.1 to 3.4 ). For none of these parameters was the critical condition $x$ measurement interaction effect found. Furthermore, no significant main effect of condition or measurement appeared. A closer look at figure 3.2 shows that, after conditioning, the insulin response of the experimental group was slightly stronger than that of the control group. Exploratory t-tests revealed that the group difference was significant only at 5 minutes after CS presentation $(t(16)=2.52 ; p<.03$ ).

Subjective state during the test sessions Neither main nor interaction effects were found on hunger, thirst, tension and craving for either salty or sweet food. The craving-forsweet-food scores during the post-conditioning test session is depicted in figure 3.5.

Intake and subjective ratings of glucose test drinks For intake of the glucose test drinks, the critical measurement $x$ condition interaction effect was not found. However, a main effect was found for measurement $(F(1,16)=15.7 ; p=.001)$, reflecting increased intake of glucose after conditioning in both conditions (see figure 3.6). For hedonic rating a trend towards a condition $x$ measurement interaction effect was found $(F(1,16)$ $=3.36 ; \mathrm{p}<.09$ ) reflecting increased ratings after conditioning in the experimental condition (table 3.2). No effects were found on sweetness ratings of the test drinks.

${ }^{2}$ Analysis of the blood samples taken, however, revealed no abnormalities in both blood sugar and insulin levels. 


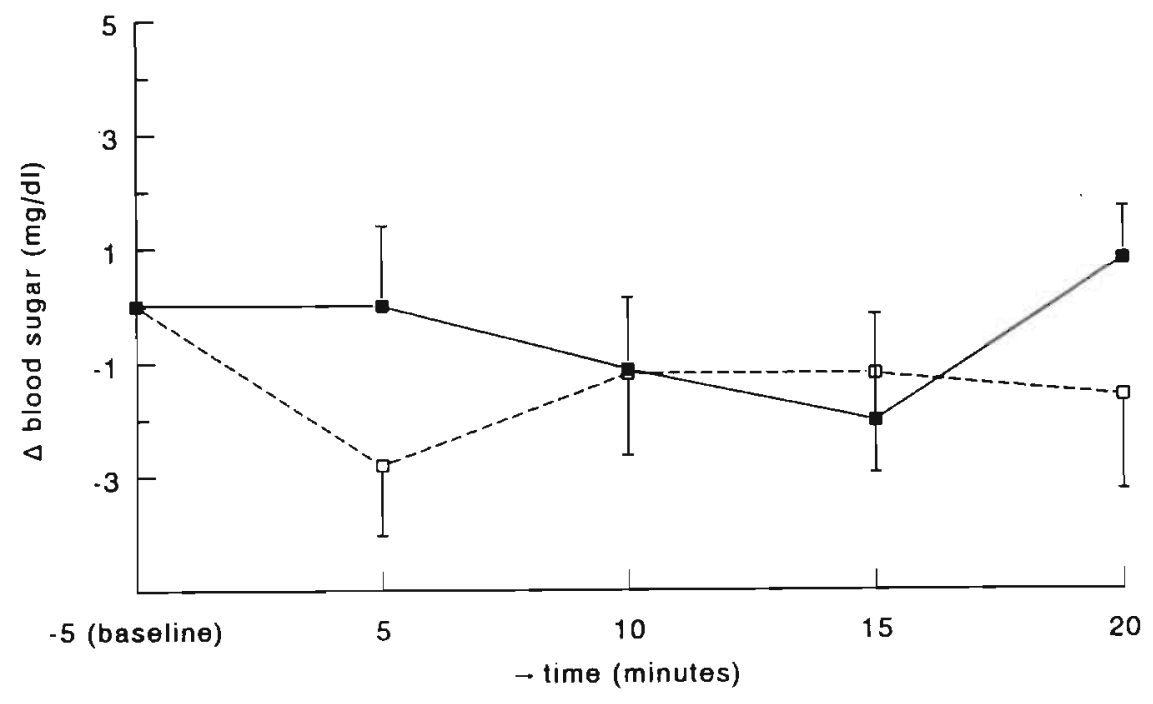

- - EXP. CONDITION --Q-- CONTR. CONDITION

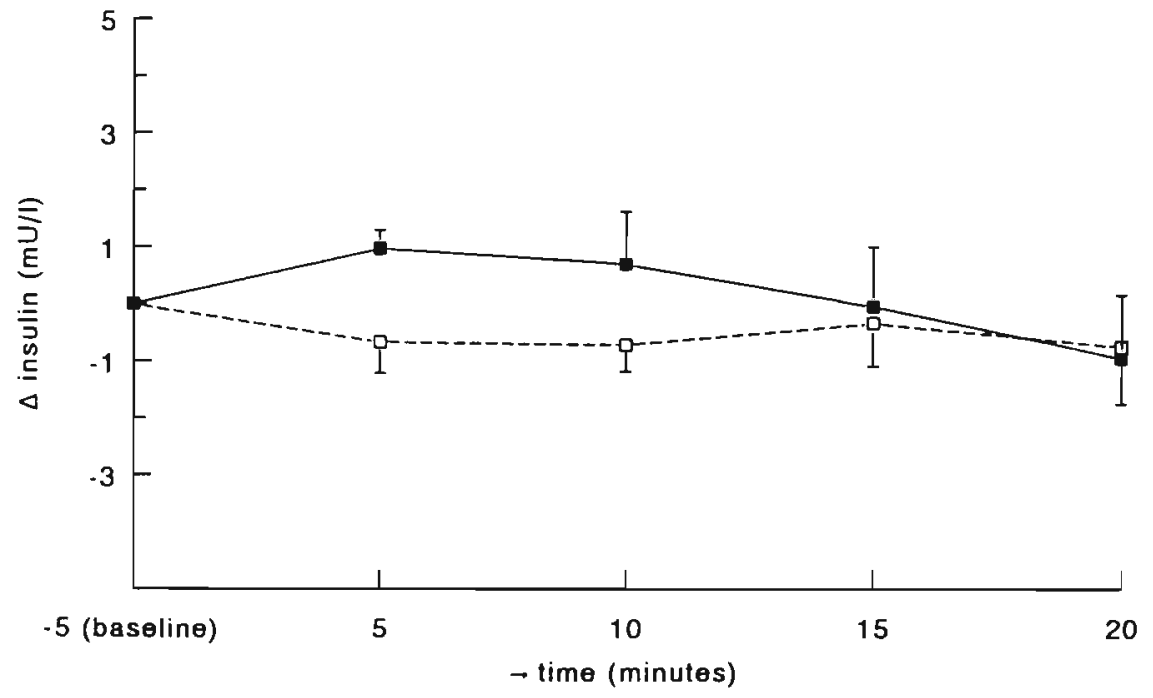

Figures 3.1. and 3.2. Blood sugar and insulin responses (mean \pm s.e.m) to CS presentation during the post-conditioning test session. The values represent deviation from baseline levels, which were $79.8 \pm 2.0 \mathrm{mg} / \mathrm{dl}$ for blood sugar and $9.4 \pm 1.2 \mathrm{mU} / \mathrm{l}$ for insulin. 


$$
\text { - - EXP. CONDITION } \quad--\square \text { CONTR. CONDITION }
$$

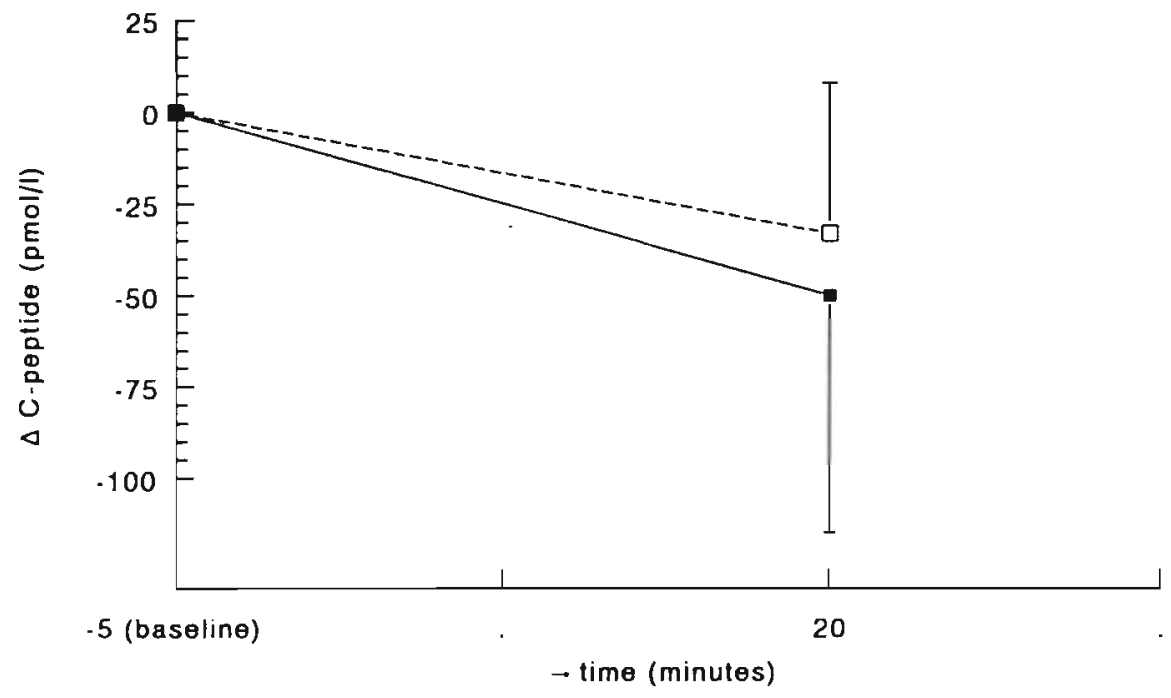

- - EXP. CONDITION --口-- CONTR. CONDITION

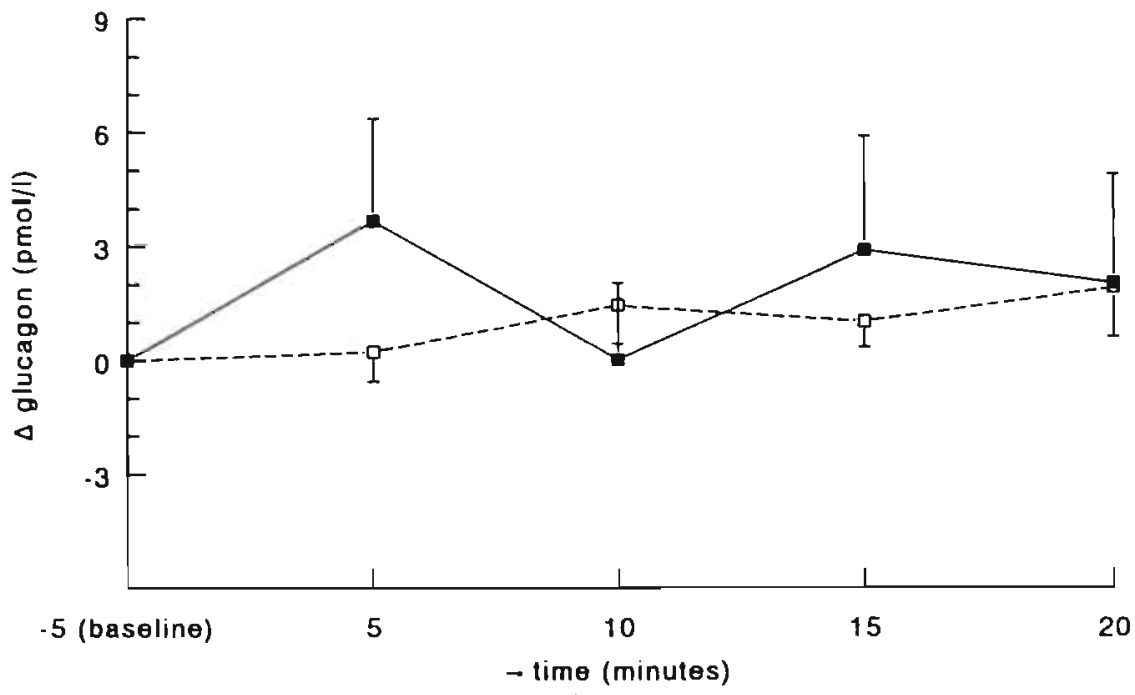

Figures 3.3. and 3.4. C-Peptide and glucagon responses (mean \pm s.e.m) to CS presentation during the post-conditioning test session. The values represent deviation from baseline levels, which were $830 \pm 90 \mathrm{pmol} / \mathrm{l}$ for C-Peptide, and 54.2 $\pm 2.2 \mathrm{pmod} / \mathrm{l}$ for glucagon. 


\section{Preliminary discussion and data from an added condition}

By and large, the CBE prediction of conditioned physiological responses in the experimental group was not confirmed. Nevertheless, some interesting results were found in the experimental condition: a larger insulin response in the experimental condition at 5 minutes after CS presentation, and a trend towards increased hedonic rating of the glucose drinks. However, the mean size of the insulin response in the experimental condition (approximately $1 \mathrm{mUnit} / \mathrm{l}$ ) was not impressive, given the fact that the range of spontaneously occurring fluctuations in insulin level tends to be larger than this (see e.g. Lucas, Bellisle \& de Maio, 1987). To investigate the possibility that the increased insulin secretion co-occurred with the trend towards increased hedonic ratings of the glucose test drinks, the correlation between the shifts in insulin secretion and hedonic rating was computed. This correlation turned out to be -0.32 (n.s.), suggesting a discordance between insulin secretion and hedonic value. Furthermore, a second series of ANOVAs was employed to account for possible inter-individual differences in the peak latency of the putative conditioned responses. In these analyses the baseline level was compared with the most extreme of the post-stimulus values in the direction of our hypotheses, i.e. maximum values for insulin level, and minimal values of blood sugar and glucagon levels post-stimulus and these values were subjected to the same $2 \times 2$ ANOVA as above. None of these analyses revealed significant effects. Further, the expected larger levels of craving and glucose intake in the experimental than in the control condition failed to be confirmed too.

Table 3.2. Hedonic and sweetness ratings (mean \pm sd) of test drinks before and after conditioning (range: $0-100$ )

\begin{tabular}{cccc}
$\begin{array}{c}\text { hedonic value } \\
\text { before } \\
\text { after }\end{array}$ & $34.2(23.5)$ & $31.5(24.4)$ & water condition \\
& $46.7(21.3)$ & $31.1(17.4)$ & $40.8(19.1)$ \\
$\begin{array}{c}\text { sweetness } \\
\text { before } \\
\text { after }\end{array}$ & $52.4(15.7)$ & $67.6(17.7)$ & $32.1(18.4)$ \\
\hline
\end{tabular}

The exact reason for the striking increase in glucose intake found at the post-conditioning test session in nearly all subjects remained unclear: it might be an effect related either to experience with sweet taste or acquaintance with laboratory procedure. In order to further elucidate this issue, a third condition was run: 9 subjects (BMI: 21.0, sd 1.4; 


\section{Chapter 3}

age: 23.1 , sd 2.9) were selected according to the same criteria as in the previous conditions. The procedure was equal to that in the other conditions except for the following: 1) in the conditioning sessions $30 \mathrm{cl}$ of plain water served as the "UCS"; 2) no blood samples were drawn 3) no random control procedure was carried out, i.e. no take away drinks were given.

Once again, it appeared that the glucose intake after conditioning was larger than before conditioning in all but one of the subjects (figure 3.6). On the average, glucose intake was $69 \%$ larger after than before conditioning (paired $t(8)=-3.36 ; p=.01$ ). After adding the intake data of this condition to those of the other two conditions, a 3 (condition) $\times 2$ (pre-vs post conditioning) repeated measurements ANOVA was run. This test did not reveal a condition $x$ measurement interaction effect, whereas the significant main effect of measurement $(F(1,24)=25.0 ; p<.001)$ reflected the increased intake in all three conditions. This result suggests that mere exposure to sweet drinks was not the critical factor in the increased glucose intake observed in the experimental (glucose UCS) and control (aspartame UCS) conditions.

\section{DISCUSSION}

The present procedure involved sixfold pairing of a peppermint flavor/fragrance CS with a UCS ingestion of glucose (experimental condition) or aspartame (control condition) drinks in human female subjects. In contrast with the hypothesis, during the postconditioning test session, the experimental subjects did not show significant conditioned blood sugar and glucagon decreases or $\mathrm{C}$-peptide increases relative to either the preconditioning session or the control subjects. Separate t-tests showed that the insulin response at 5 minutes after CS presentation was larger in the experimental than in the control condition. This difference was in the direction predicted by the CBE model, although the effect size did not exceed the range of spontaneous occurring baseline fluctuations. The prediction of especially increased craving for sweet substances in experimental subjects after CS presentation after conditioning was not confirmed. Mean hedonic rating of glucose drinks increased somewhat more in the experimental than in the control condition, but the condition $x$ measurement interaction did not reach significance. The most impressive effect, found in both conditions as well as a later added condition in which plain water was presented as the UCS, was that ad lib glucose intake during the post-conditioning test session was $78 \%$ larger than during the pre-conditioning test.

To start with the most impressive effect, the increase in ad lib glucose intake would fit 


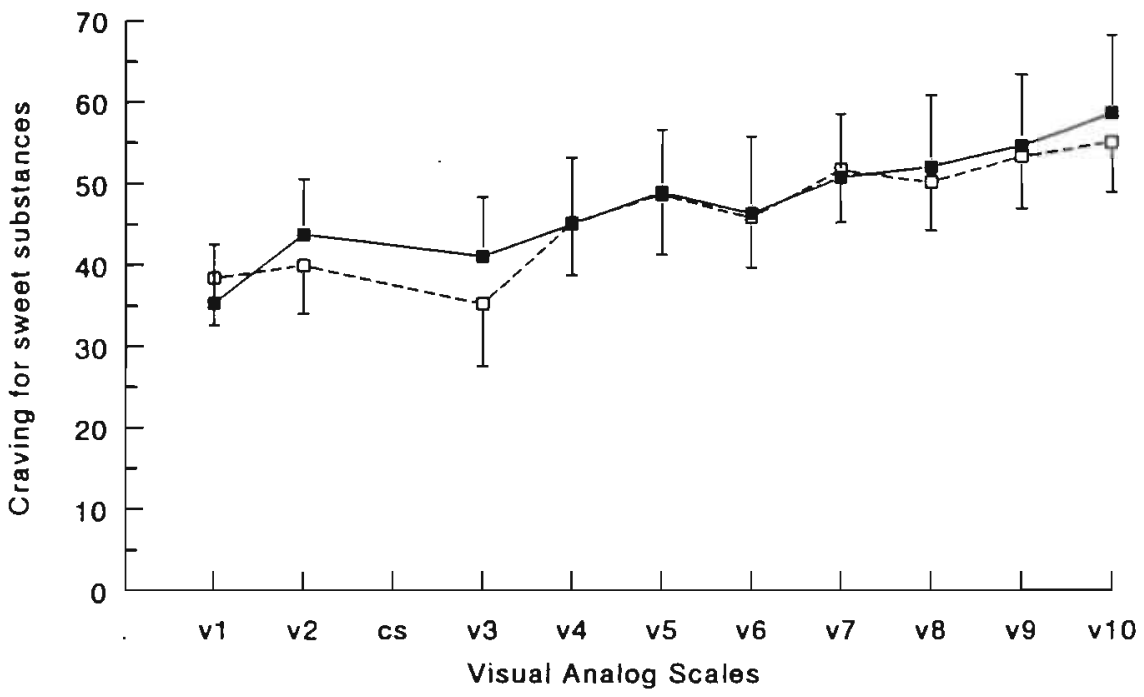

Figure 3.5. Mean \pm s.e.m. of subjective craving scores for sweet foods during the post-conditioning test session. CS presentation was between $v 2$ and $v 3$.

BEFORE CONDITIONING

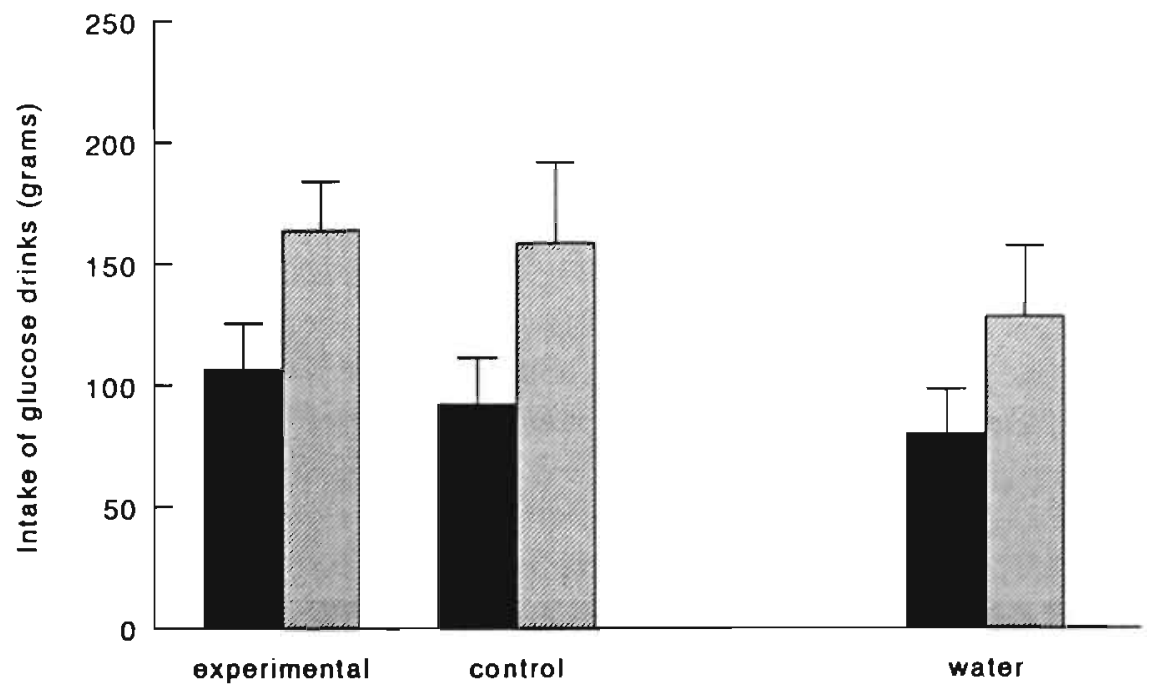

Figure 3.6. Mean \pm s.e.m. of grams of ingested glucose test drinks during the preand post-conditioning test sessions. 


\section{Chapter 3}

the predictions of the CBE model, were it not that the aspartame and plain water conditioned control subjects showed a comparable increase. It is therefore plausible that another behavioral mechanism was at work, for example "neophobia" or "bait shyness", a well-known phenomenon from animal research. Although, in general, neophobia refers to initial reluctance to ingest unfamiliar foods, it may also occur whenever animals are placed in a new environment. An initial decrease or inhibition of spontaneous food intake is shown which only gradually disappears; it can take days before intake has returned to the prior level (Barnett, 1958; Williams, 1968; Woods, 1991). Such a phenomenon may have occurred during our experiment: at the first test session (day 1) the subjects were still unadapted to the lab and procedure, and they might have been "reluctant" to drink. On the second test session ( 9 sessions later), however, the initial reluctance could have faded, thus clearing the way for the observed larger glucose intake. A further physiological explanation of the faded neophobia, i.e. by higher insulin levels (Woods, 1991) seems not warranted in the present case because of the weak insulinemic effects found. Admittedly, however, the possibility that exaggerated insulin secretion occurred after oral contact with the glucose cannot be ruled out (blood samples were taken only before but not during the glucose taste test).

As to the absent physiological and subjective effects of the present conditioning procedure, one might conclude that the CBE model has been refuted. However, the basic prediction we made was that once conditioned hypoglycemia had occurred, craving and increased intake of sweet drinks would follow. Unfortunately, since the first step, i.e., evoking conditioned glycemic and insulinemic changes, failed, the present data are inconclusive with regard to the hypothesized impact of (learned) hypoglycemia/hyperinsulinemia on craving.

The remainder of this discussion will focus on characteristics of the CS, UCS and the conditioning procedure in the present study, that may have underlain the present results and deserve consideration in future glycemic conditioning research.

First, it might be argued that our subjects' pre-experimental experience with the peppermint CSs like tooth paste, chewing gum, and peppermint, i.e. stimuli associated with only weak internal physiological effects ${ }^{3}$, might have slowed down their rate of conditioning through latent inhibition (Schwartz \& Robbins, 1995). However, in as far as latent inhibition acts by means of reduced attention to the presented stimuli (Miller et al., 1995; Schwartz \& Robbins, 1995), the inclusion of both the unique pre-ingestive ritual and explicit identification of the CS, UCS and their contingency will have

\footnotetext{
${ }^{3}$ Note that our subjects did not have an exceptional like of peppermint (see subject selection criteria); thus experience with larger peppermint intake was probably absent.
} 
diminished such an effect during our procedure. Moreover, it has been shown that in a successful instance of glycemic conditioning with large UCSs, latent inhibition by preexposure to the CS failed to thwart rapid acquisition of the conditioned response (Mityushov, 1954).

Second, one might wonder whether the UCS intensity (i.e. 50 grams of glucose) was sufficiently strong to lead to conditioning. When looking at the literature on the physiological effects of glucose, this suspicion seems unjustified. The blood sugar rise in response to 50 grams of glucose (i.e. between 46 and $61 \mathrm{mg} / \mathrm{dl}$; Förster et al., 1972; Tse et al., 1983) overwhelmingly larger than the usual "spontaneous" baseline fluctuations (being between 1 and $2 \mathrm{mg} / \mathrm{dl}$; Lucas, Bellisle \& Di Maio, 1987). Also, though glucose is a powerful blood sugar raiser, ingestion of doses above 50 grams would not have led to a large additional blood sugar rise (e.g. Förster et al., 1972). Still, previous successful instances of glycemic conditioning used an intravenous glucose UCS, involving larger blood sugar rises (up to $200 \mathrm{mg} / \mathrm{dl}$; Mityushov, 1954) and leading to more rapid acquisition of a significant $C R$ than when oral glucose UCS are used. Therefore, in the present study, it may be not so much the UCS, but its combination with a limited number of conditioning trials that has been responsible for the present failure to find conditioned hypoglycemia.

Finally, it might be that both extinction and blocking have occurred during the present procedure, and were relatively important, given the small number of conditioning trials. Extinction of the conditioned response could have occurred as a result of the random control procedure (ingesting the take-away drink) in the experimental group. Although care was taken to maximize its sensory difference with the lab-UCS drinks, the takeaway drink may still have been perceived as a "tin full of sweet drink" CS, and, as it contained metabolically inert aspartame, its ingestion may have extinguished conditioned responses already established during lab-sessions. A perhaps more important explanation of our finding of no conditioning may have been blocking (Schwartz \& Robbins, 1995): prior to the experiment our subjects (like anybody) may have had an already firmly established association between oral sweetness (CS) and subsequent blood sugar increases (UCS). Our conditioning procedure involved association of an odor/fragrance CS with oral glucose. Thus, during conditioning sessions two CSs may have been operating: the experimental, "intended" CS (CS1), and the sweet taste of the UCS glucose beverage itself (CS2). Hence, during the conditioning trials, relative to CS2, CS1 did not provide additional information about an impending blood sugar rise. Hence, it might be argued that the CSI alone would not able to trigger conditioned responses during the post-conditioning test session. Although we had good reason for not applying a glucose probe to the tongue as a CS on test days (oral glucose is known to elicit a 


\section{Chapter 3}

(mainly unconditioned) insulin secretion through glucose sensitive receptors located in the mouth and upper gastrointestinal tract (Grill, Berridge \& Ganster, 1984; Nicolaïdis 1977), it may be valuable to present a small sweet tasting stimulus (e.g. aspartame solution, Bruce et al., 1987) at the test session.

In conclusion, research remains to be done which elucidates the role of conditioned glycemic responses in craving for food. As a first step, procedures must be developed to which human subjects acquire significant conditioned glycemic and insulin responses. Then, using these procedures, while controlling for neophobic interference, one could assess the contribution of physiological learning to craving and binge eating. 


\section{Chapter 4}

\section{INTRODUCTION AND COMMENTARY TO: M.I. MITYUSHOV (1954), "CONDITIONED REFLEX SECRETION OF INSULIN"1}

As has been known for decades now, the physiological impact of Pavlovian conditioning extends beyond salivation, skin conductance and heartrate (Ádám, 1967; Dworkin, 1993; Razran, 1961). Current research on the role of conditioned responses in homeostatic regulation ${ }^{2}$ (e.g. Dworkin, 1993) has once again demonstrated the potential importance of the orginal studies on classical conditioning by the Eastem European Pavlovians, carried out several decades ago. The present article contains the translation of a Russian paper on blood sugar conditioning, which still appears to be highly relevant for today's conditioning research. The paper was first published in 1954 by the physiologist M.I. Mityushov. Before presenting the paper, we will describe its relevance for current research. The paper will be followed by a reanalysis and discussion of the presented data.

\section{INTRODUCTION}

At first glance, it seems as if today's Pavlovian conditioning research has become a science of stimuli rather than responses. For example, in a recent review of the predominant RescorlaWagner theory, the terms CS and US are used approximately 560 times, whereas the response terms CR or UR are altogether lacking (Miller et al., 1995) ${ }^{3}$. The frequent use of "CS" and "UCS" can be attributed to the description of conditioning principles pertaining to stimulus interactions, for instance, latent inhibition, blocking, and overshadowing. Meanwhile, the roles

1 This paper, together with the translation of Mityushov (1954; see Appendix of this thesis) will be submitted for publication by J. Overduin, B.R. Dworkin \& A. Jansen. The authors gratefully acknowledge Professor Gyōrgy Ádám, Eōtvōs University, Budapest, for his helpful comments on both our article and the translation of Mityushov's paper.

${ }^{2}$ Strikingly, the potential importance of conditioning for "The Wisdom of the Body" appears to have been overlooked by W.B. Cannon, the father of modern regulatory physiology, notwithstanding the fact that Pavlov and Cannon were acquainted and met each other on several occasions (sce Cannon, 1939; Dworkin, 1993).

${ }^{3}$ Garcia et al. (1985) already noted that in the classic paper by Rescorla (1967), the terms CS and US were mentioned 130 times, whereas "CR" was only used ten times, and "UR" not once. Hence, it appears that the situation has grown even more asymmetrical ever since. 


\section{Chapter 4}

of CR and UCR have received less attention. The few discussions about the nature of conditioned responses have focussed on the "survival value" for the organism. Nevertheless, the prevalent view is that nothing is gained by alluding to functionality of conditioned responses. For instance, it may often seem gratuitous to argue that an increase in skin conductance CRs serves the "purpose" of preventing the organism from injury to the skin (e.g. Furedy, 1989; Turkann, 1989). Overall, such skepticisms may be justified in the case where the CR measured is peripheral or multi-faceted (e.g., skin conductance fluctuations, gross skeletal activity; Dworkin, 1989; 1993).

However, in the domain of homeostatic regulation, reference to the adaptive value of conditioned responses may certainly be elucidating (Dworkin, 1989; 1993). Pavlov himself held this opinion. His earlier physiological work (1897/1910) has been fully open to the possibility of conditioned responses serving regulatory purposes ${ }^{4}$. After describing the digestive system, with its secretions so well-attuned to the chemical compostion of the ingestive, Pavlov goes on to say that also the composition of conditioned salivation and other secretions is in accordance with the composition of the signalled substance (UCS). For example, CSs signalling the presentation of dry meat powder (UCS) trigger a more abundant watery salivation than CSs predictive of moist real meat (Babkin, 1949; Pavlov, 1897/1910). Today, the regulatory view of classical conditioning is especially in vogue in the field of drug conditioning. According to the prevalent view, drug intake (UCS) poses a disturbance of the internal milieu, which prompts regulatory physiological responses (UCRs). If the drug intake is reliably signalled by cues like the injection ritual in a specific environment, these CSs acquire the power to trigger conditioned responses that attenuate the physiological disturbance caused by intake of the drug (Eikelboom \& Stewant, 1982; MacRae, Scoles \& Siegel, 1987; Siegel, 1989). In most cases, the CR appears earlier than, or simultaneously with the UCR. In this way, the CR may enhance physiological regulation by mitigating a physiological disturbance in its very early stages, or by strengthening the homeostatic UCR (see Dworkin, 1993). It will be proposed here that conditioning of blood sugar level can be understood by similar principles.

\section{Principles of blood sugar conditioning}

Before introducing contemporary blood sugar conditioning research, a brief overview of blood

${ }^{4}$ In his later years (from 1903 onwards), Pavlov began to regard salivation rather as a tool to measure the strength of conditioning. As appears from his writings, Pavlov less and less considered the chemical composition or physiological impact of his dogs" conditioned secretions as a research object to focus upon (Dworkin, 1993). 
sugar regulation is in place. Blood sugar (more precisely, blood glucose) is the main fuel of e.g. the brain and the heart. Not surprisingly, the level of blood sugar is tightly controlled by a multitude of the nervous and endocrine activities. Under normal circumstances, two organs, the pancreas and liver, play a predominant role in blood sugar regulation. The pancreas is able to secrete insulin, a powerful blood sugar lowering hormones. The trigger for insulin secretion can be a high level of blood sugar and/or activity in the parasympathetic vagus nerve which innervates the pancreas (Niijima, 1989; Strubbe \& Steffens, 1993). The liver is able to lower or heighten blood sugar level, depending on circumstances. In the former case, glucose is withdrawn from the blood and stored as glycogen. In the latter, e.g. glycogen is converted to glucose and released into the blood stream. The hepatic blood sugar regulation is under hormonal and nervous control: catecholamines, glucagon and sympathetic nervous activity increase glucose production, whereas insulin and parasympathetic nervous activity decreases the glucose output from the liver (Brodsky \& Devlin, 1994). Interestingly, the autonomic nervous influences on pancreas and liver tend to be coordinated and synergistic. The source of these coordinated actions apparently lies within the hypothalamus (Niijima, 1989). In general, blood sugar regulation, supported by many more physiological factors than described here, acts so as to limit fluctuations in blood sugar during normal state, mobilize blood sugar in case of need (e.g., during stress or vigorous exercise), and store sugars in case of abundance (e.g. after a meal).

Conditioning of blood sugar level appears to be possible, and has systematically been studied since the 1960s (for reviews see Dworkin, 1993; Woods \& Kulkosky, 1976). The studies were all carried out in rats, given repeated injections with insulin (the UCS) in the course of days or weeks. Generally, the conditioned stimuli used in these studies have been complex, consisting of a stereotyped injection ritual within a salient environment. From a simple homeostatic viewpoint, the expected result of these procedures would be a hyperglycemic CR, i.e., a conditioned blood sugar rise that counters the hypoglycemia caused by the insulin UCS. However, the results found within this line of research were more subtle. Presentation of the CS (i.e. a saline injection in the conditioning environment) led to hypoglycemic CRs in some studies, and hyperglycemic CRs in others. These results have been explained in two ways.

${ }^{5}$ Although somewhat beyond the focus of the present article, glucagon deserves mentioning as the most powerful blood sugar raising hormone. Like insulin, glucagon is a pancreatic hormone, and its rate of secretion is inversely proportional to that of insulin. Because of the major impact of these hormones, the insulin/glucagon ratio has been used as an index of physiological state in organisms (Brodsky \& Devlin, 1994). 


\section{Chapter 4}

First, the insulin dose used as UCS has not been equally large in different studies. In several studies, the UCS was a huge insulin dose (leading to blood insulin levels that were 200 times larger than baseline level; N.B. under normal circumstances, after a meal, insulin levels rise to a peak of between 2 to 20 times baseline, see e.g. Guyton, 1991). In these cases, the insulin was thought to stimulate certain brain receptors involved in a positive feedback loop leading to additional insulin secretion (UCR) ${ }^{6}$. The unconditioned insulin reflex eventually re-emerged as the conditioned response which in its turn was responsable for the observed hypoglycemia. In contrast, studies in which smaller insulin doses were used as the UCS, have found a conditioned hyperglycemia (e.g. Siegel, 1972; Woods \& Shogren, 1972). These studies may have more relevance for blood sugar regulation under normal physiological condition. The physiological disturbance, caused by these smaller insulin UCS was a moderate fall in blood sugar level, which prompts compensatory UCRs (e.g. glucagon and catecholamine response, inhibition of insulin secretion) in order to restore baseline levels of blood sugar. After conditioning, these UCRs apparently were copied in CRs which could be elicted by the CS. Consequently, CS presentation lead to a hyperglycemic response (see Dworkin, 1993).

A second explanation, put forward to account for the findings of both conditioned hyper- and hypoglycemia, has been that the procedures differed in the amount of stress imposed on the rats. Notably, handling, drawing blood, and using unfamiliar cages to run the conditioning trials might impose stress upon the animals. Stress may increase blood sugar (by e.g. catecholamine, corticosteroid and glucagon release), thus overshadowing possible hypoglycemic CRs. Indeed, it has appeared that conditioned hyperglycemia appeared if the conditioning environment differed strongly from the normal home cage. Conditioning in a cage resembling the home cage led to conditioned hypoglycemia (see Dworkin, 1993; Flaherty, Grigson \& Brady, 1987).

Although the above studies are certainly informative about the role of conditioning in blood sugar regulation, insulin injections do not strike as naturally occurring physiological UCSs. It might be that using UCSs containing glucose would be a more natural stimulus: after food intake the blood glucose level generally shows a distinctive rise, to which ample homeostatic reactions are emitted (Guyton, 1991). Therefore, it is puzzling that glucose has been seldomly used as the UCS.

We are aware of only four reports on conditioning procedures with pure glucose as the UCS.

${ }^{6}$ For further explanations of the physiological mechanisms probably involved, see Woods (1983) and Dworkin (1993). 
Deutsch (1974) who used rats, found. a $3.5 \%$ hypoglycemia to a caffein flavour CS, after repeatedly pairing of a flavour with a glucose dose (UCS) which was intubated into the stomach. Deutsch claimed that the observed conditioned hypoglycemia had been the result of a conditioned insulin secretion. Matysiak and Green (1984) camied out three experiments in which an injection ritual (CS) was repeatedly paired with intravenous glucose UCS in rats. Post conditioning testing in all three experiments showed a conditioned blood sugar decrease (CR) of approximately $3 \%$ in response to a saline injections; a control group of rats that had previously received saline UCSs showed a 4-6\% hyperglycemia. These data were somewhat problematic however, because baseline levels were measured on a day different from the test day. Woods (1976), after conditioning two groups of rats with either glucose or saline injections as UCS, did find an insignificant hyperglycemia on the test trial (injecting saline), but there were no differences between glucose and saline-conditioned rats. It is possible that the hyperglycemia resulted from stress. Woods attributed the lacking hypoglycemia to the glucose UCS dose not being large enough. Overduin and Jansen (submitted) used $50 \mathrm{gr}$ of oral glucose as a UCS in humans, and peppermint taste/odor as CS in humans, but found no significant hypoglycemia. This was probably explained by the limited number of six conditioning trials and the - comparatively - small physiological impact of oral dose of glucose which was used.

Taken together, procedures using glucose as the UCS have produced either hypo- or euglycemic, but not hyperglycemic CRs. Although none of these results contradict a homeostatic, i.e., regulatory explanation, replications of the conditioned hypoglycemic effect surely would add to make this explanation more convincing. In this respect, the study by Mityushov has an interesting contribution to make.

\section{The article}

Although M.I. Mityushov's article, of which a translation will follow next, was entitled Conditioned Reflex Secretion of Insulin, it only reported direct measurements of blood sugar. The article was first published in 1954 in the Russian Journal Zhurnal Visshey Nervnoi Deyatel'nosti, which can be translated as "Journal of Higher Nervous Activity". The Zhumal was the main Eastern-European journal of the Pavlovians and, in the 1950s and 1960s, excerpts from it were published in German and English as well. For reasons unknown, Mityushov's paper never appeared in translation.

Several features of Mityushov's experiment contribute to its relevance for contemporary research. First, a large glucose injection was used as a UCS and an (albeit indirect) attempt 
Chapter 4

was made to assess the influence of insulin in the conditioned hypoglycemia. Second, humans and dogs, rather than rodents served as subjects. Third, relatively many conditioning trials were conducted. Last, but not least, although Mityushov's article does not contain rigorous statistical analyses, it appears that unequivocal and interesting results were found.

TRANSLATION OF MITYUSHOV'S ARTICLE (SEE APPENDIX)

\section{COMMENTARY}

\section{Abstract}

In a conditioning procedure, Mityushov repeatedly administered intravenous glucose (UCS) to four dogs and nine humans. The injection procedure was carried out in a specific room served as the CS. At the post conditioning test session, a saline injection (CS) was given at $t=0$. The blood sugar response (CR) was measured by repeated blood sampling at $t=-10,-5,5,15$ and 45 minutes for humans, and at $-10,-5,5,15,30,60$ and 90 minutes for dogs. Explicit data were presented for several responses of 4 humans and 2 dogs. These subjects had been subjected to between 5 and 52 conditioning trials. In all cases, the saline injection elicited a conditioned blood sugar fall of between 5 and $20 \%$ below baseline. The nadir of the response occurred at 15 minutes after the saline injection. As the CR was measured repeatedly within subjects, it could be seen that $C R$ intensity tended to increase as a function of the number of previous conditioning trials. Although no direct insulin measurements could be made at the time, Mityushov collected evidence that the hypoglycemia observed in humans and dogs, was secondary to a conditioned secretion of insulin. Mice that were injected with blood that was drawn from dogs during their conditioned hypoglycemia, showed a subsequent fall in their blood sugar level. Mityushov claimed that he observed the conditioned hypoglycemic response in all of his human and canine subjects.

\section{Additional analyses}

Mityushov's article contains neither graphs nor statistical tests of the blood sugar data. Nevertheless, it was possible to further analyze the data depicted in tables A.1 to A.3 of Mityushov's article (see appendix). For this purpose, data for dogs and humans were treated separately. Only data were considered of the "correct" test trials, i.e., those taking place in the 


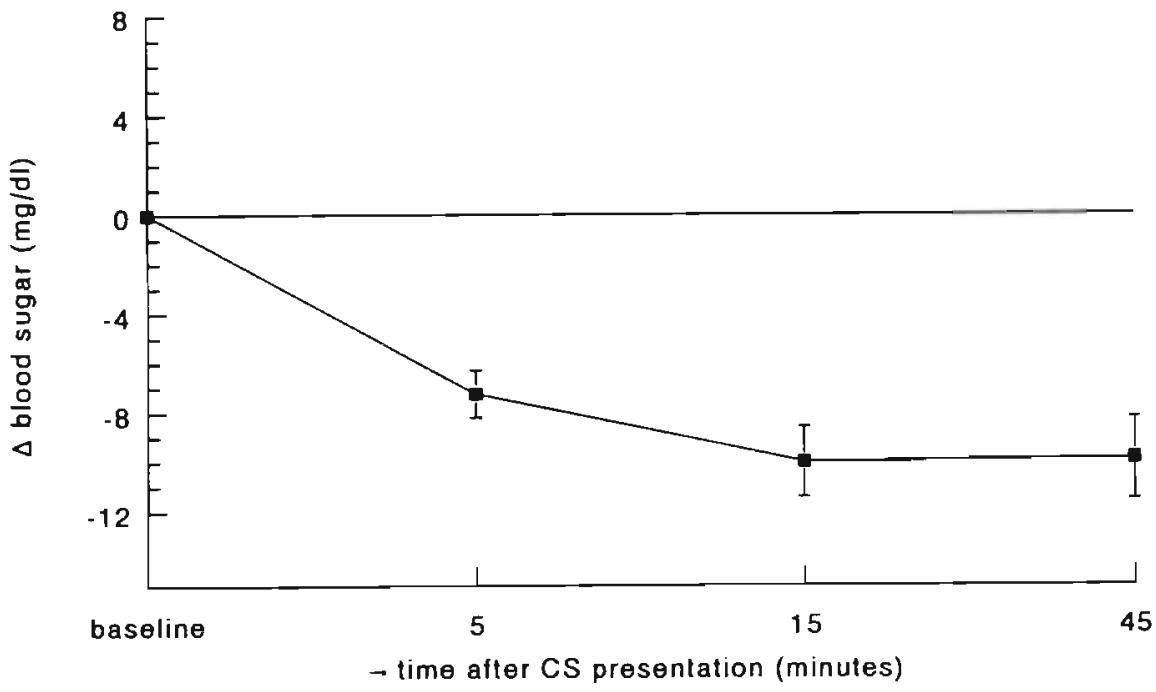

Figure 4.1. Post-conditioning blood sugar responses (mean \pm s.e.m.) to saline injections in 4 humans, based on a total of 17 responses. Responses expressed as deviation from baseline.
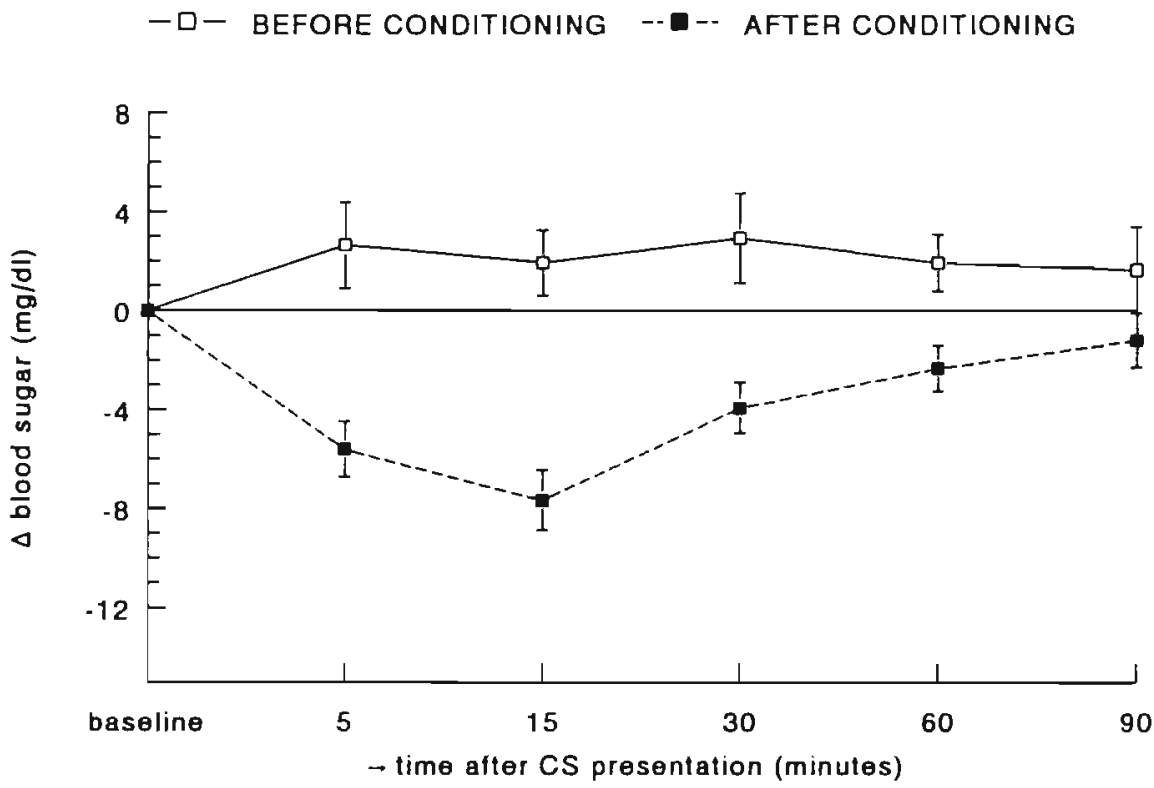

Figure 4.2. Pre- and post-conditioning blood sugar responses (mean \pm s.e.m.) to saline injections in 2 dogs, based on a total of 7 responses before, and 15 after conditioning. Responses expressed as deviation from baseline. 


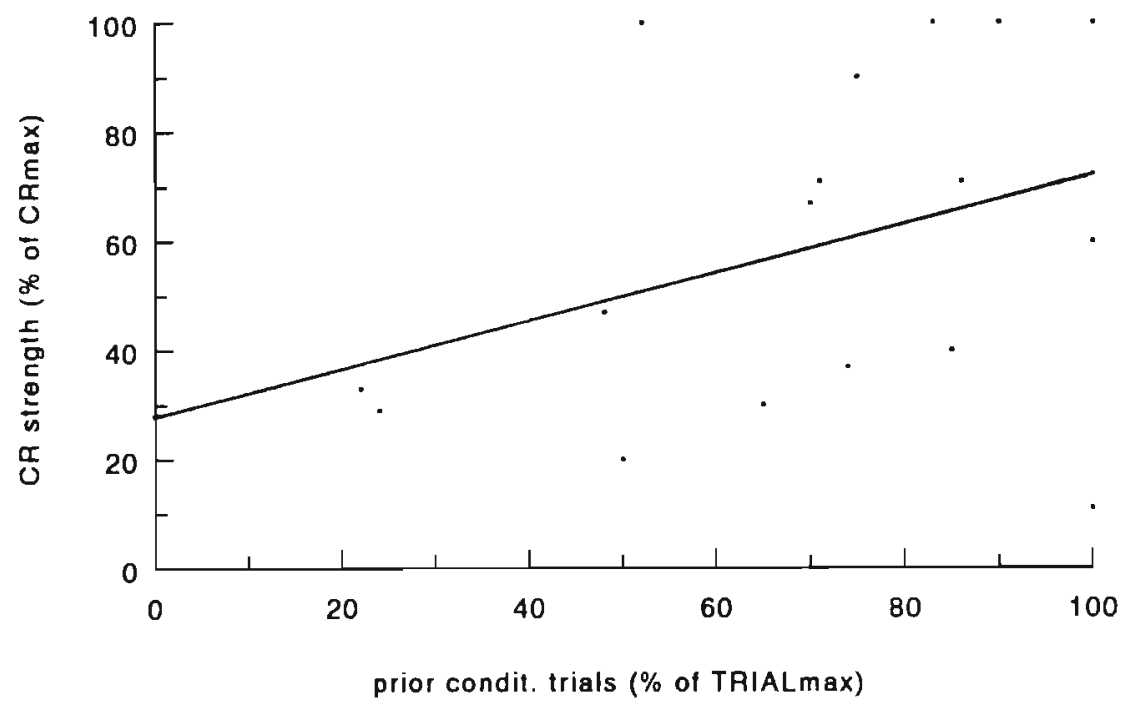

Figure 4.3. Acquisition curve of conditioned hypoglycemia in humans. For each subject, CR strength has been expressed as the percentage of the maximum CR intensity shown throughout the experiment.

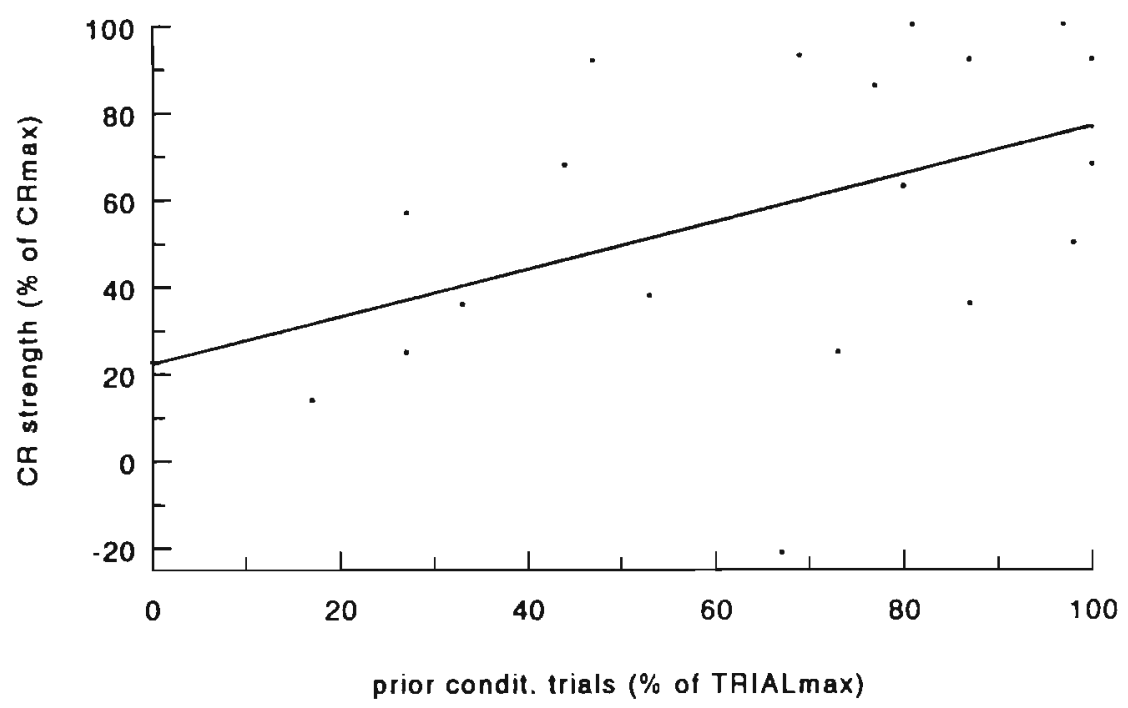

Figure 4.4. Acquisition curve of conditioned hypoglycemia in dogs. See figure 4.3. for further explanation. 
conditioning chamber with the usual experimenter.

Means Figures 4.1 and 4.2, depicting the glycemic responses to saline injections for humansand dogs respectively, were construed as follows. First, baseline level was computed as the mean of the two pre-injection baseline values. Then, the blood sugar values after each saline injection were expressed as the deviation from baseline. Finally, all responses of all subjects were taken together, mean and s.e.m. were computed and entered into the figures. For dogs, separate graphs were drawn for responses before and after conditioning.

The differences between baseline and post injection blood sugar levels were tested separately within three data subsets: for canine pre- and post-conditioning, and human post-conditioning data respectively. For each data set, a paired t-test was done comparing the mean baseline level with the mean blood sugar level after saline injection. For dogs' responses prior to conditioning (9 responses), no difference was found $(\mathrm{t}(8)=.17$; ns). After conditioning, for dogs $(\mathrm{t}(18)=$ 5.35; $\mathrm{p}<0.001 ; 19$ responses), and humans ( $\mathrm{t}(18)=7.83 ; \mathrm{p}<0.001 ; 19$ responses) highly significant differences were found, reflecting a hypoglycemia with its nadir at 15 minutes after the saline injection. Additional t-tests, comparing baseline with the individual post-injection blood sugar levels, revealed that after conditioning, all blood sugar levels measured within 45 minutes after saline injection, were significantly below baseline (table 4.1 ).

Table 4.1. Tests for differences between blood sugar levels before and after saline injection.

\begin{tabular}{cccc}
\hline & Before conditioning & \multicolumn{2}{c}{ After conditioning } \\
& dogs & $\operatorname{dogs}$ & humans \\
minutes & & & \\
$\mathbf{5}$ & $\mathrm{ns}$ & $<.001$ & $<.001$ \\
$\mathbf{1 5}$ & .05 & $<.001$ & $<.001$ \\
$\mathbf{3 0}$ & $<.05$ & $<.003$ & $<.001$ \\
$\mathbf{4 5}$ & & & \\
$\mathbf{6 0}$ & $\mathrm{ns}$ & $\mathrm{ns}$ & \\
$\mathbf{9 0}$ & $\mathrm{ns}$ & $\mathrm{ns}$ & \\
\hline
\end{tabular}

To summarize, both canine and human subjects consistently showed conditioned hypoglycemic responses to the saline injection. Prior to conditioning, saline responses lead to significantly elevated blood sugar level at 15 and 30 minutes for dogs (no human data were reported for these pre-conditioning trials).

Acquisition curves of the conditioned hypoglycemia Because the blood sugar response to saline 


\section{Chapter 4}

injections was assessed after different numbers of previous conditioning trials within subjects, an acquisition curve of the hypoglycemic response could be construed for humans and dogs (figures 4.3 and 4.4). Given the individual differences in the size of the largest $C R$ and the number of conditioning trials that were nn, the following method was adopted ${ }^{7}$. For each individual, the most intense hypoglycemic CR of all test trials (CRmax) was determined. Also the maximum number of conditioning trials for the given indiviudal was computed as Trialmax. Then, for each test trial, two values were computed: $x$, the number of conditioning trials run before the trial in question expressed as a percentage of Trialmax, and $y$, the strength of the CR expressed as a percentage of CRmax. After that, the values $(x, y)$ for all reported $C R s$ in the experiment were entered in a scatter plot $(x, y)$. Finally, a provisional leaming curve was constructed by drawing a linear regression line through the scatter plot $^{8}$. As can be seen from the positive slope of the learning curve, CR-strength tended to increase as more conditioning trials were run. The correlation between $x$ and $y$ were significantly different from zero: 0.43 for canine, and 0.35 for human subjects.

Habituation of the hyperglycemic response to repeated saline injections in dogs before conditioning As shown in figure 4.5, saline injections before conditioning evoked a slight hyperglycemic response in dogs. To investigate whether after repeated injections a waning of the hyperglycemic response did appear, the $(x, y)$ plot was made of glycemic responses at 15 minutes after injection, in the same way as was done above (see figures 4.3 and 4.4). As above, the glycemic responses were expressed as a percentage of CRmax for the given dog (i.e. his largest hypoglycemic response occurring after subsequent conditioning; see above). Also, a linear regression line was drawn through the plot. Given the positive slope of the regression line it appears that the hyperglycemic response tended to decline as more saline injections had been received by the dog. The correlation between number of previous saline trials and the strength of the hyperglycemia was -0.32 . Evidently, the hyperglycemic response did not disappear completely after many saline injections. As Mityushov observed, the hyperglycemia to saline may have been caused by stress related to the injection procedure. Modern research has confirmed that stress may increase blood sugar level e.g. by catecholamine and glucagon release as well as an inhibition of insulin secretion (Brodsky \& Devlin, 1994; Dworkin, 1993).

\footnotetext{
${ }^{7}$ Only blood sugar data at 15 minutes after saline injections were used.

${ }^{8}$ Because of the limited number of datapoints, no higher order curve fitting was attempted.
} 


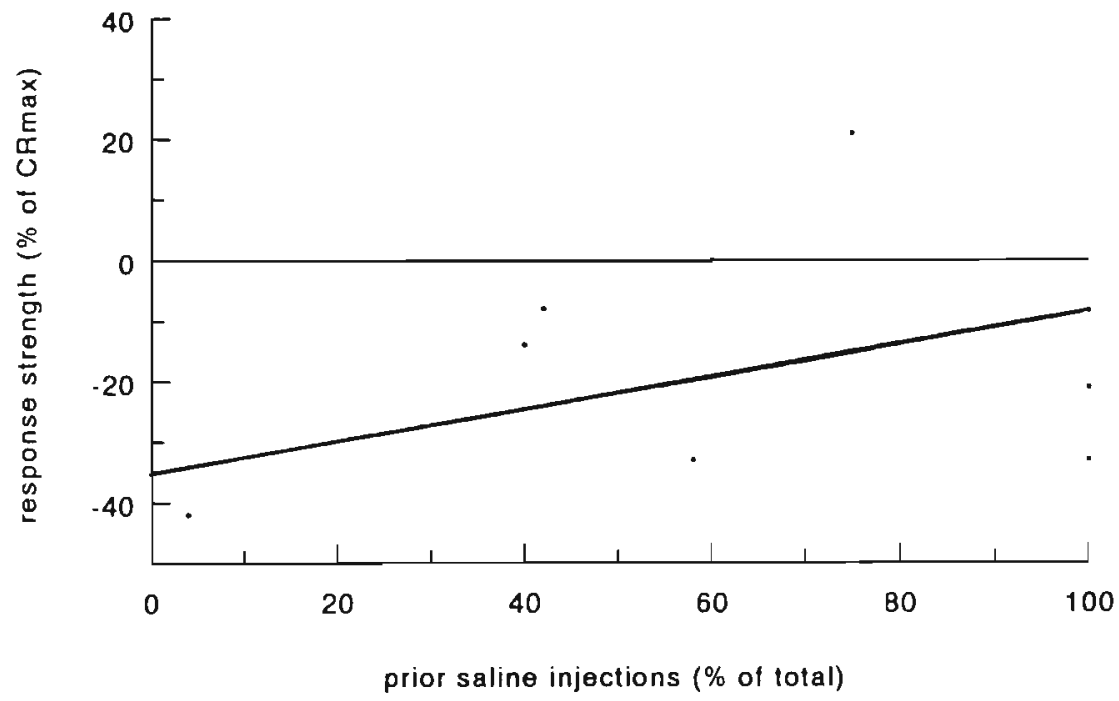

Figure 4.5. Habituation of the hyperglycemic response to repeated saline injections in 2 dogs, based on 7 responses. For each subject, response strength was expressed as percentage of the maximum $\mathrm{CR}$ shown throughout the experiment.

\section{DISCUSSION}

To our knowledge, Mityushov's study is the first successful demonstration of blood sugar conditioning in humans using a glucose UCS. The results are in line with other studies in which a glucose UCS was used (Deutsch, 1974; Matysiak \& Green, 1984). Furthermore, the data from this study are in line with a homeostatic explanation: a CS which has come to predict a blood sugar rise provokes a compensatory, hypoglycemic CR (see Dworkin, 1993).

Before discussing the results further, three problems of Mityushov's study should be mentioned. First, although Mityushov claims that he found a conditioned hypoglycemia in all subjects, he has only presented data for a small subset of his subjects. Unfortunately, overall means or statistical tests are lacking. A second problem is the lack of control group receiving e.g. repeated saline UCS injections. Of course, it can be said that this problem is mitigated because the blood sugar response to saline was measured also before conditioning began. In other words, the subjects served as their own controls. However, although Mityushov claims 


\section{Chapter 4}

that all subjects responded alike during these "baseline trials", his reporting only part of the data would not meet today's methodological standards. A third problem of Mityushov's procedure concerns the unnaturally high blood sugar levels $(250 \mathrm{mg} / \mathrm{dl}$ for humans; $420 \mathrm{mg} / \mathrm{dl}$ for dogs) provoked by the injected glucose UCS. One might wonder if the present results should be extrapolated to normal circumstances in which blood sugar levels do not exceed 190 $\mathrm{mg} / \mathrm{dl}$ (Guyton, 1991). However, observations in the literature suggest no discontinuities in blood sugar regulation under extreme hyperglycemia. Firstly, blood sugar levels of approximately $300 \mathrm{mg} / \mathrm{dl}$ after intravenous glucose injections in humans have been found to retum to baseline in slightly over one hour ${ }^{9}$, and this has been found to be related with increased insulin secretion and a suppression of glucagon release ${ }^{10}$ (Blouin et al., 1993). During sustained hyperglycemia in humans $(300 \mathrm{mg} / \mathrm{dl})$, insulin secretion rate remains at a high rate for hours (Polonsky et al., 1988). The rate of insulin secretion in dogs' and rats' pancreas is linearly correlated to at least blood sugar levels up to $300 \mathrm{mg} / \mathrm{dl}$ in dogs (Girardier et al., 1976), and $500 \mathrm{mg} / \mathrm{dl}$ in rats (Gerich, Charles \& Grodsky, 1976). Furthermore, blood sugar storage in the liver (through glycogen synthesis) increases linearly with blood sugar level, up to levels of $450 \mathrm{mg} / \mathrm{dl}$ in rats (Niijima, 1989). One might derive from these observations that the results found by Mityushov could be extrapolated to less extreme physiological conditions as well".

When looking at the data presented by Mityushov, it appears that his conditioning procedure has been effective in producing significant hypoglycemic conditioned responses after saline injection. As mentioned earlier, these results fit well with a homeostatic explanation. The CS (saline injection) that had come to signal a blood sugar increase (UCS), evoked CRs aimed at lowering blood sugar. That is, the CRs could be understood as adding to the nullification of the expected homeostatic disturbance. Which physiological pathways might then be involved? Mityushov suggests that insulin secretion was basic to the observed conditioned hypoglycemia (hence the title of his paper). This is a plausible explanation given the finding of a blood sugar fall in mice after an injection with blood drawn from the hypoglycemic dogs: some blood factor

${ }^{9}$ Mityushov reported the same blood sugar response in his subjects after the glucose UCS injection.

10 Like insulin, glucagon secretion is under the influence of autonomic nervous activity (Brodsky \& Devlin, 1994; Niijima, 1989).

11 This is of course in contrast with the conditioning studies using large insulin injections as the UCS (see the introduction of this article). 
may have been responsible, and insulin is one of the very few blood sugar lowering factors. Additionally, there is by now substantial evidence that the endocrine pancreas is capable of secreting insulin in response to naturally occurring food signals, like the smell, taste and sight of food (the cephalic responses, Bruce et al., 1987; Powley \& Berthoud, 1985) and learned signals predicting meals (Strubbe, 1992; Woods et al., 1977). These responses have been found to be mediated by parasympathetic nervous activity (Strubbe, 1992; Strubbe \& Steffens, 1993). What Mityushov probably did, is to bring insulin secretion under control of the injection ritual. A second mechanism underlying the hypoglycemia observed by Mityushov, might be a nervously regulated inhibition of glucagon secretion. Thirdly, the possibility exists that the saline injection (CS) induced a conditioned inhibition of the glucose production in the liver. This might have occurred through activity in the hepatic branch of the parasympathetic vagus nerve (Niijima, 1989). Whether this pathway is indeed susceptible to conditioning remains to be established.

Two alternative explanations of the observed hypoglycemia should be mentioned. First, the saline injection might have diluted the blood, and consequently blood sugar level would appear to have fallen (this problem appears in research in rats, see, for example, Deutsch, 1974). A second, alternative explanation might be that the hypoglycemia resulted from a temporary depletion of blood sugar, caused by a transient increase in utilization due to injection-related stress. However, both explanations are unlikely, because, as can be seen in figure 4.2 , saline control injections given to dogs before conditioning, in all cases led to slight hyperglycemias. Besides its physiological implications, the paper by Mityushov bears upon procedural issues in glycemic conditioning. Mityushov's finding of rapid blood sugar and insulin conditioning in humans (after 5 trials) is highly remarkable because of the lacking natural belongingness of an injection-CS to blood sugar level ${ }^{12}$. Moreover, no odors were added to the CS-compound, although odors and blood sugar level fluctuations can be said to have natural "belongingness" (consider food ingestion), and Woods et al. (1969) discovered that glycemic conditioning was considerably speeded up in rats if odors formed part of the CS. Another remarkable feature of the Mityushov report is that, whilst today's conditioning literature would not recommend preexposure to the CS, as this is generally understood to retard conditioning (e.g. MacRae, Scoles \& Siegel, 1987; Miller et al., 1995), Mityushov was able to find a rapid acquisition of the CR

12 Of course, also the appearance of the experimenter and the conditioning room seem to bave served as additional CSs, as Mityushov's hypoglycemic CRs failed to appear as soon as the saline injection took place in a different room or with a different experimenter (see the appendix of this thesis, tables A.2 and A.3). 


\section{Chapter 4}

after giving repeated saline injections before conditioning ${ }^{13}$.

The broader relevance of Mityushov's data is that the magnitude of the conditioned hypoglycemias (5-20\%) overlaps with that of the hypoglycemias that have been recently found to precede meal initiation in rats (Campfield \& Smith, 1990), and humans (Campfield et al., 1996). Although Campfield and Smith have always emphasized that the origin of the pre-meal hypoglycemias are endogenous, Mityushov's results suggest that the responses might be conditioned to external cues, a suggestion also made by Woods (1990). In fact, it has been recently proposed that conditioned insulin secretion and hypoglycemia play a role in normal and binge eating (Jansen, 1994a; Woods, 1991)

It is obvious that Mityushov's study on blood sugar conditioning deserves replication with control groups, direct insulin measurement, and statistic tests added. Furthermore this study may serve as an illustration of the potential importance of investigations carried out decades ago by Pavlovian physiologists in Eastern Europe, who found conditioned response interesting in their own right. When this large data base, embodied in several thousands of non-English publications (Dworkin, 1993; Razran, 1961) would be explored, provoking and interesting results might be found, many of which await replication and extension.

In the Eastera-European Pavovian tradition, pre-exposure to the CS used in subsequent conditioning was a standsrd procedure. Pre-exposure was meant to habiruate orienting reflex to the $C S$, because orienting was confidert antagonistic to conditioning, habituation was thought to be a prereģisite to successful conditioning. 
Chapter 5

\section{CUE REACTIVITY TO \\ FOOD- AND BODY-RELATED STIMULI \\ IN RESTRAINED AND UNRESTRAINED EATERS ${ }^{1}$}

\section{INTRODUCTION}

The symptomatology of bulimia nervosa includes recurrent binge eating and an overconcern regarding body shape and weight (APA, 1994). During binge eating, large amounts of food are ingested while the subject experiences an irresistable food craving and loss of control over food intake. Recently, classical conditioning models, derived from addiction research (e.g. Siegel, 1983; Drummond, Tiffany, Glautier \& Remington, 1995) have been applied to binge eating (Jansen, 1990, 1994; Wardle, 1990). In these models it is assumed that through a learning process, cues predictive of binge eating (e.g. the sight and smell of snacks, or states of depression and boredom) eventually come to elicit anticipatory physiological responses. One of these is a secretion of insulin which counters the post-binge blood sugar rise. According to the conditioned binge eating theory, anticipatory responses, when intense, are experienced as a craving for food.

In aldietion research, an interesting pperationalization of conditioning theories has emerged: cue reactivity measurement, a method in which drug-related cues are presented and psychophysiological measures are monitored. The intensity of these reactions (i.e. cue reactivity) is supposed to be a reflection of the responses underlying craving. For example, alcoholics and smokers display heart acceleration and increased skin conductance responses when presented with substance-related cues, an effect that was not seen in normals Niaura, Rohsenow, Binkoff, Monti, Pedraza, Abrams, 1988; Childress, Ehrman, Rohsenow, Robbins \& O'Brien, 1992). An important reason for assessing cue reactivity is that it probably forms a risk factor in relapse of treated addicts (Childress et al., 1992; Drumond et al., 1995). Moreover, measurement of cue reactivity could be used to study the effect of exposure therapy in addicts (Jansen, 1994). In contrast to addiction research, binge eaters" psychophysiological cue reactivity to food stimuli has been investigated rarely, perhaps with the exception of salivation (e.g. Klajner, Herman, Polivy o Chabra, 1981; LeGoff \& Spigelman, 1987).

In the present study, an experimental, non-clineal approach (see Abramson \& Seligman, 1977) was adopted by studying cue reachivity in restrained eaters. The restraint concept

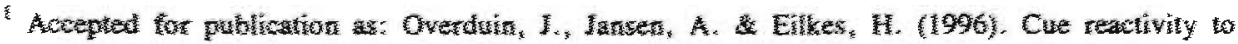

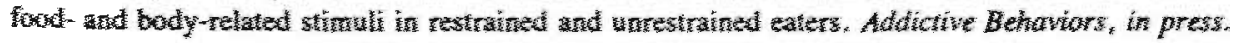


was originally developed to describe the occasionally disinhibitive eating behavior of dieters, and it has often been used as an analogue of binge eating tendency (Wardle \& Beinart, 1981; Herman \& Polivy, 1988). Two similarities between restrained eaters and bulimics can be mentioned.

The first is that the eating style of restrained subjects, selected by Herman \& Polivy's Restraint Scale (Herman \& Polivy, 1980) is characterized by unsuccessful attempts to restrict food intake and an increased frequency of binge eating (Wardle, 1980). Within the laboratory, restrained eaters have been found to increase their intake of high-caloric foods after a range of manipulations like, for example, ingestion of a "preload" (for example, a high-caloric pudding; Herman \& Polivy, 1980; Lowe, 1993), merely smelling a preload (Jansen \& Van den Hout, 1991; Rogers \& Hill, 1989), or induction of certain mood states (Greeno \& Wing, 1994; Lowe, 1993; Ruderman, 1986). In line with these findings, the present study was aimed at testing the hypothesis of increased cue reactivity to attractive food cues in high vs. low restrained eaters.

A second similarity between bulimics and restrained eaters is the existence of an overconcern (albeit to a different extent) with body shape and weight. As a rule, bulimics and restrained eaters consider themselves too fat and they are afraid of getting fatter (Herman \& Polivy, 1988). In this study, we tested the hypothesis that restrained eaters show more intense emotional responses than unrestrained eaters when viewing slides depicting their own body.

In the present study, food and body cue reactivity was assessed by monitoring autonomic physiological responses. These were considered relevant for three reasons: their role in anticipation and processing of ingested food (Woods, 1991), their being sensitive measures in cue reactivity research of various addictions (Niaura et al., 1988; Childress et al., 1992), and their suitability as indices of affective responses to stimuli (Lang, Greenwald, Bradley, \& Hamm, 1993).

In short, two hypotheses were tested in the present study. First, all subjects will show more intense appetitive responses to slides depicting binge food than to slides with a neutral content; restrained eaters show this effect to a larger extent than do unrestrained eaters. Second, restrained eaters will show more intense emotional arousal to pictures of their own body, than unrestrained eaters.

\section{METHOD}

\section{Subjects}

Female subjects were called for by means of an advertisement in a local newspaper. The 75 individuals that responded were invited to fill in the Restraint Scale (RS; Herman et 
al., 1978). Of these, 26 individuals having either a RS-score below 10 or above $15^{2}$ were asked to participate in the experiment. Characteristics of the two subject groups thus formed are shown in table 5.1. After the experiment, it appeared that two subjects did have an extreme Body Mass Index (BMI resp. 16.5 and 26.5). Data from these subjects were excluded from statistical analysis.

Table 5.1. Characteristics of Restrained and Unrestrained subjects in this study

\begin{tabular}{|c|c|c|c|c|c|c|}
\hline & \multicolumn{2}{|c|}{$\begin{array}{l}\text { Unrestrained } \\
\qquad(N=13)\end{array}$} & \multicolumn{2}{|c|}{$\begin{array}{l}\text { Restrained } \\
\quad(N=11)\end{array}$} & \multicolumn{2}{|c|}{ t-test } \\
\hline & mean & $\mathrm{sd}$ & mean & sd & $\mathrm{t}$ & p-value \\
\hline Body Mass Index & 19.9 & 1.4 & 21.8 & 1.7 & 2.90 & .01 \\
\hline Age & 20.3 & 1.7 & 21.5 & 3.1 & 1.14 & $\mathrm{~ns}$ \\
\hline Restraint Scale & 5.2 & 2.2 & 20.2 & 2.8 & 14.6 & $<.001$ \\
\hline
\end{tabular}

\section{Psychophysiological parameters as related to hypotheses}

Hypothesis one: larger reactivity to food cues than for neutral cues, a fortiori so in restrained eaters.

Skin conductance response, amplitude Enlarged SCRs have been found in alcohol and nicotine addicts who where presented substance related cues (Niaura et al., 1988; Childress et al., 1992). We expected all subjects to show larger SCR-amplitudes in response to bingefood than to neutral slides; furthermore this effect was expected to be stronger in restrained than in unrestrained eaters.

Heartrate (HR) The heart is innervated by both sympathetic and parasympathetic nerves, and reactions to stimuli are often multiphasic and not easily interpreted (Venables, 1991). So far, cue reactivity research has scarcely addressed this issue: heartrate data are usually averaged over longer time intervals. Overall HR-increase after cue presentation has been found in smoking-addicts, but responses in alcoholics have been contradictory (Childress et al., 1992). Kostarczyk (1986) has found HR acceleration in dogs, presented with a conditioned stimulus which had been previously paired with a food delivery. The accelaration occurred during the 10 post-stimulus seconds. Given the above findings, we expected a larger HR acceleration in restrained than in unrestrained eaters watching slides with binge

\footnotetext{
${ }^{2}$ That is, 0.5 standarddeviations below and above the mean RS-score of a group of 482 Dutch female students respectively; Overduin \& Jansen, 1996; Jansen \& Overduin, unpublished data).
} 


\section{Chapter 5}

foods.

Startle reflex, amplitude Lang and co-workers (Lang et al., 1990, Bradley et al., 1990) have demonstrated that changes in the intensity of startle eyeblink reflexes, elicited during picture viewing, covary with the subjective emotional valence of the picture. Viewing positive slides has an inhibitory, whereas viewing negative pictures has a facilitating effect on the reflex amplitude (Lang et al., 1990). Lang et al. have suggested that startle modulation is bases on the activation of either appetitive or defensive behavior systems. It should be noted that predictions about the reaction of restrained eaters to food slides are problematic. On one side, one would expect increases in startle amplitude because of negative emotional valence of the slide content (due to likely associations with guilt and loss of control), on the other one should expect less intense startle responses because food slides induce an appetitive state. Therefore, in the case of restrained subjects, no strict directional prediction was formulated for startle reflex modulation to food slides. Unrestrained subjects were expected to display a small decrease in startle amplitude, related to the craving elicited by the slides.

Startle reflex, onset latency The startle onset latency appears to covary inversely with startle amplitude (Bradley, Cuthbert \& Lang, 1990). That is, increases in amplitude go hand in hand with decreases in onset latency and vice versa. Thus, during presentations of binge food pictures we expected unrestrained eaters to show increased onset latencies, while for restrained eaters no specific predictions were made.

Facial EMG In emotion research, EMG activity of facial muscles has been demonstrated to be specifically indicative of emotional valence. Two important examples are the Zygomatic 'cheek-muscle', which contributes to the act of smiling and becomes increasingly active when a person views pictures with a positive emotional valence, and the Corrugator 'frownmuscle' which is active during experience of negative emotion (Lang et al., 1993; Dimberg, 1987). For the same reasons explained above for the startle response, no explicit predictions could be made for facial muscle EMG responses to food slides in restrained eaters.

Hypotheses two: Increased emotional arousal in restrained eaters to slides depicting their own body.

Skin conductance reaction, recovery time SCR-recovery time reflects the speed with which the SC-level retums to baseline after having reached its peak. Repeatedly, it has been suggested that increased SCR recovery time is indicative of a defensive response (Dimberg, 1987; Edelberg, 1972). Accordingly, in the present study we expected increased SCR recovery time in restrained, but not in unrestrained eaters presented with slides depicting 
their own body.

Heart rate HR acceleration at between 3-5 seconds after stimulus onset is due to sympathetic activity and has been interpreted as reflecting a defensive reaction (Venables, 1991). We therefore expected restrained eaters to show larger heart rate accelerations than unrestrained eaters during presentation of pictures of their own body.

Startle reflex. amplitude Because of the negative emotional valence of body slides, we expected these slides to induce a larger increase in startle amplitude in restrained than in unrestrained subjects (Lang et al., 1990).

Startle reflex, onset latency Presentation of body slides was expected to lead to larger decreases in startle onset (relative to responses to neutral slides) in restrained than in unrestrained subjects.

Facial EMG Because the negative emotional valence of body pictures, restrained eaters were expected to show larger decreases in zygomatic, and larger increases in corrugator EMG-activity than were unrestrained eaters.

\section{Equipment, and processing of the psychophysiological signals}

All psychophysiological signals were fed to Beckman couplers and subsequently stored on multichannel magnetic tape for further off-line analysis. One of the channels contained information about times and types of stimulus presentation. Only the Skin Conductance signal was recorded (and subsequently scored) on paper using of a Beckman R711 polygraph with multi-channel writer.

SCR was recorded using two $\mathrm{Ag}-\mathrm{AgCl}$ electrodes (diameter $8 \mathrm{~mm}$ ) attached to the middle fingers of the non-dominant hand. Two parameters were drawn from the SCR-signal: amplitude (SCR-amp) and recovery time (SCR-rec). SCR-amp was defined as the (baseline corrected) maximum SC-level within 6 seconds after slide onset (baseline was defined as the SC level before a significant post-stimulus increase began). SCR-rec was defined as the time interval between the moment the SC level reached its peak and the moment the signal had returned to a level of $(0.5 *$ SCR-amp) above baseline.

Startle reflexes were elicited by a pulse of $90 \mathrm{~dB}$ white noise with instantaneous rise time through headphones (Anthony, 1985). Part of the startle reflex is the eyeblink, and the relevant EMG signal was detected using two $\mathrm{Ag}-\mathrm{AgCl}$ electrodes applied on the orbicularis oculi, just beneath the lower left eyelid. The EMG-signal was sampled from tape with a frequency of $1000 \mathrm{~Hz}$ and filtered by a digital, 255-point, $60 \mathrm{~Hz}$ highpass FIR-filter. Then the signal was rectified and integrated using a time constant of 20 milliseconds. Under the condition that a typical reflex shape could be distinguished, two parameters were extracted from the signal: amplitude and onset latency. Startle amplitude was defined as the highest EMG-level within $150 \mathrm{msec}$ after presentation of the noise. The amplitude was corrected 


\section{Chapter 5}

for pre-stimulus baseline level, defined as the mean EMG level in the 50 pre-stimulus milliseconds. Startle onset latency was defined as the time interval between presentation of the startle stimulus and the moment when EMG level began to rise sharply and significantly above baseline level. Reactions with onset latency earlier than 18 or later than $70 \mathrm{msec}$ or with no apparent reflex shape were excluded from further data processing.

Facial muscle EMG activity from the zygomatic and corrugator muscles was recorded using two $\mathrm{Ag}-\mathrm{AgCl}$ electrodes with a diameter of $2 \mathrm{~mm}$, placed according to suggestions by Fridlund and Cacioppo (1986). Signals from both channels were treated likewise. After $1000 \mathrm{~Hz}$ sampling from the tape the signals were respectively lead through a digital, 255point, $90 \mathrm{~Hz}$ high-pass FIR-filter, rectified, and integrated by a contour-following integrator with a time constant of $500 \mathrm{msec}$ (Fridlund, 1979). Baseline EMG was defined as the mean intensity of the signal in the 5 seconds preceding slide presentation (Dimberg, 1987). The reaction was defined as the mean EMG level during the 5 seconds after stimulus onset, corrected for baseline.

Heartrate was recorded by means of two Blue Sensor disposable ECG electrodes (type R$00-S$ ) attached on a diagonal axis over the heart region and a reference electrode on the shoulder. From magnetic tape the signal was sampled with a frequency of $1000 \mathrm{~Hz}$ and led through a digital, 255-point, $35 \mathrm{~Hz}$ lowpass FIR-filter. After that, times of R-peaks in the signal were detected by a special devised computerprogram and checked for artifacts and electronic interference. In a next step second-to-second heartrate for the 12 post-stimulus seconds were computed by a specially devised software program employing the weighing procedure proposed by both Velden and Graham (1988). Baseline was defined as the mean heartrate in the 5 seconds preceeding stimulus onset. Eventually two values were extracted: 'overall acceleration', i.e. the mean second-to-second heartrate for the 12 seconds after stimulus onset, and defensive acceleration, defined as the highest of the second-to-second heartrate for seconds 3, 4 and 5 after stimulus onset (Laberg, Wilson, Eldredge \& Nordby, 1991).

\section{Materials}

All auditory stimuli (music and startle noises) were presented through Beyer DT 109 headphones. The music used for the depressive mood induction (see procedure) was a piece by the classical composer Prokoviev, entitled 'Russia under the Mongolian Yoke' played at half speed (Clark, 1983). Slide projection was carried out with a Kodak carousel projector, which was automatically driven by a computer program on a PDP Minc Microcomputer.

There were three slide categories, 'neutral', 'food' and 'body'. The neutral pictures were the same for all subjects and showed pieces of office equipment. Body and food slides were selected individually for each subject. Care was taken that the food stimuli would reflect 
each subject's preference. To achieve this, a rating scale was administered 3 weeks before the experiment on which the subjects had to rate 5 snack categories on preference and 'binge provoking characteristics'. From each of the three categories ranked highest by the subject, 2 slides were included in the slide series. The 5 categories were potato chips, chocolate bars, candy bars, licorice, and pies. The set of body slides consisted of pictures that had been taken of the subject three weeks prior to the experiment (see procedure). The subject was visible wearing a standard set of underwear that would leave belly, arms, legs, neck and face uncovered. In a set of six slides the body was shown from different standardized angles: two pictures were taken frontally, two en profil, and two from an angle of 45 degrees.

\section{Questionnaires of the experimental session}

During the experimental session subjective data were mostly collected by means of Visual Analog Scales (VASs) on which subjects score between 0 ("not at all") and 100 ("extremely").

Two times a VAS containing the following three items was administered (see procedure): "At this moment I feel tense", "At this moment I feel peckish" and "At this moment I feel depressed". Further, each member of the presented slide-series (see procedure) was evaluated by two questions: "how agreeable do you find this slide?" and "How much food craving does this slide evoke?" The final questionnaire contained questions about subjects' food intake prior to the experimental session and the questions: "How satisfied are you with your body appearance?", "Are you currently dieting in order to lose weight?"

\section{Procedure}

Pre-experimental session Three weeks before the experimental session, six pictures were taken of the subjects for use in the experiment (see materials). After the photo-session a questionnaire was handed out in which subjects could rate a number of food items on their craving arousing properties. These ratings were eventually used for selection of the food slides (see materials).

Experimental session Each subject was asked to eat a standard meal at 3 hours prior to the experimental session and to refrain from eating thereafter. The subject was welcomed and informed about the course of the experiment. The first VAS was handed out. Each subject was seated in a comfortable chair, electrodes were attached, and a headphone was put on. She was asked to remain in her chair quietly so as to not disturb the psychophysiological measurements. In order to ensure that full-fledged cue reactivity would be registred a mood induction procedure was employed, as craving in restrained eaters has been repeatedly reported to be mood dependent (Greeno \& Wing, 1994; Cools, Schotte \& McNally, 1992; 


\section{Chapter 5}

Ruderman, 1985). First, it was announced that music would be played. The subject was asked to concentrate on sad thoughts as long as the music would play. The experimenter left the room, lights were dimmed and the music began playing for 5 minutes. After the music the experimenter reentered the room and administered the second VAS. After that the slide series was announced. It was emphasized that the subject should watch the slides carefully, because later some questions would be asked about them. Then the experimenter left the room, which was darkened now and the slide presentation began. Two slides were used as a introduction, and after that the six neutral, six food and six body slides were presented in random order. Each slide was shown for a period of twelve seconds. Inter slide intervals differed and were either ten, fifteen, twenty or twenty-five seconds. During half of the slides a startle eliciting noise was presented. To preclude subjects' expectations on when the startle noise would sound the noise was presented at variable times (either 1, 3 or 7 seconds) after slide onset for the different slides. Following the first slide presentation all slides were shown again, and each slide was rated. After the removal of the electrodes the final questionnaire was administered. The subject was weighted and paid for her participation. The six personal body slides were handed over to the subject.

\section{RESULTS}

\section{Statistical method}

Data were processed using SPSS-PC statistical software. In most instances, repeated measurement ANCOVAs were conducted, with Body Mass Index as a covariate, as the two subject groups differed on this variable (see table 5.1). Due to malfunctioning of the psychophysiological equipment and interference, part of the data of some subjects were excluded from further analysis.

\section{Manipulation check of the mood induction}

The mood induction proved to be successful in both groups. A 2 (before/after mood induction) $\times 2$ (restraint) ANCOVA showed a main effect for mood induction, indicating a significant increase in negative $\operatorname{mood}$ scores $(F(1,22)=36.2, p<.001)$. Furthermore the mood induction led to decreased craving ratings $(F(1,22)=7.85 ; p=.01)$. No main effect of mood induction was found on subjective tension level. None of the subjective ratings showed a main effect of group or a group $x$ mood induction interaction effect. 


\section{Subjective and psychophysiological responses to the slides}

Results of the statistical tests are presented according to the main hypotheses of the experiment.

Hypothesis 1: cue reactivity is larger for food than for neutral slides in all subjects, but to a larger extent in restrained subjects.

Data on SCR amplitude, Heartrate, startle reflex parameters, and craving ratings for the food slides were subjected to separate 2 (restraint) $\times 2$ (slide type) repeated measurement ANCOVAs with Body Mass Index as the covariate. The predicted restraint $x$ slidetype interaction was found only for corrugator EMG activity $(F(1,17)=5.95 ; p<.03)$. The pattern of cell-means (not shown here) revealed that the average EMG response to both slide types was a decrease in corrugator (frown) activity, which suggests a positive emotional response in both subject groups.

Main effects of slidetype were found for food craving $(F(1,22)=133.9 ; p<.001)$ and startle onset latency $(F(1,22)=8.68 ; p<.008)$. These effects reflected a more intense appetitive response to foodslides than to neutral slides. No main effects of restraint were found.

In sum, the results showed that, although food slides triggered more intense psychophysiological reactions and craving than did neutral slides, restrained and unrestrained eaters differed only in corrugator EMG activity, albeit to a modest extent.

Hypothesis 2: body slides elicit more negative emotional arousal in restrained than in unrestrained subjects.

Data on SCR recovery time, HR during the 2-5 seconds post-stimulus time window, Corrugator and Zygomatic EMG changes, Startle reflex onset and amplitude, and emotional ratings of the slides were subjected to separate 2 (restraint) $\times 2$ (slide type) ANCOVAs with repeated measures and Body Mass Index as a covariate.

No main or interaction effect on emotional rating was found, reflecting that the restrained subjects did not rate their body-pictures more negatively than did unrestrained subjects. None of the psychophysiological parameters showed the predicted restraint $\mathrm{x}$ slide type interaction effect. Also no main effect was found for restraint. A main effect for slide type was found only for Heartrate acceleration $(F(1,14)=8.98 ; p<.01)$ and for SCR recovery time $(F(1,18)=5.09 ; p<.04)$. These effects reflected more intense negative emotional arousal during presentation of the body slides. 


\section{Chapter 5}

Again, it can be said that none of the predicted differences between restrained and unrestrained eaters was found.

\section{Body satisfaction and current dieting}

Restrained and unrestrained eaters did not differ in body satisfaction $(\mathrm{t}(20)=.47$; ns). This was somewhat surprising because the restrained eating style is often assumed to originate from body dissatisfaction. Of the unrestrained subjects, nobody was currently dieting, whereas of the restraints $38 \%$ was.

\section{DISCUSSION}

In the present investigation it was found that restrained and unrestrained eaters did not differ in psychophysiological reactivity to slides of either their preferred binge food or their own body. Moreover, no group differences were found on subjective ratings of food slides (craving), or body slides (negative emotional valence). Nevertheless, a main effect of slide type on several psychophysiological parameters was found. On the average, responses to the body slides were the most intense, followed by the food and neutral slides respectively. The present failure to find a group difference in psychophysiological reactivity to food and body cues may result either from peculiarities of the present procedure, or from more fundamental problems with a pure conditioning explanation of cue reactivity.

The former explanation is based on the assumption that the classical conditioning interpretation of cue reactivity is valid, but that the present procedure should be adjusted to demonstrate differential cue reactivity. This possibility should be taken seriously in the light of the small existing empirical data base on psychophysiological food cue reactivity in humans. Five adjustments of the experimental procedure might be thought of. First, the present study involved non-clinical subjects. It might be that significantly increased cue reactivity is confined to clinical binge eaters, because of their occasional huge food intake. As to body cue reactivity, a analogous argument could be made, based on the different body esteem of e.g. bulimia nervosa patients and non-clinical subjects. Second, the data indicate that the (individually selected) food slides did have had some arousing effect on the subjects. However, it might be that because of their mere visual nature, food slides lack in truly disinhibitive potency. Accordingly, investigation of the effects of real food items providing additional olfactory/gustatory stimulation should deserve attention. Third, the subjective ratings show that the food slides elicited increased arousal and increased craving in both restrained and unrestrained subjects. This might be the consequence of a basic characteristic in which food cue reactivity study differ from studies into addictions. Not only had both 
subject groups some learning experience with the presented food cues, also their habitual intake may have been comparably large (indeed, the slides were individually selected on craving arousing value). This might have lead to equal food cue reactivity. Future research might better collect quantitative data on subjects' usual craving and intake of food associated with the cues presented during the lab procedure rather than relying on a personality questionnaire (i.e. the Restraint Scale) only. Fourth, the psychophysiological parameters monitored in the present study may not have been sufficiently "food-specific". Heightened cue-reactivity to disinhibiting food cues might be found only in physiological domains more tightly linked to food ingestion, e.g. blood sugar or insulin responses (Jansen, 1994). And fifth, there is a possibility that notable differences between restrained and unrestrained eaters in food cue reactivity begin to appear at more than 12 seconds after cue perception, i.e. beyond the time window in which we monitored our subjects' responses.

The second explanation for our failure to find differential cue reactivity in restrained and unrestrained eaters may be more fundamental in nature: the conditioning account of cue reactivity may not be adequate. As a matter of fact, Tiffany (1995) has proposed that the expression of cue-reactivity (in addicts) should be understood as a reflection of behavioral and cognitive demands of a situation rather than as a mere residual of learning history. Specifically, cue-elicited responses might be especially intense in cases where an encounter with the substance itself is clearly forthcoming. This issue may not have been sufficiently addressed in the present procedure: although the subjects were to watch the slides attentively, no explicit attempts were included to arouse subjects' expectations of encounters with the depicted food items, e.g. of ingesting or abstaining from ingestion in the presence of the food). It might be that differential food cue reactivity may be demonstrated only after procedures in which the psychosocial concomitants described by Tiffany are explicitely manipulated.

Research on cue reactivity in the field of binge eating has only recently started. At present, assessment of the contributions of situational vs. conditioning influences, as well as identification of the appropriate response parameters appear to be the most central issues in food cue reactivity research. 



\section{FOOD CUE REACTIVITY IN FASTING AND NON-FASTING SUBJECTS ${ }^{1}$}

\section{INTRODUCTION}

A recently proposed theory of binge eating holds that cues that reliably precede intake of binge food can acquire the ability to elicit a range of anticipatory physiological responses. These classically conditioned responses facilitate bodily processing of food and help in maintaining homeostasis (Jansen, 1990; 1994a; Woods, 1991). Additionally, the theory states that conditioned physiological responses to binge cues are subjectively experienced as craving and as such can trigger food intake. Research on the nature of conditioned anticipatory responses could be helpful in both controlling them, and in preventing relapse in treated patients (Childress, Ehrman, Rohsenow, Robbins \& O'Brien, 1992; Glautier, 1994; Jansen, Broekmate \& Heijmans, 1992). Until now, however, the exact nature of the conditioned physiological responses to binge cues remains unclear (Overduin, Jansen \& Eilkes, 1996). A major aim of the present study was the determination of psychophysiological and subjective responses to food cues (sight and smell) in normal, non-clinical subjects. Moreover, as dietary restriction is supposedly linked to the development of binge eating (e.g. Polivy \& Herman, 1985; Tuschl, 1990) and short-term deprivation was expected to increase reactivity to food cues, also responses of 24-hour fasting subjects were studied. Studies in the addiction field show that increases in salivation, skin conductance and subjectively reported craving to cue presentation are the most consistent findings, whereas heart rate acceleration was found less often (Childress et al., 1992; Glautier, 1994). In the present study it was hypothesized that presentation of cues signalling desired food will elicit more salivation and larger increases in heartrate and frequency of skin conductance fluctuations than presentation of control (i.e. soap) stimuli. Furthermore, food cue reactivity was hypothesized to be larger in fasting than in non-fasting subjects.

1 Accepted for publication as: Overduin, J. \& Jansen, A. (1996). Food cue reactiviy in fasting and non-fasting subjects. European Eating Disorders Review, in press. Heiny Eilkes is gratefully acknowledged for her assistance in carrying out the present experiment. 


\section{METHOD}

\section{Design}

Each of the fasting and non-fasting subjects was exposed to both food- and soap stimuli. The order of stimulus presentation was counterbalanced within each condition. Psychophysiological cue reactivity was determined by measuring salivation, spontaneous skin conductance fluctuations and heartrate, before (baseline) and during stimulus presentations.

\section{Subjects}

Twenty-one healthy normal weight students without a history of eating disorders ( 9 females and 12 males; mean age 21.6 years) participated in the experiment. The subjects were randomly allocated to the fasting condition $(n=11)$ and to the non-fasting condition $(\mathrm{n}=10)$. Mean BMI in the fasting group $(21.9$, sd 2.8$)$ did not differ from the normal group $(21.4$, sd $2.0 ; \mathrm{t}(19)=.55 ; \mathrm{ns})$. It was told to the subjects that the experiment involved an investigation of "attentional and sensory processes".

\section{Questionnaires and psychophysiological parameters}

Questionnaires At the beginning of the experiment hunger and craving ratings were collected by means of on Visual Analogue Scales (VASs) ranging from 0 ("not at all") to 100 ("very much"). After the end of presentation of each stimulus a VAS was administered on food craving and hedonic evaluation of the stimulus1.

All electronic signals, including the markers for stimulus presentation, were registered by Beckman couplers and a Beckman R711 polygraph with a multi-channel writer.

Skin Conductance was recorded by means of two $\mathrm{Ag}-\mathrm{AgCl}$ electrodes (diameter $8 \mathrm{~mm}$ ) attached to the middle fingers of the non-dominant hand. The number of non-specific fluctuations in skin conductance level (further to be called "SC-fluctuations") were counted to acquire an index of the frequency of sympathetic nervous "bursts" both before and during presentation of the stimuli. They were defined as SC-level increases exceeding 0.05 micromhos within 2 seconds (Boucsein, 1992) and taking place at least 6 seconds after breathing artefacts or stimulus onset. The number of fluctuations was counted for each minute.

Heartrate was recorded by means of two Blue Sensor disposable ECG electrodes (type R-00-S) attached on a diagonal axis over the heart region and a reference electrode on the shoulder. For each minute the mean heartrate was established.

Saliva was collected by means of three dental rolls, according to the method of LeGoff, 
Leichner \& Spigelman (1988). The subject inserted a first roll underneath the tongue, and two rolls laterally, into each side of the lower jaw between teeth and gums. Salivation collection time was exactly three minutes. The mean salivation per minute was computed for statistical analysis.

Breathing amplitude Because the number of SC-fluctuations is influenced by irregular breathing (sighing, coughing and so on; Boucsein, 1992), breathing amplitude was monitored using a Beckman respiratory belt connected to a Beckman Voltage/Pressure Coupler. The mean breathing amplitude during the 6 second interval preceding each $\mathrm{SC}$-fluctuation was established and they were averaged for the minute under consideration. In this way the mean breathing amplitude preceding SC-fluctuation was computed for each of the 14 one-minute intervals of the experiment. Then, for each minute, control periods of 6 seconds not followed by SC-fluctuations were inspected and mean breathing amplitudes were computed in the same way as above. Finally, the difference between pre SC-fluctuation breathing amplitudes and the control breathing amplitudes was assessed by t-tests.

\section{Stimuli}

The food stimuli consisted of two candy bars (Twix) stripped from their paper and one for each subject freshly prepared healthy brown roll, with salat, cheese, ham and tomato.

The control stimuli consisted of four small pieces of round, lightly flowerishly perfumed bathing soap. Two of the pieces were white-, and two were green-coloured. All stimuli were presented on a plate with a blinded removable cover over it. After lifting the cover, the items, resting on colored paper napkins, became visible.

\section{Procedure}

The fasting group was asked to refrain from eating for a period of 24 hours prior to the experimental session. To ensure that fasting really would take place, the subjects were instructed to collect their morning urine and bring it with them, so as to "control for fasting". In reality no further analyses were carried out on these urine-samples. The non-fasting group was instructed to have a normal meal at 3 hours prior to the experimental session and to refrain from eating until after the experiment. After entering the lab, each subject first completed the hunger and craving VAS. Then she was informed about the general procedure and how to insert the dental rolls. She was seated in a comfortable chair and instructed not to move exaggerately during the experiment, so as not to disturb the measurements. The explanation of the procedure was repeated while the electrodes were attached. During the first two minutes of the experiment, 


\section{Chapter 6}

psychophysiological baseline measurements were carried out with the subject sitting still in the chair and the experimenter sitting in front of her. Thereafter the subject was asked to insert the dental rolls. Within 6 seconds after the insertion of the dental rolls, the first stimulus was presented in front of the subject and the cover of the dish was lifted by the experimenter. The instruction was given to attend (look and smell) to the food intensely so that the subject would be able to correctly complete the VASs later on. The dish was arranged so that the subject's nose was close to it, so that no bending down was required to smell and watch. Three minutes after the onset of stimulus presentation, the subject was told to remove the dental rolls from his mouth, to put them in the container and to close it. Stimulus presentation and physiological measurement continued for another two minutes after which the hedonic evaluation and craving VAS was administred with the stimulus still within sight. Then there was a pause of eight minutes in which they were distracted by the projection of six neutral slides with office equipment. The subject was told to watch them attentively and to memorize them as good as possible so as to be able to answer questions about them later on. After this pause the second stimulus was presented. The procedure was identical to the first stimulus presentation. Finally, the subject was weighed, measured, debriefed, paid for participation and offered the sandwich presented during the experiment.

\section{RESULTS}

\section{Data processing}

Because fasting has been found to lead to decreased tonic sympathetic nervous activity (Landsberg \& Young, 1978; Webber \& MacDonald, 1993), phasic (cue-elicited) physiological activity was corrected for tonic (baseline) levels: for heartrate and skin conductance the mean levels of seven (i.e. two pre-stimulus and five post-stimulus) minutes were computed. Then, tonic level was defined as the average of the two pre-stimulus minute levels. Phasic reactivity score was defined as the difference between the average of the 5 post-stimulus minutes levels and tonic level. The tonic and phasic reactivity scores for heartrate and skin conductance, salivation rate and the VAS-ratings of the stimuli were subjected to a 2 (condition; fasting vs non-fasting) $\times 2$ (stimulus-type; food vs soap) MANOVA with stimulus-type as a within-subjects factor.

Unfortunately, due to malfunctioning of the equipment, for some people skin conductance and heartrate data were lost. 
Table 6.1. Subjective state prior to presentation of stimuli (scoring range: $0-100$ )

\begin{tabular}{|c|c|c|c|c|c|c|}
\hline & \multicolumn{2}{|c|}{$\begin{array}{c}\text { non-fasting subjects } \\
\qquad(\mathrm{N}=10)\end{array}$} & \multicolumn{2}{|c|}{$\begin{array}{l}\text { fasting subjects } \\
\qquad(\mathrm{N}=11)\end{array}$} & \multicolumn{2}{|c|}{ t-test } \\
\hline & mean & sd & mean & sd & $t(23)$ & pule \\
\hline hunger-VAS & 50.4 & 20.0 & 84.2 & 10.5 & -4.9 & $<\infty \mathrm{ll}$ \\
\hline food craving-VAS & 47.6 & 19.6 & 83.6 & 16.9 & -4.6 & $<.001$ \\
\hline
\end{tabular}

\section{Manipulation checks}

t-Tests demonstrated that at the beginning of the experiment, the fasting subjects experienced significantly more hunger $(\mathrm{t}(19)=4.92 ; \mathrm{p}<.001)$ and craving for food $(\mathrm{t}(19)$ $=6.46 ; \mathrm{p}<.001$ ) than non-deprived subjects (see table 6.1). Also, the food stimuli were not only considered significantly tastier in appearance $[F(1,18)=148.2 ; p<.001]$ and more agreeable in smell $[\mathrm{F}(1,18)=6.58 ; \mathrm{p}<.02]$, but also leading to larger food craving $[F(1,18)=39.9 ; \mathrm{p}<.001]$ than the soap stimuli (table 6.2).

Thus, both manipulations succeeded: fasting subjects experienced more hunger and craving than non-fasting subjects and the food stimuli turned out to be considerably appetitive.

Table 6.2. Subjective ratings during food and soap stimulus presentation (scoring range: 0-100)

\begin{tabular}{|c|c|c|c|c|c|c|c|c|}
\hline & \multicolumn{3}{|c|}{ Non-fasting subjects } & \multicolumn{3}{|c|}{ Fasting subjects } & \multicolumn{2}{|c|}{ t-test } \\
\hline & mean & sd & $\mathrm{N}$ & mean & sd & $\mathrm{N}$ & i & p \\
\hline \multicolumn{9}{|l|}{ Hedonic rating of stimulus } \\
\hline food & 68.6 & 19.3 & 10 & 88.6 & 15.0 & 11 & 2.66 & $<.02$ \\
\hline soap & 15.0 & 19.8 & 10 & 19.3 & 30.2 & 11 & .38 & ns \\
\hline \multicolumn{9}{|l|}{ Craving for food } \\
\hline food & 62.7 & 17.8 & 10 & 91.4 & 9.8 & 10 & 4.47 & $<.001$ \\
\hline soap & 24.4 & 18.2 & 10 & 56.3 & 30.2 & 11 & 2.89 & $<.01$ \\
\hline
\end{tabular}

\section{Responses to stimulus presentation}

The minute-to-minute courses of heartrate and skin conductance fluctuations are shown in figures 6.1 and 6.2. Data on the tonic (baseline) levels showed that fasting subjects displayed a smaller number of SC-fluctuations $[F(1,17)=4.75 ; p<.05]$ and a trend towards lower heartrate $[F(1,18)=4.34 ; p<.06]$ than non-fasting subjects.

$t$-Tests on the difference between the mean breathing amplitude before SC-fluctuations and that of control periods (see method section) failed to reach significance. Thus it can be concluded that the SC-fluctuations did not result from breathing irregularities during stim- 
Table 6.3. Psychophysiological responses to food and soap stimulus presentation

\begin{tabular}{|c|c|c|c|c|c|c|c|c|}
\hline & \multicolumn{3}{|c|}{ Non-fasting subjects } & \multicolumn{3}{|c|}{ Fasting subjects } & \multicolumn{2}{|c|}{ t-test } \\
\hline & mean & sd & $\mathbf{N}$ & mean & sd & $\mathbf{N}$ & t & $p$ \\
\hline \multicolumn{9}{|l|}{ Salivation (mg/min) } \\
\hline food & 575 & 359 & 10 & 609 & 280 & 11 & .24 & ns \\
\hline soap & 651 & 397 & 10 & 536 & 272 & 10 & .76 & ns \\
\hline \multicolumn{9}{|l|}{$\Delta$ Heartrate (BPM) } \\
\hline food & 3.6 & 4.1 & 10 & 6.8 & 4.9 & 10 & 1.56 & $\mathbf{n s}$ \\
\hline soap & 4.0 & 3.3 & 10 & 4.4 & 6.4 & 9 & .17 & ns \\
\hline \multicolumn{9}{|c|}{$\Delta S C$-fluctuations/minute } \\
\hline food & 1.8 & 1.5 & 9 & 0.5 & 1.1 & 11 & 2.31 & $<.04$ \\
\hline soap & 0.6 & 2.2 & 10 & 0.0 & 1.2 & 10 & .81 & ns \\
\hline
\end{tabular}

ulus presentation.

On phasic reactivity scores (see table 6.3), a main effect of condition was found only for frequency of SC-fluctuations $[F(1,17)=4.44 ; p<.05]$, based on fasting subjects showing smaller increases in SC fluctuation frequency in response to both stimuli. However, the hypothesized condition $\mathrm{x}$ stimulus-type interaction did not reach significance. This implies that fasting subjects did not show especially strong reactivity to food cues. Finally, neither main effects of stimulus-type, nor stimulus-type $\mathrm{x}$ condition interaction effects were found for the other physiological parameters.

\section{DISCUSSION}

In the present study, food cue reactivity was examined in fasting and non-fasting subjects. Firstly, it was found that fasting subjects showed a lower baseline frequency of skin conductance fluctuations and a trend towards lower baseline heartrate. Secondly, although subjects gave higher appetitive ratings to food than to soap stimuli, this was not reflected in stronger psychophysiological reactivity (i.e. salivation, SC-fluctuations, heartrate). Finally, fasting subjects reported more craving for the food stimuli than did non-fasting subjects, but they did not show the hypothesized enlarged food cue reactivity. In fact, their SCfluctuations increase to food cue appeared to be even smaller than non-fasting subjects. The smaller baseline frequency of spontaneous skin conductance fluctuations and trend towards lower baseline heartrate in fasting subjects fits with earlier documented findings on lowered sympathetic nervous activity in fasting subjects (Schultz-Gambard, 1988; Landsberg 
\& Young, 1978; Webber \& MacDonald, 1993). Probably, this should be seen as a general bodily adaptation to deprivation that is aimed at sparing energy (Landsberg \& Young, 1978), rather than as a reflection of readiness for food intake.

The finding of no larger food cue reactivity to food than to soap, as well as the approximately equal cue reactivity in fasting and non-fasting subjects is surprising. Even if one takes into consideration that the exact nature of anticipatory responses to food stimuli (for example, the critical parameters and their directionality) is as yet largely unknown, one is struck by the finding that fasting subjects did not show stronger salivation. Salivation, a response obviously related to food ingestion, has been claimed to be an objective index of hunger (e.g. Le Goff et al., 1988). Indeed, besides being hungry, our fasting subjects craved and highly appreciated the food presented to them. But why, then, didn't they salivate more?

A first possibility might lie in the procedure of the present study. It might be that not all the subjects have complied with the fasting instruction, thereby obscuring differences in cue reactivity with the non-fasting subjects. Indeed, the present study did not involve objective checks on deprivation level (for example, increased levels of circulating ketone bodies; Hoffer, 1988). Nevertheless, relevant information pertaining to this issue can be drawn from the present psychophysiological data. If true cue reactivity after fasting were larger in fasting than in non-fasting subjects, and not all subject in the fasting condition were complying with their instructions, enlarged variability in responses would be expected among subjects in the fasting condition. However, the standard deviations in table 6.3 do not consistently confirm this prediction. Thus, although we have no waterproof evidence that all subjects in the fasting condition did stick to their regimen, no convincing indication could be found of heightened cue reactivity in a subset of our fasting subjects.

Secondly, it is possible that increased cue reactivity occurs only after fasting durations exceeding 24 hours. Data from the literature on appetitive parameters related to cue reactivity may be relevant in this respect. For humans, the plateau of perceived hunger intensity appears to occur after two days of fasting (Le Magnen, 1985). However, the additional impact of presenting food cues has, to our knowledge, not been investigated. For rats, the literature is inconclusive as to whether the size of the first meal after fasting is strongly correlated with fasting duration if fasting continues for more than 24 hours (Le Magnen, 1985). All in all, the possibility that increased cue reactivity could indeed be found after fasting periods longer than 24 hours deserves future attention.

A third explanation for the lack of differences in cue reactivity may be related to our subjects' learning history, which, unlike hunger level, was not manipulated in the present study. Jansen $(1990,1994)$ has argued that food cue reactivity will be particularly strong in cases of intermittent dieting and overeating, a common pattem among binge eaters. 


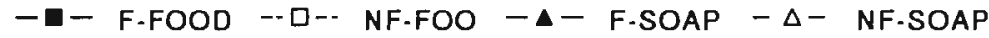

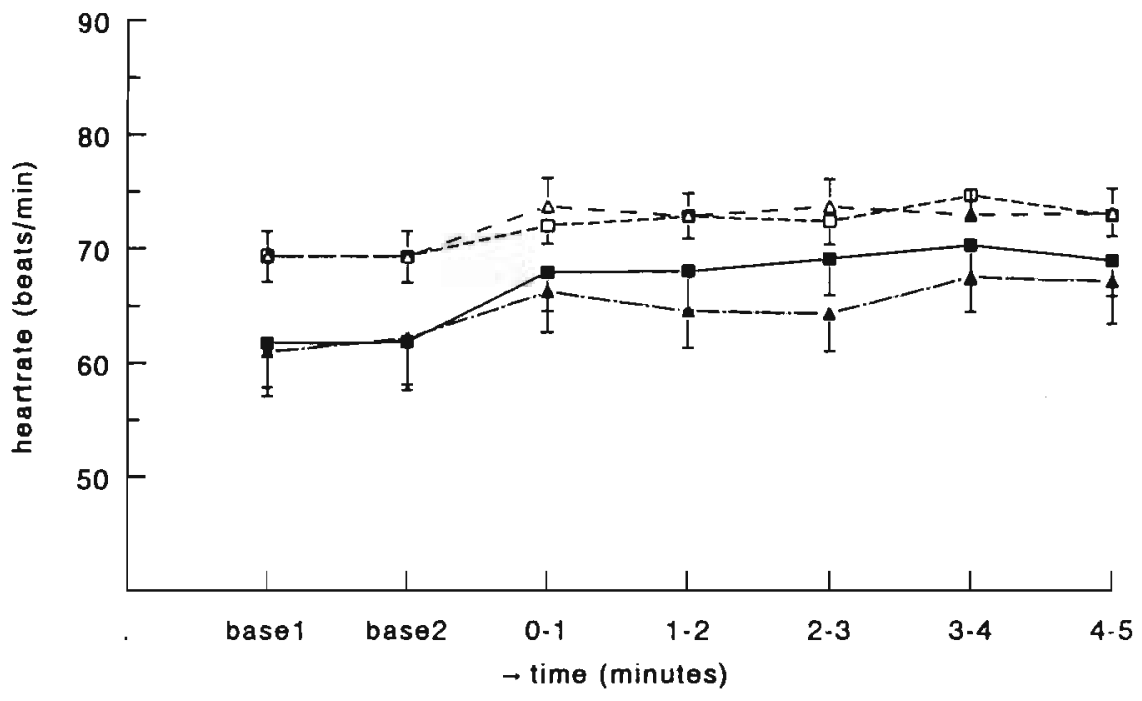

Figure 6.1. Minute-to-minute heartrates (mean \pm s.e.m.) before and during stimulus presentations.

-ם- F-FOOD --D-- NF-FOO - A- F-SOAP - $\triangle-$ NF-SOAP

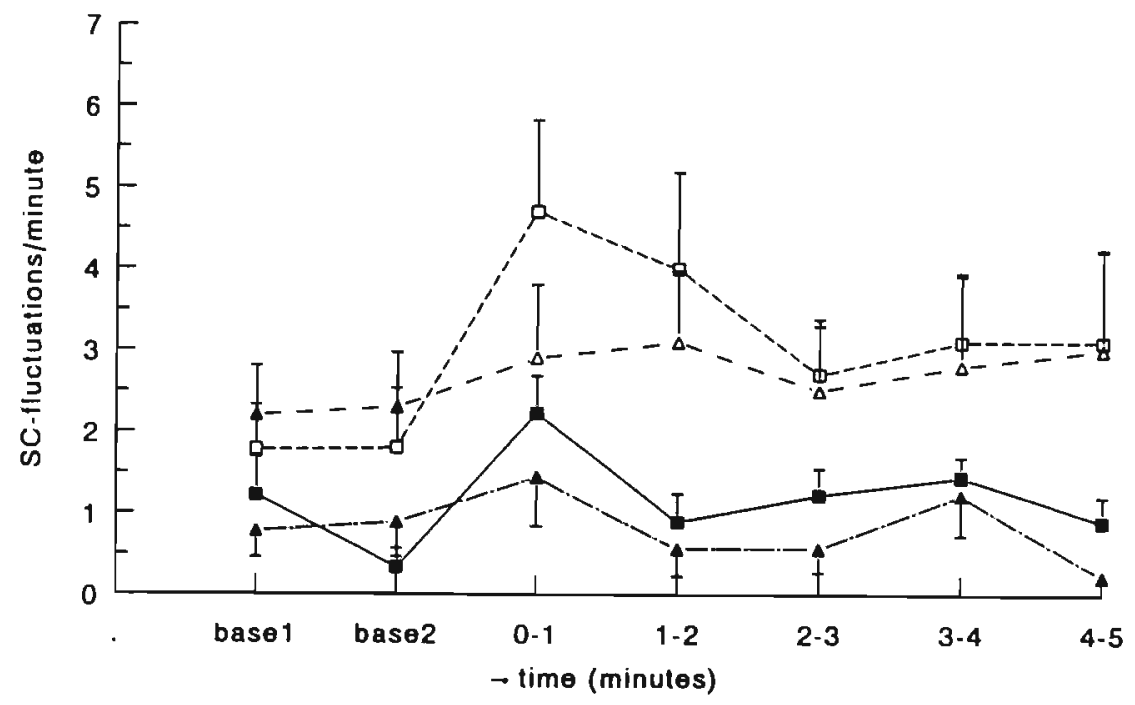

Figure 6.2. Minute-to-minute frequency of skin conductance fuctuations (mean \pm s.e.m.) before and during stimulus presentations. 
Indeed, animal studies show that a food intake scheme of one or two meals a day elicit significantly larger anticipatory responses to food cues than an intake scheme of six meals a day (Woods et al., 1977; Strubbe, 1992).

An experimental manipulation of learning history then, would require a conditioning procedure in which a set of food cues (CS) reliably predicts subsequent large intake (US). Also, it might critically be investigated whether deprivation level in its own right facilitates such learning, i.e., whether deprivation acts as an 'occasion setter' of the conditioning process (Davidson, 1993; Catania, 1991). Jansen, Boon, Nauta \& van den Hout (1992) have shown that such a Pavlovian conditioning procedure elicits increased salivation to the food cues in undeprived subjects. Altematively, it might be worthwhile to investigate whether cue reactivity intensity decreases if subjects are exposed repeatedly to food cues while being prevented actual intake. Prolonged exposure to binge cues with response prevention has been shown to be successful in decreasing craving and binge frequency in binge eaters (Jansen, Heymans \& Broekmate, 1992), but psychophysiological parameters were not monitored in these studies.

In sum, visual and olfactory cues related to food and non-food items have again been demonstrated to trigger various changes in subjective and psychophysiological parameters, but the logic underlying the intensity of these changes needs further exploration. We argue that future cue reactivity research may benefit from focusing more on the role of conditioning. It might be hypothesized that the intensity of cue reactivity covaries with the magnitude of the learned, hence expected, subsequent food ingestion. 



\section{Chapter 7 \\ STROOP INTERFERENCE AND FOOD INTAKE ${ }^{1}$}

\section{INTRODUCTION}

Preoccupation with food and eating, as well as the tendency to be overly concerned with one's body weight and shape, are key symptoms of the eating disorders bulimia nervosa and anorexia nervosa (APA, 1987; Walsh, 1992). Concerns about body shape and weight are considered pivotal to the maintenance of chaotic eating behaviour such as dieting, fasting and splurging (Fairburn, Cooper, Cooper, McKenna \& Anastasiades, 1991; Garfinkel, 1992).

In order to assess information processing in eating-disordered subjects some authors recently used the Stroop colour-naming task. In the Stroop task words printed in various colours are presented to the subject who then is required to name the colour as quickly as possible, while ignoring the meaning of the words. In studies on eating disorders reaction times to neutral words and words about food or body shape (so called target words) are compared. A relatively longer reaction time for target words indicates that the subject is distracted by the meaning of the target words, and this is supposed to be indicative of distorted information processing.

Until now several studies have shown Stroop interference for target words in eating disordered subjects. Significant interference for a combination of eating and shaperelated words were found in normal weight bulimic subjects (Fairburn et al., 1991; Cooper, Anastasiades \& Fairburn, 1992; Cooper \& Fairburn, 1993). Other studies report interference effects with separately presented eating-related words in anorexic and/or bulimic patients (Channon, Hemsley \& de Silva, 1988; Ben-Tovim, Walker, Fok \& Yap, 1989; Ben-Tovim \& Walker, 1991). Most of the studies undertaken document an interference effect for eating-related words in eating disordered subjects.

How can these data be explained? Ben-Tovim et al. (1989) hypothesized that the attentional bias for eating-related words reflects anxiety evoked by the meaning of these words and can thus be regarded as a measure of psychopathology. However, in the light of the finding that fasting subjects are hyperattentive to non-aversive appetitive stimuli (Channon \& Hayward, 1990; Lavy \& van den Hout, 1993), Stroop interference does not necessarily seem to indicate anxiety. In line with this, Channon and Hayward (1990) explained the observed attentional bias for food cues of fasting and eating-disordered subjects in terms of restriction. They state that the Stroop task provides "an objective

1 Published as: Overduin, J., Jansen, A. \& Louwerse, E. (1995). Stroop interference and food intake. International Journal of Eating Disorders 18, 277-285. 
index of current food restriction" (p. 451). But this conclusion might be a bit premature. For example, Green and Rogers (1993) found slower colournaming of foodwords in restrained eaters, irrespectively of whether the subjects were currently dieting or not. Therefore, although food restriction may result in hyperattention to food cues, hyperattention to food cues presumably isn't exclusively related to food restriction.

Another explanation of hyperattention was offered by Lavy and van den Hout (1993). According to them hyperattention might be related to an increased urge to act that may be reflected either in the avoidance of threatening, or in the approach of appetitive cues. From this one could derive the hypothesis that hyperattention to food words may not only be triggered by deprivation but, more generally, by stimuli that are desired strongly.

In the present study we wanted to investigate whether it is possible to instantaneously induce an attentional bias in subjects by providing them with an appetizer, i.e. a small amount of food that tastes "moreishly". Secondly, conceming the urge-to-act hypothesis, we wanted to assess the relation between Stroop interference and subsequent ad libitum food intake.

Results found on Stroop interference for separately presented body shape-related words appear to be more equivocal. Channon et al. (1988) failed to find an enlarged interference effect for shape-related words in anorexic subjects. Ben-Tovim et al. (1989) reported a trend for Stroop interference on shape-related words in anorexic and bulimic subjects whereas Ben-Tovim and Walker (1991) demonstrated a significant interference effect in bulimic as well as anorexic subjects. Mahamedi and Heatherton (1993) found that eating a preload lead to an increase in attentional bias for body shape words, especially in restrained eaters. However, after a comparison of the post-preload attentional bias in restrained and unrestrained eaters one cannot conclude that bias was reliably stronger in restrained eaters. Results from a within subjects study by Ogden and Greville (1993) in which attentional biases before and after a preload were assessed point in a similar direction.

The subjects in our experiment were non-clinical restrained and unrestrained eaters. If the emotional concerns explanation of Stroop interference for body shape words were true, one would expect larger reaction times for restrained eaters whether they would have eaten an appetizer or not. The urge-to-act explanation of attentional bias, on the other hand, is based on approach and withdrawal behavior and does not lead to strong expectations about Stroop reaction times for body shape words. 


\section{METHOD}

\section{Subjects}

Initially, around 100 female university students were approached to participate in a 'study on life-style' by filling out a 'life-style questionnaire'. Four key eating behaviour items were included in the list (e.g. "I repeatedly find it necessary to engage in dieting" and "My eating behaviour is normal when I'm in the presence of others but uncontrolled when I'm alone"). After that, 51 of the most extreme scorers (high and low) on these items were invited to participate in the experiment. At the end of the experiment the subjects completed the Restraint Scale (Herman, Polivy, Pliner, Threlkeld \& Munic, 1978) in order to prevent priming concerns about weight, shape and food intake. Every subject was termed high or low restrained on the basis of the median RS-score (being a score of 10 ) of the sample. Characteristics of the two groups are shown in Table 7.1.

Table 7.1. Characteristics of Restrained and Unrestrained subjects in this study

\begin{tabular}{lccccccc}
\hline & \multicolumn{2}{c}{ Unrestrained } & \multicolumn{2}{c}{ Restrained } \\
& \multicolumn{2}{c}{$(\mathrm{N}=25)$} & \multicolumn{2}{c}{$(\mathrm{N}=26)$} & \multicolumn{2}{c}{ t-test } \\
mean & sd & mean & sd & t & p-value \\
Body Mass Index & 20.7 & 1.3 & 22.3 & 2.7 & 2.64 & .01 \\
Age & 23.3 & 2.4 & 22.6 & 1.9 & 1.16 & ns \\
Restraint Scale & 6.8 & 2.5 & 15.0 & 3.5 & 9.66 & $<.001$ \\
\hline
\end{tabular}

\section{Materials}

Food The appetizer consisted of 60 grams of bavaroise pudding. Three flavours of ice cream (vanilla, strawberry, and chocolate) were used for the ad lib taste-test. The ice was arranged on a plate in such a way that eating would leave only a marginal visual trace.

Stroop program The Stroop task was run on a personal computer. In the middle of the screen words were shown one by one in 8 -mm block letters against a black background. The subject's voice was detected by a microphone connected with a sounddetector which registered response latencies in milliseconds. As soon as a sound was detected, the word disappeared from the screen. The time interval between presentation of consecutive words was 2 seconds. In case of wrong answers or disturbing sounds a 'missing value' was recorded.

Stroop words For every subject a total of 120 words was presented. The words were picked from four arrays (Table 7.2) in such a way that at the end of the task each of the 40 words had been shown three times. The colour of every word (either red, blue, green or yellow) was randomly chosen by the computer program. 


\section{Chapter 7}

There were two categories of 'neutral' words. The office equipment words had been added to establish a baseline with which reaction times for the target words were compared. The second category involved life-style words, so as to strengthen the impression that the experiment indeed was a study on life-style and perception. Response latencies for these words were not used in the data analysis.

Table 7.2. Words used in the modified Stroop task

\begin{tabular}{llll}
\hline \multicolumn{2}{c}{ TARGET WORDS } & \multicolumn{2}{c}{ NEUTRAL WORDS } \\
eating & shape & office & life-style \\
bun & balance & desk & ambition \\
cake & belly & envelope & appointment \\
candy & bikinis & felt-pen & bonus \\
chips & cheeks & file & career \\
chocolate & fat & paper & dedication \\
ice cream & hips & pen & manager \\
liquorice & legs & pencil & promotion \\
pie & slim & ruler & study \\
pudding & thighs & scissors & success \\
tart & thin & tape & working \\
\hline
\end{tabular}

\section{Procedure}

Subjects were told that we were studying influences of life-style on perceptual processes such as taste, smell, and visual perception. They were not informed that the test would involve highly palatable food.

The subjects were asked to have a standard meal of two sandwiches 3 hours before the experiment, and to refrain from eating in the period thereafter. They were randomly assigned to either the appetizer or the no-appetizer condition and were tested individually. After entering the laboratory, each subject received information about how to carry out the Stroop task. First, a short Stroop task with neutral words (the number one to ten) was run for practice. Then, in the appetizer condition, the pudding was presented and the subject was asked to eat it while concentrating on its colour, fragrance, and taste. Subjects in the control, no-appetizer-condition continued immediately with the Stroop task.

After the task three pre-weighed plates of ice cream were presented together with an icecream taste-evaluation questionnaire. The experimenter announced that he would leave the room for 15 minutes, during which the subject should complete the questionnaire. It was pointed out that tasting the ice cream would be helpful in completing the question- 
naire and that the subject was allowed to eat as much as she wanted or thought necessary. After the taste test the experimenter returned to administer the Restraint Scale. The ice cream that was left was taken to another room and re-weighed by the second experimenter. Finally, the subject was debriefed and paid for participation.

\section{RESULTS}

The Stroop interference scores for every target (i.e. eating and shape) category was defined as the median of response latencies for that category minus the median of response latencies for the neutral category. Reaction times above 1500 and below 250 milliseconds were excluded from the analysis. Standard deviations are shown between parentheses.

\section{Manipulation check}

Subjects scored the palatability of the bavaroise pudding (i.e. the appetizer, on a Visual Analogue Scale, ranging from 0 "not good at all" to 100 "extremely tasty". The overall mean score was 57.9 (s.d. 24.8) meaning that, although the pudding was not rated as extremely tasteful, at least it was considered reasonably palatable. No difference between restrained and unrestrained subjects in this respect was found $[\mathrm{t}(22)=1.08, \mathrm{NS})]$.

Table 7.3. Interference times for food words (milliseconds)

\begin{tabular}{|c|c|c|c|c|c|c|}
\hline & \multicolumn{3}{|c|}{ Unrestrained Eaters } & \multicolumn{3}{|c|}{ Restrained Eaters } \\
\hline & mean & sd & $\mathrm{N}$ & mean & sd & $\mathbf{N}$ \\
\hline No-appetizer & -6.0 & 30.3 & 13 & 17.1 & 29.4 & 14 \\
\hline Appetizer & 25.8 & 34.6 & 12 & 20.9 & 30.1 & 12 \\
\hline
\end{tabular}

\section{Food Words}

Table 7.3 shows the Stroop interference times for food words. A $2 \times 2$ ANOVA revealed a main effect of condition $(F(1,49)=4.18 ; p<.05)$, but no group effect. The condition by group interaction showed a weak trend $F(1,49)=2.57 ; p=.12$ ). It appeared that in the no-appetizer condition restrained subjects showed a significantly greater Stroop interference than unrestrained subjects $[\mathrm{t}(25)=2.01, \mathrm{p}<0.03]$.

Table 7.4. Ad lib intake of ice-cream (grams) 


\begin{tabular}{lcccccc}
\hline & \multicolumn{3}{c}{ Unrestrained Eaters } & \multicolumn{3}{c}{ Restrained Eaters } \\
& mean & sd & $\mathrm{N}$ & mean & sd & $\mathrm{N}$ \\
No-appetizer & 95.1 & 54 & 13 & 136.1 & 83 & 14 \\
Appetizer & 121.5 & 90 & 12 & 74.3 & 61 & 12 \\
\hline
\end{tabular}

It can be further seen that among unrestrained subjects, the ones in the appetizer condition showed significantly greater Stroop interference than those in the no-appetizer condition $(t(23)=2.44 ; p=.01$, one-tailed). In restrained subjects, no effect of condition was found $t(24)=.33$; NS).

\section{Food intake}

The food intake data are presented in Table 7.4. A $2 \times 2$ ANOVA showed no main effects for group or condition. However, a significant group by condition interaction was found $(F(1,49)=4.58 ; p<.04)$. Oddly enough, in the appetizer condition only the restrained subjects appeared to have lowered (i.e. regulated) their food intake relative to subjects in the no- appetizer condition $(\mathrm{t}(24)=2.19 ; \mathrm{p}=.04)$; among unrestrained subjects no effect of condition was found $(\mathrm{t}(23)=.88$; NS). For all subjects taken together, there was no strong $\mathrm{P}-\mathrm{M}$ correlation $(\mathrm{r}=0.27 ; \mathrm{n}=51$; NS) between the Stroop interference score for food words and ad libitum food intake. Interestingly, when correlation coefficients were calculated for the restrained and unrestrained subjects separately, the unrestrained group showed a firm correlation of 0.58 ( $\mathrm{n}=25$; $\mathrm{p}<$ $0.01)$, whereas in the restrained group no correlation was found $(r=-0.06 ; n=26$; NS).

Table 7.5. Interference times for shape-related words (milliseconds)

\begin{tabular}{lllllll}
\hline & \multicolumn{3}{c}{ Unrestrained Eaters } & \multicolumn{3}{c}{ Restrained Eaters } \\
& mean & sd & N & mean & sd & N \\
No-appetizer & 12.3 & 32.8 & 13 & 25.9 & 45.6 & 14 \\
Appetizer & 23.9 & 39.3 & 12 & 28.8 & 28.2 & 12 \\
\hline
\end{tabular}

\section{Body shape-related words}

In Table 7.5, Stroop interference times for shape-related words are presented. A $2 \times 2$ ANOVA did not show any main effects for condition and group or a condition $x$ group interaction. Clearly, restrained and unrestrained eaters did not differ in their level of 
Stroop interference for shape-related words. Moreover, for both unrestrained and restraint subjects eating the appetizer did not influence Stroop response latencies for shape-related words.

\section{DISCUSSION}

The main finding of the present study is that eating a small amount of palatable food triggers an attentional bias to eating-related stimuli in normal unrestrained subjects. This attentional bias strongly correlated with the amount of subsequent ad libitum food intake. In contrast to the unrestrained subjects, the restrained eaters were shown to be continually hyperattentive to eating-related words. Their subsequent food intake however appeared to be independent of hyperattention. Finally, restrained eaters did not show a larger attentional bias for body shape-related stimuli than non-restrained eaters.

What do these findings mean? In the first place, our data contradict the idea that an attentional bias to food related stimuli reflects a specific psychopathology of clinical eating disorders (Ben-Tovim \& Walker, 1991; Cooper \& Fairburn, 1993). Our non-clinical restrained subjects showed a permanent attentional bias to eating-related stimuli.

Secondly, the attentional bias in unrestrained subjects after ingestion of the appetizer suggests that Stroop interference per se does not reflect fear, emotional concerns, or deprivation. The firm correlation between Stroop latencies and food intake in unrestrained subjects suggests a selective attention to cues related to desires, cravings or urges. Interestingly, no correlation was found between Stroop food interference and food intake in restrained eaters. This could be because restrained subjects, by definition, normally tend to suppress their food intake. One could expect that Stroop interference scores and food intake will correlate strongly when restrained eaters show disinhibition. Unfortunately, in the present study restrained eaters in the appetizer condition seem to have regulated their intake because they ate less than those in the non appetizer condition. This lack of disinhibition might be explained by the size of our pudding, which was considerably smaller than the preloads in traditional counterregulation experiments (Jansen, Merckelbach, Oosterlaan, Tuiten \& van den Hout, 1988; Herman \& Mack, 1975; Hibscher \& Herman, 1977; Herman \& Polivy, 1980). It would thus be highly interesting to examine the relationship between Stroop interference and subsequent food intake in restrained eaters who indeed show disinhibited eating after a large preload.

A third interesting finding is that compared with unrestrained subjects, restrained subjects didn't show an especially large attentional bias toward body shape words, neither in the no-appetizer nor in the appetizer condition. These data seem to be in contrast with reports 


\section{Chapter 7}

of Stroop interference in bulimics (Fairburn et al., 1991; Cooper et al., 1992; Cooper \& Fairbum, 1993). In these studies however, it was not tested to which extend either shapeor the food-related target stimuli contributed to the effect. Also our findings are not entirely in accordance with findings of Ogden and Greville (1993) and Mahamedi and Heatherton (1993). The reason for this could have to do with the difference in methods. Mahamedi and Heatherton (like Fairburn et al.) used a card Stroop in which target words from the same category are presented together. In the Ogden and Greville study a computerized Stroop task was used, but again the target words were presented in blocks. Both methods introduce the danger that the subjects will ruminate about the meaning of the words during the task, and this could lead to longer reaction times, especially in restrained subjects who have just eaten a high-caloric milkshake. In the present study, words from target and neutral categories were presented one by one in a mixed fashion, which presumably diminishes the rumination effect. Undoubtedly, restrained eaters and eating-disordered subjects are overly concerned about their body shape. If interference as found by a computer-run Stroop task indeed reflects selective hyperattention to stimuli of personal or emotional valence, one would expect that restrained subjects show a greater Stroop interference for body shape-related words than unrestrained subjects do. However, the data do not support this hypothesis. If, however, the attentional bias reflects immediate avoidance or approach tendencies, one would not strongly expect an attentional bias for shape-related words: it is rather difficult to avoid or approach shape or body. In conclusion, it could be quite possible that card- or blocked versions of the Stroop measure rather emotional rumination on body words than attentional bias.

Finally, some remarks can be made about the value of the Stroop task in the assessment of treatment outcome for eating disordered patients. In spider phobics, Lavy, van den Hout and Amtz (1993) found a marginal correlation between pretreatment attentional bias and treatment (exposure) success. It might be hypothesized that a sustained post-treatment attentional bias for eating-related words could be a predictor of relapse in bulimic subjects. If that prediction is found to be true, the Stroop task may serve as an instrument for the assessment and predict of (long-term) treatment success. Recently, it has been shown that craving extinguishes during prolonged exposure with response prevention to cues that have become associated with the desired substance (Jansen, Broekmate \& Heymans, 1992; Powell, Gray \& Bradley, 1993). It is well-known too that craving can be triggered by exposure to appetitive cues (Weingarten \& Elston, 1990; Jansen et al., 1992) and therefore could constitute a causal factor in relapse. It could very well be that the conceptualization of craving as an approach behaviour leads to a promising application of the computerized Stroop technique in the study of eating disorders. 


\section{A NEW SCALE FOR USE IN NON-CLINICAL RESEARCH INTO DISINHIBITIVE EATING ${ }^{1}$}

\section{INTRODUCTION}

The food intake pattern of all bulimic and about half of the anorexic patients includes recurrent binge eating: during binges, control of food intake is lost and quantities of food are ingested that are larger than most people would eat under similar circumstances (Walsh, 1993). Lab paradigms of disinhibitive eating behaviour have been developed and studied in non-clinical subjects in order to unravel the underlying mechanisms (For the rationale behind this non-clinical approach, see Abramson \& Seligman, 1977). Questionnaire scores often provide the criterion of subject selection. In lab research into disinhibitive eating, the most widely used questionnaire has been the Restraint Scale (RS; Herman \& Polivy, 1980). This questionnaire has been shown to identify subjects prone to a form of disinhibitive eating called counterregulation. In the basic counterregulation paradigm subjects are "preloaded" by having them ingest a high-caloric food item, after which the food intake is assessed in an ad lib ice cream taste test. Subjects with high RS scores have been found to counterregulate, i.e. they show larger food intake when preloaded than when unpreloaded, whereas low scorers regulate their food intake and eat less when they have been preloaded previously (Herman \& Polivy, 1980). The replicable nature of counterregulation allowed for more extensive research on disinhibition through various modifications of the basic paradigm (for overviews see Lowe, 1993; Greeno \& Wing, 1994).

Although of all available questionnaires in the field, the Restraint Scale has been demonstrated the most effective predictor of counterregulation (Heatherton et al., 1988; Lowe, 1993), its use in disinhibition research has stirred up at least four critical issues.

The first issue relates to criticisms about some psychometric characteristics of the RS. Although the internal consistency of the scale is satisfactory, doubts have been raised about the stability of the internal structure of the scale. Different studies have shown factors to be composed of different items or items to be loading significantly on two factors at the same time. A most prominent example of the factorial instability is the collapse of the factor structure within a obese subject sample (e.g. Ruderman, 1983; for

${ }^{1}$ To be publisbed as: Overduin, J. \& Jansen, A. (1996). A new scale for use in non-clinical research into disinhibitive eating. Personality and Individual Differences, in press. Ellen Louwerse, Susan Bōgels and Peter de Jong are gratefully acknowledged for their aid in administering the questionnaires. Harrie Vorst (Methodology Department, University of Amsterdam) is thanked for commenting on some of our data. 
a reference of these criticisms and a reply see Heatherton et al., 1988). The instability of the factor structure may very well be a consequence of the wide array of themes tapped by relatively small numbers of items (i.e. weight fluctuations, diet frequency, negative evaluation of weight gain, binge eating). The factor instability suggests that not all constituent themes within the questionnaire are clearly mapped (see McDonald \& Mulaik, 1979). One solution to this problem might be to extend the scale with items highly specific to each of the themes; in this way each theme will eventually be covered specifically by one factor (Briggs et al., 1986). Indeed, such an approach has been employed in the development of new questionnaires, notably the Dutch eating Behaviour Questionnaire (DEBQ; van Strien, Frijters, Bergers \& Defares, 1986) and the Three factor eating behaviour Questionnaire (TFEQ; Stunkard \& Messick, 1985). However, although these questionnaires appear to have more clearly discernable subscales, recommendations have been given to use only one subscale at one time, either because the total scale was not intended to measure one construct (DBEQ; van Strien et al., 1986), or because inter-subscale correlations turned out to be insufficiently high (TFEQ; see Heatherton et al., 1988).

The second issue in using the RS bears upon the well-established finding that counterregulation of high RS-scorers appears mainly after ingestion of large, and not after small preloads. Importantly however, in recent years it has become clear that also small preloads or food cues (e.g. sight, smell and taste of food) can act as triggers of craving and increased subsequent food intake (Jansen \& van den Hout, 1991; Rogers \& Hill, 1989; Weingarten \& Elston, 1990). Analogously to developments in addiction research (e.g. Siegel, 1983; Childress, 1992; Drummond, Tiffany, Glautier \& Remington, 1995), new models of binge eating have been put forward emphasizing leamed cue reactivity as a maintenance factor (Jansen, 1990; 1994; Wardle, 1990). Cue reactivity means that stimuli predictive of food intake trigger a physiological state that is experienced as craving (Jansen, 1994). As to the predictive value of the Restraint Scale, disinhibitive eating after presentation of food cues has been found only in subjects with extreme RS scores (Jansen \& van den Hout, 1991). Hence, a subclass of high restrained eaters (i.e. those scoring slightly above the median RS score) did not show changes in eating behaviour after cue presentation. This may be a direct consequence of these subjects endorsing of the "concern for dieting" items in the Restrained scale (high RS scores can result from either strong concern for dieting or high disinhibition, or both; Westenhoeffer et al., 1994). Specific measures of concern for dieting, such as TFEQ and DEBQ have been found to identify successful dieters, i.e. those who do not frequently disininhibit (Heatherton et al., 1988; Lowe, 1993). This renders it likely that many high restrained eaters in this experiment deliberately inhibited their food intake during the taste test. Thus, although high scorers on the RS might possess considerable food cue reactivity, high "concern for dieting" may lessen chances on observable changes in food 
intake after presentation of food cues other than large preload.

Finally, neither in the RS nor in the DEBQ or TFEQ reference is made to self-esteem. Recently however, it has been shown that in particular high restrained eaters whose selfesteem is low do counterregulate (Polivy, Heatherton \& Herman, 1988). It has been proposed that the larger variability among high RS scorers in food intake within the lab can be attributed to different levels of self-esteem among individuals within this group.

The aim of the present study is to alleviate the above problems with using the Restraint Scale, which admittedly remains the questionnaire most predictive of at least one form of disinhibition, i.e. counterregulation. We attempted to develop a questionnaire for selection of subjects maximally prone to lab disinhibition to a broad range of cues, and which would have the following characteristics: a clearer factor structure than the RS, attenuation of the unwanted impact of concern for dieting items on food intake, and reference to self-esteem. Moreover, the scale should be internally consistent so that total scores could be used. We compared the new developed questionnaire with the Restraint Scale on two characteristics: psychometric properties, and characteristics of extreme groups selected on basis of scores.

\section{GENERAL METHODOLOGY}

All questionnaires were administered within a non-clinical population of students participating in courses, and volunteering university employees. All statistical analyses were carried out using the SPSS-X software program.

\section{DEVELOPMENT OF A NEW DISINHIBITION SCALE}

\section{Purpose and rationale}

A rational, construct-oriented approach (see Nunnally, 1978; Wilde, 1977) was adopted to develop a new scale for selecting individuals maximally susceptible to triggered disinhibitive eating in the lab. We reasoned that lab-disinhibition may be best predicted by items referring to the occurrence of disinhibition in daily life. Furthermore we considered that disinhibition occurs in three forms that should all be mapped to get a full picture: 1) eating, at times, too much "objectively" 2) eating too much "subjectively", i.e. cases in which the individual judges that self-imposed restrictions are overruled; 3 ) objective consequences of repeated disinhibition, i.e. weight fluctuations. To these three factors thought to predict lab disinhibition we added self-esteem as a fourth "risk" 


\section{Chapter 8}

factor. Our objective was to end up with a questionnaire containing subscales for each of the above factors clearly defined both semantically and psychometrically (see Briggs et al., 1986; Comrey, 1978; Nunnally, 1978).

\section{Construction of the initial item pool}

An initial pool of items addressing the above four themes (binge eating, failed restriction, weight fluctuations and self-esteem) was composed as follows.

Binge eating Item 6 of the Restraint Scale (see table 8.1), which evidently refers to binge eating practices, was extended with related items on overeating behaviours thought to be "socially somewhat unacceptable". Occurrence of binge eating in daily life, whatever its cause, was assumed to be a good predictor of disinhibition in the lab.

Failed restriction These items tapped on the inability to hold on to dietary restriction each time it's imposed. All of these items had a general format "If I try to impose restriction on my eating I fail". Our items deal very generally with the occurrence of disinhibition after attempts to restrict food intake. Putative causes of the disinhibition were not addressed. High scores on failed restriction items were assumed to contribute to disinhibition in the lab. Matters were arranged so that people who report to never self-impose eating restrictions received a zero score on these items.

Weight fluctuations High scores on weight fluctuation items of the Restraint Scale have been claimed to result from a chaotic eating pattern in which disinhibition follows attemps at dieting, a jojo-effect (Heatherton, Polivy, \& Herman, 1991; Herman \& Polivy, 1982; Lowe, 1993). Our weight fluctuations items were Restraint Scale items (nos. 2, 3 and 4), and a newly conceived item. A modification of Restraint scale item no. 10 was included as well: instead of the difference between prior maximum and currently desired weight, the difference between prior maximum and minimum weight was computed (see also Laessle et al., 1989). High scores on the weight fluctuations items were assumed to increase susceptibility to lab disinhibition.

Self-esteem Items on self-esteem were added not because self-esteem as such is part of the disinhibition concept, but because, as said earlier, low self-esteem has been found to facilitate the occurrence of disinhibitive eating in the lab (Polivy et al., 1988). The items were picked from the Rosenberg Self-esteem Scale (Rosenberg, 1965), and consisted of evaluations of the self in comparison with other people. The items were scored in such a way that answers in the direction of lower self-esteem received higher scores.

Answering format Most items were self-referring descriptions of behaviours or attitudes (self-esteem items). Respondents were to indicate the frequency with which each description applied to their daily situation. Except for items on weight fluctuations this was done on a 5-point Likert scale ranging from 0 points ("never") to 4 ("always"). To 
diminish the possibility of acquiescence (Nunnally, 1978) some items were phrased inversely to disinhibition and its scoring direction should be reversed. The "failed restriction" items had a sixth alternative added, to allow for the possibility that a respondent never self-imposes restrictive eating. This alternative was scored zero.

\section{Item analysis and construction of the Disinhibitive Eating Scale}

The item pool consisting of 36 items was administered to 350 female students and university employees who were approached within the area of the university building (Body Mass Index $20.93 \pm 2.2$; age $23.33 \pm 4.4$. A negligible proportion (about $3 \%$ ) of those approached, declined to participate in the investigation. Item analysis was conducted on basis of a principal components analysis with orthogonal rotation of the extracted factors, as well as assessment of item-total correlations. Items loading poorly on the extracted factors and/or having low item-total correlations were removed from the pool. Some of the binge eating items were removed to prevent relative overrepresentation of these items in the remaining item pool. Finally, the item phrasing were reconsidered and in some cases simplified. The thus formed Disinhibition Scale (DIS) consisted of 17 items divided within 4 subscales (see table 8.2 for the subscale structure and item phrasings): "binge eating" (5 items), "failed restriction" (4 items), "weight fluctuations" (4 items) and "self-esteem" (4 items). The 5-point Likert-format of items was maintained.

\section{TESTING THE RESTRAINT AND DISINHIBITION SCALES}

\section{Subjects and method}

Both Restraint Scale and the newly developed disinhibition scale (DIS) were administered to 269 females (BMI $21.03 \pm 2.4$; age $19.8 \pm 3.0$ ) who were participating in a freshman course at the psychology department. The Restraint Scale was scored according to the requirements introduced by Herman \& Polivy (1980), the DIS according to those in table 8.2. After the establishment of the normative data, three psychometric properties of the questionnaires were investigated: the factor structure (using a principal components analysis with subsequent rotation of the extracted factors confirming to the eigenvalue $>1$ as well as the scree criterion), level of internal consistency (as revealed by Cronbach's Alpha), and item-total correlations and correlations between subscales (only for the DIS). Then, the relationship between the two questionnaires was established by correlations.

Finally the relationship between dieting frequency and the DIS was explored. The 


\section{Chapter 8}

formulation of the DIS items on failed restriction is conditional: "if I try to restrict my eating then I fail". No explicit reference is made to the frequency with which attempts are made to restrict food intake, although "diet frequency" has been claimed to be influential in disinhibition after exposure to binge cues (Lowe, 1993). To elucidate how diet frequency fits the structure of the DIS, a new factor analysis was carried out on all DIS items extended with the Restraint Scale item no. 1 (i.e., the frequency of dieting item).

\section{Table 8.1. Factor structure of the Restraint Scale}

\begin{tabular}{|c|c|c|}
\hline & F-1 & F-2 \\
\hline 1. How often are you dieting? & .75 & .34 \\
\hline $\begin{array}{l}\text { 2. What is the maximum amount of weight (in kilos) } \\
\text { that you have ever lost in one month? }\end{array}$ & .15 & .65 \\
\hline 3. What is your maximum weight gain within a week? & .14 & .81 \\
\hline 4. In a typical week, how much does your weight fluctuate? & .07 & .79 \\
\hline $\begin{array}{l}\text { 5. Would a weight fluctuation of } 5 \mathrm{lbs} \text { affect the way } \\
\text { you live your life? }\end{array}$ & .76 & -.08 \\
\hline 6. Do you eat sensibly in front of others and splurge alone? & .39 & .44 \\
\hline 7. Do you give too much time and thought to food? & .67 & .32 \\
\hline 8. Do you have feelings of guilt after overeating? & .71 & .41 \\
\hline 9. How conscious are you of what you're eating? & .58 & .08 \\
\hline $\begin{array}{l}\text { 10. How many pounds over your desired weight were you at } \\
\text { your maximum weight? }\end{array}$ & .48 & .49 \\
\hline
\end{tabular}

\section{Results}

Restraint Scale The factor analysis revealed a structure with two factors with eigenvalue larger than 1, accounting for $54.0 \%$ of the common itemvariance, the structure of which is shown in table 8.1. As can be seen, three items had considerable (i.e. $>.40$ ) loadings on both factors. Cronbach's alpha for the total scale was .83 which reflects a fully satisfactory level of internal consistency. The item-total correlations ranged from .38 to .70 (mean .53).

Disinhibitive Eating Scale The factor structure (table 8.2) shows four factors with eigenvalue larger than 1 , accounting for $67.7 \%$ of the common itemvariance and having each item loading considerably (above .40) only on the factor prototypical of its subscale, and not on the other factors. Thus the factor pattern approached the Thurstonian 'simple' factor structure (Nunnally, 1978) Internal consistency of the total scale (alpha $=.88$ ) and subscales (alpha's ranging from .76 to .88 ) were good, as were the it- 
Subscale bingeing $(\alpha=0.88)$

2 . At times I lose control over my eating behaviour"

5. I eat sensibly when with others, but I gorge myself

$\begin{array}{llll}.67 & .29 & .27 & .23 \\ .75 & .22 & .25 & .08 \\ & & & \\ .82 & .15 & .17 & .19 \\ .77 & .13 & .19 & .06 \\ .81 & .14 & .06 & .24\end{array}$

8. If others saw how much I ate, then I'd feel ashamed

11. I eat so much at one go that it is actually disgusting other people

Subscale self-esteem $\quad(\alpha=0.85)$

3. I have the feeling that other people do things better than me

6. There are many things about myself that I would change if possible

9. I'd rather be a different person than 1 am now

12. I'm very satisfied with the way I am (-)

$\begin{array}{llll}.20 & .77 & -.03 & -.08 \\ .11 & .86 & .06 & -.02 \\ & & & \\ .21 & .84 & .12 & .06 \\ .17 & .75 & .13 & .18 \\ & & & \\ .16 & .07 & .77 & .11 \\ & & & \\ .14 & -.05 & .82 & .13 \\ .13 & .13 & .83 & .13 \\ .37 & .16 & .73 & .15\end{array}$

Subscale unsuccessful dieting $(\alpha=0.85)$

1. When I'm watching my weight, I succeed in not touching food items (-)

4. When I'm dieting, I manage not to eat between meals (-)

10. When I'm dieting, $I$ can't control myself and start eating 'forbidden foods'

\section{Subscale Weight fluctuations $(\alpha=0.76)$}

14. How strongly has your weight fluctuated in the past few years?

15. What is the maximum weight you have lost in one month?

16. What is the average variation in your weight in one week? I

Difference score of:

17. What has been your highest weight since you were 18 (excluding pregnancy)?

18. What has been your lowest weight since you were 18 ?

$\begin{array}{llll}.34 & .08 & .18 & .73 \\ .08 & -.07 & .08 & .83 \\ .10 & .05 & .19 & .60\end{array}$

(-) negatively keyed item

answering format: $0=$ never; $1=$ sometimes; $2=$ regularly; $3=$ often; $4=$ always; (items 1 through 13)

answering format: $0=$ not at all; $1=$ bardly at all; $2=$ moderately; $3=$ considerably; $4=$ extremely

answering format: $0=$ less than $0.5 \mathrm{~kg} ; 1=0.5$ to $1 \mathrm{~kg} ; 2=1$ to $2 \mathrm{~kg} ; 3=2$ to $4 \mathrm{~kg} ; 4=4 \mathrm{~kg}$ or more

I answering format: $0=$ less than $0.5 \mathrm{~kg} ; 1=0.5$ to $1 \mathrm{~kg} ; 2=1$ to $1.5 \mathrm{~kg} ; 3=1.5$ to $2.5 \mathrm{~kg} ; 4=2.5 \mathrm{~kg}$ or more

answering format: $0=$ less than $3 \mathrm{~kg} ; 1=3$ to $5 \mathrm{~kg} ; 2=5$ to $7 \mathrm{~kg} ; 3=7$ to $10 \mathrm{~kg} ; 4=10 \mathrm{~kg}$ or more 


\section{Chapter 8}

em-total correlations (range .33 to .69 , mean .51). In general, correlations between subscales were moderately positive (table 8.3 ).

Correlation and comparison of the Restraint and Disinhibition Scales The correlations between RS and DIS total scores was .78. Factor analysis of the DIS items together with the diet frequency showed the original DIS factor structure, with the diet frequency item loading .37 on the failed restriction, and .57 on the weight fluctuations factors. This shows that not so much the failed restriction subscale (with items phrased conditionally), but the weight fluctuations subscale accounted for the frequency of failed attempts at dietary restriction.

Table 8.3. Inter-subscale correlations of the Disinhibitive Eating Scale

\begin{tabular}{lccc} 
& Bingeing & Failed restriction & Self-esteem \\
Bingeing & - & & \\
Failed restriction & .49 & - & - \\
Self-esteem & .44 & .24 & .13 \\
Weight fluctuations & .41 & .35 & \\
\hline
\end{tabular}

\section{NORMATIVE DATA AND CHARACTERISTICS OF SUBJECT GROUPS WITH EXTREME DIS AND RS SCORES}

\section{Subjects and method}

First, we established the population distribution of DIS and RS total scores within our sample of 269 female students. Then, for each questionnaire three pairs of extreme scoring groups were formed according to the following criteria. 1) below vs. above the median; 2) below 25 th vs. above 75 th percentile; 3 ) below 10 th vs. above 90 th percentile.

For each of the three criteria, we assessed to what extent the groups formed on basis of DIS and RS consisted of the same individuals. This comparison bears upon divergent validity of the DIS: if the DIS would select the same individuals as the RS, then no purpose would be served by using it.

For each questionnaire separately, high and low scoring groups were compared on BMI and current dieting. The current dieting item was phrased simply: "are you presently dieting to lose weight?" and could be answered yes or no. Three such comparisons, i.e. one for each selection criterion, were made. Difference in BMI were tested with t-tests; percentage of individuals dieting by Chi square tests. 


\section{Results}

Normative data of DIS and RS total scores are presented in table 8.4. Mean score for the RS was 11.66 (sd 5.5) and for the DIS 22.93 (sd 10.9). Strikingly, the RS median was 11, i.e. considerably lower than in Northern American samples. The overlap of extreme groups formed by selection by DIS and RS are shown in figure 8.1. It is clear that the composition of groups selected by either DIS or RS differed, and that the difference was larger when more extreme criteria are used for selection.

Table 8.4. Population distribution of Restraint Scale and Disinhibitive Eating Scale total scores ( $N=269$ females)

\begin{tabular}{ccc}
\hline Percentile & RS & DIS \\
$\mathbf{5}$ & 3 & 7 \\
$\mathbf{1 0}$ & 4 & 9 \\
$\mathbf{2 0}$ & 6 & 12 \\
$\mathbf{2 5}$ & 7 & 14 \\
$\mathbf{3 0}$ & 8 & 15 \\
$\mathbf{4 0}$ & 9 & 18 \\
$\mathbf{5 0}$ & 10 & 21 \\
$\mathbf{6 0}$ & 12 & 24 \\
$\mathbf{7 0}$ & 13 & 27 \\
$\mathbf{7 5}$ & 14 & 29 \\
$\mathbf{8 0}$ & 16 & 31 \\
$\mathbf{9 0}$ & 19 & 37 \\
95 & 22 & 43 \\
\hline
\end{tabular}

Differences in current dieting an BMI of extremely high and low scoring groups on DIS and RS are shown in table 8.5. The results for DIS and RS were comparable and consistent: high scorers had larger BMI and higher prevalence of current dieting than low scorers. Interestingly, among individuals scoring above the RS median i.e. the group traditionally selected for lab research on counterregulation, less than half was currently dieting ${ }^{2}$. Even of the individuals with RS scores within the highest ten percentiles, only $65 \%$ reported to be currently dieting.

Also for BMI, significant and large group differences appeared irrespectively of the used group allocation criterion. The size of differences were as large as between 1.5 population standard deviations of BMI. The correlation of the BMI with RS and DIS scores were respectively 0.48 and 0.52 .

\footnotetext{
${ }^{2}$ Parenthetically, only $16.5 \%$ of all subjects reported to be currently dieting.
} 
Table 8.5. Comparisons of extreme scoring subject groups, using three criteria for Restraint Scale and Disinhibitive Eating Scale scores: a. median split; b. $<25$ th vs $>75$ th percentile; c. $<10$ th vs $>90$ th percentile. $(N=269$ lemales)

\begin{tabular}{|c|c|c|c|c|c|c|c|c|}
\hline \multirow{2}{*}{\multicolumn{3}{|c|}{$\begin{array}{l}\text { RESTRAINT SCALE } \\
\text { cut-off scores }\end{array}$}} & \multicolumn{4}{|c|}{ Body Mass index } & \multicolumn{2}{|c|}{$\%$ currently dieting } \\
\hline & & & \multicolumn{2}{|c|}{ low scorers } & \multicolumn{2}{|c|}{ high scorers } & \multirow{2}{*}{$\begin{array}{c}\text { low scorers } \\
4.7\end{array}$} & \multirow{2}{*}{$\begin{array}{c}\text { high scorers } \\
44.7^{4}\end{array}$} \\
\hline $\mathbf{a}$ & $\leq 11$ & $>11$ & 19.9 & 1.7 & 22.0 & $2.6^{\circ}$ & & \\
\hline b & $<8$ & $>14$ & 19.3 & 1.4 & 22.7 & $2.9^{-}$ & 1.6 & $56.9^{+}$ \\
\hline c & $<5$ & $>19$ & 19.0 & 1.3 & 22.1 & $2.7^{\circ}$ & 0 & $65.2^{+}$ \\
\hline \multicolumn{4}{|c|}{ DISINHIBITIVE EATING SCALE } & \multicolumn{3}{|c|}{ Body Mass index } & \multicolumn{2}{|c|}{ \% currently dieting } \\
\hline & \multicolumn{2}{|c|}{ cut-off scores } & \multicolumn{2}{|c|}{ low scorers } & \multicolumn{2}{|c|}{ high scorers } & low scorers & high scor \\
\hline $\mathbf{a}$ & $\leq 21$ & $>21$ & 20.0 & 1.7 & 22.1 & $2.6^{\circ}$ & 10.3 & $41.4^{+}$ \\
\hline b & $<15$ & $>29$ & 19.5 & 1.7 & 22.9 & $2.8^{\circ}$ & 2.9 & $44.1^{\dagger}$ \\
\hline c & $<10$ & $>37$ & 19.2 & 1.7 & 23.3 & $3.5^{\circ}$ & 3.6 & $57.1^{+}$ \\
\hline
\end{tabular}

- t-test for group differences: $\mathrm{p}<.001$

' chi-square test: $\mathrm{p}<.001$

\section{DISCUSSION}

The results of the psychometric comparison of DIS and the RS in a non-clinical sample of 269 female students showed comparably good internal consistency in the two questionnaires. Factor analyses yielded a two-factor solution in the RS, and a four-factor solution in the DIS. The RS total score distribution in the sample had a median of 11. The correlation between the two scales was 0.78 . It was found that high scorers on either RS or DIS have a considerably higher Body Mass Index and a higher prevalence of current dieting than did low scorers. Finally, it appeared that groups formed on basis of extreme DIS scores were partly composed of different individuals than groups formed on basis of extreme RS scores, and that this difference grew larger when more extreme scores were used as allocation criterion.

The psychometric analysis of the RS confirms earlier reports (for example, Heatherton et al., 1988) of a good value of Cronbach's alpha. Once again the existence of two factors has been demonstrated, which in this case do not differ from the concern for dieting and weight fluctuations factors. It should be noted however that it can be suspected on statistical grounds ( $46 \%$ of the item variance was not explained by the two factors; 3 items loaded > .40 on both factors) as well as on the relatively wide ranging item content, that the RS 
scores potentially encompasses more than two concepts, but that these concepts would emerge as factors if more items were added to the RS. Of course there is little news to this conclusion and discussions on the most fruitful interpretation of restraint scores continue (Charnock, 1989a, 1989b; Dritschel, Cooper \& Charnock, 1993; Heatherton et al., 1988; Lowe, 1993; Ogden, 1993; Polivy \& Herman 1989). Our finding of a median RS score of 11 in our sample of Dutch female students was a confirmation of our earlier experience (Overduin \& Jansen, unpublished data) and of other reports on European subject samples (Wardle, 1986; Laessle, 1989). The implication is that subject selection on basis of an assumed median score of 15 (as found in Northern American samples) may not be appropriate in research within the average European female population.

The DIS was found to show the psychometric characteristics for which it was developed. In the first place a good internal consistency while at the same time all items can be said to be referring to disinhibition or a risk factor for disinhibition. Said otherwise, the total DIS scores appear to be both statistically and semantically meaningful. Secondly, a fair level of common item variance $(67.7 \%$ ) was explained by a well-interpretable four-factor structure with each item loading considerably on only one factor. Importantly, all factors were concordant with the a priori conceived subscales. The resulting advantage is that the DIS total score is based upon a transparant set of meaningful subscale scores. The relative contribution of each subscale to the total DIS score can thus be analyzed for each individual. Finally, the added self-esteem subscale formed an independent factor and the correlation with the rest of the DIS turned out to be moderately positive $(r=0.35$ ). Thus, addition of self-esteem items, expected to heighten the predictive power of the DIS as to lab disinhibition succeeded without compromising the internal consistency or factor structure of the DIS.

Two similarities between the DIS and RS are evident. In the first place a high correlation between the two scales was found, which is not too surprising because of a considerable overlap of item content (e.g. weight fluctuations subscale and RS item no. 6). Secondly, both scales have moderate to strong positive correlations with Body Mass Index. As a consequence in comparisons of high and low scoring groups on food intake in the lab, these differences in BMI should not be neglected. The same applies for the prevalence of current dieting which differs for high and low scorers on both scales. This covariation once again appears to be a fundamental problem in research on disinhibitive eating. Moreover, the fact that among high scorers on DIS and RS only a slight majority (i.e. far from 100\%) is currently dieting, indicates the necessity of carefully distinguishing between general dieting frequency and current dieting when studying behaviours of subjects selected by means of these questionnaires (see e.g. Lowe, 1993).

Also differences between the DIS and RS were found, the most important of which is 


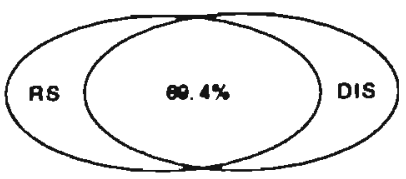

a

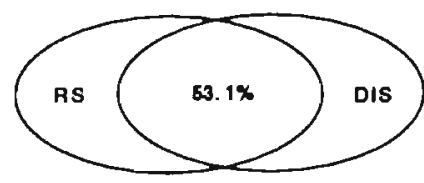

b

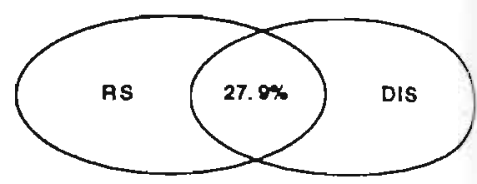

c

Figure 8.1. Percentage of subjects selected by both RS and DIS when applying different group selection criteria: a. median split; b. $<25$ vs. $>75$ th percentile; c. $<10$ th vs. $>90$ th percentile.

practical: in part, different individuals are selected by the two scales when extreme scores are used as a criterion. Both scales have appeared to be equally internally consistent, so that the reasons and possible consequences of selection on basis of DIS scores must be sought for in the item content. Firstly, in contrast to the RS, the DIS contains no concern for dieting items. The aim of the two scales differed: the RS was designed to identify dieters (Polivy \& Herman, 1989; Heatherton, 1988), whereas the DIS was designed for prediction of maximal proneness to lab disinhibition. Of course, the absence of explicit concern for dieting items in the DIS does not at all imply that high DIS-scorers are unconcerned with dieting. What it does imply however, is that relative to the RS, the impact of concern for dieting has been attenuated in the DIS total scores. And, as has been pointed out in the introduction, because individuals high on concern for dieting will have the inclination to inhibit their food intake in the lab, attenuation of its contribution to DIS-scores has the advantage that subjects are selected whose reactivity to food cues will be reflected in eating behaviour also in procedures in which small preloads or food cues are presented. In other words, the chances of false negative findings (i.e. inhibition in subjects with high cue reactivity) will be decreased. Secondly, in contrast to the RS, self-esteem items are included in the DIS. High DIS scorers will have low self-esteem, whereas among high restaint scorers there could be either high or low in self-esteem, thus accounting for different composition of extreme scoring groups. A last relevant issue is that of content validity: to what extent does the DIS address factors related to pathological eating behaviour? Content validity is relevant if one wishes to eventually apply lab results to clinical cases. The content validity of the DIS is suggested by research of Tobin, Johnson, Steinberg, Staats and Dennis (1991), who factor-analyzed a large and complete self-report battery on bulimic symptoms in patients: four factors of the six factors found, i.e., weight fluctuations, binge eating, self-esteem and failed restriction are also present within the DIS. The remaining two factors found by Tobin et al. were family history, and drive for thinness, the latter being similar to 
concern for dieting which was not included in the DIS for reasons explained carlier. All in all, personality factors important in pathological disinhibitive eating can be found in DIS scores to a reasonable extent.

The DIS has been shown to be a conceptually and psychometrically sound selection instrument which taps a construct that is related, but not equal to that measured by the RS. Different emphases exist in the DIS and RS, which partly makes for selection of subject groups of different composition. The DIS was constructed to alleviate some of the issues related to use of the RS in cue reactivity research. Naturally, its predictive power as to lab disinhibition remains to be demonstrated empirically. 



\section{Chapter 9}

\section{CONCLUSIONS AND GENERAL DISCUSSION}

In this final chapter, the results of the empirical studies of the present thesis will be summarized (9.1). After a reappraisal of its physiological assumptions (9.2), a final conclusion will be formulated about the classical conditioning theory of binge eating (9.3). Finally, theoretical and practical suggestions for future research will be made in sections 9.4 and 9.5 respectively.

\subsection{OVERVIEW OF THE EMPIRICAL WORK IN THIS THESIS}

After the description of the conditioning theory of binge eating (CBE) in chapter 2, subsequent chapters were devoted to empirical tests of related hypotheses. In two chapters, the results of conditioning procedures were described. Chapter 3 described a classical conditioning procedure that was run in order to evoke a physiological state which, according to $\mathrm{CBE}$ theory, precedes binge eating. On six occasions, olfactory/taste cues (i.e., the conditioned stimuli, CSs) were paired with glucose ingestion (the unconditioned stimulus, UCS). Subjects in the control condition received the metabolically inert sweetener aspartame as the UCS. At test trials (before and after the conditioning procedure) the CS compound was presented without the UCS, and levels of subjective craving, insulin and blood sugar measured. Subsequently, ad lib glucose intake was measured in a taste test. CBE hypotheses for the post-conditioning test day were that CS presentation would elicit the following responses: conditioned hypoglycaemia, conditioned hyperinsulinaemia, increased craving for sweet snacks, and increased glucose intake. These hypotheses could not be confirmed, except for a small group difference in insulin response, which, however, did not exceed baseline fluctuations. Furthermore, in both conditions, intake of glucose was considerably larger after than before conditioning. As this behaviour was not mirrored in ratings of subjective craving, it was attributed to a "neophobia" or "bait shyness" that had occurred during the pre-conditioning test session, but faded over the subsequent conditioning trials, a phenomenon which has been described previously in the animal literature. In order to investigate whether repeated exposure to sweet drinks (UCS) was a critical factor, a similar conditioning procedure was run additionally with plain water as a UCS. Also in this condition, glucose intake was increased after the procedure. It was therefore concluded that the disappearance of the subjects' "neophobia" had been related to their getting acquainted with the lab procedure, rather than with the taste of sweet drinks. Chapter 4 contained a comment on, as well as a re-analysis of a not widely known study on hypoglycaemic conditioning 


\section{Chapter 9}

by the Russian physiologist Mityushov (1954; Appendix, this thesis). In contrast to our conditioning study described in chapter three, Mityushov was able to demonstrate a statistically as well as physiologically significant conditioned hypoglycaemia in humans and dogs, after a procedure involving repeated pairing of an injection ritual (CS) with intravenous glucose (UCS). Further observations suggested that the hypoglycaemia was secondary to a conditioned secretion of insulin. Although Mityushov's report would not fully meet today's methodological standards (e.g. no control group was run; no overall statistics were reported), it was concluded that his conditioning procedure evoked a physiological state that, according to the CBE theory, occurs prior to bingeing.

The next two chapters investigated psychophysiological cue reactivity to food related stimuli. In CBE terms, cue reactivity could be considered as a residual of learning history. In chapter 5 reactions to slides with binge-related food items were studied. The CBE prediction in this study was that cue reactivity would be larger in restrained than in unrestrained eaters. This was hypothesized because restrained eaters can be considered "unsuccessful dieters" who regularly engage in (non-clinical) binge eating. Hence, they should display especially intense conditioned responses to food cues. A manipulation check revealed that subjective hedonic ratings of the food slides were higher than those for control slides (depicting office equipment). Nevertheless, no increased psychophysiological cue reactivity was found in restrained subjects, so that the CBE hypothesis was not confirmed. The study in chapter 6 assessed psychophysiological reactivity to the sight and smell of attractive food. Two groups of normal subjects were compared, one of which had been fasting for the previous 24 hours. It was thought that the fasting state would form a baseline to which food craving could be easily elicited by food cues, and that the related physiological responses would be more clearly discernible. The hypotheses were that subjective craving to food cues would be larger in fasting than nonfasting subjects, and, more specifically, that these differences would be mirrored in the intensity of the psychophysiological responses. As expected, it was found that food item presentation elicited more craving in fasted than in non-fasted subjects. However, no group differences were found in psychophysiological cue reactivity, a finding which was not in line with the CBE hypothesis.

Chapter 7 contained an experimental test of the cognitive effects of cue-elicited craving. Restrained and unrestrained eaters were compared on their attentional bias to food words measured in a modified Stroop colour-naming test. There were two conditions. In the appetizer condition, subjects had to eat a spoonful of palatable pudding, whereas in the control condition no appetizer was ingested. Then, the modified Stroop test was run, comparing, among other things, colour naming latency for food and neutral words. After the Stroop test, ad lib ice cream intake was measured during a "taste test". The 
hypotheses were that ingestion of the appetizer would result in stronger Stroop interference for food words, and larger food intake in restrained than in unrestrained subjects. This was not confirmed: in the appetizer condition, no especially strong Stroop interference or disinhibitive eating could be found in restrained subjects. However, it was also observed that whereas restrained subjects did not counterregulate, unrestrained subjects did. Interestingly, for unrestrained subjects, the magnitude of the Stroop interference for food words appeared to correlate 0.58 with the subsequent intake of ice-cream. This was taken to suggest that attentional bias to food words may be a potential predictor of increased eating after cue presentation.

Finally, in chapter $\mathbf{8}$, the development was described of the DIS, a new questionnaire that might be used as a selection tool in future research. The DIS aims at selecting persons who are maximally prone to disinhibitive eating. In this respect, the DIS differs from the widely used Restraint Scale, that was originally developed for identifying dieters. The psychometric analysis of the DIS showed good internal consistency, as well as a clear four-factor structure tapping binge eating, failed restriction, weight fluctuations, and self-esteem, i.e. a factor structure resembling that found in earlier studies of large item pools tapping a full spectrum of bulimic characteristics. It was concluded that the DIS could be used as a tool in the development of new models of binge eating.

Taken together, none of our novel studies specifically testing hypotheses derived from $\mathrm{CBE}$ theory produced confirmative results. What should the implications of these results be for the CBE theory? One might suppose that particulars of the studies were responsible. Our non-clinical restrained subjects may have represented too weak cases of binge eating; also, the food slides used as cues in our cue reactivity experiment may not have been overly "realistic"; finally, our conditioning study may have included too few trials to demonstrate conditioning.

However, one might also rebut these arguments with the following considerations. Of the available questionnaires in the field, the Restraint Scale has been generally recognized as the most predictive of lab-overeating and binge eating in daily life (Heatherton et al., 1988; Wardle, 1980). Further, our food slides have probably been sufficiently realistic, which is suggested by the fact that, for all subjects taken together, psychophysiological reactions and subjective craving were stronger for food than did for neutral (office equipment) slides (Chapter 5). Finally, in other contexts, conditioned hypoglycaemia (using intravenous UCSs) have been demonstrated after two to five conditioning trials (Fehm-Wolfsdorf et al., 1993; Mityushov, 1954; Appendix, this thesis; Woods et al., 1969), so that after our six trials (using oral UCSs) one should expect at least a significant trace of evolving hypoglycaemia. All in all, it appears that the present studies have been fairly critical and suggest reconsideration of the CBE theory. This reconsider- 


\section{Chapter 9}

ation can be found in the following section.

\subsection{QUANTITATIVE REAPPRAISAL OF THE CBE THEORY}

In the original formulations of the conditioning theory of binge eating (chapter 2 this thesis; Jansen, $1990 ; 1994 \mathrm{a}$ ), no quantitative estimates were given of the size of putative conditioned physiological responses. Considering the general lack of confirmation for the CBE theory in the present thesis, it seems worthwhile to reconsider CBE theory's physiological predictions in a quantitative and critical way. Below, five key physiological CBE hypotheses (see chapter 2 for background explanation) will be assessed, using data from general physiology, cephalic phase response studies, conditioning studies, and comparative studies of physiological responding in bulimics and normals.

\subsubsection{Hypothesis one: binge eating causes exceptionally large blood sugar rises (UCS)}

Analysis: The CBE line of reasoning is that bingeing provokes a very large blood sugar rise, requiring that the size of the (compensatory) conditioned hypoglycaemia (CR) be proportionally large. The large intensity $C R$ would then be reflected in the subjective experience of craving.

No direct measurements of blood sugar rises after bingeing are available. Nevertheless, it is doubtful that beyond moderate meal size, additional intake leads to higher blood sugar rises. With fasting baseline typically lying between 70 and $100 \mathrm{mg} / \mathrm{dl}$, a normal meal will lead to blood sugar levels of between 100-150 mg/dl (Bantle et al., 1983; Oettlé, Emmett \& Heaton, 1988; Shively, Apgar \& Tarka, 1986; Teff, Levin \& Engelman, 1993; Weingarten, Hendler \& Rodin, 1988). Empirical data show that the upper boundaries of blood sugar level do not lie considerably higher. First, ingestion of doses of glucose (i.e. $1200 \mathrm{kcal}$ of a extremely potent blood sugar raiser; Brand Miller, Pang \& Broomhead, 1995) have been found to induce blood sugar levels not exceeding ${ }^{1}$

1 The physiological reason for the limited blood sugar rise to large meals in healthy (non-diabetic) persons depends on at least four inborn, homeostatic mechanisms. First, large meals will slow down gastric emptying, meaning that the food stays longer in the stomach and is allowed slower access to the small intestine, the place whence nutrients appear in the blood (McHugh, 1983). Second, if food is eaten hurriedly (this may be seen in binge eaters; section 1.2) the subsequent blood sugar rise is blunted importantly, due to several slowening effects on digestion (Read et al, 1986). Third, as soon as the glucose enters the blood, an (unconditioned) post-absorptive insulin response directs sugar from the blood into cells and tissues. Fourth, if, despite the aforementioned mechanisms, blood sugar level did exceed $150-190 \mathrm{mg} / \mathrm{dl}$, the sugar will not be reabsorbed by the kidneys and appears in the urine (this so-called glucosuria is responsible for the large volumes of high sugar urine which can be found in diabetics; Guyton, 1991). 
$180 \mathrm{mg} / \mathrm{dl}$ (Förster et al., 1972). Second, the ingestion of large amounts of favourite "real food" items appears to lead to moderate blood sugar rises. Kaye, Gwirtsman and George (1988) encouraged normal subjects to eat a buffet of favourite foods until their limits were reached. The recorded intake was 1482 (sd 430) $\mathrm{kcal}$, whereas blood sugar had only risen to $122 \mathrm{mg} / \mathrm{dl}$. A third - specifically bulimic - factor blunting the postmeal blood sugar rise is self-induced vomiting. Johnson et al. (1994) observed a rapid and strong blood sugar fall in bulimics who expelled ingested food (Johnson et al., 1994). In the Kaye et al. (1988) study, bulimics were encouraged to binge on a buffet of favourite food items, and given the possibility to purge. After a mean intake of 3022 (sd 1084) kcal and self-induced vomiting, bulimcs' average blood sugar level was found to lie at $120 \mathrm{mg} / \mathrm{dl}$. After this, subsequent bingeing and purging was allowed, and eventually the mean amount of ingested food was 7688 (sd 3971) kcal. In spite of this huge intake, blood sugar levels at subsequent l-hour intervals remained between 90 and 100 $\mathrm{mg} / \mathrm{dl}$.

Conclusion: Although no direct data are available on binge eating bulimics, ingesting large amounts of foods is unlikely to provoke an extreme blood sugar rise, because of the action of various homeostatic controls and the effects of self-induced vomiting.

\subsubsection{Hypothesis two: The conditioned blood sugar fall compensates substantially for the anticipated post-binge blood sugar rise}

Analysis: The CBE theory posits that conditioned hypoglycaemia compensates for the expected blood sugar rise (UCRs) ${ }^{2}$. How effective is this compensation?

To quantify the amount of compensation provided by the $\mathrm{CR}$, the $|\mathrm{CR} / \mathrm{UCR}|$ ratio can be computed. For example, if this ratio is 1 , full compensation of the post meal blood sugar rise occurs; if the ratio is 0.10 , this would mean that the conditioned hypoglycaemia would compensate for $10 \%$ of the expected blood sugar rise. The successful blood sugar conditioning studies using glucose as the UCS (Deutsch, 1974; Mityushov, 1954/Appendix, this thesis) provide insight into the |CR/UCR | ratio. In Deutsch's study, the blood sugar rise (UCR) to the glucose UCS in rats was $31.3 \%$; whereas the CR hypoglycaemic response to placebo intubation was $3.3 \%$ from baseline. Thus the

${ }^{2}$ For the sake of simplicity, blood sugar rise and fall are called responses here. Physiologically speaking, however, this may not be entirely correct. The blood sugar rise to ingested food ("UCR") is the result of passive absorption of glucose from the gut. A similar consideration may apply to the conditioned hypoglycemia which probably results from an (active) insulin secretion (see chapter 4 , this thesis; Dworkin, 1993; Eikelboom \& Stewart, 1982, for further discussions). 


\section{Chapter 9}

|CR/UCR | ratio in this case was approximately $0.11 .^{3}$ In Mityushov's study, the observed hypoglycaemic $\mathrm{CR}$ in humans was $10 \mathrm{mg} / \mathrm{dl}$, whereas the blood sugar rise to the UCS in humans was about $165 \mathrm{mg} / \mathrm{dl}$, making the $|\mathrm{CR} / \mathrm{UCR}|$ ratio $10 / 165=0.06$. Conclusion: Judging from the scanty data available, hypoglycaemic CRs appear to be far from compensatory.

\subsubsection{Hypothesis three: cephalic insulin responses are, physiologically speaking, substantial responses}

A substantial part of the data basis underlying CBE theory concerns cephalic phase responses to food cues (section 2.2). These responses are put forward as illustrations of substantial responses causing a fall in blood sugar level and underlying feelings of craving.

Analysis The available data show that the magnitude of the cephalic insulin response are rather small when compared with either baseline fluctuations or post-meal insulin secretion (Bellisle et al., 1983; Le Magnen, 1992; Moyer, Rodin \& Cummings, 1993). Whereas the range of reported cephalic insulin increases from baseline has been between 0-250\% of baseline levels in rats (Louis-Sylvestre, 1976; Powley \& Berthoud, 1985; Strubbe \& Steffens, 1975), and between 0 and $95 \%$ for humans (Lucas, Bellisle \& de Maio, 1987; Bruce et al., 1987; Sjöstrom et al., 1980; Moyer et al., 1993; Powley \& Berthoud, 1985; Teff et al., 1991; 1993; 1995), the post-absorptive increase of insulin after moderate (i.e. $100-700 \mathrm{kcal}$ ) food intake in humans lies between 200 and $1000 \%$ of baseline (Bantle et al., 1983; Bellisle et al., 1983; Shively et al., 1986; Rodin, Reed \& Jamner, 1988; Weingarten et al., 1988). Importantly for the CBE theory, two studies (Broberg \& Bernstein, 1989; Moyer, Rodin \& Cummings, 1993) have been conducted in bulimic patients who were to smell palatable food attentively. Neither study reported cephalic insulin responses exceeding baseline; moreover, the standard deviations of responses reported by Broberg and Bernstein (1989) were too small to suggest outliers among the subjects. In their discussion these authors have suggested that these results may have been due to their subjects not being in the "proper mood" that usually accompanies binge episodes. This remark applies to the vast majority of lab studies with bulimic subjects.

Conclusion Cephalic insulin secretion to food cues appears to be modestly sized when

${ }^{3}$ Note that another provisional computation could be made which adjusts for hyperglycaemia that occurred because of the rats' stress during the stomach intubation procedure (Deutsch reported that prior to the conditioning procedure, the rats had shown a $3.1 \%$, stress-related blood sugar rise when intubated with water). If one subtracts this blood sugar rise from both UCR and CR, then the $\mid$ CR/UCR $\mid$ ratio becomes $(3.3+3.1) /(31.3-3.1)=6.4 / 28.2=0.23$. 
compared with either baseline fluctuations or with post-meal insulin secretion. This observation applies to bulimics as well.

\subsubsection{Hypothesis four: cues related to binge food trigger especially large conditioned hypoglycaemia/hyperinsulinaemia in bulimics}

Analysis: As noted earlier, a potent elicitor of anticipatory insulin secretion and hypoglycaemia is sweet taste (section 2.2; Deutsch, 1974; Louis-Sylvestre, 1976, Powley \& Berthoud, 1985). Furthermore, binge eaters have been found to avoid snacks and other high caloric food items in between binges (e.g. Rosen et al., 1986; Walsh, 1993). If, as predicted by CBE theory, considerable conditioned hypoglycaemia or hyperinsulinaemia to binge food cues (CS) occurred in binge eaters, it follows that these responses should be observed in binge eaters and normals after the ingestion some fixed amount of binge-related food. Specifically, bulimics should show a retardation and/or a blunting of post-ingestive blood sugar rise, as well as a simultaneously enlarged insulin secretion.

First of all, two studies have been carried out comparing insulin and blood sugar responses to intravenous glucose in bulimics and normals (i.e., no food cues were involved in these studies). Blouin et al. (1991) found a smaller peak blood sugar value, but also a smaller insulin/glugacon ratio in bulimics than in normals. These findings were not replicated by Blouin et al. (1993), who did not find differences between responses of bulimics and normals. Studies which did involve oral ingestion of carbohydrates showed no irregularities in responses of bulimia patients: after ingestion of a sweet glucose drink (Hohlstein et al., 1986; Casper et al., 1989), or a 1200 kcal lemonflavoured carbohydrate drink (Turner et al., 1991), equal blood sugar and insulin responses were found in bulimics and normals. Addition of further "binge-relevant" sensory cues did not alter this pattern of results. Weingarten et al. (1988) provided bulimics and normals with equicaloric $(334 \mathrm{kcal})$ meals in a forbidden version (milkshake, and french toast with sirup) and an unforbidden version (cottage cheese and fruit). Again no between-group differences were found in blood sugar and insulin response. Moreover, the unforbidden meal evoked a somewhat larger insulin response in both subject groups, and this is contrary to CBE prediction. In one study (Schweiger et al., 1987) a difference between bulimics and normals was found: ingestion of a pudding rich in carbohydrates led indeed to a larger insulin secretion in bulimics (in line with $\mathrm{CBE}$ predicitions). However, this enlarged insulin secretion in bulimics appeared to be secondary to an enlarged blood sugar rise, a result which is in contrast with the $\mathrm{CBE}$ predictions. Jansen (1994a) has questioned the relevance of the above studies for CBE theory, because this theory only predicts pre-meal responses (however, see section 9.2.5 
for a further discussion on this issue). Furthermore, the cited studies neither involved individually chosen binge foods, nor were they conducted in the usual bingeing environment of the binge eating subjects.

Conclusion In contrast with the CBE hypothesis, ingestion of sweet or binge relevant food items has not been found to result in enlarged insulin or (hypoglycaemic) blood sugar responses in bulimics.

\subsubsection{Hypothesis five: conditioned hypoglycaemia underlies the urge to binge}

Analysis: The CBE theory posits that conditioned hypoglycaemias to food cues are experienced subjectively as an urge to eat.

Importantly, any hypoglycaemia explanation of binge eating should take into account that food intake itself will increase blood sugar level as nutrients start being absorbed from the small intestine. If hypoglycaemia underlies the urge to eat, an important question concerns the speed with which the hypoglycaemia will subside once eating has started. Probably, hypoglycaemias of the largest reliable size reported in the conditioning literature (i.e. $10 \mathrm{mg} / \mathrm{dl}$; Mityushov, 1954; Broberg \& Bernstein, 1989) would dissipate soon after the commencement of eating. This can be concluded from the available literature on blood sugar rise after ingestion of typical binge foods (candy bars and potato crisps). Ingestion of one candybar and a cup of tea (296 kcal) was found to lead to a $24 \mathrm{mg} / \mathrm{dl}$ rise within 20 minutes after meal onset; a serving of potato crisps and some cola drink (292 kcal) lead to a $29 \mathrm{mg} / \mathrm{dl}$ rise within the same time (Oettle et al., 1987). Other important data are given by Broberg \& Bernstein (1989) who did indeed find a considerable cephalic hypoglycaemia of $8-9 \mathrm{mg} / \mathrm{dl}$ after having their subjects smell palatable cinnamon rolls. Both in their bulimic and dieting subjects subsequent intake of the cookies was allowed. The bulimics who ate 36 grams of the rolls (perhaps about $150-200 \mathrm{kcal}$ ) returned to blood sugar baseline at between 10 and 15 minutes after the beginning of eating. These data show that after merely moderate intake hypoglycaemia is lifted long before the ending of the average binge, which has been reported to lie at 55.7 (sd 32.4) minutes (Jansen, Van den Hout \& Griez, 1990). It is only to be expected that bingeing is even more effective in lifting hypoglycaemia ${ }^{4}$.

Conclusion Hypoglycaemias of the size reported in the conditioning literature can be expected to subside soon after meal onset. The fact that the duration of the average binge appears to be considerably longer than that of hypoglycaemia, suggests that the role of conditioned hypoglycaemia may be limited, and that additional mechanisms

4 Compare the $\pm 300 \mathrm{kcal}$ snack, used in the Oettle et al. (1987) investigation, with the rate of food intake in binge eaters (between 30 and $80 \mathrm{kcal} / \mathrm{min}$; Rossiter et al., 1992; Walsh, 1993). 
should be postulated to fully explain binge eating.

\subsubsection{Conclusions}

Of course, the above discussions give only hints about the size of conditioned responses predicted by CBE theory. The true response sizes await revelation by monitoring of patients during full-fledged binge attacks. Until further notice, the following can be concluded:

a. the presently available data do not endorse CBE theory's assumption of physiologically significant conditioned hypoglycaemias/hyperinsulinaemias that "should be eaten away";

b. in bulimic patients, no enlarged hyperinsulinaemias or hypoglycaemias ("CRs") have been demonstrated in response to the taste or smell of binge related foods ("CSs");

c. ingestion of snacks provokes a rise in blood sugar that can be expected to lift hypoglycaemias of the size reported in the conditioning literature within a considerably shorter time than the generally reported duration of binges.

In sum, on basis of the known empirical data, profound scepticism seems warranted about the role of conditioned hypoglycaemia/hyperinsulinaemia in binge eating.

\subsection{THE CONDITIONING THEORY OF BINGE EATING: FINAL QUALIFICATION}

A conditioning theory of binge eating (CBE theory) was described in chapter two. By and large, none of the $C B E$ hypotheses empirically tested in this thesis could be confirmed. Furthermore, a quantitative reappraisal of the physiological assumptions of the CBE theory (section 9.2) cast considerable doubt on the premise that conditioned hypoglycaemia/hyperinsulinaemia is central to binge eating. In sharp contrast, CBE theory also has inspired a cue exposure procedure that promises to be an effective treatment of binge eating (section 2.2.6). It is not immediately clear what should be concluded from a case where an (as yet) unproven theory inspires effective therapy. Interestingly enough, similar dilemmas have been harassing conditioning research of panic disorder and addiction. We will examine the commentaries made by two major workers in these fields, McNally (1994) and Siegel (1989), before drawing final conclusions for binge eating.

McNally (1994) views classical conditioning theory starkly as a "misleading metaphor for the mechanisms underlying panic" while admitting that "(..) it has inspired effective treatments of panic disorder" (p. 108). McNally's main criticism of the conditioning 


\section{Chapter 9}

account of panic is that theoreticians have failed to clearly identify stimuli (CS, UCS) and responses (CR, UCR), often with awkward implications such as bodily sensations in panic being CS,CR and UCS at the same time. McNally thinks that this vagueness does not allow conditioning accounts to be very fruitful in basic research of panic disorder.

Siegel (1989) comments on several studies in which conditioned drug tolerance was found in the absence of demonstrable conditioned physiological responses. He concurs with the view that "failures to demonstrate compensatory CRs do not represent major challenges to the conditioning account of tolerance" (p. 163), and points to present knowledge deficits in classically conditioned drug tolerance. More specifically, too little is known yet about when and how physiological responses are triggered by environmental and drug stimuli, and how physiological CRs exactly relate to behavioural phenomena like tolerance and drug intake.

To what extent do the above considerations apply to the conditioning theory of binge eating? Concerning McNally's remarks, it should be emphasized that, unlike the panic theories, CBE theory's stimuli and responses have been well-defined. Equally truly, however, other investigators of binge eating have proposed an entirely different learning mechanism in bingeing that, while involving different responses and stimuli, has also inspired a therapeutical procedure that leads to important reductions in binge eating (Leitenberg et al., 1984; Schmidt \& Marks, 1989; for explanation see section 9.4.2). Nevertheless, the "vagueness" intrinsic to the existence of two different theories may have less to do with misleading metaphors, than with the possible existence of more than one mechanism underlying binge eating (Schmidt \& Marks, 1989). Siegel's commentary distinguishes between the physiological and behavioural levels. As to the physiology related to binge eating, we would not adopt Siegel's opinion that failures to demonstrate compensatory responses are minor challenges to the conditioning theory of binge eating. The physiological events (if any) underlying the urge to eat might turn out to be entirely different from those hypothesized, prompting reformulation or even rejection of CBE theory ${ }^{5}$. Nevertheless, on the behavioural level CBE theory appears to be working well, given the success of cue exposure. There is by now accumulating data suggesting this (section 2.2.6), so that the situation for binge eating is no different than that for conditioned drug tolerance. For therapeutic purposes this situation need not be disastrous: CS (binge cues) and UCS (binge eating) and their relationship are transparent.

$S$ For example, it might be that the peripheral, conditioned compensatory responses that have been previously observed in addiction research (see the overview presented in Macrae, Scoles \& Siegel, 1987), can be understood as serving homeostatic regulation (see e.g. chapter 4, this thesis), whereas craving and other behavioural effects critically depend on as yet unknown central effects (occurring within the brain). 
The therapist's position could be compared with that of a radio owner who, although fairly skilled at operating the knobs, remains unaware of the radio's interior electronics. For both therapist and basic researcher, however, uncertainty remains about the mechanism through which the habit of binge eating was acquired in the first place. To summarize and conclude, the physiological mechanism of urge (if any) remains elusive; the major advance relative to the "urge-less" theory of binge eating (e.g. counterregulation theory, section 1.3), is that a maintenance factor appears to have been identified, that can be cast in conditioning terms and used for therapeutical purposes; the contribution of learning to binge eating has not been exhaustively studied. Important questions remain about the physiological and acquisition mechanisms in binge eating.

Next, theoretical (section 9.4) and practical (section 9.5) directions for future research will be proposed.

\subsection{FUTURE RESEARCH: THEORETICAL CONSIDERATIONS}

In this section, two directions ${ }^{6}$ in research on the role of learning in binge eating will be proposed. The first approach should be considered an update of the conditioned hypoglycaemia/hyperinsulinaemia explanation that was originally offered by CBE theory. In the second approach, repeated post-binge vomiting and restriction are identified as key factors in binge eating. It may well be that both mechanisms are simultaneously active in binge eaters, judging from reports that exposure therapies directed at either mechanism have been effective in reducing binge eating (Schmidt \& Marks, 1989). Practical research implications of the theorizing will be described in sections 9.5.3 and 9.5.4.

\subsubsection{Maintaining a blood sugar/insulin-related explanation}

Reliable pre-meal blood sugar fall: characteristics When one still looks for a role of learned insulin and glucose dynamics in craving and binge eating, a recent development may provide clues. Campfield and Smith (1990a; 1990b) have reported a robust role of insulin and blood sugar signals in meal initiation. Blood sugar levels were continuously monitored in free moving rats with ad lib food available. Campfield and Smith claim

6 A third, social learning approach, which is in current upswing in cue-reactivity research (Tiffany 1995a; 1995b) will not be elaborated further here. First, because it explicitly rejects the idea that craving is a central mechanism in relapse (Tiffany, 1995b, p. 155; this might be the case for drug and alcohol addictions, but is rather unlikely for binge eating), and second, because apparently, the social learning account of relapse has not yet reached a stage at which convincing lab models may be developed (Bradizza, Stasiewicz \& Maisto, 1994). 
that almost without any exception, ad lib meal intake in the rats was preceded by a transient blood sugar fall of at least $6 \%$ below baseline, lasting for more than 6 minutes. Meal initiation occurred at 12.1 (sd 1.7) minutes after the beginning of the blood sugar fall, i.e. 5.4 (sd 1.5) minutes after the nadir of the hypoglycaemia. Other characteristics of the robust hypoglycaemia-food intake sequence were revealed in numerous experiments: if food became available at 5 minutes after the hypoglycaemia had disappeared, no food intake occurred; if the hypoglycaemia was countered by a rapid glucose infusion, no feeding occurred; if the experimenters mimicked a hypoglycaemia by infusion of other biochemicals, food intake occurred if the characteristics of the glycaemic curve resembled those of the spontaneous hypoglycaemias; no within-subjects correlation was found between the depth of the hypoglycaemia and the amount of subsequent intake. One origin of the spontaneous hypoglycaemias was found to lie in a prior short-lived insulin increase (50\% above baseline).

The results have been confirmed in humans whose blood sugar level was monitored continuously for a prolonged period (Campfield et al., 1992; 1996). The subjects could require a meal if they wanted. It was found that hypoglycaemias of $6-10 \%$ below baseline preceded meal request. These blood sugar dynamics also correlated with selfreported hunger.

Commentary Exactly how the hypos described by Campfield and Smith might relate to learning and conditioning is not clear yet. The following comments can be made.

1. In contrast to the $\mathrm{CBE}$ theory account, the hypoglycaemias (and the preceding hyperinsulinaemias) described by Campfield and Smith are short-lived and signal meal initiation rather than compensate for homeostatic disturbances.

2. The size of cue elicited (cephalic and conditioned) hypoglycaemias reported in some of the human studies (Broberg \& Bernstein, 1989; Mityushov, 1954/Appendix this thesis) is within the range of the meal-preceding hypoglycaemias found by Campfield and Smith. Moreover, the hypos were preceded by a small 50\% insulin "spike", the size of which is that of the typical (cue-elicited) insulin responses (see sections 2.2 and 9.2, this thesis).

3. Are the observed hypoglycaemias endogenous and/or learned in nature? In his commentary, Woods (1990) has argued against Campfield and Smith's purely endogenous explanation. He points to the fact that the rats in the Campfield \& Smith studies were kept in a very stable monotonous environment, whereas in a sensorily richer environment the rats' behaviour (e.g. the observed hypoglycaemia and food intake!) might become more easily attuned to external (meal-related) events and other cues. Interestingly, the only meals not preceded by hypoglycaemia concerned foods that were novel to the rats. As the rats got acquainted with the food items, gradually the hypoglyc- 
aemias began to appear as with other foods (Dr. Campfield, personal communication). This suggests a role of learning in the meal preceding hypoglycaemias.

4. Now that the moderately sized, short-lived hypoglycaemias found by Campfield and Smith appear to be playing a role in normal food intake, it still remains elusive in what way these responses might contribute to binge eating. Two possibilities may be mentioned. First, one might speculate that in binge eaters, for some reason the frequency of transient hypoglycaemias exceeding some critical threshold is increased (relative to healthy normals) before or during binge eating. Frequent hypoglycaemic dips might be experienced subjectively as "waves of craving" leading to temptation to binge (or continuing the binge if the hypos were superimposed on the post-prandial blood sugar rise $^{7}$ ). Considering the turmoil reigning in the blood sugar and insulin domain after food intake, the latter speculation remains problematic. A related possibility might be that the hypoglycaemic mechanism only induces binge-initiation, and that during the binge another principle is at work (see for instance section 9.4.2).

5. Finally, it also could turn out that hypoglycaemias occur equally in normals and binge eaters, but that binge eaters are especially inclined to interpret small hypoglycaemias as hunger. In fact, from research on hypoglycaemic episodes in diabetic patients, it is known that there is an individual stereotype in the experienced symptoms. There are "trembling/sweating" individuals, whereas others report predominantly hunger during hypoglycaemia (Hepburn, 1993; Service, 1995). Nevertheless, an explanation based merely on differential interpretation of body signals is likely to be too weak to account for the particular vigour with which binge eating proceeds (see section 1.2).

\subsubsection{Learning perspective based on post-meal deprivation and vomiting}

There is consensus among food intake researchers that meal size is correlated not only with momentary, but also with expected level of deprivation (see Collier, 1986; Davey, 1989; Le Magnen, 1992; Weingarten, 1985; Woods \& Strubbe, 1994). Moreover, binges of bulimics and anorexics of the binge eating/purging type (APA, 1994) are part of a eating pattern of restriction, fasting and vomiting. While these behaviours are meant to be "rational" means of weight restriction, the possibility of their becoming maintenance factors in binge eating should not be ruled out. This section will review various studies to suggest that yet another learning mechanism may contribute to bingeing: learned cues that reliably predict the occurrence of deprivation after a meal might

7 As noted in section 2.2, Louis-Sylvestre (1984) did demonstrate short insulin bursts after introduction of new food items during meals in rats. It is equally true that changing the foods during a meal increases total intake. 
increase the size of that meal. The data presented below will be used as a starting-point for a new lab model of binge eating (section 9.5.3).

Repeated fasting after meals may increase meal size Although no systematic data have been collected, it seems safe to say that binge eating episodes are frequently followed by a period of self-imposed restriction and/or vigorous exercise (APA, 1994). There is one report that suggests that meal size might increase if deprivation reliably follows that meal (Le Magnen, 1992, ch. 1). Rats were adjusted to a diet of 3 daily one-hour meals a day at fixed times for 18 days. Then one of the three meals was omitted daily, thus creating the following schedule: meal I - 7 hours - meal II -- 15 hours -- meal I, and so on. Food intake was monitored for another 18 days. After several days, a clear meal size pattern developed: meal II, i.e. the meal preceding the 15 -hour deprivation, became considerably larger than meal I, and ultimately increased with about $90 \%$ of the calories of the now omitted third meal. This effect, found irrespectively of the time of day, was taken to suggest that rats adjust their meal size by using information of future availability of food. Meal size increases when post-meal deprivation is expected. At least until recently, no human research had been done on this mechanism (Le Magnen, 1992). The implication is that if bingeing is reliably followed by prolonged restriction or fasting, this might lead to a learned increase of the pre-fasting (i.e. binge) meal.

Repeated vomiting after meals may increase meal size Binges are often followed by vomiting in $80-90 \%$ of the bulimic patients (APA, 1994; Rosen et al., 1986). Post-binge vomiting appears to have an enhancing impact on bingeing itself. Firstly, the speed of eating appears to be larger in purging than in non-purging bulimics (compare Rossiter et al., 1992 with Walsh, 1993). Secondly, the binge size of purging bulimics is twice of that of non-purging bulimics (compare Rossiter \& Agras, 1990 with Rossiter et al., 1992) ${ }^{8}$. It will be suggested here that, if bingeing is reliably followed by vomiting, a learning mechanism might induce learned increase of the fast-preceding meal (i.e. the binge) itself. This allegation will be substantiated by data on physiological state after vomiting, operant conditioning, conditioned sham feeding, and conditioned desatiation studies.

First, post-binge vomiting may lead to a temporary state of deprivation that bears upon food intake. Johnson et al. (1994) had 12-hour fasted bulimic subjects binge and vomit subsequently during which blood sugar and insulin levels were monitored. After

${ }^{8}$ Almost certainly, this can be explained by the simple fact that vomiting creates space for yet more binge food. Hence, it becomes understandable how the largest binge sizes reported in the literature could have been in the $20,000-55,000 \mathrm{kcal}$ range, i.e., between 10 and 28 times the average daily food intake in the normal population and, literally, impossible to stomach (Russell, 1979; Walsh, 1993). 
vomiting a steep decline of blood sugar level was found (12 $\mathrm{mg} / \mathrm{dl}$ within 15 minutes) bringing blood sugar back at baseline level. Concurrently, however, the insulin level remained twice as high as baseline ${ }^{9}$. A similar overshoot of insulin level after a 3000 kcal binge and subsequent vomiting in bulimics was found by Kaye et al. (1989), and it might imply a momentary glycaemic state of deprivation ${ }^{10}$. Direct evidence that expected insulin overshoot may produce instrumental food seeking and eating comes from a study by Siegel and Nettleton (1970). Four groups of rats were housed in Skinner boxes. Group A received repeated insulin injections and had the possibility to bar-press for food, while Group B received insulin injections without this possibility. Control groups $C$ and $D$ received saline injections with and without bar pressing possibility respectively. During the post-conditioning test session, saline was injected in all groups and bar pressing frequency was monitored: group A rats clearly showed the largest bar pressing rate of all groups, while at a pre-conditioning test session the rates had been equal for all groups. Siegel and Nettleton (1970) concluded that the rats of group $\mathrm{A}$ had acquired an instrumental feeding response to reduce expected "insulininduced distress".

Second, sham feeding studies may be relevant as an analogue of post-binge vomiting: large food-intake is signalled orally, but the amount of nutrients subsequently absorbed does not follow suit. Van Vort and Smith (1987) have shown that flavours associated with sham feeding enhance meal size. Rats were provided with a fistula that could be opened (sham feeding) as well as closed (real feeding). During a conditioning procedure, the rats were given a diet of milk. In alternating sequence, the milk had either an added flavour which signalled sham feeding (CS-) or a flavour that preceded real feeding $(\mathrm{CS}+)$. A post-conditioning test with fistulas closed (the real feeding situation) showed that the amount of milk flavoured with the CS- taste was drunk in larger amounts (33$50 \%$ more) than the previous CS + flavoured milk. The effect was immune to extinction for at least three post-conditioning test trials. Similar results have been found in other

${ }^{9}$ Methodologically unfortunately, in the Johnson et al. procedure, the vomiting was followed by renewed ingestion so that no data are available of completed binge-purge cycle that ends with purgeing.

${ }^{10}$ See also Davidson (1993). Rats were trained to display a set of motor acts. Each motor act was acquired under a unique and fixed deprivation level. After training, insulin injections led the rats to display the motor act acquired during 24-hour deprivation. As a comparison, injections with the satiety hormone cholecystokinine led the rats to display the motor act which had been learned under 0 -hour deprivation. These observations are in line with the idea that an excess of insulin (or its physiological consequences) and deprivation may share a common interoceptive quality. 


\section{Chapter 9}

studies as well (see Le Magnen, 1992, ch. 6). For binge eating this might imply that oral stimulation by binge food ("CS"), reliably followed by disproportionally small nutrient absorption (because of vomiting), may lead to conditioned meal enlargement.

A final data source is conditioned desatiation research in rats. Booth $(1985 ; 1991)$ has advocated that meal size is under influence of expected post-meal signals of satiety (e.g. nutrients appearing in the blood). After repeatedly pairing a flavour with carbohydrate dense food (strongly satiating), Booth added this flavour to different foods and saw decreased meal size in rats. In contrast, a dramatic increase in meal size was found in rats when during a meal involving high caloric food, a flavour was introduced that had been previously associated with low post-ingestive satiety. This increased intake was coined "conditioned desatiation"11 (Booth, 1985; 1991; Le Magnen, 1992, ch. 6). For binge eaters, the taste of binge foods might become associated with relatively low satiation (due to vomiting relatively little is actually absorbed ${ }^{12}$ ). Hence, conditioned desatiation to binge cues might act to increase intake of typical binge foods.

Breaking the binge-deprivation association decreases binge eating This has become apparent from the results of a therapeutic procedure called binge-exposure with prevention of vomiting (Rosen \& Leitenberg, 1982; Leitenberg et al., 1984; Schmidt \& Marks, 1989). The procedure involves several sessions in which the patient ingests binge food past the point where the urge to vomit develops. However, the patient is prevented from vomiting and the therapist remains with the patient until the urge to vomit has declined. Several studies have reported that this procedure leads to important reductions of binge eating ${ }^{13}$ (Rosen \& Leitenberg, 1982; Leitenberg et al., 1984; Schmidt \& Marks, 1989;

11 Two critical remarks are in place here. A vast literature exists in which flavour CSs predicting bigh nutrient value (UCS) increase preference of the flavour (for a review see Sclafani, 1995). Just how to reconcile these robust findings with the data supporting conditioned desatiation constitutes an as yet unresolved issue. Second, conditioned desatiation certainly must have its limits. The implication that non-nutritive sweeteners, as used in diet drinks, should lead to an ever-increasing intake of these drinks, does not stand closer scrutiny (e.g. Jansen, 1994b).

12 There are at least four indications suggesting a modest intestinal absorption of nutrients from binge foods expelled by self-induced vomiting. Firstly, vomiting generally takes place soon after the binge, before the food has entered the small intestine. Secondly, when large amounts of foods are ingested, gastric emptying (i.e. the transit of foods from the stomach into the small intestine) slowens down (McHugh, 1983). Thirdly, rapid eating (such as especially bulimics of the purging type do (Rossiter et al., 1992; Walsh, 1993) with little chewing slowens down the speed with which ingested nutrients appear in the blood (Read et al, 1986). Fourthly, bulimic patients' gastric emptying of small amounts of food has been found to be slower than that of healthy individuals (Geliebter et al., 1992).

${ }^{13}$ Parenthetically, the surplus value of exposure with prevention of vomiting relative to cognitive behavioural treatments of binge eating has been the subject of continuing discussion (Agras et al., 1989; Leitenberg \& Rosen, 1989; Cooper \& Steere, 1995). 
Cooper \& Steere, 1995). Note that these results are contrary to predictions of CBE theory, because, according to CBE theory, substantial ingestion of binge foods would be equal to a conditioning trial in which binge cues (CS) are paired with bingeing (UCS; see section 2.2, this thesis). A common characteristic of this therapy and CBE inspired cue-exposure with binge prevention procedure (section 2.2.6) is that the unrolling of a stereotypic behaviour chain during the binge episode is thwarted. Although the rationale of the Rosen and Leitenberg procedure was derived from anxiety research (Leitenberg et al., 1984), it might be considered an example of extinction of a learned association between binge cues (or bingeing itself) ("CS") and post-binge deprivation state ("UCS"). Additional remarks If the mechanism of signalled post-binge deprivation indeed exists and contributes to binge eating, it might be enhanced by pre-binge deprivation. Repeated deprivation experience may induce learned increase in food intake to deprivation-related cues (Weingarten, 1985; Revusky \& Garcia, 1970). Theoretically, the classical conditioning terminology may not be entirely appropriate in this case: intake of certain foods (a response accompanied by sensory stimulation) is regarded as a cue (the CS), and postmeal deprivation (a state following active responses) is considered the UCS. It may be that the mechanism might be better described by regarding binge eating as an instrumental response, performed in anticipation of deprivation, i.e., an "economic" property of the feeding system that can be found in most animals (Davey, 1989, ch. 7). Because of the present lack of rigorous terminology, and for practical purposes (e.g. the modelling of binge eating; section 9.5.3), we will, until further notice, stick to the CS-UCS terminology derived from classical conditioning.

\subsection{FUTURE RESEARCH: PRACTICAL CONSIDERATIONS}

Three lab models of binge eating will be presented in this final section. As will be explained, the models serve two practical purposes. Firstly, to provide a framework for basic research on learning mechanisms in binge eating. Secondly, as procedures to establish "miniature binges" in the lab, to be utilized for pre-clinical research on the effectiveness of newly developed treatments.

\subsubsection{The necessity of modelling}

In chapter one, it was explained that experimental psychopathology involves the hypothesizing and investigation of mechanisms that underlie behavioural disorders. This is the fundamental approach. Additionally, a more practical mission of experimental 


\section{Chapter 9}

psychopathology research is (or rather, could be) the testing of new behavioural techniques proposed by the therapeutic or scientific community. Not unlike the screening of newly developed psychopharmaceuticals (Willner, 1991a), behavioural techniques might be tested for their preventive or curative value. But how might this be accomplished in practice? One method that will be proposed here is the development of "lab models". As explained in chapter one, "modelling" refers to the reliable production of behaviours in the lab that can be considered a "miniature" analogue of the psychopathology under study.

In this final section, three possible lab models of binge eating will be described: the cue reactivity model, the conditioned hypoglycaemia model, and the post-meal deprivation model. All rely on the theoretical CBE notion that binge eating is a learned, cue-driven, and self-perpetuating behaviour ${ }^{14}$. Each lab model requires fundamental, preparatory research (see section 1.1). First, some essential parameters of binge eating should be identified in patients (type $P$ research) and/or in specifically selected non-clinical subjects (type $Q$ research). Once type $Q$ experiments have demonstrated replicable responses related to binge eating, a potential lab model may be within reach. Modelling the disorder is not a final goal, but the beginning of the practical stage (Willner, 1991b): in subsequent type $\mathrm{R}$ research, one attempts to "treat" the lab bingeing using the new behavioural technique. If proven effective in this way, the behavioural technique might be passed on for use in clinical trials.

Importantly, a workable lab model of binge eating should include some core manipulation that produces a significant and reliable increase of craving and/or food intake. In more lively terms, the proof of a binge eating model, like the proverbial pudding, should be in the eating (Herman \& Polivy, 1980; Jansen, 1990), and interpretation of the effects of the basic manipulation should be straightforward and require no "harking" whatsoever ${ }^{15}$.

Note that the empirical data in the present thesis have not been very encouraging as to the feasibility of model development on the basis of $C B E$ principles. Thus, the three models presented next should also be considered as coordinated and ultimate attempts to test the validity of $\mathrm{CBE}$ related theorizing.

${ }^{14}$ Human models of binge eating, especially if inspired by learning theory, have been rare: the counterregulation model has dominated the field (see section 1.3). In general, models relevant for binge eating have been largely confined to animal research: they are stress models (Greeno \& Wing, 1994), nutritional reward models (Sclafani, 1995) and biochemical and surgical models of overeating (Montgomery, 1991).

${ }^{15}$ Harking = Hypothesizing After the Results are Known (van der Heijden, 1995). 


\subsubsection{Model I: the cue reactivity model of binge eating}

Backeround One interesting aspect of psychophysiological/cognitive cue reactivity is its possible use as a diagnostic index of relapse danger in treated patients. In basic research there are three facets of cue reactivity to be studied: its dependency on learning history, its occurrence after cue presentation, and its influence on food intake. A practical advantage of this type of approach in modelling is that demonstration of cue reactivity requires no painstaking learning procedures. For this reason cue reactivity experiments are sometimes referred to as "opportunistic" experiments (Glautier \& Tiffany, 1995) Type $\mathrm{P}$ research Comparing patients and controls; searching for parameters.

* Cognitive changes after cue presentation: Stroop food word interference has already been amply demonstrated in binge eaters (see Chapter 7); However, such interference has not yet been demonstrated in clinical subjects after exposure to cues, and/or before the onset of a binge ${ }^{16}$.

* Increased psychophysiological food cue reactivity should be demonstrated in binge eaters. Parenthetically, it might well turn out to be intrinsically impossible to disentangle appetitive and emotional factors in responses to food cues in bulimic patients (compare e.g. Niaura et al., 1988).

* It should be demonstrated that food cue presentation increases subsequent ad lib food intake in patients. This is relevant even if no sensitive psychophysiological cue reactivity parameters might be identified. It could also be investigated whether high reactivity to food cues influences food intake of the items itself or also generalizes to the food item itself, or generalizes to other foods as well (compare Cornell, Rodin \& Weingarten, 1989).

Type $\mathrm{Q}$ research Comparing non-clinical subjects with different learning history; withinsubject comparisons of reactivity to foods differing in habitual intake.

* Subjects should be selected on the basis of either scores on scales specifically tapping disinhibitive eating (e.g. the DIS, see chapter 8), or more rigorously, on the basis of explicit self-reports on eating habits. These might include frequency estimates of bingeing and specification of meal size and particular foods involved.

* Foods could be allocated to a small number categories according to the frequency and/or habitual amount that is ingested. Cue reactivity to food cues belonging to these

${ }^{16}$ If the urge-to-act explanation of Stroop interference (Chapter 7, this thesis) is valid, it might be interesting to use an adapted version (with weight concern/vomiting-related words) within the context of Rosen \& Leitenberg's binge food exposure procedure (section 9.4.2). In patients who have just eaten typical binge foods, such Stroop tests might be used to assess suddenly aroused weight concems and tendencies towards self-induced vomiting. 


\section{Chapter 9}

categories could be compared in a within-subjects design.

* Sensitive physiological parameters of food cue reactivity should be sought for.

* As in type P research, another next step is to assess the impact on intake of a subsequently offered food. Intake should be larger after cue-presentation than after no cue presentation.

* If possible, at least two separate sessions (one with and one without cue presentation) should be held, to ensure equal deprivation level during cue presentation. Care should then be taken that no intake neophobia appears (Chapter 3). A prerequisite would be balancing of the order of cue and non-cue sessions. Furthermore, as intake-neophobia appears to fade due to exposure to the lab environment (Chapter 3 ), it might also be considered to run one or several habituation sessions, before running the experimental sessions.

Type $\mathrm{R}$ research Establishing and using the cue reactivity model of binge eating.

* Core manipulation: cues are presented and food intake and craving measured. A within-subject design might be employed with non-clinical binge prone subjects. Responses to different categories of food cues (see under type $Q$ research), or between food cues and neutral or no cues are compared.

* Core results: food intake and craving should be larger after cues predicting large food intake, than after cues predicting small or no food intake.

* Using the model: once the model is established, practical questions about binge eating might be answered by introducing additional manipulations on the normal subjects (e.g. instructions, mood inductions, prolonged cue exposure, instructed eating patterns, homework), and an assessment of their impact on the lab-overeating produced by the core manipulation.

\subsubsection{Model II: the conditioned hypoglycaemia model of binge eating}

Background In contrast to the cue reactivity model, the conditioned hypoglycaemia model of binge eating requires an explicit learning procedure. A starting point for model development might be a procedure resembling Mityushov's (1954; chapter 4). Before describing the model, a practical consideration is in place here. Conditioning procedures like Mityushov's are laborious and time-consuming. Because, at this moment, the role of hypoglycaemia/hyperinsulinaemia in binge eating remains to be established (see section 9.3), top priority should be given to research which demonstrates reliable and significant (conditioned) hypoglycaemia or hyperinsulinaemia during fully-fledged binge attacks in patients. If these responses cannot be demonstrated, then hypoglycaemia/hyperinsulinaemia clearly do not tap the essence of binge eating and there would seem little point - at least for binge eating research - to make the extensive effort of modelling binge eating in 
humans by glycaemic conditioning. Alternatively, if binge eating attacks are indecd preceded by significant hypoglycaemia/hyperinsulinaemia, then a powerful model of binge eating might be within reach.

Type $\mathrm{P}$ research Studies in patients.

* Blood sugar and insulin levels should be monitored on line during binge attacks. The same should be done when, after exposure to binge foods, craving is high up. These measurements should preferably be carried out at patients' typical binge locations.

Type Q research Comparing subject groups with different learning history.

* Possible research would resemble cue reactivity research type II, but in this case blood sugar and insulin responses are measured. The Broberg and Bernstein (1989) study, which as far as the present author is aware, is the only convincing report of cephalic hypoglycaemia in a sample of healthy individuals (albeit dieting), should be replicated.

* The relationship between cephalic hypoglycaemia and subsequent food intake should be established.

Type $\mathrm{R}$ research Establishing and using the conditioned hypoglycaemia model of binge eating.

* Core manipulation: the unconditioned stimuli must involve a strong blood sugar rise. Conditioning with oral glucose has failed (Chapter 3). Effective conditioning with food would probably require even more trials, given the moderate glycaemic index of common foods (Brand Miller et al., 1995). The procedure used in the study by Mityushov (1954; appendix A) might offer more possibility and should be replicated. If the hypoglycaemic state could be conditioned, the procedure should first be extended to food. This could be done by introducing intake-related CS to the injection ritual, and measure ad lib food intake during the hypoglycaemic state.

* Core results: a robust binge eating model would exist if $\mathrm{CS}^{*}$ presentation were found to enhance craving and food intake (via hypoglycaemia), relative to a $\mathrm{CS}^{-}$or neutral situation, and/or unconditioned subjects.

* Using the model: see under the cue reactivity model type $\mathrm{R}$ research.

\subsubsection{Model III: the post-meal deprivation model of binge eating}

Background In section 9.4.2, an overview was given of data suggesting that meal size can be increased by associating meal cues with post-meal deprivation. These data suggests practical ways in which a lab analogue of bingeing in non-clinical subjects might be evoked after an explicit conditioning procedure.

Type $\mathrm{P}$ research Studies in patients.

* Data are needed on the probability relationship between bingeing and fasting in patients on the individual level. What is the probability that bingeing is followed by 


\section{Chapter 9}

prolonged fasting? Are there specific cues (i.e. certain foods, moods or circumstances) that predict whether binge eaters go on a prolonged fast after bingeing? Such data might be collected more systematically through the use of intake diaries.

* Similar data to that above must be acquired on the probability relationship between bingeing and self-induced vomiting within individuals.

* The short-term physiological consequences of self-induced vomiting after bingeing have received too little attention. To what extent does a metabolic state resembling deprivation occur after expelling large food quantities?

Type $O$ research Studies in non-clinical subjects.

* no strong suggestion can be made at this point.

Type $\mathrm{R}$ research Establishing and using the signalled deprivation model of binge eating.

* core manipulation: this procedure would consist of repeatedly pairing cues $\left(\mathrm{CS}^{+}\right)$with a "compound UCS" of a meal followed by an 8-9 hour deprivation. A within-subject design could be employed, comparing craving and food intake after $\mathrm{CS}^{+}$with no $\mathrm{CS}$ or a $\mathrm{CS}^{-}$. The $\mathrm{CS}^{-}$should be a signal for "no post-meal deprivation", for example by pairing the same meal followed by a period of 8-9 hours in which food is repeatedly ingested by subjects. The CSs could lie within the food itself (flavouring, colouring) or in the way it has to be ingested (typical room, small spoons; strange plates).

* Core results: after conditioning, ad lib ingestion of test food should be robustly larger after $\mathrm{CS}^{+}$than after $\mathrm{CS}^{-}$presentation.

* Using the model: see the cue reactivity model, type $\mathrm{R}$ research.

\section{Finis}






\section{REFERENCES}

A.P.A. (1994). Diagnostic and Statistical Manual of Mental Disorders 4th ed. Washington: American Psychiatric Association.

Abraham, S.F. \& Beumont, P.J.V. (1982). How patients describe bulimia or binge eating. Psychological Medicine 12, 625-635.

Abramson, L.Y. \& Seligman, M.E.P. (1977). Modeling psychopathology in the laboratory. In: J.D. Maser \& M.E. Seligman (Eds.), Psychopathology: Experimental Models, pp 1-26. San Francisco, W.H. Freeman.

Ádám, G. (1967). Interoception and Behaviour. Budapest: Hungarian Academy of Sciences.

Agras, W.S., Schneider, J.A., Arnow, B., Raeburn, S.D. \& Telch, C.F. (1989). Cognitive-behavioral and response prevention treatments for bulimia nervosa. Journal of Consulting and Clinical Psychology 57, 215-221.

American Psychiatric Association (1987). Diagnostic and Statistical Manual of Mental Disorders (3rd Revised. Ed.). Washington, DC: Author.

Anthony, B.J. (1985). In the blink of an eye: implications of reflex modification for information processing. Advances in Psychophysiology 1, 167-218.

Babkin, B.P. (1949). Pavlov: a Biography. Chicago/London: University of Chicago Press

Bantle, J.P., Laine, D.C., Castle, G.W., Thomas, J.W., Hoogwerf, B.J. \& Goetz, F.G. (1983). Post prandial glucose and insulin responses to meals containing different carbohydrates in normal and diabetic subjects. New England Journal of Medicine 309, 7-12.

Barnett, S.A. (1958). Experiments on "neophobia" in wild and laboratory rats. British Journal of Psychology 49, 195-201.

Beck, A.T, Rusch, A.J., Shaw, B.F. \& Emery, G. (1979). Cognitive Therapy of Depression. New York: Guilford Press.

Beebe, D.W. (1994). Bulimia nervosa and depression: a theoretical and clinical appraisal in light of the binge-purge cycle. British Journal of Clinical Psychology 33, 259-276.

Bellisle, F., Louis-Sylvestre, J. Demozay, F., Blsazy, D. \& Le Magnen, J (1983). Reflex insulin response associated to food intake in human subjects. Physiology and Behavior 31, 515-521.

Ben-Tovim, D.I., Walker, M.K., Fok, D. \& Yap, E. (1989). An adaptation of the Stroop Test for measuring shape and food concerns in eating disorders: a quantitative measure of psychopathology? International Journal of Eating disorders 8, 681-687.

Ben-Tovim, D.I. \& Walker, M.K. (1991). Further evidence for the Stroop Test as a quantitative measure of psychopathology in eating disorders. International Journal of Eating Disorders 10, $609-613$.

Berthoud, H.-R., Bereiter, D.A., Trimble, E.R., Siegel, E.G. \& Jeanrenaud, B. (1981). Cephalic phase, reflex insulin secretion: neuroanatomical and physiological characterization. Diabetologia $20,393-401$.

Beumont, P.J.V. (1995). The clinical presentation of anorexia and bulimia nervosa. In, K.D. Brownell \& Ch. G. Fairburn (Eds), Eating Disorders and Obesity: a Comprehensive Handbook, pp. 151-158. New York, London: Guilford Press.

Birch, L.L., McPhee, L., Sullivan, S. \& Johnson, S. (1989). Conditioned meal initiation in young children. Appetise 13, 105-113.

Blouin, A.G., Blouin, J.H., Braaten, J.T., Sarwar, G., Bushnik, T. \& Walker, J. (1991). Physiological and psychological responses to a glucose challenge in bulimia. International Journal of Easing Disorders 10, 285-296.

Blouin, A.G., Blouin, J., Bushnik, T., Braaten, J., Goldstein, C. \& Sarwar, G. (1993). A doubleblind placebo-controlled glucose challenge in bulimia nervosa: psychological effects. Biological Psychiarry 33, 160-168.

Booth, D.A. (1985). Food-conditioned eating preferences and aversions with interoceptive elements: conditioned appetites and satieties. Annals of the New York Academy of Sciences 443, 22-41. 


\section{References}

Booth, D.A. (1991). Protein- and carbohydrate specific cravings: neuroscience and sociology. In, M.I. Friedman, M.G. Tordoff and M.R. Kare (Eds.), Chemical Senses, Vol. 4: Appetile and Nurrition, pp. 261-276. New York: Marcel Dekker.

Boucsein, W. (1992). Electrodermal Activity. New York: Plenum Press.

Bouwmeester, D. (1995). Quantum Mechanics and Classical Optics. Thesis. Leiden: University of Leiden.

Bradizza, C.M., Stasiewicz, P.R. \& Maisto, S.A. (1994). A conditioning reinterpretation of cognitive events in alcohol and drug cue exposure. Journal of Behavior Therapy and Experimental Psychiatry 25, 15-22.

Bradley, M.M., Cuthbert, B.N. \& Lang, P.J. (1990). Startle reflex modification: emotion or attention? Psychophysiology 27, 513-522.

Brand Miller, J., Pang, E. \& Broomhead, L. (1995). The glycaemic index of foods containing sugars: comparison of foods with naturally-occurring vs. added sugars. British Journal of Nutrition $73,613-623$.

Briggs, S.R. \& Cheek, J.M. (1986). The role of factor analysis in the development and evaluation of personality scales. Journal of Personality 54, 106-148.

Broberg D.J., Bernstein, I.L. (1989). Preabsorptive insulin release in bulimic women and chronic dieters. Appetite 13, 161-169.

Brodsky, I.G. \& Devlin, J.T. (1994). Hormone and nutrient interactions. In, M.E. Shils, J.A. Olson \& M. Shike (Eds), Modern Nutrition in Health and Disease, 8th ed., 603-622. Philadelphia Baltimore, etc: Lea \& Febiger.

Bruce, B. \& Agras, W.S. (1990). Binge eating in females: a population-based investigation. International Journal of Eating Disorders 12, 365-373.

Bruce. D.G., Storlien, L.H., Furler, S.M. \& Chisholm, D.J. (1987). Cephalic phase metabolic responses in normal weight adults. Metabolism 36, 721-725.

Campfield, L.A. \& Smith, F.J. (1990a). Transient declines in blood glucose signal meal initiation. International Journal of Obesity 14 (suppl 3), 15-31.

Campfield, L.A. \& Smith, F.J. (1990b). Systemic factors in the control of food intake: evidence for patterns as signals. In E.M. Stricker (Ed), Handbook of Behavioral Neurobiology vol. 10: Neurobiology of Food and Fluid Intake, pp. 183-206.

Campfield, L.A., Smith, F.J., Rosenbaum, M. \& Geary, N. (1992). Human hunger: is there a role for blood glucose dynamics? Appetite 18, 244.

Campfield, L.A., Smith, F.J., Rosenbaum, M. \& Hirsch, J. (1996). Human eating: evidence for a physiological basis using a modified paradigm. Neuroscience and Biobehavioral Reviews 20, 133 . 137.

Cannon, W.B. (1939). The Wisdom of the Body 2nd ed. New York: W.W. Norton.

Carlson, H.E. \& Shah, J.H. (1989). Aspartame and its constituent amino acids, effects on prolactin, cortisol, growth hormone, insulin, and glucose in normal humans. American Journal of Clinical Nutrision 49, 427-432.

Casper, R.C., Pandy, G.N., Jaspan, J.B. \& Rubenstein, A.H. (1988). Hormone and metabolite plasma levels after oral glucose in bulimia and healthy controls. Biological Psychiarry 24, 663-674.

Catania, A.C. (1991). Glossary. In I.H. Iversen \& K.A. Lattal (Eds.), Experimental Analysis of Behavior, vol. 2. Amsterdam etc: Elsevier.

Channon, S., Hemsley, D. \& de Silva, P. (1988). Selective processing of food words in anorexia nervosa. British Journal of Clinical Psychology 27, 259-260.

Channon, S. \& Hayward, A. (1990). The effect of short-term fasting on processing of food cues in normal subjects. International Journal of Earing Disorders 9, 447-452.

Charnock, D.J.K. (1989a). A comment on the role of dietary restraint in the development of bulimia nervosa. British Journal of Clinical Psychology 28, 329-340.

Charnock, D.J.K. (1989b). Exercising restraint: a response to Polivy \& Herman. British Journal of 
Clinical Psychology 28, 343-346.

Childress, A.R, Ehrman, R., Rohsenow, D.J., Robbins, S.J. \& O'Brien, Ch.P., 1992. Classically conditioned factors in drug dependence. In J.H. Lowinson, P. Ruiz, R.B. Millman \& J.G. Langrod (Ed.), Substance Abuse: a Comprehensive Texibook, 2nd Ed. pp 56-69. Baltimore etc: Williams \& Wilkins.

Christensen, C.M. \& Navazesh, M. (1984). Anticipatory salivary flow to the sight of different foods. Appetite 5, 307-315.

Clark, D.M. (1983). On the induction of depressed mood in the laboratory: evaluation and comparison of the Velten and musical procedures. Advances in Behaviour Research \& Therapy 5, 27-49.

Collier, G. (1986). The dialogue between the house economist and the resident physiologist. Nutrition \& Behavior 3 9-26.

Comrey, A.L. (1978). Common methodological problems in factor analytic studies. Journal of Counseling and Clinical Psychology 46, 648-659.

Cools, J., Schotte, D.E. \& McNally, R.J. (1992). Emotional arousal and overeating in restrained eaters. Journal of Abnormal Psychology 101, 348-351.

Cooper, M.J., Anastasiades, P. \& Fairburn, C.G. (1992). Selective processing of eating-, shape-, and weight-related words in persons with bulimia nervosa. Journal of Abnormal Psychology 101, 352 . 355.

Cooper, M.J. \& Fairburn, C.G. (1993). Demographic and clinical correlates of selective information processing in patients with bulimia nervosa. International Journal of Eating Disorders 13, 109 116.

Cooper, P.J. (1995). Eating disorders and their relationship to mood and anxiety disorders. In, K.D. Brownell \& Ch. G. Fairburn (Eds), Eating Disorders and Obesiry: a Comprehensive Handbook, pp. 159-164. New York, London: Guilford Press.

Cooper, P.J. \& Steere, J. (1995). A comparison of two psychological treatments for bulimia nervosa: implications for models of maintenance. Behaviour Research \& Therapy 33, 875-885.

Cornell, C.E., Rodin, J. \& Weingarten, H. (1989). Stimulus-induced eating when satiated. Physiology \& Behavior 45, 695-704.

Cryer, Ph. (1992). Glucose homeostasis and hypoglycemia. In, J.D. Wilson \& D.W. Forster (Eds.), Williams Textbook of Endocrinology 8th ed., 1223-1253. Philadelphia etc.: W.B. Saunders.

Davey, G. (1989). Ecological Learning Theory. Londen, New York: Routledge.

Davidson, R.L. (1993). The nature and function of interoceptive signals to feed: toward integration of physiological and leaming perspectives. Psychological Review 100, 640-657.

Deutsch, R. (1974). Conditioned hypoglycemia: a mechanism for saccharin-induced sensitivity to insulin in the rat. Journal of Comparative and Physiological Psychology 86, 350-358.

Dimberg, U. (1987). Facial reactions, autonomic activity and experienced emotion: a three component model of emotional conditioning. Biological Psychology 24, 105-122.

Dritschel, B., Cooper, P.J. \& Charnock, D. (1993). A problematic counter-regulation experiment: implications for the link between dietary restraint and overeating. International Journal of Eating Disorders 13, 297-304.

Drummond, D.C., Tiffany, S.T., Glautier, S. \& Remington, B. (Eds.) (1995). Addictive Behaviour: Cue Exposure, Theory and Practice. Chichester etc: Wiley \& Sons.

Dworkin, B.R. (1989). Learning and functional utility. Behavioral and Brain Sciences 12, 139-141.

Dworkin, B.R. (1993). Learning and Physiological Regulation. Chicago, London: University of Chicago Press.

Edelberg, R. (1972) Electrodermal recovery rate, goal-orientation, and aversion. Psychophysiology 9, 512-520.

Eikelboom, R. \& Stewart, J. (1982). Conditioning of drug-induced physiological responses. Psychological Review 89, 507-528.

Elmore, D.K. \& de Castro, J.M. (1991). Meal patterns of normal, untreated bulimia nervosa and recove- 
red bulimic women. Physiology and Behavior 49, 99-105.

Fairburn, C.G. (1987). The definition of bulimia nervosa: guidelines for clinicians and research workers. Annals of Behavioral Medicine 9, 3-7.

Fairburn, C.G. \& Beglin, S.J. (1990). Studies of the epidemiology of bulimia nervosa. American Journal of Psychiarry 147, 401-408.

Fairburn, C.G., Cooper, P.J., Cooper, M.J., McKenna, F.P. \& Anastasiades, M. (1991). Selective information processing in bulimia nervosa. International Journal of Eating Disorders 10, 415-422.

Fairburn, C.G. \& Wilson, G.T. (1993). Binge eating: definition and classification. In, C.G. Fairburn \& G.T. Wilson (Eds.). Binge Eating: Nature, Assessment, and Treatment, 3-14. New York, London: Guilford Press.

Fairburn, C.G. (1995). Short-term psychological treatments for bulimia nervosa. In, K.D. Brownell \& Ch. G. Fairburn (Eds), Eating Disorders and Obesin: a Comprehensive Handbook, pp. 344-348. New York, London: Guilford Press.

Fehm-Wolfsdorf, G., Gnadler, M., Kern, W., Klosterhalfen, W., \& Kerner, W. (1993). Classically conditioned changes of blood glucose level in humans. Physiology and Behavior 54, 155-160.

Flaherty, C.F., Grigson, P.S. \& Brady, A. (1987). Relative novelty of conditioning context influences directionality of glycemic conditioning. Journal of Experimental Psychology 13, 144-149.

Förster, H., Haslbeck, M. \& Mehnert, H., 1972. Metabolic studies following the oral ingestion of different doses of glucose. Diabetes 21, 1102-1108.

Fridlund, A.J. (1979). Contour-following integrator for dynamic tracking of electromyographic data. Psychophysiology 16, 491-493.

Fridlund, A.J. \& Cacioppo, J.T. (1986). Guidelines for human electromyographic research. Psychophysiology 23, 567-589.

Furedy, J.J. (1989). Flights of teleological fancy about classical conditioning do not produce valid science or useful technology. Behavioral and Brain Sciences 12, 142-143.

Garcia, J., Lasiter, P.S., Bermudez-Rattoni, F. \& Deems, D.A. (1985). A general theory of aversion learning. Annals of the New York Academy of Sciences 443, 8-22.

Garfinkel, P.E. (1992). Evidence in support of attitudes to shape and weight as a diagnostic criterion of bulimia nervosa. International Journal of Eating Disorders, 4, 321-325.

Garner, D.M, Olmsted, M.P., Davis, R., Rockert, W., Goldbloom, D. \& Eagle, M. (1990). The association between bulimic symptoms and reported psychopathology. Insernational Journal of Earing disorders 9, 1-15.

Geliebter, A., Melton, P.M., McCray, R.S., Gallagher, D.R., Gage, D. \& Hashim, S.A. (1992). Gastric capacity, gastric emptying, and test-meal intake in normal and bulimic women. American Journal of Clinical Nutrition 56, 656-661.

Gerich, J.E., Charles, M.A., Grodsky, G.M. (1976). Regulation of pancreatic insulin and glucagon secretion. Annual Review of Physiology 38, 353-388.

Giduck, S.A., Threatte, R.M. \& Kare, M.R. (1987). Cephalic reflexes: their role in digestion and possible roles in absorption and metabolism. Journal of Nutrition 117, 1191-1196.

Girardier, L., Seydoux J. \& Campfield, L.A. (1976). Control of A and B cells in vivo by sympathetic nervous input and selective hyper- or hypoglycemia in dog pancreas. Journal of Physiology (Paris), $801-814$.

Glautier, S. (1994). Classical conditioning, drug cues and drug addiction. In, Ch. R. Legg \& D. Booth (Eds.), Appetite: Neural and Behavioural Bases, pp 165-192. Oxford etc, Oxford University Press.

Glautier, S. \& Tiffany, S.T. (1995). Methodological issues in cue reactivity research. In, D.C. Drum mond, S.T. Tiffany, S. Glautier \& B. Remington (Eds.), Addictive Behaviour: Cue Exposure Theory and Practice, 75-98. Chichester etc.: Wiley.

Goldfine, I.D., Abraira, C., Gruenewald, D. \& Goldstein, M.S. (1970). Plasma insulin levels during imaginary food ingestion under hypnosis. Proceedings of the Society for Experimental Biology and 
Medicine, 133, 274-276.

Green, M.W. \& Rogers, P.J. (1993). Selective attention to food and body shape words in dieters and restrained nondieters. International Journal of Eating Disorders 14, 515-517.

Greeno, C.G. \& Wing, R.R. (1994). Stress-induced eating. Psychological Bulletin 115, 444-464.

Grill, H.J., Berridge, K.C., Ganster, D.J., 1984. Oral glucose is the prime elicitor of preabsorptive insulin secretion. American Journal of Physiology 248, R88-R95.

Grilo, C.M, Shiffman, S. \& Wing, R.R. (1989). Relapse crises and coping among dieters. Journal of Consulting and Clinical Psychology 57, 488-495.

Grossman, S.P. (1986). The role of glucose, insulin and glucagon in the regulation of food intake and body weight. Neuroscience \& Biobehavioral Reviews 10, 295-315.

Guyton, A.C. (1991). Textbook of Medical Physiology, 8th ed. Philadelphia etc: Saunders.

Hadigan, C.M., Kissileff, H.R. \& Walsh, B.T. (1989). Patterns of food selection during meals in women with bulimia. American Journal of Clinical Nutrition 50, 759-766.

Hansen, A. \& de Haan, E. (1995). De behandeling van bulimia nervosa met cue-exposure; ervaringen uit de praktijk. Directieve Therapie 15, 279-291.

Hardy, R.N. (1981). Endocrine Physiology. London: Edward Arnold.

Heatherton, T.F., Herman, C.P., Polivy, J., King, G.A., \& McGree, S.T., 1988. The (mis)measure ment of restraint: an analysis of conceptual and psychometric issues. Journal of Abnormal Psychology 97, 19-28.

Hepburn, D.A. (1993). Symptoms of hypoglycaemia. In, B.M. Frier \& B.M. Fisher (Eds.), Hypoglycaemia and Diaberes: Clinical and Physiological Aspects, 93-103. London, etc.: Edward Arnold.

Herman, C.P. \& Mack, D. (1975). Restrained and unrestrained eating. Journal of Personaliry, 43. 647-660.

Herman, C.P., Polivy, J., Pliner, P., Threlkeld, J. \& Munic, D. (1978). Distractability in dieters and nondieters: an alternative view of "externality". Journal of Personaliry and Social Psychology 36, 536-548.

Herman, C.P. \& Polivy, J. (1980). Restrained eating. In, A.B. Stunkard (Ed)., Obesity, pp. 208-225. Philadelphia: Saunders.

Herman, C.P. \& Polivy, J. (1982). Weight change and dietary concern in the overweight: are they really independent? Appetite 3, 280-281.

Herman, C.P. \& Polivy, J. (1984). A boundary model for the regulation of eating. In A.B. Stunkard \& E.Stellar (Eds). Eating and its disorders, pp. 141-156. New York: Raven Press.

Herman, C.P., Polivy, J. \& Esses, V.M. (1987). The illusion of counter-regulation. Appetite 9, 161 169.

Herman, C.P. \& Polivy, J. (1988). Restraint and excess in dieters and bulimics. In K.M. Pirke, W. Vandereycken and D. Ploog (Eds.), The Psychobiology of Bulimia Nervosa, pp 33-41. Berlin etc: Springer-Verlag.

Herman, C.P. \& Polivy, J. (1990). From dietary restraint to binge eating: attaching causes to effects. Appetite 14, 123-125.

Hibscher, J.A. \& Herman, C.P. (1977). Obesity, dieting, and the expression of 'obese' characteristics. Journal of Comparative Physiology and Psychology 91, 374-380.

Hoek, H.W. (1993). Review of the epidemiological studies of eating disorders. International Review of Psychiarry 5, 61-74

Hoek, H.W. (1995). The distribution of eating disorders. In, K.D. Brownell \& Ch. G. Fairburn (Eds.), Easing Disorders and Obesiry: a Comprehensive Handbook, pp. 207-211. New York, London: Guilford Press.

Hoffer, L.J. (1988). Starvation. In, M.E. Shils \& V.R. Young (eds.). Modern Nutrition in Health and Disease, 7th ed., pp. 774-794. Philadelphia: Lea \& Febriger.

Hohlstein, L.A., Gwirtsman, H.E., Whalen, F. \& Enns, M.P. (1986). Oral glucose tolerance in bulimia. International Journal of Eating Disorders 5, 157-160. 


\section{References}

Horwitz, D.L., McLane, M. \& Kobe, P., 1988. Response to a single dose of aspartame or saccharin by niddm patients. Diabetes Care 11, 230-234.

Jansen, A. (1990). Binge earing: Notes and Data. Doctoral dissertation. Maastricht: University of Limburg.

Jansen, A. (1994a). The learned nature of binge eating. In Ch. R. Legg \& D.A. Booth (Ed.), Apperire: Neural and Behavioural Bases, pp 193-211. Oxford: Oxford University Press.

Jansen, A. (1994b). Notitie over het aanleren en veranderen van smaakpreferenties. Suikerstichting Nederland.

Jansen, A., Oosterlaan, J., Merckelbach, H. \& Van den Hout, M.A. (1988a). Non-regulation of food intake in restrained, emotional, and external eaters. Journal of Psychopashology and Behavioral Assessment 10, 345-354.

Jansen, A., Merckelbach, H., Oosterlaan, J., Tuiten, A. \& Van den Hout, M.A. (1988b). Cognitions and self-talk during food intake of restrained and unrestrained eaters. Behaviour Research and Therapy, 26, 393-398.

Jansen, A., Van den Hout, M.A., De Loof, C. Zandbergen, J. \& Griez, E. (1989). A case of Bulimia successfully treated by cue exposure. Journal of Behaviour Therapy and Experimental Psychiatry $20,327-332$.

Jansen, A., Van den Hout, M.A. \& Griez, E. (1990). Clinical and non-clinical binges. Behaviour Research and Therapy 28, 439-444.

Jansen, A. \& Van den Hout, M.A. 1991. On being led into temptation: "counterregulation" of dieters after smelling a "preload". Addictive Behaviors 5, 247-253.

Jansen, A., Merckelbach, H. \& Van den Hout, M.A. (1992a). Experimentele Psychopathologie. As sen, Maastricht: van Gorcum.

Jansen, A., Boon, B., Nauta, H. \& van den Hout, M.A. (1992b). Salivation discordant with hunger. Behaviour Research and Therapy 30, 163-166.

Jansen, A., Broekmate, J. \& Heymans, M. (1992c). Cue-exposure vs self-control in the treatment of binge eating: a pilot study. Behaviour Research and Therapy 30, 235-241.

Johnson, W.G. \& Wildman, H.E. (1983). Influence of external and covert food stimuli on insulin secretion in obese and normal persons. Behavioral Neuroscience 97, 1025-1028.

Johnson, W.G., Jarrell, M.P., Chupurdia, K.M. \& Williamson, D.A. (1994). Repeated binge/purge cycles in bulimia nervosa: Role of glucose and insulin. International Journal of Eating Disorders $15,331-341$.

Joravsky, D. (1989). Russinn Psychology: A Critical Appraisal. Oxford etc.: Blackwell.

Kaye, W.H., Gwirtsman, H.E. \& George, D.T. (1989). The effect of bingeing and vomiting on hormonal secretion. Biological Psychiatry 25, 768-780.

Kennedy, S.H., Katz, R., Neitzer, C.S., Ralevski, E. \& Mendlowitz, S. (1995). Exposure with response prevention treatment of anorexia nervosa bulimic subtype and bulimia nervosa. Behaviour Research and Therapy 33, 685-689.

Klajner, F., Herman, P.H., Polivy, J. \& Chhabra, R. (198I). Human obesity, dieting, and anticipatory salivation to food. Physiology and Behavior 27, 195-198.

Kostarczyk, E., 1986. Autonomic correlates of alimentary conditioned and unconditioned reactions in the dog. Journal of the Ausonomic Nervous System 817, 279-288.

Laberg, J.C., Wilson, G.T., Eldredge, K. \& Nordby, H. (1991). Effects of mood on heart rate reactivity in bulimia nervosa. International Journal of Eating Disorders 10, 169-178.

Lacey, J.H. \& Gibson, E. (1985). Controlling weight by purgation and vomiting: a comparative study of bulimics. Journal of Psychiarric Research 19, 337-341

Laessle, R.G., Tuschl, R.J, Kotthaus, B.C. \& Pirke, K.M. (1989). A comparison of the validity of three scales for the assessment of dietary restraint. Journal of Abnormal Psychology 98, 504-507.

Landis, C. \& Hunt, W.A. (1939). The Startle Pantern. New York: Farrar \& Rinehart.

Landsberg, L. \& Young, J.B. (1978) Fasting, feeding and regulation of the sympathetic nervous system. 
New England Journal of Medicine 298, 1295-1301.

Lang, P.J., Bradley, M.M. \& Cuthbert, B.N. (1990). Emotion, attention, and the startle reflex. Psychological Review 97, 377-398.

Lang, P.J., Greenwald, M.K., Bradley, M.M. \& Hamm, A.O. (1993). Looking at pictures: affective, facial visceral and behavioral reactions. Psychophysiology 30, 261-273.

Lavy, E., van den Hout, M. \& Amtz, A. (1993). Attentional bias and spider phobia: conceptual and clinical issues. Behaviour Research and Therapy 31, 17. 24.

Lavy, E. \& van den Hout, M. (1993). Attentional bias for appetitive cues: effects of fasting in normal subjects. Behavioural and Cognitive Psychotherapy 21, 297-310.

LeGoff, D.B. \& Spiegelman, M.N. (1987). Salivary response to olfactory food stimuli as a function of dietary restraint and body weight. Appetite 12, 83-94.

LeGoff, D.B., Leichner, P. \& Spigelman, M.N. (1988). Salivary response to olfactory stimuli in anorexics and bulimics. Apperite $11,15-25$.

Le Magnen, J. (1985). Hunger. Cambridge etc: Cambridge University Press.

Le Magnen, J. (1992). Neurobiology of Feeding and Nutrision. San Diego etc: Academic Press.

LeBlanc, J. (1992). Cephalic phase response to food and its effect on feeding behavior. In, G.H. Anderson and S.H. Kennedy (Eds), The Biology of Feast and Famine: Relevance to Eating Disorders, 4760. San Diego etc., Academic Press.

Leitenberg, H., Gross, J., Peterson, J. \& Rosen, J.C. (1984) Analysis of an anxiety model and the process of change during exposure plus response prevention treatment of bulimia nervosa. Behavior Therapy 15, 3-20.

Leitenberg, H. \& Rosen, J. (1989). Cognitive-behavioral therapy with and without exposure plus response prevention in treatment of bulimia nervosa: comment on Agras, Schneider, Arnow, Raeburn, and Telch. Journal of Consulting and Clinical Psychology 57, 776-777.

Louis-Sylvestre, J. (1976). Preabsorptive insulin release and hypoglycemia in rats. American Journal of Physiology 230, 56-60.

Louis-Sylvestre, J. \& Le Magnen J. (1983). Vagotomy abolishes the differential palatability of food. Appetite 4, 295-299.

Louis-Sylvestre, J. (1984). Meal size: role of reflexly induced insulin release. Journal of the Autonomic Nervous System 10, 317-324.

Lowe, M.R. (1993). The effects of dieting on eating behavior: a three-factor model. Psychological Bulletin 114, 100-121.

Lucas, F., Bellisle, F. \& Di Maio, A. (1987). Spontaneous insulin fluctuations and the preabsorptive insulin response to food ingestion in humans. Physiology and Behavior 40, 631-636.

MacRae, J.R., Scoles, M.T. \& Siegel, S. (1987). The contribution of Pavlovian conditioning to drug tolerance and dependence. British Journal of Addicrion 82, 371-380.

Mahamedi, F. \& Heatherton, T.F. (1993). Effects of high calorie preloads on selective processing of food and body shape stimuli among dieters and nondie ters. International Journal of Eating Disorders 13, 305-314.

Marcus, M.D. (1993). Binge eating in obesity. In, C.G. Fairburn \& G.T. Wilson (Eds.). Binge Earing: Nature, Assessment, and Treatment, 77-96. New York, London: Guilford Press.

Marcus, M.D. (1995). Binge eating and obesity. In, K.D. Brownell \& Ch. G. Fairburn (Eds), Eating Disorders and Obesity: a Comprehensive Handbook, pp. 441-444. New York, London: Guilford Press.

Matysiak, J. \& Green, L. (1984). On the directionality of classically-conditioned glycemic responses. Physiology and Behavior 32, 5-9.

McDonald, R.P. \& Mulaik, S.A. (1979). Determinacy of common factors: a nontechnical review. Psychological Bulletin 86, 297-306.

McHugh, P.R. (1983). The control of gastic emptying. In, J.G. Kral, T.L. Powley \& C.McC. Brooks (Eds.), Vagal Nerve Function: Behavioural and Methodological Considerations, 221-231. 


\section{References}

Amsterdam, etc.: Elsevier.

McNally, R.J. (1994). Panic Disorder: a Critical Analysis. New York etc.: Guilford Press.

Miller, R.R., Barnet, R.C. \& Grahame, N.J. (1995). Assessment of the Rescorla-Wagner Model. Psychological Bullerin 117, 363-386.

Mitchell, J.E., Hatsukami, D., Eckert, E.D. \& Pyle, R.L. (1985). Characteristics of 275 patients with Bulimia. American Journal of Psychiatry 142, 482-485.

Montgomery, A.M.J. (1991). Animal models of eating disorders. In, P. Willner (Ed.), Behavioural Models in Psychopharmacology, pp. 177-214. Cambridge etc.: Cambridge University Press.

Moyer, A., Rodin, J. \& Cummings, N. (1993). Cephalic phase insulin release in Bulimia. International Journal of Eating Disorders 14, 331-339.

Mityushov, M.I. (1954). Conditioned reflex insulin secretion. Journal of Higher Nervous Activiry (in Russian) 4, 206-212.

Niaura, R.S., Rohsenow, D.J., Binkoff, J.A., Monti, P.M., Pedraza, M. \& Abrams, D.B. (1988). Relevance of cue reactivity to understanding alcohol and smoking relapse. Journal of Abnormal Psychology 97, 133-152.

Nicolaĩdis, S. (1977). Sensory-neuroendocrine reflexes and their anticipatory and optimizing role on metabolism. In M.R. Kare and O. Maller (Eds.), The Chemical Senses and Nurrition, 123-143. New York etc.: Academic Press.

Niijima, A. (1989). Nervous regulation of metabolism. Progress in Neurobiology 33, 135-147.

Nunnally, J.C. (1978). Psychometric Theory, 2nd ed. New York etc. McGraw-Hill.

Oettle, G.J., Emmett, P.M. \& Heaton, K.W. (1987). Glucose and insulin responses to manufactured and whole-food snacks. American Journal of Clinical Nutrition 45, 86-91.

Ogden, J. (1993). The measurement of restraint: confounding success and failure? International Journal of Ealing Disorders 13, 69-76.

Ogden, J. \& Greville, L., (1993). Cognitive changes to preloading in restrained and unrestrained eaters as measured by the Stroop task. International Journal of Eating Disorders 14, 185-195.

Overduin, J. (1993). Het voordeel van de schrik: over het onvolledig gebruik van de reflex in emotieonderzoeken. De Psycholoog 28, 193-200.

Overduin, J. \& Jansen, A. (1996). A new scale for use in non-clinical research into disinhibitive eating. Personaling and Individual Differences, in press.

Overduin, J., Jansen, A. \& Eilkes, H. (1996). Cue reactivity to food- and body stimuli in restrained and uarestrained eaters. Addictive Behaviors in press.

Overduin, J. \& Jansen, A. submitted. Conditioned insulin and blood sugar responses in bumans in relation to binge eating.

Pavlov, I.P. (1897/1910). The Work of the Digestive Glands. London: Griffin.

Peterson, C., Maier, S.F. \& Seligman, M.E.P., 1993. Learned Helplessness: a Theory for the Age of Personal Control. New York, Oxford: Oxford University Press.

Pirke, K.M. (1995). Physiology of Bulimia Nervosa. In, C.G. Fairburn \& G.T. Wilson (Eds.). Binge Eating: nature, assessment, and trearment, 261-265. New York, London: Guilford Press.

Polivy, J. \& Herman, C.P. (1985). Dieting and binging: a causal analysis. American Psychologist 40, 193-201.

Polivy, J., Heatherton, T.F. \& Herman, C.P. (1988). Self-esteem, restraint, and eating behavior. Journal of Abnomal Psychology 97, 354-356.

Polivy, J. \& Herman, C.P. (1989). Dietary restraint and binge eating: Response to Charnock. British Journal of Clinical Psychology 28, 341-343.

Polonsky, K.S., Given, D., Hirsch, L. et al. (1988). Quantitative study of insulin secretion and clear ance in normal and obese subject. Journal of Clinical Investigation 81, 435-441.

Polonsky, K.S. \& Rubenstein, A.H., 1984. C-peptide as a measure of the secretion and hepatic extraction of insulin. Diabetes 33, 486-494.

Powell, J., Gray, J. \& Bradley, B. (1993). Subjective craving for opiates: evaluation of a cue exposure 
protocol for use with detoxified opiate addicts. British Journal of Clinical Psychology 32, 39-53.

Powley, T.L. \& Berthoud, H-R. (1985). Diet and cephalic phase insulin responses. American Journal of Clinical Nutrition 42, 991-1002.

Razran, R. (1961). The observable unconscious and the inferable conscious in current Soviet psychophysiology. Psychological Review 68, 81-147.

Read, N.W., Welch, I. McL., Austen, C.J., Barnish, C. et al. (1986). Swallowing food without chewing: a simple way to reduce postprandial glycaemia. British Journal of Nutrition 55, 43-47.

Rescorla, R.A. (1967). Pavlovian conditioning and its proper control procedures. Psychological Review $74,71-80$.

Revusky, S. \& Garcia, J. (1970). Leamed associations over long delays. In, G.H. Bower (Ed), The Psychology of Learning and Motivation vol 4., pp. 1-84. New York, London: Academic Press.

Robbins, S.J. \& Ehrman, R.N. (1992). Designing studies of drug conditioning in humans. Psychopharmacology $106,143-153$.

Rodin, J., Bach, J., Peranini, E. \& De Fronzo, F. (1985). Effect of insulin and glucose on feeding behavior. Metabolism 34, 827-833.

Rodin, J., Reed, R. \& Jamner, L. (1988). Metabolic effects of fructose and glucose: implications for food intake. American Journal of Clinical Nutrition 47, 683-689.

Rodin, J. (1990). Comparative effects of fructose, aspartame, glucose, and water preloads on calorie and macronutrient intake. American Journal of Clinical Nutrition, 51, 428-435.

Rogers, P.J. \& Hill, A.J. (1989). Breakdown of dietary restraint following mere exposure to food stimuli: interrelationships between restraint, hunger, salivation, and food intake. Addictive Behaviors 14, 387-397.

Rosen, J.C. \& Leitenberg, H. (1982). Bulimia Nervosa, treatment with exposure and response prevention. Behavior Therapy 13,117-124.

Rosen, J.C., Leitenberg, H., Fisher, C. \& Khazam, C. (1986). Binge-eating episodes in bulimia nervosa: the amount and type of food consumed. International Journal of Eating Disorders 5, 255-267.

Rosenberg, M. (1965). Society and the Adolescent Self-image. Princeton, N.J.: Princeton University Press.

Rossiter, E.M, \& Agras, W.S. (1990). An empirical test of the DSM-III-R definition of binge. Inter national Journal of Eating Disorders 9, 513-518.

Rossiter, E.M., Agras, W.S., Telch, C.F. \& Bruce, B. (1992). The eating patterns of non-purging bulimic subjects. International Journal of Eating Disorders 11, 111-120.

Ruderman, A.J. (1983). The Restraint Scale: a psychometric investigation. Behaviour Research and Therapy 21, 253-258.

Ruderman, A.J. (1985). Dysphoric mood and overeating: a test of restraint theory's disinhibition hypothesis. Journal of Abnormal Psychology, 94, 78-85.

Ruderman, A. J. (1986). Dietary restraint: a theoretical and empirical review. Psychological Bulletin $99,247-262$.

Russell, G. (1979). Bulimia nervosa: an ominous variant of anorexia nervosa. Psychological Medicine 9, 429-448.

Sapolsky, R.M. (1994). Why Zebras Don't Get Ulcers: A Guide To Stress, Stress-related Diseases, and Coping. New York: Freeman \& Co.

Schmidt, U. \& Marks, I.M. (1988). Cue exposure to food plus response prevention of binges for bulimia: a pilot study. International Journal of Eating Disorders 7, 663-672.

Schmidt, U. \& Marks, I.M. (1989). Exposure plus prevention of bingeing vs. exposure plus prevention of vomiting in bulimia nervosa: a crossover study. Journal of Nervous and Menial Disease 177, 259-266.

Schultz-Gambard, E. (1988). Indikatoren von Hunger: Psychophysiologische Untersuchung zur Wirkung einer 24-stündigen Nahrungsdeprivation. Doctoral Dissertation. Bielefeld: University of Bielefeld.

Schwartz, B. \& Robbins (1995). Psychology of Learning \& Behavior, 4th ed. New York etc: Norton.

Schweiger, U., Poellinger, J., Laessle, RF., Wolfram, G., Fichter, M.M. \& Pirke K.-M. (1987). 


\section{References}

Altered insulin response to a balanced test meal in bulimic patients. International Journal of Eating Disorders 6, 551-556.

Sclafani, A. (1995). How food preferences are learned: laboratory animal models. Proceedings of the Nutrition Society 54, 419-427.

Service, F.J. (1995). Hypoglycemic disorders. New England Journal of Medicine 332, 1144-1152.

Shively, C.A., Apgar, J.L. \& Tarka, S.M. (1986). Postprandial glucose and insulin responses to various snacks of equivalent carbohydrate content in normal subjects. American Journal of Clinical Nurrition 43, 335-342.

Siegel, S. (1972). Conditioning of insulin-induced glycemia. Journal of Compararive and Physiological Psychology 78, 233-241.

Siegel, S. (1983). Classical conditioning, drug tolerance and drug dependence. In, R. Smart, F. Glaser, Y. Israel, H. Kalant, R. Popham, and W. Schmidt (Eds.), Research Advances in Alcohol and Drug Problems, pp 207-246. New York: Plenum Press.

Siegel, S. (1989). Pharmacological conditioning and drug effects. In, A.J. Goudie \& M.W. EmmettOglesby (Eds.), Psychoactive Drugs: Tolerance and Sensitization, pp 115-180. Clifton, New Jersey: Humana Press.

Siegel, S. \& Nettleton, N. (1970). Conditioning of insulin-induced hyperphagia. Journal of Comparative and Physiological Psychology 72, 390-393.

Simon, C., Schlienger, J.L, Sapin, R. \& Imler, M. (1986). Cephalic phase insulin secretion in relation to food presentation in normal and overweight subjects. Physiology and Behavior 36, 463-469.

Sjōstrōm, L., Garellick, G., Krotkiewski, M. \& Luyckx, A. (1980). Peripheral insulin in response to the sight and smell of food. Metabolism 29, 901-909.

Steffens, A.B., Strubbe, J.H., Scheurink, A.J.W. \& Balkan, B. (1991). Neuroendocrine activity during food intake modulates secretion of the endocrine pancreas and contributes to the regulation of body weight. In: M.I. Friedman, M.G. Tordoff and M.R. Kare (Eds.), Chemical Senses Vol. 4: Apperite and Nutrition, pp 405-426. New York: Marcel Dekker.

Strubbe, J.H. (1992). Parasympathetic involvement in rapid meal-associated conditioned insulin secretion in the rat. American Journal of Physiology 263, R615-R618.

Strubbe, J.H. \& Steffens, A.B. (1975). Rapid insulin release after ingestion of a meal in the unanes thetized rat. American Journal of Physiology 229, 1019-1022.

Strubbe, J.H. \& Steffens, A.B. (1993). Neural control of insulin secretion. Hormones and Metabolic Research 25, 507-512.

Stunkard, A.J. \& Messick, S. (1985). The Three-Factor Eating Questionnaire to measure dietary restraint, disinhibition and hunger. Journal of Psychosomatic Research 29, 71-83.

Teff, K.L., Mattes, R.D. \& Engelman, K. (1991). Cephalic phase insulin release in normal weight males: verification and reliability. American Journal of Physiology 261, E430-E436.

Teff, K.L., Levin, B.E. \& Engelman, K. (1993). Oral sensory stimulation in men: effects on insulin, C-peptide, and catecholamines. American Journal of Physiology 265, R1223-R1230.

Teff, K.L., Devine, J., Engelman, K. (1995). Sweet taste: effect on cephalic phase insulin release in men. Physiology and Behavior 57, 1089-1095.

Thompson, J.P., Palmer, R.L. \& Petersen, S.A. (1988). Is there a metabolic component to counter regulation? International Journal of Eating Disorders 7, 307-319.

Tiffany, S.T. (1995a). Potential functions of classical conditioning in drug addiction. In, D.C. Drummond, S.T. Tiffany, S. Glautier \& B. Remington (Eds.), Addictive Behaviour: Cue Exposure Theory and Practice, pp 47-71. Chichester etc: Wiley.

Tiffany, S.T. (1995b). The role of cognitive factors in reactivity to drug cues. In, D.C. Drummond, S.T. Tiffany, S. Glautier \& B. Remington (Eds.), Addictive Behaviour: Cue Exposure Theory and Practice, pp 137-165. Chichester etc: Wiley.

Tobin, D.L., Johnson, C, Steinberg, S., Staats, M. \& Dennis, A.B. (1991). Multifactorial assessment of bulimia nervosa. Journal of Abnormal Psychology 100, 14-21. 
Tse, Th. F., Clutter, W.E., Shah, S.D., Miller, J. Ph. \& Cryer, Ph. E., 1983. Neuroendocrine responses to glucose ingestion in man. Journal of Clinical Investigation 72, 270-277.

Turkkan, J.S. (1989). Classical conditioning: the new hegemony. Behavioral and Brain Sciences 12 , 121-179.

Turner, M.St.J., Foggo, M., Bennie, J., Carroll, S., Dick, H. \& Goodwin, G.M. (1991). Psychological, hormonal and biochemical changes following carbohydrate bingeing: a placebo controlled study in bulimia nervosa and matched controls. Psychological Medicine 21, 123-133.

Tuschl, R.J. (1990). From dietary restraint to binge eating: some theoretical considerations. Apperite 14, 105-109.

Van Der Heijden, P.G.M. (1995). Hoe te leven met statistiek. Nederlands Tijdschriff voor de Psychologie $50,134-146$.

Van Het Reve, K. (1979). Een Dag uit het Leven van de Reuzenkoeskoes. Amsterdam: G.A. van Oorschot.

Van Strien, T., Frijters, J.E.R., Bergers, G.P.A. \& Defares, P.B. (1986). The Dutch Eating Behaviour Questionnaire (DEBQ) for assessment of restrained, emotional, and external eating behavior. International Journal of Eating Disorders 2, 295-315.

Van Vort, W. \& Smith, G.P. (1987). Sham feeding experience produces a conditioned increase of meal size. Appetite 9, 21-29.

Velden, M \& Graham, F.K. (1988). Depicting heart rate over real time: two procedures that are mathematically identical. Journal of Psychophysiology 2, 291-292.

Venables, P.H. (1991). Autonomic activity. Annals of the New York Academy of Sciences 620, 191207.

Walsh, B.T. (1992). Diagnostic criteria for eating disorders in DSM-IV: work in progress. Internasional Journal of Eating Disorders, 4, 301-304.

Walsh, B.T. (1993). Binge eating in bulimia nervosa. In: C.G. Fairburn \& G.T. Wilson (Eds), Binge Eating: Nature, Assessment, and Treatment, pp 37-49. New York, London: Guilford Press.

Wardle, J. (1980). Dietary restraint and binge eating. Behavioural Analysis and Modification 4, 201 209.

Wardle, J. (1986). The assessment of restrained eating. Behaviour Research and Therapy 24, 213-215.

Wardle, J. (1990). Conditioning processes and cue exposure in the modification of excessive eating. Addictive Behaviors 15, 387-393.

Wardle, J. \& Beinart, H. (1981). Binge eating: a theoretical review, British Journal of Clinical Psychology $20,97-109$.

Webber, J. \& MacDonald, I.A. (1993). Metabolic actions of catecholamines in man. Bailliere's Clinical Endocrinology and Metabolism 7, 393-413.

Weingarten, H.P. (1984). Meal initiation controlled by learned cues: basic behavioral properties. Appetise $5,147-158$.

Weingarten, H.P. (1985). Stimulus control of eating: implications for a two-factor theory of hunger. Appetite 6, 387-401.

Weingarten, H.P., Hendler, R. \& Rodin, J. (1988). Metabolism and endocrine secretion in response to a test meal in normal-weight bulimic women. Psychosomatic Medicine 50, 273-285.

Weingarten, H.P. \& Elston, D. (1990). The phenomenology of food cravings. Appetile 15, 231-246.

Westenhoeffer, J. (1991). Dietary restraint and disinhibition: is restraint a homogeneous construct? Appetite 16, 45-55.

Westenhoeffer, J., Broeckmann, P., Muench, A-K. \& Pudel, V. (1994). Cognitive control of eating behaviour and the disinhibition effect. Appetite 23, 27-41.

Wilde, G.J.S. (1977). Trait description and measurement by personality questionnaires. In, R.B. Cartell \& R.M. Dreger (Eds.), Handbook of Modern Personality Theory, 69-103. New York, etc: Wiley \& Sons.

Williams, R.A. (1968). Effects of repeated food deprivations and repeated feeding tests on feeding 


\section{References}

behavior. Journal of Comparative and Physiological Psychology 65, 222-226.

Willner, P. (1991a). Behavioural Models in Psychopharmacology: Theoretical, Industrial and Clinical Perspectives. Cambridge etc: Cambridge University Press.

Willner, P. (1991b). Methods for assessing the validity of animal models of human psychopathology. In, A.A. Boulton, G.B. Baker \& M.T. Martin-Iverson (Eds.), Animal Models in Psychiarry vol. I. , 1-23. Clifton, N.J.: Humana Press.

Woods, S.C. (1976). Conditioned hypoglycemia. Journal of Comparative and Physiological Psychology $90,1164-1168$.

Woods, S.C. (1981). Abstract from discussion with H.-R. Berthoud. Diabetologia 20, 401.

Woods, S.C. (1983). Conditioned hypoglycemia and conditioned insulin secretion. Advances in Metabolic Disorders 10, 485-495.

Woods, S.C. (1990). On blood glucose and eating. International Journal of Obesity 14 (suppl 3), $33-$ 34.

Woods, S.C. (1991). The eating paradox: how we tolerate food. Psychological Review 98, 488-505.

Woods, S.C. \& Kulkosky, P.J. (1976). Classically conditioned changes of blood glucose level. Psychosomatic Medicine 38, 201-219.

Woods, S.C., Makous, W. \& Hutton, R.A. (1969). Temporal parameters of conditioned hypoglycemia. Journal of Comparative and Physiological Psychology 69, 301-307.

Woods, S.C. \& Shogren, R.E. (1972). Glycemic responses following conditioning with different doses of insulin in rats. Journal of Comparative and Physiological Psychology, 81, 220-235.

Woods, S.C., Vasselli, J.R., Kaestner, E., Szakmary, G.A., Milburn, P. \& Vitiello, M.V. (1977). Conditioned insulin secretion and meal feeding in rats. Journal of Comparative and Physiological Psychology 91, 128-133.

Woods, S.C. \& Strubbe, J.H. (1994). The psychobiology of meals. Psychonomic Bulletin \& Review 1, $141-155$.

Wooley O.W., Wooley S.C. \& Williams B.S. (1976). Salivation as a measure of appetite: studies of the anorectic effects of calories and amphetamine. In, D. Novin, W. Wyrwicka \& G.A. Bray, Hunger: Basic Mechanisms and Clinical Implications, ppn 421-429).

Zamble, E. (1973). Augmentation of eating following a signal for feeding in rats. Learning and Motivation 4, 138-147. 


\section{Appendix}

\section{TRANSLATION ${ }^{1}$ OF M.I. MTTYUSHOV ${ }^{2}$ (1954) \\ "CONDITIONED REFLEX SECRETION OF INSULIN"}

From the viewpoint of today's Pavlovian physiology, the internal secretory glands form a powerful lever which enables the nervous system to change all physiological functions over a broad range. The central nervous system regulates various functions of the organism, a process that not only involves nervous impulses through specific pathways to the organs, but also a hormonal, viz. humoral link.

The mechanism by which endocrine glands participate in the nervous regulation of physiological functions, has until now been scantily studied. The main reason for this lies in the fact that until recently, even in the Soviet Union, a majority of the endocrine physiologists has adopted a methodologically unsound approach towards the study of internal secretory glands.

The internal glands were considered in isolation from the nervous system and ascribed an autonomous and often predominant role in physiological regulation. K.M. Bykov (2) in his speech at the plenary session of the Academy of Sciences of the USSR and the Academy of Medical Sciences, dedicated to the problems in Pavlovian physiological studies has stated: "In recent years, the study of hormones has secluded itself from general physiology and pathology into the specific discipline of endocrinology. The consequent isolation of specific subproblems from the more general study of organisms has lead many endocrinologists and medics to the erroneous conclusion that the various hormones play a self-supporting, autonomous role in the life of the organism".

After the plenary session the attention of many researchers was drawn towards the study of nervous regulation of endocrine glands. The study of these issues opens new vistas for the understanding of the mechanisms as well as the significance of the various glandular contributions to the physiological regulation in the organism.

Up until now the nervous regulation of the endocrine glands has been very scantly investigated. In the literature one can find a large number of facts about nervous regulation of the pituitary, the adrenal cortex as well as of the thyroid gland. The nervous regulation

\footnotetext{
'Originally published as: Матошов, М. И. (1954). Усповнорефлекторнан инкреция инсулнна. Журвал Высшеа Нервнов Деятельвостн 4, 206-212. Translation: Joost Overduin, Limburg University, Maastricht, The Netherlands; Prof. Gyōrgy Ádám, Eōtvōs University, Budapest, Hungary. About a dozen idioms have been adopted from an earlier translation by L.J. Shein. Prof. Shepard Siegel, McMaster University, Hamilton, Ontario is acknowledged for providing us with this translation.
}

${ }^{2}$ M.I. Mityushov, Laboratory of Nervous Regulation of Endocrine Functions, I.P. Pavlov Physiological lnstitute, USSR Academy of Sciences. 
of other endocrine glands, specifically the pancreatic islets, has until now received considerably less attention. Different authors have made quite contradictory assertions on this subject.

The insular apparatus of the pancreas plays an exceptionally important role in the regulation of carbohydrate metabolism. That is why the study of the nervous regulation of this activity is of great interest. The purpose of our study is an investigation of the cortical regulation of insulin secretion.

In the literature we found very few data on the cortical regulation of the insular apparatus of the pancreas. Observations by many clinical scientists suggest a role of psychic trauma in the development of diabetes in humans. As an example, the case described by B.G. Baranov can be quoted, who observed the development of severe form of diabetes in a woman who had suffered a nervous breakdown. Moreover, experimental research by N.S. Sedina (7) demonstrated a conditioned hypoglycemia to subcutaneous and intraperitoneal injections of saline, following previous repeated glucose administration through subcutaneous and intraperitoneal injections. She attributes the development of hypoglycemia to a conditioned increase of insulin production. Her attempt to induce conditioned hypoglycemia through intravenous glucose injections did not succeed however.

In our research we used the conditioned reflex method likewisely. The experiments were carried out on 4 dogs as well as on 9 human subjects. The unconditioned stimulus in our experiment was a sharp change in blood sugar following the intravenous glucose injection. As is well-known, a change in blood sugar level is an adequate stimulus for the insular apparatus including all further mechanisms regulating blood sugar level. In our opinion, the intravenous glucose injection appears to be the most rational means of influencing the insular apparatus, because intravenous injections allow for precise adjustments of the stimulus intensity (glucose instantaneously enters the blood stream in a concentration unhampered by a slow rate of absorption such as seen after subcutaneous and intraperitoneal injections.

The absence of conditioned reflex hypoglycemia in the study of N.S. Sedina (7) who used intravenous glucose injections as a UCS, does not seem crucial to the issue of whether conditioned reflex hypoglycemia is possible. Sedina conducted her experiments in two dogs. In one of them, a total of 10 trials was run. The CS was paired with the UCS merely in 7 cases within a two-month period. In the second dog, 9 trials were run in which CS and US were paired only 6 times in the course of 11 days. Clearly, after such a small number of pairings it would be unfeasible to conclude the impossibility of producing a conditioned reflex.

The conditioned stimulus in our study consisted of the intravenous injection procedure as well as the circumstances under which it took place (the glucose was injected regularly at 
Table A.1. Control trials (saline injections) in dogs

Data (1952)

Number of before the injection previous saline (2 measurements with injections $10 \mathrm{~min}$. in between)

$$
\text { minutes after the saline injection }
$$

$\begin{array}{lllll}5 & 15 & 30 & 60 & 90\end{array}$

Dog Baikal

11 III

18 III

22 III

27 III

3 IV

9 IV

$\begin{array}{lllll}99 & 102 & 102 & 97 & - \\ 107 & 103 & 105 & 96 & 98 \\ 94 & 89 & 89 & 91 & 89 \\ 103 & 108 & 108 & 106 & 99 \\ 92 & 89 & 89 & 91 & 94 \\ - & 88 & 88 & 85 & 83\end{array}$

$97: 97$
$94 ; 96$
$91 ; 89$
$103 ; 105$
$92 ; 91$
$85 ; 83$

$74 ; 74$

$77 ; 77$

$70 ; 72$

Dog Dik

$\begin{array}{llllllll}8 \mathrm{I} & 2 & 74 ; 74 & 76 & 76 & 80 & 80 & 80 \\ 10 \mathrm{I} & 3 & 77 ; 77 & 75 & 77 & 75 & 75 & 75 \\ 12 \mathrm{I} & 5 & 70 ; 72 & 74 & 74 & 77 & 77 & 79\end{array}$

Table A.2. Conditioned changes in blood sugar level after repeated glucose injections

\begin{tabular}{|c|c|c|c|c|c|c|c|}
\hline \multirow{3}{*}{ Data (1952) } & \multicolumn{7}{|c|}{ BLOOD SUGAR LEVEL $(\mathrm{mg} / \mathrm{dl})$} \\
\hline & \multirow{2}{*}{$\begin{array}{l}\text { Number of } \\
\text { previous glu- } \\
\text { cose injections }\end{array}$} & \multirow{2}{*}{$\begin{array}{l}\text { before the injection } \\
\text { ( } 2 \text { measurements with } \\
10 \text { min. in between) }\end{array}$} & \multicolumn{5}{|c|}{ minutes after the saline injection } \\
\hline & & & 5 & 15 & 30 & 60 & 90 \\
\hline \multicolumn{8}{|l|}{ Dog Baikal } \\
\hline $9 \mathrm{IV}$ & 8 & $83 ; 83$ & 82 & 80 & 82 & 83 & 87 \\
\hline $15 \mathrm{IV}$ & 14 & $96 ; 96$ & 94 & 85 & 87 & 92 & 90 \\
\hline $22 \mathrm{~V}$ & 16 & $87 ; 92$ & 79 & 85 & 87 & 88 & 90 \\
\hline $3 \mathrm{VI}$ & 20 & $94 ; 95$ & 92 & 97 & 99 & 97 & 92 \\
\hline $10 \mathrm{VI}$ & 22 & $95 ; 97$ & 91 & 93 & 99 & 100 & - \\
\hline $19 \mathrm{VI}$ & 24 & $97 ; 100$ & 95 & 91 & 88 & 97 & \\
\hline $26 \mathrm{VI}$ & 26 & $96 ; 96$ & 90 & 85 & 90 & 96 & 96 \\
\hline 8 VII & 29 & $92 ; 92$ & 87 & 80 & 87 & 89 & 98 \\
\hline $14 \mathrm{VII}$ & 30 & $88 ; 88$ & 86 & 77 & 88 & 104 & - \\
\hline \multicolumn{8}{|l|}{ Dog Dik } \\
\hline $5 \Pi$ & $9^{3}$ & $77 ; 79$ & 72 & 76 & 79 & 77 & 77 \\
\hline 19 II & 14 & $79 ; 77$ & 72 & 70 & 76 & 74 & 72 \\
\hline $28 \mathrm{II}$ & 17 & $76 ; 78$ & 72 & 72 & 74 & 78 & 74 \\
\hline $13 \mathrm{III}$ & 23 & $81 ; 80$ & 74 & 71 & 78 & 78 & 8 \\
\hline $23 \mathrm{~V}$ & 36 & $88 ; 96$ & 85 & 79 & 83 & 85 & 8 \\
\hline $3 \mathrm{VI}$ & 40 & $90 ; 92$ & 79 & 79 & 86 & 88 & 88 \\
\hline $10 \mathrm{VI}$ & 42 & $88 ; 86$ & 73 & 73 & 81 & 75 & 81 \\
\hline $19 \mathrm{VI}$ & 45 & $90 ; 84$ & 90 & 82 & 84 & 88 & 82 \\
\hline 8 VII & 51 & $85 ; 85$ & 80 & 78 & 83 & 83 & - \\
\hline 14 VII & 52 & $82 ; 85$ & 82 & 74 & 82 & 82 & \\
\hline
\end{tabular}

${ }^{3}$ From the first to the fiftheenth trial the UCS was an injection of $100 \mathrm{ml} 40 \%$ glucose solution. From the 15 th trial onwards, the UCS became $150 \mathrm{ml} 40 \%$ glucose solution. 
Table A.3. Conditioned changes in blood sugar level after injection of saline in humans

Data (1952)

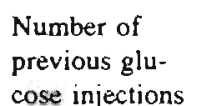

before the injection

BLOOD SUGAR LEVEL ( $\mathrm{mg} / \mathrm{dl}$ )

cose injections

(2 measurements with

minutes after the saline injection remarks

$10 \mathrm{~min}$. in between)

$5 \quad 15 \quad 45$

Subject S.G.

$\begin{array}{llllll}7 \text { III } & 5 & 93 ; 93 & 86 & 88 & 77 \\ \text { I4 III } & 10 & 80 ; 78 & 76 & 71 & 76 \\ 21 \text { III } & 15 & 83 ; 85 & 78 & 72 & 72 \\ 25 \text { III } & 17 & 89 ; 78 & 84 & 78 & 84 \\ & & & & & \\ 28 \text { III } & 18 & 99 ; 87 & 85 & 81 & 79 \\ \text { 3I III } & 19 & 105 ; 103 & 91 & 87 & 83 \\ 4 \text { IV } & 21 & 83 ; 87 & 83 & 85 & 83\end{array}$

Subject G.A.

7 III

$82 ; 82$

$\begin{array}{lll}79 & 73 & 66\end{array}$

77

$60 \mathrm{ml} 40 \%$ glucose

solution as a US

14 III

$74 ; 78$

$73 ; 69$

$91 ; 85$

$101 ; 101$

$83 ; 87$

$71 \quad 71$

$61 \quad 63$

7
8

$84 \quad 78$

$85 \quad 74$

$82 \quad 82$

76

72

84 injection occurred

in other room

28 III

19

90; 92

$\begin{array}{ll}88 & 88\end{array}$

injection occurred

in other room

4 IV

23

91; -

$\begin{array}{lll}82 & 76 & 78\end{array}$

$40 \mathrm{ml} 40 \%$ glucose

injection as US

25 IV

17

84; -

$\begin{array}{lll}76 & - & 78\end{array}$

$81 ; 83$

$\begin{array}{lll}74 & 72 & 80\end{array}$

$28 \mathrm{~V}$

19
27

94; 94

$\begin{array}{lll}87 & 85 & 85\end{array}$

Subject P.F.

14 IV

10

74; -

$74 \quad 72$

69

$40 \mathrm{ml} 40 \%$ glucose

21 IV

15

83; -

$\begin{array}{lll}74 & 74 & 76\end{array}$

25 IV

91; -

28 lV

20

$81 ; 79$

$\begin{array}{lll}84 & 87 & 89\end{array}$

$\begin{array}{lll}70 & 70 & 72\end{array}$ 
identical hours of the day by the same persons). On certain days, instead of glucose injections, intravenous saline of equal volume was given in the same manner, i.e., only the $\mathrm{CS}$ was presented. Prior to the glucose injections, several control trials were run to ascertain whether injections of $40-150 \mathrm{ml}$ saline in itself would have any effect. It was shown that the intravenous saline injections either did not change the blood sugar level, or did cause an unsignificant rise of $5-7 \mathrm{mg} \%$. More often, an increase in bloodsugar was seen, and this may be explained by certain emotional arousal that results from the injections procedure. This blood sugar rise in response to saline injections does not disappear even if intravenous injection have been given more often (in our experiment until 25 times, in dog Baikal).

The sharp rise of bloodsugar level after intravenous glucose injections appears to be a strong stimulus to which the insular apparatus must respond with an enhanced release of insulin. Repeated injections of glucose under definite stereotyped circumstances had to yield conditioned reflex secretion of insulin in response to intravenous saline injected under the same circumstances. Daily, or every second day, we infused from 40 to $150 \mathrm{ml} 40 \%$ glucose solution in the $v$. saphena of the dogs. Intravenous injections of the above glucose doses caused an increase of blood sugar concentration up to $550 \mathrm{mg} \%$ (during 5 minutes after the injection) that generally returned to baseline levels during the first hour. Blood sugar levels were assessed by means of the method of Hagedorn and Jensen. The blood to be analyzed was drawn from the marginal vein of the ear. Our experiments have shown that it is sufficient to present the dogs 8 to 10 times with the abovementioned dose of glucose, before saline injections carried out in the same manner started to cause a blood sugar fall of $5-15 \%$. The experimental results in all 4 dogs were of the same type. For the purpose of brevity we will only present the results of 2 dogs.

Dog Baikal, male, weight $10.5 \mathrm{~kg}$, received intravenous injections of saline in 25 sessions that were run before the start of sessions with glucose presentation. On six occasions bloodsugar concentrations were determined, and not once a lowering of blood sugar level was found after the intravenous saline injection (table A.1). After 8 glucose injections (40 $\mathrm{ml} 40 \%$ solution), i.e., 8 pairings of CS and UCS, saline injections in the same manner started to cause decreases in blood sugar level. The blood sugar decrease now was elicited by the action of the CS alone. Usually the blood sugar level returned to its initial value during the first hour. The level of blood sugar in the glucose injection trials rose to 380 $420 \mathrm{mg} \%$.

Dog Dik, male, weight $26,5 \mathrm{~kg}$. Prior to the administration of glucose, control injections with saline did not cause a noticeable decrease in bloodsugar level. Six trials were conducted with saline injections, and in three cases the blood sugar levels were determined (table A.1). After 9 glucose injections (100 $\mathrm{ml} 40 \%$ solution) causing increases in blood 
sugar level up to $450-520 \mathrm{mg} \%$, saline injections carried out in the same manner started to elicit a fall in blood sugar level (table A.2). In these sessions, a return to baseline levels of blood sugar occurred in the course of the first hour, just as had been the case in dog Baikal. The experimental results in the other two dogs, Zhuchka and Bek, were completely analogous to the cases described above. We carried out 39 trials with the dogs, in which after an initial 7-11 glucose injections, a saline injection was given instead, i.e. only the CS was presented by us. In 35 of these trials we got a noticeable fall of blood sugar level after saline injection, in 4 trials the aforementioned effect was not seen (in 2 of the latter 4 sessions a violation of the usual procedure had occurred: the regular experimenter was absent, and had been replaced by another person. Our observations on humans ( 9 in fact healthy subjects aged between 31 and 56) confirm the results of the trials run with animals. We injected $40-60 \mathrm{ml} 40 \%$ glucose solution, in the $v$. cubitalis.

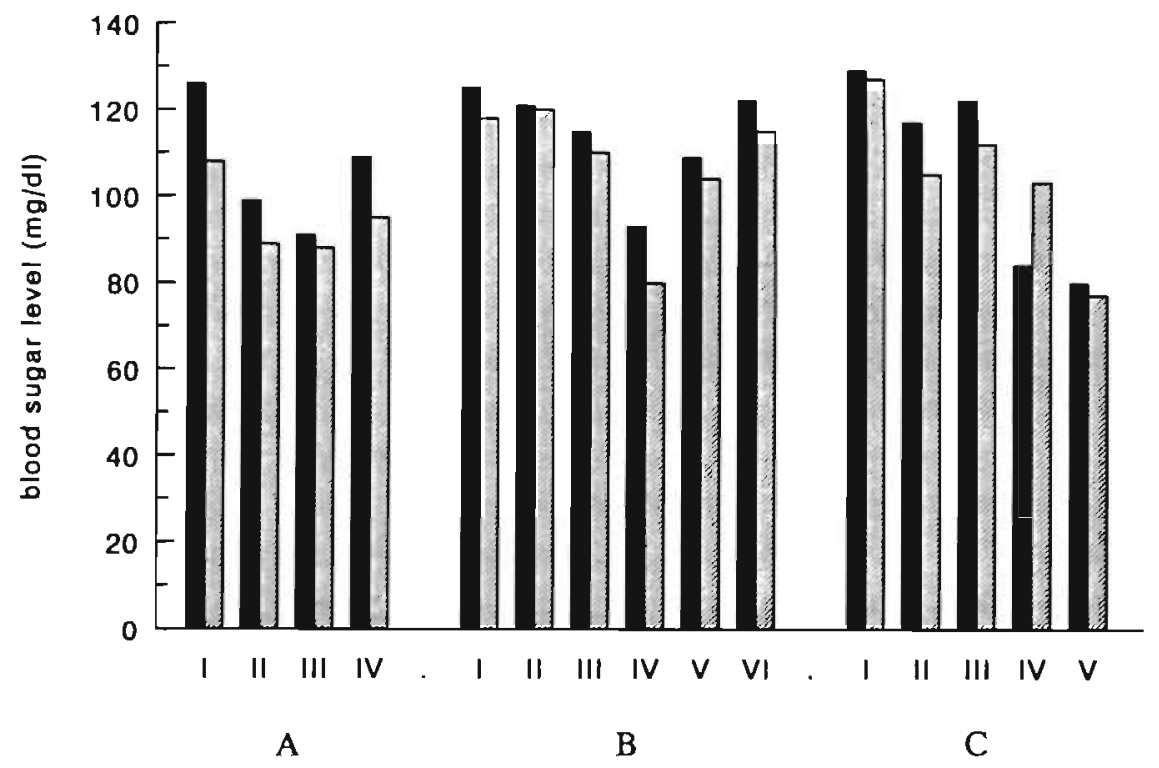

figure A.1: Determination of insulin content in dogs' blood by the bloodsugar test in white mice.

- black-colored bars: blood sugar concentration in milligram\% in the mice at 2 hours after injecting them with dog blood taken just before the saline injection;

- gray-colored bars: the same, but laken from dogs at 15 minutes after the saline injection, being the CS to insulin secretion (The US consisted of a glucose injection). Each bar represents the mean blood sugar level in mice after infusion of $0,6 \mathrm{ml}$ blood of the dogs A-Dik; B- Baikal; C- Bek. For each dog, blood was tested on a different number of occasions. 
At five minutes after the injection, the blood sugar level increased to values up to $250 \mathrm{mg} \%$ and a return to baseline level appeared in the course of the first hour. Blood for the analyses was drawn from the finger according to the usual method.

Preliminary control trials showed that an intravenous saline injection caused either no noticeable change or a non-significant increase in blood sugar level. After 5-10 fold repeated glucose injections, saline injections, i.e. presentation of the CS alone, began to cause a decrease in blood sugar level of between 5 and $20 \mathrm{mg} \%$ (table A.3). The data collected in all 9 subjects appeared to be alike: therefore, in table A. 3 we only show the results of 4 subjects. In humans, 33 sessions were run in which, after a preceding 5 - to 14 fold glucose administration, the same quantity of saline was injected under the same circumstances, i.e. the CS was presented alone. In 30 of these sessions we found a clear drop in blood sugar level after the saline injections. In 3 sessions the blood sugar level did not change. In those cases we had not followed the usual experimental procedure: for reasons beyond our influence, the saline injections had to be given in a different room. Thus, in the overwhelming majority of investigations in both animals and humans we found a conditioned fall in blood sugar in response to a saline injection. As indicated above, saline by itself did not evoke similar changes in blood sugar level.

The conditioned fall in blood sugar found by us, as well as that found by H.C. Sedina (7) with subcutaneous and intraperitoneal glucose injections, may depend on two factors: first, on a conditioned insulin secretion, and second, on a conditioned increase of glucose utilization by the tissues of the organism. In the literature there are reports by a number of authors on conditioned lowering of blood sugar level after of systematic subcutaneous injections of insulin in a stereotyped manner (J.A. Povorinski (5), V.A. Savchenko (6), I.J. Maleva (4), and C.M Leites (3). In the investigations of these authors the conditioned decrease of blood sugar appeared to be based exclusively on a conditioned enhancement of glucose utilization by the tissues.

We would be interested in first place in the degree in which the insular apparatus contributed to the observed conditioned fall in blood sugar. Therefore, we decided to carry out a series of experiments which included an assessment of insulin levels in the animals' blood. Only changes of insulin concentration in blood would be able to demonstrate a clearcut contribution of glandular secretion to conditioned reflex lowering of blood sugar level. Unfortunately, we do not yet possess a reliable method of chemical determination of insulin in the blood and presently the most suitable method appears to be the biological test.

We used the method by Brooks and Horsters, widely used in laboratory of E.S. London. We drew $0.6 \mathrm{ml}$ blood of a dog and administred $0,2 \mathrm{ml}$ to each of three mice (subcutaneously to the belly skin). After 2 hours an analysis was conducted on the blood sugar concentration of the mice. In the laboratory of E.S. London, it was established that $0.2 \mathrm{ml}$ 


\section{Appendix}

of the alien blood had not changed the blood sugar level in the mice, but that larger quantities did evoke changes.

To render the conditions of the experiment as constant as possible, the mice in our experiment were moved to a separate cage and deprived of food at one hour before the start of the experiment. In dogs, two blood samples were drawn from the marginal vein of the ear. The first was drawn immediately before the saline (control) injection, and the second at 15 minutes after the injection, i.e. at the time of the conditioned decrease of blood sugar. The latter time was chosen because in the majority of our experimental sessions the conditioned decrease of blood sugar had been the strongest then (see tables).

The results of this series of experiments are shown in figure A.1 from which one can see that in the majority of cases (14 out of 15) the blood sugar level in the mice that had been given the dog's blood from the period of the conditioned blood sugar decrease was less then of mice who had received the blood of the dogs collected before the saline injection.

Consequently, the dogs' blood during the conditioned decrease of blood sugar level contained considerably more insulin than the blood collected prior to CS presentation.

Thus, the biological tests of insulin level show that in the conditioned fall in blood sugar level as found by us, an important role is played by the secretion of insulin. Consequently, on basis of all experimental data from our investigations on animals and humans, we conclude that after systematic (8-10 times repeated) intravenous glucose injections, a conditioned fall in blood sugar level occurred when instead of glucose saline was given in the same manner.

This fall in blood sugar level was caused to a certain degree by a conditioned secretion of insulin, as confirmed by our results with tests of the insulin level in the blood.

received 6-X-1952

\section{References}

1. B.G. Baranov (1952). Sovietskaya Vrachebnaya Gazeta 13;1.

2. K.M. Bykov (1950). Stenographic report of the united scientific session of the USSR Academy of Sciences and Academy of Medical Sciences SSSR, devoted to the problems of Pavlovian Physiological Studies.

3. C.M. Leites (1951). Abstract of the lecture at the united meeting of the All-Soviet and Ukrainian Institutes of Experimental Endocrinology 97.

4. I.J. Maleva (1951). Klinicheskaya Meditsina 29, 9, 41.

5. J.A. Povorinski (1939). Reports of the IX. Scientific Meeting of the Central Psychoneurological Institute, Kharkov.

6. Savchenko, V.A. (1946). On the mechanism of action of insulin and adrenalin.

7. Sedina, N.A. (1949). Mechanisms of pathological reactions, issue 11-15, 267. 
. 



\section{SUMMARY OF THIS THESIS}

Probably between one and five percent of the female adult population is suffering from binge eating. Binge eating is part of the DSM-IV diagnoses bulimia nervosa, anorexia nervosa (binge-eating/purging subtype) and binge-eating disorder, and is characterized by recurrent ingestion, within a discrete period of time (e.g. within a 2-hour period), of an amount of food that is definitely larger than most people would eat during a similar period of time and under similar circumstances. During binges there is a sense that control over eating is lacking. Chapter 1 highlights the prevalence, size and frequency of binge eating, as well as its social impact. Strikingly, binge eating often occurs in women who attempt to lower their body weight. In the past two decades, binge eating has been the object of much theorizing and research. Notably, the influential restraint theory maintains that binge eating can be explained by the existence of "disinhibitors" (for example, low moods or catastrophic thoughts) breaking an ongoing attempt to restrict food intake (the "restraint"). A laboratory model of overeating, called "counterregulation", has been put forward as the operationalization of this theory. However, doubts can be raised about the validity of the restraint theory, firstly, because counterregulation has been shown to be a behavior that is only modestly replicable, and secondly, because the restraint theory does not postulate an explicit urge-to-eat mechanism.

In chapter 2, the Conditioning Theory of Binge Eating ( $\mathrm{CBE}$ theory), pivotal to the work in this thesis, is described. $\mathrm{CBE}$ theory posits that underlying binge eating are classically (Pavlovian) conditioned responses. These conditioned responses (CR) occur in response to "binge cues" (CSs; conditioned stimuli), which, for the individual, have previously been reliable predictors of a binge (UCS, unconditioned stimulus). Binge cues may be, for example, the sight, smell or taste of certain foods, or the individual's presence in an environment in which binges usually occur. The perception of binge cues is supposed to lead to a conditioned secretion of the hormone insulin, and an accompanying fall in blood sugar level (CR). Although these responses are, homeostatically speaking, adaptive because they attenuate the post-prandial blood sugar rise, according to $\mathrm{CBE}$ theory they are subjectively experienced as a craving for food. A review of the relevant literature is given. Interestingly, CBE theory has given rise to the development of a therapeutic procedure for binge eating called cue-exposure. In this procedure, patients are presented with the odour and sight (CS) of binge foods for a prolonged time (for example one hour long), without the possibility of ingesting these foods (UCS). The extinction of craving (and bingeing), predicted by CBE theory, has indeed been demonstrated in several studies. 


\section{Summary}

Subsequent chapters report empirical studies in which several CBE hypotheses have been tested.

Chapter 3 describes a test of the CBE hypothesis that perception of stimuli that reliably predict a blood sugar rise will lead to insulin secretion, hypoglycaemia and increased craving for sweet substances. In six conditioning trials, subjects were asked to ingest a glucose drink (UCS) in a room with a peppermint odour (CS) hanging in the air. Glucose was chosen as the UCS because it leads to a large blood sugar rise and prompts a strong insulin response. During test sessions, before and after conditioning, only the CS was presented, after which blood samples were taken, and craving was monitored by questionnaire. Subsequently, subjects participated in a "taste test", during which they were to compare several glucose drinks and in which the amount of ingested glucose was measured. Subjects in a control condition, in which the inert sweetener aspartame was presented during conditioning, were hypothesized to show no conditioned responses. The results showed no differences between conditions on blood sugar level or subjective craving. A slightly increased secretion of insulin was observed in the experimental condition. However, the size of the observed difference did not exceed the range of normal spontaneous fluctuations. Interestingly, in both conditions, a strong increase in the amount of ingested glucose was observed after conditioning. This finding was explained as a consequence of a neophobia or "bait shyness" during the initial test session: the subjects would be reluctant to drink freely in the novel lab situation. Subsequently, repeated lab-sessions could have led to a gradual fading of the reluctance to drink. To investigate the phenomenon further, an additional condition was run. This time, the conditioning procedure involved a normal tap water UCS (instead of sweet drinks). Once again, a large increase in glucose intake was observed after conditioning. It was concluded that a key factor in the increased glucose intake was an increased experience with carrying out a lab-procedure, rather than repeated exposure to sweet drinks. These results may have implications for future food intake research using repeated measurements.

The CBE prediction that blood sugar conditioning is possible using a glucose-containing UCS is corroborated in chapter 4. A commentary and re-analysis is given of a 1954 study by the Russian physiologist Mityushov (see also the appendix of this thesis). Mityushov found a physiologically significant hypoglycaemia after injecting saline in humans and dogs who had been given repeated glucose injections previously. The observed results suggest a physiological state which, according to CBE theory, occurs prior to bingeing. Mityushov's procedure might, in case of replication of the results, be employed in future research into binge eating.

The next two chapters test CBE hypotheses about food cue reactivity, i.e., the intensity 
with which people respond to presentation of food-related cues. The CBE theory predicts that cue reactivity is especially intense in individuals with occasional large food intake. In chapter 5, slides depicting subjects' favourite binge foods were used as cues. During presentation of the slides, psychophysiological responses (e.g. skin conductance, heartrate and facial EMG) were monitored. Two groups of subjects, restrained and unrestrained eaters (selected by Herman \& Polivy's Restraint Scale) were compared. Because high Restraint scores suggest a food intake pattern characterized by alternate dieting and overeating, the CBE-derived prediction was that restrained eaters would show larger cue reactivity than unrestrained eaters. The results did not confirm this prediction. Whereas slides with binge food were indeed rated higher on craving-arousing property than were control slides (with office equipment), no differences were found in psychophysiological responses of restrained and unrestrained subjects.

In chapter 6, real food items (sandwiches and candy bars) were presented to subjects given the instruction to smell and look towards these food items attentively. Skin conductance responses, heart rate and salivation were monitored in subjects who had fasted either for 3 , or for 24 hours. The CBE-related hypothesis was that 24-hour fasting subjects would show larger craving and (accordingly) cue reactivity and craving than 3hour fasting subjects. The results showed no group differences in cue reactivity, although, as expected, the 24-hour fasting subjects reported increased craving and a higher hedonic rating of the presented food items.

Chapter 7 features a comparison between restrained and unrestrained eaters (as selected by the Restraint Scale, see chapter 5). Given the CBE theory, it was predicted that ingestion of an appetizer would lead restrained eaters to disinhibit during a subsequent taste test with ice-cream. The present study tried to assess the preoccupation (attentional bias) for food-related stimuli in disinhibiting individuals by means of the Stroop colournaming test. In the control condition, no appetizer was presented. The results showed that, contrary to expectations, no disinhibitive eating was found among the restrained subjects. A remarkable finding was the significant correlation between the level of attentional bias and the amount of subsequently ingested ice-cream in unrestrained eaters. In restrained eaters the correlation was near zero. A possible clinical application of the Stroop test as a predictor of relapse in treated bulimics is discussed.

In Chapter 8 some problems are noted in using the Restraint Scale in lab studies of binge eating. Doubts are raised about the ability of the Restraint Scale (which was originally developed to identify dieters) to optimally predict overeating in the lab (see also section 1.3, and chapters 5 and 7 of this thesis). Furthermore, the Restraint Scale has been criticized for some psychometric weaknesses. Given these problems, a new selection instrument was developed the Disinhibitive Eating Scale (DIS), which contains 
four subscales: failing diets, binge eating, weight fluctuations and self-esteem. A psychometric analysis of the 17-item DIS revealed generally satisfying characteristics. Although DIS and RS total scores intercorrelated strongly, it was shown that both scales select subject groups which differ in composition. It remains to be established whether the DIS indeed possesses the predictive validity for disinhibition in the lab, for which it was developed.

Chapter 9 concludes the present thesis, with the observation that none of the novel studies reported here have produced results confirmative of the hypotheses derived from $\mathrm{CBE}$ theory. In an attempt to elucidate why, a critical and quantitative re-assessment was conducted of the literature considered as supportive of the CBE assumptions (see chapter 2 ). The following picture emerged. First, there is little support for the existence of physiologically speaking significant conditioned blood sugar and insulin responses. Second, at present there are no indications that bulimics show increased insulin and blood sugar reactions to food-related cues. Finally, ingestion of only one candy bar or a few biscuits results in a blood sugar rise that will nullify conditioned hypoglycaemias of the size reported thus far within 10-15 minutes; nevertheless the average binge has been reported to last two to three times longer than that.

Based on these considerations, profound scepticism seems warranted about the role of conditioned blood sugar and insulin responses in binge eating. Considering the success of the cue exposure therapy which was derived from $\mathrm{CBE}$ theory (section 2.2.6), a curious, but not disastrous situation has arisen.

The present dissertation concludes with theoretical and practical suggestions for future research on binge eating. Two important aspects of $C B E$ theory that have not been rejected, i.e., the role of learning, and stimulus-driven character of binge eating, are given emphasis.

Two theoretical directions are discussed. In the first, a possible (and modest) role of learned blood sugar and insulin responses is proposed for the minutes immediately preceding a binge. In the second, it is proposed that an additional mechanism is active in binge eating. Perception of cues that have come to reliably predict a period of fasting and/or self-induced vomiting will lead to increased food intake. In this view, people would not so much binge in order to lift a momentary deprivation (i.e. the hypoglycemia predicted by CBE theory), but rather to preclude a forthcoming deprivation. Several studies, in line with the existence of such a mechanism, are reviewed.

To conclude, several practical suggestions are made for future binge eating research. Emphasis is put on the development of convincing and robust lab models of binge eating. A model of binge eating can be said to exist if some core manipulation reliably produces increased craving and food intake. Subsequent knowledge growth may then 
occur by introducing additional manipulations, and evaluating their effects on eating behaviour. Three possible lab models of binge eating are described. First, the cue reactivity model, in which food intake and craving are increased by presentation of stimuli related to food items previously ingested in daily life. Second, the conditioned hypoglycaemia model, in which, after a conditioning procedure resembling that of Mityushov (see chapter 4; appendix), cue presentation leads to increased food intake, mediated by a conditioned hypoglycaemia. And third, the post-meal deprivation model, in which food intake is increased by presentation of stimuli that, in a previous learning procedure, have become predictive of a fasting period. Relevant experiments, and results needed for the development of the models are described in some detail. The research proposals may give food for research activities for many years to come. 



\section{SAMENVATTING}

Vermoedelijk lijdt tussen de één en vijf procent van de vrouwelijke volwassenen op dit ogenblik aan eetbuien (Engels: binge eating). Het vertonen van eetbuien vormt een onderdeel van de DSM-IV diagnoses 'Bulimia Nervosa', 'Anorexía Nervosa (vreetbuien/purgerende type)', en de 'Vreetbuienstoornis', en wordt omschreven als het frequent, binnen een beperkte tijd (bijvoorbeeld twee uur) eten van een hoeveelheid voedsel die beslist groter is dan wat de meeste mensen in eenzelfde periode en onder dezelfde omstandigheden zouden eten. Karakteristiek daarbij is het gevoel de beheersing over de voedselinname kwijt te zijn. In hoofdstuk 1 worden prevalentie, grootte, frequentie, samenstelling en sociale gevolgen van eetbuien nader beschreven. Opvallend is dat eetbuien vaak voorkomen bij vrouwen die het uiterst belangrijk vinden om slank te zijn. De vraag werpt zich dan ook op hoe het optreden van eetbuien kan worden verklaard. De invloedrijke restraint-theorie stelt dat overeten wordt veroorzaakt door 'ontremmers', (zoals bijvoorbeeld somberheid of catastrofale gedachten) die een tot dan toe bewust volgehouden lijnpoging (de restraint) torpederen. Deze theorie werd geoperationaliseerd en in het lab bestudeerd onder de naam counterregulatie. Twijfel is echter gerezen over de houdbaarheid van de restraint/counterregulatie theorie: ten eerste blijkt counterregulatie matig repliceerbaar in het lab, ten tweede geeft de restraint theorie geen expliciete verklaring voor de eetdrang.

In hoofdstuk 2 is de Conditioning Theory of Binge Eating (CBE-theorie) beschreven, welke centraal staat in deze dissertatie. De CBE-theorie stelt dat er bij eetbuien sprake is van aangeleerde, zgn. klassiek (of: Pavloviaans) geconditioneerde responsen. De geconditioneerde responsen (CR) treden op zodra een persoon geleerd heeft dat bepaalde stimuli (CS, ook wel eetbui-cues) betrouwbare voorspellers zijn van eetbuien (ongeconditioneerde stimuli, UCS). Eetbui-cues kunnen zijn het zien, ruiken of proeven van voedsel, of het verblijf in ruimtes waar eetbuien gewoonlijk plaatsvinden. De waarneming van eetbui-cues leidt automatisch tot afscheiding van het hormoon insuline en een bloedsuikerdaling (CR), die anticiperen op de grote bloedsuikerstijging na de eetbui. Enerzijds is dit een zinvolle, natuurlijke reactie (het lichaam streeft een evenwichtige bloedsuiker huishouding na). Anderzijds wordt de anticipatieve bloedsuikerdaling volgens de CBE-theorie subjectief beleefd als een drang tot eten. Een Iiteratuuroverzicht beschrijft de evidentie voor CBE-assumpties. Interessant is dat intussen enkele succesvolle effectstudies zijn verricht naar een therapie genaamd cue exposure. Hierbij worden patiënten langdurig geconfronteerd met de geur en uiterlijk (CS) van het eetbuien voedsel, echter zonder de mogelijkheid het voedsel (UCS) in te nemen. De door de CBE-theorie voorspelde uitdoving van de eetdrang bleek in deze studies uit te komen. 
In de volgende hoofdstukken worden verscheidene $\mathrm{CBE}$-hypotheses empirisch getoetst. In Hoofdstuk 3 wordt de CBE-voorspelling getest dat insuline secretie, bloedsuikerdaling en toegenomen drang tot inname van zoetigheid kunnen worden opgewekt door een stimulus die een glucose inname voorspelt. Gedurende zes conditioneringssessies dronken proefpersonen een glucosedrank in een kamer waar een pepermuntgeur (CS) was verspreid. Glucose verhoogt het bloedsuikerniveau aanzienlijk en leidt tot insulineafscheiding. In twee sessies, één voorafgaand en één volgend op de reeks conditioneringssessies, vonden de gedragsmetingen plaats. Alleen de CS werd gepresenteerd, vervolgens werden bloed en vragenlijsten afgenomen. Hierna kregen de proefpersonen een smaaktest, waarbij glucose dranken moesten worden vergeleken; de hoeveelheid daarbij ingenomen drank werd gemeten. Voor de controle groep - geconditioneerd met de inerte zoetstof aspartaam - werden geen effecten van conditionering verwacht. De resultaten van deze studie toonden niet de verwachte effecten op bloedsuiker en craving. Wel werd er een iets vergrote insulinerespons gevonden in proefpersonen van de experimentele groep op vijf minuten na CS-aanbieding. Op basis van de relevante literatuur werd echter geconcludeerd dat de grootte van het gevonden effect die van spontane fluctuaties niet oversteeg. Opvallend resultaat, in beide condities, was de sterk toegenomen hoeveelheid gedronken glucosedrank na conditionering. Gehypothetiseerd werd dat dit resultaat samenhing met zgn. neophobia en bait shyness tijdens de eerste, onwennige testsessie, waardoor proefpersonen terughoudend waren bij het drinken van de glucose drank. De dagenlange conditioneringsprocedure zou hebben geleid tot het wegebben van de terughoudendheid. Teneinde dit verschijnsel nader te onderzoeken werd een derde conditie toegevoegd, waarbij proefpersonen deze keer werden geconditioneerd met kraanwater in plaats van zoete drankjes. Na conditionering bleek ook in deze conditie de consumptie van glucosedrank toegenomen. Geconcludeerd werd dat, meer nog dan toegenomen vertrouwdheid met de lab procedure, de ervaring met zoete drankjes hiervoor verantwoordelijk was. Deze conclusie is relevant voor toekomstig onderzoek waarin herhaalde metingen van voedselinname aan de orde zijn.

De CBE-assumptie dat bloedsuikerconditionering met behulp van glucose bevattende UCS-sen wel degelijk mogelijk is, wordt in Hoofdstuk 4 ondersteund. Dit bevat een commentaar op, en heranalyse van een studie van de Russische fysioloog Mitjoesjov uit 1954 (zie de Appendix). Dr. Mitjoesjov slaagde erin een fysiologisch significante hypoglykaemie aan te tonen na het injecteren van een 'placebo' (saline) bij mensen en honden die eerder herhaaldelijk glucose injecties hadden ontvangen. De resultaten suggereren een fysiologische toestand die volgens de CBE theorie voorafgaat aan eetbuien. Mitjoesjov's procedure zou - na replicatie van de uitkomsten - wellicht benut kunnen worden in toekomstig eetbui-onderzoek. 
De volgende twee hoofdstukken bevatten studies over zgn. cue-reactiviteit: d.w.z. de intensiteit van psychofysiologische reacties tijdens het waarnemen van aan voedsel gerelateerde stimuli. Uit de CBE-theorie volgt dat voedselcue-reactiviteit vergroot is bij personen met een geschiedenis van grote voedselinnames.

In hoofdstuk 5 werden als stimuli dia's gebruikt met daarop voor elke proefpersoon geselecteerd 'eetbuien-voedel'. Tijdens het dia-kijken werden psychofysiologische reacties als huidgeleiding, hartslagfrequentie, en gezichtsspierspanningen gemeten. De reacties van twee groepen proefpersonen - geselecteerd met behulp van Herman en Polivy's Restraint Scale - werden met elkaar vergeleken. Aangezien een hoge score op de Restraint Scale duidt op een eetpatroon met afwisselend lijnen en overeten, was de CBE-hypothese dat hoge scorers (lijners) sterkere cue-reactiviteit zouden vertonen dan lage scorers (niet-lijners). Hoewel een manipulatiecheck toonde dat de dia's met eetbuivoedsel als smakelijker werden gewaardeerd dan controle dia's (met kantoorartikelen), werden geen verschillen gevonden in psychofysiologische reacties van lijners en nietlijners.

In hoofdstuk 6 werden de proefpersonen voedingswaren (broodjes gezond en candybars) voorgezet, met de opdracht er verscheidene minuten aandachtig aan te blijven ruiken en naar te kjjken. Huidgeleiding, hartslag en salivatie werden geregistreerd van proefpersonen die vooraf drie dan wel vierentwintig uur hadden gevast. De CBE hypothese was de waarneming van het voedsel een intensere eetdrang en (hiermee) psychofysiologische reacties zou teweegbrengen bij degenen die vierentwintig, dan bij degenen die drie uur hadden gevast. Hoewel de vierentwintig uur inderdaad een vergrote subjectieve eetdrang (en waardering van het voedsel) rapporteerden, kon de gestelde hypothese niet worden bevestigd. In dit geval was door het voedsel opgewekte eetdrang dus niet zichtbaar in intense fysiologische reacties.

In Hoofdstuk 7 werd opnieuw het gedrag vergeleken van lijners en niet-lijners (geselecteerd mbv de Restraint Scale, zie hoofdstuk 5). Uit de CBE-theorie en de bestaande literatuur werd de voorspelling afgeleid dat het aanbieden van een 'appetizer' bij lijners zou leiden tot disinhibitief eetgedrag tijdens een smaaktest met roomijs. In dit experiment werden hierbij optredende cognitieve effect onderzocht, met behulp van de Stroop reactietaak. Met deze taak werd gehoopt inzicht te krijgen in de mate van preoccupatie (ook wel attentionele bias) voor voedselstimuli. In de controle conditie werd geen appetizer aangeboden. Tegen de hypothese in bleek dat lijners in de appetizer conditie geen disinhibitief eetgedrag vertoonden. Opmerkelijk was de significante correlatie tussen de attentionele bias voor voedselwoorden en de vervolgens genuttigde hoeveelheid ijs bij niet-lijners. Bij lijners was deze correlatie afwezig. Gewezen werd op een mogelijke klinische toepassing van de Stroop taak als voorspeller van terugval bij 


\section{Samenvatting}

bulimische patiënten.

Hoofdstuk 8 gaat in op enkele problemen bij het gebruik van de Restraint Scale als selectieinstrument voor eetbui-onderzoek in het lab. Men kan betwijfelen of de Restraint Scale, orspronkelijk ontwikkeld ter selectie van lijners, optimaal geschikt is om overeten in het lab te voorspellen (zie ook 1.3, en hoofdstukken 5 en 7). Daarenboven is de Restraint Scale in het verleden wegens enkele psychometrische zwakheden bekritiseerd. Vandaar dat een nieuw selectie-instrument wordt gepresenteerd, de Disinhibitive Eating Scale (DIS), met vier subschalen: mislukkende dieten, eetbuien, gewichtsfluctuaties en self-esteem. Een psychometrische analyse van de 17 items tellende DIS leverde alleszins bevredigende resultaten. Hoewel de DIS en Restraint Scale vrij sterk correleerden, werd aangetoond dat beide vragenlijsten verschillende groepen proefpersonen selecteren. Het wachten is nu op een bevestiging van de voorspellende waarde van de DIS voor (over)eetgedrag in het lab.

In hoofdstuk 9 wordt de slotbalans opgemaakt. Geconstateerd wordt dat geen van de hier gerapporteerde, nieuw uitgevoerde studies resultaten hebben opgeleverd die bevestigend waren voor de CBE-theorie. In een poging tot nadere verklaring wordt de literatuur die eerder als ondersteuning voor de CBE-assumpties is gepresenteerd (hoofdstuk 2) kritisch en quantitatief hergeëvalueerd. Het volgende beeld rijst hieruit op. Ten eerste blijkt in de literatuur weinig ondersteuning te vinden voor het bestaan van fysiologisch gezien - aanzienlijke geconditioneerde bloedsuiker en insuline responsen. Ten tweede is uit onderzoek tot nog toe niet geleken dat bulimia nervosa patiënten in versterkte mate insuline en bloedsuikerresponsen vertonen na waarneming van aan voedsel gerelateerde stimuli. Tot slot blijkt dat inname van slechts één candy bar of een paar koekjes na 10-15 minuten tot een bloedsuikerstijging leidt die geconditioneerde hypoglykaemieën van de in de literatuur beschreven grootte volledig opheft; tegelijkertijd is uit ander onderzoek gebleken dat eetbuien gemiddeld twee tot drie keer zo lang duren. Op basis van bovenstaande overwegingen wordt geconcludeerd dat omtrent de rol van geconditioneerde bloedsuikerdaling en insuline secretie in eetbuien diepgaand scepticisme gerechtvaardigd lijkt. Gezien de vermoedelijke effectiviteit van uit de CBE-theorie afgeleide cue-exposure therapie (zie paragraaf 2.2 .6 ) is een merkwaardige, doch nietdesastreuze situatie ontstaan.

De disseratie sluit af met theoretische en practische suggesties voor toekomstig eetbuionderzoek. Twee aspecten van de CBE-theorie die niet zijn weerlegd (nl. de rol van conditionering en aan voedsel gerelateerde stimuli) worden hierbij benadrukt.

Een tweetal theoretische benaderingen wordt geschetst. In de eerste wordt gewezen op een mogelijke (bescheiden) rol van aangeleerde bloedsuiker en insuline reacties, vooral gedurende de minuten voorafgaand aan een eetbui. In de tweede benadering is een 
mechanisme geschetst waarin de rol van vasten en zelf-opgewekt braken na een eetbui centraal staat. Waarneming van eetbui-gerelateerde signalen die braken of een periode van vasten voorspellen, zou de voedselinname doen toenemen. Eenvoudig gezegd zou een eetbui niet zozeer voortduren teneinde een momentane deprivatie (zie het lage bloedsuiker-niveau uit de CBE-theorie) 'weg te eten', maar om een toekomstige deprivatie te voorkómen. Een literatuuroverzicht gaat in op aanwijzingen voor het bestaan van dit mechanisme.

Tot slot worden practische aanbevelingen voor toekomstig eetbui-onderzoek gedaan. Gesteld wordt dat de ontwikkeling van overtuigende en robuuste laboratorium modellen van eetbuien bij mensen cruciaal is. Van een eetbui-model is sprake indien men beschikt over een procedure waarmee betrouwbaar eetdrang en vergrote voedselinname worden bewerkstelligd. Door het vervolgens toevoegen van extra manipulaties aan deze basisprocedure, en evaluatie van de consequenties voor de voedselinname, kunnen eetbuien verder in het lab worden onderzocht.

Drie mogelijke eetbui modellen worden beschreven, het cue reactivity model, waarbij de eetdrang en voedselinname worden vergroot door aanbieding van stimuli gerelateerd aan voedsel dat de proefpersoon kent uit het dagelijks leven, het condirioned hypoglycaemia model, waarbij, volgend op een conditioneringsprocedure à la Mitjoesjov (zic hoofdstuk 4; appendix), presentatie van een CS via een geconditioneerde hypoglykaemie leidt tot een toegenomen voedselinname, en het post-meal deprivation model, waarbij de voedselinname wordt vergroot door aanbieding van stimuli die in een voorafgaande leerprocedure een periode van vasten voorspelden. Relevant onderzoek en resultaten, nodig voor de ontwikkeling van bovenstaande modellen, worden in enig detail beschreven. De uitvoering van deze onderzoeksvoorstellen kan voor vele jaren werk opleveren. 



\section{DANKWOORD}

Hoewel op het omslag slechts mijn eigen naam prijkt, hebben ook anderen een rol gespeeld bij het voltooien van deze dissertatie. Welke rol? Lastige vraag. Men denke eens aan alle krachten die een pingpongballetje de oceaan doen oversteken: niet alleen stromingen in de eindrichting, ook zijstromingen, tegenstroming en stiltes in de stroming hebben onderweg bijgedragen (zie Karel van het Reve, 1979). Zo voel ik het ook een beetje bij dit proefschrift. Enkelen wil ik met name genoemd hebben.

Anita Jansen, 'cheffin', jouw intrigerende theorie vormde de basis voor het project waaraan ik heb mogen werken. Onze samenwerking was voor mij een boeiende, prikkelende, soms echter ook onzachte ervaring. Leerzaam was het zeker: ik voel me thans niet langer 'in opleiding'. Ik hoop dat de toekomstige eetbui-wetenschap nog vaak door nieuwe ideeën van jou zal worden opgefrist.

Marcel van den Hout, promotor, bedankt voor de gastvrijheid van jouw instituten DEP en EPP, en voor je bereikbaarheid die ik als buitengewoon practisch heb ervaren. Steeds weer als ik klopte, bleek jouw deur niet gesloten.

Heiny Eilkes, Ellen Louwerse en Annie Raven: hartelijke dank voor jullie inzet en zorgvuldigheid bij het helpen uitvoeren van de experimenten die in dit proefschrift staan. Hetzelfde wil ik mutatis mutandis kwijt aan alle proefpersonen die hebben meegewerkt, maar die geen exemplaar van mijn proefschrift hebben gekregen.

Theo van Aerts, dank voor het programmeren van de reeks psychofysiologische signaalverwerkers, een puike familie; Erik Schouten, je was een stoïcijns beantwoorder van telkens weer dezelfde vragen over mijn Manova-uitdraai; Bob Wilkinson, vriendelijke dank voor je bijdrage aan mijn Engels. Angela Verweij, vaak wist je op onnavolgbare wijze te informeren of ik wel goed had geslapen; Marja Hortulanus, dank-je voor het verscheuren van dat bierviltje. Alle collega's van DEP/EPP met wie ik heb gepingpongd, alsmede degenen met wie ik niet heb gepingpongd: het ga jullie goed. Welmoet Gerritsen, bedankt voor enkele vriendschappelijke telefoongesprekken; Casper Schoemaker, dat ik me jouw volkomen terechte commentaren nooit voor $100 \%$ ter harte heb genomen, komt ongetwijfeld doordat ik mijn arikels al gedeeltelijk af meende te hebben. Mijn paranimfen: Sabine Kroeze, ik heb echt geboft met jouw kamergenoot- en kameraadschap. Instemmend wil ik je hier citeren: "Gén gepeiger meer!" ; Bart Leunissen, een frequent en langdurig Horecabezoek - voor zover Maastricht dit toeliet - was ons niet vreemd. Ik vertrouw erop dat we een vorm zullen vinden om deze belangwekkende bezigheid in de toekomst voort te zetten.

Mijn ouders: lieve Ma en $\mathrm{Pa}$, dit boekwerk is aan jullie opgedragen. Opvoeden is vast veeleisender dan het schrijven van wel XX dissertaties. Mochten jullie enkele zaken uit deze dissertatie niet geheel kunnen navoelen, wees dan niet bezorgd: het is waarschijnlijk een teken van mijn nu aangebroken 'Jaren des Onderscheids'. 



\section{CURRICULUM VITAE}

Barend Jan Joost Overduin werd op 25 oktober 1964 geboren in Enschede. Daar doorliep hij het Atheneum-B van het Kottenpark College. Vervolgens ging hij natuurkunde studeren aan de Rijksuniversiteit Leiden totdat hij in september 1984 zijn propedeuse diploma (met genoegen) ontving. Aansluitend studeerde hij psychologie, eerst aan de Rijksuniversiteit Utrecht (richtingen Klinische Psychologie en Functieleer), later aan de Universiteit van Amsterdam waar hij in september 1991 cum laude afstudeerde bij de vakgroep Psychonomie, afdeling Emotieleer (Prof. N.H. Frijda). Vijf dagen later begon hij als onderzoeker in opleiding te werken bij de vakgroep Differentiële en Experimentele Psychologie (toen nog GGK) van de Rijksuniversiteit Limburg. Deze dissertatie is een neerslag van dit werk. Momenteel wordt hij dermate geboeid door de combinatie van pharmacologie en ethologie, dat hij overweegt om weer te gaan studeren.

\section{Publicaties}

Overduin, J. (1992). Passie. Bespreking van 'Dieptepsychologie' van J.H. van den Berg. De Psycholoog 27, 450.

Overduin, J. (1993). Het voordeel van de schrik. Over het onvolledige gebruik van de reflex in emotieonderzoeken. De Psycholoog 28, 193-201.

Merckelbach, H. \& Overduin, J. (1993). Een Nederlandse Pavloviaan: J. ten Cate (1887-1967). De Psycholoog 29 67-71.

Overduin, J. (1994). Leidens Ontzet in de ethologie. Herwaardering van de dierlijke subjectiviteit. De Psycholoog 29, 279-284.

Overduin, J., Jansen, A. \& Louwerse, E. (1995). Stroop interference and food intake. International Journal of Eating Disorders 18, 277-285. (Chapter 7, this thesis)

Overduin, J. \& Jansen, A. (1995). The conditioning model of binge eating. In: I. Florin \& B. Tuschen (eds), Current Research in Eating Disorders, 16-27. Münster: Verlag für Psychotherapie.

Jansen, A., Overduin, J. \& Meijboom, A. (1995). Controlled and automatic cognitive processes in eating disorders. In: 1. Florin \& B. Tuschen (eds), Current Research in Eating disorders, 74-81. Münster: Verlag für Psychotherapie.

Overduin, J. (1996). Empirisch onderzoek van de eetbui: uitgangspunten en enkele verkenningen. Psychoscoop 7, 4-11.

Overduin, J. \& Jansen, A. (1996). A new scale for use in non-clinical research into disinhibitive eating. Personality and Individual Differences, in press. (Chapter 8 , this thesis)

Overduin, J. \& Jansen, A. (1996). Food cue reactivity in fasting and non-fasting subjects. European Eating Disorders Review, in press. (Chapter 6, this thesis)

Overduin, J., Jansen, A. \& Eilkes, H. (1996). Cue-reactivity to food- and body stimuli in restrained and unrestrained eaters. Addictive Behaviors, in press. (Chapter 8 , this thesis) 



\section{Pavlovian Conditioning and Binge Eating}

Some Empirical Explorations

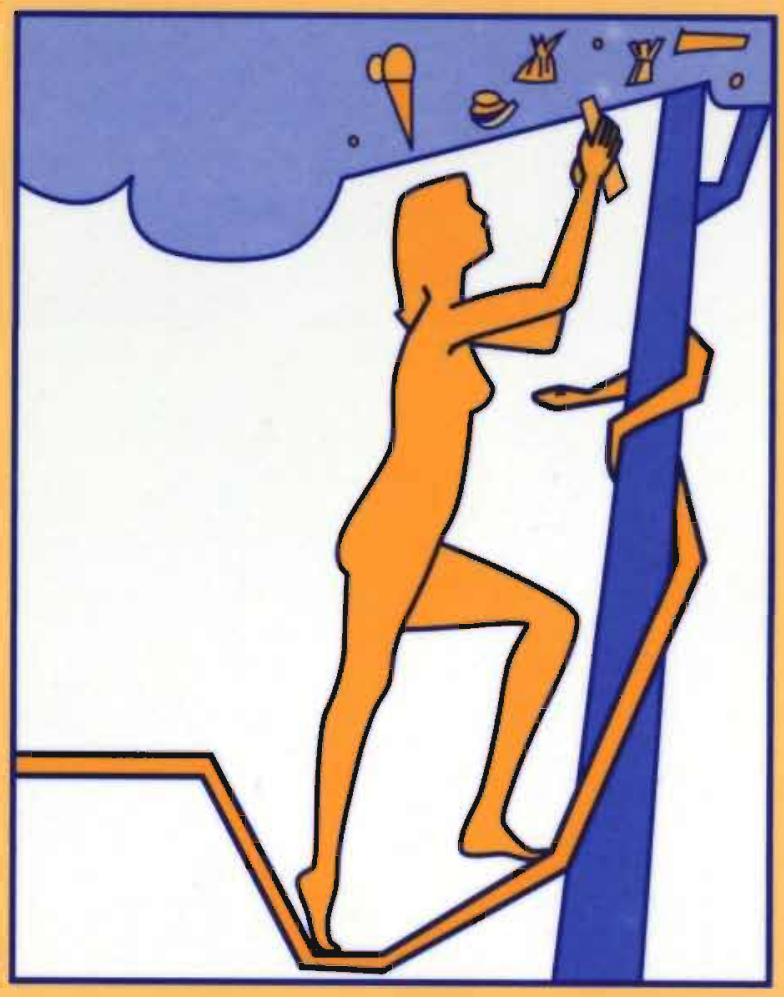

\section{Joost Overduin}




\section{Pavlovian Conditioning and Binge Eating}

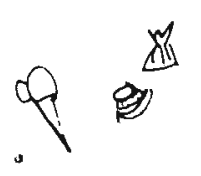


Cover Design: Mariëtte Strik

Printed by: Quick Print, Nijmegen

Overduin, Barend Jan Joost

Pavlovian conditioning and Binge Eating: Some Empirical Explorations/ Barend Jan Joost Overduin.

Thesis University of Limburg, Maastricht. - With ref. With summary in Dutch.

ISBN $90-9009-606-X$

Subject headings: binge eating/ classical conditioning lexperimental psychopathology. 


\title{
Pavlovian Conditioning and Binge Eating Some Empirical Explorations
}

\author{
PROEFSCHRIFT \\ ter verkrijging van de graad van doctor \\ aan de Rijksuniversiteit Limburg te Maastricht, \\ op gezag van de Rector Magnificus, Prof. Mr. M.J. Cohen, \\ volgens het besluit van het College van Dekanen, \\ in het openbaar te verdedigen op \\ vrijdag 5 juli 1996 om 14.00 uur
}

door

Barend Jan Joost Overduin 


\section{Promotor:}

Prof. Dr. M.A. van den Hout

\section{Co-promotor:}

Dr. A. Jansen

\section{Beoordelingscommissie:}

Prof. Dr. H. Merckelbach (voorzitter)

Prof. Dr. N.H. Frijda (Universiteit van Amsterdam)

Prof. Dr. J. Jolles

Dr. N.A. Nicolson

Dr. J.H. Strubbe (Rijksuniversiteit Groningen)

The research in this thesis was financially supported by grant no. 560-268-052 from the Netherlands Organization for Scientific Research, section Behavioural Sciences (NWO-SGW)

The following organizations are gratefully acknowledged for financially supporting the publication of this thesis:

- Faculty of Health Sciences, University of Limburg, Maastricht

- Hoechst-Marion-Roussel, Hoevelaken

- Holland Sweetener Company, Maastricht

- N.W.O., Stichting Gedragswetenschappen, Den Haag

- Quest International, Naarden

- Suikerstichting Nederland, Baarn 
Aan mijn ouders 



\section{Contents}

1.1 On the experimental psychopathology approach

types of investigation - rationale of modelling psychopathology

1.2 Binge eating: definition and characteristics

diagnosistic criteria - prevalence - binge size - binge frequency binge ingredients - mood and binge eating - impact on social functioning and well-being - summary

1.3 Herman \& Polivy's counterregulation model of binge eating description of the counterregulation model - critique of the counterregulation model - final remarks

Chapter 2: A Pavlovian Conditioning Theory of Binge Eating (CBE)

2.1 Description of the conditioning theory of binge eating

five assumptions

2.2 Overview of relevant data

binge cues - homeostatic regulation of blood sugar level -

anticipatory responses to sensory food cues - classical conditioning

of food intake and physiological responses - hyperinsulinaemial

hypoglycaemia experienced as craving - extinction of conditioned physiological responses and binge eating

2.3 Summary and implications for the present thesis

Summary - implications for the empirical work of this thesis

\section{Part II: Empirical Studies}

Chapter 3: Conditioned Insulin and Blood Sugar Responses in Humans in Relation to Binge Eating

Chapter 4: Introduction and commentary to Mityushov (1954):

Conditioned reflex secretion of insulin

Chapter 5: Cue Reactivity to Food-and Body-Related Stimuli in Restrained and Unrestrained Eaters 
Chapter 6: Food Cue Reactivity in Fasting and Non-fasting Subjects

Chapter 7: Stroop Interference and Food Intake

Chapter 8: A New Scale for Use in Non-clinical Research into

Disinhibitive Eating

\section{Part III: Conclusion}

Chapter 9: Conclusions and General Discussion

9.1 Overview of the empirical work in this thesis

9.2 Quantitative reappraisal of the CBE theory

five hypotheses reanalyzed

9.3 The conditioning theory of binge eating: final qualification

9.4 Future research: theoretical considerations maintaining a blood sugar/insulin-related explanation - learning perspective based on post-meal deprivation

9.5 Future research: practical considerations necessity of modelling - three models of binge eating - I. the cue-reactivity model - II. the conditioned hypoglycaemia model III. the post-meal deprivation model

References

Appendix: Mityushov (1954): "Conditioned Reflex Secretion of Insulin" (translation)

Summary

Samenvatting

Dankwoord 


\section{Chapter 1 \\ EXPERIMENTAL PSYCHOPATHOLOGY AND BINGE EATING}

This thesis is an investigation into binge eating. The term "binge eating" refers to a pathology consisting of repeated bursts of excessive food intake. The approach that will be taken throughout this thesis goes under the name of experimental psychopathology. Experimental psychopathology involves the development and empirical testing of hypotheses about underlying mechanisms in psychopathology (Abramson \& Seligman, 1977; Jansen, Merckelbach \& Van den Hout, 1992). After a brief introduction of methods and rationale of the experimental psychopathology approach (section 1.1), essential features of binge eating will be described (section 1.2). The epithet "experimental" by no means implies that experimental psychopathology is non-theoretical: in fact, theories play a pivotal role in experimenting. Section 1.3 contains a critical discussion of counterregulation, a paradigm that has dominated binge eating research in the last two decades. In chapter two, a conditioning theory of binge eating will be described. It is this theory which has inspired the empirical work found in subsequent chapters.

\subsection{ON THE EXPERIMENTAL PSYCHOPATHOLOGY APPROACH}

\subsubsection{Types of investigation}

Within experimental psychopathology, investigations may take numerous forms, three of which will be described here as a frame of reference. In "type P" investigations, the behaviour of patients and non-patients is compared in structured situations, so as to demonstrate or identify crucial parameters of the pathology. An example in the field of eating disorders would be to provide bulimia nervosa patients and healthy subjects with various foods and compare their food choice or rate of ingestion in the lab (e.g. Hadigan, Kissileff \& Walsh, 1989). A problem with this type of research is that recruitment of large samples of well-defined patients is often time consuming and meets various practical difficulties. For pragmatic reasons, researchers may choose to switch to "type Q" investigations, in which two groups of non-clinical subjects are compared. The two groups differ in their extra-experimental history, as for for example reflected by low or high scores on questionnaires tapping habits or traits related to the pathology. An example would be the comparison of the eating behaviour of frequent dieters vs. nondieters in a structured lab situation. A problem with type $P$ and $Q$ research is that individual history may escape rigorous experimental control. Hence a third type of 


\section{Chapter 1}

investigation exists, "type R", involving the study of lab models of the pathology". "Modelling" refers to the "production, under controlled conditions, of phenomena analogous to naturally occurring mental disorders" (Abramson \& Seligman, 1977). Thus, a model is a lab-analogue, or a reversible "miniature version" of the real pathology, to be evoked in non-clinical subjects. A lab-model of binge eating may be at hand as soon as non-clinical subjects have been found repeatedly to increase their food intake after some experimental manipulation. Although the evocation of the lab behaviour may tell us something about the origins of the real pathology, it is not the final goal of modelling (Willner, 1991b): important knowledge derives from subsequent investigation of how the lab model is influenced by various manipulations. For instance, it might be tested whether prodigious food intake by non-clinical subjects in the lab can be suppressed by having the subject smelling the food for a prolonged time. As the development and testing of models is central to experimental psychopathology research, its rationale will be more closely discussed next.

\subsubsection{Rationale of modelling psychopathology}

Ultimate goals of experimental psychopathology are to promote understanding and, perhaps, to suggest new treatments of psychopathology. As such, working with lab models requires a more formal approach than does clinical observation, which admittedly is also a source of knowledge and ideas (cf. Beck et al., 1979). There are advantages and disadvantages to the lab approach.

Many workers have received the endeavour of developing and testing lab models of psychopathology rather coolly. To them, modelling appears to lead researchers astray, that is away from the "full blown pathology of real life". For example, learned helplessness, often used as a lab model of depression, was criticized by the noted British clinician and scientist I. Marks as follows:

\section{"..) present behavioral approaches are not specially promising for the} management of depression.(.) it has not so far been shown that learned helplessness is associated with anything more than mild mood change without the concomitants of serious clinical depression such as guilt, nihilism, suicidal ideas, anorexia, and insomnia lasting at least several weeks. " (cited in Peterson, Maier \& Seligman, 1993, p. 13)

1 The term "model" will be used here only in connection with the experimental procedure of producing miniature psychopathological behaviour in the lab. For the theoretical model of pathological behaviour guiding the experiment, the term "theory" will be reserved. 
Admittedly, lab models can involve stylized forms of behaviour, which only remotely resemble the clinical phenomenon. However, the similarity of lab model and pathology is one, but not always the major concern in the development and testing of models of pathological behaviour, as M.E.P. Seligman, the major proponent of the learned helplessness paradigm, noted in his reply to Marks' criticism:

(the thought that) ". any model of depression in the laboratory must somehow produce suicide, crying, weight loss, guilt and nihilism (..) does not bear scientific scrutiny. Model airplanes do not need to make transatlantic flights; they only need to embody the essence of flying in an airplane. A laboratory model of anxiety need not produce screaming, defecation, and panic antacks - it need only isolate the essential properties of anxiety" (ibid.)

In his reply, Seligman describes the isolation of essential properties of the psychopathology as the crux to model building and testing. The advantage of the isolation of symptoms is understandable if one considers that a given psychopathological symptom hardly ever comes alone. Comorbidity is common and often it remains unclear which symptom precedes, modulates or maintains the other in a given patient. For example, depression and anxiety often coincide with binge eating (APA, 1994; Beebe, 1994; Cooper, 1995; Garner et al., 1990). Thus, isolation of specific symtoms for study becomes especially difficult in patients. Hence, studying a lab model in non-clinical subjects may allow a more efficient investigation of mechanisms underlying the pathology.

Abramson and Seligman (1977) present a list of criteria to evaluate lab models of psychopathology. The important questions to be asked are: a. Are the essential features of pathology's causes as well as its prevention and cures validly described by the model? b. To what extent is there similarity of symptoms between the model and "naturally occurring" psychopathology? $\underline{c}$. To what extent is there similarity between pathology and model regarding physiology, cause, cure and prevention? $\mathrm{d}$. Is the lab analogue a model of one specific psychopathology or of a feature occurring in a range of psychopathologies? Importantly, it should be noted that if all the above criteria are fully met, then so much knowledge is available that testing the model would become superfluous (Jansen et al., 1992a).

In conclusion, there are both limitations and advantages in studying lab models. The limitations arise from the consideration that the functioning of humans is embedded in a complex social cultural context. Hence, the isolation of putatively "essential" characteris- 


\section{Chapter 1}

tics reduces ecological validity. On the other hand, modelling of isolated aspects of the pathology, may elucidate causal mechanisms, which might otherwise remain hidden amongst a myriad of intertwined factors.

\subsection{BINGE EATING: DEFINITION AND CHARACTERISTICS}

"Binge eating", the object of investigation in this thesis, is not to be understood as simple overeating. In this section, the definition, main characteristics and concomitants of binge eating will be described.

\subsubsection{Definition and diagnosis}

The fourth edition of the highly influential Diagnostic and Statistical Manual of Mental Disorders (DSM IV) includes the following definition of binge eating (APA, 1994):

1. eating, in a discrete period of time (e.g., within an two-hour period), an amount of food that is definitely larger than most people would eat during a similar period of time under similar circumstances; 2 . a sense of lack of control over eating during the episode (e.g., a feeling that one cannot stop eating or control what or how much one is eating).

Binge eating is an important symptom in bulimia nervosa, binge eating disorder ${ }^{2}$ and in anorexia nervosa, binge-eating/purging type. For the diagnosis of bulimia nervosa an average binge frequency of at least twice weekly for a period of three months is required; for the diagnosis of binge eating disorder the binge episodes should occur on at least two days a week for a period of six months (APA, 1994). Especially because of its quality of control-loss, binge eating cannot simply be equated with gluttony in obese individuals: less than half of the obese suffer from binge eating (Marcus, 1993; 1995).

Binge eaters with a diagnosis of bulimia nervosa or anorexia nervosa (binge eating/purging type) try to counter the caloric effects of binges by dieting, fasting, and/or doing sports excessively. Self-induced vomiting or using laxatives are also among the means used to lose the weight. In bulimic patients who present for treatment, $80-90 \%$ engage in self-induced vomiting. Because of these behaviours, bulimic binge eaters are not especially obese (APA, 1994). In both bulimia and anorexia nervosa, the weightreducing behaviours coincide with morbid, often unwarranted fears of being fat and getting fatter. Moreover, self-esteem is seen in terms of slimness and amount of body

${ }^{2}$ The diagnose Binge-Eating Disorder has been introduced only recently. In contrast to bulimics, patients diagnosed with Binge-Eating Disorder do not display so-called inappropriate compensatory hehaviours such as self-induced vomiting, misuse of laxatives, fasting and/or excessive exercise (APA, 1994). 
fat. Irrational ideas about eating and distorted body images (to be addressed only marginally in this dissertation) indeed form an important target of successful cognitive behavioural therapeutic interventions in eating disorders (Fairburn, 1995).

\subsubsection{Prevalence of binge eating}

Throughout the 1980 s, different studies estimated the point prevalence of binge eating in the female population ${ }^{3}$ at between 1 and $79 \%$ (Fairburn \& Beglin, 1990; Jansen, 1990). However, these wide ranging figures clearly reflect different definitions of binge eating that have been adopted. Importantly, if one relies on subjective criteria, individuals may refer to intake of merely one chocolate biscuit as "binge eating" (e.g. Rosen, Leitenberg, Fisher \& Khazam, 1986; Rossiter \& Agras, 1990). It is only logical that with the gradual development of more stringent criteria (e.g. the DSM-IV definition) the reported prevalences of binge eating have shown a decline through the years (Jansen, 1990). To get a full picture one should combine recent prevalence estimates for bulimia nervosa, binge eating disorder and anorexia nervosa (binge eating/purging type). Firstly, for bulimia nervosa the prevalence in the adult female population was estimated to lie at around $1 \%$. (Fairburn \& Beglin, 1990; Hoek, 1993; 1995). Secondly, the prevalence of anorexia nervosa (binge eating/purging type) should be expected to be about half of that for anorexia nervosa, which has been estimated at $0.3 \%$ (Hoek, 1993; 1995) or 0.5-1 $\%$ (APA, 1994). Thirdly, binge eating disorder has a prevalence between 0.7 and $4 \%$ (APA, 1994) or $2 \%$ (Marcus, 1993). These figures add up to a total prevalence of binge eating in the female population of somewhere between 1.85 and $5.5 \% .^{4}$.

${ }^{3}$ The prevalence of eating disorders among males has been reported as only a tenth or a twentieth of that for females (Hoek, 1993).

${ }^{4}$ It should be noted that the present calculations are provisional. Fairburn \& Beglin (1990) bave voiced doubts about the possibility of ever obtaining the "true" prevalence of binge eating (at least in bulimia nervosa) in the general population. Furthermore, indications that only a small subset of binge eaters bas been detected and is receiving treatment mean that patient groups may not be representative of binge eaters in the general population (Fairburn \& Beglin, 1990). Therefore, the data on binge eating presented in this section should be considered with some caution. 


\section{Chapter 1}

\subsubsection{Binge sizes}

The DSM-IV criteria for binge eating do not specify an absolute caloric value per binge. This has to do with the large variability of binge size both inter-individually (range: more than 5000 kcalories; Rossiter \& Agras, 1990; Rossiter et al, 1992) and intraindividually (range: approximiately 2000 kcalories; Rosen et al., 1986; Rossiter \& Agras, 1990) in the amount of food ingested during self-reported binges. Whilst there is no doubt that huge quantities of food can be ingested in binge eating, several factors may hamper precise estimations of binge size. First, patients may be prone to refer to any intake of "forbidden food" as a binge, even if only 50 kcalories (the caloric value of one chocolate biscuit) are involved (e.g. Rosen et al., 1986; Rossiter \& Agras, 1990). Inclusion of these so-called "subjective binges" (Fairburn \& Wilson, 1993) may lead to underestimation of the problem. As a provisional solution of this issue, Fairburn (1987) has proposed to count only "uncontrollable" eating episodes larger than $1000 \mathrm{kcal}$ as binges. It appears that about $45-50 \%$ of the self-reported binges (in bulimic patients) conform to this criterion (Rossiter \& Agras, 1990; Walsh, 1993).

Another difficulty in establishing binge size is the underreporting: diary studies typically revealed average binge size of slightly above $1000 \mathrm{kcal}$,, while in lab or otherwise controlled circumstances typically average values of between 1500 and $5000 \mathrm{kcal}$ have been found (Walsh, 1993).

In bulimia patients who do practice self-induced vomiting after bingeing, the average binge size is about twice that of those who do not vomit (Lacey \& Gibson, 1985; Rossiter \& Agras, 1990; Rossiter et al., 1992).

\subsubsection{Binge frequency}

Included in the DSM-IV criteria for the bulimia nervosa and binge eating disorders is the requirement that bingeing should occur at an average frequency of twice a week (bulimia nervosa) or on two different days per week (binge eating disorder) for a period of three and six months respectively (APA, 1994). In spite of these criteria it appears to be difficult to make accurate estimates of binge frequency. As with binge size estimations, frequency estimates are hampered by the phenomenon of "subjective bingeing", i.e. labelling of small or normal meals involving "forbidden foods", as a binge (Fairburn

${ }^{5}$ Note that the following data were mostly drawn from studies on bulimic patients. For reference, the caloric value of one candy bar is between 200 and 250 kilocalories, 200 grams of potato crisps contain about $1080 \mathrm{kcal}$, and mean daily intake is about $2000 \mathrm{kcal}$ for adults, males somewhat more than females (Oettle et al., 1987; Rossiter et al., 1992). The largest binge reported has been 55000 $\mathrm{kcal}$ (this binge included several pauses in which self-induced vomiting occurred; Walsh, 1993). 
\& Wilson, 1993). Several studies in bulimics have reported an average binge frequency of 10-11 per week (Walsh, 1993; Rosen et al., 1986; Rossiter \& Agras, 1990). It should be emphasized that these figures reflect both individuals who binge at least daily and those that do so less often. When counting only binges exceeding $1000 \mathrm{kcal}$ (Fairburn, 1987), the picture becomes different: Rosen et al. (1986) followed a group of 20 Bulimia patients (with bingeing and self-induced vomiting more than three times a week) for one week, and found an average of five binges above 1100 calories. Rossiter and Agras (1990) asked 32 Bulimia patients of the purging type to keep a food intake diary, and found an average of about 4.8 binges above 1000 kcalories in one week (Rossiter \& Agras, 1990).

\subsubsection{Ingredients of a binge}

Often it is reported that during binges, the choice of foods is no longer guided by pleasant taste: raw wheat or pure gravy are ingested if nothing else is available to satisfy the craving. The sensory quality of the binge food does, however, play a role, especially at the commencement of the binge: food types with a special mouth feel, i.e., sweet, soft and creamy, appear to have a special attraction (Abraham \& Beumont, 1982). This might also have to do with the ease with which such food can be expelled after bingeing (Beumont, 1995).

More importantly, binges tend to consist of snacks like chocolate, ice-cream, cakes and pie, and other "forbidden foods" for anyone with the ambition to lose weight (e.g. Abraham \& Beumont, 1982; Rosen et al., 1986; Walsh, 1993). This fact has lead to the idea that binges may gratify a carbohydrate-specific hunger aimed at replenishing brain serotonin (e.g. Pirke, 1995). However, in the early 1980s the alleged overrepresentation of carbohydrate in binges was already discarded (Abraham \& Beumont, 1982). Furthermore, subsequent lab and self-report studies have not found a different macro-nutrient composition in binges and normal meals (Elmore \& de Castro, 1991; Jansen, Van den Hout \& Griez, 1990; Rossiter et al., 1992; Walsh, 1993). Clearly, this does not deny the fact that large amounts of all macro-nutrients may be ingested during binges.

\subsubsection{Mood and binge eating}

As to the affective states surrounding binge episodes, it might be best to say that binge episodes constitute a mixed blessing. First, tension, depression, frustration, loneliness, anxiety and boredom are among the most frequently mentioned precipitants of binges (Abraham \& Beumont, 1982; Bruce \& Agras, 1992; Mitchell, Hatsukami, Eckert \& Pyle, 1985; Beebe, 1994). Once bingeing starts, levels of reported anxiety, depression, etc., appear to be lowered in comparison with the pre binge state (Beebe, 1994). 


\section{Chapter 1}

Second, however, bingeing itself also gives rise to negative moods (Beebe, 1994; Mitchell et al., 1985). Indeed, a range of studies reviewed by Beebe (1994) shows that what is "gained" in mood during bingeing (i.e. lower anxiety, depression) is "lost" by the emergence of new negative feelings, like guilt, loss of control, helplessness, and again depression after the binge, a negative outcome which may lead the patient into purging. Bingeing in patients can thus be said to be consoling for a very limited time.

\subsubsection{Relationship of bingeing to social functioning and well-being}

Whatever the particular background of the individual, it is clear that chronic binge eating co-occurs with impaired social functioning and well-being. Binge eating and purging, which are often kept secret from close relatives and spouse, negatively affect social relationships (Beumont, 1995; Mitchell et al., 1985). Also work impairment as well as financial problems caused by the high cost of binge foods have been reported (Mitchell et al., 1985). Often, bulimia nervosa coincides with depression. There has been ample debate about the causal status of depression in binge eating (Beebe, 1994). An important observation is that overall severity of psychopathology seems unpredictive of treatment success in bulimia nervosa, and that psychopathology diminishes significantly after treatment of the bulimic symptoms (Garner et al., 1990). This may indicate that psychopathological symptoms are secondary to the binge eating/purging itself (Beumont, 1995; Cooper, 1995).

\subsubsection{Summary}

The diagnosis of binge eating includes frequent large food intakes accompanied by feelings of loss of control. Phenomena closely related to binge eating are self-imposed food restriction and post-binge vomiting, related to morbid fears of gaining weight. Binge eating is more prevalent in the female than in the male population. The prevalence of binge eating in the adult female population has been estimated to lie between one and five percent. Binge size varies widely intra- and inter-individually, but in different studies averages have been reported of between 600 and 3000 kilocalories. Average frequency of substantial binge episodes (i.e., $1000 \mathrm{kcal}$ or more) in bulimics have been reported to lie at five per week. Although typical binge food consists for an important part of high-caloric snacks, they do not appear to satisfy specific hungers for carbohydrates: binges do not differ significantly from normal meals in their macro-nutrient composition. Affective consequences of bingeing are mixed: binges appear temporarily to alleviate depression and tension, but also cause shame, guilt and feelings of controlloss. Binge eating and vomiting coincide with impaired well being and social functioning of the individual. There is some consensus among researchers that depression and other 
psychopathology are a consequence rather than a cause of binge eating.

\subsection{HERMAN \& POLIVY'S COUNTERREGULATION MODEL OF B BINGE EATING}

As counterregulation has been the predominant lab model in research of human binge eating for many years, it will be described and evaluated below. The conclusions will provide a background for our decision to adopt a different (conditioning) paradigm in chapter 2 .

\subsubsection{Description of the counterregulation model}

In the mid 1970s, hunger and weight were no longer seen as the only factors governing food intake. Research on normal and deranged eating turned towards the concept of eating style. Two prominent workers in the new research area were C.P. Herman and J. Polivy at the University of Toronto psychology department, who developed the "preload-counterregulation" paradigm (Herman \& Polivy, 1980; 1984; Ruderman, 1986). The basic procedure is as follows: subjects participate in a "taste test" involving a questionnaire-guided comparison of different sorts of ice cream. Each subject has ad lib access to plates with abundant ice cream for a period of, for example, 10 minutes, and is kept unaware of the fact that her/his intake will be measured. There are two conditions: in the "preload" condition a high caloric item (e.g. a milkshake) must be eaten prior to the taste test, whereas the control condition involves no preloading. Furthermore, in the basic design the ice cream intake of dieters is compared with that of non-dieters. Dieting is identified by high scores on a questionnaire, the Herman and Polivy's Restraint Scale $^{6}$. The pattern of food intake that has been repeatedly observed by Herman and Polivy is as follows. Unrestrained eaters (the "non-dieters") act reasonably by diminishing their food when they have already eaten a preload. That is, they "regulate" their food intake. Surprisingly, restrained eaters (i.e. the "dieters") have been observed to "counterregulate: they eat more in the preload than in the control condition?

The explanation given by Herman and Polivy $(1980 ; 1984)$ is based on two elements. First, restrained eaters are said to impose a deliberate, "cognitive" restraint on their food intake. In the no-preload condition, this prompts them to eat less ice cream than

\footnotetext{
${ }^{6}$ For a more detailed discussion of the Restraint Scale see chapter 8 of this thesis.
}

${ }^{7}$ Note that an important criterion of a lab model of binge eating thus was fulfilled: after some experimental manipulation, an increase in food intake was observed. 


\section{Chapter 1}

unrestrained eaters. Second, restrained eaters are likely to see their restraint broken by the "forced" ingestion of the high-caloric preload. Herman and Polivy have reasoned that broken restraint prompts catastrophic thoughts (e.g. "I've blown my diet, so I might as well go on eating"), that will pave the way for excessive eating. Contributing to the larger food intake in these dieters are "biological pressures" acting to compensate for previous chronic restriction of food intake ${ }^{8}$.

Later studies have extended the basic findings by demonstrating broken restraint after other experimental manipulations. It was shown that restrained eaters also increase their food intake after low-mood inductions, or after information that an already eaten preload was of high caloric content. Also the mere prospect of forced ingestion of high caloric food items has been found to trigger increases in food intake (see Herman \& Polivy 1984; Ruderman, 1986). Although the original purpose of the preload/counterregulation paradigm was to investigate dieters' eating behaviour, counterregulation has increasingly been labelled as a form of disinhibition (Herman \& Polivy, 1988; Polivy \& Herman, 1989).

\subsubsection{Critique of the counterregulation model}

Twenty years have past since the concepts of restraint and counterregulation were introduced. The gathered data and related theorizing have been effective in drawing researchers' attention from mere hunger and body weight towards cognitions and behavioural mechanisms active in the disinhibiting eater. Also, this line of research has underscored the multitude of problems encountered by people attempting to lose weight. It has become clear that various stimuli, mood states, thoughts and ideas may have an instantaneous and facilitating effect on food intake.

Despite these advantages, several data suggest that the mechanism of (broken) restraint cannot fully explain why people counterregulate or, more generally, indulge in binge eating. Also, methodological criticisms have been raised about key explanations in counterregulation research. Some of these critical notes (see for example Charnock, 1989 a,b; Dritschel, Cooper \& Chamock, 1993; Jansen, 1990; Lowe, 1993; for replies see Heatherton, Herman, Polivy, King \& McGree, 1988; Polivy \& Herman, 1989) will be briefly discussed below, followed by an overall evaluation.

Counterregulation as a lab-analogue of overeating The fact that in counterregulation experiments the preload manipulation (ingestion of a 420 -gram milkshake) has been

8 The "biological pressure" explanation of urge was only initially defended by Herman \& Polivy. Later, it was discarded (compare the two papers by Herman \& Polivy from 1980 and 1984); see also section 1.3.2 of this thesis). 
between 6 and 13.5 times as large as the increase in ice-cream consumption by restrained eaters (i.e., 31 grams (Herman \& Mack, 1975), and 68 grams (Herman, Polivy \& Esses, 1987) respectively) should not be considered a major blow to the validity of the paradigm: counterregulation is a model that need only embody the essence of overeating (section 1.1), and indeed, the preload manipulation appears to lead to an increase of subsequent food intake. But is this reliably the case? Some authors have claimed that it is not (Chamock, 1989a; 1989b; Dritschel et al., 1993; Jansen, Oosterlaan, Merckelbach \& Van den Hout, 1988). The result often presented under the heading "counterregulation" is based statistically on a restraint $x$ preload interaction effect. However, closer inspection often reveals that the restraint $x$ preload interaction effect is caused by unrestrained eaters eating less when preloaded than when unpreloaded (a reliable, but trivial finding), and restrained eaters eating equal amounts of food in both conditions. Clearly, for counterregulation to reflect "overeating", it would be required that restrained eaters eat more when preloaded than when unpreloaded, but this crucial test has seldomly been conducted. In sum, counterregulation proper seems to be a somewhat less robust finding than has often been realized. Although non-regulation admittedly is an interesting phenomenon, it does not strike one as a convincing analogue of binge eating.

Broken restraint as a prerequisite for counterregulation In a critical test of the claim that breaking cognitive restraint is responsible for lab disinhibition, Jansen et al. (1988b) monitored the thought stream of restrained and unrestrained eaters in a preload study. A 26-item questionnaire with thoughts like "I've blown my diet so I might as well continue to eat" and "I feel out of control", etc., was completed by subjects. The results showed that, although ad lib ice-cream intake showed the usual restraint $x$ preload interaction effect, preloaded restrained eaters showed as many catastrophic thoughts as preloaded unrestrained eaters.

Another issue concerns the identification of the diet boundary, i.e. the critical amount that should be eaten in order to break cognitive restraint, leading to disinhibitive eating (Herman \& Polivy, 1984). Herman and Polivy themselves have experienced considerable difficulty in objectifying the diet boundary. In their 1984 article (p. 151) they suggest that a 210 -gram milkshake preload was too small to break the restraint in many dieters; preloads of 420 grams would be more effective. However, three years later Herman, Polivy \& Esses (1987) set out to determine the critical preload size required for breaking restraint, by simply asking their subjects. It appeared that restrained eaters were unable to estimate their diet boundary, a fact which led the investigators to drop this theme from investigation. In retrospect, Herman et al. (1987) suggested that "an increasing number of dieters consider their diet to be broken by any forced preload at all" (p. 164). What is more, two subsequent studies (Rogers \& Hill, 1989; Jansen \& 


\section{Chapter 1}

Van den Hout, 1991) have shown that mere smelling of palatable food (i.e. zero intake) was sufficient to lead to subsequent disinhibition. It is hard to see why restraint would be broken by smelling palatable food. All in all, disinhibitive eating may well occur without a preceding break of "cognitive restraint".

Explanation of the urge to eat Disinhibitive eating has been said to originate in a loss of cognitive restraint (Herman \& Polivy, 1980; 1984). When analysing this statement, one notices that it does not clarify why the loss of cognitive restraint should induce overeating: no additional urge-mechanism is postulated. The authors (Herman \& Polivy, 1984) remain remarkably uninformative on this point, when they state that their counterregulating subjects ate until their "satiety boundary" was reached. The satiety boundary is described as a state in which further food intake would give rise to feelings of discomfort. To explain counterregulation, Herman and Polivy (1984) claim that the satiety boundary in restrained eaters lies at higher level than that in unrestrained eaters, thus "allowing if nor guaranteeing (..) prodigious consumption" (p.150) once restraint has been broken. One substantiation of the higher satiety boundary alluded to (albeit rather unpersistingly), is that restraint eaters, as a consequence of their dieting might experience an increased biological pressure to eat, so as to restore their biological set point of weight (p. 145). In addition to ideal weight being an ill-defined construct (Greeno \& Wing, 1994), empirical findings make this explanation less likely. First, only between $37 \%$ and $50 \%$ of high restraint subjects report they are currently dieting (Dritschel et al., 1993 Lowe, 1993; chapter 5 and 8 this thesis). Second, a study in which dieting status was checked showed that currently dieting restrained eaters did not counterregulate after a preload, whereas non-dieting restrained eaters did. This finding has been replicated (Lowe, 1993, p.104), and is contrary to the below-set point explanation of counterregulation'. Third, there is the robust finding that restrained eaters generally have a higher BMI than unrestrained eaters do (see the studies in Jansen, 1990; this thesis chapters 5, 7, and 8). Apparently, the "higher satiety boundary" is no explanation of urge, but rather a stately way of saying that more food has been eaten (Jansen, 1990).

9 Apparently in line with a set point explanation of counterregulation is the finding that in the strongest example of counterregulation reported (Herman \& Mack, 1975) normal weight but not overweight restrained eaters did counterregulate (for the reanalysis of these data, see Ruderman, 1986). However, if disinhibition were caused by being below the biological set point, it would follow that 1 . the counterregulating restrained eaters' biological set point is at overweight level; 2 . overweight restrained eaters do not show disinhibitive eating in daily life. These (rather unlikely) allegations still need to be proven. 


\subsubsection{Final remarks}

In sum, the preload-counterregulation paradigm has led to increased knowledge and has set the issues in experimental research on overeating. However, the underlying notion of the counterregulation theoreticians has always been that disinhibitive eating can only occur if prior inhibition ("restraint") existed (Heatherton et al., 1988; Herman \& Polivy, 1990). Of course, when taken literally, this notion is overwhelmingly logical. In practice, however, it is becoming increasingly clear that the boundaries of knowledge growth have been reached in counterregulation research. A striking illustration is the recent report that restrained eaters will only counterregulate if they score high on a scale measuring "tendency to overeat" (Westenhoeffer, Broeckmann, Muench \& Pudel, 1994). In one of their more recent papers, Herman and Polivy $(1990 ;$ p. 123) themselves have suggested that researchers of binge eating "(..) are not in a sirong position to posit causes or mechanisms, since we are not sure what exactly we are trying to explain" (..). To be sure, it appears that the view that binge eating is a lifting of inhibition may discourage the search for mechanisms that explain the urge. This has been a reason for our adopting a different approach in the present thesis: in chapter two, a conditioning theory of binge eating will be described. This theory involves an urge mechanism that may be the "final common pathway" of the different ways in which stimuli may trigger binge eating. Additionally, it describes a mechanism through which binge eating may be self-maintaining. 



\section{A CONDITIONING THEORY OF BINGE EATING}

A conditioning theory of binge eating will be described in section 2.1 , followed by relevant empirical data (section 2.2). Section 2.3 contains an introduction to hypotheses derived from the conditioning theory. These hypotheses will be tested in subsequent chapters.

\subsection{DESCRIPTION OF THE THEORY}

The conditioning theory of binge eating (henceforth CBE theory; Jansen, 1990; 1994a) posits that bingeing is a learned and self-perpetuating behaviour. CBE theory derives from addiction research, where classical (i.e., Pavlovian) conditioning has been accredited an important role in tolerance and relapse (e.g. Childress et al., 1992; Siegel, 1983; Tiffany, 1995a). In short, cues (CS) that signal large food intake (UCS) will, through a conditioning process, become triggers of physiological responses (CRs). These CRs are experienced subjectively as food craving, thereby encouraging binge eating. A subtle version of this mechanism is well-known to us all: when walking past a bakery and sensing the fragrance of freshly baked pies, we will often feel the water running into our mouths, suddenly experiencing an appetite that was absent only moments ago. In binge eating the mechanism has got considerably out of hand. While salivation is one of the CRs, emphasis will be placed on conditioned blood sugar decrease and insulin secretion. Underlying the CBE theory are five basic assumptions, which will be described next.

\subsubsection{First assumption: binges are signalled by cues}

The conditioned stimuli (CSs) are formed by cues that invariably precede binge eating. Examples are the sight, smell, and taste of typical binge foods. The association between between binge cues and binge eating is especially strong because binge eaters tend to restrict their food intake in between binges, thereby actively avoiding the high-caloric "forbidden" binge foods. Binge cues may also be affective and situational: feelings of loneliness, anger, depression or boredom all may reliably precede bingeing, as may certain times of the day or the environment in which binges occur (cf. being home alone, in the kitchen near the fridge, etc.).

1 Related, but not formally developed ideas can be found in Wardle (1990); see also Woods (1991) for an eloquent and inspiring review of related ideas in the field of normal food intake. 


\subsubsection{Second assumption: food intake provokes corrective physiological responses}

Food ingestion leads to a temporary disturbance of the internal physiological state. However, because the body generally strives towards stability of its internal milieu, it will emit regulatory, homeostaric responses. For example, a meal generally causes blood sugar levels to rise considerably. As a reaction, the hormone insulin is secreted from the pancreas, with the effect that blood sugar levels are redirected towards the pre-meal baseline. In conditioning terminology, the physiological disturbance following food intake is the unconditioned stimulus (UCS), and the homeostatic responses form the unconditioned responses (UCRs).

\subsubsection{Third assumption: through conditioning, binge cues come to trigger anticipatory} physiological responses

By means of a conditioning process, binge cues become conditioned stimuli (CS) that will trigger conditioned salivation, insulin secretion, blood sugar decreases, etc., (CRs) before food intake has taken place. The conditioned physiological responses are called anticipative and compensatory because they counter the physiological disturbance that usually follows food intake. Moreover, the intensity of the CRs will be proportional to the amount of expected food intake. Specifically, the CBE theory predicts large insulin secretion and large hypoglycaemia to cues that signal binge eating.

\subsubsection{Fourth assumption: conditioned physiological responses are subjectively experienced as food craving}

According to CBE theory, perception of binge cues induces insulin secretion and a fall in blood sugar level. The CBE theory posits that this body state will be subjectively experienced as food craving. Consequently, food ingestion, serving as "drug-taking behaviour" becomes more likely. A somewhat picturesque way of describing this would be that bingeing is an attempt to "eat away" the conditioned hypoglycaemia.

\subsubsection{Fifth assumption: binge eating is a self-maintaining process}

Evidently, craving heightens the likelihood of bingeing. In binge eaters, it could be said that each binge acts as a conditioning trial in which binge cues (CS) are paired with a binge (UCS), thus strengthening the cue-binge association. From the body's "viewpoint", the homeostatic "necessity" of emitting strong compensatory CRs to binge cues is "reconfirmed" each time a binge occurs. The individual thus may enter a vicious circle: a next encounter with binge cues will again elicit the "necessary" conditioned responses that again, through craving, facilitate bingeing. 


\subsection{OVERVIEW OF RELEVANT DATA}

\subsubsection{Existence of binge cues}

In binge eating research, the probability relationship between binge cues (CS) and binges (UCS) has received little systematic attention. This is probably due to its apparent triviality: there seems little need to argue that food-intrinsic cues, like sight, smell and taste of binge food, precede bingeing. As a matter of fact, retrospective self-reports never fail to include food stimuli as important precipitants of binge eating (Abraham \& Beumont, 1982; Bruce \& Agras, 1992; Mitchell et al., 1985) and of temptation and relapse in dieters (Grilo, Shiffman \& Wing, 1989). However, as far as the present author knows, no quantitative reports exist about the probability relationship between specific cues and bingeing at the individual level. Nevertheless, some indications can be found that binge eating is generally preceded by cues unique to binge episodes. First, it has been found that bingers with bulimia or anorexia show intermediate dieting that is often accomplished by avoiding "forbidden" high-caloric foods that are typically ingested during binges (Walsh, 1993). Therefore, especially cues pertaining to binge food rather than other foods could be expected to be uniquely confined to binge contexts. Second, it has become clear that in therapeutic contexts, patients can, when asked, identify typically tempting binge foods, circumstances and locations. These cues may be used in cue-exposure procedures (e.g. Jansen et al., 1992).

To find evidence that affective binge precipitants (low moods, negative thoughts and stressful social interactions) act as "cues" seems more problematic. Although emotional states are among the most frequently mentioned precipitants of bingeing by patients (Abraham \& Beumont, 1982; Bruce \& Agras, 1992; Mitchell et al., 1985), it remains unclear whether mood states act as the actual binge cues, or as so-called "occasion setters", i.e. the background against which the food cue-binge (CS-UCS) association is established (Catania, 1991; Davidson, 1993).

\subsubsection{Homeostatic regulation of blood sugar level}

The idea of homeostatic responses correcting disturbances of the internal milieu is, of course, not new. It was developed in the 1920s by W.B. Cannon, the father of 20th century physiology. The concept of homeostasis offered an explanatory framework for better understanding the multitude of co-occurring and interacting body processes (Cannon, 1939; Guyton, 1991, ch. 1). A comprehensive treatment of homeostatic physiology related to food intake is beyond the scope of this thesis (and of its author too). A bird eye's view will only be given of blood sugar regulation. When ingested 


\section{Chapter 2}

food has passed through the stomach, it will appear in the small intestine, where its various nutrients are absorbed and appear in the blood stream. Consequently, the level of blood glucose (i.e. blood sugar) begins to rise. However, blood sugar is tightly regulated: several homeostatic responses are emitted to counter the blood sugar rise. One of these responses is an excessive insulin release by the pancreas. Insulin, sometimes also called "the hormone of plenty", lowers blood sugar levels by, for example, making cell walls more permeable for glucose. As a consequence, the glucose formerly contained in the food will enter the various body cells and tissues where it is stored for later use or utilized at once ${ }^{2}$. In sum, whatever the disturbance imposed on the body's internal milieu, the interaction between nutrients, nervous activity and hormones will act to keep blood sugar within a restricted range. For reviews see for example Brodsky \& Devlin (1994), Cryer (1992), Guyton (1991), Hardy (1981), Steffens et al. (1991).

\subsubsection{Existence of anticipatory responses to sensory food cues}

Description. Food-anticipatory responses to cues (as described in the CBE theory) abound in the mammalian body, and have first been described as "psychic reflexes" by Pavlov (1910). In the last twenty years, the term cephalic phase responses (cephalos = head) has come into general use. Cephalic phase response (Berthoud et al., 1981; Giduck, Threatte \& Kare, 1987; Le Blanc, 1992; Powley \& Berthoud 1985, Teff et al., 1993) is a general term referring to physiological responses that are triggered by foodrelated, visual, olfactory, gustatory or oropharyngal-mechanical cues. Thus, cephalic responses may occur both before and during food ingestion. Besides salivation, studied so thoroughly by Pavlov, secretion of gastric and pancreatic juice and of a range of hormones like insulin, pancreatic polypeptide, glucagon can be secreted cephalically. Also other responses have been reported, like increases in metabolic rate (i.e. the body's energy production) and glucose absorption by the liver (Le Blanc, 1992). Many, but not all of these cephalic responses peak at about five minutes and return to baseline at ten minutes after food cue perception (Le Blanc, 1992; Berthoud \& Powley, 1985). Physiologically speaking, the early cephalic phase responses should be distinguished from post-absorptive, homeostatic (see section 2.2.2) reactions to nutrients entering the blood. In particular, the hormonal response to food intake often shows two phases: a smaller cephalic wave in the first few minutes after intake followed by the onset of a post-absorptive response at 5-15 minutes (e.g. Guyton, 1991; Teff et al., 1993). This is

\footnotetext{
2 A reverse mechanism comes into play if the blood sugar level becomes too low, or fuel demands increase, e.g., during stress or vigorous exercise. In these cases, various blood sugat enhancing hormones (glucagon, growth hormone, catecholamines and corticosteroids) are secreted. Generally, they suppress insulin secretion, and mobilize sugars and fat previously stored in cells and tissues.
} 
not to say that the impact of cephalic responses could not extend to longer periods than 10 minutes; the functioning of organs and tissues can be altered for more prolonged periods, when the initial cephalically triggered hormones no longer circulate in the blood (Le Blanc, 1992; Thompson et al., 1988; Bruce et al., 1987). Moreover, cephalic responding is not confined to the pre-ingestive period; it may also occur when new food items are introduced during an ongoing meal (Louis-Sylvestre, 1984). It is generally held that the efferent nervous pathway of cephalic responses is primarily parasympathetic, involving the different branches of the vagus nerve connected to the various glands and organs (Powley \& Berthoud, 1985; Louis-Sylvestre, Giachetti \& Le Magnen, 1983; Strubbe \& Steffens, 1975; Strubbe \& Steffens, 1993).

Functions Because of the very existence of cephalic responses, the issue arises about their possible biological function (e.g. Powley \& Berthoud, 1985). Several functions have been proposed.

First, cephalic responses may attenuate the physiological disturbances that follow the ingestion of food (Powley \& Berthoud, 1985; Woods, 1991). In fact, it has been shown that the nutrient intake leads to larger physiological disturbances when the cephalic phase is by-passed (e.g. by infusion of nutrients) whereas, conversely, the blood sugar rise in response to glucose infusions is decreased if a cephalic response is evoked concurrently (Bruce et al., 1987, Le Magnen, 1992; Strubbe \& Steffens, 1993).

Second, cephalic responses may prepare the various body structures for the breaking up, storage and metabolism of food (Le Blanc, 1992; Powley \& Berthoud, 1985; Woods, 1991). In addition, drawing upon the finding of increased metabolic rate during the first 30 minutes after the beginning of cephalic responses, Le Blanc (1992) has speculated that cephalic responses might aid in mobilizing energy for the pursuit and capture of prey.

A third function of cephalic responses may be to induce or enhance appetite. This might be no less advantageous in an evolutionary sense than the regulatory functions mentioned earlier: appetite instigates food intake at the very moments food is available (Woods, 1991). This is neatly expressed in the French proverb L'appetit vient en mangeant (Le Magnen, 1992).

The fourth function, proposed by Louis-Sylvestre (1984), is that cephalic responses may stimulate versatility of food intake, a function which becomes apparent during meals. In rats turning from one food item to another, an additional insulin secretion super-imposed on the normal post-prandial insulin level is seen, with the effect of lowering postprandial blood sugar rise, and probably, maintaining the hedonic value of intake (Louis- 
Sylvestre, 1984) ${ }^{3}$. A perhaps related effect has been shown in humans. After having eaten a lunch of considerable size, subjects were sensorily primed by a small bite of either pizza or ice cream. A subsequent ad lib intake test with both foods abundantly available showed that intake of the primed food item always exceeded that of the unprimed, although both items had been regarded equally highly by the subjects (Cornell, Rodin \& Weingarten, 1989).

Modulating factors Cephalic phase responses appear in different forms and intensities both intra- and inter-individually. Several sources of variation in cephalic responding exist, mainly related to the nature of the signalled food, and the learning history of the subject.

First, the chemical composition of the anticipated food may matter. Already Pavlov (1910) pointed at the adaptiveness of cephalic responses. In his dogs, the chemical characteristics of the various anticipatory secretions were specifically attuned to those of the expected intake. For example, thickness, amount and chemical composition of the cephalically aroused saliva and pancreatic juice were most appropriate to deal with physiological consequences of the ingestive, even if the (learned) food cue was so alien to food as the ticking of a metronome or the sight of a bottle (see Babkin, 1949; Dworkin, 1993).

A second factor influencing the intensity of cephalic responses is the palatability and perceived palatability of the expected food. Apparently, the most potent elicitors of salivary and insulin responses are sensorily rich food items such as odorous apple pie or spicy pizza, especially when they are brought in contact with the oral cavity (Christensen \& Navazesh, 1984; Teff et al., 1991). The role of visual attractiveness of food items has been neatly demonstrated by Lucas et al. (1987). Half of a freshly baked quiche was mashed in a food processor, after which it was mixed with an odourless blue dye. Both this item and an original (attractive) piece of quiche was presented to normal weight subjects. The cephalic insulin response to the attractive dish was more intense than that to the unattractively mashed variant.

Third, the anticipated amount of food or the expected metabolic consequences play a role in the intensity of the cephalic insulin response (Powley \& Berthoud, 1985): in rats sipping from a saccharin solution, the intensity of the cephalic insulin response has been found to increase proportionally with the quantity ingested, a remarkable finding as saccharin is a metabolically inert artificial sweetener. The effect might be dependent

3 Parenthetically, one should be careful to attribute such behavioural effects to the action of insulin alone, because cephalically induced increases of insulin secretion are usually small compared with those found after food intake (see also sections 9.2 and 9.3 of this thesis). 
upon the rats' previous intake experience with real sugars. When drinking the sweet saccharin, the rats "anticipated" a blood sugar rise proportional to the amount of saccharin ingested. Accordingly, for a larger intake of the sweet solution, a proportionally larger insulin response appeared.

Fourth, cognitive factors have been proposed to account for occurrence or non-occurrence of cephalic responses (e.g. Sjöström et al., 1980). The expectation that food items presented will be actually ingested appears to be especially important (e.g. Pavlov, 1910). Bruce et al. (1987) found a 0.90 correlation between a questionnaire score of intake expectation and the cephalic hypoglycaemic response to food cues. An interesting example showing the impact of cognition has been provided by Goldfine, Abraira, Gruenewald and Goldstein (1970), who were able to induce a cephalic insulin rise in their subjects through hypnotical suggestions of a palatable meal.

Finally, habitual eating pattern appears to modulate the intensity of the cephalic responses. A robust finding is that obese persons show larger cephalic insulin responses than do normal weight persons (Simon et al., 1980; Sjöström et al., 1980). In absolute levels the intensity of cephalic responses has been found to be nearly four times as large in the obese; expressed in percentage increase from baseline levels (obese humans are known to have increased baseline levels of insulin), the obese showed twice as strong cephalic insulin responses (Sjöström et al., 1980). Another study found a trend towards larger cephalic insulinaemic response in obese (Johnson et al., 1983). Although these results are most likely due to hypertrophy of the insulin producing Beta-cells of the pancreas (Dr. Strubbe, personal communication), there remains an additional possibility (which would be in line with CBE theory) that the larger average meal size of the obese in daily life contributes to enlarged cephalic insulin responses.

There is also some indication that cephalic salivation is modified by food intake experience. Le Goff, Leichner \& Spigelman (1988) compared salivary flow to food cues in bulimics and anorexics before and after therapy. Before therapy salivation to food stimuli was more intense in bulimics than in anorexics. After therapy, a re-assessment showed that bulimics salivated less, and anorexics salivated more to food cues than they had originally. This could be explained accordingly by assuming that a bulimic eating style, involving occasional large food intake (binge UCSs), results in stronger salivation (CR), whereas in anorexic patients, food cues predicting small food intake, lead to modest salivary responses (Jansen, Boon, Nauta \& Van den Hout, 1992).

In contrast with the above results, Broberg and Bernstein (1989) and Moyer, Rodin and Cummings (1993) did not find enlarged cephalic insulin responses or hypoglycaemia in bulimic patients exposed to palatable foods. In these cases the CBE predictions were not confirmed. 


\subsubsection{Classical conditioning of food intake and related physiological responses}

Although it is true that cephalic responses to food cues, especially those occurring during food ingestion, may be understood partly as expressions of "hard-wired" reflex loops which are activated when the ingested food strikes various receptors while travelling through the mouth, throat and stomach (Berthoud et al., 1981; Nicolaïdis, 1977), this does not at all imply that the influence of learning history should be discarded (e.g. Dworkin, 1993; Jansen, 1994a; Jansen, Boon, Nauta \& Van den Hout, 1992; Woods, 1981; 1991). On the physiological level, learning may act in at least two ways: by modulation of the existing hard-wired reflex loops (Dworkin, 1993), and by bringing the efferent part of the reflex loop under arbitrary stimulus control (Woods \& Kulkosky, 1976). On the behavioural level, learned cues may activate food seeking and intake. Clearly, the strongest support for these allegations (at the heart of the CBE theory) would be provided if acquisition of the responses described could be demonstrated by means of a classical conditioning procedure. In the following overview such studies will be discussed. It has indeed proven possible to bring meal initiation, meal size, salivation, insulin secretion and hypoglycaemia under the control of arbitrary cues.

Conditioned meal initiation and meal size increase Weingarten $(1984 ; 1985)$ has provided evidence that meal initiation in rats can be classically conditioned to arbitrary cues. In his procedure, which extended over several days, feeding in rats was always preceded by a 4-minute CS (light-buzzer compound), after which a food tray was filled. Then, during the test session, rats were first given ad lib access to food. After they gave signs of being satiated (cessation of intake; grooming), the light-buzzer CS was switched on. It was observed that the rats started eating again, and doing so ingested an amount equalling about $20 \%$ of their daily intake prior to the experiment. It seems that conditioned physiological responses may have contributed to the meal initiation. In a similar procedure to Weingarten's, Strubbe (1992) used the slamming of a food tray as a CS for food delivery. At the test session, conditioned insulin appeared to the slamming of the tray. In humans, surprisingly little research has been conducted in this field. Birch et al. (1989) found in children an increase in candy intake in presence of a candy-predicting light, but only if the children had been aware of the CS-UCS (light-candy) contingency. One might wonder what purpose would be served by increasing food intake after perception of signals predictive of food. Zamble (1973) has provided clues to this problem. He showed that rats that were allowed one-hour food access daily (each day at a different time) ate more if their meals were signalled 15 minutes in advance, than if food was unsignalled. The fact that these eating behaviours resulted in smaller weight loss in the signalled rats, suggests that conditioned enhancement of food intake may be 
of survival value if food is scarce and/or "patchily" distributed in the organism's environment (see Collier, 1986).

Conditioned hypoglycaemia. Blood sugar level can be brought under arbitrary CS control of odours and injection rituals (for reviews see Dworkin, 1993; Woods \& Kulkosky, 1976). In many but not all instances, insulin injections have been used as the UCS, yielding mixed results of either conditioned hypo- or hyperglycaemia, depending on the doses of insulin used and the specifics of the procedure (for detailed discussions see Dworkin, 1993; Woods, 1983; Chapter 4 of this thesis).

More relevant for the CBE-model are procedures in which pure glucose or normal food have been used as a UCS. Some will be discussed here (see also chapters 3 and 4 of this thesis. A strong demonstration of learning in hypoglycaemia using a glucose UCS has been provided by Deutsch (1974) who fed his rats with the sweetener saccharin and observed a hypoglycaemia although this chemical, as noted earlier, does not contribute directly to blood sugar level. Deutsch suggested the observed hypoglycaemia was a compensatory response reflecting the rats' learning history with natural (real) sugars in which sweet taste always had predicted a blood sugar rise. To test this suspicion, Deutsch first investigated whether the hypoglycaemic response could be extinguished. This indeed happened after offering the rats free access to saccharin for a prolonged time. Next, Deutsch argued that it should be possible to established conditioned hypoglycaemia to an arbitrary taste CS as well. Thus, he repeatedly presented his rats with a (non-sweet) cafeine solution to drink followed by the UCS, intubation of glucose into the rats'stomachs. After ten such pairings, the taste of the cafeine itself was able to elicit a hypoglycaemia. Matysiak \& Green (1984) have also found conditioned hypoglycaemia in rats after a learning procedure involving glucose injection UCSs. In contrast, Woods (1976) failed to find this result. This was probably due to the small glucose dose involved. In humans and dogs, a strong example of hypoglycaemic conditioning, leading to about $15 \%$ fall in blood sugar using UCS glucose injections, was reported by Russian physiologist Mityushov (1954; see Chapter 4 and the appendix of this thesis).

Conditioned insulin secretion The blood sugar conditioning studies referred to above, did not include direct measurements of insulin. Nevertheless, it has often been suggested that the observed blood sugar fall was secondary to a conditioned insulin secretion (Dworkin, 1993; Mityushov, 1954; Woods, 1983). Direct evidence has come from studies by Woods et al. (1977) and Strubbe (1992) who demonstrated insulin secretion to meal predicting cues. These authors meal-fed rats at a limited and fixed number of times each day. Woods et al. (1977), giving their rats unlimited excess to food for one hour daily, found an increased insulin secretion at the time of day the rats were usually fed. Strubbe 


\section{Chapter 2}

(1992) used slamming of a food tray as the CS. Two procedures were tested: rats had either two or six times daily free access to food (UCS). On the test day, slamming the food tray alone evoked significant insulin secretion, but only so in rats who had been receiving two meals daily. One of the explanations was that the meal size (i.e. UCS intensity) during the conditioning procedure had been larger in rats fed twice, than in those fed six times daily. Also deprivation in itself might have played a facilitating role (see Weingarten, 1985).

The data presented are in line with the CBE notion that meal initiation and food intakerelated physiological responses can be conditioned to learned cues in people and animals. Food deprivation is not a prerequisite for these effects to occur.

\subsubsection{Hyperinsulinaemia/hypoglycaemia experienced as craving}

The CBE theory assumes that conditioned insulin secretion and hypoglycaemia will be subjectively experienced as craving for food. This assumption receives support from different sources. First, research on hypoglycaemic episodes in diabetic patients shows that hypoglycaemia is often accompanied by feelings of hunger (Hepburn, 1993; Service, 1995). Second, Rodin, Bach, Peranini \& De Fronzo (1985) used the clamp technique, enabling them to keep insulin and blood sugar at fixed levels. Several combinations of blood glucose/insulin levels were established in human subjects who were asked each time to report the intensity of their hunger feelings. It appeared that hunger scores were highest during combined hyperinsulinaemia/hypoglycaemia. Equally, large (Grossmann, 1986) and small (Campfield et al., 1996) insulin injections in humans have led to increases in reported hunger. Fourth, in rats, a short-lived insulin secretion followed by a hypoglycaemia has been found to reliably precede meal intake (Campfield \& Smith, $1990 \mathrm{a} ; 1990 \mathrm{~b})$. Finally, there are indications that conditioned meal initiation in rats (see section 2.2.4) is accompanied by a blood sugar decline in rats (Weingarten et al., quoted in Le Magnen, 1992 p. 84).

\subsubsection{Extinction of conditioned physiological responses and binge eating}

The CBE-hypothesis that craving and binge eating follow the acquisition of conditioned insulin secretion and hypoglycaemia, has not been tested directly. However, animal and human data are available about the reverse mechanism, that is, extinction of conditioned responses by repeated CS presentations not followed by the UCS.

Animal studies First, Deutsch (1974) has observed that rats' cephalic hypoglycaemic response to the sweet taste of saccharin disappeared after 60 days of ad lib access to the 
(metabolically inert) saccharin ${ }^{4}$. Second, extinction of explicitly conditioned hypoglycaemias has been demonstrated after repeatedly exposing the rats to the gustatory or olfactory CS (Deutsch, 1974; Woods, 1976).

Studies in humans Exposure to binge food cues (CS) with prevention of subsequent food intake (UCS) has been demonstrated to reduce binge eating. The procedure is as follows. First, therapist and patient will map cues and acts which are characteristic of binge episodes. This information is used during the exposure sessions when the binge situation is mimicked as realistically as possible. Under supervision of the therapist, the patient is encouraged to go through the ritual of selecting and preparing the typical binge food, thereby handling, smelling or licking the food, and tasting morsels of it. To increase realism, the exposure will be often carried out at the typical binge location, i.e. the patient's home. Although strong food craving arises, the patient is prevented from ingesting food. Prolonged exposure, for example 45 minutes or more, will result in the craving being significantly decreased. Several studies have been published about the effects of the cue exposure procedure in bulimic patients. Overall, the results have been promising in that a reduction of binge frequency was found (Hansen \& De Haan, 1995; Jansen, Van den Hout, De Loof, Zandbergen \& Griez, 1989, Jansen, Broekmate \& Heymans, 1992; Kennedy et al., 1995; Schmidt \& Marks, 1988, 1989) which appeared to have persisted at 6-months (Schmidt \& Marks, 1989) or 1-year (Jansen et al., 1992b) follow-up. Interestingly, also levels of depression and unrealistic cognitions about food and body shape had diminished after treatment (Jansen et al., 1992b). Taken together, these findings fit well into the CBE theory's conceptualization of binge cues as CS and binge eating as a UCS.

4 The issue of whether saccharin-induced hypoglycaemia is learned or inborn has been much debated. For instance, Berthoud et al. (1981) have claimed that saccharin-induced insulin secretion in rats should be regarded an inborn response, because 10 -fold exposure to mere saccharin did not lead to its extinction. In a verbatim commentary on Dr. Berthoud's paper, Woods (1981), referring to Deutsch's results, has argued that 10 extinction trials are insufficient to refute a learning interpretation. Dr. Berthoud's reply that Deutsch's results were not to the point, because Deutsch only measured blood sugar at two hours after saccharin presentation, is valid. Indeed, more convincing data should involve measurement at five minutes after exposure to saccharin, and such measurements still need to be done. Note that already in Pavlov's times, essentially the same debate evolved about the allegedly learned nature of salivation to meat in dog puppies (Joravsky, 1989). 


\subsection{SUMMARY AND IMPLICATIONS FOR THE PRESENT THESIS}

\subsubsection{Summary}

A conditioning theory of binge eating (CBE theory) was presented in this chapter. CBE theory regards binge eating as an acquired behaviour. Binge cues acting as conditioned stimuli (CSs) that reliably predict large food intake (UCS) will eventually acquire the power to elicit physiological responses (CRs). The CRs are subjectively experienced as craving. Once established, binge eating is a self-maintaining process. The binge eating individual may enter a vicious circle: each binge implies a pairing of binge cues (CS) with large food intake (UCS). Consequently, future encounters with binge cues will trigger intense CRs, that in their turn favour binge eating, and so on. Although different physiological CRs are triggered by the CS, especially insulin secretion and hypoglycaemia are hypothesized to be important in craving. Data relevant to the assumptions of the CBE theory were discussed. Although it remains to be demonstrated whether binge eaters are conditioned in the hypothesized way, a cue exposure procedure, implied by CBE theory, appears to be an effective treatment of binge eating. The procedure, involving presentation of binge cues with simultaneous prevention of food ingestion, has been reported more than once to reduce binge eating. These findings are compatible with a hypothesized extinction of the learned CS-UCS (cue-binge) association.

\subsubsection{Implications for the research in this thesis}

So far, little direct research of CBE-predictions has been carried out. The non-clinical lab investigations to be presented in the following chapters have been inspired by the CBE theory and focus on various aspects of the theory.

The hypothesis of the investigation in chapter 3 is directed at the etiological level: if a set of cues CS is made to predict a considerably large intake of sugar (UCS), then eventually the cues themselves will elicit conditioned insulin and blood sugar responses, craving and heightened sugar intake. Chapter 4 discusses the results of a remarkable, but not widely known Russian study (first published in 1954) on conditioned hypoglycaemia. The data from this study are re-analysed, and put into the perspective of modern research (including the $\mathrm{CBE}$ theory). Chapter 5 contains an investigation of the proximal stimuli of bingeing: restrained eaters (i.e. unsuccessful dieters who occasionally binge) and unrestrained eaters (normals) are compared with regard to their psychophysiological reactions to food cues. This study tries to identify relevant parameters of food cue reactivity, while testing the hypotheses that restrained eaters, because of their 
learning history show increased food cue reactivity. A related study is reported in chapter 6. Here it is hypothesized that fasting subjects will show larger psychophysiological reactivity to food cues than non-fasting subjects do. Once again, the principal aim is to identify sensitive parameters of psychophysiological cue reactivity, the fasting manipulation being an attempt to induce maximal food cue reactivity. Chapter 7 focuses on the cognitive level. Restrained and unrestrained eaters are exposed to an appetizer (i.e. the food cue), and subsequently compared with regard to their attentional bias for food words in the Stroop task. As restrained eaters are hypothesized to show disinhibitive eating after the appetizer, it is hypothesized that they will display larger attentional bias for food words then do unrestrained eaters. Also, the relationship between attentional bias and subsequent ice cream intake in an ad lib taste test is assessed. Finally, Chapter 8 serves a more methodological purpose. It describes the development of a new disinhibitive eating questionnaire which may be helpful in selecting non-clinical binge eaters, and in the development of new "lab-models" of binge eating (section 1.1.). 



\section{Chapter 3}

\section{CONDITIONED INSULIN AND BLOOD SUGAR RESPONSES IN HUMANS IN RELATION TO BINGE EATING ${ }^{1}$}

\section{INTRODUCTION}

An important feature of bulimia nervosa is recurrent binge eating. During binge eating, an amount of food is ingested that is definitely larger than most people would eat during a similar period of time under similar circumstances (APA, 1994). A typical characteristic of binge eating also included in the DSM IV binge definition is a sense that control over food intake is lacking (APA, 1994). Furthermore, bingers often report an irresistable food craving just before a binge (Abraham \& Beumont, 1982; Jansen, Van den Hout \& Griez, 1990; Mitchell, Hatsukami, Eckert \& Pyle, 1985).

Hence, binge eating can be said to bear resemblance to alcohol and drug addiction: both are characterized by the intake of large amounts of substances, craving and loss of control over intake. Addiction research of the last two decades has elucidated the role of conditioning in drug tolerance and craving (Siegel, 1983; Childress et al., 1992). Specific cues (CS) that signal drug intake (UCS) eventually become able to elicit physiological responses (CR) that compensate for the anticipated bodily effects of drug intake. Thus a behavioral tolerance to the drug develops, guided by cues like the injection ritual or the usual environment in which the drug is used. Additionally, the drug-signalling cues are supposed to trigger craving and drug seeking behavior. Recently, an analogous conditioning theory of binge eating was proposed (Jansen 1990; 1994a; Wardle, 1990). In the present article the conditioning model of binge eating (CBE model) is outlined and a first test of it reported.

The CBE model (Jansen, 1990; 1994a) is based on five assumptions: (1) each episode of (binge) eating disrupts the body's internal milieu; (2) to counter the disruption, physiological, homeostatic reactions occur; (3) binges are preceded by specific cues; (4) these cues can become elicitors of the homeostatic responses by a process of classical conditioning; (5) the conditioned reactions are subjectively experienced as craving for food.

1 Submitted for publication by J. Overduin \& A. Jansen. The authors gratefully acknowledge Mrs. Annie Raven for her assistance in carrying out the present experiment, Dr. Paul Menheere for his biotechnical advice, Mr. Ton Teerling (Quest International, Naarden) for supplying us with the benzaldehyde and maltol flavors, and the Holland Sweetener Company (Mastricht) for providing us with aspartame. Dr. Barry Dworkin (Penn State University) is acknowledged for commenting on some of our data. 
The first two assumptions imply that bingeing (as food intake in general) has disruptive effects on the internal milieu of the body (for an overview see Woods, 1991). The body tries to restore equilibrium by regulatory, homeostatic reactions. As an important example, a meal causes blood sugar levels to rise, and this prompts the pancreas to release the blood sugar lowering hormone insulin. The third assumption is that binges tend to be preceded by so-called binge cues like the sight, smell, and taste of typical binge food. One could also think of times of the day, particular moods, or the environment in which binges usually occur (cf. being home and feeling lonely in the afternoon). The basic notion is that the person has learned that these cues uniquely and reliably predict binges. Fourthly, the $\mathrm{CBE}$ model proposes that through classical conditioning binge cues can acquire the power to trigger responses like insulin secretion before any food intake has taken place. In conditioning terms, the cues come to act as a CS triggering an insulin secretion (CR) that leads to a drop in blood sugar level. The blood sugar fall is called both anticipative and compensatory because it occurs prior to food intake and compensates for expected physiological disturbances (i.e. post-prandial blood sugar rise). The last assumption in the CBE model is that the cue elicited responses like hyperinsulinemia and hypoglycemia are subjectively experienced as craving. Evidently, craving heightens the chances that a binge will take place. When a binge indeed occurs, the association between cues (CS) and binges (UCS) is strengthened. In this manner the individual can enter a vicious circle, in that the next encounter with binge cues will elicit larger CRs again leading to bingeing and so forth.

Data relevant to the last two assumptions of the CBE model, implying that homeostatic responses and craving can be conditioned to food predicting cues, can be found scattered throughout the literature. The sight, smell and taste of food are known to evoke secretion of insulin and other hormones known under the name cephalic phase responses (e.g. Le Blanc, 1992; Powley \& Berthoud, 1985). In some cases a hypoglycemia has been observed as well (Louis-Sylvestre, 1976). The intensity of cephalic phase responses are said to be correlated to the palatability of the food and the amount of subsequently ingested food (Louis-Sylvestre, 1984; Lucas, Bellisle \& Di Maio, 1987).

Explicit conditioning procedures have shown that insulin secretion hypoglycemia and meal initiation can become conditioned to arbitrary cues like odors, an injection procedure and the usual time of feeding. The UCS consisted of either food or a glucose solution (Deutsch, 1974; Mityushov, 1954; Strubbe, 1992; Woods \& Kulkosky, 1976; Woods et al., 1977; Weingarten, 1984). Finally, the CBE model has been clinically applied to form a therapy for binge eating. The procedure, involving exposure to binge cues with simultaneous prevention of bingeing, appears to be promising (Jansen, Broekmate \& Heymans, 1992).

The aim of the present study was to test the CBE model in normal healthy subjects. A conditioning procedure was run in which a peppermint odor/flavor CS was repeatedly 
paired with the ingestion of glucose solution. Responses were monitored on the physiological, behavioral and subjective level. The hypothesis under scrutiny was that after conditioning, presentation of the CS alone would trigger a) conditioned hypoglyce$\mathrm{mia} /$ hyperinsulinemia coinciding with b) increased subjectively experienced craving, leading subsequently to $\mathrm{c}$ ) increased ad lib intake of glucose drinks.

\section{METHOD}

\section{Design}

The experiment was run according to a 2 (condition: control vs. experimental) $\times 2$ (measurement: pre- vs. post-conditioning) design, and carried out in a double-blind fashion. In the experimental condition subjects were conditioned by sixfold pairing a peppermint smell/taste CS with a subsequent ingestion of a glucose dose (UCS). Glucose was chosen as the UCS, because its ingestion causes a sharp increase in blood sugar level, therewith provoking a strong insulin response (Rodin, 1990; Tse et al., 1983). In the control condition an aspartame solution was used as the UCS. Aspartame tastes sweet (like glucose) but is metabolically inert so that no blood sugar change occurs after ingestion (Rodin, 1990; Carlson \& Shah, 1989; Horwitz, McLane and Kobe, 1988). Two sessions were included to encourage the association of the UCS presentation only with the CS proper, and not with other stimuli related to the lab visit. To control for the non-associative effect of experience with the UCS, a random control procedure (Robbins \& Ehrman, 1992) was added: on each conditioning day all subjects received a take-away drink to be ingested at home. This drink contained aspartame for the experimental, and glucose for the control group. Due to this arrangement, at the time of the second test session (i.e. the last day of the experiment), subjects in both conditions had been exposed to same doses of aspartame and glucose drinks.

\section{Blood Parameters}

Blood sugar and insulin were monitored before and after CS presentation on test days in order to test the CBE hypotheses of conditioned hypoglycemia/hyperinsulinemia. Cpeptide was measured as a control on insulin secretion. C-Peptide and insulin are always secreted simultaneously in equimolar quantities, but $\mathrm{C}$-peptide circulates in the blood for a longer period than shorter-lived insulin. Thus C-peptide measurement could reveal rapid, otherwise unnoticed fluctuations in insulin (Polonsky \& Rubenstein, 1984). Finally, levels of the pancreatic hormone glucagon were measured. Glucagon increases the blood sugar level and is a powerful antagonist of insulin: its secretion is stimulated 
when levels of blood sugar become too low, and inhibited when, for example, sugar is ingested (Brodsky \& Devlin, 1994). The glucagon was measured mainly as a control hormone: heightened glucagon levels could provide an explanation if hyperinsulinemia but no simultaneous hypoglycemia should be found (a phenomenon not uncommon in insulin secretion to food stimuli (Bruce, Storlien, Furler \& Chisholm, 1987; Powley \& Berthoud, 1985). Glucagon was also monitored because it was speculated that conditioned hypoglycemia might not only be secondary to insulin secretion, but also to a conditioned inhibition of glucagon secretion.

\section{Subjects}

In a first step, 100 first- and second-year female university students who had responded to an advertisement in the university paper were given a selection questionnaire on personal health and taste preference. Eventually, 20 subjects (age $19.9 \pm 1.9$ years; BMI $20.1 \pm 1.2$ ) were selected using the following criteria: healthy state, no history of diabetes in the subject or her direct relatives, normal weight (i.e. a Body Mass Index between 18.5 and 23.5), current use of oral contraceptives (so as to blunt natural menstrual cyclic fluctuations of progesterone and appetite; Lissner et al., 1988), no history of bingeing, or dieting to lose weight (implying no eating disorders), and no extreme like or dislike of sweet substances and peppermint. It was announced that the research involved assessments of the impact of repeated tasting on sensitivity for subtle taste differences of sweet drinks, and that relevant blood parameters would be monitored. Allocation to either experimental $(\mathrm{N}=10)$ or control group $(\mathrm{N}=10)$ was done pseudo-randomly, taking care that the neither BMI nor age differed between groups. For each subject the procedure started between 6-12 days after the onset of the last menstruation, i.e. 1-7 days after contraceptive pill use was resumed. In this way, care was taken that the last day in the experiment would be at least 3 days before the onset of the next menstruation. At the end of the experiment each subject was paid the Dutch equivalent of US $\$ 300$.

\section{Materials}

Questionnaires Most questionnaires used Visual Analog Scales (VAS) with scores between 0 ("not at all") and 100 ("very much"). During the test sessions a 5-item VAS on the levels of hunger, thirst, tension, craving for salty as well as sweet food was administered 10 times (see table 3.1 for the time schedule of presentation). Further, the taste test questionnaire included ratings of the sensory qualities of the three test drinks. Some of these items were in fact "time fillers" (see procedure); only hedonic and sweetness ratings of the test drinks were considered as relevant data. The conditioning 
sessions each included two 5-item VASs identical to those described above, as well as questions on the sensory characteristics of the three UCS drinks and the pepper$\mathrm{mint} / \mathrm{mentholatum} \mathrm{CS}$. These questions were included solely to stimulate conscious identification of the CS and UCS; no further use was made of the data from these questionnaires.

Conditioned stimulus The conditioned stimulus was equal in both conditions and consisted of a compound of peppermint/mentholatum odor and flavor, presented in combination with a pre-ingestive ritual in an CS-room. The odor/flavor combination was used, as its "belongingness" with ingestion (UCS) was thought to accelerate the acquisition of the conditioned response (e.g. Schwartz \& Robbins, 1995; Woods, Makous \& Hutton, 1969). The peppermint-odor was dispersed by an electronic device in which peppermint/mentholatum oil was continuously evaporated. The flavor consisted of a solution of peppermint $(3 \mathrm{gr} / \mathrm{l})$ combined with mentholatum flavor $(1 \mathrm{gr} / \mathrm{l})$ in water. The subject would apply $2 \mathrm{ml}$ of this solution to the tongue resulting in the typically refreshing oral sensation of peppermint. The stereotyped acts the subject was instructed to perform before ingestion of the UCS (see procedure) completed the CS compound.

Unconditioned stimulus The UCS for the experimental condition was a solution of 50 grams of glucose (dextrose; Janssen Chimica, Tilburg) in $30 \mathrm{cl}$ of water. Fifty grams of oral glucose contain about $200 \mathrm{kcal}$ (Rodin, 1990) and generally lead to a blood sugar rise between 46 and $61 \mathrm{mg} / \mathrm{dl}$ with subsequent insulin levels between 60 and 110 micro Units/ml (Förster, Haslbeck \& Mehnert, 1972; Tse et al., 1983). In our control condition the UCS was an aspartame solution $(0.5$ grams dissolved in $30 \mathrm{cl}$ of water; Holland Sweetener Company, Maastricht). During a previous pilot study in other subjects, this aspartame solution had been rated as being equally sweet as the glucose UCS of the experimental condition. For both conditions a small amount $(3 \mathrm{ml} / \mathrm{l})$ of almond flavor (a $1 \%$ benzaldehyde-in-alcohol solution) was added for taste in the UCS solutions.

Test drinks The drinks presented during the taste test of the two test sessions were of equal composition in both conditions, namely a $150 \mathrm{~g} / \mathrm{l}$ glucose in water solution, again flavored with almond $(3 \mathrm{ml} / \mathrm{l})$. A total of 3 liters of the test drink was available to the subject, i.e., considerably more than the expected ad lib intake during the taste test.

Take-away drinks It was attempted to maximize the difference in appearance of the takeaway and lab drinks, so that CS perception would remain strictly limited to the lab situation. The quantity of sweeteners (glucose for the control group and aspartame for the experimental group) was the same as in the UCSs. Instead of almond, a "marshmallow" (maltol) flavor was added, and the drink was handed out in a dark-colored bottle (contents: $30 \mathrm{cl}$ ). 


\section{Chapter 3}

Urine jar This $25 \mathrm{cl}$ jar, made of transparent glass, was to be taken home for collection of the first morning urine, and brought back to the lab the next session as "a measure of metabolism of the take-away drinks". No further analysis was made of the urine samples (see procedure).

The drinking box The box from which all lab-beverages were drunk was made of whitecolored solid material. Its top contained three holes through which the straws (diameter $5 \mathrm{~mm}$; length $30 \mathrm{~cm}$ ) protruded. The straws did not require strong sucking to get the drink up from below. At conditioning sessions three cylinders, each filled with $10 \mathrm{cl}$ (i.e. one-third of the UCS), were placed in the box. However, for the ad lib taste tests during test sessions, three one liter jars filled with the test drinks were used. The subjects were not able to see the contents of the box or the color of the drinks.

The pipette The pipette, used for applying the peppermint flavor CS on the tongue, was an open glas laboratory tube (length $15 \mathrm{~cm}$ ) with a rubber balloon on top. The subject was to squeeze the balloon with the pipette tube below the fluid surface, and then let loose; thus the pipette would fill with approximately $2 \mathrm{ml}$ of the peppermint solution (CS), which was ingested by again squeezing the balloon while aiming at the tongue.

Biochemical analysis Blood glucose was measured in venous whole blood by an automated glucoseoxidase method performed on the EBIO analyzer (Eppendorf, Hamburg). Serum insulin and $C$-peptide were measured by radioimmunoassay after polyethyleneglycol (6000) pretreatment. For insulin materials were used provided by Pharmacia (Uppsala, Sweden), while the kit for C-peptide was manifactured by Byk-Sangtec (Dietzenbach, Germany). The interassay coefficients of variations were better than 8.4 \% for the insulin assay and better than $9.7 \%$ for the $C$-peptide assay at the concentration levels of interest in this article. Glucagon was measured in EDTA plasma by sequential radioimmunoassay using the glucagon double antibody kit from D.P.C. (Los Angeles, U.S.A.). Interassay coefficients of variations are better than $15 \%$ at the concentration level of $35 \mathrm{pmol} / \mathrm{l}$.

\section{Procedure}

A few days prior to the experiment an introductory meeting was held. The experimental procedure itself encompassed 10 daily sessions within a period of 12 days; between the fifth and sixth sessions there was a two-day weekend pause. There were two test sessions (day 1 and 10), two "no-conditioning" lab sessions to avoid association of UCS presentation and lab stimuli different from the proper CS (days 4 and 7) and 6 conditioning sessions (days 2,3,5,6,8 and 9). For each subject all sessions were planned at the same time of day. Blood parameters and ad lib glucose intake were measured only on test days. 
Introductory session This session was held to provide the subjects with preliminary information on the procedure and to get them acquainted with the lab, as well as handling the experimental attributes (pipette, straws, etc.). These materials were demonstrated in a room different from the CS room. Subjects were informed that on the first and the last days a longer procedure than normal would take place in which blood samples would be taken. The subject signed a statement in which she promised to refrain from ingesting peppermint or peppermint-related chewing gum, and sweetened liquor during the two weeks of the experiment. Furthermore, instructions were given to eat a meal of two sandwiches with one glass of drink three hours before each session, and to refrain from further eating and drinking until the session.

Conditioning sessions. CS+ (days 2,3.5.6.8 and 9) The subject was welcomed in a waiting room. It was pointed out to the subject that she was to enter a room in which a peppermint odor would be hanging in the air, and that a taste test involving sweet drinks would have to be carried out there. Upon entering the CS room the subject sat down at a table on which the drinking box and pipette were within reach. The experimenter was seated at an angle of 90 degrees within sight of the subject at a distance of 1.20 meters. Then the subject was instructed to apply the peppermint/mentholatum (CS) solution to her tongue. She was encouraged to taste the solution attentively and let it rinse through her mouth for one minute before actually swallowing it. During this minute the experimenter repeated the instruction to concentrate on the peppermint-taste, and added that soon the sweet beverage taste test would begin. It had been explained beforehand that the test would involve three slightly differently composed drinks, and that the subject had to detect the just noticeable differences between the drinks (in reality, each of the three straws was resting in $10 \mathrm{cl}$ of the same UCS solution). After the minute of tasting the peppermint flavor, the subject was instructed to swallow it, and there was a pause of 15 seconds. Then the experimenter told the subject to put the leftmost straw in to her mouth, but not yet start drinking. After 10 seconds the experimenter stood up, said "start drinking now; drink all three drinks from left to right in a steady tempo and concentrate on their taste differences", and left the room. In general the $30 \mathrm{cl}$ drink was finished in 2-3 minutes, after which the subject remained in the room for another 20 minutes. At regular intervals she was instructed by intercom to fill out a questionnaire (see materials). After 20 minutes the session was ended, the subject was given the take home drink, questionnaire and urine jar and then was allowed to leave. See table 3.1 for the time schedule of the conditioning sessions.

The "no-conditioning" sessions (day 4 and 7) During these sessions the subject was welcomed in the waiting room. It was announced that this time something different from usual would happen, i.e. that no taste test would be carried out. Next, the subject was 


\section{Chapter 3}

led to another room where a paper and pencil test was carried out of $2 \times 6$ minutes as "a check for concentration". After that the session was terminated.

Table 3.1. Schedule of conditioning and test trials

Conditioning trials

$\begin{array}{llllll} & |\operatorname{cs}| j \text { us } \mid & & & \\ -5 & 0 & 5 & 10 & 15 & 20 \text { minutes }\end{array}$

Test trials

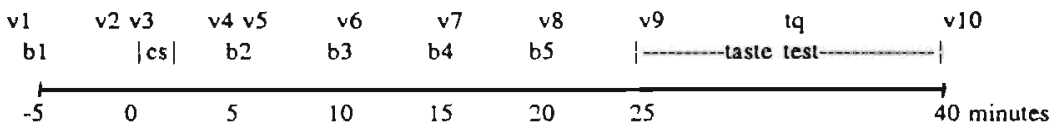

\footnotetext{
$\mathrm{cs}=$ conditioned stimulus (peppermint taste and odor)

us $=$ unconditioned stimulus (glucose or aspartame drinks)

$\mathrm{b}=$ blood sampling

$\mathrm{v}=$ visual analogue scale

tq $=$ tsste questionnaire
}

Home sessions The take-away drinks (see materials) were ingested at home between 7 and 10 p.m.. The instructions were to drink the beverage at a self chosen rate, on the condition that after the onset of drinking, the bottle should be emptied by the subject within 10 minutes. Subsequently, a questionnaire on which hedonic and sensory characteristics of the drinks were rated. No use was made of these data. The subject was instructed to collect and bring along a sample of morning urine at the next session for "analysis of the metabolism of the take-away drinks". In reality, the only purpose of this was to ensure that the subjects would drink the take-away drinks at home and no analyses were conducted.

Test-sessions (day 1 and 10 ) After a welcome the subject was seated in a nursing room where an indwelling catheter (venflon) was applied to the cubital vein of the left arm, a few centimeters distal of the elbow. Next, the subject was brought into the standard waiting room for adaptation and relaxation for a period of 30 minutes. Then the actual procedure began: the experimenter appeared, and explained that the forthcoming taste test would take place in the room where a peppermint odor would be hanging in the air. It was explained that after entering the room the taste test would begin after a pause the length of which might be $0,5,10,15$ or 20 minutes, depending on the outcome of a 
special "lottery". Then, the drawing of the first (baseline) blood samples was carried out, and experimenter and subject entered the CS room at about 4 minutes after the drawing of the first blood samples. After the subject was seated, the presentation of the CS proceded in exactly the same manner as during the conditioning sessions. However, after the subject had put the leftmosi straw in her mouth, the experimenter, instead of giving the "go" signal (as in the conditioning session), looked at his "lottery documents" and said: "no, you can't begin now, please leave the straw, fill out the next questionnaire, and wait". After the subject completed the questionnaire a second round of bloodsamples was taken (in reality the "lottery" was a sham procedure aimed at actively maintaining the subject's expectation of the UCS while blood samples were taken over a period of 20 minutes). The lottery was repeated four minutes later: the subject again had to put the left straw in her mouth and after 10 seconds she had to leave the straw, blood was taken. This action sequence was repeated 4 times. Matters were arranged in a way that blood samples were drawn at 5, 10,15 and 20 minutes after the presentation of the CS (according to Mityushov (1954; see also Woods \& Kulkosky, 1976) the hypoglycemia was expected to reach its nadir at $20-45$ minutes after CS presentation). At 24.5 minutes after entering in the $\mathrm{CS}$ room the subject again put the left straw in her mouth. This time the experimenter did indeed give the "go" signal, and told the subject to commence the taste test, and left the room. First one sip of each drink should be taken from the left to the right (this had been explained earlier). After that, the subject was allowed to drink as much as she wanted or needed to complete the questionnaire.

After 15 minutes the experimenter re-entered the room, and after removal of the venflon the subject was allowed to leave (first test session), or was taken to another room in which the subject was weighed, debrieved and thanked for participation (second test session). The amount of ingested glucose drink was weighed after the subject had left. See table 3.1 for the time schedule of these sessions.

\section{ANALYSIS OF DATA}

Statistical analyses were carried out using a SPSS-PC software program.

In a first step, an effect score was computed for each of the physiological parameters and the scores on all Visual Analog Scales of the two test sessions. For blood sugar, insulin and glucagon the effect score was defined as the difference between the mean level of samples 2-5 (i.e. the post-stimulus values) and the level of sample 1 (baseline). For C-peptide (which was analyzed only in the first and last blood samples) the difference between the post- and pre-stimulus sample was computed. 


\section{Chapter 3}

Similarly, effect scores for hunger and craving were defined as the difference between the mean of post-stimulus scores ( 8 scores) and the mean of baseline scores ( 2 scores). Further, for both the pre- and post-conditioning test session, the amount of ingested glucose test drink, and the mean sweetness and hedonical rating was computed.

Finally, all the computed physiological effect scores, quantities of drinks and subjective ratings were subjected to a 2 (experimental vs. control condition) $\times 2$ (pre/post conditioning) repeated measures ANOVA.

As one of the subjects (experimental condition) fainted during the blood sampling during both test sessions, her physiological ${ }^{2}$ and subjective data were exluded from further analysis. Furthermore, in one subject (control condition) the venflon jammed during the first test session, so that no blood data were acquired. Finally, one subject (control condition) showed a peculiar (exceptionally high and rapidly falling) baseline insulin level in the second test session. She was considered an outlier and her physiological data were excluded from further analysis.

\section{RESULTS}

Blood parameters (figures 3.1 to 3.4 ). For none of these parameters was the critical condition $x$ measurement interaction effect found. Furthermore, no significant main effect of condition or measurement appeared. A closer look at figure 3.2 shows that, after conditioning, the insulin response of the experimental group was slightly stronger than that of the control group. Exploratory t-tests revealed that the group difference was significant only at 5 minutes after CS presentation $(t(16)=2.52 ; p<.03$ ).

Subjective state during the test sessions Neither main nor interaction effects were found on hunger, thirst, tension and craving for either salty or sweet food. The craving-forsweet-food scores during the post-conditioning test session is depicted in figure 3.5.

Intake and subjective ratings of glucose test drinks For intake of the glucose test drinks, the critical measurement $x$ condition interaction effect was not found. However, a main effect was found for measurement $(F(1,16)=15.7 ; p=.001)$, reflecting increased intake of glucose after conditioning in both conditions (see figure 3.6). For hedonic rating a trend towards a condition $x$ measurement interaction effect was found $(F(1,16)$ $=3.36 ; \mathrm{p}<.09$ ) reflecting increased ratings after conditioning in the experimental condition (table 3.2). No effects were found on sweetness ratings of the test drinks.

${ }^{2}$ Analysis of the blood samples taken, however, revealed no abnormalities in both blood sugar and insulin levels. 


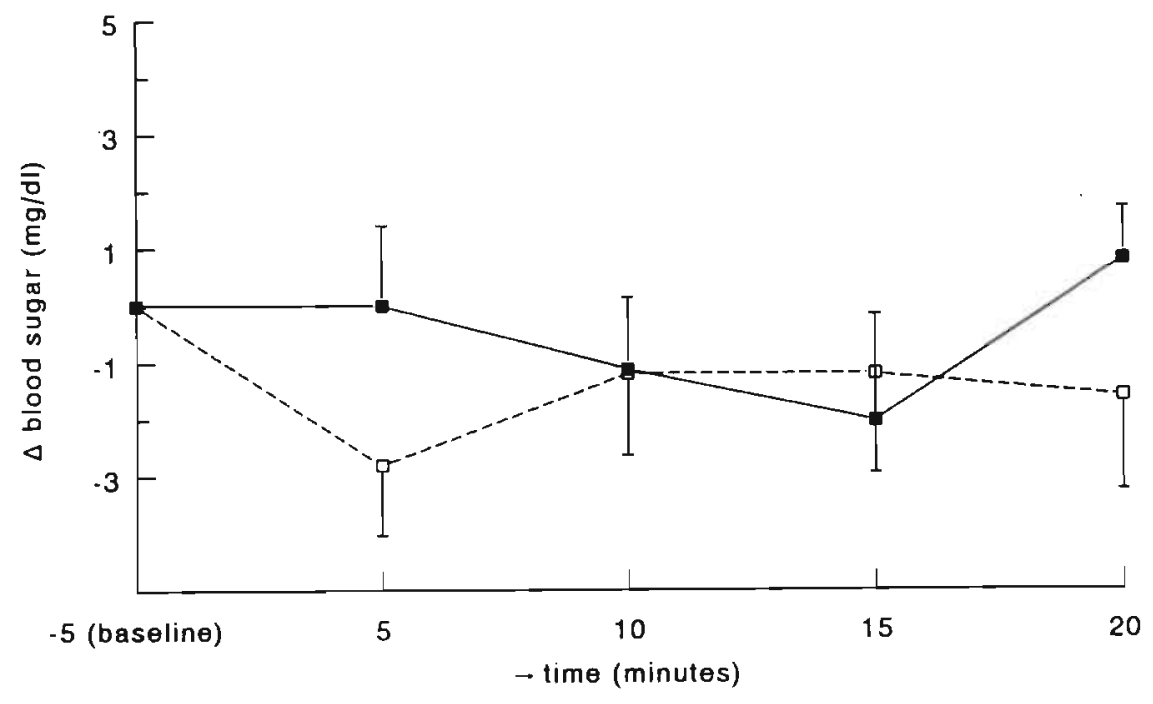

- - EXP. CONDITION --Q-- CONTR. CONDITION

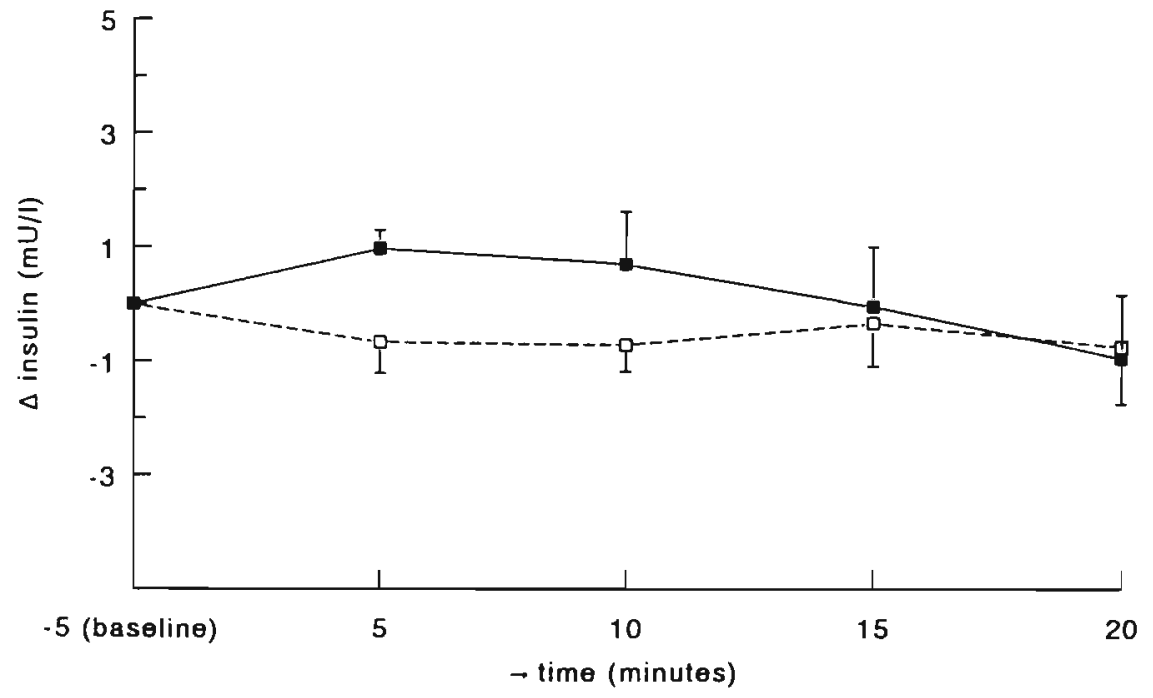

Figures 3.1. and 3.2. Blood sugar and insulin responses (mean \pm s.e.m) to CS presentation during the post-conditioning test session. The values represent deviation from baseline levels, which were $79.8 \pm 2.0 \mathrm{mg} / \mathrm{dl}$ for blood sugar and $9.4 \pm 1.2 \mathrm{mU} / \mathrm{l}$ for insulin. 


$$
\text { - - EXP. CONDITION } \quad--\square \text { CONTR. CONDITION }
$$

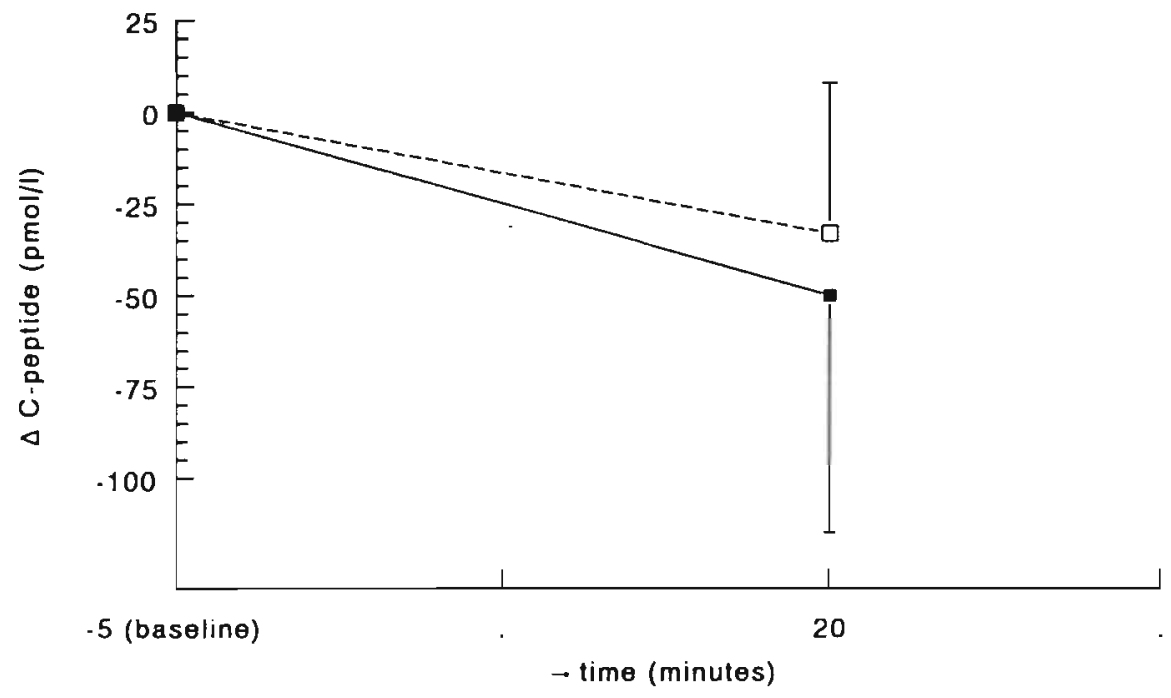

- - EXP. CONDITION --口-- CONTR. CONDITION

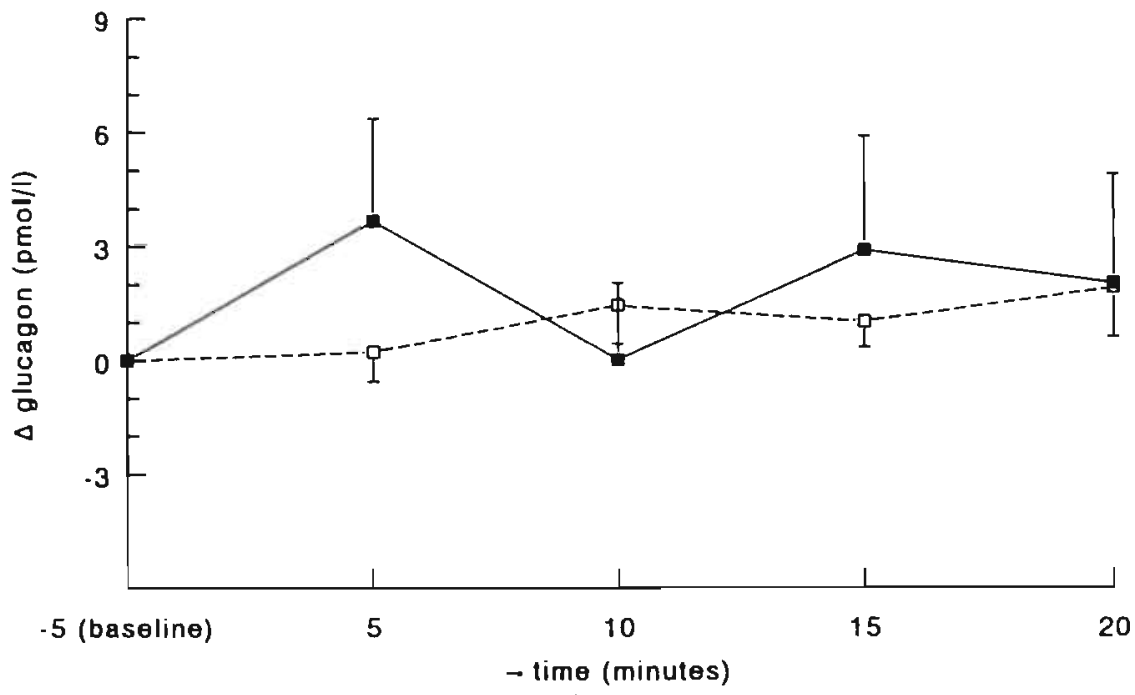

Figures 3.3. and 3.4. C-Peptide and glucagon responses (mean \pm s.e.m) to CS presentation during the post-conditioning test session. The values represent deviation from baseline levels, which were $830 \pm 90 \mathrm{pmol} / \mathrm{l}$ for C-Peptide, and 54.2 $\pm 2.2 \mathrm{pmod} / \mathrm{l}$ for glucagon. 


\section{Preliminary discussion and data from an added condition}

By and large, the CBE prediction of conditioned physiological responses in the experimental group was not confirmed. Nevertheless, some interesting results were found in the experimental condition: a larger insulin response in the experimental condition at 5 minutes after CS presentation, and a trend towards increased hedonic rating of the glucose drinks. However, the mean size of the insulin response in the experimental condition (approximately $1 \mathrm{mUnit} / \mathrm{l}$ ) was not impressive, given the fact that the range of spontaneously occurring fluctuations in insulin level tends to be larger than this (see e.g. Lucas, Bellisle \& de Maio, 1987). To investigate the possibility that the increased insulin secretion co-occurred with the trend towards increased hedonic ratings of the glucose test drinks, the correlation between the shifts in insulin secretion and hedonic rating was computed. This correlation turned out to be -0.32 (n.s.), suggesting a discordance between insulin secretion and hedonic value. Furthermore, a second series of ANOVAs was employed to account for possible inter-individual differences in the peak latency of the putative conditioned responses. In these analyses the baseline level was compared with the most extreme of the post-stimulus values in the direction of our hypotheses, i.e. maximum values for insulin level, and minimal values of blood sugar and glucagon levels post-stimulus and these values were subjected to the same $2 \times 2$ ANOVA as above. None of these analyses revealed significant effects. Further, the expected larger levels of craving and glucose intake in the experimental than in the control condition failed to be confirmed too.

Table 3.2. Hedonic and sweetness ratings (mean \pm sd) of test drinks before and after conditioning (range: $0-100$ )

\begin{tabular}{cccc}
$\begin{array}{c}\text { hedonic value } \\
\text { before } \\
\text { after }\end{array}$ & $34.2(23.5)$ & $31.5(24.4)$ & water condition \\
& $46.7(21.3)$ & $31.1(17.4)$ & $40.8(19.1)$ \\
$\begin{array}{c}\text { sweetness } \\
\text { before } \\
\text { after }\end{array}$ & $52.4(15.7)$ & $67.6(17.7)$ & $32.1(18.4)$ \\
\hline
\end{tabular}

The exact reason for the striking increase in glucose intake found at the post-conditioning test session in nearly all subjects remained unclear: it might be an effect related either to experience with sweet taste or acquaintance with laboratory procedure. In order to further elucidate this issue, a third condition was run: 9 subjects (BMI: 21.0, sd 1.4; 


\section{Chapter 3}

age: 23.1 , sd 2.9) were selected according to the same criteria as in the previous conditions. The procedure was equal to that in the other conditions except for the following: 1) in the conditioning sessions $30 \mathrm{cl}$ of plain water served as the "UCS"; 2) no blood samples were drawn 3) no random control procedure was carried out, i.e. no take away drinks were given.

Once again, it appeared that the glucose intake after conditioning was larger than before conditioning in all but one of the subjects (figure 3.6). On the average, glucose intake was $69 \%$ larger after than before conditioning (paired $t(8)=-3.36 ; p=.01$ ). After adding the intake data of this condition to those of the other two conditions, a 3 (condition) $\times 2$ (pre-vs post conditioning) repeated measurements ANOVA was run. This test did not reveal a condition $x$ measurement interaction effect, whereas the significant main effect of measurement $(F(1,24)=25.0 ; p<.001)$ reflected the increased intake in all three conditions. This result suggests that mere exposure to sweet drinks was not the critical factor in the increased glucose intake observed in the experimental (glucose UCS) and control (aspartame UCS) conditions.

\section{DISCUSSION}

The present procedure involved sixfold pairing of a peppermint flavor/fragrance CS with a UCS ingestion of glucose (experimental condition) or aspartame (control condition) drinks in human female subjects. In contrast with the hypothesis, during the postconditioning test session, the experimental subjects did not show significant conditioned blood sugar and glucagon decreases or $\mathrm{C}$-peptide increases relative to either the preconditioning session or the control subjects. Separate t-tests showed that the insulin response at 5 minutes after CS presentation was larger in the experimental than in the control condition. This difference was in the direction predicted by the CBE model, although the effect size did not exceed the range of spontaneous occurring baseline fluctuations. The prediction of especially increased craving for sweet substances in experimental subjects after CS presentation after conditioning was not confirmed. Mean hedonic rating of glucose drinks increased somewhat more in the experimental than in the control condition, but the condition $x$ measurement interaction did not reach significance. The most impressive effect, found in both conditions as well as a later added condition in which plain water was presented as the UCS, was that ad lib glucose intake during the post-conditioning test session was $78 \%$ larger than during the pre-conditioning test.

To start with the most impressive effect, the increase in ad lib glucose intake would fit 


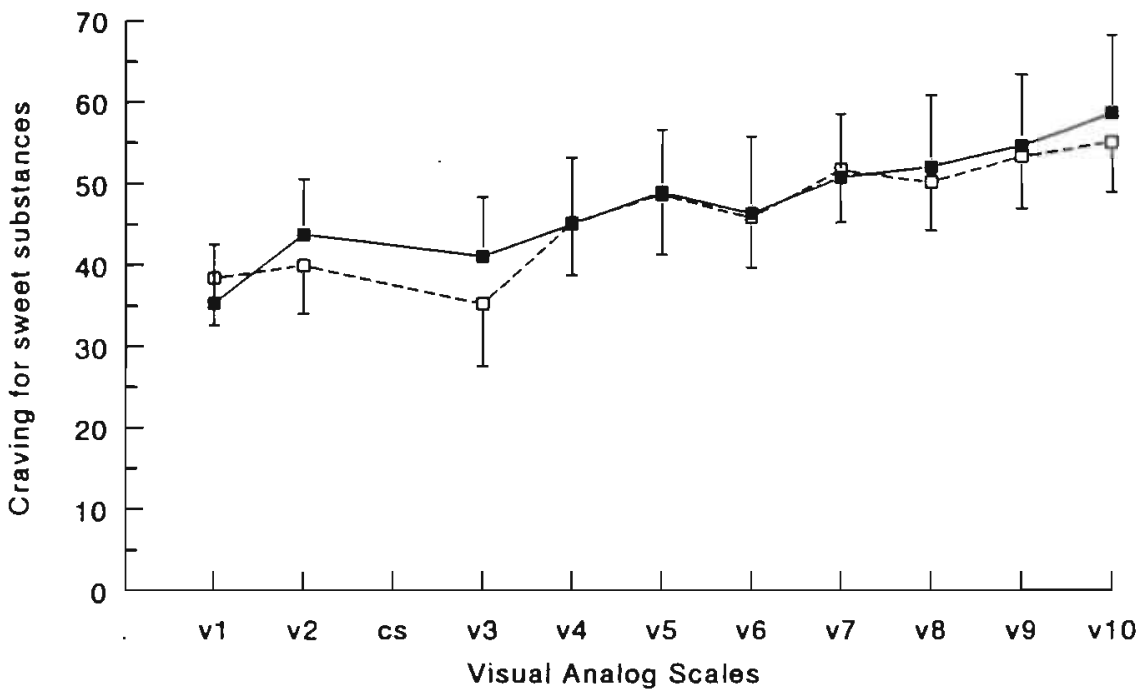

Figure 3.5. Mean \pm s.e.m. of subjective craving scores for sweet foods during the post-conditioning test session. CS presentation was between $v 2$ and $v 3$.

BEFORE CONDITIONING

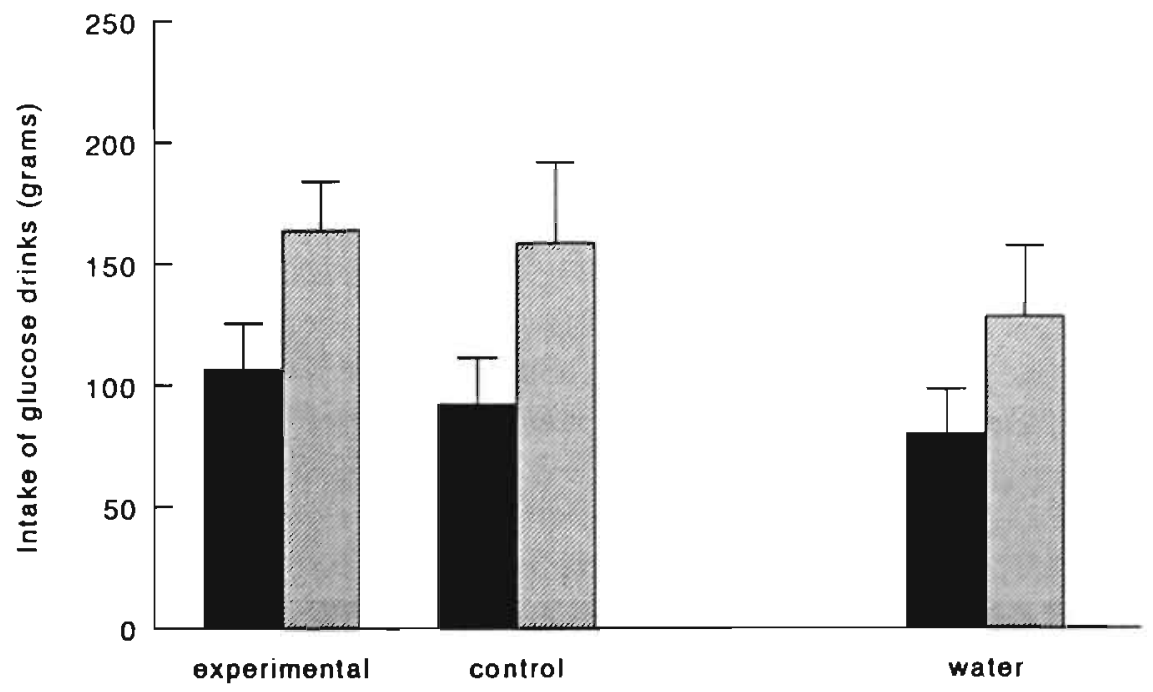

Figure 3.6. Mean \pm s.e.m. of grams of ingested glucose test drinks during the preand post-conditioning test sessions. 


\section{Chapter 3}

the predictions of the CBE model, were it not that the aspartame and plain water conditioned control subjects showed a comparable increase. It is therefore plausible that another behavioral mechanism was at work, for example "neophobia" or "bait shyness", a well-known phenomenon from animal research. Although, in general, neophobia refers to initial reluctance to ingest unfamiliar foods, it may also occur whenever animals are placed in a new environment. An initial decrease or inhibition of spontaneous food intake is shown which only gradually disappears; it can take days before intake has returned to the prior level (Barnett, 1958; Williams, 1968; Woods, 1991). Such a phenomenon may have occurred during our experiment: at the first test session (day 1) the subjects were still unadapted to the lab and procedure, and they might have been "reluctant" to drink. On the second test session ( 9 sessions later), however, the initial reluctance could have faded, thus clearing the way for the observed larger glucose intake. A further physiological explanation of the faded neophobia, i.e. by higher insulin levels (Woods, 1991) seems not warranted in the present case because of the weak insulinemic effects found. Admittedly, however, the possibility that exaggerated insulin secretion occurred after oral contact with the glucose cannot be ruled out (blood samples were taken only before but not during the glucose taste test).

As to the absent physiological and subjective effects of the present conditioning procedure, one might conclude that the CBE model has been refuted. However, the basic prediction we made was that once conditioned hypoglycemia had occurred, craving and increased intake of sweet drinks would follow. Unfortunately, since the first step, i.e., evoking conditioned glycemic and insulinemic changes, failed, the present data are inconclusive with regard to the hypothesized impact of (learned) hypoglycemia/hyperinsulinemia on craving.

The remainder of this discussion will focus on characteristics of the CS, UCS and the conditioning procedure in the present study, that may have underlain the present results and deserve consideration in future glycemic conditioning research.

First, it might be argued that our subjects' pre-experimental experience with the peppermint CSs like tooth paste, chewing gum, and peppermint, i.e. stimuli associated with only weak internal physiological effects ${ }^{3}$, might have slowed down their rate of conditioning through latent inhibition (Schwartz \& Robbins, 1995). However, in as far as latent inhibition acts by means of reduced attention to the presented stimuli (Miller et al., 1995; Schwartz \& Robbins, 1995), the inclusion of both the unique pre-ingestive ritual and explicit identification of the CS, UCS and their contingency will have

\footnotetext{
${ }^{3}$ Note that our subjects did not have an exceptional like of peppermint (see subject selection criteria); thus experience with larger peppermint intake was probably absent.
} 
diminished such an effect during our procedure. Moreover, it has been shown that in a successful instance of glycemic conditioning with large UCSs, latent inhibition by preexposure to the CS failed to thwart rapid acquisition of the conditioned response (Mityushov, 1954).

Second, one might wonder whether the UCS intensity (i.e. 50 grams of glucose) was sufficiently strong to lead to conditioning. When looking at the literature on the physiological effects of glucose, this suspicion seems unjustified. The blood sugar rise in response to 50 grams of glucose (i.e. between 46 and $61 \mathrm{mg} / \mathrm{dl}$; Förster et al., 1972; Tse et al., 1983) overwhelmingly larger than the usual "spontaneous" baseline fluctuations (being between 1 and $2 \mathrm{mg} / \mathrm{dl}$; Lucas, Bellisle \& Di Maio, 1987). Also, though glucose is a powerful blood sugar raiser, ingestion of doses above 50 grams would not have led to a large additional blood sugar rise (e.g. Förster et al., 1972). Still, previous successful instances of glycemic conditioning used an intravenous glucose UCS, involving larger blood sugar rises (up to $200 \mathrm{mg} / \mathrm{dl}$; Mityushov, 1954) and leading to more rapid acquisition of a significant $C R$ than when oral glucose UCS are used. Therefore, in the present study, it may be not so much the UCS, but its combination with a limited number of conditioning trials that has been responsible for the present failure to find conditioned hypoglycemia.

Finally, it might be that both extinction and blocking have occurred during the present procedure, and were relatively important, given the small number of conditioning trials. Extinction of the conditioned response could have occurred as a result of the random control procedure (ingesting the take-away drink) in the experimental group. Although care was taken to maximize its sensory difference with the lab-UCS drinks, the takeaway drink may still have been perceived as a "tin full of sweet drink" CS, and, as it contained metabolically inert aspartame, its ingestion may have extinguished conditioned responses already established during lab-sessions. A perhaps more important explanation of our finding of no conditioning may have been blocking (Schwartz \& Robbins, 1995): prior to the experiment our subjects (like anybody) may have had an already firmly established association between oral sweetness (CS) and subsequent blood sugar increases (UCS). Our conditioning procedure involved association of an odor/fragrance CS with oral glucose. Thus, during conditioning sessions two CSs may have been operating: the experimental, "intended" CS (CS1), and the sweet taste of the UCS glucose beverage itself (CS2). Hence, during the conditioning trials, relative to CS2, CS1 did not provide additional information about an impending blood sugar rise. Hence, it might be argued that the CSI alone would not able to trigger conditioned responses during the post-conditioning test session. Although we had good reason for not applying a glucose probe to the tongue as a CS on test days (oral glucose is known to elicit a 


\section{Chapter 3}

(mainly unconditioned) insulin secretion through glucose sensitive receptors located in the mouth and upper gastrointestinal tract (Grill, Berridge \& Ganster, 1984; Nicolaïdis 1977), it may be valuable to present a small sweet tasting stimulus (e.g. aspartame solution, Bruce et al., 1987) at the test session.

In conclusion, research remains to be done which elucidates the role of conditioned glycemic responses in craving for food. As a first step, procedures must be developed to which human subjects acquire significant conditioned glycemic and insulin responses. Then, using these procedures, while controlling for neophobic interference, one could assess the contribution of physiological learning to craving and binge eating. 


\section{Chapter 4}

\section{INTRODUCTION AND COMMENTARY TO: M.I. MITYUSHOV (1954), "CONDITIONED REFLEX SECRETION OF INSULIN"1}

As has been known for decades now, the physiological impact of Pavlovian conditioning extends beyond salivation, skin conductance and heartrate (Ádám, 1967; Dworkin, 1993; Razran, 1961). Current research on the role of conditioned responses in homeostatic regulation ${ }^{2}$ (e.g. Dworkin, 1993) has once again demonstrated the potential importance of the orginal studies on classical conditioning by the Eastem European Pavlovians, carried out several decades ago. The present article contains the translation of a Russian paper on blood sugar conditioning, which still appears to be highly relevant for today's conditioning research. The paper was first published in 1954 by the physiologist M.I. Mityushov. Before presenting the paper, we will describe its relevance for current research. The paper will be followed by a reanalysis and discussion of the presented data.

\section{INTRODUCTION}

At first glance, it seems as if today's Pavlovian conditioning research has become a science of stimuli rather than responses. For example, in a recent review of the predominant RescorlaWagner theory, the terms CS and US are used approximately 560 times, whereas the response terms CR or UR are altogether lacking (Miller et al., 1995) ${ }^{3}$. The frequent use of "CS" and "UCS" can be attributed to the description of conditioning principles pertaining to stimulus interactions, for instance, latent inhibition, blocking, and overshadowing. Meanwhile, the roles

1 This paper, together with the translation of Mityushov (1954; see Appendix of this thesis) will be submitted for publication by J. Overduin, B.R. Dworkin \& A. Jansen. The authors gratefully acknowledge Professor Gyōrgy Ádám, Eōtvōs University, Budapest, for his helpful comments on both our article and the translation of Mityushov's paper.

${ }^{2}$ Strikingly, the potential importance of conditioning for "The Wisdom of the Body" appears to have been overlooked by W.B. Cannon, the father of modern regulatory physiology, notwithstanding the fact that Pavlov and Cannon were acquainted and met each other on several occasions (sce Cannon, 1939; Dworkin, 1993).

${ }^{3}$ Garcia et al. (1985) already noted that in the classic paper by Rescorla (1967), the terms CS and US were mentioned 130 times, whereas "CR" was only used ten times, and "UR" not once. Hence, it appears that the situation has grown even more asymmetrical ever since. 


\section{Chapter 4}

of CR and UCR have received less attention. The few discussions about the nature of conditioned responses have focussed on the "survival value" for the organism. Nevertheless, the prevalent view is that nothing is gained by alluding to functionality of conditioned responses. For instance, it may often seem gratuitous to argue that an increase in skin conductance CRs serves the "purpose" of preventing the organism from injury to the skin (e.g. Furedy, 1989; Turkann, 1989). Overall, such skepticisms may be justified in the case where the CR measured is peripheral or multi-faceted (e.g., skin conductance fluctuations, gross skeletal activity; Dworkin, 1989; 1993).

However, in the domain of homeostatic regulation, reference to the adaptive value of conditioned responses may certainly be elucidating (Dworkin, 1989; 1993). Pavlov himself held this opinion. His earlier physiological work (1897/1910) has been fully open to the possibility of conditioned responses serving regulatory purposes ${ }^{4}$. After describing the digestive system, with its secretions so well-attuned to the chemical compostion of the ingestive, Pavlov goes on to say that also the composition of conditioned salivation and other secretions is in accordance with the composition of the signalled substance (UCS). For example, CSs signalling the presentation of dry meat powder (UCS) trigger a more abundant watery salivation than CSs predictive of moist real meat (Babkin, 1949; Pavlov, 1897/1910). Today, the regulatory view of classical conditioning is especially in vogue in the field of drug conditioning. According to the prevalent view, drug intake (UCS) poses a disturbance of the internal milieu, which prompts regulatory physiological responses (UCRs). If the drug intake is reliably signalled by cues like the injection ritual in a specific environment, these CSs acquire the power to trigger conditioned responses that attenuate the physiological disturbance caused by intake of the drug (Eikelboom \& Stewant, 1982; MacRae, Scoles \& Siegel, 1987; Siegel, 1989). In most cases, the CR appears earlier than, or simultaneously with the UCR. In this way, the CR may enhance physiological regulation by mitigating a physiological disturbance in its very early stages, or by strengthening the homeostatic UCR (see Dworkin, 1993). It will be proposed here that conditioning of blood sugar level can be understood by similar principles.

\section{Principles of blood sugar conditioning}

Before introducing contemporary blood sugar conditioning research, a brief overview of blood

${ }^{4}$ In his later years (from 1903 onwards), Pavlov began to regard salivation rather as a tool to measure the strength of conditioning. As appears from his writings, Pavlov less and less considered the chemical composition or physiological impact of his dogs" conditioned secretions as a research object to focus upon (Dworkin, 1993). 
sugar regulation is in place. Blood sugar (more precisely, blood glucose) is the main fuel of e.g. the brain and the heart. Not surprisingly, the level of blood sugar is tightly controlled by a multitude of the nervous and endocrine activities. Under normal circumstances, two organs, the pancreas and liver, play a predominant role in blood sugar regulation. The pancreas is able to secrete insulin, a powerful blood sugar lowering hormones. The trigger for insulin secretion can be a high level of blood sugar and/or activity in the parasympathetic vagus nerve which innervates the pancreas (Niijima, 1989; Strubbe \& Steffens, 1993). The liver is able to lower or heighten blood sugar level, depending on circumstances. In the former case, glucose is withdrawn from the blood and stored as glycogen. In the latter, e.g. glycogen is converted to glucose and released into the blood stream. The hepatic blood sugar regulation is under hormonal and nervous control: catecholamines, glucagon and sympathetic nervous activity increase glucose production, whereas insulin and parasympathetic nervous activity decreases the glucose output from the liver (Brodsky \& Devlin, 1994). Interestingly, the autonomic nervous influences on pancreas and liver tend to be coordinated and synergistic. The source of these coordinated actions apparently lies within the hypothalamus (Niijima, 1989). In general, blood sugar regulation, supported by many more physiological factors than described here, acts so as to limit fluctuations in blood sugar during normal state, mobilize blood sugar in case of need (e.g., during stress or vigorous exercise), and store sugars in case of abundance (e.g. after a meal).

Conditioning of blood sugar level appears to be possible, and has systematically been studied since the 1960s (for reviews see Dworkin, 1993; Woods \& Kulkosky, 1976). The studies were all carried out in rats, given repeated injections with insulin (the UCS) in the course of days or weeks. Generally, the conditioned stimuli used in these studies have been complex, consisting of a stereotyped injection ritual within a salient environment. From a simple homeostatic viewpoint, the expected result of these procedures would be a hyperglycemic CR, i.e., a conditioned blood sugar rise that counters the hypoglycemia caused by the insulin UCS. However, the results found within this line of research were more subtle. Presentation of the CS (i.e. a saline injection in the conditioning environment) led to hypoglycemic CRs in some studies, and hyperglycemic CRs in others. These results have been explained in two ways.

${ }^{5}$ Although somewhat beyond the focus of the present article, glucagon deserves mentioning as the most powerful blood sugar raising hormone. Like insulin, glucagon is a pancreatic hormone, and its rate of secretion is inversely proportional to that of insulin. Because of the major impact of these hormones, the insulin/glucagon ratio has been used as an index of physiological state in organisms (Brodsky \& Devlin, 1994). 


\section{Chapter 4}

First, the insulin dose used as UCS has not been equally large in different studies. In several studies, the UCS was a huge insulin dose (leading to blood insulin levels that were 200 times larger than baseline level; N.B. under normal circumstances, after a meal, insulin levels rise to a peak of between 2 to 20 times baseline, see e.g. Guyton, 1991). In these cases, the insulin was thought to stimulate certain brain receptors involved in a positive feedback loop leading to additional insulin secretion (UCR) ${ }^{6}$. The unconditioned insulin reflex eventually re-emerged as the conditioned response which in its turn was responsable for the observed hypoglycemia. In contrast, studies in which smaller insulin doses were used as the UCS, have found a conditioned hyperglycemia (e.g. Siegel, 1972; Woods \& Shogren, 1972). These studies may have more relevance for blood sugar regulation under normal physiological condition. The physiological disturbance, caused by these smaller insulin UCS was a moderate fall in blood sugar level, which prompts compensatory UCRs (e.g. glucagon and catecholamine response, inhibition of insulin secretion) in order to restore baseline levels of blood sugar. After conditioning, these UCRs apparently were copied in CRs which could be elicted by the CS. Consequently, CS presentation lead to a hyperglycemic response (see Dworkin, 1993).

A second explanation, put forward to account for the findings of both conditioned hyper- and hypoglycemia, has been that the procedures differed in the amount of stress imposed on the rats. Notably, handling, drawing blood, and using unfamiliar cages to run the conditioning trials might impose stress upon the animals. Stress may increase blood sugar (by e.g. catecholamine, corticosteroid and glucagon release), thus overshadowing possible hypoglycemic CRs. Indeed, it has appeared that conditioned hyperglycemia appeared if the conditioning environment differed strongly from the normal home cage. Conditioning in a cage resembling the home cage led to conditioned hypoglycemia (see Dworkin, 1993; Flaherty, Grigson \& Brady, 1987).

Although the above studies are certainly informative about the role of conditioning in blood sugar regulation, insulin injections do not strike as naturally occurring physiological UCSs. It might be that using UCSs containing glucose would be a more natural stimulus: after food intake the blood glucose level generally shows a distinctive rise, to which ample homeostatic reactions are emitted (Guyton, 1991). Therefore, it is puzzling that glucose has been seldomly used as the UCS.

We are aware of only four reports on conditioning procedures with pure glucose as the UCS.

${ }^{6}$ For further explanations of the physiological mechanisms probably involved, see Woods (1983) and Dworkin (1993). 
Deutsch (1974) who used rats, found. a $3.5 \%$ hypoglycemia to a caffein flavour CS, after repeatedly pairing of a flavour with a glucose dose (UCS) which was intubated into the stomach. Deutsch claimed that the observed conditioned hypoglycemia had been the result of a conditioned insulin secretion. Matysiak and Green (1984) camied out three experiments in which an injection ritual (CS) was repeatedly paired with intravenous glucose UCS in rats. Post conditioning testing in all three experiments showed a conditioned blood sugar decrease (CR) of approximately $3 \%$ in response to a saline injections; a control group of rats that had previously received saline UCSs showed a 4-6\% hyperglycemia. These data were somewhat problematic however, because baseline levels were measured on a day different from the test day. Woods (1976), after conditioning two groups of rats with either glucose or saline injections as UCS, did find an insignificant hyperglycemia on the test trial (injecting saline), but there were no differences between glucose and saline-conditioned rats. It is possible that the hyperglycemia resulted from stress. Woods attributed the lacking hypoglycemia to the glucose UCS dose not being large enough. Overduin and Jansen (submitted) used $50 \mathrm{gr}$ of oral glucose as a UCS in humans, and peppermint taste/odor as CS in humans, but found no significant hypoglycemia. This was probably explained by the limited number of six conditioning trials and the - comparatively - small physiological impact of oral dose of glucose which was used.

Taken together, procedures using glucose as the UCS have produced either hypo- or euglycemic, but not hyperglycemic CRs. Although none of these results contradict a homeostatic, i.e., regulatory explanation, replications of the conditioned hypoglycemic effect surely would add to make this explanation more convincing. In this respect, the study by Mityushov has an interesting contribution to make.

\section{The article}

Although M.I. Mityushov's article, of which a translation will follow next, was entitled Conditioned Reflex Secretion of Insulin, it only reported direct measurements of blood sugar. The article was first published in 1954 in the Russian Journal Zhurnal Visshey Nervnoi Deyatel'nosti, which can be translated as "Journal of Higher Nervous Activity". The Zhumal was the main Eastern-European journal of the Pavlovians and, in the 1950s and 1960s, excerpts from it were published in German and English as well. For reasons unknown, Mityushov's paper never appeared in translation.

Several features of Mityushov's experiment contribute to its relevance for contemporary research. First, a large glucose injection was used as a UCS and an (albeit indirect) attempt 
Chapter 4

was made to assess the influence of insulin in the conditioned hypoglycemia. Second, humans and dogs, rather than rodents served as subjects. Third, relatively many conditioning trials were conducted. Last, but not least, although Mityushov's article does not contain rigorous statistical analyses, it appears that unequivocal and interesting results were found.

TRANSLATION OF MITYUSHOV'S ARTICLE (SEE APPENDIX)

\section{COMMENTARY}

\section{Abstract}

In a conditioning procedure, Mityushov repeatedly administered intravenous glucose (UCS) to four dogs and nine humans. The injection procedure was carried out in a specific room served as the CS. At the post conditioning test session, a saline injection (CS) was given at $t=0$. The blood sugar response (CR) was measured by repeated blood sampling at $t=-10,-5,5,15$ and 45 minutes for humans, and at $-10,-5,5,15,30,60$ and 90 minutes for dogs. Explicit data were presented for several responses of 4 humans and 2 dogs. These subjects had been subjected to between 5 and 52 conditioning trials. In all cases, the saline injection elicited a conditioned blood sugar fall of between 5 and $20 \%$ below baseline. The nadir of the response occurred at 15 minutes after the saline injection. As the CR was measured repeatedly within subjects, it could be seen that $C R$ intensity tended to increase as a function of the number of previous conditioning trials. Although no direct insulin measurements could be made at the time, Mityushov collected evidence that the hypoglycemia observed in humans and dogs, was secondary to a conditioned secretion of insulin. Mice that were injected with blood that was drawn from dogs during their conditioned hypoglycemia, showed a subsequent fall in their blood sugar level. Mityushov claimed that he observed the conditioned hypoglycemic response in all of his human and canine subjects.

\section{Additional analyses}

Mityushov's article contains neither graphs nor statistical tests of the blood sugar data. Nevertheless, it was possible to further analyze the data depicted in tables A.1 to A.3 of Mityushov's article (see appendix). For this purpose, data for dogs and humans were treated separately. Only data were considered of the "correct" test trials, i.e., those taking place in the 


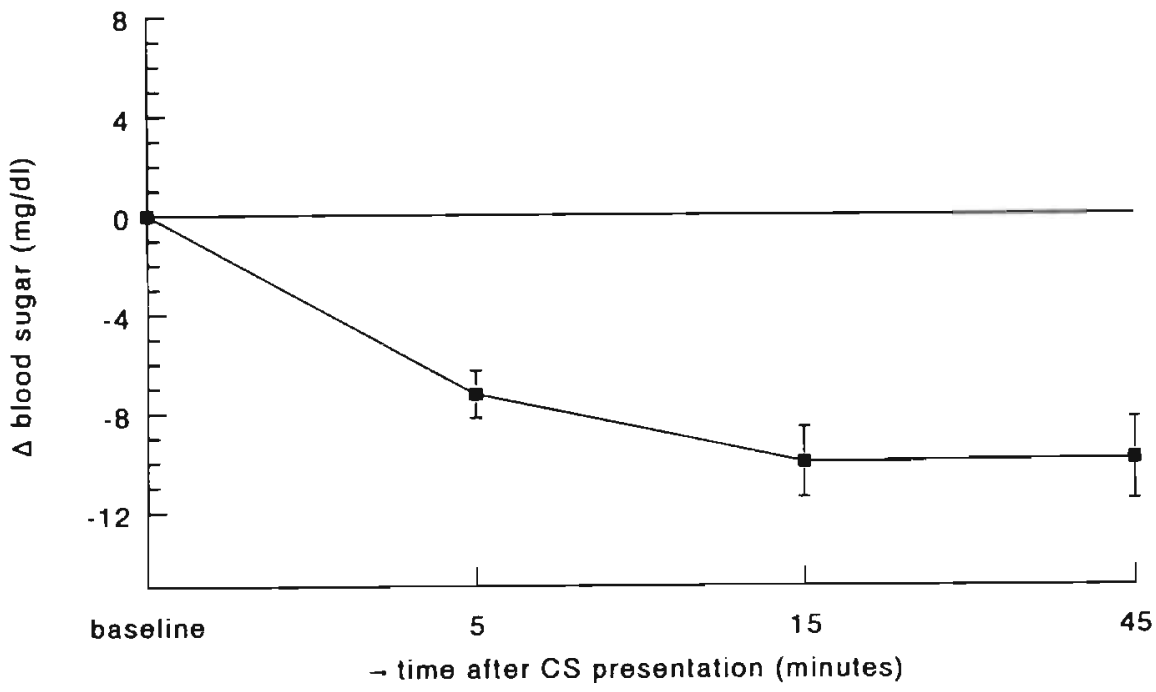

Figure 4.1. Post-conditioning blood sugar responses (mean \pm s.e.m.) to saline injections in 4 humans, based on a total of 17 responses. Responses expressed as deviation from baseline.
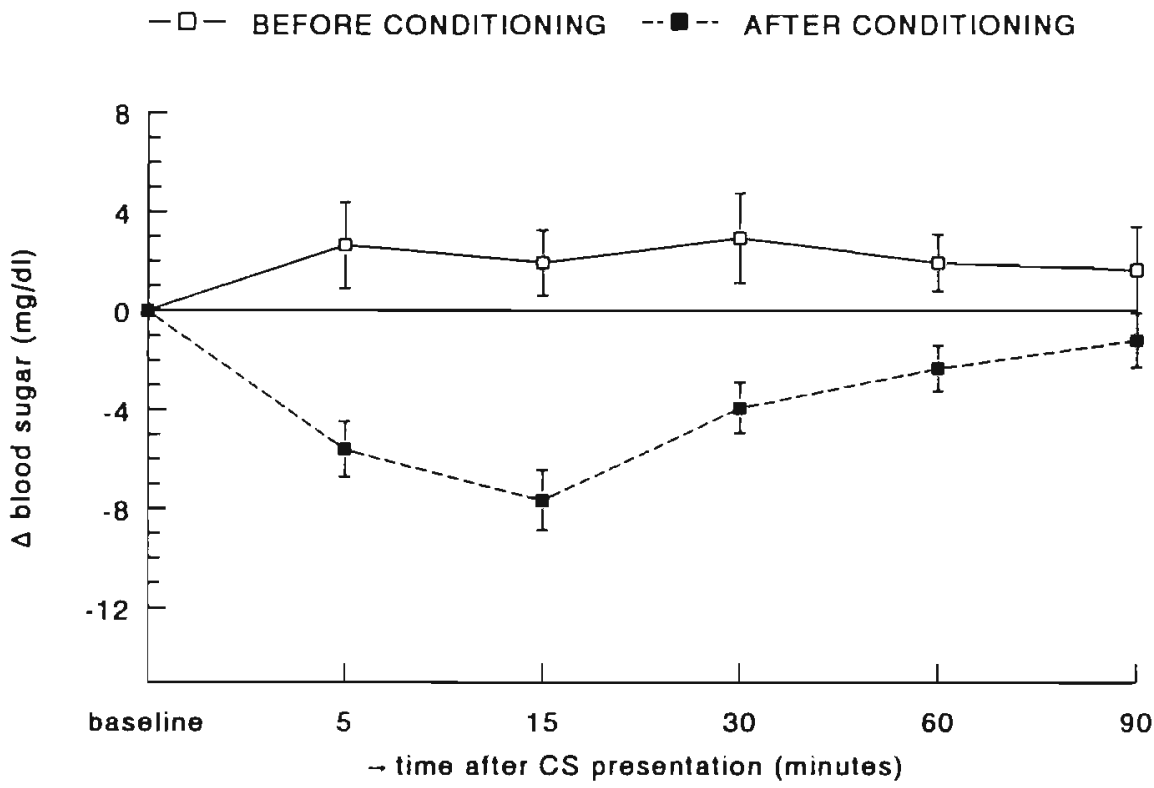

Figure 4.2. Pre- and post-conditioning blood sugar responses (mean \pm s.e.m.) to saline injections in 2 dogs, based on a total of 7 responses before, and 15 after conditioning. Responses expressed as deviation from baseline. 


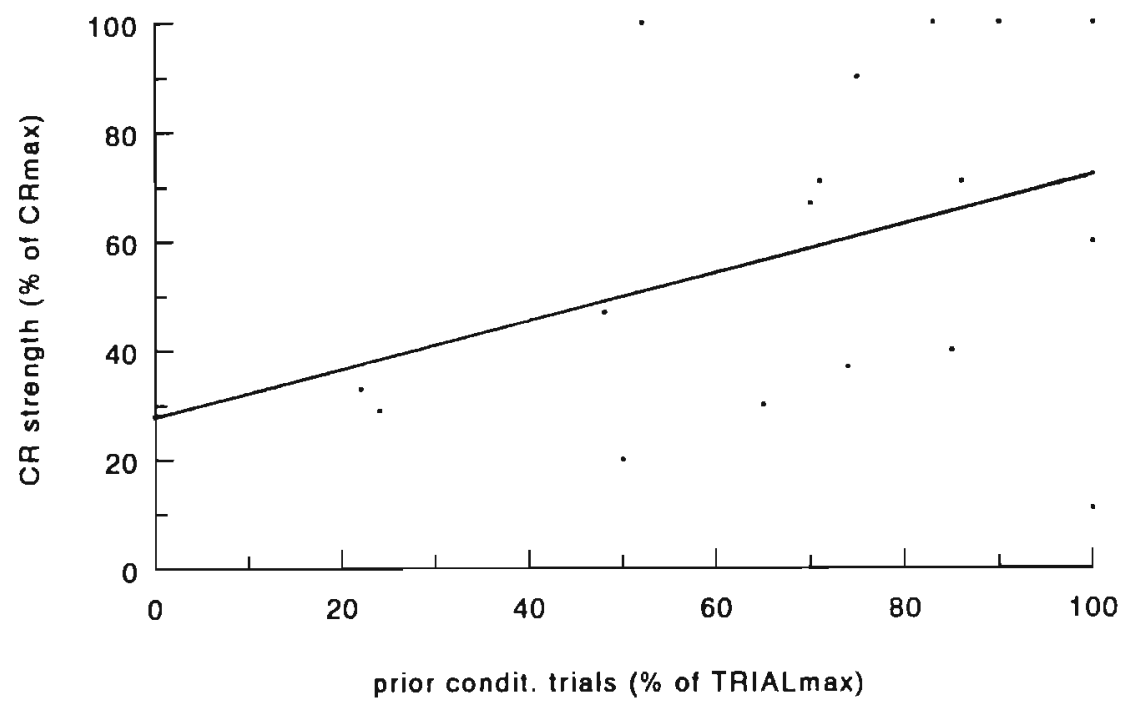

Figure 4.3. Acquisition curve of conditioned hypoglycemia in humans. For each subject, CR strength has been expressed as the percentage of the maximum CR intensity shown throughout the experiment.

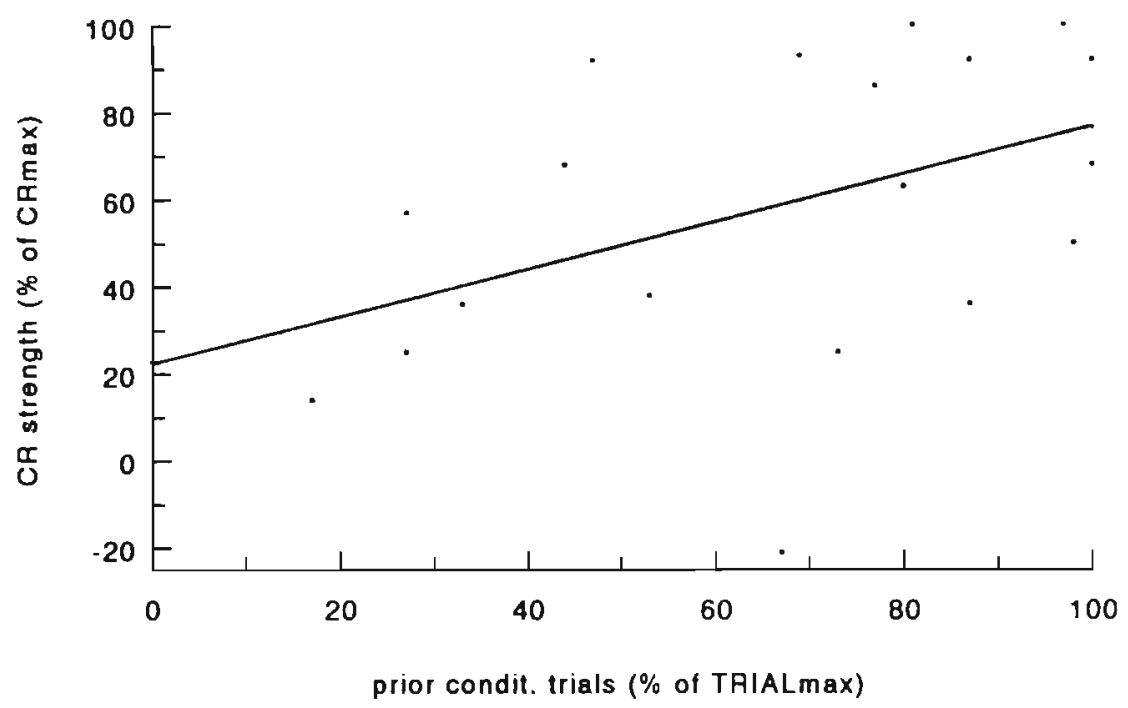

Figure 4.4. Acquisition curve of conditioned hypoglycemia in dogs. See figure 4.3. for further explanation. 
conditioning chamber with the usual experimenter.

Means Figures 4.1 and 4.2, depicting the glycemic responses to saline injections for humansand dogs respectively, were construed as follows. First, baseline level was computed as the mean of the two pre-injection baseline values. Then, the blood sugar values after each saline injection were expressed as the deviation from baseline. Finally, all responses of all subjects were taken together, mean and s.e.m. were computed and entered into the figures. For dogs, separate graphs were drawn for responses before and after conditioning.

The differences between baseline and post injection blood sugar levels were tested separately within three data subsets: for canine pre- and post-conditioning, and human post-conditioning data respectively. For each data set, a paired t-test was done comparing the mean baseline level with the mean blood sugar level after saline injection. For dogs' responses prior to conditioning (9 responses), no difference was found $(\mathrm{t}(8)=.17$; ns). After conditioning, for dogs $(\mathrm{t}(18)=$ 5.35; $\mathrm{p}<0.001 ; 19$ responses), and humans ( $\mathrm{t}(18)=7.83 ; \mathrm{p}<0.001 ; 19$ responses) highly significant differences were found, reflecting a hypoglycemia with its nadir at 15 minutes after the saline injection. Additional t-tests, comparing baseline with the individual post-injection blood sugar levels, revealed that after conditioning, all blood sugar levels measured within 45 minutes after saline injection, were significantly below baseline (table 4.1 ).

Table 4.1. Tests for differences between blood sugar levels before and after saline injection.

\begin{tabular}{cccc}
\hline & Before conditioning & \multicolumn{2}{c}{ After conditioning } \\
& dogs & $\operatorname{dogs}$ & humans \\
minutes & & & \\
$\mathbf{5}$ & $\mathrm{ns}$ & $<.001$ & $<.001$ \\
$\mathbf{1 5}$ & .05 & $<.001$ & $<.001$ \\
$\mathbf{3 0}$ & $<.05$ & $<.003$ & $<.001$ \\
$\mathbf{4 5}$ & & & \\
$\mathbf{6 0}$ & $\mathrm{ns}$ & $\mathrm{ns}$ & \\
$\mathbf{9 0}$ & $\mathrm{ns}$ & $\mathrm{ns}$ & \\
\hline
\end{tabular}

To summarize, both canine and human subjects consistently showed conditioned hypoglycemic responses to the saline injection. Prior to conditioning, saline responses lead to significantly elevated blood sugar level at 15 and 30 minutes for dogs (no human data were reported for these pre-conditioning trials).

Acquisition curves of the conditioned hypoglycemia Because the blood sugar response to saline 


\section{Chapter 4}

injections was assessed after different numbers of previous conditioning trials within subjects, an acquisition curve of the hypoglycemic response could be construed for humans and dogs (figures 4.3 and 4.4). Given the individual differences in the size of the largest $C R$ and the number of conditioning trials that were nn, the following method was adopted ${ }^{7}$. For each individual, the most intense hypoglycemic CR of all test trials (CRmax) was determined. Also the maximum number of conditioning trials for the given indiviudal was computed as Trialmax. Then, for each test trial, two values were computed: $x$, the number of conditioning trials run before the trial in question expressed as a percentage of Trialmax, and $y$, the strength of the CR expressed as a percentage of CRmax. After that, the values $(x, y)$ for all reported $C R s$ in the experiment were entered in a scatter plot $(x, y)$. Finally, a provisional leaming curve was constructed by drawing a linear regression line through the scatter plot $^{8}$. As can be seen from the positive slope of the learning curve, CR-strength tended to increase as more conditioning trials were run. The correlation between $x$ and $y$ were significantly different from zero: 0.43 for canine, and 0.35 for human subjects.

Habituation of the hyperglycemic response to repeated saline injections in dogs before conditioning As shown in figure 4.5, saline injections before conditioning evoked a slight hyperglycemic response in dogs. To investigate whether after repeated injections a waning of the hyperglycemic response did appear, the $(x, y)$ plot was made of glycemic responses at 15 minutes after injection, in the same way as was done above (see figures 4.3 and 4.4). As above, the glycemic responses were expressed as a percentage of CRmax for the given dog (i.e. his largest hypoglycemic response occurring after subsequent conditioning; see above). Also, a linear regression line was drawn through the plot. Given the positive slope of the regression line it appears that the hyperglycemic response tended to decline as more saline injections had been received by the dog. The correlation between number of previous saline trials and the strength of the hyperglycemia was -0.32 . Evidently, the hyperglycemic response did not disappear completely after many saline injections. As Mityushov observed, the hyperglycemia to saline may have been caused by stress related to the injection procedure. Modern research has confirmed that stress may increase blood sugar level e.g. by catecholamine and glucagon release as well as an inhibition of insulin secretion (Brodsky \& Devlin, 1994; Dworkin, 1993).

\footnotetext{
${ }^{7}$ Only blood sugar data at 15 minutes after saline injections were used.

${ }^{8}$ Because of the limited number of datapoints, no higher order curve fitting was attempted.
} 


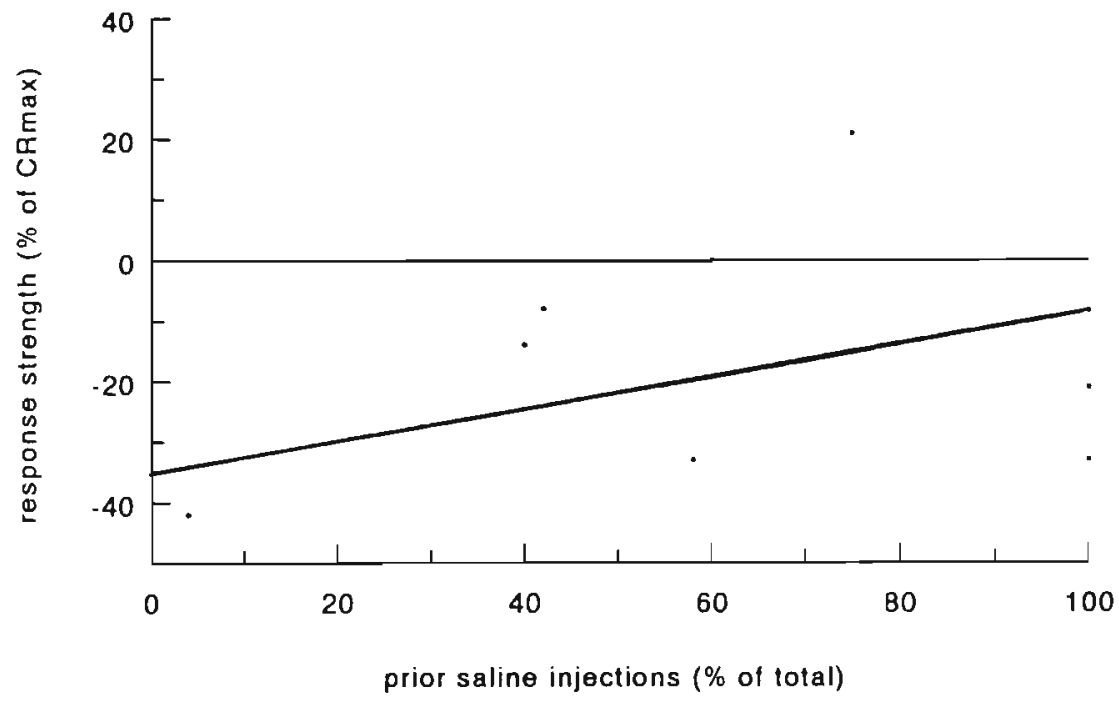

Figure 4.5. Habituation of the hyperglycemic response to repeated saline injections in 2 dogs, based on 7 responses. For each subject, response strength was expressed as percentage of the maximum $\mathrm{CR}$ shown throughout the experiment.

\section{DISCUSSION}

To our knowledge, Mityushov's study is the first successful demonstration of blood sugar conditioning in humans using a glucose UCS. The results are in line with other studies in which a glucose UCS was used (Deutsch, 1974; Matysiak \& Green, 1984). Furthermore, the data from this study are in line with a homeostatic explanation: a CS which has come to predict a blood sugar rise provokes a compensatory, hypoglycemic CR (see Dworkin, 1993).

Before discussing the results further, three problems of Mityushov's study should be mentioned. First, although Mityushov claims that he found a conditioned hypoglycemia in all subjects, he has only presented data for a small subset of his subjects. Unfortunately, overall means or statistical tests are lacking. A second problem is the lack of control group receiving e.g. repeated saline UCS injections. Of course, it can be said that this problem is mitigated because the blood sugar response to saline was measured also before conditioning began. In other words, the subjects served as their own controls. However, although Mityushov claims 


\section{Chapter 4}

that all subjects responded alike during these "baseline trials", his reporting only part of the data would not meet today's methodological standards. A third problem of Mityushov's procedure concerns the unnaturally high blood sugar levels $(250 \mathrm{mg} / \mathrm{dl}$ for humans; $420 \mathrm{mg} / \mathrm{dl}$ for dogs) provoked by the injected glucose UCS. One might wonder if the present results should be extrapolated to normal circumstances in which blood sugar levels do not exceed 190 $\mathrm{mg} / \mathrm{dl}$ (Guyton, 1991). However, observations in the literature suggest no discontinuities in blood sugar regulation under extreme hyperglycemia. Firstly, blood sugar levels of approximately $300 \mathrm{mg} / \mathrm{dl}$ after intravenous glucose injections in humans have been found to retum to baseline in slightly over one hour ${ }^{9}$, and this has been found to be related with increased insulin secretion and a suppression of glucagon release ${ }^{10}$ (Blouin et al., 1993). During sustained hyperglycemia in humans $(300 \mathrm{mg} / \mathrm{dl})$, insulin secretion rate remains at a high rate for hours (Polonsky et al., 1988). The rate of insulin secretion in dogs' and rats' pancreas is linearly correlated to at least blood sugar levels up to $300 \mathrm{mg} / \mathrm{dl}$ in dogs (Girardier et al., 1976), and $500 \mathrm{mg} / \mathrm{dl}$ in rats (Gerich, Charles \& Grodsky, 1976). Furthermore, blood sugar storage in the liver (through glycogen synthesis) increases linearly with blood sugar level, up to levels of $450 \mathrm{mg} / \mathrm{dl}$ in rats (Niijima, 1989). One might derive from these observations that the results found by Mityushov could be extrapolated to less extreme physiological conditions as well".

When looking at the data presented by Mityushov, it appears that his conditioning procedure has been effective in producing significant hypoglycemic conditioned responses after saline injection. As mentioned earlier, these results fit well with a homeostatic explanation. The CS (saline injection) that had come to signal a blood sugar increase (UCS), evoked CRs aimed at lowering blood sugar. That is, the CRs could be understood as adding to the nullification of the expected homeostatic disturbance. Which physiological pathways might then be involved? Mityushov suggests that insulin secretion was basic to the observed conditioned hypoglycemia (hence the title of his paper). This is a plausible explanation given the finding of a blood sugar fall in mice after an injection with blood drawn from the hypoglycemic dogs: some blood factor

${ }^{9}$ Mityushov reported the same blood sugar response in his subjects after the glucose UCS injection.

10 Like insulin, glucagon secretion is under the influence of autonomic nervous activity (Brodsky \& Devlin, 1994; Niijima, 1989).

11 This is of course in contrast with the conditioning studies using large insulin injections as the UCS (see the introduction of this article). 
may have been responsible, and insulin is one of the very few blood sugar lowering factors. Additionally, there is by now substantial evidence that the endocrine pancreas is capable of secreting insulin in response to naturally occurring food signals, like the smell, taste and sight of food (the cephalic responses, Bruce et al., 1987; Powley \& Berthoud, 1985) and learned signals predicting meals (Strubbe, 1992; Woods et al., 1977). These responses have been found to be mediated by parasympathetic nervous activity (Strubbe, 1992; Strubbe \& Steffens, 1993). What Mityushov probably did, is to bring insulin secretion under control of the injection ritual. A second mechanism underlying the hypoglycemia observed by Mityushov, might be a nervously regulated inhibition of glucagon secretion. Thirdly, the possibility exists that the saline injection (CS) induced a conditioned inhibition of the glucose production in the liver. This might have occurred through activity in the hepatic branch of the parasympathetic vagus nerve (Niijima, 1989). Whether this pathway is indeed susceptible to conditioning remains to be established.

Two alternative explanations of the observed hypoglycemia should be mentioned. First, the saline injection might have diluted the blood, and consequently blood sugar level would appear to have fallen (this problem appears in research in rats, see, for example, Deutsch, 1974). A second, alternative explanation might be that the hypoglycemia resulted from a temporary depletion of blood sugar, caused by a transient increase in utilization due to injection-related stress. However, both explanations are unlikely, because, as can be seen in figure 4.2 , saline control injections given to dogs before conditioning, in all cases led to slight hyperglycemias. Besides its physiological implications, the paper by Mityushov bears upon procedural issues in glycemic conditioning. Mityushov's finding of rapid blood sugar and insulin conditioning in humans (after 5 trials) is highly remarkable because of the lacking natural belongingness of an injection-CS to blood sugar level ${ }^{12}$. Moreover, no odors were added to the CS-compound, although odors and blood sugar level fluctuations can be said to have natural "belongingness" (consider food ingestion), and Woods et al. (1969) discovered that glycemic conditioning was considerably speeded up in rats if odors formed part of the CS. Another remarkable feature of the Mityushov report is that, whilst today's conditioning literature would not recommend preexposure to the CS, as this is generally understood to retard conditioning (e.g. MacRae, Scoles \& Siegel, 1987; Miller et al., 1995), Mityushov was able to find a rapid acquisition of the CR

12 Of course, also the appearance of the experimenter and the conditioning room seem to bave served as additional CSs, as Mityushov's hypoglycemic CRs failed to appear as soon as the saline injection took place in a different room or with a different experimenter (see the appendix of this thesis, tables A.2 and A.3). 


\section{Chapter 4}

after giving repeated saline injections before conditioning ${ }^{13}$.

The broader relevance of Mityushov's data is that the magnitude of the conditioned hypoglycemias (5-20\%) overlaps with that of the hypoglycemias that have been recently found to precede meal initiation in rats (Campfield \& Smith, 1990), and humans (Campfield et al., 1996). Although Campfield and Smith have always emphasized that the origin of the pre-meal hypoglycemias are endogenous, Mityushov's results suggest that the responses might be conditioned to external cues, a suggestion also made by Woods (1990). In fact, it has been recently proposed that conditioned insulin secretion and hypoglycemia play a role in normal and binge eating (Jansen, 1994a; Woods, 1991)

It is obvious that Mityushov's study on blood sugar conditioning deserves replication with control groups, direct insulin measurement, and statistic tests added. Furthermore this study may serve as an illustration of the potential importance of investigations carried out decades ago by Pavlovian physiologists in Eastern Europe, who found conditioned response interesting in their own right. When this large data base, embodied in several thousands of non-English publications (Dworkin, 1993; Razran, 1961) would be explored, provoking and interesting results might be found, many of which await replication and extension.

In the Eastera-European Pavovian tradition, pre-exposure to the CS used in subsequent conditioning was a standsrd procedure. Pre-exposure was meant to habiruate orienting reflex to the $C S$, because orienting was confidert antagonistic to conditioning, habituation was thought to be a prereģisite to successful conditioning. 
Chapter 5

\section{CUE REACTIVITY TO \\ FOOD- AND BODY-RELATED STIMULI \\ IN RESTRAINED AND UNRESTRAINED EATERS ${ }^{1}$}

\section{INTRODUCTION}

The symptomatology of bulimia nervosa includes recurrent binge eating and an overconcern regarding body shape and weight (APA, 1994). During binge eating, large amounts of food are ingested while the subject experiences an irresistable food craving and loss of control over food intake. Recently, classical conditioning models, derived from addiction research (e.g. Siegel, 1983; Drummond, Tiffany, Glautier \& Remington, 1995) have been applied to binge eating (Jansen, 1990, 1994; Wardle, 1990). In these models it is assumed that through a learning process, cues predictive of binge eating (e.g. the sight and smell of snacks, or states of depression and boredom) eventually come to elicit anticipatory physiological responses. One of these is a secretion of insulin which counters the post-binge blood sugar rise. According to the conditioned binge eating theory, anticipatory responses, when intense, are experienced as a craving for food.

In aldietion research, an interesting pperationalization of conditioning theories has emerged: cue reactivity measurement, a method in which drug-related cues are presented and psychophysiological measures are monitored. The intensity of these reactions (i.e. cue reactivity) is supposed to be a reflection of the responses underlying craving. For example, alcoholics and smokers display heart acceleration and increased skin conductance responses when presented with substance-related cues, an effect that was not seen in normals Niaura, Rohsenow, Binkoff, Monti, Pedraza, Abrams, 1988; Childress, Ehrman, Rohsenow, Robbins \& O'Brien, 1992). An important reason for assessing cue reactivity is that it probably forms a risk factor in relapse of treated addicts (Childress et al., 1992; Drumond et al., 1995). Moreover, measurement of cue reactivity could be used to study the effect of exposure therapy in addicts (Jansen, 1994). In contrast to addiction research, binge eaters" psychophysiological cue reactivity to food stimuli has been investigated rarely, perhaps with the exception of salivation (e.g. Klajner, Herman, Polivy o Chabra, 1981; LeGoff \& Spigelman, 1987).

In the present study, an experimental, non-clineal approach (see Abramson \& Seligman, 1977) was adopted by studying cue reachivity in restrained eaters. The restraint concept

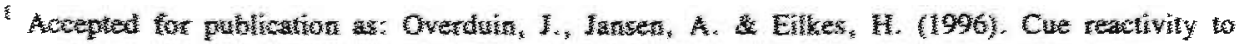

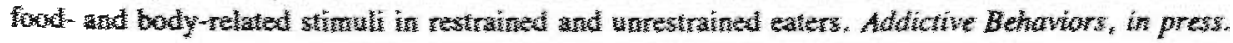


was originally developed to describe the occasionally disinhibitive eating behavior of dieters, and it has often been used as an analogue of binge eating tendency (Wardle \& Beinart, 1981; Herman \& Polivy, 1988). Two similarities between restrained eaters and bulimics can be mentioned.

The first is that the eating style of restrained subjects, selected by Herman \& Polivy's Restraint Scale (Herman \& Polivy, 1980) is characterized by unsuccessful attempts to restrict food intake and an increased frequency of binge eating (Wardle, 1980). Within the laboratory, restrained eaters have been found to increase their intake of high-caloric foods after a range of manipulations like, for example, ingestion of a "preload" (for example, a high-caloric pudding; Herman \& Polivy, 1980; Lowe, 1993), merely smelling a preload (Jansen \& Van den Hout, 1991; Rogers \& Hill, 1989), or induction of certain mood states (Greeno \& Wing, 1994; Lowe, 1993; Ruderman, 1986). In line with these findings, the present study was aimed at testing the hypothesis of increased cue reactivity to attractive food cues in high vs. low restrained eaters.

A second similarity between bulimics and restrained eaters is the existence of an overconcern (albeit to a different extent) with body shape and weight. As a rule, bulimics and restrained eaters consider themselves too fat and they are afraid of getting fatter (Herman \& Polivy, 1988). In this study, we tested the hypothesis that restrained eaters show more intense emotional responses than unrestrained eaters when viewing slides depicting their own body.

In the present study, food and body cue reactivity was assessed by monitoring autonomic physiological responses. These were considered relevant for three reasons: their role in anticipation and processing of ingested food (Woods, 1991), their being sensitive measures in cue reactivity research of various addictions (Niaura et al., 1988; Childress et al., 1992), and their suitability as indices of affective responses to stimuli (Lang, Greenwald, Bradley, \& Hamm, 1993).

In short, two hypotheses were tested in the present study. First, all subjects will show more intense appetitive responses to slides depicting binge food than to slides with a neutral content; restrained eaters show this effect to a larger extent than do unrestrained eaters. Second, restrained eaters will show more intense emotional arousal to pictures of their own body, than unrestrained eaters.

\section{METHOD}

\section{Subjects}

Female subjects were called for by means of an advertisement in a local newspaper. The 75 individuals that responded were invited to fill in the Restraint Scale (RS; Herman et 
al., 1978). Of these, 26 individuals having either a RS-score below 10 or above $15^{2}$ were asked to participate in the experiment. Characteristics of the two subject groups thus formed are shown in table 5.1. After the experiment, it appeared that two subjects did have an extreme Body Mass Index (BMI resp. 16.5 and 26.5). Data from these subjects were excluded from statistical analysis.

Table 5.1. Characteristics of Restrained and Unrestrained subjects in this study

\begin{tabular}{|c|c|c|c|c|c|c|}
\hline & \multicolumn{2}{|c|}{$\begin{array}{l}\text { Unrestrained } \\
\qquad(N=13)\end{array}$} & \multicolumn{2}{|c|}{$\begin{array}{l}\text { Restrained } \\
\quad(N=11)\end{array}$} & \multicolumn{2}{|c|}{ t-test } \\
\hline & mean & $\mathrm{sd}$ & mean & sd & $\mathrm{t}$ & p-value \\
\hline Body Mass Index & 19.9 & 1.4 & 21.8 & 1.7 & 2.90 & .01 \\
\hline Age & 20.3 & 1.7 & 21.5 & 3.1 & 1.14 & $\mathrm{~ns}$ \\
\hline Restraint Scale & 5.2 & 2.2 & 20.2 & 2.8 & 14.6 & $<.001$ \\
\hline
\end{tabular}

\section{Psychophysiological parameters as related to hypotheses}

Hypothesis one: larger reactivity to food cues than for neutral cues, a fortiori so in restrained eaters.

Skin conductance response, amplitude Enlarged SCRs have been found in alcohol and nicotine addicts who where presented substance related cues (Niaura et al., 1988; Childress et al., 1992). We expected all subjects to show larger SCR-amplitudes in response to bingefood than to neutral slides; furthermore this effect was expected to be stronger in restrained than in unrestrained eaters.

Heartrate (HR) The heart is innervated by both sympathetic and parasympathetic nerves, and reactions to stimuli are often multiphasic and not easily interpreted (Venables, 1991). So far, cue reactivity research has scarcely addressed this issue: heartrate data are usually averaged over longer time intervals. Overall HR-increase after cue presentation has been found in smoking-addicts, but responses in alcoholics have been contradictory (Childress et al., 1992). Kostarczyk (1986) has found HR acceleration in dogs, presented with a conditioned stimulus which had been previously paired with a food delivery. The accelaration occurred during the 10 post-stimulus seconds. Given the above findings, we expected a larger HR acceleration in restrained than in unrestrained eaters watching slides with binge

\footnotetext{
${ }^{2}$ That is, 0.5 standarddeviations below and above the mean RS-score of a group of 482 Dutch female students respectively; Overduin \& Jansen, 1996; Jansen \& Overduin, unpublished data).
} 


\section{Chapter 5}

foods.

Startle reflex, amplitude Lang and co-workers (Lang et al., 1990, Bradley et al., 1990) have demonstrated that changes in the intensity of startle eyeblink reflexes, elicited during picture viewing, covary with the subjective emotional valence of the picture. Viewing positive slides has an inhibitory, whereas viewing negative pictures has a facilitating effect on the reflex amplitude (Lang et al., 1990). Lang et al. have suggested that startle modulation is bases on the activation of either appetitive or defensive behavior systems. It should be noted that predictions about the reaction of restrained eaters to food slides are problematic. On one side, one would expect increases in startle amplitude because of negative emotional valence of the slide content (due to likely associations with guilt and loss of control), on the other one should expect less intense startle responses because food slides induce an appetitive state. Therefore, in the case of restrained subjects, no strict directional prediction was formulated for startle reflex modulation to food slides. Unrestrained subjects were expected to display a small decrease in startle amplitude, related to the craving elicited by the slides.

Startle reflex, onset latency The startle onset latency appears to covary inversely with startle amplitude (Bradley, Cuthbert \& Lang, 1990). That is, increases in amplitude go hand in hand with decreases in onset latency and vice versa. Thus, during presentations of binge food pictures we expected unrestrained eaters to show increased onset latencies, while for restrained eaters no specific predictions were made.

Facial EMG In emotion research, EMG activity of facial muscles has been demonstrated to be specifically indicative of emotional valence. Two important examples are the Zygomatic 'cheek-muscle', which contributes to the act of smiling and becomes increasingly active when a person views pictures with a positive emotional valence, and the Corrugator 'frownmuscle' which is active during experience of negative emotion (Lang et al., 1993; Dimberg, 1987). For the same reasons explained above for the startle response, no explicit predictions could be made for facial muscle EMG responses to food slides in restrained eaters.

Hypotheses two: Increased emotional arousal in restrained eaters to slides depicting their own body.

Skin conductance reaction, recovery time SCR-recovery time reflects the speed with which the SC-level retums to baseline after having reached its peak. Repeatedly, it has been suggested that increased SCR recovery time is indicative of a defensive response (Dimberg, 1987; Edelberg, 1972). Accordingly, in the present study we expected increased SCR recovery time in restrained, but not in unrestrained eaters presented with slides depicting 
their own body.

Heart rate HR acceleration at between 3-5 seconds after stimulus onset is due to sympathetic activity and has been interpreted as reflecting a defensive reaction (Venables, 1991). We therefore expected restrained eaters to show larger heart rate accelerations than unrestrained eaters during presentation of pictures of their own body.

Startle reflex. amplitude Because of the negative emotional valence of body slides, we expected these slides to induce a larger increase in startle amplitude in restrained than in unrestrained subjects (Lang et al., 1990).

Startle reflex, onset latency Presentation of body slides was expected to lead to larger decreases in startle onset (relative to responses to neutral slides) in restrained than in unrestrained subjects.

Facial EMG Because the negative emotional valence of body pictures, restrained eaters were expected to show larger decreases in zygomatic, and larger increases in corrugator EMG-activity than were unrestrained eaters.

\section{Equipment, and processing of the psychophysiological signals}

All psychophysiological signals were fed to Beckman couplers and subsequently stored on multichannel magnetic tape for further off-line analysis. One of the channels contained information about times and types of stimulus presentation. Only the Skin Conductance signal was recorded (and subsequently scored) on paper using of a Beckman R711 polygraph with multi-channel writer.

SCR was recorded using two $\mathrm{Ag}-\mathrm{AgCl}$ electrodes (diameter $8 \mathrm{~mm}$ ) attached to the middle fingers of the non-dominant hand. Two parameters were drawn from the SCR-signal: amplitude (SCR-amp) and recovery time (SCR-rec). SCR-amp was defined as the (baseline corrected) maximum SC-level within 6 seconds after slide onset (baseline was defined as the SC level before a significant post-stimulus increase began). SCR-rec was defined as the time interval between the moment the SC level reached its peak and the moment the signal had returned to a level of $(0.5 *$ SCR-amp) above baseline.

Startle reflexes were elicited by a pulse of $90 \mathrm{~dB}$ white noise with instantaneous rise time through headphones (Anthony, 1985). Part of the startle reflex is the eyeblink, and the relevant EMG signal was detected using two $\mathrm{Ag}-\mathrm{AgCl}$ electrodes applied on the orbicularis oculi, just beneath the lower left eyelid. The EMG-signal was sampled from tape with a frequency of $1000 \mathrm{~Hz}$ and filtered by a digital, 255-point, $60 \mathrm{~Hz}$ highpass FIR-filter. Then the signal was rectified and integrated using a time constant of 20 milliseconds. Under the condition that a typical reflex shape could be distinguished, two parameters were extracted from the signal: amplitude and onset latency. Startle amplitude was defined as the highest EMG-level within $150 \mathrm{msec}$ after presentation of the noise. The amplitude was corrected 


\section{Chapter 5}

for pre-stimulus baseline level, defined as the mean EMG level in the 50 pre-stimulus milliseconds. Startle onset latency was defined as the time interval between presentation of the startle stimulus and the moment when EMG level began to rise sharply and significantly above baseline level. Reactions with onset latency earlier than 18 or later than $70 \mathrm{msec}$ or with no apparent reflex shape were excluded from further data processing.

Facial muscle EMG activity from the zygomatic and corrugator muscles was recorded using two $\mathrm{Ag}-\mathrm{AgCl}$ electrodes with a diameter of $2 \mathrm{~mm}$, placed according to suggestions by Fridlund and Cacioppo (1986). Signals from both channels were treated likewise. After $1000 \mathrm{~Hz}$ sampling from the tape the signals were respectively lead through a digital, 255point, $90 \mathrm{~Hz}$ high-pass FIR-filter, rectified, and integrated by a contour-following integrator with a time constant of $500 \mathrm{msec}$ (Fridlund, 1979). Baseline EMG was defined as the mean intensity of the signal in the 5 seconds preceding slide presentation (Dimberg, 1987). The reaction was defined as the mean EMG level during the 5 seconds after stimulus onset, corrected for baseline.

Heartrate was recorded by means of two Blue Sensor disposable ECG electrodes (type R$00-S$ ) attached on a diagonal axis over the heart region and a reference electrode on the shoulder. From magnetic tape the signal was sampled with a frequency of $1000 \mathrm{~Hz}$ and led through a digital, 255-point, $35 \mathrm{~Hz}$ lowpass FIR-filter. After that, times of R-peaks in the signal were detected by a special devised computerprogram and checked for artifacts and electronic interference. In a next step second-to-second heartrate for the 12 post-stimulus seconds were computed by a specially devised software program employing the weighing procedure proposed by both Velden and Graham (1988). Baseline was defined as the mean heartrate in the 5 seconds preceeding stimulus onset. Eventually two values were extracted: 'overall acceleration', i.e. the mean second-to-second heartrate for the 12 seconds after stimulus onset, and defensive acceleration, defined as the highest of the second-to-second heartrate for seconds 3, 4 and 5 after stimulus onset (Laberg, Wilson, Eldredge \& Nordby, 1991).

\section{Materials}

All auditory stimuli (music and startle noises) were presented through Beyer DT 109 headphones. The music used for the depressive mood induction (see procedure) was a piece by the classical composer Prokoviev, entitled 'Russia under the Mongolian Yoke' played at half speed (Clark, 1983). Slide projection was carried out with a Kodak carousel projector, which was automatically driven by a computer program on a PDP Minc Microcomputer.

There were three slide categories, 'neutral', 'food' and 'body'. The neutral pictures were the same for all subjects and showed pieces of office equipment. Body and food slides were selected individually for each subject. Care was taken that the food stimuli would reflect 
each subject's preference. To achieve this, a rating scale was administered 3 weeks before the experiment on which the subjects had to rate 5 snack categories on preference and 'binge provoking characteristics'. From each of the three categories ranked highest by the subject, 2 slides were included in the slide series. The 5 categories were potato chips, chocolate bars, candy bars, licorice, and pies. The set of body slides consisted of pictures that had been taken of the subject three weeks prior to the experiment (see procedure). The subject was visible wearing a standard set of underwear that would leave belly, arms, legs, neck and face uncovered. In a set of six slides the body was shown from different standardized angles: two pictures were taken frontally, two en profil, and two from an angle of 45 degrees.

\section{Questionnaires of the experimental session}

During the experimental session subjective data were mostly collected by means of Visual Analog Scales (VASs) on which subjects score between 0 ("not at all") and 100 ("extremely").

Two times a VAS containing the following three items was administered (see procedure): "At this moment I feel tense", "At this moment I feel peckish" and "At this moment I feel depressed". Further, each member of the presented slide-series (see procedure) was evaluated by two questions: "how agreeable do you find this slide?" and "How much food craving does this slide evoke?" The final questionnaire contained questions about subjects' food intake prior to the experimental session and the questions: "How satisfied are you with your body appearance?", "Are you currently dieting in order to lose weight?"

\section{Procedure}

Pre-experimental session Three weeks before the experimental session, six pictures were taken of the subjects for use in the experiment (see materials). After the photo-session a questionnaire was handed out in which subjects could rate a number of food items on their craving arousing properties. These ratings were eventually used for selection of the food slides (see materials).

Experimental session Each subject was asked to eat a standard meal at 3 hours prior to the experimental session and to refrain from eating thereafter. The subject was welcomed and informed about the course of the experiment. The first VAS was handed out. Each subject was seated in a comfortable chair, electrodes were attached, and a headphone was put on. She was asked to remain in her chair quietly so as to not disturb the psychophysiological measurements. In order to ensure that full-fledged cue reactivity would be registred a mood induction procedure was employed, as craving in restrained eaters has been repeatedly reported to be mood dependent (Greeno \& Wing, 1994; Cools, Schotte \& McNally, 1992; 


\section{Chapter 5}

Ruderman, 1985). First, it was announced that music would be played. The subject was asked to concentrate on sad thoughts as long as the music would play. The experimenter left the room, lights were dimmed and the music began playing for 5 minutes. After the music the experimenter reentered the room and administered the second VAS. After that the slide series was announced. It was emphasized that the subject should watch the slides carefully, because later some questions would be asked about them. Then the experimenter left the room, which was darkened now and the slide presentation began. Two slides were used as a introduction, and after that the six neutral, six food and six body slides were presented in random order. Each slide was shown for a period of twelve seconds. Inter slide intervals differed and were either ten, fifteen, twenty or twenty-five seconds. During half of the slides a startle eliciting noise was presented. To preclude subjects' expectations on when the startle noise would sound the noise was presented at variable times (either 1, 3 or 7 seconds) after slide onset for the different slides. Following the first slide presentation all slides were shown again, and each slide was rated. After the removal of the electrodes the final questionnaire was administered. The subject was weighted and paid for her participation. The six personal body slides were handed over to the subject.

\section{RESULTS}

\section{Statistical method}

Data were processed using SPSS-PC statistical software. In most instances, repeated measurement ANCOVAs were conducted, with Body Mass Index as a covariate, as the two subject groups differed on this variable (see table 5.1). Due to malfunctioning of the psychophysiological equipment and interference, part of the data of some subjects were excluded from further analysis.

\section{Manipulation check of the mood induction}

The mood induction proved to be successful in both groups. A 2 (before/after mood induction) $\times 2$ (restraint) ANCOVA showed a main effect for mood induction, indicating a significant increase in negative $\operatorname{mood}$ scores $(F(1,22)=36.2, p<.001)$. Furthermore the mood induction led to decreased craving ratings $(F(1,22)=7.85 ; p=.01)$. No main effect of mood induction was found on subjective tension level. None of the subjective ratings showed a main effect of group or a group $x$ mood induction interaction effect. 


\section{Subjective and psychophysiological responses to the slides}

Results of the statistical tests are presented according to the main hypotheses of the experiment.

Hypothesis 1: cue reactivity is larger for food than for neutral slides in all subjects, but to a larger extent in restrained subjects.

Data on SCR amplitude, Heartrate, startle reflex parameters, and craving ratings for the food slides were subjected to separate 2 (restraint) $\times 2$ (slide type) repeated measurement ANCOVAs with Body Mass Index as the covariate. The predicted restraint $x$ slidetype interaction was found only for corrugator EMG activity $(F(1,17)=5.95 ; p<.03)$. The pattern of cell-means (not shown here) revealed that the average EMG response to both slide types was a decrease in corrugator (frown) activity, which suggests a positive emotional response in both subject groups.

Main effects of slidetype were found for food craving $(F(1,22)=133.9 ; p<.001)$ and startle onset latency $(F(1,22)=8.68 ; p<.008)$. These effects reflected a more intense appetitive response to foodslides than to neutral slides. No main effects of restraint were found.

In sum, the results showed that, although food slides triggered more intense psychophysiological reactions and craving than did neutral slides, restrained and unrestrained eaters differed only in corrugator EMG activity, albeit to a modest extent.

Hypothesis 2: body slides elicit more negative emotional arousal in restrained than in unrestrained subjects.

Data on SCR recovery time, HR during the 2-5 seconds post-stimulus time window, Corrugator and Zygomatic EMG changes, Startle reflex onset and amplitude, and emotional ratings of the slides were subjected to separate 2 (restraint) $\times 2$ (slide type) ANCOVAs with repeated measures and Body Mass Index as a covariate.

No main or interaction effect on emotional rating was found, reflecting that the restrained subjects did not rate their body-pictures more negatively than did unrestrained subjects. None of the psychophysiological parameters showed the predicted restraint $\mathrm{x}$ slide type interaction effect. Also no main effect was found for restraint. A main effect for slide type was found only for Heartrate acceleration $(F(1,14)=8.98 ; p<.01)$ and for SCR recovery time $(F(1,18)=5.09 ; p<.04)$. These effects reflected more intense negative emotional arousal during presentation of the body slides. 


\section{Chapter 5}

Again, it can be said that none of the predicted differences between restrained and unrestrained eaters was found.

\section{Body satisfaction and current dieting}

Restrained and unrestrained eaters did not differ in body satisfaction $(\mathrm{t}(20)=.47$; ns). This was somewhat surprising because the restrained eating style is often assumed to originate from body dissatisfaction. Of the unrestrained subjects, nobody was currently dieting, whereas of the restraints $38 \%$ was.

\section{DISCUSSION}

In the present investigation it was found that restrained and unrestrained eaters did not differ in psychophysiological reactivity to slides of either their preferred binge food or their own body. Moreover, no group differences were found on subjective ratings of food slides (craving), or body slides (negative emotional valence). Nevertheless, a main effect of slide type on several psychophysiological parameters was found. On the average, responses to the body slides were the most intense, followed by the food and neutral slides respectively. The present failure to find a group difference in psychophysiological reactivity to food and body cues may result either from peculiarities of the present procedure, or from more fundamental problems with a pure conditioning explanation of cue reactivity.

The former explanation is based on the assumption that the classical conditioning interpretation of cue reactivity is valid, but that the present procedure should be adjusted to demonstrate differential cue reactivity. This possibility should be taken seriously in the light of the small existing empirical data base on psychophysiological food cue reactivity in humans. Five adjustments of the experimental procedure might be thought of. First, the present study involved non-clinical subjects. It might be that significantly increased cue reactivity is confined to clinical binge eaters, because of their occasional huge food intake. As to body cue reactivity, a analogous argument could be made, based on the different body esteem of e.g. bulimia nervosa patients and non-clinical subjects. Second, the data indicate that the (individually selected) food slides did have had some arousing effect on the subjects. However, it might be that because of their mere visual nature, food slides lack in truly disinhibitive potency. Accordingly, investigation of the effects of real food items providing additional olfactory/gustatory stimulation should deserve attention. Third, the subjective ratings show that the food slides elicited increased arousal and increased craving in both restrained and unrestrained subjects. This might be the consequence of a basic characteristic in which food cue reactivity study differ from studies into addictions. Not only had both 
subject groups some learning experience with the presented food cues, also their habitual intake may have been comparably large (indeed, the slides were individually selected on craving arousing value). This might have lead to equal food cue reactivity. Future research might better collect quantitative data on subjects' usual craving and intake of food associated with the cues presented during the lab procedure rather than relying on a personality questionnaire (i.e. the Restraint Scale) only. Fourth, the psychophysiological parameters monitored in the present study may not have been sufficiently "food-specific". Heightened cue-reactivity to disinhibiting food cues might be found only in physiological domains more tightly linked to food ingestion, e.g. blood sugar or insulin responses (Jansen, 1994). And fifth, there is a possibility that notable differences between restrained and unrestrained eaters in food cue reactivity begin to appear at more than 12 seconds after cue perception, i.e. beyond the time window in which we monitored our subjects' responses.

The second explanation for our failure to find differential cue reactivity in restrained and unrestrained eaters may be more fundamental in nature: the conditioning account of cue reactivity may not be adequate. As a matter of fact, Tiffany (1995) has proposed that the expression of cue-reactivity (in addicts) should be understood as a reflection of behavioral and cognitive demands of a situation rather than as a mere residual of learning history. Specifically, cue-elicited responses might be especially intense in cases where an encounter with the substance itself is clearly forthcoming. This issue may not have been sufficiently addressed in the present procedure: although the subjects were to watch the slides attentively, no explicit attempts were included to arouse subjects' expectations of encounters with the depicted food items, e.g. of ingesting or abstaining from ingestion in the presence of the food). It might be that differential food cue reactivity may be demonstrated only after procedures in which the psychosocial concomitants described by Tiffany are explicitely manipulated.

Research on cue reactivity in the field of binge eating has only recently started. At present, assessment of the contributions of situational vs. conditioning influences, as well as identification of the appropriate response parameters appear to be the most central issues in food cue reactivity research. 



\section{FOOD CUE REACTIVITY IN FASTING AND NON-FASTING SUBJECTS ${ }^{1}$}

\section{INTRODUCTION}

A recently proposed theory of binge eating holds that cues that reliably precede intake of binge food can acquire the ability to elicit a range of anticipatory physiological responses. These classically conditioned responses facilitate bodily processing of food and help in maintaining homeostasis (Jansen, 1990; 1994a; Woods, 1991). Additionally, the theory states that conditioned physiological responses to binge cues are subjectively experienced as craving and as such can trigger food intake. Research on the nature of conditioned anticipatory responses could be helpful in both controlling them, and in preventing relapse in treated patients (Childress, Ehrman, Rohsenow, Robbins \& O'Brien, 1992; Glautier, 1994; Jansen, Broekmate \& Heijmans, 1992). Until now, however, the exact nature of the conditioned physiological responses to binge cues remains unclear (Overduin, Jansen \& Eilkes, 1996). A major aim of the present study was the determination of psychophysiological and subjective responses to food cues (sight and smell) in normal, non-clinical subjects. Moreover, as dietary restriction is supposedly linked to the development of binge eating (e.g. Polivy \& Herman, 1985; Tuschl, 1990) and short-term deprivation was expected to increase reactivity to food cues, also responses of 24-hour fasting subjects were studied. Studies in the addiction field show that increases in salivation, skin conductance and subjectively reported craving to cue presentation are the most consistent findings, whereas heart rate acceleration was found less often (Childress et al., 1992; Glautier, 1994). In the present study it was hypothesized that presentation of cues signalling desired food will elicit more salivation and larger increases in heartrate and frequency of skin conductance fluctuations than presentation of control (i.e. soap) stimuli. Furthermore, food cue reactivity was hypothesized to be larger in fasting than in non-fasting subjects.

1 Accepted for publication as: Overduin, J. \& Jansen, A. (1996). Food cue reactiviy in fasting and non-fasting subjects. European Eating Disorders Review, in press. Heiny Eilkes is gratefully acknowledged for her assistance in carrying out the present experiment. 


\section{METHOD}

\section{Design}

Each of the fasting and non-fasting subjects was exposed to both food- and soap stimuli. The order of stimulus presentation was counterbalanced within each condition. Psychophysiological cue reactivity was determined by measuring salivation, spontaneous skin conductance fluctuations and heartrate, before (baseline) and during stimulus presentations.

\section{Subjects}

Twenty-one healthy normal weight students without a history of eating disorders ( 9 females and 12 males; mean age 21.6 years) participated in the experiment. The subjects were randomly allocated to the fasting condition $(n=11)$ and to the non-fasting condition $(\mathrm{n}=10)$. Mean BMI in the fasting group $(21.9$, sd 2.8$)$ did not differ from the normal group $(21.4$, sd $2.0 ; \mathrm{t}(19)=.55 ; \mathrm{ns})$. It was told to the subjects that the experiment involved an investigation of "attentional and sensory processes".

\section{Questionnaires and psychophysiological parameters}

Questionnaires At the beginning of the experiment hunger and craving ratings were collected by means of on Visual Analogue Scales (VASs) ranging from 0 ("not at all") to 100 ("very much"). After the end of presentation of each stimulus a VAS was administered on food craving and hedonic evaluation of the stimulus1.

All electronic signals, including the markers for stimulus presentation, were registered by Beckman couplers and a Beckman R711 polygraph with a multi-channel writer.

Skin Conductance was recorded by means of two $\mathrm{Ag}-\mathrm{AgCl}$ electrodes (diameter $8 \mathrm{~mm}$ ) attached to the middle fingers of the non-dominant hand. The number of non-specific fluctuations in skin conductance level (further to be called "SC-fluctuations") were counted to acquire an index of the frequency of sympathetic nervous "bursts" both before and during presentation of the stimuli. They were defined as SC-level increases exceeding 0.05 micromhos within 2 seconds (Boucsein, 1992) and taking place at least 6 seconds after breathing artefacts or stimulus onset. The number of fluctuations was counted for each minute.

Heartrate was recorded by means of two Blue Sensor disposable ECG electrodes (type R-00-S) attached on a diagonal axis over the heart region and a reference electrode on the shoulder. For each minute the mean heartrate was established.

Saliva was collected by means of three dental rolls, according to the method of LeGoff, 
Leichner \& Spigelman (1988). The subject inserted a first roll underneath the tongue, and two rolls laterally, into each side of the lower jaw between teeth and gums. Salivation collection time was exactly three minutes. The mean salivation per minute was computed for statistical analysis.

Breathing amplitude Because the number of SC-fluctuations is influenced by irregular breathing (sighing, coughing and so on; Boucsein, 1992), breathing amplitude was monitored using a Beckman respiratory belt connected to a Beckman Voltage/Pressure Coupler. The mean breathing amplitude during the 6 second interval preceding each $\mathrm{SC}$-fluctuation was established and they were averaged for the minute under consideration. In this way the mean breathing amplitude preceding SC-fluctuation was computed for each of the 14 one-minute intervals of the experiment. Then, for each minute, control periods of 6 seconds not followed by SC-fluctuations were inspected and mean breathing amplitudes were computed in the same way as above. Finally, the difference between pre SC-fluctuation breathing amplitudes and the control breathing amplitudes was assessed by t-tests.

\section{Stimuli}

The food stimuli consisted of two candy bars (Twix) stripped from their paper and one for each subject freshly prepared healthy brown roll, with salat, cheese, ham and tomato.

The control stimuli consisted of four small pieces of round, lightly flowerishly perfumed bathing soap. Two of the pieces were white-, and two were green-coloured. All stimuli were presented on a plate with a blinded removable cover over it. After lifting the cover, the items, resting on colored paper napkins, became visible.

\section{Procedure}

The fasting group was asked to refrain from eating for a period of 24 hours prior to the experimental session. To ensure that fasting really would take place, the subjects were instructed to collect their morning urine and bring it with them, so as to "control for fasting". In reality no further analyses were carried out on these urine-samples. The non-fasting group was instructed to have a normal meal at 3 hours prior to the experimental session and to refrain from eating until after the experiment. After entering the lab, each subject first completed the hunger and craving VAS. Then she was informed about the general procedure and how to insert the dental rolls. She was seated in a comfortable chair and instructed not to move exaggerately during the experiment, so as not to disturb the measurements. The explanation of the procedure was repeated while the electrodes were attached. During the first two minutes of the experiment, 


\section{Chapter 6}

psychophysiological baseline measurements were carried out with the subject sitting still in the chair and the experimenter sitting in front of her. Thereafter the subject was asked to insert the dental rolls. Within 6 seconds after the insertion of the dental rolls, the first stimulus was presented in front of the subject and the cover of the dish was lifted by the experimenter. The instruction was given to attend (look and smell) to the food intensely so that the subject would be able to correctly complete the VASs later on. The dish was arranged so that the subject's nose was close to it, so that no bending down was required to smell and watch. Three minutes after the onset of stimulus presentation, the subject was told to remove the dental rolls from his mouth, to put them in the container and to close it. Stimulus presentation and physiological measurement continued for another two minutes after which the hedonic evaluation and craving VAS was administred with the stimulus still within sight. Then there was a pause of eight minutes in which they were distracted by the projection of six neutral slides with office equipment. The subject was told to watch them attentively and to memorize them as good as possible so as to be able to answer questions about them later on. After this pause the second stimulus was presented. The procedure was identical to the first stimulus presentation. Finally, the subject was weighed, measured, debriefed, paid for participation and offered the sandwich presented during the experiment.

\section{RESULTS}

\section{Data processing}

Because fasting has been found to lead to decreased tonic sympathetic nervous activity (Landsberg \& Young, 1978; Webber \& MacDonald, 1993), phasic (cue-elicited) physiological activity was corrected for tonic (baseline) levels: for heartrate and skin conductance the mean levels of seven (i.e. two pre-stimulus and five post-stimulus) minutes were computed. Then, tonic level was defined as the average of the two pre-stimulus minute levels. Phasic reactivity score was defined as the difference between the average of the 5 post-stimulus minutes levels and tonic level. The tonic and phasic reactivity scores for heartrate and skin conductance, salivation rate and the VAS-ratings of the stimuli were subjected to a 2 (condition; fasting vs non-fasting) $\times 2$ (stimulus-type; food vs soap) MANOVA with stimulus-type as a within-subjects factor.

Unfortunately, due to malfunctioning of the equipment, for some people skin conductance and heartrate data were lost. 
Table 6.1. Subjective state prior to presentation of stimuli (scoring range: $0-100$ )

\begin{tabular}{|c|c|c|c|c|c|c|}
\hline & \multicolumn{2}{|c|}{$\begin{array}{c}\text { non-fasting subjects } \\
\qquad(\mathrm{N}=10)\end{array}$} & \multicolumn{2}{|c|}{$\begin{array}{l}\text { fasting subjects } \\
\qquad(\mathrm{N}=11)\end{array}$} & \multicolumn{2}{|c|}{ t-test } \\
\hline & mean & sd & mean & sd & $t(23)$ & pule \\
\hline hunger-VAS & 50.4 & 20.0 & 84.2 & 10.5 & -4.9 & $<\infty \mathrm{ll}$ \\
\hline food craving-VAS & 47.6 & 19.6 & 83.6 & 16.9 & -4.6 & $<.001$ \\
\hline
\end{tabular}

\section{Manipulation checks}

t-Tests demonstrated that at the beginning of the experiment, the fasting subjects experienced significantly more hunger $(\mathrm{t}(19)=4.92 ; \mathrm{p}<.001)$ and craving for food $(\mathrm{t}(19)$ $=6.46 ; \mathrm{p}<.001$ ) than non-deprived subjects (see table 6.1). Also, the food stimuli were not only considered significantly tastier in appearance $[F(1,18)=148.2 ; p<.001]$ and more agreeable in smell $[\mathrm{F}(1,18)=6.58 ; \mathrm{p}<.02]$, but also leading to larger food craving $[F(1,18)=39.9 ; \mathrm{p}<.001]$ than the soap stimuli (table 6.2).

Thus, both manipulations succeeded: fasting subjects experienced more hunger and craving than non-fasting subjects and the food stimuli turned out to be considerably appetitive.

Table 6.2. Subjective ratings during food and soap stimulus presentation (scoring range: 0-100)

\begin{tabular}{|c|c|c|c|c|c|c|c|c|}
\hline & \multicolumn{3}{|c|}{ Non-fasting subjects } & \multicolumn{3}{|c|}{ Fasting subjects } & \multicolumn{2}{|c|}{ t-test } \\
\hline & mean & sd & $\mathrm{N}$ & mean & sd & $\mathrm{N}$ & i & p \\
\hline \multicolumn{9}{|l|}{ Hedonic rating of stimulus } \\
\hline food & 68.6 & 19.3 & 10 & 88.6 & 15.0 & 11 & 2.66 & $<.02$ \\
\hline soap & 15.0 & 19.8 & 10 & 19.3 & 30.2 & 11 & .38 & ns \\
\hline \multicolumn{9}{|l|}{ Craving for food } \\
\hline food & 62.7 & 17.8 & 10 & 91.4 & 9.8 & 10 & 4.47 & $<.001$ \\
\hline soap & 24.4 & 18.2 & 10 & 56.3 & 30.2 & 11 & 2.89 & $<.01$ \\
\hline
\end{tabular}

\section{Responses to stimulus presentation}

The minute-to-minute courses of heartrate and skin conductance fluctuations are shown in figures 6.1 and 6.2. Data on the tonic (baseline) levels showed that fasting subjects displayed a smaller number of SC-fluctuations $[F(1,17)=4.75 ; p<.05]$ and a trend towards lower heartrate $[F(1,18)=4.34 ; p<.06]$ than non-fasting subjects.

$t$-Tests on the difference between the mean breathing amplitude before SC-fluctuations and that of control periods (see method section) failed to reach significance. Thus it can be concluded that the SC-fluctuations did not result from breathing irregularities during stim- 
Table 6.3. Psychophysiological responses to food and soap stimulus presentation

\begin{tabular}{|c|c|c|c|c|c|c|c|c|}
\hline & \multicolumn{3}{|c|}{ Non-fasting subjects } & \multicolumn{3}{|c|}{ Fasting subjects } & \multicolumn{2}{|c|}{ t-test } \\
\hline & mean & sd & $\mathbf{N}$ & mean & sd & $\mathbf{N}$ & t & $p$ \\
\hline \multicolumn{9}{|l|}{ Salivation (mg/min) } \\
\hline food & 575 & 359 & 10 & 609 & 280 & 11 & .24 & ns \\
\hline soap & 651 & 397 & 10 & 536 & 272 & 10 & .76 & ns \\
\hline \multicolumn{9}{|l|}{$\Delta$ Heartrate (BPM) } \\
\hline food & 3.6 & 4.1 & 10 & 6.8 & 4.9 & 10 & 1.56 & $\mathbf{n s}$ \\
\hline soap & 4.0 & 3.3 & 10 & 4.4 & 6.4 & 9 & .17 & ns \\
\hline \multicolumn{9}{|c|}{$\Delta S C$-fluctuations/minute } \\
\hline food & 1.8 & 1.5 & 9 & 0.5 & 1.1 & 11 & 2.31 & $<.04$ \\
\hline soap & 0.6 & 2.2 & 10 & 0.0 & 1.2 & 10 & .81 & ns \\
\hline
\end{tabular}

ulus presentation.

On phasic reactivity scores (see table 6.3), a main effect of condition was found only for frequency of SC-fluctuations $[F(1,17)=4.44 ; p<.05]$, based on fasting subjects showing smaller increases in SC fluctuation frequency in response to both stimuli. However, the hypothesized condition $\mathrm{x}$ stimulus-type interaction did not reach significance. This implies that fasting subjects did not show especially strong reactivity to food cues. Finally, neither main effects of stimulus-type, nor stimulus-type $\mathrm{x}$ condition interaction effects were found for the other physiological parameters.

\section{DISCUSSION}

In the present study, food cue reactivity was examined in fasting and non-fasting subjects. Firstly, it was found that fasting subjects showed a lower baseline frequency of skin conductance fluctuations and a trend towards lower baseline heartrate. Secondly, although subjects gave higher appetitive ratings to food than to soap stimuli, this was not reflected in stronger psychophysiological reactivity (i.e. salivation, SC-fluctuations, heartrate). Finally, fasting subjects reported more craving for the food stimuli than did non-fasting subjects, but they did not show the hypothesized enlarged food cue reactivity. In fact, their SCfluctuations increase to food cue appeared to be even smaller than non-fasting subjects. The smaller baseline frequency of spontaneous skin conductance fluctuations and trend towards lower baseline heartrate in fasting subjects fits with earlier documented findings on lowered sympathetic nervous activity in fasting subjects (Schultz-Gambard, 1988; Landsberg 
\& Young, 1978; Webber \& MacDonald, 1993). Probably, this should be seen as a general bodily adaptation to deprivation that is aimed at sparing energy (Landsberg \& Young, 1978), rather than as a reflection of readiness for food intake.

The finding of no larger food cue reactivity to food than to soap, as well as the approximately equal cue reactivity in fasting and non-fasting subjects is surprising. Even if one takes into consideration that the exact nature of anticipatory responses to food stimuli (for example, the critical parameters and their directionality) is as yet largely unknown, one is struck by the finding that fasting subjects did not show stronger salivation. Salivation, a response obviously related to food ingestion, has been claimed to be an objective index of hunger (e.g. Le Goff et al., 1988). Indeed, besides being hungry, our fasting subjects craved and highly appreciated the food presented to them. But why, then, didn't they salivate more?

A first possibility might lie in the procedure of the present study. It might be that not all the subjects have complied with the fasting instruction, thereby obscuring differences in cue reactivity with the non-fasting subjects. Indeed, the present study did not involve objective checks on deprivation level (for example, increased levels of circulating ketone bodies; Hoffer, 1988). Nevertheless, relevant information pertaining to this issue can be drawn from the present psychophysiological data. If true cue reactivity after fasting were larger in fasting than in non-fasting subjects, and not all subject in the fasting condition were complying with their instructions, enlarged variability in responses would be expected among subjects in the fasting condition. However, the standard deviations in table 6.3 do not consistently confirm this prediction. Thus, although we have no waterproof evidence that all subjects in the fasting condition did stick to their regimen, no convincing indication could be found of heightened cue reactivity in a subset of our fasting subjects.

Secondly, it is possible that increased cue reactivity occurs only after fasting durations exceeding 24 hours. Data from the literature on appetitive parameters related to cue reactivity may be relevant in this respect. For humans, the plateau of perceived hunger intensity appears to occur after two days of fasting (Le Magnen, 1985). However, the additional impact of presenting food cues has, to our knowledge, not been investigated. For rats, the literature is inconclusive as to whether the size of the first meal after fasting is strongly correlated with fasting duration if fasting continues for more than 24 hours (Le Magnen, 1985). All in all, the possibility that increased cue reactivity could indeed be found after fasting periods longer than 24 hours deserves future attention.

A third explanation for the lack of differences in cue reactivity may be related to our subjects' learning history, which, unlike hunger level, was not manipulated in the present study. Jansen $(1990,1994)$ has argued that food cue reactivity will be particularly strong in cases of intermittent dieting and overeating, a common pattem among binge eaters. 


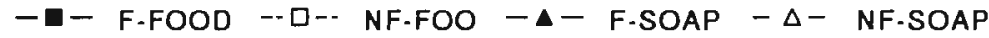

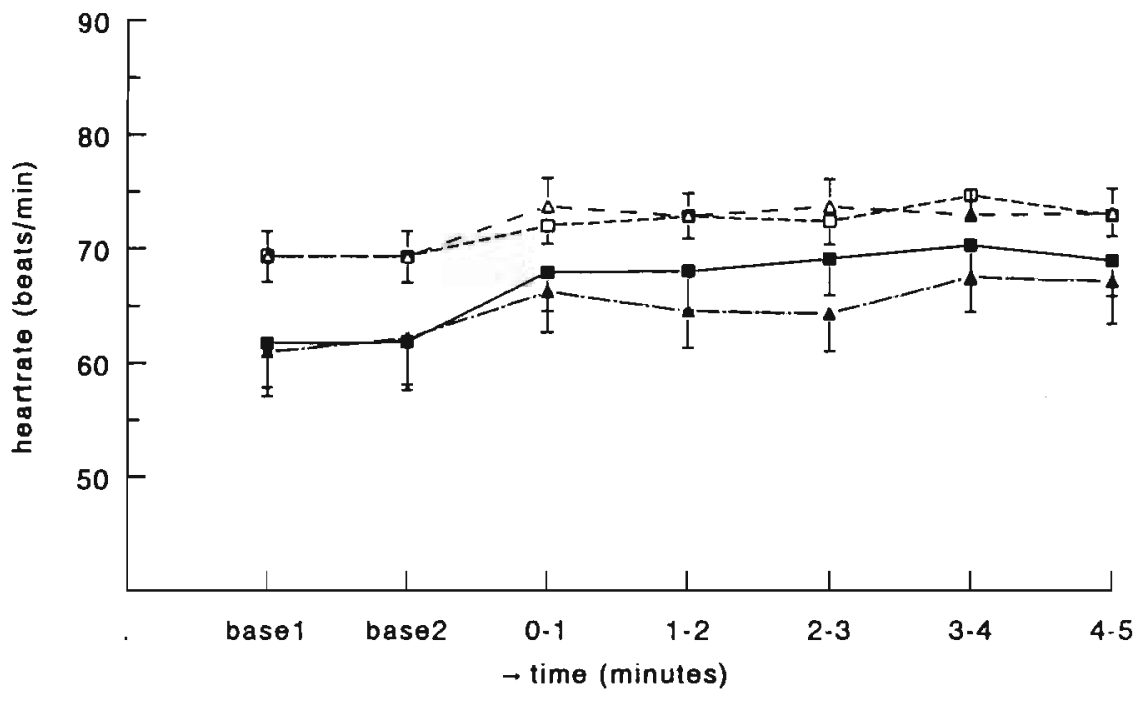

Figure 6.1. Minute-to-minute heartrates (mean \pm s.e.m.) before and during stimulus presentations.

-ם- F-FOOD --D-- NF-FOO - A- F-SOAP - $\triangle-$ NF-SOAP

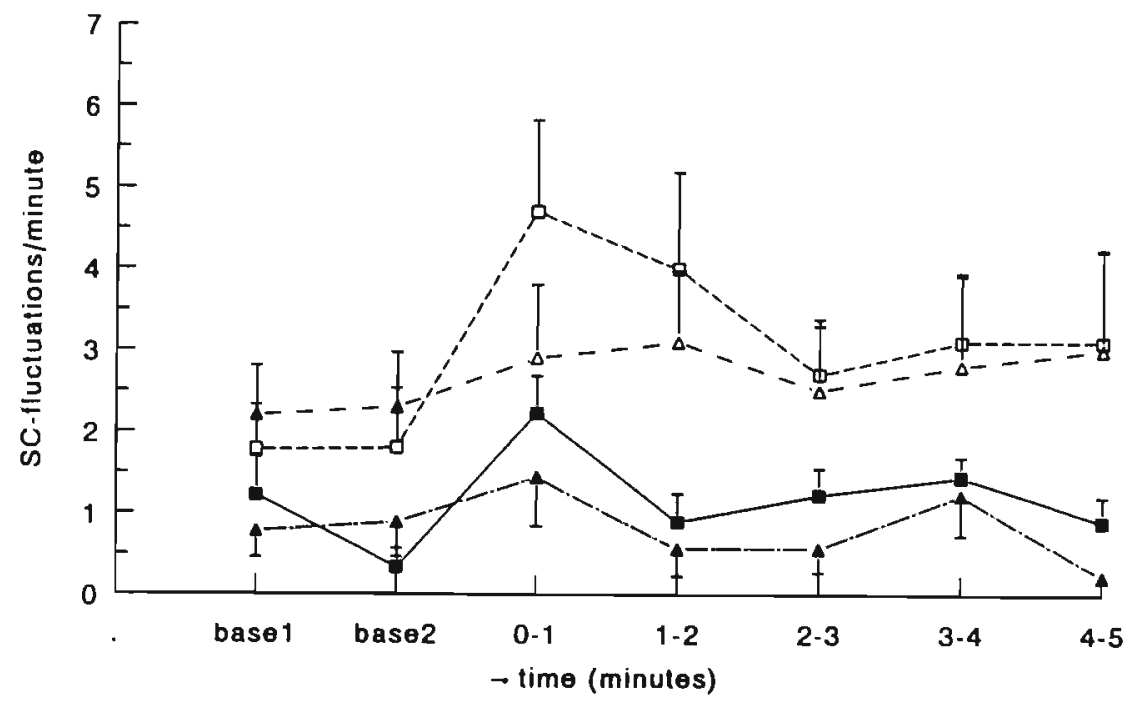

Figure 6.2. Minute-to-minute frequency of skin conductance fuctuations (mean \pm s.e.m.) before and during stimulus presentations. 
Indeed, animal studies show that a food intake scheme of one or two meals a day elicit significantly larger anticipatory responses to food cues than an intake scheme of six meals a day (Woods et al., 1977; Strubbe, 1992).

An experimental manipulation of learning history then, would require a conditioning procedure in which a set of food cues (CS) reliably predicts subsequent large intake (US). Also, it might critically be investigated whether deprivation level in its own right facilitates such learning, i.e., whether deprivation acts as an 'occasion setter' of the conditioning process (Davidson, 1993; Catania, 1991). Jansen, Boon, Nauta \& van den Hout (1992) have shown that such a Pavlovian conditioning procedure elicits increased salivation to the food cues in undeprived subjects. Altematively, it might be worthwhile to investigate whether cue reactivity intensity decreases if subjects are exposed repeatedly to food cues while being prevented actual intake. Prolonged exposure to binge cues with response prevention has been shown to be successful in decreasing craving and binge frequency in binge eaters (Jansen, Heymans \& Broekmate, 1992), but psychophysiological parameters were not monitored in these studies.

In sum, visual and olfactory cues related to food and non-food items have again been demonstrated to trigger various changes in subjective and psychophysiological parameters, but the logic underlying the intensity of these changes needs further exploration. We argue that future cue reactivity research may benefit from focusing more on the role of conditioning. It might be hypothesized that the intensity of cue reactivity covaries with the magnitude of the learned, hence expected, subsequent food ingestion. 



\section{Chapter 7 \\ STROOP INTERFERENCE AND FOOD INTAKE ${ }^{1}$}

\section{INTRODUCTION}

Preoccupation with food and eating, as well as the tendency to be overly concerned with one's body weight and shape, are key symptoms of the eating disorders bulimia nervosa and anorexia nervosa (APA, 1987; Walsh, 1992). Concerns about body shape and weight are considered pivotal to the maintenance of chaotic eating behaviour such as dieting, fasting and splurging (Fairburn, Cooper, Cooper, McKenna \& Anastasiades, 1991; Garfinkel, 1992).

In order to assess information processing in eating-disordered subjects some authors recently used the Stroop colour-naming task. In the Stroop task words printed in various colours are presented to the subject who then is required to name the colour as quickly as possible, while ignoring the meaning of the words. In studies on eating disorders reaction times to neutral words and words about food or body shape (so called target words) are compared. A relatively longer reaction time for target words indicates that the subject is distracted by the meaning of the target words, and this is supposed to be indicative of distorted information processing.

Until now several studies have shown Stroop interference for target words in eating disordered subjects. Significant interference for a combination of eating and shaperelated words were found in normal weight bulimic subjects (Fairburn et al., 1991; Cooper, Anastasiades \& Fairburn, 1992; Cooper \& Fairburn, 1993). Other studies report interference effects with separately presented eating-related words in anorexic and/or bulimic patients (Channon, Hemsley \& de Silva, 1988; Ben-Tovim, Walker, Fok \& Yap, 1989; Ben-Tovim \& Walker, 1991). Most of the studies undertaken document an interference effect for eating-related words in eating disordered subjects.

How can these data be explained? Ben-Tovim et al. (1989) hypothesized that the attentional bias for eating-related words reflects anxiety evoked by the meaning of these words and can thus be regarded as a measure of psychopathology. However, in the light of the finding that fasting subjects are hyperattentive to non-aversive appetitive stimuli (Channon \& Hayward, 1990; Lavy \& van den Hout, 1993), Stroop interference does not necessarily seem to indicate anxiety. In line with this, Channon and Hayward (1990) explained the observed attentional bias for food cues of fasting and eating-disordered subjects in terms of restriction. They state that the Stroop task provides "an objective

1 Published as: Overduin, J., Jansen, A. \& Louwerse, E. (1995). Stroop interference and food intake. International Journal of Eating Disorders 18, 277-285. 
index of current food restriction" (p. 451). But this conclusion might be a bit premature. For example, Green and Rogers (1993) found slower colournaming of foodwords in restrained eaters, irrespectively of whether the subjects were currently dieting or not. Therefore, although food restriction may result in hyperattention to food cues, hyperattention to food cues presumably isn't exclusively related to food restriction.

Another explanation of hyperattention was offered by Lavy and van den Hout (1993). According to them hyperattention might be related to an increased urge to act that may be reflected either in the avoidance of threatening, or in the approach of appetitive cues. From this one could derive the hypothesis that hyperattention to food words may not only be triggered by deprivation but, more generally, by stimuli that are desired strongly.

In the present study we wanted to investigate whether it is possible to instantaneously induce an attentional bias in subjects by providing them with an appetizer, i.e. a small amount of food that tastes "moreishly". Secondly, conceming the urge-to-act hypothesis, we wanted to assess the relation between Stroop interference and subsequent ad libitum food intake.

Results found on Stroop interference for separately presented body shape-related words appear to be more equivocal. Channon et al. (1988) failed to find an enlarged interference effect for shape-related words in anorexic subjects. Ben-Tovim et al. (1989) reported a trend for Stroop interference on shape-related words in anorexic and bulimic subjects whereas Ben-Tovim and Walker (1991) demonstrated a significant interference effect in bulimic as well as anorexic subjects. Mahamedi and Heatherton (1993) found that eating a preload lead to an increase in attentional bias for body shape words, especially in restrained eaters. However, after a comparison of the post-preload attentional bias in restrained and unrestrained eaters one cannot conclude that bias was reliably stronger in restrained eaters. Results from a within subjects study by Ogden and Greville (1993) in which attentional biases before and after a preload were assessed point in a similar direction.

The subjects in our experiment were non-clinical restrained and unrestrained eaters. If the emotional concerns explanation of Stroop interference for body shape words were true, one would expect larger reaction times for restrained eaters whether they would have eaten an appetizer or not. The urge-to-act explanation of attentional bias, on the other hand, is based on approach and withdrawal behavior and does not lead to strong expectations about Stroop reaction times for body shape words. 


\section{METHOD}

\section{Subjects}

Initially, around 100 female university students were approached to participate in a 'study on life-style' by filling out a 'life-style questionnaire'. Four key eating behaviour items were included in the list (e.g. "I repeatedly find it necessary to engage in dieting" and "My eating behaviour is normal when I'm in the presence of others but uncontrolled when I'm alone"). After that, 51 of the most extreme scorers (high and low) on these items were invited to participate in the experiment. At the end of the experiment the subjects completed the Restraint Scale (Herman, Polivy, Pliner, Threlkeld \& Munic, 1978) in order to prevent priming concerns about weight, shape and food intake. Every subject was termed high or low restrained on the basis of the median RS-score (being a score of 10 ) of the sample. Characteristics of the two groups are shown in Table 7.1.

Table 7.1. Characteristics of Restrained and Unrestrained subjects in this study

\begin{tabular}{lccccccc}
\hline & \multicolumn{2}{c}{ Unrestrained } & \multicolumn{2}{c}{ Restrained } \\
& \multicolumn{2}{c}{$(\mathrm{N}=25)$} & \multicolumn{2}{c}{$(\mathrm{N}=26)$} & \multicolumn{2}{c}{ t-test } \\
mean & sd & mean & sd & t & p-value \\
Body Mass Index & 20.7 & 1.3 & 22.3 & 2.7 & 2.64 & .01 \\
Age & 23.3 & 2.4 & 22.6 & 1.9 & 1.16 & ns \\
Restraint Scale & 6.8 & 2.5 & 15.0 & 3.5 & 9.66 & $<.001$ \\
\hline
\end{tabular}

\section{Materials}

Food The appetizer consisted of 60 grams of bavaroise pudding. Three flavours of ice cream (vanilla, strawberry, and chocolate) were used for the ad lib taste-test. The ice was arranged on a plate in such a way that eating would leave only a marginal visual trace.

Stroop program The Stroop task was run on a personal computer. In the middle of the screen words were shown one by one in 8 -mm block letters against a black background. The subject's voice was detected by a microphone connected with a sounddetector which registered response latencies in milliseconds. As soon as a sound was detected, the word disappeared from the screen. The time interval between presentation of consecutive words was 2 seconds. In case of wrong answers or disturbing sounds a 'missing value' was recorded.

Stroop words For every subject a total of 120 words was presented. The words were picked from four arrays (Table 7.2) in such a way that at the end of the task each of the 40 words had been shown three times. The colour of every word (either red, blue, green or yellow) was randomly chosen by the computer program. 


\section{Chapter 7}

There were two categories of 'neutral' words. The office equipment words had been added to establish a baseline with which reaction times for the target words were compared. The second category involved life-style words, so as to strengthen the impression that the experiment indeed was a study on life-style and perception. Response latencies for these words were not used in the data analysis.

Table 7.2. Words used in the modified Stroop task

\begin{tabular}{llll}
\hline \multicolumn{2}{c}{ TARGET WORDS } & \multicolumn{2}{c}{ NEUTRAL WORDS } \\
eating & shape & office & life-style \\
bun & balance & desk & ambition \\
cake & belly & envelope & appointment \\
candy & bikinis & felt-pen & bonus \\
chips & cheeks & file & career \\
chocolate & fat & paper & dedication \\
ice cream & hips & pen & manager \\
liquorice & legs & pencil & promotion \\
pie & slim & ruler & study \\
pudding & thighs & scissors & success \\
tart & thin & tape & working \\
\hline
\end{tabular}

\section{Procedure}

Subjects were told that we were studying influences of life-style on perceptual processes such as taste, smell, and visual perception. They were not informed that the test would involve highly palatable food.

The subjects were asked to have a standard meal of two sandwiches 3 hours before the experiment, and to refrain from eating in the period thereafter. They were randomly assigned to either the appetizer or the no-appetizer condition and were tested individually. After entering the laboratory, each subject received information about how to carry out the Stroop task. First, a short Stroop task with neutral words (the number one to ten) was run for practice. Then, in the appetizer condition, the pudding was presented and the subject was asked to eat it while concentrating on its colour, fragrance, and taste. Subjects in the control, no-appetizer-condition continued immediately with the Stroop task.

After the task three pre-weighed plates of ice cream were presented together with an icecream taste-evaluation questionnaire. The experimenter announced that he would leave the room for 15 minutes, during which the subject should complete the questionnaire. It was pointed out that tasting the ice cream would be helpful in completing the question- 
naire and that the subject was allowed to eat as much as she wanted or thought necessary. After the taste test the experimenter returned to administer the Restraint Scale. The ice cream that was left was taken to another room and re-weighed by the second experimenter. Finally, the subject was debriefed and paid for participation.

\section{RESULTS}

The Stroop interference scores for every target (i.e. eating and shape) category was defined as the median of response latencies for that category minus the median of response latencies for the neutral category. Reaction times above 1500 and below 250 milliseconds were excluded from the analysis. Standard deviations are shown between parentheses.

\section{Manipulation check}

Subjects scored the palatability of the bavaroise pudding (i.e. the appetizer, on a Visual Analogue Scale, ranging from 0 "not good at all" to 100 "extremely tasty". The overall mean score was 57.9 (s.d. 24.8) meaning that, although the pudding was not rated as extremely tasteful, at least it was considered reasonably palatable. No difference between restrained and unrestrained subjects in this respect was found $[\mathrm{t}(22)=1.08, \mathrm{NS})]$.

Table 7.3. Interference times for food words (milliseconds)

\begin{tabular}{|c|c|c|c|c|c|c|}
\hline & \multicolumn{3}{|c|}{ Unrestrained Eaters } & \multicolumn{3}{|c|}{ Restrained Eaters } \\
\hline & mean & sd & $\mathrm{N}$ & mean & sd & $\mathbf{N}$ \\
\hline No-appetizer & -6.0 & 30.3 & 13 & 17.1 & 29.4 & 14 \\
\hline Appetizer & 25.8 & 34.6 & 12 & 20.9 & 30.1 & 12 \\
\hline
\end{tabular}

\section{Food Words}

Table 7.3 shows the Stroop interference times for food words. A $2 \times 2$ ANOVA revealed a main effect of condition $(F(1,49)=4.18 ; p<.05)$, but no group effect. The condition by group interaction showed a weak trend $F(1,49)=2.57 ; p=.12$ ). It appeared that in the no-appetizer condition restrained subjects showed a significantly greater Stroop interference than unrestrained subjects $[\mathrm{t}(25)=2.01, \mathrm{p}<0.03]$.

Table 7.4. Ad lib intake of ice-cream (grams) 


\begin{tabular}{lcccccc}
\hline & \multicolumn{3}{c}{ Unrestrained Eaters } & \multicolumn{3}{c}{ Restrained Eaters } \\
& mean & sd & $\mathrm{N}$ & mean & sd & $\mathrm{N}$ \\
No-appetizer & 95.1 & 54 & 13 & 136.1 & 83 & 14 \\
Appetizer & 121.5 & 90 & 12 & 74.3 & 61 & 12 \\
\hline
\end{tabular}

It can be further seen that among unrestrained subjects, the ones in the appetizer condition showed significantly greater Stroop interference than those in the no-appetizer condition $(t(23)=2.44 ; p=.01$, one-tailed). In restrained subjects, no effect of condition was found $t(24)=.33$; NS).

\section{Food intake}

The food intake data are presented in Table 7.4. A $2 \times 2$ ANOVA showed no main effects for group or condition. However, a significant group by condition interaction was found $(F(1,49)=4.58 ; p<.04)$. Oddly enough, in the appetizer condition only the restrained subjects appeared to have lowered (i.e. regulated) their food intake relative to subjects in the no- appetizer condition $(\mathrm{t}(24)=2.19 ; \mathrm{p}=.04)$; among unrestrained subjects no effect of condition was found $(\mathrm{t}(23)=.88$; NS). For all subjects taken together, there was no strong $\mathrm{P}-\mathrm{M}$ correlation $(\mathrm{r}=0.27 ; \mathrm{n}=51$; NS) between the Stroop interference score for food words and ad libitum food intake. Interestingly, when correlation coefficients were calculated for the restrained and unrestrained subjects separately, the unrestrained group showed a firm correlation of 0.58 ( $\mathrm{n}=25$; $\mathrm{p}<$ $0.01)$, whereas in the restrained group no correlation was found $(r=-0.06 ; n=26$; NS).

Table 7.5. Interference times for shape-related words (milliseconds)

\begin{tabular}{lllllll}
\hline & \multicolumn{3}{c}{ Unrestrained Eaters } & \multicolumn{3}{c}{ Restrained Eaters } \\
& mean & sd & N & mean & sd & N \\
No-appetizer & 12.3 & 32.8 & 13 & 25.9 & 45.6 & 14 \\
Appetizer & 23.9 & 39.3 & 12 & 28.8 & 28.2 & 12 \\
\hline
\end{tabular}

\section{Body shape-related words}

In Table 7.5, Stroop interference times for shape-related words are presented. A $2 \times 2$ ANOVA did not show any main effects for condition and group or a condition $x$ group interaction. Clearly, restrained and unrestrained eaters did not differ in their level of 
Stroop interference for shape-related words. Moreover, for both unrestrained and restraint subjects eating the appetizer did not influence Stroop response latencies for shape-related words.

\section{DISCUSSION}

The main finding of the present study is that eating a small amount of palatable food triggers an attentional bias to eating-related stimuli in normal unrestrained subjects. This attentional bias strongly correlated with the amount of subsequent ad libitum food intake. In contrast to the unrestrained subjects, the restrained eaters were shown to be continually hyperattentive to eating-related words. Their subsequent food intake however appeared to be independent of hyperattention. Finally, restrained eaters did not show a larger attentional bias for body shape-related stimuli than non-restrained eaters.

What do these findings mean? In the first place, our data contradict the idea that an attentional bias to food related stimuli reflects a specific psychopathology of clinical eating disorders (Ben-Tovim \& Walker, 1991; Cooper \& Fairburn, 1993). Our non-clinical restrained subjects showed a permanent attentional bias to eating-related stimuli.

Secondly, the attentional bias in unrestrained subjects after ingestion of the appetizer suggests that Stroop interference per se does not reflect fear, emotional concerns, or deprivation. The firm correlation between Stroop latencies and food intake in unrestrained subjects suggests a selective attention to cues related to desires, cravings or urges. Interestingly, no correlation was found between Stroop food interference and food intake in restrained eaters. This could be because restrained subjects, by definition, normally tend to suppress their food intake. One could expect that Stroop interference scores and food intake will correlate strongly when restrained eaters show disinhibition. Unfortunately, in the present study restrained eaters in the appetizer condition seem to have regulated their intake because they ate less than those in the non appetizer condition. This lack of disinhibition might be explained by the size of our pudding, which was considerably smaller than the preloads in traditional counterregulation experiments (Jansen, Merckelbach, Oosterlaan, Tuiten \& van den Hout, 1988; Herman \& Mack, 1975; Hibscher \& Herman, 1977; Herman \& Polivy, 1980). It would thus be highly interesting to examine the relationship between Stroop interference and subsequent food intake in restrained eaters who indeed show disinhibited eating after a large preload.

A third interesting finding is that compared with unrestrained subjects, restrained subjects didn't show an especially large attentional bias toward body shape words, neither in the no-appetizer nor in the appetizer condition. These data seem to be in contrast with reports 


\section{Chapter 7}

of Stroop interference in bulimics (Fairburn et al., 1991; Cooper et al., 1992; Cooper \& Fairbum, 1993). In these studies however, it was not tested to which extend either shapeor the food-related target stimuli contributed to the effect. Also our findings are not entirely in accordance with findings of Ogden and Greville (1993) and Mahamedi and Heatherton (1993). The reason for this could have to do with the difference in methods. Mahamedi and Heatherton (like Fairburn et al.) used a card Stroop in which target words from the same category are presented together. In the Ogden and Greville study a computerized Stroop task was used, but again the target words were presented in blocks. Both methods introduce the danger that the subjects will ruminate about the meaning of the words during the task, and this could lead to longer reaction times, especially in restrained subjects who have just eaten a high-caloric milkshake. In the present study, words from target and neutral categories were presented one by one in a mixed fashion, which presumably diminishes the rumination effect. Undoubtedly, restrained eaters and eating-disordered subjects are overly concerned about their body shape. If interference as found by a computer-run Stroop task indeed reflects selective hyperattention to stimuli of personal or emotional valence, one would expect that restrained subjects show a greater Stroop interference for body shape-related words than unrestrained subjects do. However, the data do not support this hypothesis. If, however, the attentional bias reflects immediate avoidance or approach tendencies, one would not strongly expect an attentional bias for shape-related words: it is rather difficult to avoid or approach shape or body. In conclusion, it could be quite possible that card- or blocked versions of the Stroop measure rather emotional rumination on body words than attentional bias.

Finally, some remarks can be made about the value of the Stroop task in the assessment of treatment outcome for eating disordered patients. In spider phobics, Lavy, van den Hout and Amtz (1993) found a marginal correlation between pretreatment attentional bias and treatment (exposure) success. It might be hypothesized that a sustained post-treatment attentional bias for eating-related words could be a predictor of relapse in bulimic subjects. If that prediction is found to be true, the Stroop task may serve as an instrument for the assessment and predict of (long-term) treatment success. Recently, it has been shown that craving extinguishes during prolonged exposure with response prevention to cues that have become associated with the desired substance (Jansen, Broekmate \& Heymans, 1992; Powell, Gray \& Bradley, 1993). It is well-known too that craving can be triggered by exposure to appetitive cues (Weingarten \& Elston, 1990; Jansen et al., 1992) and therefore could constitute a causal factor in relapse. It could very well be that the conceptualization of craving as an approach behaviour leads to a promising application of the computerized Stroop technique in the study of eating disorders. 


\section{A NEW SCALE FOR USE IN NON-CLINICAL RESEARCH INTO DISINHIBITIVE EATING ${ }^{1}$}

\section{INTRODUCTION}

The food intake pattern of all bulimic and about half of the anorexic patients includes recurrent binge eating: during binges, control of food intake is lost and quantities of food are ingested that are larger than most people would eat under similar circumstances (Walsh, 1993). Lab paradigms of disinhibitive eating behaviour have been developed and studied in non-clinical subjects in order to unravel the underlying mechanisms (For the rationale behind this non-clinical approach, see Abramson \& Seligman, 1977). Questionnaire scores often provide the criterion of subject selection. In lab research into disinhibitive eating, the most widely used questionnaire has been the Restraint Scale (RS; Herman \& Polivy, 1980). This questionnaire has been shown to identify subjects prone to a form of disinhibitive eating called counterregulation. In the basic counterregulation paradigm subjects are "preloaded" by having them ingest a high-caloric food item, after which the food intake is assessed in an ad lib ice cream taste test. Subjects with high RS scores have been found to counterregulate, i.e. they show larger food intake when preloaded than when unpreloaded, whereas low scorers regulate their food intake and eat less when they have been preloaded previously (Herman \& Polivy, 1980). The replicable nature of counterregulation allowed for more extensive research on disinhibition through various modifications of the basic paradigm (for overviews see Lowe, 1993; Greeno \& Wing, 1994).

Although of all available questionnaires in the field, the Restraint Scale has been demonstrated the most effective predictor of counterregulation (Heatherton et al., 1988; Lowe, 1993), its use in disinhibition research has stirred up at least four critical issues.

The first issue relates to criticisms about some psychometric characteristics of the RS. Although the internal consistency of the scale is satisfactory, doubts have been raised about the stability of the internal structure of the scale. Different studies have shown factors to be composed of different items or items to be loading significantly on two factors at the same time. A most prominent example of the factorial instability is the collapse of the factor structure within a obese subject sample (e.g. Ruderman, 1983; for

${ }^{1}$ To be publisbed as: Overduin, J. \& Jansen, A. (1996). A new scale for use in non-clinical research into disinhibitive eating. Personality and Individual Differences, in press. Ellen Louwerse, Susan Bōgels and Peter de Jong are gratefully acknowledged for their aid in administering the questionnaires. Harrie Vorst (Methodology Department, University of Amsterdam) is thanked for commenting on some of our data. 
a reference of these criticisms and a reply see Heatherton et al., 1988). The instability of the factor structure may very well be a consequence of the wide array of themes tapped by relatively small numbers of items (i.e. weight fluctuations, diet frequency, negative evaluation of weight gain, binge eating). The factor instability suggests that not all constituent themes within the questionnaire are clearly mapped (see McDonald \& Mulaik, 1979). One solution to this problem might be to extend the scale with items highly specific to each of the themes; in this way each theme will eventually be covered specifically by one factor (Briggs et al., 1986). Indeed, such an approach has been employed in the development of new questionnaires, notably the Dutch eating Behaviour Questionnaire (DEBQ; van Strien, Frijters, Bergers \& Defares, 1986) and the Three factor eating behaviour Questionnaire (TFEQ; Stunkard \& Messick, 1985). However, although these questionnaires appear to have more clearly discernable subscales, recommendations have been given to use only one subscale at one time, either because the total scale was not intended to measure one construct (DBEQ; van Strien et al., 1986), or because inter-subscale correlations turned out to be insufficiently high (TFEQ; see Heatherton et al., 1988).

The second issue in using the RS bears upon the well-established finding that counterregulation of high RS-scorers appears mainly after ingestion of large, and not after small preloads. Importantly however, in recent years it has become clear that also small preloads or food cues (e.g. sight, smell and taste of food) can act as triggers of craving and increased subsequent food intake (Jansen \& van den Hout, 1991; Rogers \& Hill, 1989; Weingarten \& Elston, 1990). Analogously to developments in addiction research (e.g. Siegel, 1983; Childress, 1992; Drummond, Tiffany, Glautier \& Remington, 1995), new models of binge eating have been put forward emphasizing leamed cue reactivity as a maintenance factor (Jansen, 1990; 1994; Wardle, 1990). Cue reactivity means that stimuli predictive of food intake trigger a physiological state that is experienced as craving (Jansen, 1994). As to the predictive value of the Restraint Scale, disinhibitive eating after presentation of food cues has been found only in subjects with extreme RS scores (Jansen \& van den Hout, 1991). Hence, a subclass of high restrained eaters (i.e. those scoring slightly above the median RS score) did not show changes in eating behaviour after cue presentation. This may be a direct consequence of these subjects endorsing of the "concern for dieting" items in the Restrained scale (high RS scores can result from either strong concern for dieting or high disinhibition, or both; Westenhoeffer et al., 1994). Specific measures of concern for dieting, such as TFEQ and DEBQ have been found to identify successful dieters, i.e. those who do not frequently disininhibit (Heatherton et al., 1988; Lowe, 1993). This renders it likely that many high restrained eaters in this experiment deliberately inhibited their food intake during the taste test. Thus, although high scorers on the RS might possess considerable food cue reactivity, high "concern for dieting" may lessen chances on observable changes in food 
intake after presentation of food cues other than large preload.

Finally, neither in the RS nor in the DEBQ or TFEQ reference is made to self-esteem. Recently however, it has been shown that in particular high restrained eaters whose selfesteem is low do counterregulate (Polivy, Heatherton \& Herman, 1988). It has been proposed that the larger variability among high RS scorers in food intake within the lab can be attributed to different levels of self-esteem among individuals within this group.

The aim of the present study is to alleviate the above problems with using the Restraint Scale, which admittedly remains the questionnaire most predictive of at least one form of disinhibition, i.e. counterregulation. We attempted to develop a questionnaire for selection of subjects maximally prone to lab disinhibition to a broad range of cues, and which would have the following characteristics: a clearer factor structure than the RS, attenuation of the unwanted impact of concern for dieting items on food intake, and reference to self-esteem. Moreover, the scale should be internally consistent so that total scores could be used. We compared the new developed questionnaire with the Restraint Scale on two characteristics: psychometric properties, and characteristics of extreme groups selected on basis of scores.

\section{GENERAL METHODOLOGY}

All questionnaires were administered within a non-clinical population of students participating in courses, and volunteering university employees. All statistical analyses were carried out using the SPSS-X software program.

\section{DEVELOPMENT OF A NEW DISINHIBITION SCALE}

\section{Purpose and rationale}

A rational, construct-oriented approach (see Nunnally, 1978; Wilde, 1977) was adopted to develop a new scale for selecting individuals maximally susceptible to triggered disinhibitive eating in the lab. We reasoned that lab-disinhibition may be best predicted by items referring to the occurrence of disinhibition in daily life. Furthermore we considered that disinhibition occurs in three forms that should all be mapped to get a full picture: 1) eating, at times, too much "objectively" 2) eating too much "subjectively", i.e. cases in which the individual judges that self-imposed restrictions are overruled; 3 ) objective consequences of repeated disinhibition, i.e. weight fluctuations. To these three factors thought to predict lab disinhibition we added self-esteem as a fourth "risk" 


\section{Chapter 8}

factor. Our objective was to end up with a questionnaire containing subscales for each of the above factors clearly defined both semantically and psychometrically (see Briggs et al., 1986; Comrey, 1978; Nunnally, 1978).

\section{Construction of the initial item pool}

An initial pool of items addressing the above four themes (binge eating, failed restriction, weight fluctuations and self-esteem) was composed as follows.

Binge eating Item 6 of the Restraint Scale (see table 8.1), which evidently refers to binge eating practices, was extended with related items on overeating behaviours thought to be "socially somewhat unacceptable". Occurrence of binge eating in daily life, whatever its cause, was assumed to be a good predictor of disinhibition in the lab.

Failed restriction These items tapped on the inability to hold on to dietary restriction each time it's imposed. All of these items had a general format "If I try to impose restriction on my eating I fail". Our items deal very generally with the occurrence of disinhibition after attempts to restrict food intake. Putative causes of the disinhibition were not addressed. High scores on failed restriction items were assumed to contribute to disinhibition in the lab. Matters were arranged so that people who report to never self-impose eating restrictions received a zero score on these items.

Weight fluctuations High scores on weight fluctuation items of the Restraint Scale have been claimed to result from a chaotic eating pattern in which disinhibition follows attemps at dieting, a jojo-effect (Heatherton, Polivy, \& Herman, 1991; Herman \& Polivy, 1982; Lowe, 1993). Our weight fluctuations items were Restraint Scale items (nos. 2, 3 and 4), and a newly conceived item. A modification of Restraint scale item no. 10 was included as well: instead of the difference between prior maximum and currently desired weight, the difference between prior maximum and minimum weight was computed (see also Laessle et al., 1989). High scores on the weight fluctuations items were assumed to increase susceptibility to lab disinhibition.

Self-esteem Items on self-esteem were added not because self-esteem as such is part of the disinhibition concept, but because, as said earlier, low self-esteem has been found to facilitate the occurrence of disinhibitive eating in the lab (Polivy et al., 1988). The items were picked from the Rosenberg Self-esteem Scale (Rosenberg, 1965), and consisted of evaluations of the self in comparison with other people. The items were scored in such a way that answers in the direction of lower self-esteem received higher scores.

Answering format Most items were self-referring descriptions of behaviours or attitudes (self-esteem items). Respondents were to indicate the frequency with which each description applied to their daily situation. Except for items on weight fluctuations this was done on a 5-point Likert scale ranging from 0 points ("never") to 4 ("always"). To 
diminish the possibility of acquiescence (Nunnally, 1978) some items were phrased inversely to disinhibition and its scoring direction should be reversed. The "failed restriction" items had a sixth alternative added, to allow for the possibility that a respondent never self-imposes restrictive eating. This alternative was scored zero.

\section{Item analysis and construction of the Disinhibitive Eating Scale}

The item pool consisting of 36 items was administered to 350 female students and university employees who were approached within the area of the university building (Body Mass Index $20.93 \pm 2.2$; age $23.33 \pm 4.4$. A negligible proportion (about $3 \%$ ) of those approached, declined to participate in the investigation. Item analysis was conducted on basis of a principal components analysis with orthogonal rotation of the extracted factors, as well as assessment of item-total correlations. Items loading poorly on the extracted factors and/or having low item-total correlations were removed from the pool. Some of the binge eating items were removed to prevent relative overrepresentation of these items in the remaining item pool. Finally, the item phrasing were reconsidered and in some cases simplified. The thus formed Disinhibition Scale (DIS) consisted of 17 items divided within 4 subscales (see table 8.2 for the subscale structure and item phrasings): "binge eating" (5 items), "failed restriction" (4 items), "weight fluctuations" (4 items) and "self-esteem" (4 items). The 5-point Likert-format of items was maintained.

\section{TESTING THE RESTRAINT AND DISINHIBITION SCALES}

\section{Subjects and method}

Both Restraint Scale and the newly developed disinhibition scale (DIS) were administered to 269 females (BMI $21.03 \pm 2.4$; age $19.8 \pm 3.0$ ) who were participating in a freshman course at the psychology department. The Restraint Scale was scored according to the requirements introduced by Herman \& Polivy (1980), the DIS according to those in table 8.2. After the establishment of the normative data, three psychometric properties of the questionnaires were investigated: the factor structure (using a principal components analysis with subsequent rotation of the extracted factors confirming to the eigenvalue $>1$ as well as the scree criterion), level of internal consistency (as revealed by Cronbach's Alpha), and item-total correlations and correlations between subscales (only for the DIS). Then, the relationship between the two questionnaires was established by correlations.

Finally the relationship between dieting frequency and the DIS was explored. The 


\section{Chapter 8}

formulation of the DIS items on failed restriction is conditional: "if I try to restrict my eating then I fail". No explicit reference is made to the frequency with which attempts are made to restrict food intake, although "diet frequency" has been claimed to be influential in disinhibition after exposure to binge cues (Lowe, 1993). To elucidate how diet frequency fits the structure of the DIS, a new factor analysis was carried out on all DIS items extended with the Restraint Scale item no. 1 (i.e., the frequency of dieting item).

\section{Table 8.1. Factor structure of the Restraint Scale}

\begin{tabular}{|c|c|c|}
\hline & F-1 & F-2 \\
\hline 1. How often are you dieting? & .75 & .34 \\
\hline $\begin{array}{l}\text { 2. What is the maximum amount of weight (in kilos) } \\
\text { that you have ever lost in one month? }\end{array}$ & .15 & .65 \\
\hline 3. What is your maximum weight gain within a week? & .14 & .81 \\
\hline 4. In a typical week, how much does your weight fluctuate? & .07 & .79 \\
\hline $\begin{array}{l}\text { 5. Would a weight fluctuation of } 5 \mathrm{lbs} \text { affect the way } \\
\text { you live your life? }\end{array}$ & .76 & -.08 \\
\hline 6. Do you eat sensibly in front of others and splurge alone? & .39 & .44 \\
\hline 7. Do you give too much time and thought to food? & .67 & .32 \\
\hline 8. Do you have feelings of guilt after overeating? & .71 & .41 \\
\hline 9. How conscious are you of what you're eating? & .58 & .08 \\
\hline $\begin{array}{l}\text { 10. How many pounds over your desired weight were you at } \\
\text { your maximum weight? }\end{array}$ & .48 & .49 \\
\hline
\end{tabular}

\section{Results}

Restraint Scale The factor analysis revealed a structure with two factors with eigenvalue larger than 1, accounting for $54.0 \%$ of the common itemvariance, the structure of which is shown in table 8.1. As can be seen, three items had considerable (i.e. $>.40$ ) loadings on both factors. Cronbach's alpha for the total scale was .83 which reflects a fully satisfactory level of internal consistency. The item-total correlations ranged from .38 to .70 (mean .53).

Disinhibitive Eating Scale The factor structure (table 8.2) shows four factors with eigenvalue larger than 1 , accounting for $67.7 \%$ of the common itemvariance and having each item loading considerably (above .40) only on the factor prototypical of its subscale, and not on the other factors. Thus the factor pattern approached the Thurstonian 'simple' factor structure (Nunnally, 1978) Internal consistency of the total scale (alpha $=.88$ ) and subscales (alpha's ranging from .76 to .88 ) were good, as were the it- 
Subscale bingeing $(\alpha=0.88)$

2 . At times I lose control over my eating behaviour"

5. I eat sensibly when with others, but I gorge myself

$\begin{array}{llll}.67 & .29 & .27 & .23 \\ .75 & .22 & .25 & .08 \\ & & & \\ .82 & .15 & .17 & .19 \\ .77 & .13 & .19 & .06 \\ .81 & .14 & .06 & .24\end{array}$

8. If others saw how much I ate, then I'd feel ashamed

11. I eat so much at one go that it is actually disgusting other people

Subscale self-esteem $\quad(\alpha=0.85)$

3. I have the feeling that other people do things better than me

6. There are many things about myself that I would change if possible

9. I'd rather be a different person than 1 am now

12. I'm very satisfied with the way I am (-)

$\begin{array}{llll}.20 & .77 & -.03 & -.08 \\ .11 & .86 & .06 & -.02 \\ & & & \\ .21 & .84 & .12 & .06 \\ .17 & .75 & .13 & .18 \\ & & & \\ .16 & .07 & .77 & .11 \\ & & & \\ .14 & -.05 & .82 & .13 \\ .13 & .13 & .83 & .13 \\ .37 & .16 & .73 & .15\end{array}$

Subscale unsuccessful dieting $(\alpha=0.85)$

1. When I'm watching my weight, I succeed in not touching food items (-)

4. When I'm dieting, I manage not to eat between meals (-)

10. When I'm dieting, $I$ can't control myself and start eating 'forbidden foods'

\section{Subscale Weight fluctuations $(\alpha=0.76)$}

14. How strongly has your weight fluctuated in the past few years?

15. What is the maximum weight you have lost in one month?

16. What is the average variation in your weight in one week? I

Difference score of:

17. What has been your highest weight since you were 18 (excluding pregnancy)?

18. What has been your lowest weight since you were 18 ?

$\begin{array}{llll}.34 & .08 & .18 & .73 \\ .08 & -.07 & .08 & .83 \\ .10 & .05 & .19 & .60\end{array}$

(-) negatively keyed item

answering format: $0=$ never; $1=$ sometimes; $2=$ regularly; $3=$ often; $4=$ always; (items 1 through 13)

answering format: $0=$ not at all; $1=$ bardly at all; $2=$ moderately; $3=$ considerably; $4=$ extremely

answering format: $0=$ less than $0.5 \mathrm{~kg} ; 1=0.5$ to $1 \mathrm{~kg} ; 2=1$ to $2 \mathrm{~kg} ; 3=2$ to $4 \mathrm{~kg} ; 4=4 \mathrm{~kg}$ or more

I answering format: $0=$ less than $0.5 \mathrm{~kg} ; 1=0.5$ to $1 \mathrm{~kg} ; 2=1$ to $1.5 \mathrm{~kg} ; 3=1.5$ to $2.5 \mathrm{~kg} ; 4=2.5 \mathrm{~kg}$ or more

answering format: $0=$ less than $3 \mathrm{~kg} ; 1=3$ to $5 \mathrm{~kg} ; 2=5$ to $7 \mathrm{~kg} ; 3=7$ to $10 \mathrm{~kg} ; 4=10 \mathrm{~kg}$ or more 


\section{Chapter 8}

em-total correlations (range .33 to .69 , mean .51). In general, correlations between subscales were moderately positive (table 8.3 ).

Correlation and comparison of the Restraint and Disinhibition Scales The correlations between RS and DIS total scores was .78. Factor analysis of the DIS items together with the diet frequency showed the original DIS factor structure, with the diet frequency item loading .37 on the failed restriction, and .57 on the weight fluctuations factors. This shows that not so much the failed restriction subscale (with items phrased conditionally), but the weight fluctuations subscale accounted for the frequency of failed attempts at dietary restriction.

Table 8.3. Inter-subscale correlations of the Disinhibitive Eating Scale

\begin{tabular}{lccc} 
& Bingeing & Failed restriction & Self-esteem \\
Bingeing & - & & \\
Failed restriction & .49 & - & - \\
Self-esteem & .44 & .24 & .13 \\
Weight fluctuations & .41 & .35 & \\
\hline
\end{tabular}

\section{NORMATIVE DATA AND CHARACTERISTICS OF SUBJECT GROUPS WITH EXTREME DIS AND RS SCORES}

\section{Subjects and method}

First, we established the population distribution of DIS and RS total scores within our sample of 269 female students. Then, for each questionnaire three pairs of extreme scoring groups were formed according to the following criteria. 1) below vs. above the median; 2) below 25 th vs. above 75 th percentile; 3 ) below 10 th vs. above 90 th percentile.

For each of the three criteria, we assessed to what extent the groups formed on basis of DIS and RS consisted of the same individuals. This comparison bears upon divergent validity of the DIS: if the DIS would select the same individuals as the RS, then no purpose would be served by using it.

For each questionnaire separately, high and low scoring groups were compared on BMI and current dieting. The current dieting item was phrased simply: "are you presently dieting to lose weight?" and could be answered yes or no. Three such comparisons, i.e. one for each selection criterion, were made. Difference in BMI were tested with t-tests; percentage of individuals dieting by Chi square tests. 


\section{Results}

Normative data of DIS and RS total scores are presented in table 8.4. Mean score for the RS was 11.66 (sd 5.5) and for the DIS 22.93 (sd 10.9). Strikingly, the RS median was 11, i.e. considerably lower than in Northern American samples. The overlap of extreme groups formed by selection by DIS and RS are shown in figure 8.1. It is clear that the composition of groups selected by either DIS or RS differed, and that the difference was larger when more extreme criteria are used for selection.

Table 8.4. Population distribution of Restraint Scale and Disinhibitive Eating Scale total scores ( $N=269$ females)

\begin{tabular}{ccc}
\hline Percentile & RS & DIS \\
$\mathbf{5}$ & 3 & 7 \\
$\mathbf{1 0}$ & 4 & 9 \\
$\mathbf{2 0}$ & 6 & 12 \\
$\mathbf{2 5}$ & 7 & 14 \\
$\mathbf{3 0}$ & 8 & 15 \\
$\mathbf{4 0}$ & 9 & 18 \\
$\mathbf{5 0}$ & 10 & 21 \\
$\mathbf{6 0}$ & 12 & 24 \\
$\mathbf{7 0}$ & 13 & 27 \\
$\mathbf{7 5}$ & 14 & 29 \\
$\mathbf{8 0}$ & 16 & 31 \\
$\mathbf{9 0}$ & 19 & 37 \\
95 & 22 & 43 \\
\hline
\end{tabular}

Differences in current dieting an BMI of extremely high and low scoring groups on DIS and RS are shown in table 8.5. The results for DIS and RS were comparable and consistent: high scorers had larger BMI and higher prevalence of current dieting than low scorers. Interestingly, among individuals scoring above the RS median i.e. the group traditionally selected for lab research on counterregulation, less than half was currently dieting ${ }^{2}$. Even of the individuals with RS scores within the highest ten percentiles, only $65 \%$ reported to be currently dieting.

Also for BMI, significant and large group differences appeared irrespectively of the used group allocation criterion. The size of differences were as large as between 1.5 population standard deviations of BMI. The correlation of the BMI with RS and DIS scores were respectively 0.48 and 0.52 .

\footnotetext{
${ }^{2}$ Parenthetically, only $16.5 \%$ of all subjects reported to be currently dieting.
} 
Table 8.5. Comparisons of extreme scoring subject groups, using three criteria for Restraint Scale and Disinhibitive Eating Scale scores: a. median split; b. $<25$ th vs $>75$ th percentile; c. $<10$ th vs $>90$ th percentile. $(N=269$ lemales)

\begin{tabular}{|c|c|c|c|c|c|c|c|c|}
\hline \multirow{2}{*}{\multicolumn{3}{|c|}{$\begin{array}{l}\text { RESTRAINT SCALE } \\
\text { cut-off scores }\end{array}$}} & \multicolumn{4}{|c|}{ Body Mass index } & \multicolumn{2}{|c|}{$\%$ currently dieting } \\
\hline & & & \multicolumn{2}{|c|}{ low scorers } & \multicolumn{2}{|c|}{ high scorers } & \multirow{2}{*}{$\begin{array}{c}\text { low scorers } \\
4.7\end{array}$} & \multirow{2}{*}{$\begin{array}{c}\text { high scorers } \\
44.7^{4}\end{array}$} \\
\hline $\mathbf{a}$ & $\leq 11$ & $>11$ & 19.9 & 1.7 & 22.0 & $2.6^{\circ}$ & & \\
\hline b & $<8$ & $>14$ & 19.3 & 1.4 & 22.7 & $2.9^{-}$ & 1.6 & $56.9^{+}$ \\
\hline c & $<5$ & $>19$ & 19.0 & 1.3 & 22.1 & $2.7^{\circ}$ & 0 & $65.2^{+}$ \\
\hline \multicolumn{4}{|c|}{ DISINHIBITIVE EATING SCALE } & \multicolumn{3}{|c|}{ Body Mass index } & \multicolumn{2}{|c|}{ \% currently dieting } \\
\hline & \multicolumn{2}{|c|}{ cut-off scores } & \multicolumn{2}{|c|}{ low scorers } & \multicolumn{2}{|c|}{ high scorers } & low scorers & high scor \\
\hline $\mathbf{a}$ & $\leq 21$ & $>21$ & 20.0 & 1.7 & 22.1 & $2.6^{\circ}$ & 10.3 & $41.4^{+}$ \\
\hline b & $<15$ & $>29$ & 19.5 & 1.7 & 22.9 & $2.8^{\circ}$ & 2.9 & $44.1^{\dagger}$ \\
\hline c & $<10$ & $>37$ & 19.2 & 1.7 & 23.3 & $3.5^{\circ}$ & 3.6 & $57.1^{+}$ \\
\hline
\end{tabular}

- t-test for group differences: $\mathrm{p}<.001$

' chi-square test: $\mathrm{p}<.001$

\section{DISCUSSION}

The results of the psychometric comparison of DIS and the RS in a non-clinical sample of 269 female students showed comparably good internal consistency in the two questionnaires. Factor analyses yielded a two-factor solution in the RS, and a four-factor solution in the DIS. The RS total score distribution in the sample had a median of 11. The correlation between the two scales was 0.78 . It was found that high scorers on either RS or DIS have a considerably higher Body Mass Index and a higher prevalence of current dieting than did low scorers. Finally, it appeared that groups formed on basis of extreme DIS scores were partly composed of different individuals than groups formed on basis of extreme RS scores, and that this difference grew larger when more extreme scores were used as allocation criterion.

The psychometric analysis of the RS confirms earlier reports (for example, Heatherton et al., 1988) of a good value of Cronbach's alpha. Once again the existence of two factors has been demonstrated, which in this case do not differ from the concern for dieting and weight fluctuations factors. It should be noted however that it can be suspected on statistical grounds ( $46 \%$ of the item variance was not explained by the two factors; 3 items loaded > .40 on both factors) as well as on the relatively wide ranging item content, that the RS 
scores potentially encompasses more than two concepts, but that these concepts would emerge as factors if more items were added to the RS. Of course there is little news to this conclusion and discussions on the most fruitful interpretation of restraint scores continue (Charnock, 1989a, 1989b; Dritschel, Cooper \& Charnock, 1993; Heatherton et al., 1988; Lowe, 1993; Ogden, 1993; Polivy \& Herman 1989). Our finding of a median RS score of 11 in our sample of Dutch female students was a confirmation of our earlier experience (Overduin \& Jansen, unpublished data) and of other reports on European subject samples (Wardle, 1986; Laessle, 1989). The implication is that subject selection on basis of an assumed median score of 15 (as found in Northern American samples) may not be appropriate in research within the average European female population.

The DIS was found to show the psychometric characteristics for which it was developed. In the first place a good internal consistency while at the same time all items can be said to be referring to disinhibition or a risk factor for disinhibition. Said otherwise, the total DIS scores appear to be both statistically and semantically meaningful. Secondly, a fair level of common item variance $(67.7 \%$ ) was explained by a well-interpretable four-factor structure with each item loading considerably on only one factor. Importantly, all factors were concordant with the a priori conceived subscales. The resulting advantage is that the DIS total score is based upon a transparant set of meaningful subscale scores. The relative contribution of each subscale to the total DIS score can thus be analyzed for each individual. Finally, the added self-esteem subscale formed an independent factor and the correlation with the rest of the DIS turned out to be moderately positive $(r=0.35$ ). Thus, addition of self-esteem items, expected to heighten the predictive power of the DIS as to lab disinhibition succeeded without compromising the internal consistency or factor structure of the DIS.

Two similarities between the DIS and RS are evident. In the first place a high correlation between the two scales was found, which is not too surprising because of a considerable overlap of item content (e.g. weight fluctuations subscale and RS item no. 6). Secondly, both scales have moderate to strong positive correlations with Body Mass Index. As a consequence in comparisons of high and low scoring groups on food intake in the lab, these differences in BMI should not be neglected. The same applies for the prevalence of current dieting which differs for high and low scorers on both scales. This covariation once again appears to be a fundamental problem in research on disinhibitive eating. Moreover, the fact that among high scorers on DIS and RS only a slight majority (i.e. far from 100\%) is currently dieting, indicates the necessity of carefully distinguishing between general dieting frequency and current dieting when studying behaviours of subjects selected by means of these questionnaires (see e.g. Lowe, 1993).

Also differences between the DIS and RS were found, the most important of which is 


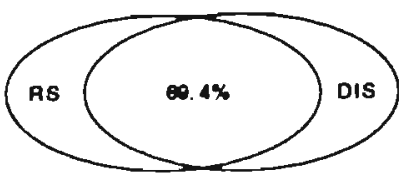

a

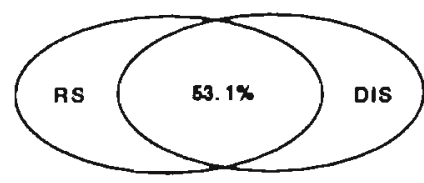

b

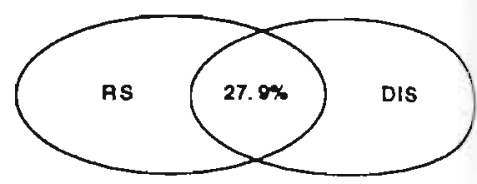

c

Figure 8.1. Percentage of subjects selected by both RS and DIS when applying different group selection criteria: a. median split; b. $<25$ vs. $>75$ th percentile; c. $<10$ th vs. $>90$ th percentile.

practical: in part, different individuals are selected by the two scales when extreme scores are used as a criterion. Both scales have appeared to be equally internally consistent, so that the reasons and possible consequences of selection on basis of DIS scores must be sought for in the item content. Firstly, in contrast to the RS, the DIS contains no concern for dieting items. The aim of the two scales differed: the RS was designed to identify dieters (Polivy \& Herman, 1989; Heatherton, 1988), whereas the DIS was designed for prediction of maximal proneness to lab disinhibition. Of course, the absence of explicit concern for dieting items in the DIS does not at all imply that high DIS-scorers are unconcerned with dieting. What it does imply however, is that relative to the RS, the impact of concern for dieting has been attenuated in the DIS total scores. And, as has been pointed out in the introduction, because individuals high on concern for dieting will have the inclination to inhibit their food intake in the lab, attenuation of its contribution to DIS-scores has the advantage that subjects are selected whose reactivity to food cues will be reflected in eating behaviour also in procedures in which small preloads or food cues are presented. In other words, the chances of false negative findings (i.e. inhibition in subjects with high cue reactivity) will be decreased. Secondly, in contrast to the RS, self-esteem items are included in the DIS. High DIS scorers will have low self-esteem, whereas among high restaint scorers there could be either high or low in self-esteem, thus accounting for different composition of extreme scoring groups. A last relevant issue is that of content validity: to what extent does the DIS address factors related to pathological eating behaviour? Content validity is relevant if one wishes to eventually apply lab results to clinical cases. The content validity of the DIS is suggested by research of Tobin, Johnson, Steinberg, Staats and Dennis (1991), who factor-analyzed a large and complete self-report battery on bulimic symptoms in patients: four factors of the six factors found, i.e., weight fluctuations, binge eating, self-esteem and failed restriction are also present within the DIS. The remaining two factors found by Tobin et al. were family history, and drive for thinness, the latter being similar to 
concern for dieting which was not included in the DIS for reasons explained carlier. All in all, personality factors important in pathological disinhibitive eating can be found in DIS scores to a reasonable extent.

The DIS has been shown to be a conceptually and psychometrically sound selection instrument which taps a construct that is related, but not equal to that measured by the RS. Different emphases exist in the DIS and RS, which partly makes for selection of subject groups of different composition. The DIS was constructed to alleviate some of the issues related to use of the RS in cue reactivity research. Naturally, its predictive power as to lab disinhibition remains to be demonstrated empirically. 



\section{Chapter 9}

\section{CONCLUSIONS AND GENERAL DISCUSSION}

In this final chapter, the results of the empirical studies of the present thesis will be summarized (9.1). After a reappraisal of its physiological assumptions (9.2), a final conclusion will be formulated about the classical conditioning theory of binge eating (9.3). Finally, theoretical and practical suggestions for future research will be made in sections 9.4 and 9.5 respectively.

\subsection{OVERVIEW OF THE EMPIRICAL WORK IN THIS THESIS}

After the description of the conditioning theory of binge eating (CBE) in chapter 2, subsequent chapters were devoted to empirical tests of related hypotheses. In two chapters, the results of conditioning procedures were described. Chapter 3 described a classical conditioning procedure that was run in order to evoke a physiological state which, according to $\mathrm{CBE}$ theory, precedes binge eating. On six occasions, olfactory/taste cues (i.e., the conditioned stimuli, CSs) were paired with glucose ingestion (the unconditioned stimulus, UCS). Subjects in the control condition received the metabolically inert sweetener aspartame as the UCS. At test trials (before and after the conditioning procedure) the CS compound was presented without the UCS, and levels of subjective craving, insulin and blood sugar measured. Subsequently, ad lib glucose intake was measured in a taste test. CBE hypotheses for the post-conditioning test day were that CS presentation would elicit the following responses: conditioned hypoglycaemia, conditioned hyperinsulinaemia, increased craving for sweet snacks, and increased glucose intake. These hypotheses could not be confirmed, except for a small group difference in insulin response, which, however, did not exceed baseline fluctuations. Furthermore, in both conditions, intake of glucose was considerably larger after than before conditioning. As this behaviour was not mirrored in ratings of subjective craving, it was attributed to a "neophobia" or "bait shyness" that had occurred during the pre-conditioning test session, but faded over the subsequent conditioning trials, a phenomenon which has been described previously in the animal literature. In order to investigate whether repeated exposure to sweet drinks (UCS) was a critical factor, a similar conditioning procedure was run additionally with plain water as a UCS. Also in this condition, glucose intake was increased after the procedure. It was therefore concluded that the disappearance of the subjects' "neophobia" had been related to their getting acquainted with the lab procedure, rather than with the taste of sweet drinks. Chapter 4 contained a comment on, as well as a re-analysis of a not widely known study on hypoglycaemic conditioning 


\section{Chapter 9}

by the Russian physiologist Mityushov (1954; Appendix, this thesis). In contrast to our conditioning study described in chapter three, Mityushov was able to demonstrate a statistically as well as physiologically significant conditioned hypoglycaemia in humans and dogs, after a procedure involving repeated pairing of an injection ritual (CS) with intravenous glucose (UCS). Further observations suggested that the hypoglycaemia was secondary to a conditioned secretion of insulin. Although Mityushov's report would not fully meet today's methodological standards (e.g. no control group was run; no overall statistics were reported), it was concluded that his conditioning procedure evoked a physiological state that, according to the CBE theory, occurs prior to bingeing.

The next two chapters investigated psychophysiological cue reactivity to food related stimuli. In CBE terms, cue reactivity could be considered as a residual of learning history. In chapter 5 reactions to slides with binge-related food items were studied. The CBE prediction in this study was that cue reactivity would be larger in restrained than in unrestrained eaters. This was hypothesized because restrained eaters can be considered "unsuccessful dieters" who regularly engage in (non-clinical) binge eating. Hence, they should display especially intense conditioned responses to food cues. A manipulation check revealed that subjective hedonic ratings of the food slides were higher than those for control slides (depicting office equipment). Nevertheless, no increased psychophysiological cue reactivity was found in restrained subjects, so that the CBE hypothesis was not confirmed. The study in chapter 6 assessed psychophysiological reactivity to the sight and smell of attractive food. Two groups of normal subjects were compared, one of which had been fasting for the previous 24 hours. It was thought that the fasting state would form a baseline to which food craving could be easily elicited by food cues, and that the related physiological responses would be more clearly discernible. The hypotheses were that subjective craving to food cues would be larger in fasting than nonfasting subjects, and, more specifically, that these differences would be mirrored in the intensity of the psychophysiological responses. As expected, it was found that food item presentation elicited more craving in fasted than in non-fasted subjects. However, no group differences were found in psychophysiological cue reactivity, a finding which was not in line with the CBE hypothesis.

Chapter 7 contained an experimental test of the cognitive effects of cue-elicited craving. Restrained and unrestrained eaters were compared on their attentional bias to food words measured in a modified Stroop colour-naming test. There were two conditions. In the appetizer condition, subjects had to eat a spoonful of palatable pudding, whereas in the control condition no appetizer was ingested. Then, the modified Stroop test was run, comparing, among other things, colour naming latency for food and neutral words. After the Stroop test, ad lib ice cream intake was measured during a "taste test". The 
hypotheses were that ingestion of the appetizer would result in stronger Stroop interference for food words, and larger food intake in restrained than in unrestrained subjects. This was not confirmed: in the appetizer condition, no especially strong Stroop interference or disinhibitive eating could be found in restrained subjects. However, it was also observed that whereas restrained subjects did not counterregulate, unrestrained subjects did. Interestingly, for unrestrained subjects, the magnitude of the Stroop interference for food words appeared to correlate 0.58 with the subsequent intake of ice-cream. This was taken to suggest that attentional bias to food words may be a potential predictor of increased eating after cue presentation.

Finally, in chapter $\mathbf{8}$, the development was described of the DIS, a new questionnaire that might be used as a selection tool in future research. The DIS aims at selecting persons who are maximally prone to disinhibitive eating. In this respect, the DIS differs from the widely used Restraint Scale, that was originally developed for identifying dieters. The psychometric analysis of the DIS showed good internal consistency, as well as a clear four-factor structure tapping binge eating, failed restriction, weight fluctuations, and self-esteem, i.e. a factor structure resembling that found in earlier studies of large item pools tapping a full spectrum of bulimic characteristics. It was concluded that the DIS could be used as a tool in the development of new models of binge eating.

Taken together, none of our novel studies specifically testing hypotheses derived from $\mathrm{CBE}$ theory produced confirmative results. What should the implications of these results be for the CBE theory? One might suppose that particulars of the studies were responsible. Our non-clinical restrained subjects may have represented too weak cases of binge eating; also, the food slides used as cues in our cue reactivity experiment may not have been overly "realistic"; finally, our conditioning study may have included too few trials to demonstrate conditioning.

However, one might also rebut these arguments with the following considerations. Of the available questionnaires in the field, the Restraint Scale has been generally recognized as the most predictive of lab-overeating and binge eating in daily life (Heatherton et al., 1988; Wardle, 1980). Further, our food slides have probably been sufficiently realistic, which is suggested by the fact that, for all subjects taken together, psychophysiological reactions and subjective craving were stronger for food than did for neutral (office equipment) slides (Chapter 5). Finally, in other contexts, conditioned hypoglycaemia (using intravenous UCSs) have been demonstrated after two to five conditioning trials (Fehm-Wolfsdorf et al., 1993; Mityushov, 1954; Appendix, this thesis; Woods et al., 1969), so that after our six trials (using oral UCSs) one should expect at least a significant trace of evolving hypoglycaemia. All in all, it appears that the present studies have been fairly critical and suggest reconsideration of the CBE theory. This reconsider- 


\section{Chapter 9}

ation can be found in the following section.

\subsection{QUANTITATIVE REAPPRAISAL OF THE CBE THEORY}

In the original formulations of the conditioning theory of binge eating (chapter 2 this thesis; Jansen, $1990 ; 1994 \mathrm{a}$ ), no quantitative estimates were given of the size of putative conditioned physiological responses. Considering the general lack of confirmation for the CBE theory in the present thesis, it seems worthwhile to reconsider CBE theory's physiological predictions in a quantitative and critical way. Below, five key physiological CBE hypotheses (see chapter 2 for background explanation) will be assessed, using data from general physiology, cephalic phase response studies, conditioning studies, and comparative studies of physiological responding in bulimics and normals.

\subsubsection{Hypothesis one: binge eating causes exceptionally large blood sugar rises (UCS)}

Analysis: The CBE line of reasoning is that bingeing provokes a very large blood sugar rise, requiring that the size of the (compensatory) conditioned hypoglycaemia (CR) be proportionally large. The large intensity $C R$ would then be reflected in the subjective experience of craving.

No direct measurements of blood sugar rises after bingeing are available. Nevertheless, it is doubtful that beyond moderate meal size, additional intake leads to higher blood sugar rises. With fasting baseline typically lying between 70 and $100 \mathrm{mg} / \mathrm{dl}$, a normal meal will lead to blood sugar levels of between 100-150 mg/dl (Bantle et al., 1983; Oettlé, Emmett \& Heaton, 1988; Shively, Apgar \& Tarka, 1986; Teff, Levin \& Engelman, 1993; Weingarten, Hendler \& Rodin, 1988). Empirical data show that the upper boundaries of blood sugar level do not lie considerably higher. First, ingestion of doses of glucose (i.e. $1200 \mathrm{kcal}$ of a extremely potent blood sugar raiser; Brand Miller, Pang \& Broomhead, 1995) have been found to induce blood sugar levels not exceeding ${ }^{1}$

1 The physiological reason for the limited blood sugar rise to large meals in healthy (non-diabetic) persons depends on at least four inborn, homeostatic mechanisms. First, large meals will slow down gastric emptying, meaning that the food stays longer in the stomach and is allowed slower access to the small intestine, the place whence nutrients appear in the blood (McHugh, 1983). Second, if food is eaten hurriedly (this may be seen in binge eaters; section 1.2) the subsequent blood sugar rise is blunted importantly, due to several slowening effects on digestion (Read et al, 1986). Third, as soon as the glucose enters the blood, an (unconditioned) post-absorptive insulin response directs sugar from the blood into cells and tissues. Fourth, if, despite the aforementioned mechanisms, blood sugar level did exceed $150-190 \mathrm{mg} / \mathrm{dl}$, the sugar will not be reabsorbed by the kidneys and appears in the urine (this so-called glucosuria is responsible for the large volumes of high sugar urine which can be found in diabetics; Guyton, 1991). 
$180 \mathrm{mg} / \mathrm{dl}$ (Förster et al., 1972). Second, the ingestion of large amounts of favourite "real food" items appears to lead to moderate blood sugar rises. Kaye, Gwirtsman and George (1988) encouraged normal subjects to eat a buffet of favourite foods until their limits were reached. The recorded intake was 1482 (sd 430) $\mathrm{kcal}$, whereas blood sugar had only risen to $122 \mathrm{mg} / \mathrm{dl}$. A third - specifically bulimic - factor blunting the postmeal blood sugar rise is self-induced vomiting. Johnson et al. (1994) observed a rapid and strong blood sugar fall in bulimics who expelled ingested food (Johnson et al., 1994). In the Kaye et al. (1988) study, bulimics were encouraged to binge on a buffet of favourite food items, and given the possibility to purge. After a mean intake of 3022 (sd 1084) kcal and self-induced vomiting, bulimcs' average blood sugar level was found to lie at $120 \mathrm{mg} / \mathrm{dl}$. After this, subsequent bingeing and purging was allowed, and eventually the mean amount of ingested food was 7688 (sd 3971) kcal. In spite of this huge intake, blood sugar levels at subsequent l-hour intervals remained between 90 and 100 $\mathrm{mg} / \mathrm{dl}$.

Conclusion: Although no direct data are available on binge eating bulimics, ingesting large amounts of foods is unlikely to provoke an extreme blood sugar rise, because of the action of various homeostatic controls and the effects of self-induced vomiting.

\subsubsection{Hypothesis two: The conditioned blood sugar fall compensates substantially for the anticipated post-binge blood sugar rise}

Analysis: The CBE theory posits that conditioned hypoglycaemia compensates for the expected blood sugar rise (UCRs) ${ }^{2}$. How effective is this compensation?

To quantify the amount of compensation provided by the $\mathrm{CR}$, the $|\mathrm{CR} / \mathrm{UCR}|$ ratio can be computed. For example, if this ratio is 1 , full compensation of the post meal blood sugar rise occurs; if the ratio is 0.10 , this would mean that the conditioned hypoglycaemia would compensate for $10 \%$ of the expected blood sugar rise. The successful blood sugar conditioning studies using glucose as the UCS (Deutsch, 1974; Mityushov, 1954/Appendix, this thesis) provide insight into the |CR/UCR | ratio. In Deutsch's study, the blood sugar rise (UCR) to the glucose UCS in rats was $31.3 \%$; whereas the CR hypoglycaemic response to placebo intubation was $3.3 \%$ from baseline. Thus the

${ }^{2}$ For the sake of simplicity, blood sugar rise and fall are called responses here. Physiologically speaking, however, this may not be entirely correct. The blood sugar rise to ingested food ("UCR") is the result of passive absorption of glucose from the gut. A similar consideration may apply to the conditioned hypoglycemia which probably results from an (active) insulin secretion (see chapter 4 , this thesis; Dworkin, 1993; Eikelboom \& Stewart, 1982, for further discussions). 


\section{Chapter 9}

|CR/UCR | ratio in this case was approximately $0.11 .^{3}$ In Mityushov's study, the observed hypoglycaemic $\mathrm{CR}$ in humans was $10 \mathrm{mg} / \mathrm{dl}$, whereas the blood sugar rise to the UCS in humans was about $165 \mathrm{mg} / \mathrm{dl}$, making the $|\mathrm{CR} / \mathrm{UCR}|$ ratio $10 / 165=0.06$. Conclusion: Judging from the scanty data available, hypoglycaemic CRs appear to be far from compensatory.

\subsubsection{Hypothesis three: cephalic insulin responses are, physiologically speaking, substantial responses}

A substantial part of the data basis underlying CBE theory concerns cephalic phase responses to food cues (section 2.2). These responses are put forward as illustrations of substantial responses causing a fall in blood sugar level and underlying feelings of craving.

Analysis The available data show that the magnitude of the cephalic insulin response are rather small when compared with either baseline fluctuations or post-meal insulin secretion (Bellisle et al., 1983; Le Magnen, 1992; Moyer, Rodin \& Cummings, 1993). Whereas the range of reported cephalic insulin increases from baseline has been between 0-250\% of baseline levels in rats (Louis-Sylvestre, 1976; Powley \& Berthoud, 1985; Strubbe \& Steffens, 1975), and between 0 and $95 \%$ for humans (Lucas, Bellisle \& de Maio, 1987; Bruce et al., 1987; Sjöstrom et al., 1980; Moyer et al., 1993; Powley \& Berthoud, 1985; Teff et al., 1991; 1993; 1995), the post-absorptive increase of insulin after moderate (i.e. $100-700 \mathrm{kcal}$ ) food intake in humans lies between 200 and $1000 \%$ of baseline (Bantle et al., 1983; Bellisle et al., 1983; Shively et al., 1986; Rodin, Reed \& Jamner, 1988; Weingarten et al., 1988). Importantly for the CBE theory, two studies (Broberg \& Bernstein, 1989; Moyer, Rodin \& Cummings, 1993) have been conducted in bulimic patients who were to smell palatable food attentively. Neither study reported cephalic insulin responses exceeding baseline; moreover, the standard deviations of responses reported by Broberg and Bernstein (1989) were too small to suggest outliers among the subjects. In their discussion these authors have suggested that these results may have been due to their subjects not being in the "proper mood" that usually accompanies binge episodes. This remark applies to the vast majority of lab studies with bulimic subjects.

Conclusion Cephalic insulin secretion to food cues appears to be modestly sized when

${ }^{3}$ Note that another provisional computation could be made which adjusts for hyperglycaemia that occurred because of the rats' stress during the stomach intubation procedure (Deutsch reported that prior to the conditioning procedure, the rats had shown a $3.1 \%$, stress-related blood sugar rise when intubated with water). If one subtracts this blood sugar rise from both UCR and CR, then the $\mid$ CR/UCR $\mid$ ratio becomes $(3.3+3.1) /(31.3-3.1)=6.4 / 28.2=0.23$. 
compared with either baseline fluctuations or with post-meal insulin secretion. This observation applies to bulimics as well.

\subsubsection{Hypothesis four: cues related to binge food trigger especially large conditioned hypoglycaemia/hyperinsulinaemia in bulimics}

Analysis: As noted earlier, a potent elicitor of anticipatory insulin secretion and hypoglycaemia is sweet taste (section 2.2; Deutsch, 1974; Louis-Sylvestre, 1976, Powley \& Berthoud, 1985). Furthermore, binge eaters have been found to avoid snacks and other high caloric food items in between binges (e.g. Rosen et al., 1986; Walsh, 1993). If, as predicted by CBE theory, considerable conditioned hypoglycaemia or hyperinsulinaemia to binge food cues (CS) occurred in binge eaters, it follows that these responses should be observed in binge eaters and normals after the ingestion some fixed amount of binge-related food. Specifically, bulimics should show a retardation and/or a blunting of post-ingestive blood sugar rise, as well as a simultaneously enlarged insulin secretion.

First of all, two studies have been carried out comparing insulin and blood sugar responses to intravenous glucose in bulimics and normals (i.e., no food cues were involved in these studies). Blouin et al. (1991) found a smaller peak blood sugar value, but also a smaller insulin/glugacon ratio in bulimics than in normals. These findings were not replicated by Blouin et al. (1993), who did not find differences between responses of bulimics and normals. Studies which did involve oral ingestion of carbohydrates showed no irregularities in responses of bulimia patients: after ingestion of a sweet glucose drink (Hohlstein et al., 1986; Casper et al., 1989), or a 1200 kcal lemonflavoured carbohydrate drink (Turner et al., 1991), equal blood sugar and insulin responses were found in bulimics and normals. Addition of further "binge-relevant" sensory cues did not alter this pattern of results. Weingarten et al. (1988) provided bulimics and normals with equicaloric $(334 \mathrm{kcal})$ meals in a forbidden version (milkshake, and french toast with sirup) and an unforbidden version (cottage cheese and fruit). Again no between-group differences were found in blood sugar and insulin response. Moreover, the unforbidden meal evoked a somewhat larger insulin response in both subject groups, and this is contrary to CBE prediction. In one study (Schweiger et al., 1987) a difference between bulimics and normals was found: ingestion of a pudding rich in carbohydrates led indeed to a larger insulin secretion in bulimics (in line with $\mathrm{CBE}$ predicitions). However, this enlarged insulin secretion in bulimics appeared to be secondary to an enlarged blood sugar rise, a result which is in contrast with the $\mathrm{CBE}$ predictions. Jansen (1994a) has questioned the relevance of the above studies for CBE theory, because this theory only predicts pre-meal responses (however, see section 9.2.5 
for a further discussion on this issue). Furthermore, the cited studies neither involved individually chosen binge foods, nor were they conducted in the usual bingeing environment of the binge eating subjects.

Conclusion In contrast with the CBE hypothesis, ingestion of sweet or binge relevant food items has not been found to result in enlarged insulin or (hypoglycaemic) blood sugar responses in bulimics.

\subsubsection{Hypothesis five: conditioned hypoglycaemia underlies the urge to binge}

Analysis: The CBE theory posits that conditioned hypoglycaemias to food cues are experienced subjectively as an urge to eat.

Importantly, any hypoglycaemia explanation of binge eating should take into account that food intake itself will increase blood sugar level as nutrients start being absorbed from the small intestine. If hypoglycaemia underlies the urge to eat, an important question concerns the speed with which the hypoglycaemia will subside once eating has started. Probably, hypoglycaemias of the largest reliable size reported in the conditioning literature (i.e. $10 \mathrm{mg} / \mathrm{dl}$; Mityushov, 1954; Broberg \& Bernstein, 1989) would dissipate soon after the commencement of eating. This can be concluded from the available literature on blood sugar rise after ingestion of typical binge foods (candy bars and potato crisps). Ingestion of one candybar and a cup of tea (296 kcal) was found to lead to a $24 \mathrm{mg} / \mathrm{dl}$ rise within 20 minutes after meal onset; a serving of potato crisps and some cola drink (292 kcal) lead to a $29 \mathrm{mg} / \mathrm{dl}$ rise within the same time (Oettle et al., 1987). Other important data are given by Broberg \& Bernstein (1989) who did indeed find a considerable cephalic hypoglycaemia of $8-9 \mathrm{mg} / \mathrm{dl}$ after having their subjects smell palatable cinnamon rolls. Both in their bulimic and dieting subjects subsequent intake of the cookies was allowed. The bulimics who ate 36 grams of the rolls (perhaps about $150-200 \mathrm{kcal}$ ) returned to blood sugar baseline at between 10 and 15 minutes after the beginning of eating. These data show that after merely moderate intake hypoglycaemia is lifted long before the ending of the average binge, which has been reported to lie at 55.7 (sd 32.4) minutes (Jansen, Van den Hout \& Griez, 1990). It is only to be expected that bingeing is even more effective in lifting hypoglycaemia ${ }^{4}$.

Conclusion Hypoglycaemias of the size reported in the conditioning literature can be expected to subside soon after meal onset. The fact that the duration of the average binge appears to be considerably longer than that of hypoglycaemia, suggests that the role of conditioned hypoglycaemia may be limited, and that additional mechanisms

4 Compare the $\pm 300 \mathrm{kcal}$ snack, used in the Oettle et al. (1987) investigation, with the rate of food intake in binge eaters (between 30 and $80 \mathrm{kcal} / \mathrm{min}$; Rossiter et al., 1992; Walsh, 1993). 
should be postulated to fully explain binge eating.

\subsubsection{Conclusions}

Of course, the above discussions give only hints about the size of conditioned responses predicted by CBE theory. The true response sizes await revelation by monitoring of patients during full-fledged binge attacks. Until further notice, the following can be concluded:

a. the presently available data do not endorse CBE theory's assumption of physiologically significant conditioned hypoglycaemias/hyperinsulinaemias that "should be eaten away";

b. in bulimic patients, no enlarged hyperinsulinaemias or hypoglycaemias ("CRs") have been demonstrated in response to the taste or smell of binge related foods ("CSs");

c. ingestion of snacks provokes a rise in blood sugar that can be expected to lift hypoglycaemias of the size reported in the conditioning literature within a considerably shorter time than the generally reported duration of binges.

In sum, on basis of the known empirical data, profound scepticism seems warranted about the role of conditioned hypoglycaemia/hyperinsulinaemia in binge eating.

\subsection{THE CONDITIONING THEORY OF BINGE EATING: FINAL QUALIFICATION}

A conditioning theory of binge eating (CBE theory) was described in chapter two. By and large, none of the $C B E$ hypotheses empirically tested in this thesis could be confirmed. Furthermore, a quantitative reappraisal of the physiological assumptions of the CBE theory (section 9.2) cast considerable doubt on the premise that conditioned hypoglycaemia/hyperinsulinaemia is central to binge eating. In sharp contrast, CBE theory also has inspired a cue exposure procedure that promises to be an effective treatment of binge eating (section 2.2.6). It is not immediately clear what should be concluded from a case where an (as yet) unproven theory inspires effective therapy. Interestingly enough, similar dilemmas have been harassing conditioning research of panic disorder and addiction. We will examine the commentaries made by two major workers in these fields, McNally (1994) and Siegel (1989), before drawing final conclusions for binge eating.

McNally (1994) views classical conditioning theory starkly as a "misleading metaphor for the mechanisms underlying panic" while admitting that "(..) it has inspired effective treatments of panic disorder" (p. 108). McNally's main criticism of the conditioning 


\section{Chapter 9}

account of panic is that theoreticians have failed to clearly identify stimuli (CS, UCS) and responses (CR, UCR), often with awkward implications such as bodily sensations in panic being CS,CR and UCS at the same time. McNally thinks that this vagueness does not allow conditioning accounts to be very fruitful in basic research of panic disorder.

Siegel (1989) comments on several studies in which conditioned drug tolerance was found in the absence of demonstrable conditioned physiological responses. He concurs with the view that "failures to demonstrate compensatory CRs do not represent major challenges to the conditioning account of tolerance" (p. 163), and points to present knowledge deficits in classically conditioned drug tolerance. More specifically, too little is known yet about when and how physiological responses are triggered by environmental and drug stimuli, and how physiological CRs exactly relate to behavioural phenomena like tolerance and drug intake.

To what extent do the above considerations apply to the conditioning theory of binge eating? Concerning McNally's remarks, it should be emphasized that, unlike the panic theories, CBE theory's stimuli and responses have been well-defined. Equally truly, however, other investigators of binge eating have proposed an entirely different learning mechanism in bingeing that, while involving different responses and stimuli, has also inspired a therapeutical procedure that leads to important reductions in binge eating (Leitenberg et al., 1984; Schmidt \& Marks, 1989; for explanation see section 9.4.2). Nevertheless, the "vagueness" intrinsic to the existence of two different theories may have less to do with misleading metaphors, than with the possible existence of more than one mechanism underlying binge eating (Schmidt \& Marks, 1989). Siegel's commentary distinguishes between the physiological and behavioural levels. As to the physiology related to binge eating, we would not adopt Siegel's opinion that failures to demonstrate compensatory responses are minor challenges to the conditioning theory of binge eating. The physiological events (if any) underlying the urge to eat might turn out to be entirely different from those hypothesized, prompting reformulation or even rejection of CBE theory ${ }^{5}$. Nevertheless, on the behavioural level CBE theory appears to be working well, given the success of cue exposure. There is by now accumulating data suggesting this (section 2.2.6), so that the situation for binge eating is no different than that for conditioned drug tolerance. For therapeutic purposes this situation need not be disastrous: CS (binge cues) and UCS (binge eating) and their relationship are transparent.

$S$ For example, it might be that the peripheral, conditioned compensatory responses that have been previously observed in addiction research (see the overview presented in Macrae, Scoles \& Siegel, 1987), can be understood as serving homeostatic regulation (see e.g. chapter 4, this thesis), whereas craving and other behavioural effects critically depend on as yet unknown central effects (occurring within the brain). 
The therapist's position could be compared with that of a radio owner who, although fairly skilled at operating the knobs, remains unaware of the radio's interior electronics. For both therapist and basic researcher, however, uncertainty remains about the mechanism through which the habit of binge eating was acquired in the first place. To summarize and conclude, the physiological mechanism of urge (if any) remains elusive; the major advance relative to the "urge-less" theory of binge eating (e.g. counterregulation theory, section 1.3), is that a maintenance factor appears to have been identified, that can be cast in conditioning terms and used for therapeutical purposes; the contribution of learning to binge eating has not been exhaustively studied. Important questions remain about the physiological and acquisition mechanisms in binge eating.

Next, theoretical (section 9.4) and practical (section 9.5) directions for future research will be proposed.

\subsection{FUTURE RESEARCH: THEORETICAL CONSIDERATIONS}

In this section, two directions ${ }^{6}$ in research on the role of learning in binge eating will be proposed. The first approach should be considered an update of the conditioned hypoglycaemia/hyperinsulinaemia explanation that was originally offered by CBE theory. In the second approach, repeated post-binge vomiting and restriction are identified as key factors in binge eating. It may well be that both mechanisms are simultaneously active in binge eaters, judging from reports that exposure therapies directed at either mechanism have been effective in reducing binge eating (Schmidt \& Marks, 1989). Practical research implications of the theorizing will be described in sections 9.5.3 and 9.5.4.

\subsubsection{Maintaining a blood sugar/insulin-related explanation}

Reliable pre-meal blood sugar fall: characteristics When one still looks for a role of learned insulin and glucose dynamics in craving and binge eating, a recent development may provide clues. Campfield and Smith (1990a; 1990b) have reported a robust role of insulin and blood sugar signals in meal initiation. Blood sugar levels were continuously monitored in free moving rats with ad lib food available. Campfield and Smith claim

6 A third, social learning approach, which is in current upswing in cue-reactivity research (Tiffany 1995a; 1995b) will not be elaborated further here. First, because it explicitly rejects the idea that craving is a central mechanism in relapse (Tiffany, 1995b, p. 155; this might be the case for drug and alcohol addictions, but is rather unlikely for binge eating), and second, because apparently, the social learning account of relapse has not yet reached a stage at which convincing lab models may be developed (Bradizza, Stasiewicz \& Maisto, 1994). 
that almost without any exception, ad lib meal intake in the rats was preceded by a transient blood sugar fall of at least $6 \%$ below baseline, lasting for more than 6 minutes. Meal initiation occurred at 12.1 (sd 1.7) minutes after the beginning of the blood sugar fall, i.e. 5.4 (sd 1.5) minutes after the nadir of the hypoglycaemia. Other characteristics of the robust hypoglycaemia-food intake sequence were revealed in numerous experiments: if food became available at 5 minutes after the hypoglycaemia had disappeared, no food intake occurred; if the hypoglycaemia was countered by a rapid glucose infusion, no feeding occurred; if the experimenters mimicked a hypoglycaemia by infusion of other biochemicals, food intake occurred if the characteristics of the glycaemic curve resembled those of the spontaneous hypoglycaemias; no within-subjects correlation was found between the depth of the hypoglycaemia and the amount of subsequent intake. One origin of the spontaneous hypoglycaemias was found to lie in a prior short-lived insulin increase (50\% above baseline).

The results have been confirmed in humans whose blood sugar level was monitored continuously for a prolonged period (Campfield et al., 1992; 1996). The subjects could require a meal if they wanted. It was found that hypoglycaemias of $6-10 \%$ below baseline preceded meal request. These blood sugar dynamics also correlated with selfreported hunger.

Commentary Exactly how the hypos described by Campfield and Smith might relate to learning and conditioning is not clear yet. The following comments can be made.

1. In contrast to the $\mathrm{CBE}$ theory account, the hypoglycaemias (and the preceding hyperinsulinaemias) described by Campfield and Smith are short-lived and signal meal initiation rather than compensate for homeostatic disturbances.

2. The size of cue elicited (cephalic and conditioned) hypoglycaemias reported in some of the human studies (Broberg \& Bernstein, 1989; Mityushov, 1954/Appendix this thesis) is within the range of the meal-preceding hypoglycaemias found by Campfield and Smith. Moreover, the hypos were preceded by a small 50\% insulin "spike", the size of which is that of the typical (cue-elicited) insulin responses (see sections 2.2 and 9.2, this thesis).

3. Are the observed hypoglycaemias endogenous and/or learned in nature? In his commentary, Woods (1990) has argued against Campfield and Smith's purely endogenous explanation. He points to the fact that the rats in the Campfield \& Smith studies were kept in a very stable monotonous environment, whereas in a sensorily richer environment the rats' behaviour (e.g. the observed hypoglycaemia and food intake!) might become more easily attuned to external (meal-related) events and other cues. Interestingly, the only meals not preceded by hypoglycaemia concerned foods that were novel to the rats. As the rats got acquainted with the food items, gradually the hypoglyc- 
aemias began to appear as with other foods (Dr. Campfield, personal communication). This suggests a role of learning in the meal preceding hypoglycaemias.

4. Now that the moderately sized, short-lived hypoglycaemias found by Campfield and Smith appear to be playing a role in normal food intake, it still remains elusive in what way these responses might contribute to binge eating. Two possibilities may be mentioned. First, one might speculate that in binge eaters, for some reason the frequency of transient hypoglycaemias exceeding some critical threshold is increased (relative to healthy normals) before or during binge eating. Frequent hypoglycaemic dips might be experienced subjectively as "waves of craving" leading to temptation to binge (or continuing the binge if the hypos were superimposed on the post-prandial blood sugar rise $^{7}$ ). Considering the turmoil reigning in the blood sugar and insulin domain after food intake, the latter speculation remains problematic. A related possibility might be that the hypoglycaemic mechanism only induces binge-initiation, and that during the binge another principle is at work (see for instance section 9.4.2).

5. Finally, it also could turn out that hypoglycaemias occur equally in normals and binge eaters, but that binge eaters are especially inclined to interpret small hypoglycaemias as hunger. In fact, from research on hypoglycaemic episodes in diabetic patients, it is known that there is an individual stereotype in the experienced symptoms. There are "trembling/sweating" individuals, whereas others report predominantly hunger during hypoglycaemia (Hepburn, 1993; Service, 1995). Nevertheless, an explanation based merely on differential interpretation of body signals is likely to be too weak to account for the particular vigour with which binge eating proceeds (see section 1.2).

\subsubsection{Learning perspective based on post-meal deprivation and vomiting}

There is consensus among food intake researchers that meal size is correlated not only with momentary, but also with expected level of deprivation (see Collier, 1986; Davey, 1989; Le Magnen, 1992; Weingarten, 1985; Woods \& Strubbe, 1994). Moreover, binges of bulimics and anorexics of the binge eating/purging type (APA, 1994) are part of a eating pattern of restriction, fasting and vomiting. While these behaviours are meant to be "rational" means of weight restriction, the possibility of their becoming maintenance factors in binge eating should not be ruled out. This section will review various studies to suggest that yet another learning mechanism may contribute to bingeing: learned cues that reliably predict the occurrence of deprivation after a meal might

7 As noted in section 2.2, Louis-Sylvestre (1984) did demonstrate short insulin bursts after introduction of new food items during meals in rats. It is equally true that changing the foods during a meal increases total intake. 
increase the size of that meal. The data presented below will be used as a starting-point for a new lab model of binge eating (section 9.5.3).

Repeated fasting after meals may increase meal size Although no systematic data have been collected, it seems safe to say that binge eating episodes are frequently followed by a period of self-imposed restriction and/or vigorous exercise (APA, 1994). There is one report that suggests that meal size might increase if deprivation reliably follows that meal (Le Magnen, 1992, ch. 1). Rats were adjusted to a diet of 3 daily one-hour meals a day at fixed times for 18 days. Then one of the three meals was omitted daily, thus creating the following schedule: meal I - 7 hours - meal II -- 15 hours -- meal I, and so on. Food intake was monitored for another 18 days. After several days, a clear meal size pattern developed: meal II, i.e. the meal preceding the 15 -hour deprivation, became considerably larger than meal I, and ultimately increased with about $90 \%$ of the calories of the now omitted third meal. This effect, found irrespectively of the time of day, was taken to suggest that rats adjust their meal size by using information of future availability of food. Meal size increases when post-meal deprivation is expected. At least until recently, no human research had been done on this mechanism (Le Magnen, 1992). The implication is that if bingeing is reliably followed by prolonged restriction or fasting, this might lead to a learned increase of the pre-fasting (i.e. binge) meal.

Repeated vomiting after meals may increase meal size Binges are often followed by vomiting in $80-90 \%$ of the bulimic patients (APA, 1994; Rosen et al., 1986). Post-binge vomiting appears to have an enhancing impact on bingeing itself. Firstly, the speed of eating appears to be larger in purging than in non-purging bulimics (compare Rossiter et al., 1992 with Walsh, 1993). Secondly, the binge size of purging bulimics is twice of that of non-purging bulimics (compare Rossiter \& Agras, 1990 with Rossiter et al., 1992) ${ }^{8}$. It will be suggested here that, if bingeing is reliably followed by vomiting, a learning mechanism might induce learned increase of the fast-preceding meal (i.e. the binge) itself. This allegation will be substantiated by data on physiological state after vomiting, operant conditioning, conditioned sham feeding, and conditioned desatiation studies.

First, post-binge vomiting may lead to a temporary state of deprivation that bears upon food intake. Johnson et al. (1994) had 12-hour fasted bulimic subjects binge and vomit subsequently during which blood sugar and insulin levels were monitored. After

${ }^{8}$ Almost certainly, this can be explained by the simple fact that vomiting creates space for yet more binge food. Hence, it becomes understandable how the largest binge sizes reported in the literature could have been in the $20,000-55,000 \mathrm{kcal}$ range, i.e., between 10 and 28 times the average daily food intake in the normal population and, literally, impossible to stomach (Russell, 1979; Walsh, 1993). 
vomiting a steep decline of blood sugar level was found (12 $\mathrm{mg} / \mathrm{dl}$ within 15 minutes) bringing blood sugar back at baseline level. Concurrently, however, the insulin level remained twice as high as baseline ${ }^{9}$. A similar overshoot of insulin level after a 3000 kcal binge and subsequent vomiting in bulimics was found by Kaye et al. (1989), and it might imply a momentary glycaemic state of deprivation ${ }^{10}$. Direct evidence that expected insulin overshoot may produce instrumental food seeking and eating comes from a study by Siegel and Nettleton (1970). Four groups of rats were housed in Skinner boxes. Group A received repeated insulin injections and had the possibility to bar-press for food, while Group B received insulin injections without this possibility. Control groups $C$ and $D$ received saline injections with and without bar pressing possibility respectively. During the post-conditioning test session, saline was injected in all groups and bar pressing frequency was monitored: group A rats clearly showed the largest bar pressing rate of all groups, while at a pre-conditioning test session the rates had been equal for all groups. Siegel and Nettleton (1970) concluded that the rats of group $\mathrm{A}$ had acquired an instrumental feeding response to reduce expected "insulininduced distress".

Second, sham feeding studies may be relevant as an analogue of post-binge vomiting: large food-intake is signalled orally, but the amount of nutrients subsequently absorbed does not follow suit. Van Vort and Smith (1987) have shown that flavours associated with sham feeding enhance meal size. Rats were provided with a fistula that could be opened (sham feeding) as well as closed (real feeding). During a conditioning procedure, the rats were given a diet of milk. In alternating sequence, the milk had either an added flavour which signalled sham feeding (CS-) or a flavour that preceded real feeding $(\mathrm{CS}+)$. A post-conditioning test with fistulas closed (the real feeding situation) showed that the amount of milk flavoured with the CS- taste was drunk in larger amounts (33$50 \%$ more) than the previous CS + flavoured milk. The effect was immune to extinction for at least three post-conditioning test trials. Similar results have been found in other

${ }^{9}$ Methodologically unfortunately, in the Johnson et al. procedure, the vomiting was followed by renewed ingestion so that no data are available of completed binge-purge cycle that ends with purgeing.

${ }^{10}$ See also Davidson (1993). Rats were trained to display a set of motor acts. Each motor act was acquired under a unique and fixed deprivation level. After training, insulin injections led the rats to display the motor act acquired during 24-hour deprivation. As a comparison, injections with the satiety hormone cholecystokinine led the rats to display the motor act which had been learned under 0 -hour deprivation. These observations are in line with the idea that an excess of insulin (or its physiological consequences) and deprivation may share a common interoceptive quality. 


\section{Chapter 9}

studies as well (see Le Magnen, 1992, ch. 6). For binge eating this might imply that oral stimulation by binge food ("CS"), reliably followed by disproportionally small nutrient absorption (because of vomiting), may lead to conditioned meal enlargement.

A final data source is conditioned desatiation research in rats. Booth $(1985 ; 1991)$ has advocated that meal size is under influence of expected post-meal signals of satiety (e.g. nutrients appearing in the blood). After repeatedly pairing a flavour with carbohydrate dense food (strongly satiating), Booth added this flavour to different foods and saw decreased meal size in rats. In contrast, a dramatic increase in meal size was found in rats when during a meal involving high caloric food, a flavour was introduced that had been previously associated with low post-ingestive satiety. This increased intake was coined "conditioned desatiation"11 (Booth, 1985; 1991; Le Magnen, 1992, ch. 6). For binge eaters, the taste of binge foods might become associated with relatively low satiation (due to vomiting relatively little is actually absorbed ${ }^{12}$ ). Hence, conditioned desatiation to binge cues might act to increase intake of typical binge foods.

Breaking the binge-deprivation association decreases binge eating This has become apparent from the results of a therapeutic procedure called binge-exposure with prevention of vomiting (Rosen \& Leitenberg, 1982; Leitenberg et al., 1984; Schmidt \& Marks, 1989). The procedure involves several sessions in which the patient ingests binge food past the point where the urge to vomit develops. However, the patient is prevented from vomiting and the therapist remains with the patient until the urge to vomit has declined. Several studies have reported that this procedure leads to important reductions of binge eating ${ }^{13}$ (Rosen \& Leitenberg, 1982; Leitenberg et al., 1984; Schmidt \& Marks, 1989;

11 Two critical remarks are in place here. A vast literature exists in which flavour CSs predicting bigh nutrient value (UCS) increase preference of the flavour (for a review see Sclafani, 1995). Just how to reconcile these robust findings with the data supporting conditioned desatiation constitutes an as yet unresolved issue. Second, conditioned desatiation certainly must have its limits. The implication that non-nutritive sweeteners, as used in diet drinks, should lead to an ever-increasing intake of these drinks, does not stand closer scrutiny (e.g. Jansen, 1994b).

12 There are at least four indications suggesting a modest intestinal absorption of nutrients from binge foods expelled by self-induced vomiting. Firstly, vomiting generally takes place soon after the binge, before the food has entered the small intestine. Secondly, when large amounts of foods are ingested, gastric emptying (i.e. the transit of foods from the stomach into the small intestine) slowens down (McHugh, 1983). Thirdly, rapid eating (such as especially bulimics of the purging type do (Rossiter et al., 1992; Walsh, 1993) with little chewing slowens down the speed with which ingested nutrients appear in the blood (Read et al, 1986). Fourthly, bulimic patients' gastric emptying of small amounts of food has been found to be slower than that of healthy individuals (Geliebter et al., 1992).

${ }^{13}$ Parenthetically, the surplus value of exposure with prevention of vomiting relative to cognitive behavioural treatments of binge eating has been the subject of continuing discussion (Agras et al., 1989; Leitenberg \& Rosen, 1989; Cooper \& Steere, 1995). 
Cooper \& Steere, 1995). Note that these results are contrary to predictions of CBE theory, because, according to CBE theory, substantial ingestion of binge foods would be equal to a conditioning trial in which binge cues (CS) are paired with bingeing (UCS; see section 2.2, this thesis). A common characteristic of this therapy and CBE inspired cue-exposure with binge prevention procedure (section 2.2.6) is that the unrolling of a stereotypic behaviour chain during the binge episode is thwarted. Although the rationale of the Rosen and Leitenberg procedure was derived from anxiety research (Leitenberg et al., 1984), it might be considered an example of extinction of a learned association between binge cues (or bingeing itself) ("CS") and post-binge deprivation state ("UCS"). Additional remarks If the mechanism of signalled post-binge deprivation indeed exists and contributes to binge eating, it might be enhanced by pre-binge deprivation. Repeated deprivation experience may induce learned increase in food intake to deprivation-related cues (Weingarten, 1985; Revusky \& Garcia, 1970). Theoretically, the classical conditioning terminology may not be entirely appropriate in this case: intake of certain foods (a response accompanied by sensory stimulation) is regarded as a cue (the CS), and postmeal deprivation (a state following active responses) is considered the UCS. It may be that the mechanism might be better described by regarding binge eating as an instrumental response, performed in anticipation of deprivation, i.e., an "economic" property of the feeding system that can be found in most animals (Davey, 1989, ch. 7). Because of the present lack of rigorous terminology, and for practical purposes (e.g. the modelling of binge eating; section 9.5.3), we will, until further notice, stick to the CS-UCS terminology derived from classical conditioning.

\subsection{FUTURE RESEARCH: PRACTICAL CONSIDERATIONS}

Three lab models of binge eating will be presented in this final section. As will be explained, the models serve two practical purposes. Firstly, to provide a framework for basic research on learning mechanisms in binge eating. Secondly, as procedures to establish "miniature binges" in the lab, to be utilized for pre-clinical research on the effectiveness of newly developed treatments.

\subsubsection{The necessity of modelling}

In chapter one, it was explained that experimental psychopathology involves the hypothesizing and investigation of mechanisms that underlie behavioural disorders. This is the fundamental approach. Additionally, a more practical mission of experimental 


\section{Chapter 9}

psychopathology research is (or rather, could be) the testing of new behavioural techniques proposed by the therapeutic or scientific community. Not unlike the screening of newly developed psychopharmaceuticals (Willner, 1991a), behavioural techniques might be tested for their preventive or curative value. But how might this be accomplished in practice? One method that will be proposed here is the development of "lab models". As explained in chapter one, "modelling" refers to the reliable production of behaviours in the lab that can be considered a "miniature" analogue of the psychopathology under study.

In this final section, three possible lab models of binge eating will be described: the cue reactivity model, the conditioned hypoglycaemia model, and the post-meal deprivation model. All rely on the theoretical CBE notion that binge eating is a learned, cue-driven, and self-perpetuating behaviour ${ }^{14}$. Each lab model requires fundamental, preparatory research (see section 1.1). First, some essential parameters of binge eating should be identified in patients (type $P$ research) and/or in specifically selected non-clinical subjects (type $Q$ research). Once type $Q$ experiments have demonstrated replicable responses related to binge eating, a potential lab model may be within reach. Modelling the disorder is not a final goal, but the beginning of the practical stage (Willner, 1991b): in subsequent type $\mathrm{R}$ research, one attempts to "treat" the lab bingeing using the new behavioural technique. If proven effective in this way, the behavioural technique might be passed on for use in clinical trials.

Importantly, a workable lab model of binge eating should include some core manipulation that produces a significant and reliable increase of craving and/or food intake. In more lively terms, the proof of a binge eating model, like the proverbial pudding, should be in the eating (Herman \& Polivy, 1980; Jansen, 1990), and interpretation of the effects of the basic manipulation should be straightforward and require no "harking" whatsoever ${ }^{15}$.

Note that the empirical data in the present thesis have not been very encouraging as to the feasibility of model development on the basis of $C B E$ principles. Thus, the three models presented next should also be considered as coordinated and ultimate attempts to test the validity of $\mathrm{CBE}$ related theorizing.

${ }^{14}$ Human models of binge eating, especially if inspired by learning theory, have been rare: the counterregulation model has dominated the field (see section 1.3). In general, models relevant for binge eating have been largely confined to animal research: they are stress models (Greeno \& Wing, 1994), nutritional reward models (Sclafani, 1995) and biochemical and surgical models of overeating (Montgomery, 1991).

${ }^{15}$ Harking = Hypothesizing After the Results are Known (van der Heijden, 1995). 


\subsubsection{Model I: the cue reactivity model of binge eating}

Backeround One interesting aspect of psychophysiological/cognitive cue reactivity is its possible use as a diagnostic index of relapse danger in treated patients. In basic research there are three facets of cue reactivity to be studied: its dependency on learning history, its occurrence after cue presentation, and its influence on food intake. A practical advantage of this type of approach in modelling is that demonstration of cue reactivity requires no painstaking learning procedures. For this reason cue reactivity experiments are sometimes referred to as "opportunistic" experiments (Glautier \& Tiffany, 1995) Type $\mathrm{P}$ research Comparing patients and controls; searching for parameters.

* Cognitive changes after cue presentation: Stroop food word interference has already been amply demonstrated in binge eaters (see Chapter 7); However, such interference has not yet been demonstrated in clinical subjects after exposure to cues, and/or before the onset of a binge ${ }^{16}$.

* Increased psychophysiological food cue reactivity should be demonstrated in binge eaters. Parenthetically, it might well turn out to be intrinsically impossible to disentangle appetitive and emotional factors in responses to food cues in bulimic patients (compare e.g. Niaura et al., 1988).

* It should be demonstrated that food cue presentation increases subsequent ad lib food intake in patients. This is relevant even if no sensitive psychophysiological cue reactivity parameters might be identified. It could also be investigated whether high reactivity to food cues influences food intake of the items itself or also generalizes to the food item itself, or generalizes to other foods as well (compare Cornell, Rodin \& Weingarten, 1989).

Type $\mathrm{Q}$ research Comparing non-clinical subjects with different learning history; withinsubject comparisons of reactivity to foods differing in habitual intake.

* Subjects should be selected on the basis of either scores on scales specifically tapping disinhibitive eating (e.g. the DIS, see chapter 8), or more rigorously, on the basis of explicit self-reports on eating habits. These might include frequency estimates of bingeing and specification of meal size and particular foods involved.

* Foods could be allocated to a small number categories according to the frequency and/or habitual amount that is ingested. Cue reactivity to food cues belonging to these

${ }^{16}$ If the urge-to-act explanation of Stroop interference (Chapter 7, this thesis) is valid, it might be interesting to use an adapted version (with weight concern/vomiting-related words) within the context of Rosen \& Leitenberg's binge food exposure procedure (section 9.4.2). In patients who have just eaten typical binge foods, such Stroop tests might be used to assess suddenly aroused weight concems and tendencies towards self-induced vomiting. 


\section{Chapter 9}

categories could be compared in a within-subjects design.

* Sensitive physiological parameters of food cue reactivity should be sought for.

* As in type P research, another next step is to assess the impact on intake of a subsequently offered food. Intake should be larger after cue-presentation than after no cue presentation.

* If possible, at least two separate sessions (one with and one without cue presentation) should be held, to ensure equal deprivation level during cue presentation. Care should then be taken that no intake neophobia appears (Chapter 3). A prerequisite would be balancing of the order of cue and non-cue sessions. Furthermore, as intake-neophobia appears to fade due to exposure to the lab environment (Chapter 3 ), it might also be considered to run one or several habituation sessions, before running the experimental sessions.

Type $\mathrm{R}$ research Establishing and using the cue reactivity model of binge eating.

* Core manipulation: cues are presented and food intake and craving measured. A within-subject design might be employed with non-clinical binge prone subjects. Responses to different categories of food cues (see under type $Q$ research), or between food cues and neutral or no cues are compared.

* Core results: food intake and craving should be larger after cues predicting large food intake, than after cues predicting small or no food intake.

* Using the model: once the model is established, practical questions about binge eating might be answered by introducing additional manipulations on the normal subjects (e.g. instructions, mood inductions, prolonged cue exposure, instructed eating patterns, homework), and an assessment of their impact on the lab-overeating produced by the core manipulation.

\subsubsection{Model II: the conditioned hypoglycaemia model of binge eating}

Background In contrast to the cue reactivity model, the conditioned hypoglycaemia model of binge eating requires an explicit learning procedure. A starting point for model development might be a procedure resembling Mityushov's (1954; chapter 4). Before describing the model, a practical consideration is in place here. Conditioning procedures like Mityushov's are laborious and time-consuming. Because, at this moment, the role of hypoglycaemia/hyperinsulinaemia in binge eating remains to be established (see section 9.3), top priority should be given to research which demonstrates reliable and significant (conditioned) hypoglycaemia or hyperinsulinaemia during fully-fledged binge attacks in patients. If these responses cannot be demonstrated, then hypoglycaemia/hyperinsulinaemia clearly do not tap the essence of binge eating and there would seem little point - at least for binge eating research - to make the extensive effort of modelling binge eating in 
humans by glycaemic conditioning. Alternatively, if binge eating attacks are indecd preceded by significant hypoglycaemia/hyperinsulinaemia, then a powerful model of binge eating might be within reach.

Type $\mathrm{P}$ research Studies in patients.

* Blood sugar and insulin levels should be monitored on line during binge attacks. The same should be done when, after exposure to binge foods, craving is high up. These measurements should preferably be carried out at patients' typical binge locations.

Type Q research Comparing subject groups with different learning history.

* Possible research would resemble cue reactivity research type II, but in this case blood sugar and insulin responses are measured. The Broberg and Bernstein (1989) study, which as far as the present author is aware, is the only convincing report of cephalic hypoglycaemia in a sample of healthy individuals (albeit dieting), should be replicated.

* The relationship between cephalic hypoglycaemia and subsequent food intake should be established.

Type $\mathrm{R}$ research Establishing and using the conditioned hypoglycaemia model of binge eating.

* Core manipulation: the unconditioned stimuli must involve a strong blood sugar rise. Conditioning with oral glucose has failed (Chapter 3). Effective conditioning with food would probably require even more trials, given the moderate glycaemic index of common foods (Brand Miller et al., 1995). The procedure used in the study by Mityushov (1954; appendix A) might offer more possibility and should be replicated. If the hypoglycaemic state could be conditioned, the procedure should first be extended to food. This could be done by introducing intake-related CS to the injection ritual, and measure ad lib food intake during the hypoglycaemic state.

* Core results: a robust binge eating model would exist if $\mathrm{CS}^{*}$ presentation were found to enhance craving and food intake (via hypoglycaemia), relative to a $\mathrm{CS}^{-}$or neutral situation, and/or unconditioned subjects.

* Using the model: see under the cue reactivity model type $\mathrm{R}$ research.

\subsubsection{Model III: the post-meal deprivation model of binge eating}

Background In section 9.4.2, an overview was given of data suggesting that meal size can be increased by associating meal cues with post-meal deprivation. These data suggests practical ways in which a lab analogue of bingeing in non-clinical subjects might be evoked after an explicit conditioning procedure.

Type $\mathrm{P}$ research Studies in patients.

* Data are needed on the probability relationship between bingeing and fasting in patients on the individual level. What is the probability that bingeing is followed by 


\section{Chapter 9}

prolonged fasting? Are there specific cues (i.e. certain foods, moods or circumstances) that predict whether binge eaters go on a prolonged fast after bingeing? Such data might be collected more systematically through the use of intake diaries.

* Similar data to that above must be acquired on the probability relationship between bingeing and self-induced vomiting within individuals.

* The short-term physiological consequences of self-induced vomiting after bingeing have received too little attention. To what extent does a metabolic state resembling deprivation occur after expelling large food quantities?

Type $O$ research Studies in non-clinical subjects.

* no strong suggestion can be made at this point.

Type $\mathrm{R}$ research Establishing and using the signalled deprivation model of binge eating.

* core manipulation: this procedure would consist of repeatedly pairing cues $\left(\mathrm{CS}^{+}\right)$with a "compound UCS" of a meal followed by an 8-9 hour deprivation. A within-subject design could be employed, comparing craving and food intake after $\mathrm{CS}^{+}$with no $\mathrm{CS}$ or a $\mathrm{CS}^{-}$. The $\mathrm{CS}^{-}$should be a signal for "no post-meal deprivation", for example by pairing the same meal followed by a period of 8-9 hours in which food is repeatedly ingested by subjects. The CSs could lie within the food itself (flavouring, colouring) or in the way it has to be ingested (typical room, small spoons; strange plates).

* Core results: after conditioning, ad lib ingestion of test food should be robustly larger after $\mathrm{CS}^{+}$than after $\mathrm{CS}^{-}$presentation.

* Using the model: see the cue reactivity model, type $\mathrm{R}$ research.

\section{Finis}






\section{REFERENCES}

A.P.A. (1994). Diagnostic and Statistical Manual of Mental Disorders 4th ed. Washington: American Psychiatric Association.

Abraham, S.F. \& Beumont, P.J.V. (1982). How patients describe bulimia or binge eating. Psychological Medicine 12, 625-635.

Abramson, L.Y. \& Seligman, M.E.P. (1977). Modeling psychopathology in the laboratory. In: J.D. Maser \& M.E. Seligman (Eds.), Psychopathology: Experimental Models, pp 1-26. San Francisco, W.H. Freeman.

Ádám, G. (1967). Interoception and Behaviour. Budapest: Hungarian Academy of Sciences.

Agras, W.S., Schneider, J.A., Arnow, B., Raeburn, S.D. \& Telch, C.F. (1989). Cognitive-behavioral and response prevention treatments for bulimia nervosa. Journal of Consulting and Clinical Psychology 57, 215-221.

American Psychiatric Association (1987). Diagnostic and Statistical Manual of Mental Disorders (3rd Revised. Ed.). Washington, DC: Author.

Anthony, B.J. (1985). In the blink of an eye: implications of reflex modification for information processing. Advances in Psychophysiology 1, 167-218.

Babkin, B.P. (1949). Pavlov: a Biography. Chicago/London: University of Chicago Press

Bantle, J.P., Laine, D.C., Castle, G.W., Thomas, J.W., Hoogwerf, B.J. \& Goetz, F.G. (1983). Post prandial glucose and insulin responses to meals containing different carbohydrates in normal and diabetic subjects. New England Journal of Medicine 309, 7-12.

Barnett, S.A. (1958). Experiments on "neophobia" in wild and laboratory rats. British Journal of Psychology 49, 195-201.

Beck, A.T, Rusch, A.J., Shaw, B.F. \& Emery, G. (1979). Cognitive Therapy of Depression. New York: Guilford Press.

Beebe, D.W. (1994). Bulimia nervosa and depression: a theoretical and clinical appraisal in light of the binge-purge cycle. British Journal of Clinical Psychology 33, 259-276.

Bellisle, F., Louis-Sylvestre, J. Demozay, F., Blsazy, D. \& Le Magnen, J (1983). Reflex insulin response associated to food intake in human subjects. Physiology and Behavior 31, 515-521.

Ben-Tovim, D.I., Walker, M.K., Fok, D. \& Yap, E. (1989). An adaptation of the Stroop Test for measuring shape and food concerns in eating disorders: a quantitative measure of psychopathology? International Journal of Eating disorders 8, 681-687.

Ben-Tovim, D.I. \& Walker, M.K. (1991). Further evidence for the Stroop Test as a quantitative measure of psychopathology in eating disorders. International Journal of Eating Disorders 10, $609-613$.

Berthoud, H.-R., Bereiter, D.A., Trimble, E.R., Siegel, E.G. \& Jeanrenaud, B. (1981). Cephalic phase, reflex insulin secretion: neuroanatomical and physiological characterization. Diabetologia $20,393-401$.

Beumont, P.J.V. (1995). The clinical presentation of anorexia and bulimia nervosa. In, K.D. Brownell \& Ch. G. Fairburn (Eds), Eating Disorders and Obesity: a Comprehensive Handbook, pp. 151-158. New York, London: Guilford Press.

Birch, L.L., McPhee, L., Sullivan, S. \& Johnson, S. (1989). Conditioned meal initiation in young children. Appetise 13, 105-113.

Blouin, A.G., Blouin, J.H., Braaten, J.T., Sarwar, G., Bushnik, T. \& Walker, J. (1991). Physiological and psychological responses to a glucose challenge in bulimia. International Journal of Easing Disorders 10, 285-296.

Blouin, A.G., Blouin, J., Bushnik, T., Braaten, J., Goldstein, C. \& Sarwar, G. (1993). A doubleblind placebo-controlled glucose challenge in bulimia nervosa: psychological effects. Biological Psychiarry 33, 160-168.

Booth, D.A. (1985). Food-conditioned eating preferences and aversions with interoceptive elements: conditioned appetites and satieties. Annals of the New York Academy of Sciences 443, 22-41. 


\section{References}

Booth, D.A. (1991). Protein- and carbohydrate specific cravings: neuroscience and sociology. In, M.I. Friedman, M.G. Tordoff and M.R. Kare (Eds.), Chemical Senses, Vol. 4: Appetile and Nurrition, pp. 261-276. New York: Marcel Dekker.

Boucsein, W. (1992). Electrodermal Activity. New York: Plenum Press.

Bouwmeester, D. (1995). Quantum Mechanics and Classical Optics. Thesis. Leiden: University of Leiden.

Bradizza, C.M., Stasiewicz, P.R. \& Maisto, S.A. (1994). A conditioning reinterpretation of cognitive events in alcohol and drug cue exposure. Journal of Behavior Therapy and Experimental Psychiatry 25, 15-22.

Bradley, M.M., Cuthbert, B.N. \& Lang, P.J. (1990). Startle reflex modification: emotion or attention? Psychophysiology 27, 513-522.

Brand Miller, J., Pang, E. \& Broomhead, L. (1995). The glycaemic index of foods containing sugars: comparison of foods with naturally-occurring vs. added sugars. British Journal of Nutrition $73,613-623$.

Briggs, S.R. \& Cheek, J.M. (1986). The role of factor analysis in the development and evaluation of personality scales. Journal of Personality 54, 106-148.

Broberg D.J., Bernstein, I.L. (1989). Preabsorptive insulin release in bulimic women and chronic dieters. Appetite 13, 161-169.

Brodsky, I.G. \& Devlin, J.T. (1994). Hormone and nutrient interactions. In, M.E. Shils, J.A. Olson \& M. Shike (Eds), Modern Nutrition in Health and Disease, 8th ed., 603-622. Philadelphia Baltimore, etc: Lea \& Febiger.

Bruce, B. \& Agras, W.S. (1990). Binge eating in females: a population-based investigation. International Journal of Eating Disorders 12, 365-373.

Bruce. D.G., Storlien, L.H., Furler, S.M. \& Chisholm, D.J. (1987). Cephalic phase metabolic responses in normal weight adults. Metabolism 36, 721-725.

Campfield, L.A. \& Smith, F.J. (1990a). Transient declines in blood glucose signal meal initiation. International Journal of Obesity 14 (suppl 3), 15-31.

Campfield, L.A. \& Smith, F.J. (1990b). Systemic factors in the control of food intake: evidence for patterns as signals. In E.M. Stricker (Ed), Handbook of Behavioral Neurobiology vol. 10: Neurobiology of Food and Fluid Intake, pp. 183-206.

Campfield, L.A., Smith, F.J., Rosenbaum, M. \& Geary, N. (1992). Human hunger: is there a role for blood glucose dynamics? Appetite 18, 244.

Campfield, L.A., Smith, F.J., Rosenbaum, M. \& Hirsch, J. (1996). Human eating: evidence for a physiological basis using a modified paradigm. Neuroscience and Biobehavioral Reviews 20, 133 . 137.

Cannon, W.B. (1939). The Wisdom of the Body 2nd ed. New York: W.W. Norton.

Carlson, H.E. \& Shah, J.H. (1989). Aspartame and its constituent amino acids, effects on prolactin, cortisol, growth hormone, insulin, and glucose in normal humans. American Journal of Clinical Nutrision 49, 427-432.

Casper, R.C., Pandy, G.N., Jaspan, J.B. \& Rubenstein, A.H. (1988). Hormone and metabolite plasma levels after oral glucose in bulimia and healthy controls. Biological Psychiarry 24, 663-674.

Catania, A.C. (1991). Glossary. In I.H. Iversen \& K.A. Lattal (Eds.), Experimental Analysis of Behavior, vol. 2. Amsterdam etc: Elsevier.

Channon, S., Hemsley, D. \& de Silva, P. (1988). Selective processing of food words in anorexia nervosa. British Journal of Clinical Psychology 27, 259-260.

Channon, S. \& Hayward, A. (1990). The effect of short-term fasting on processing of food cues in normal subjects. International Journal of Earing Disorders 9, 447-452.

Charnock, D.J.K. (1989a). A comment on the role of dietary restraint in the development of bulimia nervosa. British Journal of Clinical Psychology 28, 329-340.

Charnock, D.J.K. (1989b). Exercising restraint: a response to Polivy \& Herman. British Journal of 
Clinical Psychology 28, 343-346.

Childress, A.R, Ehrman, R., Rohsenow, D.J., Robbins, S.J. \& O'Brien, Ch.P., 1992. Classically conditioned factors in drug dependence. In J.H. Lowinson, P. Ruiz, R.B. Millman \& J.G. Langrod (Ed.), Substance Abuse: a Comprehensive Texibook, 2nd Ed. pp 56-69. Baltimore etc: Williams \& Wilkins.

Christensen, C.M. \& Navazesh, M. (1984). Anticipatory salivary flow to the sight of different foods. Appetite 5, 307-315.

Clark, D.M. (1983). On the induction of depressed mood in the laboratory: evaluation and comparison of the Velten and musical procedures. Advances in Behaviour Research \& Therapy 5, 27-49.

Collier, G. (1986). The dialogue between the house economist and the resident physiologist. Nutrition \& Behavior 3 9-26.

Comrey, A.L. (1978). Common methodological problems in factor analytic studies. Journal of Counseling and Clinical Psychology 46, 648-659.

Cools, J., Schotte, D.E. \& McNally, R.J. (1992). Emotional arousal and overeating in restrained eaters. Journal of Abnormal Psychology 101, 348-351.

Cooper, M.J., Anastasiades, P. \& Fairburn, C.G. (1992). Selective processing of eating-, shape-, and weight-related words in persons with bulimia nervosa. Journal of Abnormal Psychology 101, 352 . 355.

Cooper, M.J. \& Fairburn, C.G. (1993). Demographic and clinical correlates of selective information processing in patients with bulimia nervosa. International Journal of Eating Disorders 13, 109 116.

Cooper, P.J. (1995). Eating disorders and their relationship to mood and anxiety disorders. In, K.D. Brownell \& Ch. G. Fairburn (Eds), Eating Disorders and Obesiry: a Comprehensive Handbook, pp. 159-164. New York, London: Guilford Press.

Cooper, P.J. \& Steere, J. (1995). A comparison of two psychological treatments for bulimia nervosa: implications for models of maintenance. Behaviour Research \& Therapy 33, 875-885.

Cornell, C.E., Rodin, J. \& Weingarten, H. (1989). Stimulus-induced eating when satiated. Physiology \& Behavior 45, 695-704.

Cryer, Ph. (1992). Glucose homeostasis and hypoglycemia. In, J.D. Wilson \& D.W. Forster (Eds.), Williams Textbook of Endocrinology 8th ed., 1223-1253. Philadelphia etc.: W.B. Saunders.

Davey, G. (1989). Ecological Learning Theory. Londen, New York: Routledge.

Davidson, R.L. (1993). The nature and function of interoceptive signals to feed: toward integration of physiological and leaming perspectives. Psychological Review 100, 640-657.

Deutsch, R. (1974). Conditioned hypoglycemia: a mechanism for saccharin-induced sensitivity to insulin in the rat. Journal of Comparative and Physiological Psychology 86, 350-358.

Dimberg, U. (1987). Facial reactions, autonomic activity and experienced emotion: a three component model of emotional conditioning. Biological Psychology 24, 105-122.

Dritschel, B., Cooper, P.J. \& Charnock, D. (1993). A problematic counter-regulation experiment: implications for the link between dietary restraint and overeating. International Journal of Eating Disorders 13, 297-304.

Drummond, D.C., Tiffany, S.T., Glautier, S. \& Remington, B. (Eds.) (1995). Addictive Behaviour: Cue Exposure, Theory and Practice. Chichester etc: Wiley \& Sons.

Dworkin, B.R. (1989). Learning and functional utility. Behavioral and Brain Sciences 12, 139-141.

Dworkin, B.R. (1993). Learning and Physiological Regulation. Chicago, London: University of Chicago Press.

Edelberg, R. (1972) Electrodermal recovery rate, goal-orientation, and aversion. Psychophysiology 9, 512-520.

Eikelboom, R. \& Stewart, J. (1982). Conditioning of drug-induced physiological responses. Psychological Review 89, 507-528.

Elmore, D.K. \& de Castro, J.M. (1991). Meal patterns of normal, untreated bulimia nervosa and recove- 
red bulimic women. Physiology and Behavior 49, 99-105.

Fairburn, C.G. (1987). The definition of bulimia nervosa: guidelines for clinicians and research workers. Annals of Behavioral Medicine 9, 3-7.

Fairburn, C.G. \& Beglin, S.J. (1990). Studies of the epidemiology of bulimia nervosa. American Journal of Psychiarry 147, 401-408.

Fairburn, C.G., Cooper, P.J., Cooper, M.J., McKenna, F.P. \& Anastasiades, M. (1991). Selective information processing in bulimia nervosa. International Journal of Eating Disorders 10, 415-422.

Fairburn, C.G. \& Wilson, G.T. (1993). Binge eating: definition and classification. In, C.G. Fairburn \& G.T. Wilson (Eds.). Binge Eating: Nature, Assessment, and Treatment, 3-14. New York, London: Guilford Press.

Fairburn, C.G. (1995). Short-term psychological treatments for bulimia nervosa. In, K.D. Brownell \& Ch. G. Fairburn (Eds), Eating Disorders and Obesin: a Comprehensive Handbook, pp. 344-348. New York, London: Guilford Press.

Fehm-Wolfsdorf, G., Gnadler, M., Kern, W., Klosterhalfen, W., \& Kerner, W. (1993). Classically conditioned changes of blood glucose level in humans. Physiology and Behavior 54, 155-160.

Flaherty, C.F., Grigson, P.S. \& Brady, A. (1987). Relative novelty of conditioning context influences directionality of glycemic conditioning. Journal of Experimental Psychology 13, 144-149.

Förster, H., Haslbeck, M. \& Mehnert, H., 1972. Metabolic studies following the oral ingestion of different doses of glucose. Diabetes 21, 1102-1108.

Fridlund, A.J. (1979). Contour-following integrator for dynamic tracking of electromyographic data. Psychophysiology 16, 491-493.

Fridlund, A.J. \& Cacioppo, J.T. (1986). Guidelines for human electromyographic research. Psychophysiology 23, 567-589.

Furedy, J.J. (1989). Flights of teleological fancy about classical conditioning do not produce valid science or useful technology. Behavioral and Brain Sciences 12, 142-143.

Garcia, J., Lasiter, P.S., Bermudez-Rattoni, F. \& Deems, D.A. (1985). A general theory of aversion learning. Annals of the New York Academy of Sciences 443, 8-22.

Garfinkel, P.E. (1992). Evidence in support of attitudes to shape and weight as a diagnostic criterion of bulimia nervosa. International Journal of Eating Disorders, 4, 321-325.

Garner, D.M, Olmsted, M.P., Davis, R., Rockert, W., Goldbloom, D. \& Eagle, M. (1990). The association between bulimic symptoms and reported psychopathology. Insernational Journal of Earing disorders 9, 1-15.

Geliebter, A., Melton, P.M., McCray, R.S., Gallagher, D.R., Gage, D. \& Hashim, S.A. (1992). Gastric capacity, gastric emptying, and test-meal intake in normal and bulimic women. American Journal of Clinical Nutrition 56, 656-661.

Gerich, J.E., Charles, M.A., Grodsky, G.M. (1976). Regulation of pancreatic insulin and glucagon secretion. Annual Review of Physiology 38, 353-388.

Giduck, S.A., Threatte, R.M. \& Kare, M.R. (1987). Cephalic reflexes: their role in digestion and possible roles in absorption and metabolism. Journal of Nutrition 117, 1191-1196.

Girardier, L., Seydoux J. \& Campfield, L.A. (1976). Control of A and B cells in vivo by sympathetic nervous input and selective hyper- or hypoglycemia in dog pancreas. Journal of Physiology (Paris), $801-814$.

Glautier, S. (1994). Classical conditioning, drug cues and drug addiction. In, Ch. R. Legg \& D. Booth (Eds.), Appetite: Neural and Behavioural Bases, pp 165-192. Oxford etc, Oxford University Press.

Glautier, S. \& Tiffany, S.T. (1995). Methodological issues in cue reactivity research. In, D.C. Drum mond, S.T. Tiffany, S. Glautier \& B. Remington (Eds.), Addictive Behaviour: Cue Exposure Theory and Practice, 75-98. Chichester etc.: Wiley.

Goldfine, I.D., Abraira, C., Gruenewald, D. \& Goldstein, M.S. (1970). Plasma insulin levels during imaginary food ingestion under hypnosis. Proceedings of the Society for Experimental Biology and 
Medicine, 133, 274-276.

Green, M.W. \& Rogers, P.J. (1993). Selective attention to food and body shape words in dieters and restrained nondieters. International Journal of Eating Disorders 14, 515-517.

Greeno, C.G. \& Wing, R.R. (1994). Stress-induced eating. Psychological Bulletin 115, 444-464.

Grill, H.J., Berridge, K.C., Ganster, D.J., 1984. Oral glucose is the prime elicitor of preabsorptive insulin secretion. American Journal of Physiology 248, R88-R95.

Grilo, C.M, Shiffman, S. \& Wing, R.R. (1989). Relapse crises and coping among dieters. Journal of Consulting and Clinical Psychology 57, 488-495.

Grossman, S.P. (1986). The role of glucose, insulin and glucagon in the regulation of food intake and body weight. Neuroscience \& Biobehavioral Reviews 10, 295-315.

Guyton, A.C. (1991). Textbook of Medical Physiology, 8th ed. Philadelphia etc: Saunders.

Hadigan, C.M., Kissileff, H.R. \& Walsh, B.T. (1989). Patterns of food selection during meals in women with bulimia. American Journal of Clinical Nutrition 50, 759-766.

Hansen, A. \& de Haan, E. (1995). De behandeling van bulimia nervosa met cue-exposure; ervaringen uit de praktijk. Directieve Therapie 15, 279-291.

Hardy, R.N. (1981). Endocrine Physiology. London: Edward Arnold.

Heatherton, T.F., Herman, C.P., Polivy, J., King, G.A., \& McGree, S.T., 1988. The (mis)measure ment of restraint: an analysis of conceptual and psychometric issues. Journal of Abnormal Psychology 97, 19-28.

Hepburn, D.A. (1993). Symptoms of hypoglycaemia. In, B.M. Frier \& B.M. Fisher (Eds.), Hypoglycaemia and Diaberes: Clinical and Physiological Aspects, 93-103. London, etc.: Edward Arnold.

Herman, C.P. \& Mack, D. (1975). Restrained and unrestrained eating. Journal of Personaliry, 43. 647-660.

Herman, C.P., Polivy, J., Pliner, P., Threlkeld, J. \& Munic, D. (1978). Distractability in dieters and nondieters: an alternative view of "externality". Journal of Personaliry and Social Psychology 36, 536-548.

Herman, C.P. \& Polivy, J. (1980). Restrained eating. In, A.B. Stunkard (Ed)., Obesity, pp. 208-225. Philadelphia: Saunders.

Herman, C.P. \& Polivy, J. (1982). Weight change and dietary concern in the overweight: are they really independent? Appetite 3, 280-281.

Herman, C.P. \& Polivy, J. (1984). A boundary model for the regulation of eating. In A.B. Stunkard \& E.Stellar (Eds). Eating and its disorders, pp. 141-156. New York: Raven Press.

Herman, C.P., Polivy, J. \& Esses, V.M. (1987). The illusion of counter-regulation. Appetite 9, 161 169.

Herman, C.P. \& Polivy, J. (1988). Restraint and excess in dieters and bulimics. In K.M. Pirke, W. Vandereycken and D. Ploog (Eds.), The Psychobiology of Bulimia Nervosa, pp 33-41. Berlin etc: Springer-Verlag.

Herman, C.P. \& Polivy, J. (1990). From dietary restraint to binge eating: attaching causes to effects. Appetite 14, 123-125.

Hibscher, J.A. \& Herman, C.P. (1977). Obesity, dieting, and the expression of 'obese' characteristics. Journal of Comparative Physiology and Psychology 91, 374-380.

Hoek, H.W. (1993). Review of the epidemiological studies of eating disorders. International Review of Psychiarry 5, 61-74

Hoek, H.W. (1995). The distribution of eating disorders. In, K.D. Brownell \& Ch. G. Fairburn (Eds.), Easing Disorders and Obesiry: a Comprehensive Handbook, pp. 207-211. New York, London: Guilford Press.

Hoffer, L.J. (1988). Starvation. In, M.E. Shils \& V.R. Young (eds.). Modern Nutrition in Health and Disease, 7th ed., pp. 774-794. Philadelphia: Lea \& Febriger.

Hohlstein, L.A., Gwirtsman, H.E., Whalen, F. \& Enns, M.P. (1986). Oral glucose tolerance in bulimia. International Journal of Eating Disorders 5, 157-160. 


\section{References}

Horwitz, D.L., McLane, M. \& Kobe, P., 1988. Response to a single dose of aspartame or saccharin by niddm patients. Diabetes Care 11, 230-234.

Jansen, A. (1990). Binge earing: Notes and Data. Doctoral dissertation. Maastricht: University of Limburg.

Jansen, A. (1994a). The learned nature of binge eating. In Ch. R. Legg \& D.A. Booth (Ed.), Apperire: Neural and Behavioural Bases, pp 193-211. Oxford: Oxford University Press.

Jansen, A. (1994b). Notitie over het aanleren en veranderen van smaakpreferenties. Suikerstichting Nederland.

Jansen, A., Oosterlaan, J., Merckelbach, H. \& Van den Hout, M.A. (1988a). Non-regulation of food intake in restrained, emotional, and external eaters. Journal of Psychopashology and Behavioral Assessment 10, 345-354.

Jansen, A., Merckelbach, H., Oosterlaan, J., Tuiten, A. \& Van den Hout, M.A. (1988b). Cognitions and self-talk during food intake of restrained and unrestrained eaters. Behaviour Research and Therapy, 26, 393-398.

Jansen, A., Van den Hout, M.A., De Loof, C. Zandbergen, J. \& Griez, E. (1989). A case of Bulimia successfully treated by cue exposure. Journal of Behaviour Therapy and Experimental Psychiatry $20,327-332$.

Jansen, A., Van den Hout, M.A. \& Griez, E. (1990). Clinical and non-clinical binges. Behaviour Research and Therapy 28, 439-444.

Jansen, A. \& Van den Hout, M.A. 1991. On being led into temptation: "counterregulation" of dieters after smelling a "preload". Addictive Behaviors 5, 247-253.

Jansen, A., Merckelbach, H. \& Van den Hout, M.A. (1992a). Experimentele Psychopathologie. As sen, Maastricht: van Gorcum.

Jansen, A., Boon, B., Nauta, H. \& van den Hout, M.A. (1992b). Salivation discordant with hunger. Behaviour Research and Therapy 30, 163-166.

Jansen, A., Broekmate, J. \& Heymans, M. (1992c). Cue-exposure vs self-control in the treatment of binge eating: a pilot study. Behaviour Research and Therapy 30, 235-241.

Johnson, W.G. \& Wildman, H.E. (1983). Influence of external and covert food stimuli on insulin secretion in obese and normal persons. Behavioral Neuroscience 97, 1025-1028.

Johnson, W.G., Jarrell, M.P., Chupurdia, K.M. \& Williamson, D.A. (1994). Repeated binge/purge cycles in bulimia nervosa: Role of glucose and insulin. International Journal of Eating Disorders $15,331-341$.

Joravsky, D. (1989). Russinn Psychology: A Critical Appraisal. Oxford etc.: Blackwell.

Kaye, W.H., Gwirtsman, H.E. \& George, D.T. (1989). The effect of bingeing and vomiting on hormonal secretion. Biological Psychiatry 25, 768-780.

Kennedy, S.H., Katz, R., Neitzer, C.S., Ralevski, E. \& Mendlowitz, S. (1995). Exposure with response prevention treatment of anorexia nervosa bulimic subtype and bulimia nervosa. Behaviour Research and Therapy 33, 685-689.

Klajner, F., Herman, P.H., Polivy, J. \& Chhabra, R. (198I). Human obesity, dieting, and anticipatory salivation to food. Physiology and Behavior 27, 195-198.

Kostarczyk, E., 1986. Autonomic correlates of alimentary conditioned and unconditioned reactions in the dog. Journal of the Ausonomic Nervous System 817, 279-288.

Laberg, J.C., Wilson, G.T., Eldredge, K. \& Nordby, H. (1991). Effects of mood on heart rate reactivity in bulimia nervosa. International Journal of Eating Disorders 10, 169-178.

Lacey, J.H. \& Gibson, E. (1985). Controlling weight by purgation and vomiting: a comparative study of bulimics. Journal of Psychiarric Research 19, 337-341

Laessle, R.G., Tuschl, R.J, Kotthaus, B.C. \& Pirke, K.M. (1989). A comparison of the validity of three scales for the assessment of dietary restraint. Journal of Abnormal Psychology 98, 504-507.

Landis, C. \& Hunt, W.A. (1939). The Startle Pantern. New York: Farrar \& Rinehart.

Landsberg, L. \& Young, J.B. (1978) Fasting, feeding and regulation of the sympathetic nervous system. 
New England Journal of Medicine 298, 1295-1301.

Lang, P.J., Bradley, M.M. \& Cuthbert, B.N. (1990). Emotion, attention, and the startle reflex. Psychological Review 97, 377-398.

Lang, P.J., Greenwald, M.K., Bradley, M.M. \& Hamm, A.O. (1993). Looking at pictures: affective, facial visceral and behavioral reactions. Psychophysiology 30, 261-273.

Lavy, E., van den Hout, M. \& Amtz, A. (1993). Attentional bias and spider phobia: conceptual and clinical issues. Behaviour Research and Therapy 31, 17. 24.

Lavy, E. \& van den Hout, M. (1993). Attentional bias for appetitive cues: effects of fasting in normal subjects. Behavioural and Cognitive Psychotherapy 21, 297-310.

LeGoff, D.B. \& Spiegelman, M.N. (1987). Salivary response to olfactory food stimuli as a function of dietary restraint and body weight. Appetite 12, 83-94.

LeGoff, D.B., Leichner, P. \& Spigelman, M.N. (1988). Salivary response to olfactory stimuli in anorexics and bulimics. Apperite $11,15-25$.

Le Magnen, J. (1985). Hunger. Cambridge etc: Cambridge University Press.

Le Magnen, J. (1992). Neurobiology of Feeding and Nutrision. San Diego etc: Academic Press.

LeBlanc, J. (1992). Cephalic phase response to food and its effect on feeding behavior. In, G.H. Anderson and S.H. Kennedy (Eds), The Biology of Feast and Famine: Relevance to Eating Disorders, 4760. San Diego etc., Academic Press.

Leitenberg, H., Gross, J., Peterson, J. \& Rosen, J.C. (1984) Analysis of an anxiety model and the process of change during exposure plus response prevention treatment of bulimia nervosa. Behavior Therapy 15, 3-20.

Leitenberg, H. \& Rosen, J. (1989). Cognitive-behavioral therapy with and without exposure plus response prevention in treatment of bulimia nervosa: comment on Agras, Schneider, Arnow, Raeburn, and Telch. Journal of Consulting and Clinical Psychology 57, 776-777.

Louis-Sylvestre, J. (1976). Preabsorptive insulin release and hypoglycemia in rats. American Journal of Physiology 230, 56-60.

Louis-Sylvestre, J. \& Le Magnen J. (1983). Vagotomy abolishes the differential palatability of food. Appetite 4, 295-299.

Louis-Sylvestre, J. (1984). Meal size: role of reflexly induced insulin release. Journal of the Autonomic Nervous System 10, 317-324.

Lowe, M.R. (1993). The effects of dieting on eating behavior: a three-factor model. Psychological Bulletin 114, 100-121.

Lucas, F., Bellisle, F. \& Di Maio, A. (1987). Spontaneous insulin fluctuations and the preabsorptive insulin response to food ingestion in humans. Physiology and Behavior 40, 631-636.

MacRae, J.R., Scoles, M.T. \& Siegel, S. (1987). The contribution of Pavlovian conditioning to drug tolerance and dependence. British Journal of Addicrion 82, 371-380.

Mahamedi, F. \& Heatherton, T.F. (1993). Effects of high calorie preloads on selective processing of food and body shape stimuli among dieters and nondie ters. International Journal of Eating Disorders 13, 305-314.

Marcus, M.D. (1993). Binge eating in obesity. In, C.G. Fairburn \& G.T. Wilson (Eds.). Binge Earing: Nature, Assessment, and Treatment, 77-96. New York, London: Guilford Press.

Marcus, M.D. (1995). Binge eating and obesity. In, K.D. Brownell \& Ch. G. Fairburn (Eds), Eating Disorders and Obesity: a Comprehensive Handbook, pp. 441-444. New York, London: Guilford Press.

Matysiak, J. \& Green, L. (1984). On the directionality of classically-conditioned glycemic responses. Physiology and Behavior 32, 5-9.

McDonald, R.P. \& Mulaik, S.A. (1979). Determinacy of common factors: a nontechnical review. Psychological Bulletin 86, 297-306.

McHugh, P.R. (1983). The control of gastic emptying. In, J.G. Kral, T.L. Powley \& C.McC. Brooks (Eds.), Vagal Nerve Function: Behavioural and Methodological Considerations, 221-231. 


\section{References}

Amsterdam, etc.: Elsevier.

McNally, R.J. (1994). Panic Disorder: a Critical Analysis. New York etc.: Guilford Press.

Miller, R.R., Barnet, R.C. \& Grahame, N.J. (1995). Assessment of the Rescorla-Wagner Model. Psychological Bullerin 117, 363-386.

Mitchell, J.E., Hatsukami, D., Eckert, E.D. \& Pyle, R.L. (1985). Characteristics of 275 patients with Bulimia. American Journal of Psychiatry 142, 482-485.

Montgomery, A.M.J. (1991). Animal models of eating disorders. In, P. Willner (Ed.), Behavioural Models in Psychopharmacology, pp. 177-214. Cambridge etc.: Cambridge University Press.

Moyer, A., Rodin, J. \& Cummings, N. (1993). Cephalic phase insulin release in Bulimia. International Journal of Eating Disorders 14, 331-339.

Mityushov, M.I. (1954). Conditioned reflex insulin secretion. Journal of Higher Nervous Activiry (in Russian) 4, 206-212.

Niaura, R.S., Rohsenow, D.J., Binkoff, J.A., Monti, P.M., Pedraza, M. \& Abrams, D.B. (1988). Relevance of cue reactivity to understanding alcohol and smoking relapse. Journal of Abnormal Psychology 97, 133-152.

Nicolaĩdis, S. (1977). Sensory-neuroendocrine reflexes and their anticipatory and optimizing role on metabolism. In M.R. Kare and O. Maller (Eds.), The Chemical Senses and Nurrition, 123-143. New York etc.: Academic Press.

Niijima, A. (1989). Nervous regulation of metabolism. Progress in Neurobiology 33, 135-147.

Nunnally, J.C. (1978). Psychometric Theory, 2nd ed. New York etc. McGraw-Hill.

Oettle, G.J., Emmett, P.M. \& Heaton, K.W. (1987). Glucose and insulin responses to manufactured and whole-food snacks. American Journal of Clinical Nutrition 45, 86-91.

Ogden, J. (1993). The measurement of restraint: confounding success and failure? International Journal of Ealing Disorders 13, 69-76.

Ogden, J. \& Greville, L., (1993). Cognitive changes to preloading in restrained and unrestrained eaters as measured by the Stroop task. International Journal of Eating Disorders 14, 185-195.

Overduin, J. (1993). Het voordeel van de schrik: over het onvolledig gebruik van de reflex in emotieonderzoeken. De Psycholoog 28, 193-200.

Overduin, J. \& Jansen, A. (1996). A new scale for use in non-clinical research into disinhibitive eating. Personaling and Individual Differences, in press.

Overduin, J., Jansen, A. \& Eilkes, H. (1996). Cue reactivity to food- and body stimuli in restrained and uarestrained eaters. Addictive Behaviors in press.

Overduin, J. \& Jansen, A. submitted. Conditioned insulin and blood sugar responses in bumans in relation to binge eating.

Pavlov, I.P. (1897/1910). The Work of the Digestive Glands. London: Griffin.

Peterson, C., Maier, S.F. \& Seligman, M.E.P., 1993. Learned Helplessness: a Theory for the Age of Personal Control. New York, Oxford: Oxford University Press.

Pirke, K.M. (1995). Physiology of Bulimia Nervosa. In, C.G. Fairburn \& G.T. Wilson (Eds.). Binge Eating: nature, assessment, and trearment, 261-265. New York, London: Guilford Press.

Polivy, J. \& Herman, C.P. (1985). Dieting and binging: a causal analysis. American Psychologist 40, 193-201.

Polivy, J., Heatherton, T.F. \& Herman, C.P. (1988). Self-esteem, restraint, and eating behavior. Journal of Abnomal Psychology 97, 354-356.

Polivy, J. \& Herman, C.P. (1989). Dietary restraint and binge eating: Response to Charnock. British Journal of Clinical Psychology 28, 341-343.

Polonsky, K.S., Given, D., Hirsch, L. et al. (1988). Quantitative study of insulin secretion and clear ance in normal and obese subject. Journal of Clinical Investigation 81, 435-441.

Polonsky, K.S. \& Rubenstein, A.H., 1984. C-peptide as a measure of the secretion and hepatic extraction of insulin. Diabetes 33, 486-494.

Powell, J., Gray, J. \& Bradley, B. (1993). Subjective craving for opiates: evaluation of a cue exposure 
protocol for use with detoxified opiate addicts. British Journal of Clinical Psychology 32, 39-53.

Powley, T.L. \& Berthoud, H-R. (1985). Diet and cephalic phase insulin responses. American Journal of Clinical Nutrition 42, 991-1002.

Razran, R. (1961). The observable unconscious and the inferable conscious in current Soviet psychophysiology. Psychological Review 68, 81-147.

Read, N.W., Welch, I. McL., Austen, C.J., Barnish, C. et al. (1986). Swallowing food without chewing: a simple way to reduce postprandial glycaemia. British Journal of Nutrition 55, 43-47.

Rescorla, R.A. (1967). Pavlovian conditioning and its proper control procedures. Psychological Review $74,71-80$.

Revusky, S. \& Garcia, J. (1970). Leamed associations over long delays. In, G.H. Bower (Ed), The Psychology of Learning and Motivation vol 4., pp. 1-84. New York, London: Academic Press.

Robbins, S.J. \& Ehrman, R.N. (1992). Designing studies of drug conditioning in humans. Psychopharmacology $106,143-153$.

Rodin, J., Bach, J., Peranini, E. \& De Fronzo, F. (1985). Effect of insulin and glucose on feeding behavior. Metabolism 34, 827-833.

Rodin, J., Reed, R. \& Jamner, L. (1988). Metabolic effects of fructose and glucose: implications for food intake. American Journal of Clinical Nutrition 47, 683-689.

Rodin, J. (1990). Comparative effects of fructose, aspartame, glucose, and water preloads on calorie and macronutrient intake. American Journal of Clinical Nutrition, 51, 428-435.

Rogers, P.J. \& Hill, A.J. (1989). Breakdown of dietary restraint following mere exposure to food stimuli: interrelationships between restraint, hunger, salivation, and food intake. Addictive Behaviors 14, 387-397.

Rosen, J.C. \& Leitenberg, H. (1982). Bulimia Nervosa, treatment with exposure and response prevention. Behavior Therapy 13,117-124.

Rosen, J.C., Leitenberg, H., Fisher, C. \& Khazam, C. (1986). Binge-eating episodes in bulimia nervosa: the amount and type of food consumed. International Journal of Eating Disorders 5, 255-267.

Rosenberg, M. (1965). Society and the Adolescent Self-image. Princeton, N.J.: Princeton University Press.

Rossiter, E.M, \& Agras, W.S. (1990). An empirical test of the DSM-III-R definition of binge. Inter national Journal of Eating Disorders 9, 513-518.

Rossiter, E.M., Agras, W.S., Telch, C.F. \& Bruce, B. (1992). The eating patterns of non-purging bulimic subjects. International Journal of Eating Disorders 11, 111-120.

Ruderman, A.J. (1983). The Restraint Scale: a psychometric investigation. Behaviour Research and Therapy 21, 253-258.

Ruderman, A.J. (1985). Dysphoric mood and overeating: a test of restraint theory's disinhibition hypothesis. Journal of Abnormal Psychology, 94, 78-85.

Ruderman, A. J. (1986). Dietary restraint: a theoretical and empirical review. Psychological Bulletin $99,247-262$.

Russell, G. (1979). Bulimia nervosa: an ominous variant of anorexia nervosa. Psychological Medicine 9, 429-448.

Sapolsky, R.M. (1994). Why Zebras Don't Get Ulcers: A Guide To Stress, Stress-related Diseases, and Coping. New York: Freeman \& Co.

Schmidt, U. \& Marks, I.M. (1988). Cue exposure to food plus response prevention of binges for bulimia: a pilot study. International Journal of Eating Disorders 7, 663-672.

Schmidt, U. \& Marks, I.M. (1989). Exposure plus prevention of bingeing vs. exposure plus prevention of vomiting in bulimia nervosa: a crossover study. Journal of Nervous and Menial Disease 177, 259-266.

Schultz-Gambard, E. (1988). Indikatoren von Hunger: Psychophysiologische Untersuchung zur Wirkung einer 24-stündigen Nahrungsdeprivation. Doctoral Dissertation. Bielefeld: University of Bielefeld.

Schwartz, B. \& Robbins (1995). Psychology of Learning \& Behavior, 4th ed. New York etc: Norton.

Schweiger, U., Poellinger, J., Laessle, RF., Wolfram, G., Fichter, M.M. \& Pirke K.-M. (1987). 


\section{References}

Altered insulin response to a balanced test meal in bulimic patients. International Journal of Eating Disorders 6, 551-556.

Sclafani, A. (1995). How food preferences are learned: laboratory animal models. Proceedings of the Nutrition Society 54, 419-427.

Service, F.J. (1995). Hypoglycemic disorders. New England Journal of Medicine 332, 1144-1152.

Shively, C.A., Apgar, J.L. \& Tarka, S.M. (1986). Postprandial glucose and insulin responses to various snacks of equivalent carbohydrate content in normal subjects. American Journal of Clinical Nurrition 43, 335-342.

Siegel, S. (1972). Conditioning of insulin-induced glycemia. Journal of Compararive and Physiological Psychology 78, 233-241.

Siegel, S. (1983). Classical conditioning, drug tolerance and drug dependence. In, R. Smart, F. Glaser, Y. Israel, H. Kalant, R. Popham, and W. Schmidt (Eds.), Research Advances in Alcohol and Drug Problems, pp 207-246. New York: Plenum Press.

Siegel, S. (1989). Pharmacological conditioning and drug effects. In, A.J. Goudie \& M.W. EmmettOglesby (Eds.), Psychoactive Drugs: Tolerance and Sensitization, pp 115-180. Clifton, New Jersey: Humana Press.

Siegel, S. \& Nettleton, N. (1970). Conditioning of insulin-induced hyperphagia. Journal of Comparative and Physiological Psychology 72, 390-393.

Simon, C., Schlienger, J.L, Sapin, R. \& Imler, M. (1986). Cephalic phase insulin secretion in relation to food presentation in normal and overweight subjects. Physiology and Behavior 36, 463-469.

Sjōstrōm, L., Garellick, G., Krotkiewski, M. \& Luyckx, A. (1980). Peripheral insulin in response to the sight and smell of food. Metabolism 29, 901-909.

Steffens, A.B., Strubbe, J.H., Scheurink, A.J.W. \& Balkan, B. (1991). Neuroendocrine activity during food intake modulates secretion of the endocrine pancreas and contributes to the regulation of body weight. In: M.I. Friedman, M.G. Tordoff and M.R. Kare (Eds.), Chemical Senses Vol. 4: Apperite and Nutrition, pp 405-426. New York: Marcel Dekker.

Strubbe, J.H. (1992). Parasympathetic involvement in rapid meal-associated conditioned insulin secretion in the rat. American Journal of Physiology 263, R615-R618.

Strubbe, J.H. \& Steffens, A.B. (1975). Rapid insulin release after ingestion of a meal in the unanes thetized rat. American Journal of Physiology 229, 1019-1022.

Strubbe, J.H. \& Steffens, A.B. (1993). Neural control of insulin secretion. Hormones and Metabolic Research 25, 507-512.

Stunkard, A.J. \& Messick, S. (1985). The Three-Factor Eating Questionnaire to measure dietary restraint, disinhibition and hunger. Journal of Psychosomatic Research 29, 71-83.

Teff, K.L., Mattes, R.D. \& Engelman, K. (1991). Cephalic phase insulin release in normal weight males: verification and reliability. American Journal of Physiology 261, E430-E436.

Teff, K.L., Levin, B.E. \& Engelman, K. (1993). Oral sensory stimulation in men: effects on insulin, C-peptide, and catecholamines. American Journal of Physiology 265, R1223-R1230.

Teff, K.L., Devine, J., Engelman, K. (1995). Sweet taste: effect on cephalic phase insulin release in men. Physiology and Behavior 57, 1089-1095.

Thompson, J.P., Palmer, R.L. \& Petersen, S.A. (1988). Is there a metabolic component to counter regulation? International Journal of Eating Disorders 7, 307-319.

Tiffany, S.T. (1995a). Potential functions of classical conditioning in drug addiction. In, D.C. Drummond, S.T. Tiffany, S. Glautier \& B. Remington (Eds.), Addictive Behaviour: Cue Exposure Theory and Practice, pp 47-71. Chichester etc: Wiley.

Tiffany, S.T. (1995b). The role of cognitive factors in reactivity to drug cues. In, D.C. Drummond, S.T. Tiffany, S. Glautier \& B. Remington (Eds.), Addictive Behaviour: Cue Exposure Theory and Practice, pp 137-165. Chichester etc: Wiley.

Tobin, D.L., Johnson, C, Steinberg, S., Staats, M. \& Dennis, A.B. (1991). Multifactorial assessment of bulimia nervosa. Journal of Abnormal Psychology 100, 14-21. 
Tse, Th. F., Clutter, W.E., Shah, S.D., Miller, J. Ph. \& Cryer, Ph. E., 1983. Neuroendocrine responses to glucose ingestion in man. Journal of Clinical Investigation 72, 270-277.

Turkkan, J.S. (1989). Classical conditioning: the new hegemony. Behavioral and Brain Sciences 12 , 121-179.

Turner, M.St.J., Foggo, M., Bennie, J., Carroll, S., Dick, H. \& Goodwin, G.M. (1991). Psychological, hormonal and biochemical changes following carbohydrate bingeing: a placebo controlled study in bulimia nervosa and matched controls. Psychological Medicine 21, 123-133.

Tuschl, R.J. (1990). From dietary restraint to binge eating: some theoretical considerations. Apperite 14, 105-109.

Van Der Heijden, P.G.M. (1995). Hoe te leven met statistiek. Nederlands Tijdschriff voor de Psychologie $50,134-146$.

Van Het Reve, K. (1979). Een Dag uit het Leven van de Reuzenkoeskoes. Amsterdam: G.A. van Oorschot.

Van Strien, T., Frijters, J.E.R., Bergers, G.P.A. \& Defares, P.B. (1986). The Dutch Eating Behaviour Questionnaire (DEBQ) for assessment of restrained, emotional, and external eating behavior. International Journal of Eating Disorders 2, 295-315.

Van Vort, W. \& Smith, G.P. (1987). Sham feeding experience produces a conditioned increase of meal size. Appetite 9, 21-29.

Velden, M \& Graham, F.K. (1988). Depicting heart rate over real time: two procedures that are mathematically identical. Journal of Psychophysiology 2, 291-292.

Venables, P.H. (1991). Autonomic activity. Annals of the New York Academy of Sciences 620, 191207.

Walsh, B.T. (1992). Diagnostic criteria for eating disorders in DSM-IV: work in progress. Internasional Journal of Eating Disorders, 4, 301-304.

Walsh, B.T. (1993). Binge eating in bulimia nervosa. In: C.G. Fairburn \& G.T. Wilson (Eds), Binge Eating: Nature, Assessment, and Treatment, pp 37-49. New York, London: Guilford Press.

Wardle, J. (1980). Dietary restraint and binge eating. Behavioural Analysis and Modification 4, 201 209.

Wardle, J. (1986). The assessment of restrained eating. Behaviour Research and Therapy 24, 213-215.

Wardle, J. (1990). Conditioning processes and cue exposure in the modification of excessive eating. Addictive Behaviors 15, 387-393.

Wardle, J. \& Beinart, H. (1981). Binge eating: a theoretical review, British Journal of Clinical Psychology $20,97-109$.

Webber, J. \& MacDonald, I.A. (1993). Metabolic actions of catecholamines in man. Bailliere's Clinical Endocrinology and Metabolism 7, 393-413.

Weingarten, H.P. (1984). Meal initiation controlled by learned cues: basic behavioral properties. Appetise $5,147-158$.

Weingarten, H.P. (1985). Stimulus control of eating: implications for a two-factor theory of hunger. Appetite 6, 387-401.

Weingarten, H.P., Hendler, R. \& Rodin, J. (1988). Metabolism and endocrine secretion in response to a test meal in normal-weight bulimic women. Psychosomatic Medicine 50, 273-285.

Weingarten, H.P. \& Elston, D. (1990). The phenomenology of food cravings. Appetile 15, 231-246.

Westenhoeffer, J. (1991). Dietary restraint and disinhibition: is restraint a homogeneous construct? Appetite 16, 45-55.

Westenhoeffer, J., Broeckmann, P., Muench, A-K. \& Pudel, V. (1994). Cognitive control of eating behaviour and the disinhibition effect. Appetite 23, 27-41.

Wilde, G.J.S. (1977). Trait description and measurement by personality questionnaires. In, R.B. Cartell \& R.M. Dreger (Eds.), Handbook of Modern Personality Theory, 69-103. New York, etc: Wiley \& Sons.

Williams, R.A. (1968). Effects of repeated food deprivations and repeated feeding tests on feeding 


\section{References}

behavior. Journal of Comparative and Physiological Psychology 65, 222-226.

Willner, P. (1991a). Behavioural Models in Psychopharmacology: Theoretical, Industrial and Clinical Perspectives. Cambridge etc: Cambridge University Press.

Willner, P. (1991b). Methods for assessing the validity of animal models of human psychopathology. In, A.A. Boulton, G.B. Baker \& M.T. Martin-Iverson (Eds.), Animal Models in Psychiarry vol. I. , 1-23. Clifton, N.J.: Humana Press.

Woods, S.C. (1976). Conditioned hypoglycemia. Journal of Comparative and Physiological Psychology $90,1164-1168$.

Woods, S.C. (1981). Abstract from discussion with H.-R. Berthoud. Diabetologia 20, 401.

Woods, S.C. (1983). Conditioned hypoglycemia and conditioned insulin secretion. Advances in Metabolic Disorders 10, 485-495.

Woods, S.C. (1990). On blood glucose and eating. International Journal of Obesity 14 (suppl 3), $33-$ 34.

Woods, S.C. (1991). The eating paradox: how we tolerate food. Psychological Review 98, 488-505.

Woods, S.C. \& Kulkosky, P.J. (1976). Classically conditioned changes of blood glucose level. Psychosomatic Medicine 38, 201-219.

Woods, S.C., Makous, W. \& Hutton, R.A. (1969). Temporal parameters of conditioned hypoglycemia. Journal of Comparative and Physiological Psychology 69, 301-307.

Woods, S.C. \& Shogren, R.E. (1972). Glycemic responses following conditioning with different doses of insulin in rats. Journal of Comparative and Physiological Psychology, 81, 220-235.

Woods, S.C., Vasselli, J.R., Kaestner, E., Szakmary, G.A., Milburn, P. \& Vitiello, M.V. (1977). Conditioned insulin secretion and meal feeding in rats. Journal of Comparative and Physiological Psychology 91, 128-133.

Woods, S.C. \& Strubbe, J.H. (1994). The psychobiology of meals. Psychonomic Bulletin \& Review 1, $141-155$.

Wooley O.W., Wooley S.C. \& Williams B.S. (1976). Salivation as a measure of appetite: studies of the anorectic effects of calories and amphetamine. In, D. Novin, W. Wyrwicka \& G.A. Bray, Hunger: Basic Mechanisms and Clinical Implications, ppn 421-429).

Zamble, E. (1973). Augmentation of eating following a signal for feeding in rats. Learning and Motivation 4, 138-147. 


\section{Appendix}

\section{TRANSLATION ${ }^{1}$ OF M.I. MTTYUSHOV ${ }^{2}$ (1954) \\ "CONDITIONED REFLEX SECRETION OF INSULIN"}

From the viewpoint of today's Pavlovian physiology, the internal secretory glands form a powerful lever which enables the nervous system to change all physiological functions over a broad range. The central nervous system regulates various functions of the organism, a process that not only involves nervous impulses through specific pathways to the organs, but also a hormonal, viz. humoral link.

The mechanism by which endocrine glands participate in the nervous regulation of physiological functions, has until now been scantily studied. The main reason for this lies in the fact that until recently, even in the Soviet Union, a majority of the endocrine physiologists has adopted a methodologically unsound approach towards the study of internal secretory glands.

The internal glands were considered in isolation from the nervous system and ascribed an autonomous and often predominant role in physiological regulation. K.M. Bykov (2) in his speech at the plenary session of the Academy of Sciences of the USSR and the Academy of Medical Sciences, dedicated to the problems in Pavlovian physiological studies has stated: "In recent years, the study of hormones has secluded itself from general physiology and pathology into the specific discipline of endocrinology. The consequent isolation of specific subproblems from the more general study of organisms has lead many endocrinologists and medics to the erroneous conclusion that the various hormones play a self-supporting, autonomous role in the life of the organism".

After the plenary session the attention of many researchers was drawn towards the study of nervous regulation of endocrine glands. The study of these issues opens new vistas for the understanding of the mechanisms as well as the significance of the various glandular contributions to the physiological regulation in the organism.

Up until now the nervous regulation of the endocrine glands has been very scantly investigated. In the literature one can find a large number of facts about nervous regulation of the pituitary, the adrenal cortex as well as of the thyroid gland. The nervous regulation

\footnotetext{
'Originally published as: Матошов, М. И. (1954). Усповнорефлекторнан инкреция инсулнна. Журвал Высшеа Нервнов Деятельвостн 4, 206-212. Translation: Joost Overduin, Limburg University, Maastricht, The Netherlands; Prof. Gyōrgy Ádám, Eōtvōs University, Budapest, Hungary. About a dozen idioms have been adopted from an earlier translation by L.J. Shein. Prof. Shepard Siegel, McMaster University, Hamilton, Ontario is acknowledged for providing us with this translation.
}

${ }^{2}$ M.I. Mityushov, Laboratory of Nervous Regulation of Endocrine Functions, I.P. Pavlov Physiological lnstitute, USSR Academy of Sciences. 
of other endocrine glands, specifically the pancreatic islets, has until now received considerably less attention. Different authors have made quite contradictory assertions on this subject.

The insular apparatus of the pancreas plays an exceptionally important role in the regulation of carbohydrate metabolism. That is why the study of the nervous regulation of this activity is of great interest. The purpose of our study is an investigation of the cortical regulation of insulin secretion.

In the literature we found very few data on the cortical regulation of the insular apparatus of the pancreas. Observations by many clinical scientists suggest a role of psychic trauma in the development of diabetes in humans. As an example, the case described by B.G. Baranov can be quoted, who observed the development of severe form of diabetes in a woman who had suffered a nervous breakdown. Moreover, experimental research by N.S. Sedina (7) demonstrated a conditioned hypoglycemia to subcutaneous and intraperitoneal injections of saline, following previous repeated glucose administration through subcutaneous and intraperitoneal injections. She attributes the development of hypoglycemia to a conditioned increase of insulin production. Her attempt to induce conditioned hypoglycemia through intravenous glucose injections did not succeed however.

In our research we used the conditioned reflex method likewisely. The experiments were carried out on 4 dogs as well as on 9 human subjects. The unconditioned stimulus in our experiment was a sharp change in blood sugar following the intravenous glucose injection. As is well-known, a change in blood sugar level is an adequate stimulus for the insular apparatus including all further mechanisms regulating blood sugar level. In our opinion, the intravenous glucose injection appears to be the most rational means of influencing the insular apparatus, because intravenous injections allow for precise adjustments of the stimulus intensity (glucose instantaneously enters the blood stream in a concentration unhampered by a slow rate of absorption such as seen after subcutaneous and intraperitoneal injections.

The absence of conditioned reflex hypoglycemia in the study of N.S. Sedina (7) who used intravenous glucose injections as a UCS, does not seem crucial to the issue of whether conditioned reflex hypoglycemia is possible. Sedina conducted her experiments in two dogs. In one of them, a total of 10 trials was run. The CS was paired with the UCS merely in 7 cases within a two-month period. In the second dog, 9 trials were run in which CS and US were paired only 6 times in the course of 11 days. Clearly, after such a small number of pairings it would be unfeasible to conclude the impossibility of producing a conditioned reflex.

The conditioned stimulus in our study consisted of the intravenous injection procedure as well as the circumstances under which it took place (the glucose was injected regularly at 
Table A.1. Control trials (saline injections) in dogs

Data (1952)

Number of before the injection previous saline (2 measurements with injections $10 \mathrm{~min}$. in between)

$$
\text { minutes after the saline injection }
$$

$\begin{array}{lllll}5 & 15 & 30 & 60 & 90\end{array}$

Dog Baikal

11 III

18 III

22 III

27 III

3 IV

9 IV

$\begin{array}{lllll}99 & 102 & 102 & 97 & - \\ 107 & 103 & 105 & 96 & 98 \\ 94 & 89 & 89 & 91 & 89 \\ 103 & 108 & 108 & 106 & 99 \\ 92 & 89 & 89 & 91 & 94 \\ - & 88 & 88 & 85 & 83\end{array}$

$97: 97$
$94 ; 96$
$91 ; 89$
$103 ; 105$
$92 ; 91$
$85 ; 83$

$74 ; 74$

$77 ; 77$

$70 ; 72$

Dog Dik

$\begin{array}{llllllll}8 \mathrm{I} & 2 & 74 ; 74 & 76 & 76 & 80 & 80 & 80 \\ 10 \mathrm{I} & 3 & 77 ; 77 & 75 & 77 & 75 & 75 & 75 \\ 12 \mathrm{I} & 5 & 70 ; 72 & 74 & 74 & 77 & 77 & 79\end{array}$

Table A.2. Conditioned changes in blood sugar level after repeated glucose injections

\begin{tabular}{|c|c|c|c|c|c|c|c|}
\hline \multirow{3}{*}{ Data (1952) } & \multicolumn{7}{|c|}{ BLOOD SUGAR LEVEL $(\mathrm{mg} / \mathrm{dl})$} \\
\hline & \multirow{2}{*}{$\begin{array}{l}\text { Number of } \\
\text { previous glu- } \\
\text { cose injections }\end{array}$} & \multirow{2}{*}{$\begin{array}{l}\text { before the injection } \\
\text { ( } 2 \text { measurements with } \\
10 \text { min. in between) }\end{array}$} & \multicolumn{5}{|c|}{ minutes after the saline injection } \\
\hline & & & 5 & 15 & 30 & 60 & 90 \\
\hline \multicolumn{8}{|l|}{ Dog Baikal } \\
\hline $9 \mathrm{IV}$ & 8 & $83 ; 83$ & 82 & 80 & 82 & 83 & 87 \\
\hline $15 \mathrm{IV}$ & 14 & $96 ; 96$ & 94 & 85 & 87 & 92 & 90 \\
\hline $22 \mathrm{~V}$ & 16 & $87 ; 92$ & 79 & 85 & 87 & 88 & 90 \\
\hline $3 \mathrm{VI}$ & 20 & $94 ; 95$ & 92 & 97 & 99 & 97 & 92 \\
\hline $10 \mathrm{VI}$ & 22 & $95 ; 97$ & 91 & 93 & 99 & 100 & - \\
\hline $19 \mathrm{VI}$ & 24 & $97 ; 100$ & 95 & 91 & 88 & 97 & \\
\hline $26 \mathrm{VI}$ & 26 & $96 ; 96$ & 90 & 85 & 90 & 96 & 96 \\
\hline 8 VII & 29 & $92 ; 92$ & 87 & 80 & 87 & 89 & 98 \\
\hline $14 \mathrm{VII}$ & 30 & $88 ; 88$ & 86 & 77 & 88 & 104 & - \\
\hline \multicolumn{8}{|l|}{ Dog Dik } \\
\hline $5 \Pi$ & $9^{3}$ & $77 ; 79$ & 72 & 76 & 79 & 77 & 77 \\
\hline 19 II & 14 & $79 ; 77$ & 72 & 70 & 76 & 74 & 72 \\
\hline $28 \mathrm{II}$ & 17 & $76 ; 78$ & 72 & 72 & 74 & 78 & 74 \\
\hline $13 \mathrm{III}$ & 23 & $81 ; 80$ & 74 & 71 & 78 & 78 & 8 \\
\hline $23 \mathrm{~V}$ & 36 & $88 ; 96$ & 85 & 79 & 83 & 85 & 8 \\
\hline $3 \mathrm{VI}$ & 40 & $90 ; 92$ & 79 & 79 & 86 & 88 & 88 \\
\hline $10 \mathrm{VI}$ & 42 & $88 ; 86$ & 73 & 73 & 81 & 75 & 81 \\
\hline $19 \mathrm{VI}$ & 45 & $90 ; 84$ & 90 & 82 & 84 & 88 & 82 \\
\hline 8 VII & 51 & $85 ; 85$ & 80 & 78 & 83 & 83 & - \\
\hline 14 VII & 52 & $82 ; 85$ & 82 & 74 & 82 & 82 & \\
\hline
\end{tabular}

${ }^{3}$ From the first to the fiftheenth trial the UCS was an injection of $100 \mathrm{ml} 40 \%$ glucose solution. From the 15 th trial onwards, the UCS became $150 \mathrm{ml} 40 \%$ glucose solution. 
Table A.3. Conditioned changes in blood sugar level after injection of saline in humans

Data (1952)

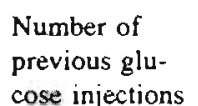

before the injection

BLOOD SUGAR LEVEL ( $\mathrm{mg} / \mathrm{dl}$ )

cose injections

(2 measurements with

minutes after the saline injection remarks

$10 \mathrm{~min}$. in between)

$5 \quad 15 \quad 45$

Subject S.G.

$\begin{array}{llllll}7 \text { III } & 5 & 93 ; 93 & 86 & 88 & 77 \\ \text { I4 III } & 10 & 80 ; 78 & 76 & 71 & 76 \\ 21 \text { III } & 15 & 83 ; 85 & 78 & 72 & 72 \\ 25 \text { III } & 17 & 89 ; 78 & 84 & 78 & 84 \\ & & & & & \\ 28 \text { III } & 18 & 99 ; 87 & 85 & 81 & 79 \\ \text { 3I III } & 19 & 105 ; 103 & 91 & 87 & 83 \\ 4 \text { IV } & 21 & 83 ; 87 & 83 & 85 & 83\end{array}$

Subject G.A.

7 III

$82 ; 82$

$\begin{array}{lll}79 & 73 & 66\end{array}$

77

$60 \mathrm{ml} 40 \%$ glucose

solution as a US

14 III

$74 ; 78$

$73 ; 69$

$91 ; 85$

$101 ; 101$

$83 ; 87$

$71 \quad 71$

$61 \quad 63$

7
8

$84 \quad 78$

$85 \quad 74$

$82 \quad 82$

76

72

84 injection occurred

in other room

28 III

19

90; 92

$\begin{array}{ll}88 & 88\end{array}$

injection occurred

in other room

4 IV

23

91; -

$\begin{array}{lll}82 & 76 & 78\end{array}$

$40 \mathrm{ml} 40 \%$ glucose

injection as US

25 IV

17

84; -

$\begin{array}{lll}76 & - & 78\end{array}$

$81 ; 83$

$\begin{array}{lll}74 & 72 & 80\end{array}$

$28 \mathrm{~V}$

19
27

94; 94

$\begin{array}{lll}87 & 85 & 85\end{array}$

Subject P.F.

14 IV

10

74; -

$74 \quad 72$

69

$40 \mathrm{ml} 40 \%$ glucose

21 IV

15

83; -

$\begin{array}{lll}74 & 74 & 76\end{array}$

25 IV

91; -

28 lV

20

$81 ; 79$

$\begin{array}{lll}84 & 87 & 89\end{array}$

$\begin{array}{lll}70 & 70 & 72\end{array}$ 
identical hours of the day by the same persons). On certain days, instead of glucose injections, intravenous saline of equal volume was given in the same manner, i.e., only the $\mathrm{CS}$ was presented. Prior to the glucose injections, several control trials were run to ascertain whether injections of $40-150 \mathrm{ml}$ saline in itself would have any effect. It was shown that the intravenous saline injections either did not change the blood sugar level, or did cause an unsignificant rise of $5-7 \mathrm{mg} \%$. More often, an increase in bloodsugar was seen, and this may be explained by certain emotional arousal that results from the injections procedure. This blood sugar rise in response to saline injections does not disappear even if intravenous injection have been given more often (in our experiment until 25 times, in dog Baikal).

The sharp rise of bloodsugar level after intravenous glucose injections appears to be a strong stimulus to which the insular apparatus must respond with an enhanced release of insulin. Repeated injections of glucose under definite stereotyped circumstances had to yield conditioned reflex secretion of insulin in response to intravenous saline injected under the same circumstances. Daily, or every second day, we infused from 40 to $150 \mathrm{ml} 40 \%$ glucose solution in the $v$. saphena of the dogs. Intravenous injections of the above glucose doses caused an increase of blood sugar concentration up to $550 \mathrm{mg} \%$ (during 5 minutes after the injection) that generally returned to baseline levels during the first hour. Blood sugar levels were assessed by means of the method of Hagedorn and Jensen. The blood to be analyzed was drawn from the marginal vein of the ear. Our experiments have shown that it is sufficient to present the dogs 8 to 10 times with the abovementioned dose of glucose, before saline injections carried out in the same manner started to cause a blood sugar fall of $5-15 \%$. The experimental results in all 4 dogs were of the same type. For the purpose of brevity we will only present the results of 2 dogs.

Dog Baikal, male, weight $10.5 \mathrm{~kg}$, received intravenous injections of saline in 25 sessions that were run before the start of sessions with glucose presentation. On six occasions bloodsugar concentrations were determined, and not once a lowering of blood sugar level was found after the intravenous saline injection (table A.1). After 8 glucose injections (40 $\mathrm{ml} 40 \%$ solution), i.e., 8 pairings of CS and UCS, saline injections in the same manner started to cause decreases in blood sugar level. The blood sugar decrease now was elicited by the action of the CS alone. Usually the blood sugar level returned to its initial value during the first hour. The level of blood sugar in the glucose injection trials rose to 380 $420 \mathrm{mg} \%$.

Dog Dik, male, weight $26,5 \mathrm{~kg}$. Prior to the administration of glucose, control injections with saline did not cause a noticeable decrease in bloodsugar level. Six trials were conducted with saline injections, and in three cases the blood sugar levels were determined (table A.1). After 9 glucose injections (100 $\mathrm{ml} 40 \%$ solution) causing increases in blood 
sugar level up to $450-520 \mathrm{mg} \%$, saline injections carried out in the same manner started to elicit a fall in blood sugar level (table A.2). In these sessions, a return to baseline levels of blood sugar occurred in the course of the first hour, just as had been the case in dog Baikal. The experimental results in the other two dogs, Zhuchka and Bek, were completely analogous to the cases described above. We carried out 39 trials with the dogs, in which after an initial 7-11 glucose injections, a saline injection was given instead, i.e. only the CS was presented by us. In 35 of these trials we got a noticeable fall of blood sugar level after saline injection, in 4 trials the aforementioned effect was not seen (in 2 of the latter 4 sessions a violation of the usual procedure had occurred: the regular experimenter was absent, and had been replaced by another person. Our observations on humans ( 9 in fact healthy subjects aged between 31 and 56) confirm the results of the trials run with animals. We injected $40-60 \mathrm{ml} 40 \%$ glucose solution, in the $v$. cubitalis.

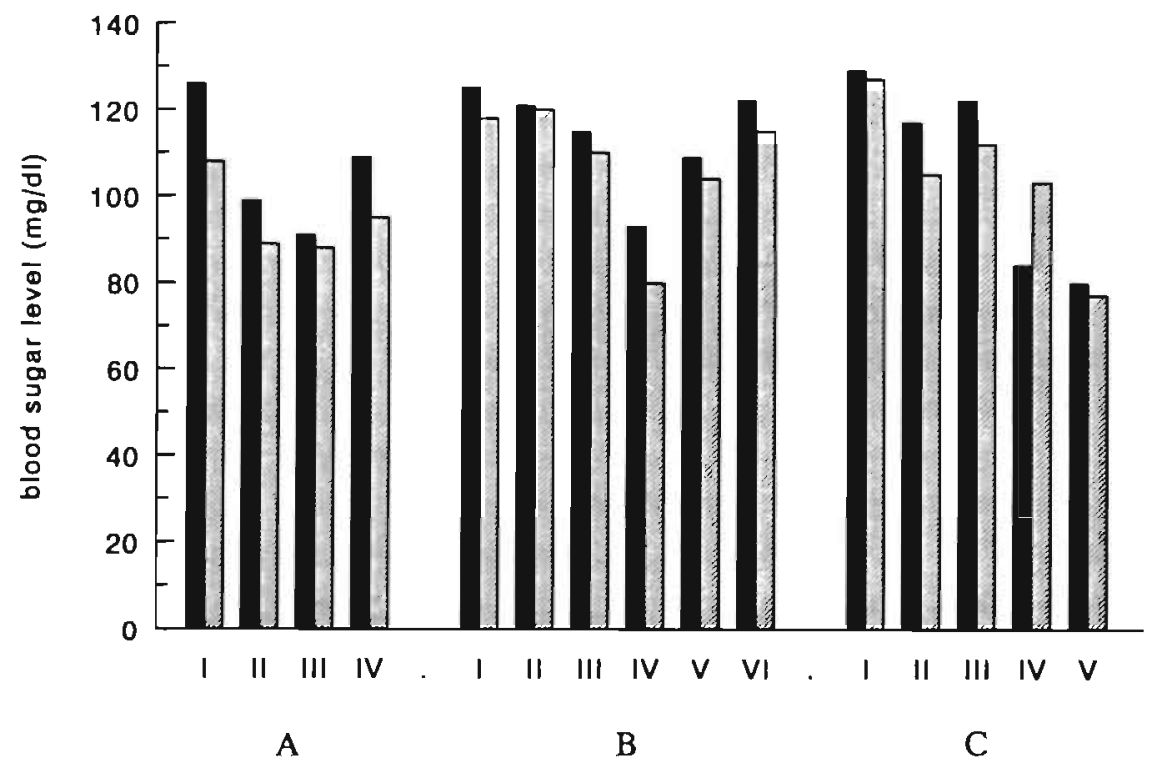

figure A.1: Determination of insulin content in dogs' blood by the bloodsugar test in white mice.

- black-colored bars: blood sugar concentration in milligram\% in the mice at 2 hours after injecting them with dog blood taken just before the saline injection;

- gray-colored bars: the same, but laken from dogs at 15 minutes after the saline injection, being the CS to insulin secretion (The US consisted of a glucose injection). Each bar represents the mean blood sugar level in mice after infusion of $0,6 \mathrm{ml}$ blood of the dogs A-Dik; B- Baikal; C- Bek. For each dog, blood was tested on a different number of occasions. 
At five minutes after the injection, the blood sugar level increased to values up to $250 \mathrm{mg} \%$ and a return to baseline level appeared in the course of the first hour. Blood for the analyses was drawn from the finger according to the usual method.

Preliminary control trials showed that an intravenous saline injection caused either no noticeable change or a non-significant increase in blood sugar level. After 5-10 fold repeated glucose injections, saline injections, i.e. presentation of the CS alone, began to cause a decrease in blood sugar level of between 5 and $20 \mathrm{mg} \%$ (table A.3). The data collected in all 9 subjects appeared to be alike: therefore, in table A. 3 we only show the results of 4 subjects. In humans, 33 sessions were run in which, after a preceding 5 - to 14 fold glucose administration, the same quantity of saline was injected under the same circumstances, i.e. the CS was presented alone. In 30 of these sessions we found a clear drop in blood sugar level after the saline injections. In 3 sessions the blood sugar level did not change. In those cases we had not followed the usual experimental procedure: for reasons beyond our influence, the saline injections had to be given in a different room. Thus, in the overwhelming majority of investigations in both animals and humans we found a conditioned fall in blood sugar in response to a saline injection. As indicated above, saline by itself did not evoke similar changes in blood sugar level.

The conditioned fall in blood sugar found by us, as well as that found by H.C. Sedina (7) with subcutaneous and intraperitoneal glucose injections, may depend on two factors: first, on a conditioned insulin secretion, and second, on a conditioned increase of glucose utilization by the tissues of the organism. In the literature there are reports by a number of authors on conditioned lowering of blood sugar level after of systematic subcutaneous injections of insulin in a stereotyped manner (J.A. Povorinski (5), V.A. Savchenko (6), I.J. Maleva (4), and C.M Leites (3). In the investigations of these authors the conditioned decrease of blood sugar appeared to be based exclusively on a conditioned enhancement of glucose utilization by the tissues.

We would be interested in first place in the degree in which the insular apparatus contributed to the observed conditioned fall in blood sugar. Therefore, we decided to carry out a series of experiments which included an assessment of insulin levels in the animals' blood. Only changes of insulin concentration in blood would be able to demonstrate a clearcut contribution of glandular secretion to conditioned reflex lowering of blood sugar level. Unfortunately, we do not yet possess a reliable method of chemical determination of insulin in the blood and presently the most suitable method appears to be the biological test.

We used the method by Brooks and Horsters, widely used in laboratory of E.S. London. We drew $0.6 \mathrm{ml}$ blood of a dog and administred $0,2 \mathrm{ml}$ to each of three mice (subcutaneously to the belly skin). After 2 hours an analysis was conducted on the blood sugar concentration of the mice. In the laboratory of E.S. London, it was established that $0.2 \mathrm{ml}$ 


\section{Appendix}

of the alien blood had not changed the blood sugar level in the mice, but that larger quantities did evoke changes.

To render the conditions of the experiment as constant as possible, the mice in our experiment were moved to a separate cage and deprived of food at one hour before the start of the experiment. In dogs, two blood samples were drawn from the marginal vein of the ear. The first was drawn immediately before the saline (control) injection, and the second at 15 minutes after the injection, i.e. at the time of the conditioned decrease of blood sugar. The latter time was chosen because in the majority of our experimental sessions the conditioned decrease of blood sugar had been the strongest then (see tables).

The results of this series of experiments are shown in figure A.1 from which one can see that in the majority of cases (14 out of 15) the blood sugar level in the mice that had been given the dog's blood from the period of the conditioned blood sugar decrease was less then of mice who had received the blood of the dogs collected before the saline injection.

Consequently, the dogs' blood during the conditioned decrease of blood sugar level contained considerably more insulin than the blood collected prior to CS presentation.

Thus, the biological tests of insulin level show that in the conditioned fall in blood sugar level as found by us, an important role is played by the secretion of insulin. Consequently, on basis of all experimental data from our investigations on animals and humans, we conclude that after systematic (8-10 times repeated) intravenous glucose injections, a conditioned fall in blood sugar level occurred when instead of glucose saline was given in the same manner.

This fall in blood sugar level was caused to a certain degree by a conditioned secretion of insulin, as confirmed by our results with tests of the insulin level in the blood.

received 6-X-1952

\section{References}

1. B.G. Baranov (1952). Sovietskaya Vrachebnaya Gazeta 13;1.

2. K.M. Bykov (1950). Stenographic report of the united scientific session of the USSR Academy of Sciences and Academy of Medical Sciences SSSR, devoted to the problems of Pavlovian Physiological Studies.

3. C.M. Leites (1951). Abstract of the lecture at the united meeting of the All-Soviet and Ukrainian Institutes of Experimental Endocrinology 97.

4. I.J. Maleva (1951). Klinicheskaya Meditsina 29, 9, 41.

5. J.A. Povorinski (1939). Reports of the IX. Scientific Meeting of the Central Psychoneurological Institute, Kharkov.

6. Savchenko, V.A. (1946). On the mechanism of action of insulin and adrenalin.

7. Sedina, N.A. (1949). Mechanisms of pathological reactions, issue 11-15, 267. 
. 



\section{SUMMARY OF THIS THESIS}

Probably between one and five percent of the female adult population is suffering from binge eating. Binge eating is part of the DSM-IV diagnoses bulimia nervosa, anorexia nervosa (binge-eating/purging subtype) and binge-eating disorder, and is characterized by recurrent ingestion, within a discrete period of time (e.g. within a 2-hour period), of an amount of food that is definitely larger than most people would eat during a similar period of time and under similar circumstances. During binges there is a sense that control over eating is lacking. Chapter 1 highlights the prevalence, size and frequency of binge eating, as well as its social impact. Strikingly, binge eating often occurs in women who attempt to lower their body weight. In the past two decades, binge eating has been the object of much theorizing and research. Notably, the influential restraint theory maintains that binge eating can be explained by the existence of "disinhibitors" (for example, low moods or catastrophic thoughts) breaking an ongoing attempt to restrict food intake (the "restraint"). A laboratory model of overeating, called "counterregulation", has been put forward as the operationalization of this theory. However, doubts can be raised about the validity of the restraint theory, firstly, because counterregulation has been shown to be a behavior that is only modestly replicable, and secondly, because the restraint theory does not postulate an explicit urge-to-eat mechanism.

In chapter 2, the Conditioning Theory of Binge Eating ( $\mathrm{CBE}$ theory), pivotal to the work in this thesis, is described. $\mathrm{CBE}$ theory posits that underlying binge eating are classically (Pavlovian) conditioned responses. These conditioned responses (CR) occur in response to "binge cues" (CSs; conditioned stimuli), which, for the individual, have previously been reliable predictors of a binge (UCS, unconditioned stimulus). Binge cues may be, for example, the sight, smell or taste of certain foods, or the individual's presence in an environment in which binges usually occur. The perception of binge cues is supposed to lead to a conditioned secretion of the hormone insulin, and an accompanying fall in blood sugar level (CR). Although these responses are, homeostatically speaking, adaptive because they attenuate the post-prandial blood sugar rise, according to $\mathrm{CBE}$ theory they are subjectively experienced as a craving for food. A review of the relevant literature is given. Interestingly, CBE theory has given rise to the development of a therapeutic procedure for binge eating called cue-exposure. In this procedure, patients are presented with the odour and sight (CS) of binge foods for a prolonged time (for example one hour long), without the possibility of ingesting these foods (UCS). The extinction of craving (and bingeing), predicted by CBE theory, has indeed been demonstrated in several studies. 


\section{Summary}

Subsequent chapters report empirical studies in which several CBE hypotheses have been tested.

Chapter 3 describes a test of the CBE hypothesis that perception of stimuli that reliably predict a blood sugar rise will lead to insulin secretion, hypoglycaemia and increased craving for sweet substances. In six conditioning trials, subjects were asked to ingest a glucose drink (UCS) in a room with a peppermint odour (CS) hanging in the air. Glucose was chosen as the UCS because it leads to a large blood sugar rise and prompts a strong insulin response. During test sessions, before and after conditioning, only the CS was presented, after which blood samples were taken, and craving was monitored by questionnaire. Subsequently, subjects participated in a "taste test", during which they were to compare several glucose drinks and in which the amount of ingested glucose was measured. Subjects in a control condition, in which the inert sweetener aspartame was presented during conditioning, were hypothesized to show no conditioned responses. The results showed no differences between conditions on blood sugar level or subjective craving. A slightly increased secretion of insulin was observed in the experimental condition. However, the size of the observed difference did not exceed the range of normal spontaneous fluctuations. Interestingly, in both conditions, a strong increase in the amount of ingested glucose was observed after conditioning. This finding was explained as a consequence of a neophobia or "bait shyness" during the initial test session: the subjects would be reluctant to drink freely in the novel lab situation. Subsequently, repeated lab-sessions could have led to a gradual fading of the reluctance to drink. To investigate the phenomenon further, an additional condition was run. This time, the conditioning procedure involved a normal tap water UCS (instead of sweet drinks). Once again, a large increase in glucose intake was observed after conditioning. It was concluded that a key factor in the increased glucose intake was an increased experience with carrying out a lab-procedure, rather than repeated exposure to sweet drinks. These results may have implications for future food intake research using repeated measurements.

The CBE prediction that blood sugar conditioning is possible using a glucose-containing UCS is corroborated in chapter 4. A commentary and re-analysis is given of a 1954 study by the Russian physiologist Mityushov (see also the appendix of this thesis). Mityushov found a physiologically significant hypoglycaemia after injecting saline in humans and dogs who had been given repeated glucose injections previously. The observed results suggest a physiological state which, according to CBE theory, occurs prior to bingeing. Mityushov's procedure might, in case of replication of the results, be employed in future research into binge eating.

The next two chapters test CBE hypotheses about food cue reactivity, i.e., the intensity 
with which people respond to presentation of food-related cues. The CBE theory predicts that cue reactivity is especially intense in individuals with occasional large food intake. In chapter 5, slides depicting subjects' favourite binge foods were used as cues. During presentation of the slides, psychophysiological responses (e.g. skin conductance, heartrate and facial EMG) were monitored. Two groups of subjects, restrained and unrestrained eaters (selected by Herman \& Polivy's Restraint Scale) were compared. Because high Restraint scores suggest a food intake pattern characterized by alternate dieting and overeating, the CBE-derived prediction was that restrained eaters would show larger cue reactivity than unrestrained eaters. The results did not confirm this prediction. Whereas slides with binge food were indeed rated higher on craving-arousing property than were control slides (with office equipment), no differences were found in psychophysiological responses of restrained and unrestrained subjects.

In chapter 6, real food items (sandwiches and candy bars) were presented to subjects given the instruction to smell and look towards these food items attentively. Skin conductance responses, heart rate and salivation were monitored in subjects who had fasted either for 3 , or for 24 hours. The CBE-related hypothesis was that 24-hour fasting subjects would show larger craving and (accordingly) cue reactivity and craving than 3hour fasting subjects. The results showed no group differences in cue reactivity, although, as expected, the 24-hour fasting subjects reported increased craving and a higher hedonic rating of the presented food items.

Chapter 7 features a comparison between restrained and unrestrained eaters (as selected by the Restraint Scale, see chapter 5). Given the CBE theory, it was predicted that ingestion of an appetizer would lead restrained eaters to disinhibit during a subsequent taste test with ice-cream. The present study tried to assess the preoccupation (attentional bias) for food-related stimuli in disinhibiting individuals by means of the Stroop colournaming test. In the control condition, no appetizer was presented. The results showed that, contrary to expectations, no disinhibitive eating was found among the restrained subjects. A remarkable finding was the significant correlation between the level of attentional bias and the amount of subsequently ingested ice-cream in unrestrained eaters. In restrained eaters the correlation was near zero. A possible clinical application of the Stroop test as a predictor of relapse in treated bulimics is discussed.

In Chapter 8 some problems are noted in using the Restraint Scale in lab studies of binge eating. Doubts are raised about the ability of the Restraint Scale (which was originally developed to identify dieters) to optimally predict overeating in the lab (see also section 1.3, and chapters 5 and 7 of this thesis). Furthermore, the Restraint Scale has been criticized for some psychometric weaknesses. Given these problems, a new selection instrument was developed the Disinhibitive Eating Scale (DIS), which contains 
four subscales: failing diets, binge eating, weight fluctuations and self-esteem. A psychometric analysis of the 17-item DIS revealed generally satisfying characteristics. Although DIS and RS total scores intercorrelated strongly, it was shown that both scales select subject groups which differ in composition. It remains to be established whether the DIS indeed possesses the predictive validity for disinhibition in the lab, for which it was developed.

Chapter 9 concludes the present thesis, with the observation that none of the novel studies reported here have produced results confirmative of the hypotheses derived from $\mathrm{CBE}$ theory. In an attempt to elucidate why, a critical and quantitative re-assessment was conducted of the literature considered as supportive of the CBE assumptions (see chapter 2 ). The following picture emerged. First, there is little support for the existence of physiologically speaking significant conditioned blood sugar and insulin responses. Second, at present there are no indications that bulimics show increased insulin and blood sugar reactions to food-related cues. Finally, ingestion of only one candy bar or a few biscuits results in a blood sugar rise that will nullify conditioned hypoglycaemias of the size reported thus far within 10-15 minutes; nevertheless the average binge has been reported to last two to three times longer than that.

Based on these considerations, profound scepticism seems warranted about the role of conditioned blood sugar and insulin responses in binge eating. Considering the success of the cue exposure therapy which was derived from $\mathrm{CBE}$ theory (section 2.2.6), a curious, but not disastrous situation has arisen.

The present dissertation concludes with theoretical and practical suggestions for future research on binge eating. Two important aspects of $C B E$ theory that have not been rejected, i.e., the role of learning, and stimulus-driven character of binge eating, are given emphasis.

Two theoretical directions are discussed. In the first, a possible (and modest) role of learned blood sugar and insulin responses is proposed for the minutes immediately preceding a binge. In the second, it is proposed that an additional mechanism is active in binge eating. Perception of cues that have come to reliably predict a period of fasting and/or self-induced vomiting will lead to increased food intake. In this view, people would not so much binge in order to lift a momentary deprivation (i.e. the hypoglycemia predicted by CBE theory), but rather to preclude a forthcoming deprivation. Several studies, in line with the existence of such a mechanism, are reviewed.

To conclude, several practical suggestions are made for future binge eating research. Emphasis is put on the development of convincing and robust lab models of binge eating. A model of binge eating can be said to exist if some core manipulation reliably produces increased craving and food intake. Subsequent knowledge growth may then 
occur by introducing additional manipulations, and evaluating their effects on eating behaviour. Three possible lab models of binge eating are described. First, the cue reactivity model, in which food intake and craving are increased by presentation of stimuli related to food items previously ingested in daily life. Second, the conditioned hypoglycaemia model, in which, after a conditioning procedure resembling that of Mityushov (see chapter 4; appendix), cue presentation leads to increased food intake, mediated by a conditioned hypoglycaemia. And third, the post-meal deprivation model, in which food intake is increased by presentation of stimuli that, in a previous learning procedure, have become predictive of a fasting period. Relevant experiments, and results needed for the development of the models are described in some detail. The research proposals may give food for research activities for many years to come. 



\section{SAMENVATTING}

Vermoedelijk lijdt tussen de één en vijf procent van de vrouwelijke volwassenen op dit ogenblik aan eetbuien (Engels: binge eating). Het vertonen van eetbuien vormt een onderdeel van de DSM-IV diagnoses 'Bulimia Nervosa', 'Anorexía Nervosa (vreetbuien/purgerende type)', en de 'Vreetbuienstoornis', en wordt omschreven als het frequent, binnen een beperkte tijd (bijvoorbeeld twee uur) eten van een hoeveelheid voedsel die beslist groter is dan wat de meeste mensen in eenzelfde periode en onder dezelfde omstandigheden zouden eten. Karakteristiek daarbij is het gevoel de beheersing over de voedselinname kwijt te zijn. In hoofdstuk 1 worden prevalentie, grootte, frequentie, samenstelling en sociale gevolgen van eetbuien nader beschreven. Opvallend is dat eetbuien vaak voorkomen bij vrouwen die het uiterst belangrijk vinden om slank te zijn. De vraag werpt zich dan ook op hoe het optreden van eetbuien kan worden verklaard. De invloedrijke restraint-theorie stelt dat overeten wordt veroorzaakt door 'ontremmers', (zoals bijvoorbeeld somberheid of catastrofale gedachten) die een tot dan toe bewust volgehouden lijnpoging (de restraint) torpederen. Deze theorie werd geoperationaliseerd en in het lab bestudeerd onder de naam counterregulatie. Twijfel is echter gerezen over de houdbaarheid van de restraint/counterregulatie theorie: ten eerste blijkt counterregulatie matig repliceerbaar in het lab, ten tweede geeft de restraint theorie geen expliciete verklaring voor de eetdrang.

In hoofdstuk 2 is de Conditioning Theory of Binge Eating (CBE-theorie) beschreven, welke centraal staat in deze dissertatie. De CBE-theorie stelt dat er bij eetbuien sprake is van aangeleerde, zgn. klassiek (of: Pavloviaans) geconditioneerde responsen. De geconditioneerde responsen (CR) treden op zodra een persoon geleerd heeft dat bepaalde stimuli (CS, ook wel eetbui-cues) betrouwbare voorspellers zijn van eetbuien (ongeconditioneerde stimuli, UCS). Eetbui-cues kunnen zijn het zien, ruiken of proeven van voedsel, of het verblijf in ruimtes waar eetbuien gewoonlijk plaatsvinden. De waarneming van eetbui-cues leidt automatisch tot afscheiding van het hormoon insuline en een bloedsuikerdaling (CR), die anticiperen op de grote bloedsuikerstijging na de eetbui. Enerzijds is dit een zinvolle, natuurlijke reactie (het lichaam streeft een evenwichtige bloedsuiker huishouding na). Anderzijds wordt de anticipatieve bloedsuikerdaling volgens de CBE-theorie subjectief beleefd als een drang tot eten. Een Iiteratuuroverzicht beschrijft de evidentie voor CBE-assumpties. Interessant is dat intussen enkele succesvolle effectstudies zijn verricht naar een therapie genaamd cue exposure. Hierbij worden patiënten langdurig geconfronteerd met de geur en uiterlijk (CS) van het eetbuien voedsel, echter zonder de mogelijkheid het voedsel (UCS) in te nemen. De door de CBE-theorie voorspelde uitdoving van de eetdrang bleek in deze studies uit te komen. 
In de volgende hoofdstukken worden verscheidene $\mathrm{CBE}$-hypotheses empirisch getoetst. In Hoofdstuk 3 wordt de CBE-voorspelling getest dat insuline secretie, bloedsuikerdaling en toegenomen drang tot inname van zoetigheid kunnen worden opgewekt door een stimulus die een glucose inname voorspelt. Gedurende zes conditioneringssessies dronken proefpersonen een glucosedrank in een kamer waar een pepermuntgeur (CS) was verspreid. Glucose verhoogt het bloedsuikerniveau aanzienlijk en leidt tot insulineafscheiding. In twee sessies, één voorafgaand en één volgend op de reeks conditioneringssessies, vonden de gedragsmetingen plaats. Alleen de CS werd gepresenteerd, vervolgens werden bloed en vragenlijsten afgenomen. Hierna kregen de proefpersonen een smaaktest, waarbij glucose dranken moesten worden vergeleken; de hoeveelheid daarbij ingenomen drank werd gemeten. Voor de controle groep - geconditioneerd met de inerte zoetstof aspartaam - werden geen effecten van conditionering verwacht. De resultaten van deze studie toonden niet de verwachte effecten op bloedsuiker en craving. Wel werd er een iets vergrote insulinerespons gevonden in proefpersonen van de experimentele groep op vijf minuten na CS-aanbieding. Op basis van de relevante literatuur werd echter geconcludeerd dat de grootte van het gevonden effect die van spontane fluctuaties niet oversteeg. Opvallend resultaat, in beide condities, was de sterk toegenomen hoeveelheid gedronken glucosedrank na conditionering. Gehypothetiseerd werd dat dit resultaat samenhing met zgn. neophobia en bait shyness tijdens de eerste, onwennige testsessie, waardoor proefpersonen terughoudend waren bij het drinken van de glucose drank. De dagenlange conditioneringsprocedure zou hebben geleid tot het wegebben van de terughoudendheid. Teneinde dit verschijnsel nader te onderzoeken werd een derde conditie toegevoegd, waarbij proefpersonen deze keer werden geconditioneerd met kraanwater in plaats van zoete drankjes. Na conditionering bleek ook in deze conditie de consumptie van glucosedrank toegenomen. Geconcludeerd werd dat, meer nog dan toegenomen vertrouwdheid met de lab procedure, de ervaring met zoete drankjes hiervoor verantwoordelijk was. Deze conclusie is relevant voor toekomstig onderzoek waarin herhaalde metingen van voedselinname aan de orde zijn.

De CBE-assumptie dat bloedsuikerconditionering met behulp van glucose bevattende UCS-sen wel degelijk mogelijk is, wordt in Hoofdstuk 4 ondersteund. Dit bevat een commentaar op, en heranalyse van een studie van de Russische fysioloog Mitjoesjov uit 1954 (zie de Appendix). Dr. Mitjoesjov slaagde erin een fysiologisch significante hypoglykaemie aan te tonen na het injecteren van een 'placebo' (saline) bij mensen en honden die eerder herhaaldelijk glucose injecties hadden ontvangen. De resultaten suggereren een fysiologische toestand die volgens de CBE theorie voorafgaat aan eetbuien. Mitjoesjov's procedure zou - na replicatie van de uitkomsten - wellicht benut kunnen worden in toekomstig eetbui-onderzoek. 
De volgende twee hoofdstukken bevatten studies over zgn. cue-reactiviteit: d.w.z. de intensiteit van psychofysiologische reacties tijdens het waarnemen van aan voedsel gerelateerde stimuli. Uit de CBE-theorie volgt dat voedselcue-reactiviteit vergroot is bij personen met een geschiedenis van grote voedselinnames.

In hoofdstuk 5 werden als stimuli dia's gebruikt met daarop voor elke proefpersoon geselecteerd 'eetbuien-voedel'. Tijdens het dia-kijken werden psychofysiologische reacties als huidgeleiding, hartslagfrequentie, en gezichtsspierspanningen gemeten. De reacties van twee groepen proefpersonen - geselecteerd met behulp van Herman en Polivy's Restraint Scale - werden met elkaar vergeleken. Aangezien een hoge score op de Restraint Scale duidt op een eetpatroon met afwisselend lijnen en overeten, was de CBE-hypothese dat hoge scorers (lijners) sterkere cue-reactiviteit zouden vertonen dan lage scorers (niet-lijners). Hoewel een manipulatiecheck toonde dat de dia's met eetbuivoedsel als smakelijker werden gewaardeerd dan controle dia's (met kantoorartikelen), werden geen verschillen gevonden in psychofysiologische reacties van lijners en nietlijners.

In hoofdstuk 6 werden de proefpersonen voedingswaren (broodjes gezond en candybars) voorgezet, met de opdracht er verscheidene minuten aandachtig aan te blijven ruiken en naar te kjjken. Huidgeleiding, hartslag en salivatie werden geregistreerd van proefpersonen die vooraf drie dan wel vierentwintig uur hadden gevast. De CBE hypothese was de waarneming van het voedsel een intensere eetdrang en (hiermee) psychofysiologische reacties zou teweegbrengen bij degenen die vierentwintig, dan bij degenen die drie uur hadden gevast. Hoewel de vierentwintig uur inderdaad een vergrote subjectieve eetdrang (en waardering van het voedsel) rapporteerden, kon de gestelde hypothese niet worden bevestigd. In dit geval was door het voedsel opgewekte eetdrang dus niet zichtbaar in intense fysiologische reacties.

In Hoofdstuk 7 werd opnieuw het gedrag vergeleken van lijners en niet-lijners (geselecteerd mbv de Restraint Scale, zie hoofdstuk 5). Uit de CBE-theorie en de bestaande literatuur werd de voorspelling afgeleid dat het aanbieden van een 'appetizer' bij lijners zou leiden tot disinhibitief eetgedrag tijdens een smaaktest met roomijs. In dit experiment werden hierbij optredende cognitieve effect onderzocht, met behulp van de Stroop reactietaak. Met deze taak werd gehoopt inzicht te krijgen in de mate van preoccupatie (ook wel attentionele bias) voor voedselstimuli. In de controle conditie werd geen appetizer aangeboden. Tegen de hypothese in bleek dat lijners in de appetizer conditie geen disinhibitief eetgedrag vertoonden. Opmerkelijk was de significante correlatie tussen de attentionele bias voor voedselwoorden en de vervolgens genuttigde hoeveelheid ijs bij niet-lijners. Bij lijners was deze correlatie afwezig. Gewezen werd op een mogelijke klinische toepassing van de Stroop taak als voorspeller van terugval bij 


\section{Samenvatting}

bulimische patiënten.

Hoofdstuk 8 gaat in op enkele problemen bij het gebruik van de Restraint Scale als selectieinstrument voor eetbui-onderzoek in het lab. Men kan betwijfelen of de Restraint Scale, orspronkelijk ontwikkeld ter selectie van lijners, optimaal geschikt is om overeten in het lab te voorspellen (zie ook 1.3, en hoofdstukken 5 en 7). Daarenboven is de Restraint Scale in het verleden wegens enkele psychometrische zwakheden bekritiseerd. Vandaar dat een nieuw selectie-instrument wordt gepresenteerd, de Disinhibitive Eating Scale (DIS), met vier subschalen: mislukkende dieten, eetbuien, gewichtsfluctuaties en self-esteem. Een psychometrische analyse van de 17 items tellende DIS leverde alleszins bevredigende resultaten. Hoewel de DIS en Restraint Scale vrij sterk correleerden, werd aangetoond dat beide vragenlijsten verschillende groepen proefpersonen selecteren. Het wachten is nu op een bevestiging van de voorspellende waarde van de DIS voor (over)eetgedrag in het lab.

In hoofdstuk 9 wordt de slotbalans opgemaakt. Geconstateerd wordt dat geen van de hier gerapporteerde, nieuw uitgevoerde studies resultaten hebben opgeleverd die bevestigend waren voor de CBE-theorie. In een poging tot nadere verklaring wordt de literatuur die eerder als ondersteuning voor de CBE-assumpties is gepresenteerd (hoofdstuk 2) kritisch en quantitatief hergeëvalueerd. Het volgende beeld rijst hieruit op. Ten eerste blijkt in de literatuur weinig ondersteuning te vinden voor het bestaan van fysiologisch gezien - aanzienlijke geconditioneerde bloedsuiker en insuline responsen. Ten tweede is uit onderzoek tot nog toe niet geleken dat bulimia nervosa patiënten in versterkte mate insuline en bloedsuikerresponsen vertonen na waarneming van aan voedsel gerelateerde stimuli. Tot slot blijkt dat inname van slechts één candy bar of een paar koekjes na 10-15 minuten tot een bloedsuikerstijging leidt die geconditioneerde hypoglykaemieën van de in de literatuur beschreven grootte volledig opheft; tegelijkertijd is uit ander onderzoek gebleken dat eetbuien gemiddeld twee tot drie keer zo lang duren. Op basis van bovenstaande overwegingen wordt geconcludeerd dat omtrent de rol van geconditioneerde bloedsuikerdaling en insuline secretie in eetbuien diepgaand scepticisme gerechtvaardigd lijkt. Gezien de vermoedelijke effectiviteit van uit de CBE-theorie afgeleide cue-exposure therapie (zie paragraaf 2.2 .6 ) is een merkwaardige, doch nietdesastreuze situatie ontstaan.

De disseratie sluit af met theoretische en practische suggesties voor toekomstig eetbuionderzoek. Twee aspecten van de CBE-theorie die niet zijn weerlegd (nl. de rol van conditionering en aan voedsel gerelateerde stimuli) worden hierbij benadrukt.

Een tweetal theoretische benaderingen wordt geschetst. In de eerste wordt gewezen op een mogelijke (bescheiden) rol van aangeleerde bloedsuiker en insuline reacties, vooral gedurende de minuten voorafgaand aan een eetbui. In de tweede benadering is een 
mechanisme geschetst waarin de rol van vasten en zelf-opgewekt braken na een eetbui centraal staat. Waarneming van eetbui-gerelateerde signalen die braken of een periode van vasten voorspellen, zou de voedselinname doen toenemen. Eenvoudig gezegd zou een eetbui niet zozeer voortduren teneinde een momentane deprivatie (zie het lage bloedsuiker-niveau uit de CBE-theorie) 'weg te eten', maar om een toekomstige deprivatie te voorkómen. Een literatuuroverzicht gaat in op aanwijzingen voor het bestaan van dit mechanisme.

Tot slot worden practische aanbevelingen voor toekomstig eetbui-onderzoek gedaan. Gesteld wordt dat de ontwikkeling van overtuigende en robuuste laboratorium modellen van eetbuien bij mensen cruciaal is. Van een eetbui-model is sprake indien men beschikt over een procedure waarmee betrouwbaar eetdrang en vergrote voedselinname worden bewerkstelligd. Door het vervolgens toevoegen van extra manipulaties aan deze basisprocedure, en evaluatie van de consequenties voor de voedselinname, kunnen eetbuien verder in het lab worden onderzocht.

Drie mogelijke eetbui modellen worden beschreven, het cue reactivity model, waarbij de eetdrang en voedselinname worden vergroot door aanbieding van stimuli gerelateerd aan voedsel dat de proefpersoon kent uit het dagelijks leven, het condirioned hypoglycaemia model, waarbij, volgend op een conditioneringsprocedure à la Mitjoesjov (zic hoofdstuk 4; appendix), presentatie van een CS via een geconditioneerde hypoglykaemie leidt tot een toegenomen voedselinname, en het post-meal deprivation model, waarbij de voedselinname wordt vergroot door aanbieding van stimuli die in een voorafgaande leerprocedure een periode van vasten voorspelden. Relevant onderzoek en resultaten, nodig voor de ontwikkeling van bovenstaande modellen, worden in enig detail beschreven. De uitvoering van deze onderzoeksvoorstellen kan voor vele jaren werk opleveren. 



\section{DANKWOORD}

Hoewel op het omslag slechts mijn eigen naam prijkt, hebben ook anderen een rol gespeeld bij het voltooien van deze dissertatie. Welke rol? Lastige vraag. Men denke eens aan alle krachten die een pingpongballetje de oceaan doen oversteken: niet alleen stromingen in de eindrichting, ook zijstromingen, tegenstroming en stiltes in de stroming hebben onderweg bijgedragen (zie Karel van het Reve, 1979). Zo voel ik het ook een beetje bij dit proefschrift. Enkelen wil ik met name genoemd hebben.

Anita Jansen, 'cheffin', jouw intrigerende theorie vormde de basis voor het project waaraan ik heb mogen werken. Onze samenwerking was voor mij een boeiende, prikkelende, soms echter ook onzachte ervaring. Leerzaam was het zeker: ik voel me thans niet langer 'in opleiding'. Ik hoop dat de toekomstige eetbui-wetenschap nog vaak door nieuwe ideeën van jou zal worden opgefrist.

Marcel van den Hout, promotor, bedankt voor de gastvrijheid van jouw instituten DEP en EPP, en voor je bereikbaarheid die ik als buitengewoon practisch heb ervaren. Steeds weer als ik klopte, bleek jouw deur niet gesloten.

Heiny Eilkes, Ellen Louwerse en Annie Raven: hartelijke dank voor jullie inzet en zorgvuldigheid bij het helpen uitvoeren van de experimenten die in dit proefschrift staan. Hetzelfde wil ik mutatis mutandis kwijt aan alle proefpersonen die hebben meegewerkt, maar die geen exemplaar van mijn proefschrift hebben gekregen.

Theo van Aerts, dank voor het programmeren van de reeks psychofysiologische signaalverwerkers, een puike familie; Erik Schouten, je was een stoïcijns beantwoorder van telkens weer dezelfde vragen over mijn Manova-uitdraai; Bob Wilkinson, vriendelijke dank voor je bijdrage aan mijn Engels. Angela Verweij, vaak wist je op onnavolgbare wijze te informeren of ik wel goed had geslapen; Marja Hortulanus, dank-je voor het verscheuren van dat bierviltje. Alle collega's van DEP/EPP met wie ik heb gepingpongd, alsmede degenen met wie ik niet heb gepingpongd: het ga jullie goed. Welmoet Gerritsen, bedankt voor enkele vriendschappelijke telefoongesprekken; Casper Schoemaker, dat ik me jouw volkomen terechte commentaren nooit voor $100 \%$ ter harte heb genomen, komt ongetwijfeld doordat ik mijn arikels al gedeeltelijk af meende te hebben. Mijn paranimfen: Sabine Kroeze, ik heb echt geboft met jouw kamergenoot- en kameraadschap. Instemmend wil ik je hier citeren: "Gén gepeiger meer!" ; Bart Leunissen, een frequent en langdurig Horecabezoek - voor zover Maastricht dit toeliet - was ons niet vreemd. Ik vertrouw erop dat we een vorm zullen vinden om deze belangwekkende bezigheid in de toekomst voort te zetten.

Mijn ouders: lieve Ma en $\mathrm{Pa}$, dit boekwerk is aan jullie opgedragen. Opvoeden is vast veeleisender dan het schrijven van wel XX dissertaties. Mochten jullie enkele zaken uit deze dissertatie niet geheel kunnen navoelen, wees dan niet bezorgd: het is waarschijnlijk een teken van mijn nu aangebroken 'Jaren des Onderscheids'. 



\section{CURRICULUM VITAE}

Barend Jan Joost Overduin werd op 25 oktober 1964 geboren in Enschede. Daar doorliep hij het Atheneum-B van het Kottenpark College. Vervolgens ging hij natuurkunde studeren aan de Rijksuniversiteit Leiden totdat hij in september 1984 zijn propedeuse diploma (met genoegen) ontving. Aansluitend studeerde hij psychologie, eerst aan de Rijksuniversiteit Utrecht (richtingen Klinische Psychologie en Functieleer), later aan de Universiteit van Amsterdam waar hij in september 1991 cum laude afstudeerde bij de vakgroep Psychonomie, afdeling Emotieleer (Prof. N.H. Frijda). Vijf dagen later begon hij als onderzoeker in opleiding te werken bij de vakgroep Differentiële en Experimentele Psychologie (toen nog GGK) van de Rijksuniversiteit Limburg. Deze dissertatie is een neerslag van dit werk. Momenteel wordt hij dermate geboeid door de combinatie van pharmacologie en ethologie, dat hij overweegt om weer te gaan studeren.

\section{Publicaties}

Overduin, J. (1992). Passie. Bespreking van 'Dieptepsychologie' van J.H. van den Berg. De Psycholoog 27, 450.

Overduin, J. (1993). Het voordeel van de schrik. Over het onvolledige gebruik van de reflex in emotieonderzoeken. De Psycholoog 28, 193-201.

Merckelbach, H. \& Overduin, J. (1993). Een Nederlandse Pavloviaan: J. ten Cate (1887-1967). De Psycholoog 29 67-71.

Overduin, J. (1994). Leidens Ontzet in de ethologie. Herwaardering van de dierlijke subjectiviteit. De Psycholoog 29, 279-284.

Overduin, J., Jansen, A. \& Louwerse, E. (1995). Stroop interference and food intake. International Journal of Eating Disorders 18, 277-285. (Chapter 7, this thesis)

Overduin, J. \& Jansen, A. (1995). The conditioning model of binge eating. In: I. Florin \& B. Tuschen (eds), Current Research in Eating Disorders, 16-27. Münster: Verlag für Psychotherapie.

Jansen, A., Overduin, J. \& Meijboom, A. (1995). Controlled and automatic cognitive processes in eating disorders. In: 1. Florin \& B. Tuschen (eds), Current Research in Eating disorders, 74-81. Münster: Verlag für Psychotherapie.

Overduin, J. (1996). Empirisch onderzoek van de eetbui: uitgangspunten en enkele verkenningen. Psychoscoop 7, 4-11.

Overduin, J. \& Jansen, A. (1996). A new scale for use in non-clinical research into disinhibitive eating. Personality and Individual Differences, in press. (Chapter 8 , this thesis)

Overduin, J. \& Jansen, A. (1996). Food cue reactivity in fasting and non-fasting subjects. European Eating Disorders Review, in press. (Chapter 6, this thesis)

Overduin, J., Jansen, A. \& Eilkes, H. (1996). Cue-reactivity to food- and body stimuli in restrained and unrestrained eaters. Addictive Behaviors, in press. (Chapter 8 , this thesis) 
